DOE/EIA-0380(96/04)

\title{
April 1996
}

With Data for January 1996
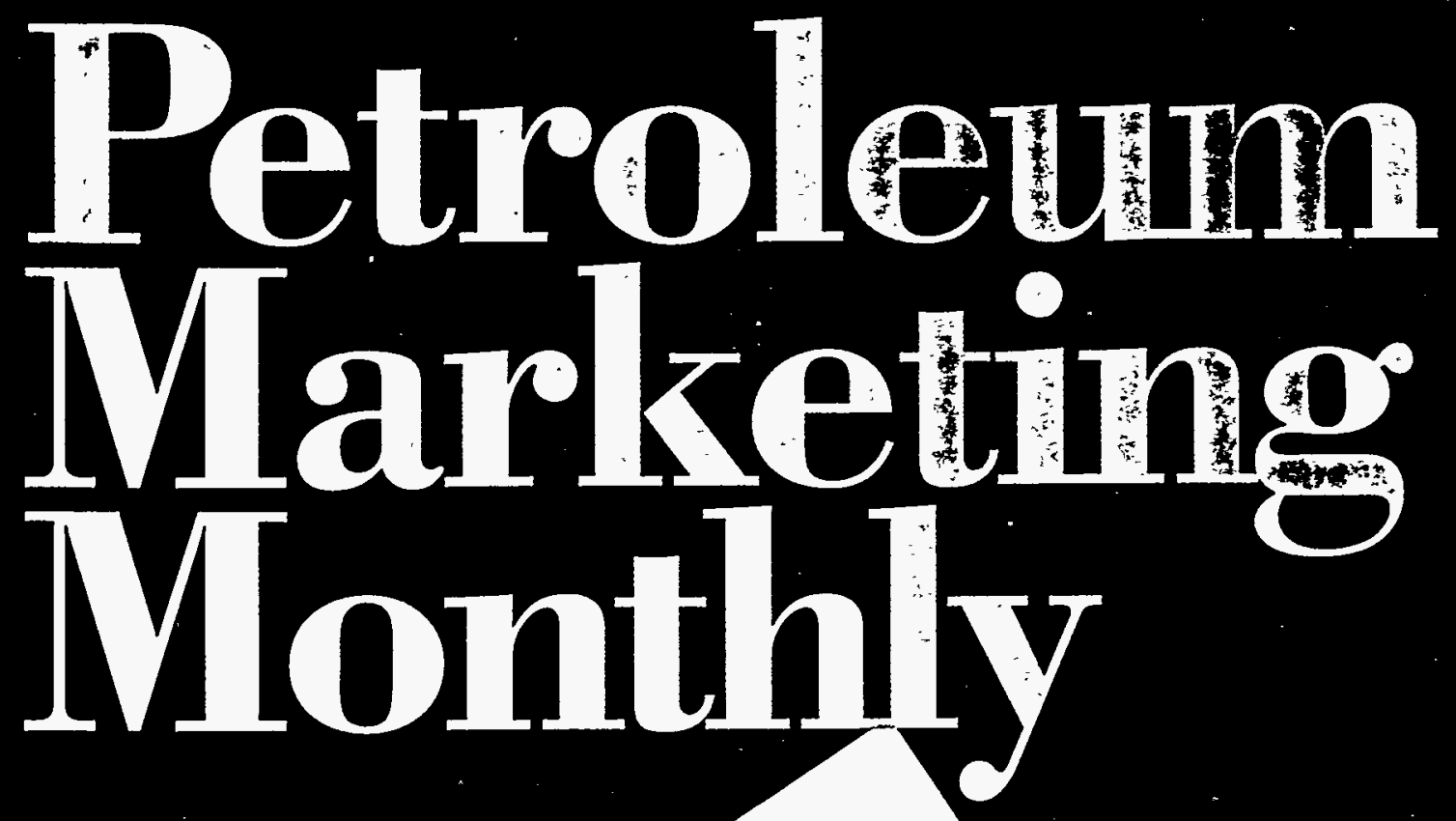


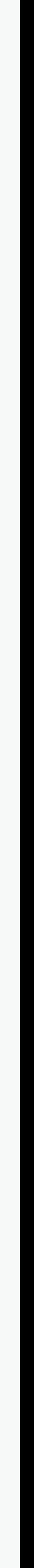


DOE/EIA-0380(96/04)

Distribution Category UC-950

\title{
Petroleum Marketing Monthly
}

April 1996

With Data for January 1996

\author{
Energy Information Administration \\ Office of Oil and Gas \\ U.S. Department of Energy \\ Washington, DC 20585
}

\section{MASTER}

This report was prepared by the Energy Information Administration, the independent statistical and analytical agency within the Department of Energy. The information contained herein should not be construed as advocating or reflecting any policy position of the Department of Energy or of any other organization. 


\section{Contacts}

The Petroleum Marketing Monthly (PMM) is prepared in the Energy Information Administration (EIA) under the general direction of Charles P. Shirkey (202) 586-6567, Petroleum Marketing Division, Office of Oil and Gas, EIA.

Detailed technical questions for specific areas of the PMM may be directed to the EIA staff listed below.

For primary program responsibility:

- Charles W. Riner (202) 586-6610

Internet E-Mail: criner@eia.doe.gov

For tables referencing the EIA-782A, the EIA-782B, and the EIA-782C:

- Charles W. Riner (202) 586-6610

Internet E-Mail: criner@eia.doe.gov
- Kenneth Platto (202) 586-6364

Internet E-Mail: kplatto@eia.doe.gov

For tables referencing the EIA-14 and EIA-856:

- Lamar Gowland (202) 586-6608

Internet E-Mail: lgowland@eia.doe.gov

- Elizabeth Scott (202) 586-1258

Internet E-Mail: escott@eia.doe.gov

For tables referencing the EIA-182:

- Alan Griffith (202) 586-7225

Internet E-Mail: agriffit@eia.doe.gov

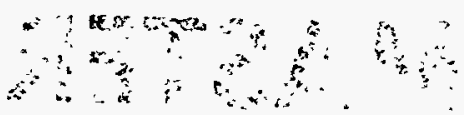




\section{Electronic Publishing System (EPUB) User Instructions}

EPUB is an electronic publishing system maintained by the Energy Information Administration (EIA) of the U.S. Department of Energy. EPUB allows the general public to electronically access selected energy data from many of EIA's statistical reports. The system is a menu-driven, bulletin board type system with extensive online help capabilities that can be accessed free-of-charge 24 hours a day by using a terminal or PC with an asynchronous modem. (EPUB will be taken down briefly at midnight for backup.)

PC users must provide the following information to their communications software in order to successfully access the EPUB system.

\section{Communications Parameters:}

Baud Rate: Up to 28,800 bps

Data Bits: 8; Stop Bits: 1

Parity: None; Duplex: Full

Terminal Type: ANSI, ANSI-BBS, VT100, etc.

Once your communications software and/or hardware has been configured, EPUB can be accessed by dialing (202) 586-2557. When a connection to the system has been made, some users may find that the menu-driven instructions and the online help capabilities will provide enough information to effectively use EPUB. More extensive information may be obtained from:

National Energy Information Center, EI-231

Energy Information Administration

Forrestal Building, Room 1F-048

Washington, DC 20585

(202) $586-8800$

Internet E-Mail: INFOCTR@EIA.DOE.GOV

TTY: For people who are deaf or hard of hearing: (202) 586-1181

Hours: 9 a.m. to 5 p.m., M-F, eastern time

For communications or technical assistance, call (202) 586-8959, 8 a.m. to 5 p.m. eastern time, Monday through Friday.
For questions about the content of EPUB reports and/or data, call (202) 586-8800, 9 a.m. to 5 p.m. eastern time, Monday through Friday.

Following is a list of some of the data and reports that are provided on EPUB:

- Heating fuel data (April through September) Updated the 2nd week of the month.

- Oxygenate data Updated approximately the 25th of the month.

- Weekly Petroleum Status Report Updated on Wednesdays (Thursdays in the event of a holiday) at 9 a.m.

- Petroleum Supply Monthly Updated between the 23rd and 26th of the month.

- Petroleum Marketing Monthly Updated on the 20th of the month.

- Natural Gas Monthly Updated on the 20th of the month.

- Weekly Coal Production Updated on Fridays by noon.

- Quarterly Coal Report Updated 40 days after the end of the quarter.

- Electric Power Monthly Updated during the first week of the month.

- Monthly Energy Review Updated the last week of the month.

- Short-Term Energy Outlook Updated 60 days after the end of the quarter.

- Winter Fuels Report (October through April) Propane inventory data updated Wednesdays at 5 p.m. All other data updated Thursdays (Friday in event of a holiday) at 5 p.m. 


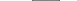




\section{Preface}

The Petroleum Marketing Monthly (PMM) provides information and statistical data on a variety of crude oils and refined petroleum products. The publication presents statistics on crude oil costs and refined petroleum products sales for use by industry, government, private sector analysts, educational institutions, and consumers. Data on crude oil include the domestic first purchase price, the f.o.b. and landed cost of imported crude oil, and the refiners' acquisition cost of crude oil. Refined petroleum product sales data include motor gasoline, distillates, residuals, aviation fuels, kerosene, and propane. The Petroleum Marketing Division, Office of Oil and Gas, Energy Information Administration ensures the accuracy, quality, and confidentiality of the published data in the Petroleum Marketing Monthly.

\section{Scope of Data}

The data within the Petroleum Marketing Monthly are compiled from six Energy Information Administration (EIA) survey forms. The crude oil statistics are calculated from data collected on the following three survey forms: Form EIA-182, "Domestic Crude Oil First Purchase Report"; Form EIA-856, "Monthly Foreign Crude Oil Acquisition Report"; and Form EIA14, "Refiners' Monthly Cost Report."

The statistics on petroleum product sales prices and volumes are derived from Form EIA-782A, "Refiners'/Gas Plant Operators' Monthly Petroleum Product Sales Report" and Form EIA-782B, "Resellers'/Retailers' Monthly Petroleum Product Sales Report."

The data presented in Tables 48 to 50 are derived from aggregations of data from Form EIA-782C, "Monthly Report of PrimeSupplier Sales of Petroleum Products Sold for Local Consumption."

\section{Sections}

Monthly statistics on purchases of crude oil and sales of petroleum products are presented in the Petroleum Marketing Monthly in five sections:

- Summary Statistics

- Crude Oil Prices

- Prices of Petroleum Products

- Volumes of Petroleum Products

- Prime Supplier Sales Volumes of Petroleum Products for Local Consumption.

The publication highlights salient statistics for the United States in the Summary Statistics section. More detailed geographic coverage occurs in the other four sections. Geographic coverage for crude oil includes country of origin for foreign crude and Petroleum Administration for Defense (PAD) Districts and individual States for domestic crude oil. Geographic coverage of the petroleum products includes PAD Districts and individual States.

Detailed statistics for crude oil, including the price of imported crude oil by country of origin, by gravity, and by crude stream, can be found in the Crude Oil Prices section.

PAD District and/or State-level statistics for petroleum products are presented in the Prices, Volumes, and Prime Supplier Sales of Petroleum Products sections. To aid the reader in determining the market changes, the majority of the tables show data for the report month and previous month for the current year, and the report month for the previous year. 


\section{Notes on the Tables}

- For the crude oil statistics referencing Form EIA182, United States includes the 50 States, the outer continental shelf, and the District of Columbia. For crude oil statistics referencing either Form EIA-14 or Form EIA-856, United States includes the 50 States, the District of Columbia, Puerto Rico, the Virgin Islands, and all American territories and possessions. For the petroleum products data, United States includes the 50 States and the District of Columbia.

- Prices exclude taxes. Refer to the Explanatory Notes for a tax table on motor fuels.

- Some of the tables use State abbreviations. Refer to the Explanatory Notes for a table of U.S. Postal State abbreviations.

- Sales of leaded gasoline are reported in the Conventional gasoline category by appropriate grade in the Prime Supplier Sales Volumes section, but are excluded from gasoline sales prices and volumes in all other sections of the publication.
Leaded gasoline is a component of averages and totals prior to October 1993.

- References to "Refiners" include gas plant operators (see the Glossary for definition of "Gas Plant Operators"). All tables whose titles do not specifically reference "Refiners" contain data from all sellers. "All Sellers" includes refiners, gas plant operators, resellers, and retailers.

- "Prime Supplier" refers to a firm that produces, imports, or transports any of the selected petroleum products across State boundaries and local marketing areas and sells the product to local distributors, local retailers, or end users.

- The category "Retail Outlet" refers to any company-operated outlet selling gasoline, on-highway low-sulfur diesel fuel, or propane for on-highway vehicle use (see Glossary).

- No. 2 distillate volumes and prices are classified in accordance with what the product was sold as, regardless of the actual specifications of that product (see definitions of No. 2 distillate in the Glossary). 


\section{Contents}

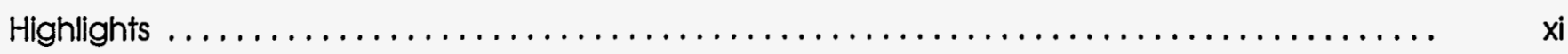

Summary Statistics $\ldots \ldots \ldots \ldots \ldots \ldots \ldots \ldots \ldots \ldots \ldots \ldots \ldots \ldots \ldots \ldots \ldots \ldots \ldots \ldots \ldots \ldots$

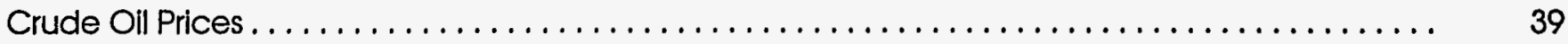

Prices of Petroleum Products $\ldots \ldots \ldots \ldots \ldots \ldots \ldots \ldots \ldots \ldots \ldots \ldots \ldots \ldots \ldots \ldots \ldots \ldots \ldots \ldots$

Volumes of Petroleum Products. . . . . . . . . . . . . . . . . 113

Prime Supplier Sales Volumes of Petroleum Products for Local Consumption. . . . . . . . . . . 139

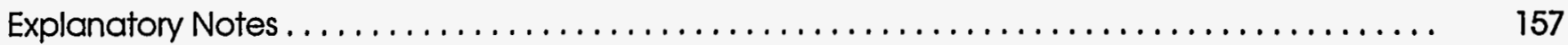

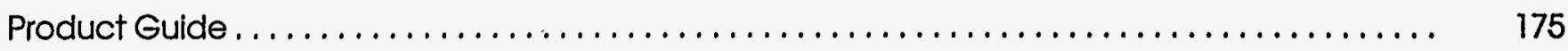

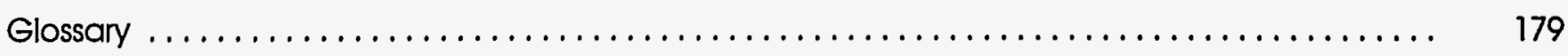

List of Articles $\ldots \ldots \ldots \ldots \ldots \ldots \ldots \ldots \ldots \ldots \ldots \ldots \ldots \ldots \ldots \ldots \ldots \ldots \ldots \ldots \ldots \ldots$ 


\section{Tables}

\section{Summary Statistics}

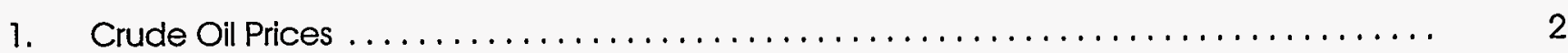

2. U.S. Refiner Prices of Petroleum Products to End Users ...................... 4

3. U.S. Refiner Volumes of Petroleum Products to End Users ...................... 6

4. U.S. Refiner Prices of Petroleum Products for Resale ........................ 8

5. U.S. Refiner Volumes of Petroleum Products for Resale ...................... 10

6. U.S. Refiner Motor Gasoline Prices by Grade and Sales Type.................... $\quad 12$

7. U.S. Refiner Motor Gasoline Volumes by Grade and Sales Type .................. 14

8. U.S. Refiner Conventional Motor Gasoline Prices by Grade and Sales Type ............ 16

9. U.S. Refiner Conventional Motor Gasoline Volumes by Grade and Sales Type .......... 18

10. U.S. Refiner Oxygenated Motor Gasoline Prices by Grade and Sales Type ............ 20

11. U.S. Refiner Oxygenated Motor Gasoline Volumes by Grade and Sales Type.......... 22

12. U.S. Refiner Reformulated Motor Gasoline Prices by Grade and Sales Type ............ 24

13. U.S. Refiner Reformulated Motor Gasoline Volumes by Grade and Sales Type.......... 26

14. U.S. Propane (Consumer Grade) Prices by Sales Type ....................... 28

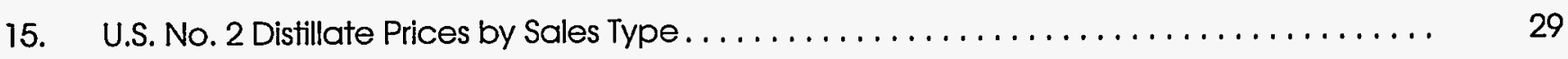

16. U.S. No. 2 Diesel Fuel Prices by Sales Type ............................ 30

17. U.S. No. 2 Diesel Fuel Prices by Sulfur Content and Sales Type .................. 31

18. Prices of No. 2 Distillate to Residences by PAD District and Selected States ........... 32

19. U.S. Refiner Residual Fuel Oil Prices. ................................ 36

20. U.S. Refiner Residual Fuel Oil Volumes $\ldots \ldots \ldots \ldots \ldots \ldots \ldots \ldots \ldots \ldots \ldots \ldots \ldots \ldots \ldots$ 


\section{Crude Oil Prices}

21. Domestic Crude Oil First Purchase Prices........................ 41

22. Domestic Crude Oil First Purchase Prices for Selected Crude Streams . . . . . . . . . . . . . 44

23. Domestic Crude Oil First Purchase Prices by API Gravity ................. 45

24. F.O.B. Costs of Imported Crude Oil by Selected Country .................. 46

25. Landed Costs of Imported Crude Oil by Selected Country . . . . . . . . . . . . . . 47

26. F.O.B. Costs of Imported Crude Oil by API Gravity. . . . . . . . . . . . . . . . . 48

27. Landed Costs of Imported Crude Oil by API Gravity ..................... 49

28. Percentages of Total Imported Crude Oil by API Gravity ................. 50

29. F,O.B. Costs of Imported Crude Oil for Selected Crude Streams . . . . . . . . . . . . . 51

30. Landed Costs of Imported Crude Oil for Selected Crude Streams . . . . . . . . . . . . 53

\section{Prices of Petroleum Products}

31. Motor Gasoline Prices by Grade, Sales Type, PAD District, and State . . . . . . . . . . 56

32. Conventional Motor Gasoline Prices by Grade, Sales Type, PAD District, and State. . . . . . . 64

33. Oxygenated Motor Gasoline Prices by Grade, Sales Type, PAD District, and State . . . . . . 72

34. Reformulated Motor Gasoline Prices by Grade, Sales Type, PAD District, and State ...... . 80

35. Refiner Motor Gasoline Prices by Grade, Sales Type, PAD District and State . . . . . . . . . . 88

36. Refiner Prices of Aviation Fuels and Kerosene by PAD District and State . . . . . . . . . 92

37. Refiner Prices of Distillate Fuels by PAD District and State .................. 96

38. Propane (Consumer Grade) Prices by Sales Type and PAD District . . . . . . . . . . . 100

39. No. 2 Distillate Prices by Sales Type, PAD District, and Selected States . . . . . . . . . . . 101

40. No. 2 Diesel Fuel Prices by Sales Type. PAD District, and Selected States . . . . . . . . . . . . 104

41. No. 2 Diesel Fuel Prices by Sulfur Content, Sales Type, and PAD District . . . . . . . . . 107

42. Residual Fuel Oil Prices by PAD District and State $\ldots \ldots \ldots \ldots \ldots \ldots \ldots \ldots \ldots \ldots$ 


\section{Volumes of Petroleum Products}

43. Refiner Motor Gasoline Volumes by Grade, Sales Type, PAD District, and State . . . . . . . . 114

44. Refiner Motor Gasoline Volumes by Formulation, Sales Type, PAD District, and State .... . 122

45. Refiner Volumes of Aviation Fuels, Kerosene, No. 1 Distillate, and Propane

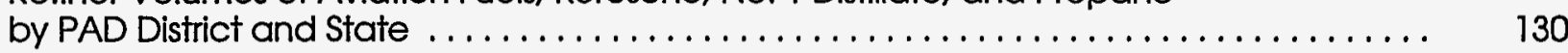

46. Refiner No. 2 Distillate, Diesel Fuel, and Fuel Oil Volumes by PAD District and State . . . . . . 134

47. Refiner Residual Fuel Oil and No. 4 Fuel Volumes by PAD District $\ldots \ldots \ldots \ldots \ldots \ldots \ldots \ldots$

Primesupplier Sales Volumes of Petroleum Products for Local Consumption

48. Prime Supplier Sales Volumes of Motor Gasoline by Grade, Formulation,

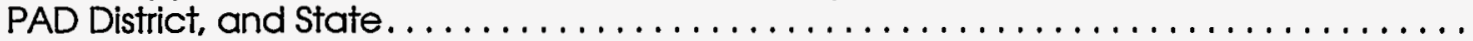

49. Prime Supplier Sales Volumes of Aviation Fuels, Propane, and Residual Fuel Oil

by PAD District and State

50. Prime Supplier Sales Volumes of Distillate Fuel Oils and Kerosene

by PAD District and State

\section{Figures}

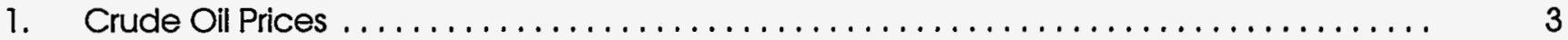

2. U.S. Refiner Retail Petroleum Product Prices . . . . . . . . . . . . . . . . . . . . . . . . . . 5

3. U.S. Refiner Retail Petroleum Product Volumes $\ldots \ldots \ldots \ldots \ldots \ldots \ldots \ldots \ldots \ldots \ldots \ldots$

4. U.S. Refiner Wholesale Petroleum Product Prices . . . . . . . . . . . . . . . . . . . . 9

5. U.S. Refiner Wholesale Petroleum Product Volumes $\ldots \ldots \ldots \ldots \ldots \ldots \ldots \ldots \ldots \ldots \ldots$

6. U.S. No. 2 Distillate Prices to Residences by PAD District ................... 35

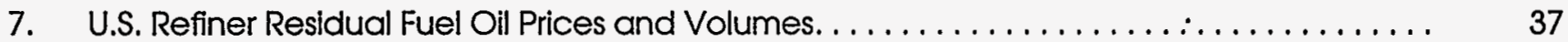




\section{Highlights}




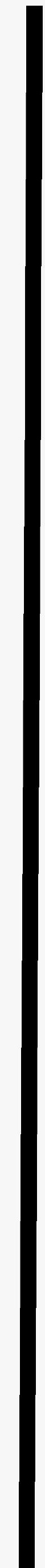




\section{Highlights}

I nternational crude oil prices were mixed during January. Driven by persistent inclement winter weather and low inventories, prices rose during the first week of the month as strong demand for heating fuels continued unabated across North America and Europe. The situation was exacerbated by crude oil production from Organization of Petroleum Exporting Countries (OPEC) remaining essentially unchanged from last month. In contrast, prices began to falter and drop by the middle of the month, to some extent as a correction to soaring price levels. Additionally, refinery production of heating fuels began to catch up to demand in the second half of the month while demand for gasoline fell, further reducing immediate demand for crude oil. Political factors also proved quite influential. The resumption of United Nations talks concerning emergency sales of Iraqi crude oil had a dampening effect on prices in world markets. While the actual volume of crude oil discussed in negotiations is comparatively small, the threat of Iraqi oil coming on-line - even for a limited amount of time - appeared to have a disproportionately negative effect on market players' perceptions.

Continuing December's trend, U.S. gasoline spot market prices rose during the first week of 1996 only to fall precipitously following the severe snow storm that struck the East Coast. As in other regions in the world, demand for gasoline diminished as bad weather affected consumers' ability to travel. However, preliminary estimates indicate imports of gasoline were unusually high for this time of year, and rose throughout the month coinciding with falling prices. In comparison, imports of distillate fuel oil remained at a level consistent with the past 2 years and preliminary estimates indicate demand was 8.5 percent higher at the end of January than at the same point in time last year while stocks were more than 17 percent lower than last year. According to heating degree-days data provided by the National Oceanic and Atmospheric Administration (NOAA), temperatures at the national level for the 1996 heating season to date were 16 percent colder than than at the same point during the 1995 heating season. More specifically, data for major cities along the East Coast and in the South were considerably colder than last year, showing differences in the 60-percent range for several cities. Preliminary estimates for refinery operations in the United States show crude oil inputs slowing and overall utilization slipping from the previous month's level with production of the major finished products only marginally higher than January 1995 levels.
Figure HL1. Crude Oil and Petroleum Product Wholesale Prices

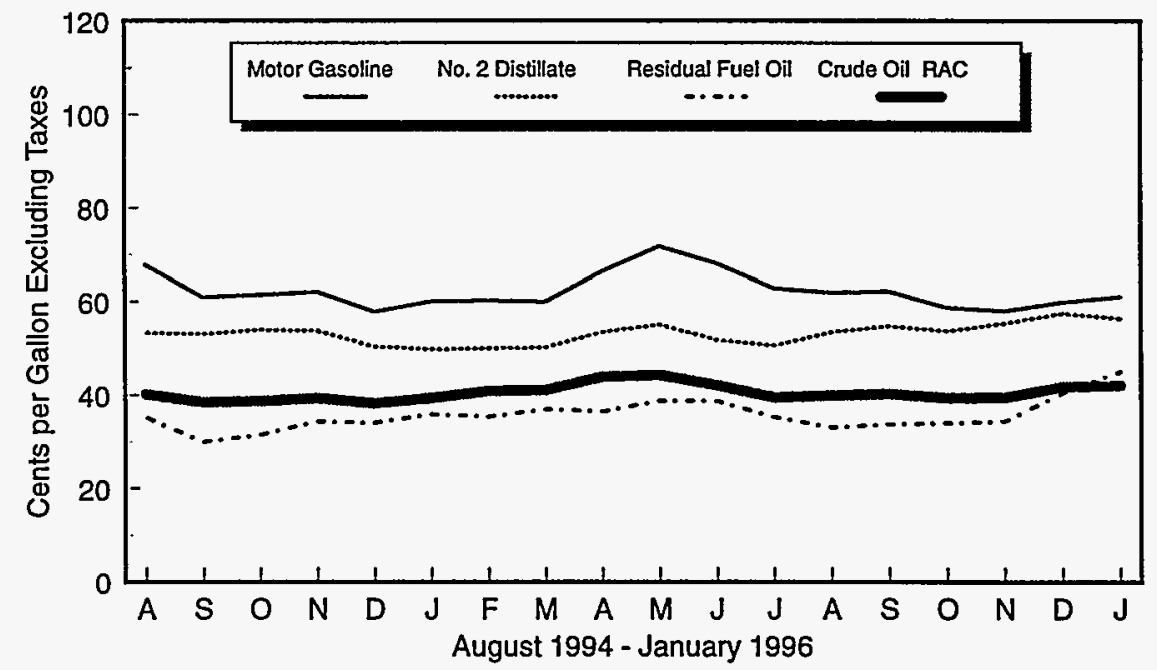

Sources: Energy Information Administration. Crude oil refiner qcquisition cost: Form ElA-14, "Refiners" Monthly Cost Report"; petroleum product prices: Form ElA-782A. "Refiners"/Gas Plant Operators' Monthly Pefroleum Product Sales Report." 
Table HL1. U.S. Refiner Prices and Volumes of Petroleum Products

(Prices: Cents per Gallon Excluding Taxes, Volumes: Million Gallons per Day)

\begin{tabular}{|c|c|c|c|c|c|c|c|c|c|c|c|c|}
\hline \multirow{3}{*}{ Products } & \multicolumn{6}{|c|}{ Sales to End Users } & \multicolumn{6}{|c|}{ Sales for Resale } \\
\hline & \multicolumn{2}{|c|}{ January 1996} & \multicolumn{2}{|c|}{ December 1995} & \multicolumn{2}{|c|}{ January 1995} & \multicolumn{2}{|c|}{ January 1996} & \multicolumn{2}{|c|}{ December 1995} & \multicolumn{2}{|c|}{ January 1995} \\
\hline & Price & Volume & Price & Volume & Price & Volume & Price & Volume & Price & Volume & Price & Volume \\
\hline 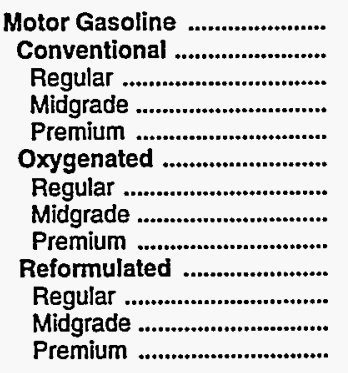 & $\begin{array}{l}74.6 \\
72.0 \\
67.6 \\
76.8 \\
84.3 \\
79.8 \\
75.5 \\
83.7 \\
94.5 \\
77.2 \\
71.1 \\
80.6 \\
89.6\end{array}$ & $\begin{array}{r}52.4 \\
30.5 \\
20.1 \\
5.2 \\
5.2 \\
8.3 \\
5.5 \\
1.5 \\
1.2 \\
13.7 \\
7.7 \\
2.9 \\
3.0\end{array}$ & $\begin{array}{l}73.0 \\
70.4 \\
65.9 \\
75.1 \\
82.6 \\
78.6 \\
74.4 \\
81.9 \\
93.0 \\
75.4 \\
69.1 \\
78.7 \\
87.6\end{array}$ & $\begin{array}{r}56.4 \\
32.8 \\
21.4 \\
5.6 \\
5.7 \\
9.0 \\
6.0 \\
1.7 \\
1.4 \\
14.6 \\
8.1 \\
3.2 \\
3.3\end{array}$ & $\begin{array}{l}74.5 \\
69.9 \\
65.0 \\
75.0 \\
83.0 \\
80.4 \\
76.3 \\
85.6 \\
95.7 \\
81.5 \\
75.2 \\
84.8 \\
94.7\end{array}$ & $\begin{array}{r}51.2 \\
30.2 \\
19.7 \\
5.1 \\
5.4 \\
8.1 \\
5.8 \\
1.1 \\
1.2 \\
12.9 \\
7.3 \\
2.8 \\
2.8\end{array}$ & $\begin{array}{l}61.1 \\
57.5 \\
55.0 \\
61.2 \\
65.6 \\
68.7 \\
65.5 \\
72.1 \\
79.0 \\
67.6 \\
63.1 \\
70.0 \\
76.3\end{array}$ & $\begin{array}{r}264.5 \\
174.2 \\
127.0 \\
15.7 \\
31.4 \\
24.8 \\
17.1 \\
3.5 \\
4.2 \\
65.5 \\
38.4 \\
9.7 \\
17.3\end{array}$ & $\begin{array}{l}59.9 \\
56.2 \\
53.7 \\
60.0 \\
64.3 \\
68.6 \\
65.5 \\
71.4 \\
78.7 \\
66.4 \\
61.9 \\
68.3 \\
74.9\end{array}$ & $\begin{array}{r}291.8 \\
193.2 \\
140.8 \\
17.0 \\
35.4 \\
26.2 \\
18.0 \\
3.8 \\
4.5 \\
72.5 \\
42.2 \\
10.6 \\
19.7\end{array}$ & $\begin{array}{l}60.1 \\
55.1 \\
52.4 \\
59.2 \\
63.9 \\
67.5 \\
64.7 \\
70.0 \\
78.2 \\
69.9 \\
65.1 \\
73.1 \\
78.9\end{array}$ & $\begin{array}{r}259.7 \\
167.8 \\
122.6 \\
15.1 \\
30.0 \\
24.5 \\
17.7 \\
2.6 \\
4.2 \\
67.5 \\
39.8 \\
9.8 \\
17.8\end{array}$ \\
\hline Aviation Gasoline ................. & 97.6 & 0.2 & 96.0 & 0.2 & 99.6 & 0.2 & 95.7 & 0.4 & 95.3 & 0.4 & 92.9 & 0.4 \\
\hline Kerosene-Type Jet Fuel ....... & 61.2 & 46.7 & 59.2 & 44.6 & 52.3 & 45.4 & 60.4 & 12.4 & 58.6 & 12.7 & 52.3 & 8.8 \\
\hline Propane (Consumer Grade) & 63.7 & 3.3 & 55.0 & 4.1 & 54.5 & 3.9 & 41.6 & 44.0 & 37.9 & 41.6 & 35.6 & 37.9 \\
\hline 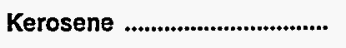 & 71.8 & 0.8 & 70.0 & 0.7 & 67.4 & 0.6 & 65.8 & 6.2 & 64.0 & 5.6 & 56.7 & 4.5 \\
\hline 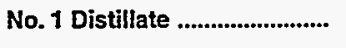 & 65.1 & 0.6 & 66.2 & 0.5 & 59.3 & 0.6 & 65.0 & 4.3 & 66.9 & 3.9 & 58.9 & 3.7 \\
\hline $\begin{array}{l}\text { No. } 2 \text { Distillate } \\
\text { No. } 2 \text { Fuel Oil ................................... } \\
\text { No. } 2 \text { Diesel Fuel ..................... } \\
\text { Low Sulfur ................................ } \\
\text { High Sulfur .......................... }\end{array}$ & $\begin{array}{l}59.7 \\
63.0 \\
59.0 \\
60.5 \\
56.1\end{array}$ & $\begin{array}{r}25.0 \\
4.7 \\
20.3 \\
13.3 \\
7.0\end{array}$ & $\begin{array}{l}59.8 \\
62.3 \\
59.3 \\
60.9 \\
56.4\end{array}$ & $\begin{array}{r}24.7 \\
4.1 \\
20.6 \\
13.0 \\
7.6\end{array}$ & $\begin{array}{l}53.9 \\
56.1 \\
53.4 \\
55.1 \\
50.6\end{array}$ & $\begin{array}{r}23.6 \\
4.3 \\
19.4 \\
12.1 \\
7.3\end{array}$ & $\begin{array}{l}56.4 \\
56.8 \\
56.2 \\
56.7 \\
54.3\end{array}$ & $\begin{array}{r}118.4 \\
46.1 \\
72.4 \\
56.8 \\
15.6\end{array}$ & $\begin{array}{l}57.5 \\
57.3 \\
57.6 \\
58.2 \\
55.2\end{array}$ & $\begin{array}{r}115.8 \\
44.4 \\
71.5 \\
56.3 \\
15.2\end{array}$ & $\begin{array}{l}49.8 \\
49.4 \\
50.1 \\
50.4 \\
48.9\end{array}$ & $\begin{array}{r}101.5 \\
36.9 \\
64.6 \\
52.1 \\
12.4\end{array}$ \\
\hline No. 4 Fuela & 60.4 & 0.9 & 57.3 & 0.6 & 50.5 & 0.9 & 60.3 & 0.5 & 51.6 & 0.6 & 45.9 & 0.7 \\
\hline $\begin{array}{l}\text { Residual Fuel Oil ................. } \\
\text { Sulfur Content not >1\% ..... } \\
\text { Sultur Content }>1 \% \ldots . . . . . .\end{array}$ & $\begin{array}{l}46.9 \\
52.9 \\
44.3\end{array}$ & $\begin{array}{r}13.4 \\
4.0 \\
9.4\end{array}$ & $\begin{array}{l}42.6 \\
48.0 \\
40.5\end{array}$ & $\begin{array}{r}12.2 \\
3.5 \\
8.7\end{array}$ & $\begin{array}{l}40.0 \\
46.0 \\
37.7\end{array}$ & $\begin{array}{r}13.0 \\
3.6 \\
9.3\end{array}$ & $\begin{array}{l}45.0 \\
49.7 \\
38.0\end{array}$ & $\begin{array}{r}16.4 \\
9.8 \\
6.7\end{array}$ & $\begin{array}{l}40.4 \\
44.5 \\
36.0\end{array}$ & $\begin{array}{r}18.2 \\
9.5 \\
8.6\end{array}$ & $\begin{array}{l}35.9 \\
38.4 \\
33.3\end{array}$ & $\begin{array}{r}15.1 \\
7.6 \\
7.5\end{array}$ \\
\hline
\end{tabular}

a Includes No. 4 fuel oil and No. 4 diesel fuel.

Notes: Motor gasoline averages and totals prior to October 1993 include leaded gasoline.

Notes: Values shown for the current month are preliminary. Values shown for previous months are revised. Data are final upon publication in the Petroleum Marketing Annual.

Source: Energy Information Administration Form EIA-782A, "Refiners'/Gas Plant Operators' Monthly Petroleum Product Sales Report."

January market and sales activity for crude oil and the principal petroleum products are summarized in the following sections.

\section{Crude Oil}

During January, the daily spot price for West Texas Intermediate (WTI) crude oil at Cushing, Oklahoma, averaged almost $\$ 19.00$ per barrel. While the average was near December's level, January's price range of more than $\$ 3.00$ per barrel was significantly more dynamic. Opening at $\$ 19.54$ per barrel, the price continued to rise until the month's high of $\$ 20.50$ per barrel was struck on Monday, January 8. However, the price plummeted over $\$ 2.00$ per barrel over the course of the week. While experiencing some modest recovery during the following 2 weeks, the price reached the low of $\$ 17.33$ per barrel on January 29 and closed the month at $\$ 17.76$ per barrel, $\$ 1.78$ lower than where it began.

- January crude oil prices showed mixed changes in level. The average domestic crude oil first purchase price gained 41 cents per barrel ( 2.7 percent), moving to $\$ 15.43$ per barrel.

- The average free-on-board (f.o.b.) cost of imported crude oil lost 22 cents (1.4 percent), and fell to $\$ 15.91$ per barrel. The average landed cost of foreign crude oil remained flat at $\$ 17.10$ per barrel.

- The average refiner acquisition cost of domestic crude oil rose 41 cents ( 2.3 percent), to $\$ 17.96$ per 
barrel. The average cost of imported crude oil to U.S. refiners lost 18 cents ( 1.0 percent), dropping to $\$ 17.40$ per barrel. The composite refiner acquisition cost of crude oil in the United States inched up 11 cents ( 0.6 percent), to $\$ 17.68$ per barrel.

\section{Petroleum Products}

\section{Motor Gasoline}

The unleaded regular gasoline spot price at New York Harbor acted in a volatile manner during January. Opening the month at 52.6 cents per gallon, it reached its high of 55.9 cents per gallon on January 8 . The price plunged after that, losing almost 9 cents per gallon by the time the low of 47.1 cents per gallon was struck on January 12 . The price took a rather erratic path for the rest of the month closing at 50.2 cents per gallon, 2.4 cents lower than its starting point.

- Unlike the more dramatic path taken in the spot market, monthly average gasoline prices showed relatively slight but positive changes. The average refiner motor gasoline retail price increased 1.6 cents to 74.6 cents per gallon, while the average wholesale price rose 1.2 cents to 61.1 cents per gallon. Including data reported by the sample of motor gasoline marketers, the national average retail price climbed 1.3 cents, to 74.5 cents per gallon at company-operated retail outlets. The average wholesale price increased 1.0 cent, to 61.4 cents per gallon. The average dealer tank wagon (DTW) price for motor gasoline rose 1.1 cents, to 68.2 cents per gallon. The average rack price showed the least growth, increasing 0.6 cent to 58.5 cents per gallon. January bulk sales rose 0.9 cent to 52.5 cents per gallon. The margin between reformulated and conventional gasoline prices barely changed from last month, rising slightly to 4.2 cents at the retail level and 8.7 cents at wholesale. The difference between conventional and oxygenated gasoline price levels dropped slightly, moving to 7.4 cents at retail and 9.9 cents at wholesale.

- Refiner sales of finished motor gasoline showed more notable and negative changes compared to prices. Total sales show a loss of 31.3 million gallons per day (9.0 percent), dropping to 316.9 million gallons per day in January. Retail sales fell 4.0 million gallons per day (7.1 percent), while wholesale sales recorded the largest drop, losing 27.3 million gallons per day (9.4 percent). Rack sales accounted for 61.0 percent of refiner wholesale gasoline volumes in January, while DTW and bulk sales made up 27.7 percent and 11.2 percent, respectively. Reformulated gasoline (RFG) constituted 25.0 percent of total motor gasoline sales, while oxygenated gasoline made up 10.4 percent of sales.

\section{No. 2 Disfillate}

Unlike December's steady gain in level, the New York Harbor spot price for No. 2 heating oil took a wide ranging path during January. Opening at 58.4 cents per gallon, the price took a course similar to gasoline and crude oil. Striking the month's high of 63.4 cents per gallon January 8 , it lost 9.7 cents by January 12 . The price behaved in an anemic fashion for the rest of the month, reaching a low of 50.4 cents per gallon on January 25. Closing at 53.6 cents per gallon, the price was 4.8 cents lower than where it started.

- Prices generally maintained their level in most categories of No. 2 distillate sales in contrast to the steep fall in prices observed in the spot market. The most noticeable change occurred in the national average residential price that rose 3.5 cents, to 94.7 cents per gallon. The average wholesale price barely changed, losing 0.6 cent to 57.3 cents per gallon. The average price of No. 2 diesel fuel rose 0.8 cent per gallon at company-operated retail outlets and lost 1.2 cents at wholesale. Low-sulfur diesel fuel prices averaged 3.2 cents per gallon more than high-sulfur diesel at retail and 2.4 cents higher for wholesale.

- Total refiner sales of No. 2 distillate gained ground again this month, rising to 143.5 million gallons per day ( 2.1 percent). No. 2 fuel oil sales accounted for most of the change, building by 2.3 million gallons per day ( 4.7 percent) while sales of No. 2 diesel fuel rose 600,000 gallons per day ( 0.7 percent). Lowsulfur diesel fuel comprised 75.6 percent of all diesel fuel sales and 48.9 percent of all refiner No. 2 distillate sales in January.

\section{Residual Fuel Oil}

- Residual fuel oil prices rose substantially again during January. Refiner prices for low-sulfur residual fuel increased 4.9 cents to 52.9 cents per gallon for retail sales and 5.2 cents to 49.7 cents per 
gallon for wholesale. Refiner high-sulfur residual fuel prices climbed 3.8 cents to 44.3 cents per gallon at the retail level and 2.0 cents to 38.0 cents per gallon at wholesale. Including data reported by the sample of residual fuel oil marketers, the average low-sulfur price jumped 5.7 cents per gallon for retail sales and 6.7 at the wholesale level. The average price for high-sulfur residual fuel increased 4.5 cents and 2.6 cents for retail and wholesale sales, respectively.

- Total refiner sales of residual fuel oil remained relatively flat, with sales totaling 29.8 million gallons per day. Low-sulfur residual fuel sales gained 800,000 gallons per day (6.2 percent), while highsulfur fell 1.2 million gallons per day (6.9 percent).

\section{Other Products}

- Almost all products falling under this heading experienced a growth in price. Refiner propane prices rose 8.7 cents per gallon and 3.7 cents per gallon at retail and wholesale, respectively. Including the sample of propane marketers, the average residential propane price jumped 4.1 cents per gallon, while the average end-user price grew 5.1 cents; the average wholesale price climbed 3.6 cents per gallon. Refiner prices for kerosene-type jet fuel increased 2.0 cents per gallon at retail and 1.8 cents at wholesale. Identical to December's trend, prices for aviation gasoline, kerosene, and No. 4 fuel rose at both levels. No. 1 distillate prices fell at both retail and wholesale.

- Refiner sales of other products were mixed during January. Retail sales of propane lost 800,000 gallons per day while wholesale gained 2.4 million gallons per day. Kerosene-type jet fuel sales rose 2.1 million gallons and dropped 300,000 million gallons per day for retail and wholesale, respectively. Kerosene, No. 1 distillate, aviation gasoline, and No. 4 distillate showed only slight changes at both retail and wholesale levels. 
Summary Statistics 
Table 1. Crude Oil Prices

(Dollars per Barrel)

\begin{tabular}{|c|c|c|c|c|c|c|}
\hline \multirow{2}{*}{$\begin{array}{l}\text { Year } \\
\text { Month }\end{array}$} & \multirow{2}{*}{$\begin{array}{c}\text { Domestic } \\
\text { First Purchase } \\
\text { Prices }\end{array}$} & \multirow{2}{*}{$\begin{array}{l}\text { Average F.O.B.a } \\
\text { Cost of Crude } \\
\text { Oil Imports }\end{array}$} & \multirow{2}{*}{$\begin{array}{l}\text { Average Landed } \\
\text { Cost of Crude } \\
\text { Oil Imports }\end{array}$} & \multicolumn{3}{|c|}{ Refiner Acquisition Cost of Crude Oil } \\
\hline & & & & Domestic & Imported & Composite \\
\hline $\begin{array}{l}1978 \\
1979 \\
1980 \\
1981 \\
1982 \\
1983 \\
1984 \\
1985\end{array}$ & $\begin{array}{r}9.00 \\
12.64 \\
21.59 \\
31.77 \\
28.52 \\
26.19 \\
25.88 \\
24.09 \\
12.51 \\
15.40 \\
12.58 \\
15.86 \\
20.03 \\
16.54 \\
15.99 \\
14.25\end{array}$ & $\begin{array}{l}13.29 \\
20.07 \\
32.37 \\
35.15 \\
32.02 \\
27.81 \\
27.60 \\
25.84 \\
12.52 \\
16.69 \\
13.25 \\
16.89 \\
20.37 \\
16.89 \\
16.77 \\
14.71\end{array}$ & $\begin{array}{l}14.35 \\
21.45 \\
33.67 \\
36.47 \\
33.18 \\
28.93 \\
28.54 \\
26.67 \\
13.49 \\
17.65 \\
14.08 \\
17.68 \\
21.13 \\
18.02 \\
17.75 \\
15.72\end{array}$ & $\begin{array}{l}10.61 \\
14.27 \\
24.23 \\
34.33 \\
31.22 \\
28.87 \\
28.53 \\
26.66 \\
14.82 \\
17.76 \\
14.74 \\
17.87 \\
22.59 \\
19.33 \\
18.63 \\
16.67\end{array}$ & $\begin{array}{l}14.57 \\
21.67 \\
33.89 \\
37.05 \\
33.55 \\
29.30 \\
28.88 \\
26.99 \\
14.00 \\
18.13 \\
14.56 \\
18.08 \\
21.76 \\
18.70 \\
18.20 \\
16.14\end{array}$ & $\begin{array}{l}12.46 \\
17.72 \\
28.07 \\
35.24 \\
31.87 \\
28.99 \\
28.63 \\
26.75 \\
14.55 \\
17.90 \\
14.67 \\
17.97 \\
22.22 \\
19.06 \\
18.43 \\
16.41\end{array}$ \\
\hline 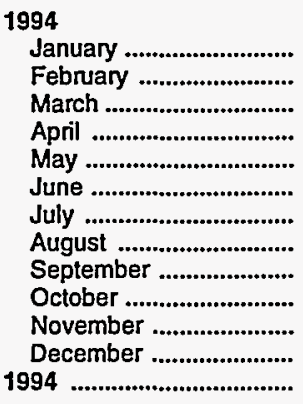 & $\begin{array}{l}10.49 \\
10.71 \\
10.94 \\
12.31 \\
14.02 \\
14.93 \\
15.34 \\
14.50 \\
13.62 \\
13.84 \\
14.14 \\
13.43 \\
13.19\end{array}$ & $\begin{array}{l}12.07 \\
12.05 \\
12.38 \\
13.55 \\
14.67 \\
15.44 \\
16.10 \\
14.94 \\
14.32 \\
14.74 \\
14.88 \\
14.46 \\
14.18\end{array}$ & $\begin{array}{l}12.74 \\
12.71 \\
13.00 \\
14.30 \\
15.62 \\
16.51 \\
17.15 \\
16.07 \\
15.47 \\
15.66 \\
15.98 \\
15.61 \\
15.18\end{array}$ & $\begin{array}{l}12.73 \\
13.24 \\
13.14 \\
14.74 \\
15.86 \\
17.38 \\
17.74 \\
17.22 \\
16.46 \\
16.35 \\
16.63 \\
16.22 \\
15.67\end{array}$ & $\begin{array}{l}12.93 \\
12.90 \\
13.18 \\
14.54 \\
15.74 \\
17.04 \\
17.52 \\
16.66 \\
15.91 \\
16.27 \\
16.46 \\
15.78 \\
15.51\end{array}$ & $\begin{array}{l}12.83 \\
13.07 \\
13.16 \\
14.64 \\
15.80 \\
17.21 \\
17.62 \\
16.92 \\
16.18 \\
16.31 \\
16.54 \\
16.03 \\
15.59\end{array}$ \\
\hline 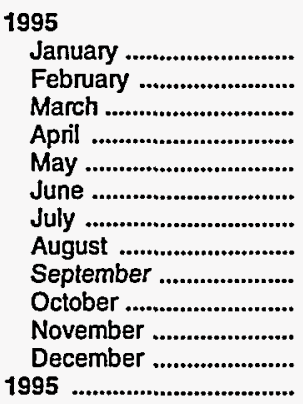 & $\begin{array}{l}14.00 \\
14.69 \\
14.68 \\
15.84 \\
15.85 \\
15.02 \\
14.01 \\
14.13 \\
14.49 \\
13.68 \\
14.03 \\
15.02 \\
14.62\end{array}$ & $\begin{array}{l}15.08 \\
15.63 \\
15.88 \\
17.28 \\
17.30 \\
15.91 \\
14.82 \\
15.05 \\
15.24 \\
14.68 \\
15.31 \\
16.13 \\
15.70\end{array}$ & $\begin{array}{l}16.23 \\
16.73 \\
17.04 \\
18.26 \\
18.18 \\
17.07 \\
15.94 \\
16.10 \\
16.38 \\
15.87 \\
16.30 \\
17.10 \\
16.78\end{array}$ & $\begin{array}{l}16.52 \\
17.16 \\
17.31 \\
18.20 \\
18.68 \\
17.94 \\
16.85 \\
16.96 \\
17.12 \\
16.82 \\
16.73 \\
17.55 \\
17.33\end{array}$ & $\begin{array}{l}16.56 \\
17.21 \\
17.22 \\
18.73 \\
18.51 \\
17.44 \\
16.50 \\
16.54 \\
16.71 \\
16.30 \\
16.50 \\
17.58 \\
17.14\end{array}$ & $\begin{array}{l}16.54 \\
17.18 \\
17.27 \\
18.44 \\
18.60 \\
17.69 \\
16.68 \\
16.75 \\
16.91 \\
16.56 \\
16.61 \\
17.57 \\
17.24\end{array}$ \\
\hline $\begin{array}{l}1996 \\
\quad \text { January ............................. }\end{array}$ & 15.43 & 15.91 & 17.10 & 17.96 & 17.40 & 17.68 \\
\hline
\end{tabular}

a Free on Board. See Glossary.

b Values through 1980 reflect the month of reporting; values since then reflect the month of acquisition, which can be the month of loading, the month of landing, or sometime between those events. Prices for crude oil can be determined at a time other than the acquisition date. See the Explanatory Notes section for additional detail.

Notes: Values for Domestic First Purchase and Refiner Acquisition for the current month, and for Average F.O.B. and Average Landed for current 2 months are preliminary. Values shown for previous months are revised. Data are final upon publication in the Petroleum Marketing Annual.

Sources: Domestic first purchase prices -- See "Sources" from Table 21. Crude oil imports costs -- See "Sources" from Table 24. Refiner acquisition costs -Energy Information Administration, Form FEA-P110-M-1, "Refiners' Monthly Cost Allocation Report," January 1978 through June 1978; Form ERA-49, "Domestic Crude Oil Entitlements Program Refiners' Monthly Report," July 1978 through December 1980; Form ElA-14, "Refiners' Monthly Cost Report," January 1981 to present. 


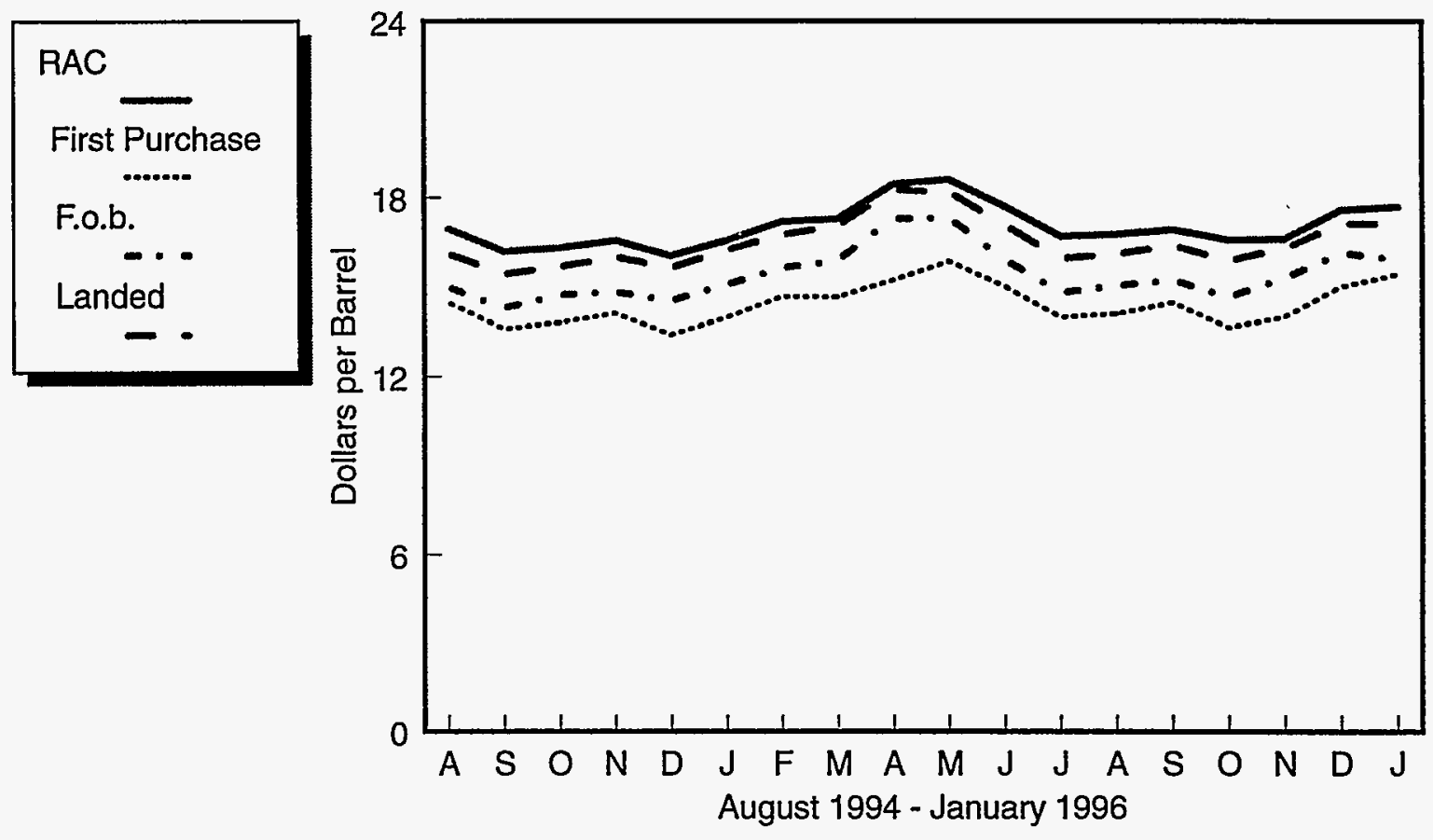

Sources: Energy Information Administration, Form ElA-182, "Domestic Crude Oil First Purchase Report": Form ElA-856, "Monthly Forelgn Crude Oil Acquisition Report"; and Form EIA-14, "Refiners' Monthly Cost Report." 
Table 2. U.S. Refiner Prices of Petroleum Products to End Users

(Cents per Gallon Excluding Taxes)

\begin{tabular}{|c|c|c|c|c|c|c|c|c|c|c|c|}
\hline \multirow[b]{2}{*}{$\begin{array}{c}\text { Year } \\
\text { Month }\end{array}$} & \multirow[b]{2}{*}{$\begin{array}{c}\text { Motor } \\
\text { Gasoline }\end{array}$} & \multirow[b]{2}{*}{$\begin{array}{l}\text { Aviation } \\
\text { Gasoline }\end{array}$} & \multirow[b]{2}{*}{$\begin{array}{c}\text { Kerosene- } \\
\text { Type } \\
\text { Jet Fuel }\end{array}$} & \multirow{2}{*}{$\begin{array}{c}\text { Propane } \\
\text { (Consumer } \\
\text { Grade) }\end{array}$} & \multirow[b]{2}{*}{ Kerosene } & \multirow[b]{2}{*}{$\begin{array}{c}\text { No. } 1 \\
\text { Distillate }\end{array}$} & \multicolumn{3}{|c|}{ No. 2 Distillate } & \multirow[b]{2}{*}{$\begin{array}{l}\text { No. } 4 \\
\text { Fuel }^{a}\end{array}$} & \multirow[b]{2}{*}{$\begin{array}{l}\text { Residual } \\
\text { Fuel Oil }\end{array}$} \\
\hline & & & & & & & $\begin{array}{c}\text { No. } 2 \\
\text { Diesel } \\
\text { Fuel }\end{array}$ & $\begin{array}{c}\text { No. } 2 \\
\text { Fuel } \\
\text { Oil } \\
\end{array}$ & Average & & \\
\hline $\begin{array}{l}1978 \\
1979 \\
1980 \\
1981 \\
1982 \\
1983 \\
1984 \\
1985 \\
1986 \\
1987\end{array}$ & $\begin{array}{r}48.4 \\
71.3 \\
103.5 \\
114.7 \\
106.0 \\
95.4 \\
90.7 \\
91.2 \\
62.4 \\
66.9 \\
67.3 \\
75.6 \\
88.3 \\
79.7 \\
78.7 \\
75.9\end{array}$ & $\begin{array}{r}51.6 \\
68.9 \\
108.4 \\
130.3 \\
131.2 \\
125.5 \\
123.4 \\
120.1 \\
101.1 \\
90.7 \\
89.1 \\
99.5 \\
112.0 \\
104.7 \\
102.7 \\
99.0\end{array}$ & $\begin{array}{r}38.7 \\
54.7 \\
86.8 \\
102.4 \\
96.3 \\
87.8 \\
84.2 \\
79.6 \\
52.9 \\
54.3 \\
51.3 \\
59.2 \\
76.6 \\
65.2 \\
61.0 \\
58.0\end{array}$ & $\begin{array}{l}33.5 \\
35.7 \\
48.2 \\
56.5 \\
59.2 \\
70.9 \\
73.7 \\
71.7 \\
74.5 \\
70.1 \\
71.4 \\
61.5 \\
74.5 \\
73.0 \\
64.3 \\
67.3\end{array}$ & $\begin{array}{r}42.1 \\
58.5 \\
90.2 \\
112.3 \\
108.9 \\
96.1 \\
103.6 \\
103.0 \\
79.0 \\
77.0 \\
73.8 \\
70.9 \\
92.3 \\
83.8 \\
78.8 \\
75.4\end{array}$ & $\begin{array}{r}41.0 \\
57.2 \\
83.4 \\
103.9 \\
102.3 \\
96.2 \\
92.7 \\
88.0 \\
62.0 \\
60.4 \\
56.4 \\
66.1 \\
81.9 \\
74.0 \\
66.6 \\
66.6\end{array}$ & $\begin{array}{l}37.7 \\
58.5 \\
81.8 \\
99.5 \\
94.2 \\
82.6 \\
82.3 \\
78.9 \\
47.8 \\
55.1 \\
50.0 \\
58.5 \\
72.5 \\
64.8 \\
61.9 \\
60.2\end{array}$ & $\begin{array}{l}40.0 \\
51.6 \\
78.8 \\
91.4 \\
90.5 \\
91.6 \\
91.6 \\
84.9 \\
56.0 \\
58.1 \\
54.4 \\
58.7 \\
73.4 \\
66.5 \\
62.7 \\
60.2\end{array}$ & $\begin{array}{l}39.6 \\
55.1 \\
80.4 \\
95.8 \\
92.5 \\
83.9 \\
83.7 \\
79.9 \\
49.1 \\
55.6 \\
50.7 \\
58.5 \\
72.6 \\
65.0 \\
62.0 \\
60.2\end{array}$ & $\begin{array}{l}31.1 \\
47.9 \\
68.2 \\
79.7 \\
75.0 \\
76.6 \\
79.6 \\
77.3 \\
48.9 \\
51.3 \\
46.1 \\
51.2 \\
62.2 \\
58.0 \\
52.6 \\
50.1\end{array}$ & $\begin{array}{l}29.8 \\
43.6 \\
60.7 \\
75.6 \\
67.6 \\
65.1 \\
68.7 \\
61.0 \\
34.3 \\
42.3 \\
33.4 \\
38.5 \\
44.4 \\
34.0 \\
33.6 \\
33.7\end{array}$ \\
\hline 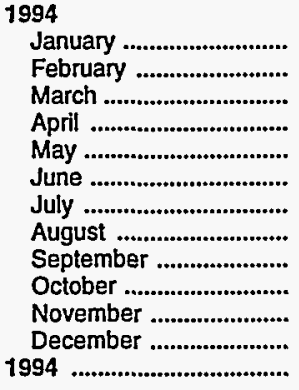 & $\begin{array}{l}66.8 \\
67.6 \\
67.3 \\
69.5 \\
71.1 \\
74.1 \\
77.0 \\
81.5 \\
79.6 \\
76.9 \\
77.5 \\
75.1 \\
73.8\end{array}$ & $\begin{array}{r}88.6 \\
88.4 \\
89.0 \\
91.3 \\
92.3 \\
95.6 \\
97.4 \\
101.7 \\
101.1 \\
100.0 \\
100.0 \\
99.2 \\
95.7\end{array}$ & $\begin{array}{l}51.5 \\
55.7 \\
51.8 \\
50.7 \\
51.0 \\
51.9 \\
53.5 \\
54.4 \\
53.9 \\
55.0 \\
57.2 \\
53.9 \\
53.4\end{array}$ & $\begin{array}{l}61.8 \\
63.5 \\
58.5 \\
54.9 \\
46.4 \\
45.5 \\
46.4 \\
48.3 \\
47.1 \\
49.4 \\
51.0 \\
51.9 \\
53.0\end{array}$ & $\begin{array}{l}79.5 \\
84.1 \\
78.2 \\
69.7 \\
55.2 \\
54.5 \\
60.4 \\
57.8 \\
58.3 \\
61.5 \\
64.0 \\
64.7 \\
66.0\end{array}$ & $\begin{array}{l}61.7 \\
67.5 \\
64.0 \\
63.5 \\
61.4 \\
61.8 \\
65.0 \\
63.9 \\
64.7 \\
65.5 \\
66.5 \\
61.3 \\
64.0\end{array}$ & $\begin{array}{l}52.5 \\
55.4 \\
54.9 \\
54.7 \\
54.3 \\
54.9 \\
55.8 \\
56.7 \\
56.6 \\
57.1 \\
57.2 \\
54.5 \\
55.4\end{array}$ & $\begin{array}{l}59.5 \\
63.9 \\
60.8 \\
58.0 \\
53.5 \\
54.0 \\
54.9 \\
55.0 \\
54.4 \\
55.7 \\
56.7 \\
56.4 \\
57.2\end{array}$ & $\begin{array}{l}53.9 \\
56.8 \\
55.6 \\
55.0 \\
54.2 \\
54.8 \\
55.7 \\
56.4 \\
56.3 \\
56.8 \\
57.1 \\
54.8 \\
55.6\end{array}$ & $\begin{array}{l}49.5 \\
55.4 \\
50.9 \\
47.6 \\
47.6 \\
47.4 \\
49.2 \\
50.1 \\
49.7 \\
49.0 \\
49.2 \\
49.4 \\
50.1\end{array}$ & $\begin{array}{l}32.5 \\
36.8 \\
32.9 \\
31.1 \\
32.6 \\
35.6 \\
38.4 \\
39.6 \\
34.4 \\
34.5 \\
36.9 \\
38.3 \\
35.2\end{array}$ \\
\hline 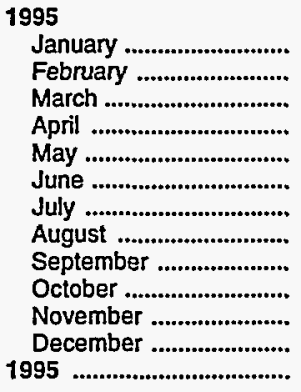 & $\begin{array}{l}74.5 \\
73.3 \\
73.1 \\
77.3 \\
83.4 \\
83.9 \\
80.0 \\
76.9 \\
75.8 \\
73.6 \\
71.8 \\
73.0 \\
76.5\end{array}$ & $\begin{array}{r}99.6 \\
99.8 \\
99.0 \\
101.3 \\
105.8 \\
106.4 \\
101.8 \\
99.2 \\
101.3 \\
96.8 \\
95.4 \\
96.0 \\
100.5\end{array}$ & $\begin{array}{l}52.3 \\
52.2 \\
50.5 \\
52.8 \\
55.0 \\
53.2 \\
51.9 \\
53.4 \\
55.7 \\
54.9 \\
57.0 \\
59.2 \\
54.0\end{array}$ & $\begin{array}{l}54.5 \\
55.1 \\
53.3 \\
46.6 \\
43.1 \\
42.9 \\
42.2 \\
44.9 \\
45.7 \\
49.2 \\
51.7 \\
55.0 \\
49.2\end{array}$ & $\begin{array}{l}67.4 \\
62.7 \\
59.4 \\
56.1 \\
51.8 \\
54.9 \\
51.3 \\
53.3 \\
57.3 \\
56.5 \\
62.8 \\
70.0 \\
58.9\end{array}$ & $\begin{array}{l}59.3 \\
59.1 \\
60.0 \\
62.5 \\
61.7 \\
59.7 \\
59.3 \\
62.4 \\
64.5 \\
62.9 \\
68.0 \\
66.2 \\
62.0\end{array}$ & $\begin{array}{l}53.4 \\
53.3 \\
53.5 \\
56.6 \\
58.1 \\
55.7 \\
54.0 \\
55.8 \\
57.4 \\
56.5 \\
58.2 \\
59.3 \\
56.0\end{array}$ & $\begin{array}{l}56.1 \\
55.9 \\
54.4 \\
55.6 \\
55.8 \\
52.8 \\
51.5 \\
53.3 \\
56.2 \\
54.1 \\
58.7 \\
62.3 \\
55.8\end{array}$ & $\begin{array}{l}\mathbf{5 3 . 9} \\
\mathbf{5 3 . 8} \\
\mathbf{5 3 . 7} \\
\mathbf{5 6 . 5} \\
\mathbf{5 7 . 7} \\
55.4 \\
\mathbf{5 3 . 7} \\
55.5 \\
\mathbf{5 7 . 3} \\
\mathbf{5 6 . 2} \\
\mathbf{5 8 . 3} \\
\mathbf{5 9 . 8} \\
\mathbf{5 6 . 0}\end{array}$ & $\begin{array}{l}50.5 \\
50.1 \\
51.0 \\
48.9 \\
50.9 \\
52.0 \\
45.6 \\
45.3 \\
49.9 \\
48.9 \\
52.2 \\
57.3 \\
50.5\end{array}$ & $\begin{array}{l}40.0 \\
39.8 \\
40.5 \\
40.3 \\
42.2 \\
42.1 \\
38.2 \\
35.1 \\
35.1 \\
35.9 \\
37.4 \\
42.6 \\
39.1\end{array}$ \\
\hline 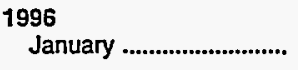 & 74.6 & 97.6 & 61.2 & 63.7 & 71.8 & 65.1 & 59.0 & 63.0 & 59.7 & 60.4 & 46.9 \\
\hline
\end{tabular}

a Inciudes No. 4 fuel oil and No. 4 diesel fuel.

Notes: Motor gasoline averages and totals prior to October 1993 include leaded gasoline.

Notes: Values shown for the current month are preliminary. Values shown for previous months are revised. Data are final upon publication in the Petroleum Marketing Annual.

Sources: Energy Information Administration Form ElA-782A, "Refiners'/Gas Plant Operators' Monthly Petroleum Product Sales Report," January 1983 forward; Form EIA-460, "Petroleum Industry Monthly Report for Product Prices," source for backcast estimates prior to January 1983. 
Figure 2. U.S. Refiner Retail Petroleum Product Prices
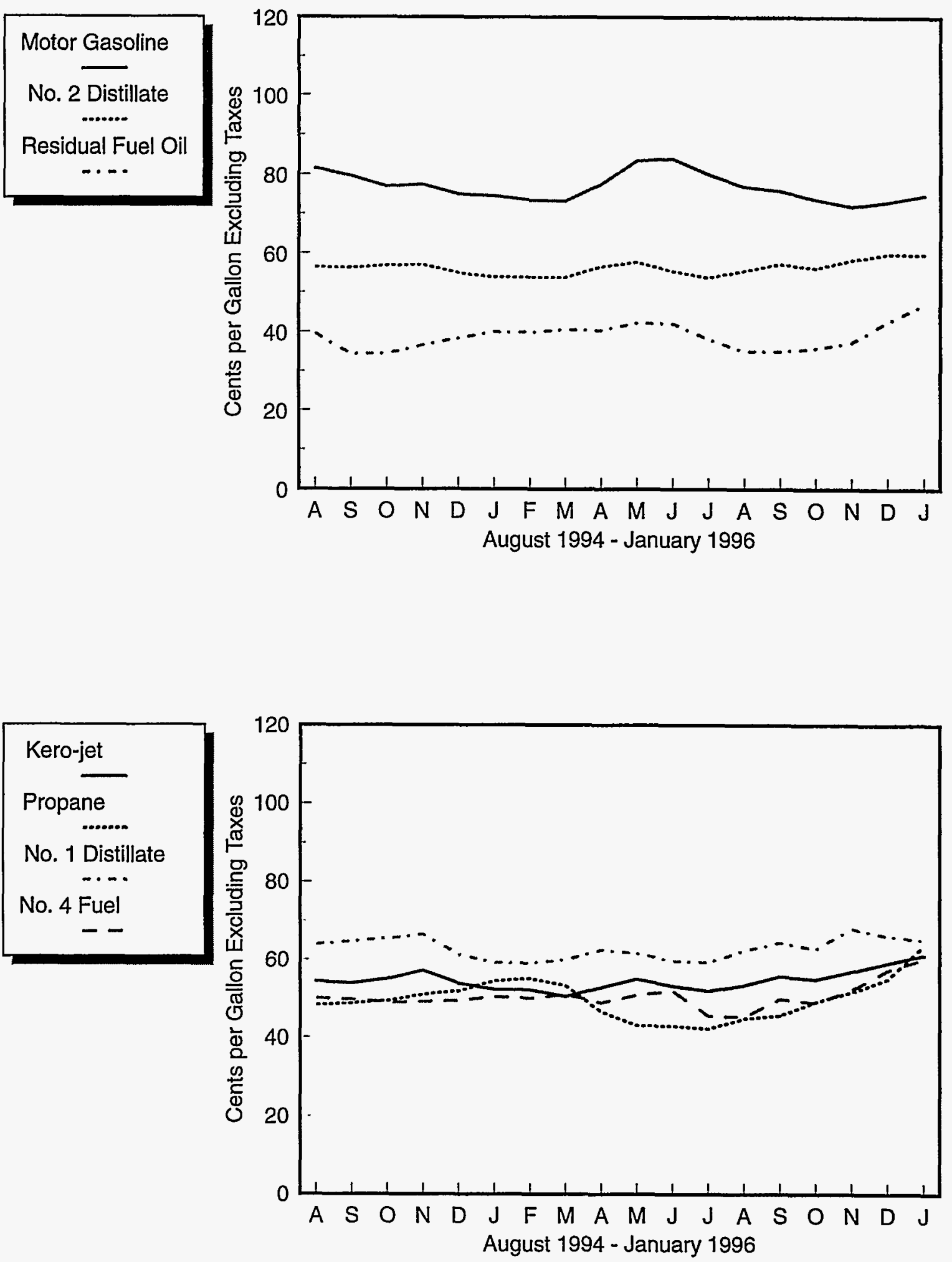

Source: Energy Information Administration, Form ElA-782A, "Refiners'/Gas Plant Operators' Monthly Petroleum Product Sales Report." 
Table 3. U.S. Refiner Volumes of Petroleum Products to End Users (Million Gallons per Day)

\begin{tabular}{|c|c|c|c|c|c|c|c|c|c|c|c|}
\hline \multirow[b]{2}{*}{$\begin{array}{c}\text { Year } \\
\text { Month }\end{array}$} & \multirow[b]{2}{*}{$\begin{array}{c}\text { Motor } \\
\text { Gasoline }\end{array}$} & \multirow[b]{2}{*}{$\begin{array}{l}\text { Aviation } \\
\text { Gasoline }\end{array}$} & \multirow[b]{2}{*}{\begin{tabular}{|c|} 
Kerosene- \\
Type \\
Jet Fuel
\end{tabular}} & \multirow[b]{2}{*}{$\begin{array}{c}\text { Propane } \\
\text { (Consumer } \\
\text { Grade) }\end{array}$} & \multirow[b]{2}{*}{ Kerosene } & \multirow[b]{2}{*}{$\begin{array}{c}\text { No. } 1 \\
\text { Distillate }\end{array}$} & \multicolumn{3}{|c|}{ No. 2 Distillate } & \multirow[b]{2}{*}{$\begin{array}{l}\text { No. } 4 \\
\text { Fuola }\end{array}$} & \multirow[b]{2}{*}{$\begin{array}{l}\text { Residua } \\
\text { Fuel Oll }\end{array}$} \\
\hline & & & & & & & $\begin{array}{c}\text { No. } 2 \\
\text { Diesel } \\
\text { Fuel }\end{array}$ & $\begin{array}{c}\text { No. } 2 \\
\text { Fuel } \\
\text { Oil }\end{array}$ & Total & & \\
\hline $\begin{array}{l}1983 \\
1984 \\
1985\end{array}$ & $\begin{array}{l}51.1 \\
57.6 \\
57.5 \\
61.2 \\
61.0 \\
61.0 \\
61.2 \\
60.3 \\
61.2 \\
59.0 \\
57.2\end{array}$ & $\begin{array}{l}0.4 \\
0.3 \\
0.3 \\
0.3 \\
0.2 \\
0.2 \\
0.2 \\
0.2 \\
0.2 \\
0.2 \\
0.2\end{array}$ & $\begin{array}{l}30.8 \\
32.9 \\
34.6 \\
35.1 \\
36.8 \\
38.2 \\
40.1 \\
39.9 \\
38.5 \\
39.8 \\
41.7\end{array}$ & $\begin{array}{l}3.1 \\
3.3 \\
3.7 \\
3.4 \\
3.8 \\
4.3 \\
2.8 \\
2.7 \\
3.1 \\
3.8 \\
3.5\end{array}$ & $\begin{array}{l}0.2 \\
0.3 \\
0.3 \\
0.3 \\
0.3 \\
0.3 \\
0.3 \\
0.2 \\
0.2 \\
0.2 \\
0.2\end{array}$ & $\begin{array}{l}0.5 \\
0.5 \\
0.5 \\
0.4 \\
0.4 \\
0.4 \\
0.5 \\
0.5 \\
0.5 \\
0.5 \\
0.4\end{array}$ & $\begin{array}{l}23.3 \\
26.3 \\
25.0 \\
24.4 \\
24.1 \\
24.5 \\
24.3 \\
22.2 \\
21.1 \\
21.5 \\
20.8\end{array}$ & $\begin{array}{l}3.7 \\
4.9 \\
5.0 \\
4.4 \\
4.5 \\
4.6 \\
4.5 \\
3.6 \\
3.2 \\
3.1 \\
2.9\end{array}$ & $\begin{array}{l}27.0 \\
31.2 \\
29.9 \\
28.8 \\
28.5 \\
29.1 \\
28.8 \\
25.9 \\
24.4 \\
24.6 \\
23.8\end{array}$ & $\begin{array}{l}0.7 \\
0.7 \\
0.5 \\
0.7 \\
0.8 \\
1.1 \\
0.9 \\
0.8 \\
0.7 \\
0.6 \\
0.6\end{array}$ & $\begin{array}{l}28.3 \\
32.9 \\
25.2 \\
31.6 \\
29.0 \\
30.2 \\
30.4 \\
25.9 \\
24.0 \\
22.4 \\
17.2\end{array}$ \\
\hline 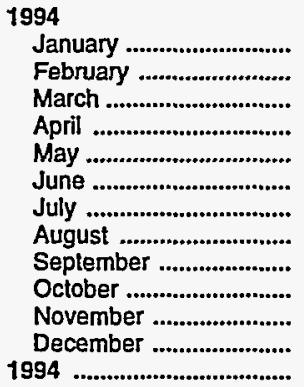 & $\begin{array}{l}51.5 \\
54.3 \\
55.4 \\
55.0 \\
55.5 \\
57.2 \\
55.8 \\
56.4 \\
55.0 \\
54.2 \\
54.5 \\
55.6 \\
\mathbf{5 5 . 0}\end{array}$ & $\begin{array}{l}0.2 \\
0.2 \\
0.2 \\
0.2 \\
0.2 \\
0.3 \\
0.2 \\
0.3 \\
0.2 \\
0.2 \\
0.2 \\
0.2 \\
0.2\end{array}$ & $\begin{array}{l}40.5 \\
41.2 \\
43.3 \\
45.6 \\
45.2 \\
46.5 \\
46.8 \\
48.1 \\
47.3 \\
46.1 \\
45.6 \\
46.6 \\
45.2\end{array}$ & $\begin{array}{l}3.3 \\
2.9 \\
2.2 \\
1.7 \\
1.9 \\
1.8 \\
1.7 \\
2.0 \\
1.9 \\
2.3 \\
2.2 \\
2.7 \\
2.2\end{array}$ & $\begin{array}{l}0.6 \\
0.6 \\
0.3 \\
0.2 \\
0.4 \\
0.4 \\
0.2 \\
0.6 \\
0.4 \\
0.5 \\
0.6 \\
0.7 \\
0.4\end{array}$ & $\begin{array}{l}0.8 \\
0.8 \\
0.5 \\
0.2 \\
0.2 \\
0.3 \\
0.2 \\
0.2 \\
0.3 \\
0.3 \\
0.4 \\
0.5 \\
0.4\end{array}$ & $\begin{array}{l}19.8 \\
21.6 \\
22.5 \\
22.1 \\
22.6 \\
22.9 \\
21.0 \\
21.5 \\
21.2 \\
20.4 \\
20.2 \\
19.8 \\
21.3\end{array}$ & $\begin{array}{l}4.7 \\
4.1 \\
3.1 \\
2.3 \\
2.3 \\
2.3 \\
2.1 \\
3.7 \\
3.7 \\
3.9 \\
4.0 \\
4.3 \\
3.4\end{array}$ & $\begin{array}{l}24.6 \\
25.7 \\
25.6 \\
24.4 \\
24.9 \\
25.2 \\
23.0 \\
25.1 \\
24.9 \\
24.2 \\
24.2 \\
24.1 \\
24.6\end{array}$ & $\begin{array}{l}1.4 \\
1.4 \\
1.1 \\
0.6 \\
0.5 \\
0.6 \\
0.4 \\
0.6 \\
0.4 \\
0.6 \\
0.8 \\
1.1 \\
0.8\end{array}$ & $\begin{array}{l}17.5 \\
15.5 \\
15.2 \\
12.6 \\
12.2 \\
14.0 \\
11.5 \\
11.6 \\
12.5 \\
13.1 \\
12.9 \\
14.0 \\
13.5\end{array}$ \\
\hline 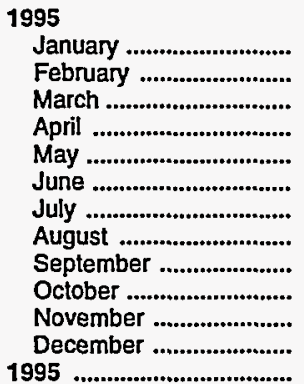 & $\begin{array}{l}51.2 \\
54.1 \\
55.6 \\
55.5 \\
56.2 \\
58.5 \\
56.9 \\
58.3 \\
56.6 \\
55.6 \\
55.9 \\
56.4 \\
55.9\end{array}$ & $\begin{array}{l}0.2 \\
0.2 \\
0.2 \\
0.2 \\
0.2 \\
0.2 \\
0.2 \\
0.3 \\
0.2 \\
0.2 \\
0.2 \\
0.2 \\
0.2\end{array}$ & $\begin{array}{l}45.4 \\
44.6 \\
44.5 \\
44.4 \\
45.9 \\
47.2 \\
46.3 \\
48.0 \\
45.4 \\
46.4 \\
47.5 \\
44.6 \\
45.9\end{array}$ & $\begin{array}{l}3.9 \\
4.1 \\
3.1 \\
3.3 \\
3.3 \\
2.8 \\
2.7 \\
2.6 \\
3.0 \\
2.9 \\
3.3 \\
4.1 \\
3.3\end{array}$ & $\begin{array}{l}0.6 \\
0.9 \\
0.6 \\
0.6 \\
0.4 \\
0.6 \\
0.8 \\
0.7 \\
0.5 \\
0.7 \\
0.7 \\
0.7 \\
0.6\end{array}$ & $\begin{array}{l}0.6 \\
0.6 \\
0.4 \\
0.2 \\
0.1 \\
0.1 \\
0.1 \\
0.2 \\
0.2 \\
0.2 \\
0.3 \\
0.5 \\
0.3\end{array}$ & $\begin{array}{l}19.4 \\
20.5 \\
20.9 \\
20.5 \\
21.2 \\
22.9 \\
20.6 \\
22.8 \\
22.1 \\
22.0 \\
22.6 \\
20.6 \\
21.3\end{array}$ & $\begin{array}{l}4.3 \\
4.9 \\
4.0 \\
3.6 \\
3.5 \\
2.9 \\
2.6 \\
3.3 \\
2.9 \\
3.1 \\
3.6 \\
4.1 \\
3.6\end{array}$ & $\begin{array}{l}23.6 \\
25.3 \\
24.9 \\
24.1 \\
24.6 \\
25.8 \\
23.1 \\
26.1 \\
25.0 \\
25.1 \\
26.2 \\
24.7 \\
24.9\end{array}$ & $\begin{array}{l}0.9 \\
0.9 \\
0.7 \\
0.4 \\
0.2 \\
0.2 \\
0.3 \\
0.4 \\
0.2 \\
0.2 \\
0.4 \\
0.6 \\
0.5\end{array}$ & $\begin{array}{l}13.0 \\
13.9 \\
12.0 \\
11.0 \\
10.5 \\
10.2 \\
10.1 \\
11.4 \\
11.8 \\
10.6 \\
11.8 \\
12.2 \\
11.5\end{array}$ \\
\hline $\begin{array}{l}1996 \\
\text { January ................................ }\end{array}$ & 52.4 & 0.2 & 46.7 & 3.3 & 0.8 & 0.6 & 20.3 & 4.7 & 25.0 & 0.9 & 13.4 \\
\hline
\end{tabular}

a Includes No. 4 fuel oil and No. 4 diesel fuel.

Notes: Motor gasoline averages and totals prior to October 1993 include leaded gasoline.

Notes: Values shown for the current month are preliminary. Values shown for previous months are revised. Data are final upon publication in the Petroleum Marketing Annual. Totals may not equal the sum of the components due to rounding.

Source: Energy Information Administration Form EIA-782A, "Refiners'/Gas Plant Operators' Monthly Petroleum Product Sales Report." 
Figure 3. U.S. Refiner Retail Petroleum Product Volumes

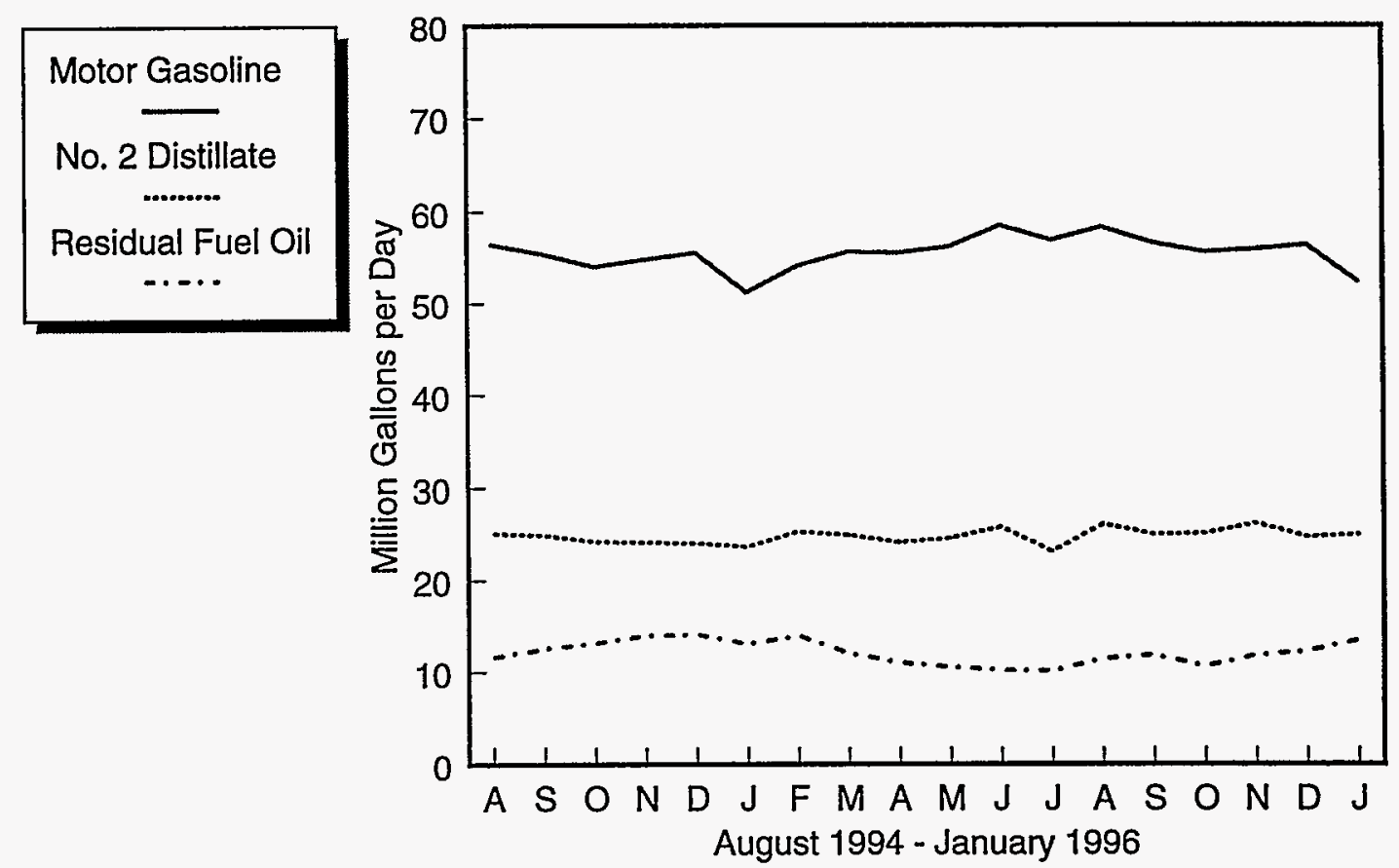

Percentages of Refiner Retail Volumes

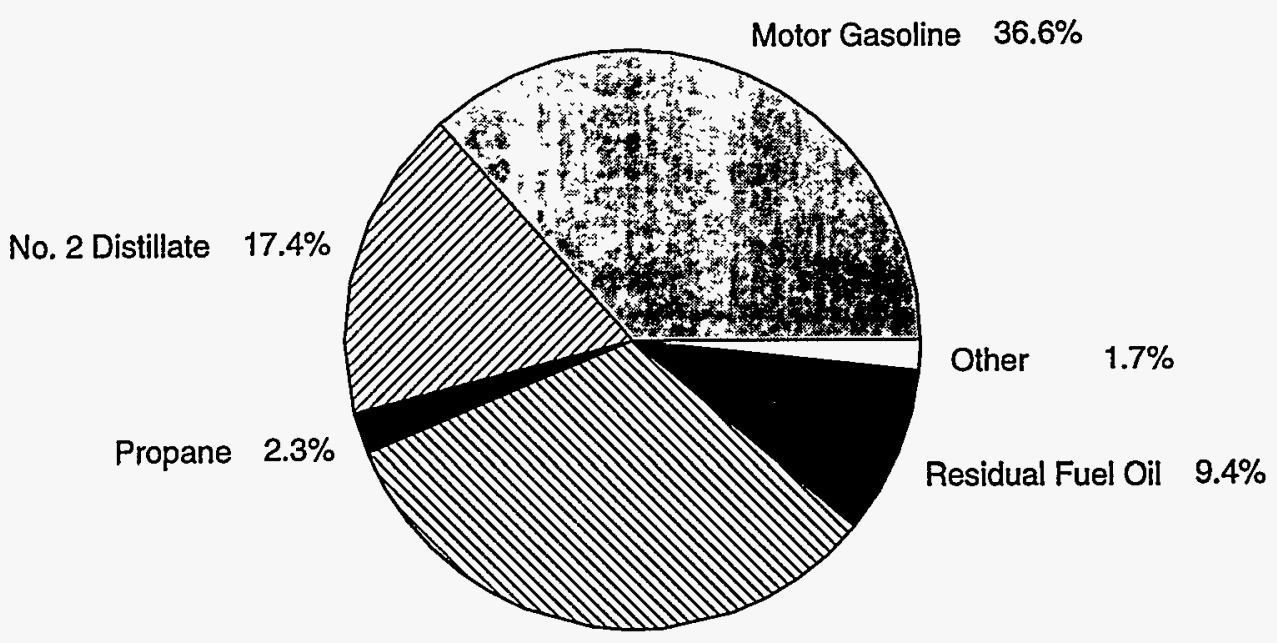

Kero-jet $32.6 \%$

Source: Energy Information Administration, Form EIA-782A, "Refiners'/Gas Plant Operators' Monthly Petroleum Product Sales Report." 
Table 4. U.S. Refiner Prices of Petroleum Products for Resale (Cents per Gallon Excluding Taxes)

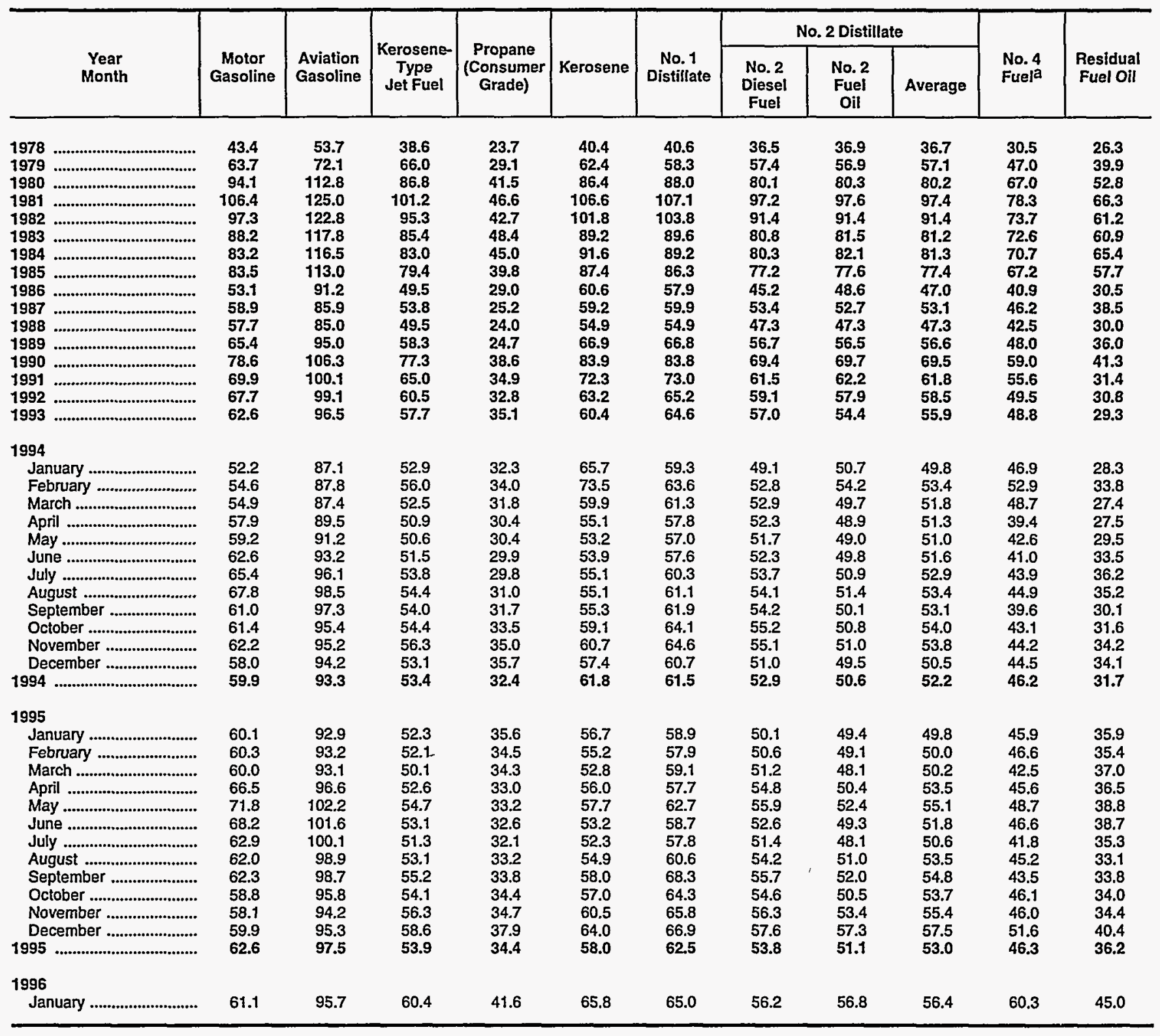

a Includes No. 4 fuel oil and No. 4 diesel fuel.

Notes: Motor gasoline averages and totals prior to October 1993 include leaded gasoline.

Notes: Values shown for the current month are preliminary. Values shown for previous months are revised. Data are final upon publication in the Petroleum Marketing Annual.

Sources: Energy Information Administration Form ElA-782A, "Refiners'/Gas Plant Operators' Monthly Petroleum Product Sales Report," January 1983 forward; Form EIA-460, "Petroleum Industry Monthly Report for Product Prices," source for backcast estimates prior to January 1983. 
Figure 4. U.S. Refiner Wholesale Petroleum Product Prices
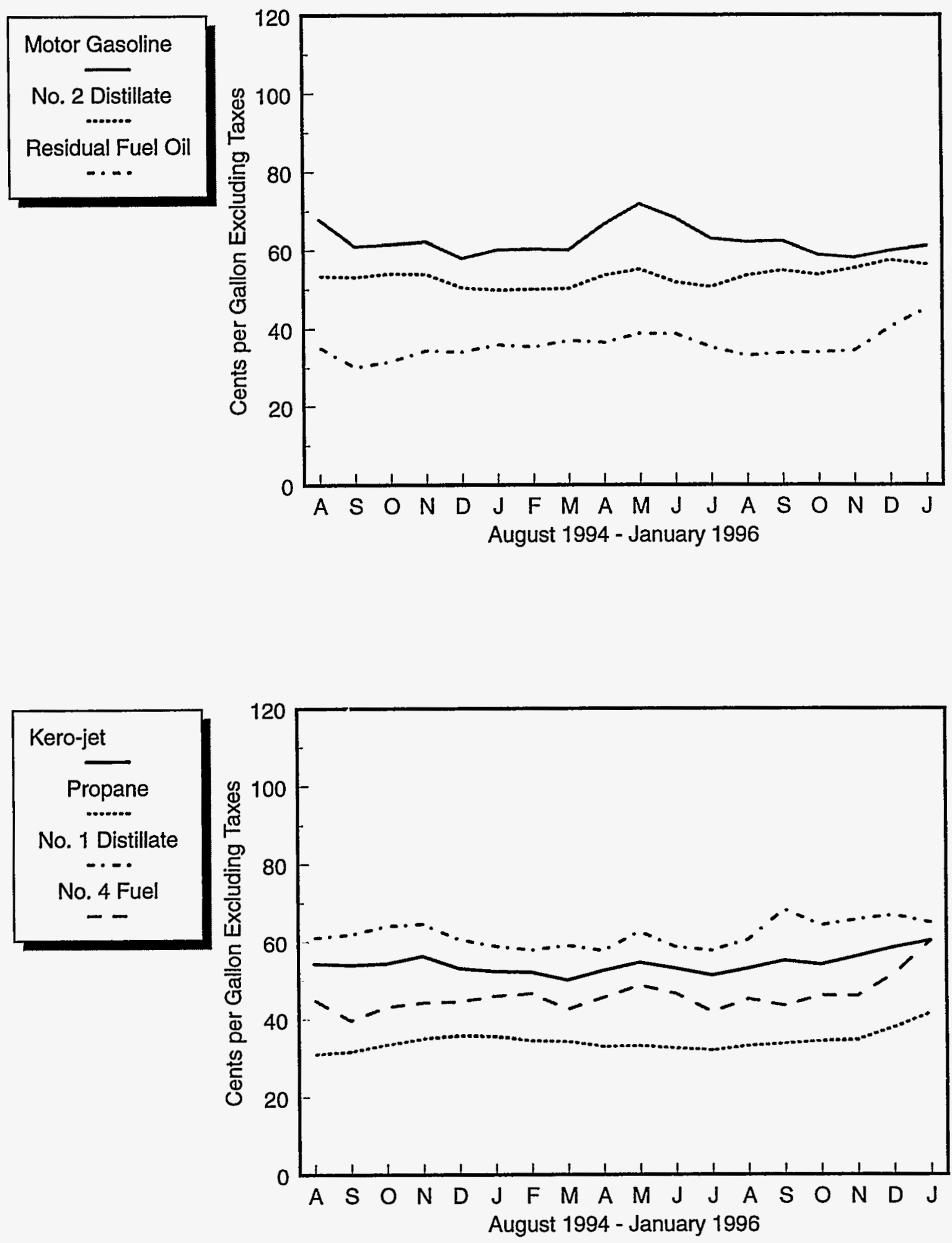

Source: Energy Information Administration, Form ElA-782A, "Refiners'/Gas Plant Operators' Monthly Petroleum Product Sales Report." 
Table 5. U.S. Refiner Volumes of Petroleum Products for Resale (Million Gallons per Day)

\begin{tabular}{|c|c|c|c|c|c|c|c|c|c|c|c|}
\hline \multirow[b]{2}{*}{$\begin{array}{l}\text { Year } \\
\text { Month }\end{array}$} & \multirow[b]{2}{*}{$\begin{array}{l}\text { Motor } \\
\text { Gasoline }\end{array}$} & \multirow[b]{2}{*}{$\begin{array}{l}\text { Aviation } \\
\text { Gasoline }\end{array}$} & \multirow[b]{2}{*}{$\begin{array}{c}\text { Kerosene- } \\
\text { Type } \\
\text { Jet Fuel }\end{array}$} & \multirow[b]{2}{*}{$\begin{array}{c}\text { Propane } \\
\text { (Consumer } \\
\text { Grade) }\end{array}$} & \multirow[b]{2}{*}{ Kerosene } & \multirow[b]{2}{*}{$\begin{array}{c}\text { No. } 1 \\
\text { Distillate }\end{array}$} & \multicolumn{3}{|c|}{ No. 2 Distillate } & \multirow[b]{2}{*}{$\begin{array}{l}\text { No. } 4 \\
\text { Fuela }\end{array}$} & \multirow[b]{2}{*}{$\begin{array}{l}\text { Residual } \\
\text { Fuel Oil }\end{array}$} \\
\hline & & & & & & & $\begin{array}{c}\text { No. } 2 \\
\text { Diesel } \\
\text { Fuel }\end{array}$ & $\begin{array}{c}\text { No. } 2 \\
\text { Fuel } \\
\text { Oil }\end{array}$ & Total & & \\
\hline $\begin{array}{l}1983 \\
1984 \\
1985 \\
1986 \\
1987 \\
1988 \\
1989 \\
1990 \\
1991\end{array}$ & $\begin{array}{l}242.5 \\
246.3 \\
256.9 \\
257.2 \\
257.2 \\
263.7 \\
260.7 \\
264.8 \\
261.3 \\
265.4 \\
266.9\end{array}$ & $\begin{array}{l}0.7 \\
0.8 \\
0.7 \\
0.7 \\
0.8 \\
0.7 \\
0.7 \\
0.7 \\
0.7 \\
0.7 \\
0.6\end{array}$ & $\begin{array}{r}5.4 \\
6.6 \\
7.6 \\
9.2 \\
10.1 \\
10.0 \\
8.6 \\
8.8 \\
8.7 \\
8.0 \\
8.3\end{array}$ & $\begin{array}{l}26.0 \\
26.7 \\
29.2 \\
26.3 \\
27.0 \\
27.7 \\
25.9 \\
25.5 \\
25.4 \\
26.7 \\
27.9\end{array}$ & $\begin{array}{l}2.5 \\
2.2 \\
2.4 \\
2.4 \\
2.3 \\
2.7 \\
2.7 \\
2.2 \\
2.1 \\
2.2 \\
2.3\end{array}$ & $\begin{array}{l}2.4 \\
2.6 \\
2.7 \\
2.5 \\
2.0 \\
2.6 \\
2.7 \\
2.4 \\
2.4 \\
2.3 \\
2.3\end{array}$ & $\begin{array}{l}38.1 \\
42.8 \\
43.3 \\
46.4 \\
44.3 \\
47.8 \\
50.7 \\
51.9 \\
51.5 \\
50.8 \\
58.0\end{array}$ & $\begin{array}{l}47.3 \\
51.4 \\
53.9 \\
53.8 \\
49.3 \\
50.1 \\
46.7 \\
45.9 \\
46.9 \\
48.9 \\
43.6\end{array}$ & $\begin{array}{r}85.5 \\
94.2 \\
97.3 \\
100.3 \\
93.6 \\
97.9 \\
97.5 \\
97.8 \\
98.3 \\
99.7 \\
101.7\end{array}$ & $\begin{array}{l}0.9 \\
1.7 \\
1.2 \\
1.2 \\
1.5 \\
1.2 \\
1.2 \\
0.8 \\
0.4 \\
0.4 \\
0.4\end{array}$ & $\begin{array}{l}20.2 \\
21.3 \\
19.9 \\
18.6 \\
16.9 \\
18.9 \\
21.0 \\
17.9 \\
17.8 \\
15.2 \\
12.6\end{array}$ \\
\hline 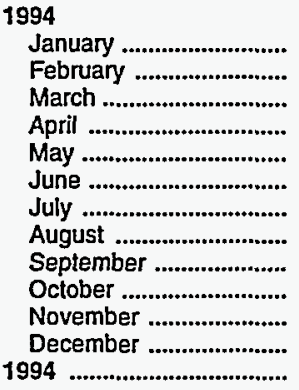 & $\begin{array}{l}254.5 \\
261.2 \\
269.0 \\
276.2 \\
279.4 \\
287.7 \\
279.2 \\
288.4 \\
282.0 \\
276.1 \\
275.9 \\
290.0 \\
276.7\end{array}$ & $\begin{array}{l}0.4 \\
0.5 \\
0.6 \\
0.6 \\
0.8 \\
0.9 \\
0.9 \\
0.9 \\
0.8 \\
0.6 \\
0.5 \\
0.4 \\
0.7\end{array}$ & $\begin{array}{r}8.2 \\
8.0 \\
7.1 \\
7.5 \\
9.1 \\
9.2 \\
10.0 \\
11.0 \\
9.7 \\
9.6 \\
9.8 \\
10.4 \\
9.1\end{array}$ & $\begin{array}{l}40.0 \\
33.7 \\
26.1 \\
21.0 \\
19.4 \\
22.6 \\
24.0 \\
26.3 \\
27.2 \\
32.6 \\
31.1 \\
36.8 \\
28.4\end{array}$ & $\begin{array}{l}7.0 \\
4.9 \\
2.7 \\
1.1 \\
0.9 \\
0.7 \\
0.7 \\
1.7 \\
2.0 \\
2.2 \\
2.5 \\
3.9 \\
2.5\end{array}$ & $\begin{array}{l}5.1 \\
3.7 \\
1.2 \\
0.7 \\
0.6 \\
0.6 \\
0.5 \\
0.7 \\
1.3 \\
1.7 \\
3.4 \\
3.9 \\
1.9\end{array}$ & $\begin{array}{l}63.4 \\
65.3 \\
71.8 \\
74.3 \\
74.9 \\
79.6 \\
69.9 \\
77.2 \\
78.6 \\
75.7 \\
69.0 \\
68.9 \\
72.4\end{array}$ & $\begin{array}{l}46.5 \\
47.2 \\
38.6 \\
29.8 \\
26.6 \\
29.3 \\
25.0 \\
26.4 \\
28.6 \\
28.2 \\
31.0 \\
38.7 \\
32.9\end{array}$ & $\begin{array}{r}109.9 \\
112.5 \\
110.4 \\
104.1 \\
101.5 \\
108.9 \\
94.9 \\
103.5 \\
107.2 \\
103.9 \\
100.0 \\
107.5 \\
105.3\end{array}$ & $\begin{array}{l}0.9 \\
0.9 \\
0.6 \\
0.3 \\
0.1 \\
0.2 \\
0.2 \\
0.2 \\
0.2 \\
0.2 \\
0.3 \\
0.6 \\
0.4\end{array}$ & $\begin{array}{r}14.0 \\
16.0 \\
16.0 \\
12.8 \\
13.4 \\
12.7 \\
11.6 \\
13.8 \\
10.5 \\
9.6 \\
11.6 \\
11.7 \\
12.8\end{array}$ \\
\hline 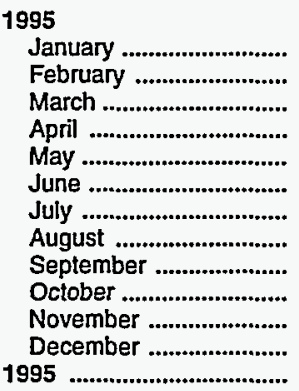 & $\begin{array}{l}259.7 \\
276.2 \\
281.8 \\
278.6 \\
290.7 \\
303.3 \\
294.5 \\
305.6 \\
294.8 \\
290.0 \\
294.2 \\
291.8 \\
288.5\end{array}$ & $\begin{array}{l}0.4 \\
0.5 \\
0.6 \\
0.6 \\
0.7 \\
0.7 \\
0.8 \\
0.8 \\
0.7 \\
0.6 \\
0.5 \\
0.4 \\
0.6\end{array}$ & $\begin{array}{r}8.8 \\
8.9 \\
8.8 \\
8.8 \\
9.2 \\
9.1 \\
9.6 \\
10.0 \\
10.2 \\
11.1 \\
11.4 \\
12.7 \\
9.9\end{array}$ & $\begin{array}{l}37.9 \\
43.7 \\
29.6 \\
24.0 \\
22.6 \\
23.7 \\
24.1 \\
27.0 \\
28.0 \\
29.9 \\
35.8 \\
41.6 \\
30.6\end{array}$ & $\begin{array}{l}4.5 \\
4.9 \\
2.0 \\
1.0 \\
0.6 \\
0.7 \\
0.7 \\
1.1 \\
1.8 \\
2.0 \\
3.9 \\
5.6 \\
2.4\end{array}$ & $\begin{array}{l}3.7 \\
3.0 \\
1.5 \\
0.5 \\
0.4 \\
0.4 \\
0.3 \\
0.7 \\
1.0 \\
1.6 \\
3.7 \\
3.9 \\
1.7\end{array}$ & $\begin{array}{l}64.6 \\
67.9 \\
72.2 \\
73.4 \\
74.8 \\
81.6 \\
75.4 \\
81.8 \\
82.1 \\
84.6 \\
77.7 \\
71.5 \\
75.6\end{array}$ & $\begin{array}{l}36.9 \\
45.7 \\
34.7 \\
31.7 \\
24.8 \\
25.7 \\
22.3 \\
22.2 \\
24.4 \\
25.6 \\
33.4 \\
44.4 \\
30.9\end{array}$ & $\begin{array}{r}101.5 \\
113.6 \\
106.9 \\
105.1 \\
99.6 \\
107.3 \\
97.7 \\
104.0 \\
106.6 \\
110.3 \\
111.1 \\
115.8 \\
106.6\end{array}$ & $\begin{array}{l}0.7 \\
0.6 \\
0.5 \\
0.2 \\
0.2 \\
0.1 \\
0.2 \\
0.1 \\
0.3 \\
0.1 \\
0.6 \\
0.6 \\
0.3\end{array}$ & $\begin{array}{l}15.1 \\
17.7 \\
14.5 \\
10.5 \\
11.0 \\
11.3 \\
11.8 \\
12.5 \\
13.4 \\
12.1 \\
12.5 \\
18.2 \\
13.4\end{array}$ \\
\hline $\begin{array}{l}1996 \\
\text { January .............................. }\end{array}$ & 264.5 & 0.4 & 12.4 & 44.0 & 6.2 & 4.3 & 72.4 & 46.1 & 118.4 & 0.5 & 16.4 \\
\hline
\end{tabular}

a Includes No. 4 fuel oil and No. 4 diesel fuel.

Notes: Motor gasoline averages and totals prior to October 1993 include leaded gasoline.

Notes: Values shown for the current month are preliminary. Values shown for previous months are revised. Data are final upon publication in the Petroleum Marketing Annual.

Source: Energy Information Administration Form ElA-782A, "Refiners'/Gas Plant Operators' Monthly Petroleum Product Sales Report." 
Figure 5. U.S. Refiner Wholesale Petroleum Product Volumes

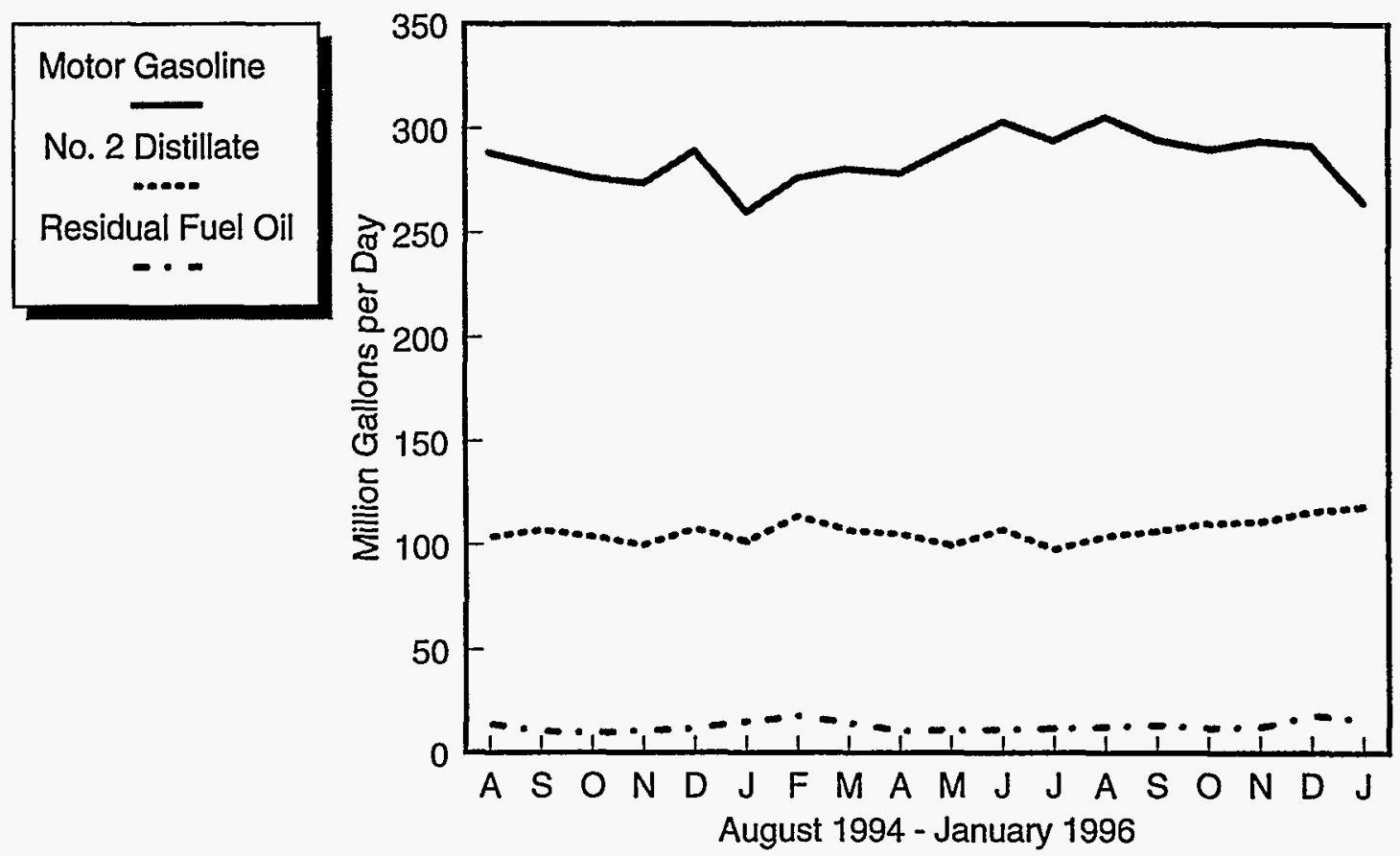

Percentages of Refiner Wholesale Volumes

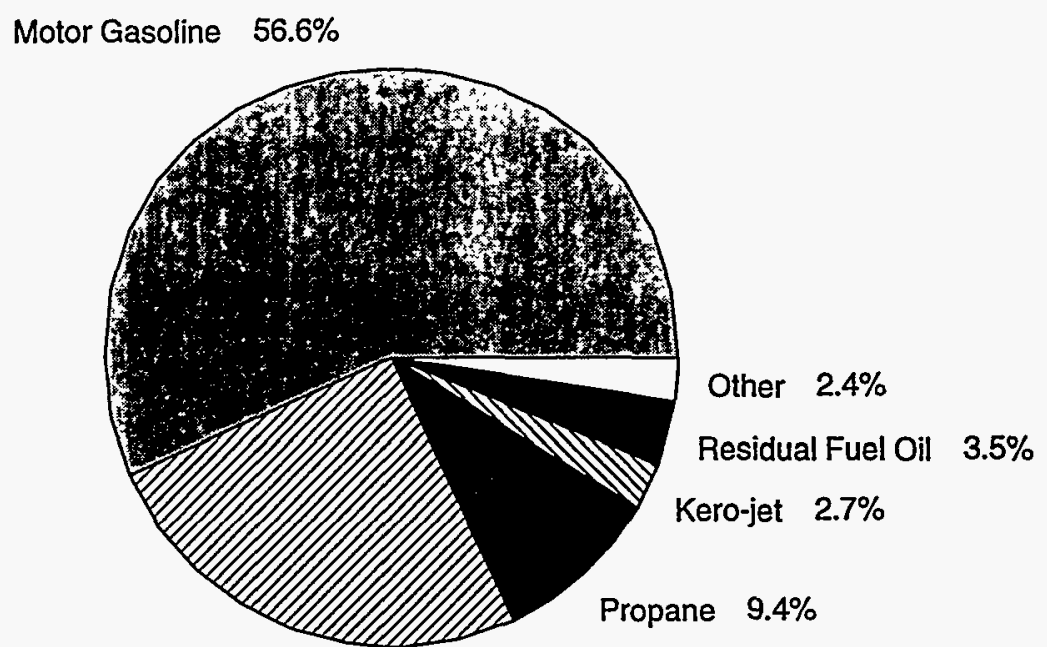

No. 2 Distillate $25.3 \%$

Source: Energy Information Administration, Form ElA-782A, "Refiners'/Gas Plant Operators' Monthly Petroleum Product Sales Report." 
Table 6. U.S. Refiner Motor Gasoline Prices by Grade and Sales Type (Cents per Gallon Excluding Taxes)

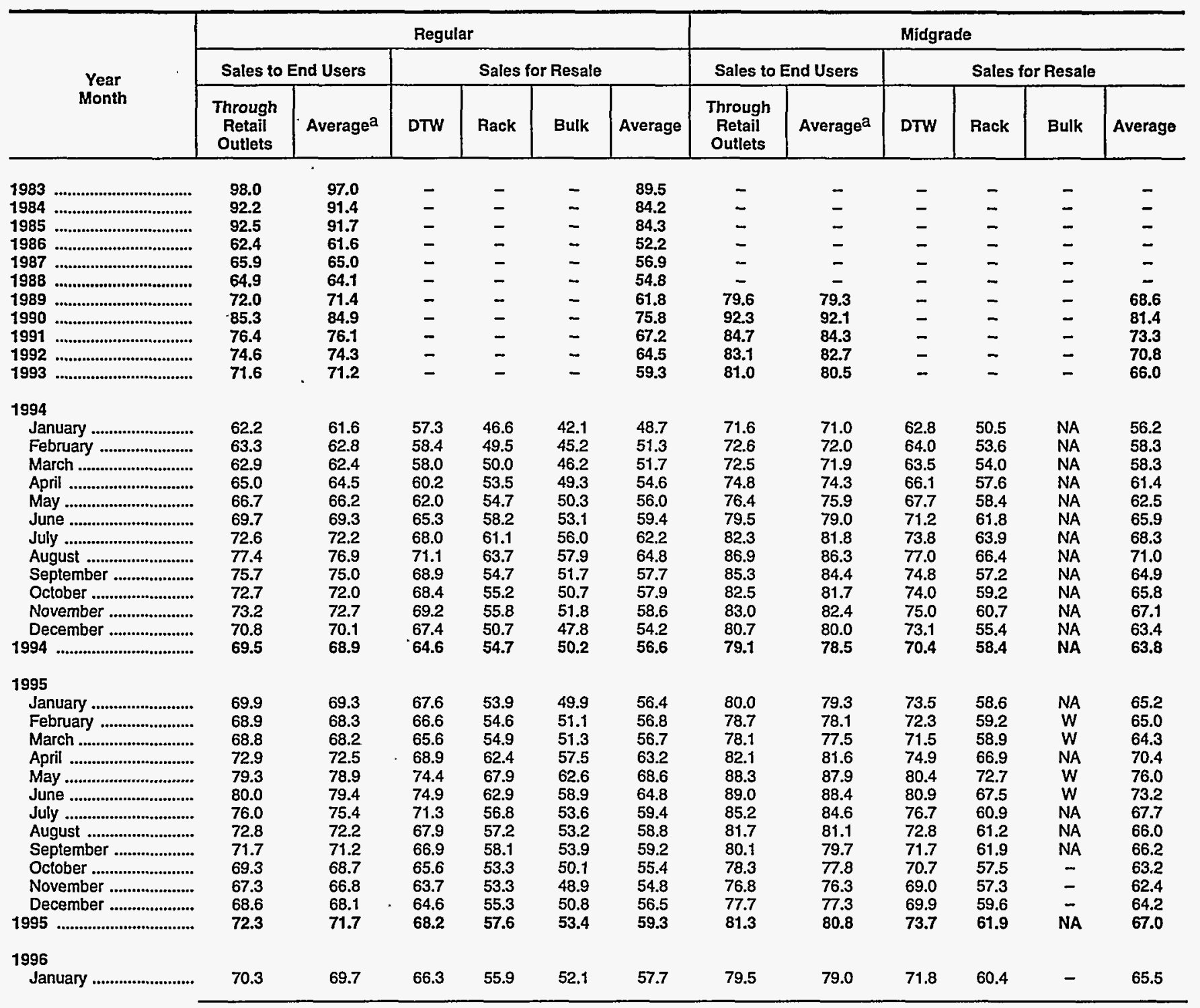

See footnotes at end of table. 
Table 6. U.S. Refiner Motor Gasoline Prices by Grade and Sales Type

(Cents per Gallon Excluding Taxes) - Continued

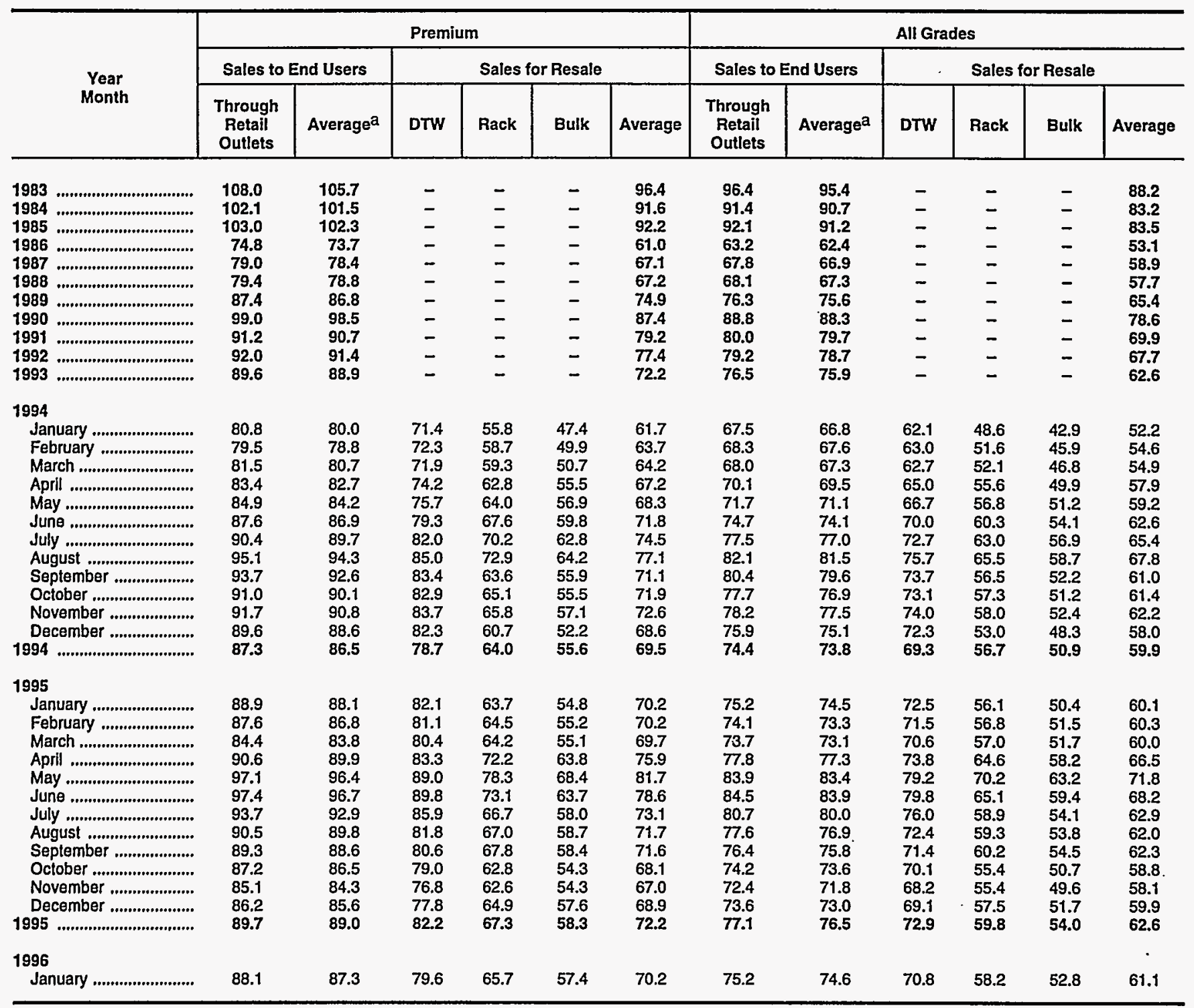

$\operatorname{Dash}(-)=$ No data reported.

NA $=$ Not avallable.

$W=$ Withheld to avoid disclosure of individual company data

a Includes sales through retail outlets as well as all direct sales to end users that were not made through company-operated retail outlets, e.g., sales to agricultural customers, commercial sales, and industrial sales.

Notes: Data for the 4 th quarter of 1993 were derived from two separate survey systems. The DTW, Rack, and Bulk components were derived from the revised EIA-782 survey system, while the End-Use and Average Resale categories were derived from the predecessor EIA-782 survey system. Therefore, the DTW, Rack, and Bulk components are not consistent with the Average Resale category. Beginning January 1994, all data-are from the revised ElA-782 survey system and are consistent.

Notes: Motor gasoline averages and totals prior to October 1993 include leaded gasoline.

Notes: Values shown for the current month are preliminary. Values shown for previous months are revised. Data are final upon publication in the Petroleum Markoting Annual.

Source: Energy Information Administration Form ElA-782A, "Refiners'/Gas Plant Operators' Monthly Petroleum Product Sales Report." 
Table 7. U.S. Refiner Motor Gasoline Volumes by Grade and Sales Type (Million Gallons per Day)

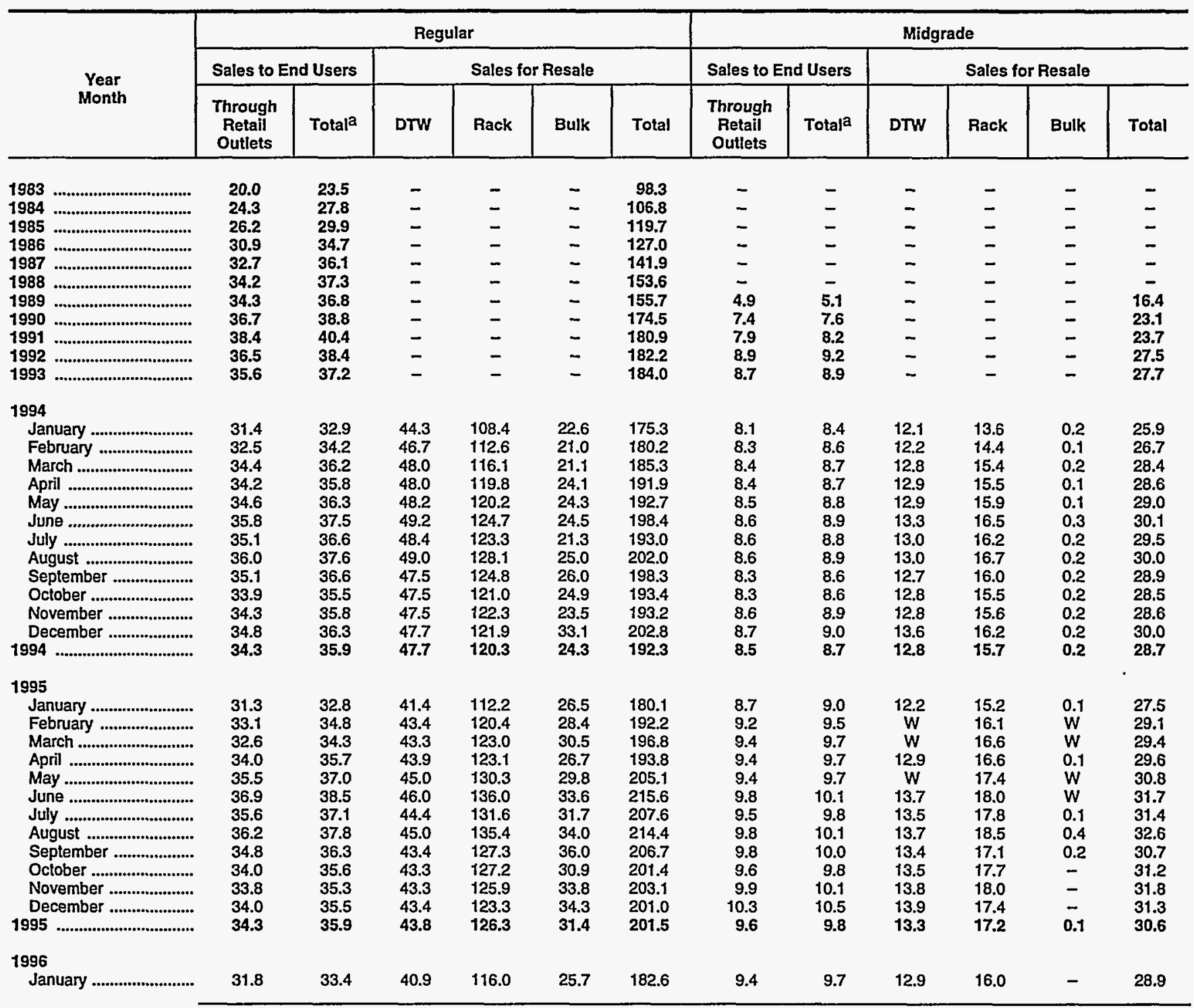

See footnotes at end of table. 
Table 7. U.S. Refiner Motor Gasoline Volumes by Grade and Sales Type (Million Gallons per Day) — Continued

\begin{tabular}{|c|c|c|c|c|c|c|c|c|c|c|c|c|}
\hline \multirow{3}{*}{$\begin{array}{l}\text { Year } \\
\text { Month }\end{array}$} & \multicolumn{6}{|c|}{ Premium } & \multicolumn{6}{|c|}{ All Grades } \\
\hline & \multicolumn{2}{|c|}{ Sales to End Users } & \multicolumn{4}{|c|}{ Sales for Resale } & \multicolumn{2}{|c|}{ Sales to End Users } & \multicolumn{4}{|c|}{ Sales for Resale } \\
\hline & $\begin{array}{c}\text { Through } \\
\text { Retail } \\
\text { Outlets }\end{array}$ & Totala & DTW & Rack & Bulk & Total & $\begin{array}{c}\text { Through } \\
\text { Retail } \\
\text { Outlets }\end{array}$ & Totala & DTW & Rack & Bulk & Total \\
\hline 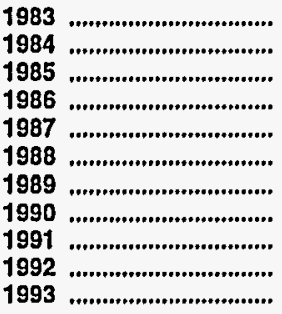 & $\begin{array}{r}5.0 \\
8.5 \\
9.2 \\
9.0 \\
11.2 \\
13.8 \\
13.5 \\
11.2 \\
10.5 \\
10.3 \\
10.3\end{array}$ & $\begin{array}{r}6.2 \\
9.2 \\
10.0 \\
9.8 \\
11.6 \\
14.5 \\
14.2 \\
11.7 \\
10.9 \\
10.7 \\
10.6\end{array}$ & $\begin{array}{l}\bar{z} \\
\bar{z} \\
\bar{z} \\
\bar{z} \\
\bar{z} \\
\overline{-}\end{array}$ & $\begin{array}{l}\bar{z} \\
\bar{z} \\
\bar{z} \\
\bar{z} \\
\bar{z} \\
\bar{z}\end{array}$ & $\begin{array}{l}\bar{z} \\
\bar{z} \\
\bar{z} \\
\bar{z} \\
\bar{z}\end{array}$ & $\begin{array}{l}28.7 \\
33.7 \\
38.0 \\
45.1 \\
53.0 \\
62.4 \\
60.8 \\
53.7 \\
48.3 \\
51.7 \\
52.8\end{array}$ & $\begin{array}{l}42.3 \\
50.3 \\
50.5 \\
54.2 \\
55.7 \\
56.7 \\
57.5 \\
57.4 \\
58.4 \\
56.4 \\
54.9\end{array}$ & $\begin{array}{l}51.1 \\
57.6 \\
57.5 \\
61.2 \\
61.0 \\
61.0 \\
61.2 \\
60.3 \\
61.2 \\
59.0 \\
57.2\end{array}$ & $\begin{array}{l}- \\
- \\
- \\
- \\
- \\
- \\
- \\
- \\
-\end{array}$ & $\begin{array}{l}- \\
\overline{-} \\
- \\
- \\
- \\
- \\
- \\
- \\
-\end{array}$ & $\begin{array}{l}- \\
- \\
- \\
- \\
- \\
- \\
- \\
- \\
-\end{array}$ & $\begin{array}{l}242.5 \\
246.3 \\
256.9 \\
257.2 \\
257.2 \\
263.7 \\
260.7 \\
264.8 \\
261.3 \\
265.4 \\
266.9\end{array}$ \\
\hline 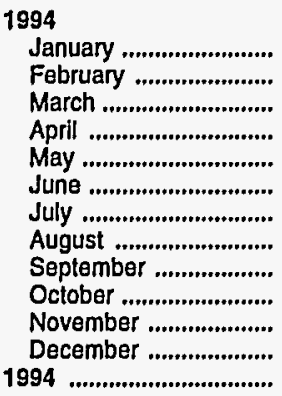 & $\begin{array}{r}9.9 \\
11.1 \\
10.1 \\
10.1 \\
10.1 \\
10.4 \\
10.1 \\
9.5 \\
9.4 \\
9.7 \\
9.4 \\
9.9 \\
10.0\end{array}$ & $\begin{array}{r}10.2 \\
11.5 \\
10.5 \\
10.5 \\
10.5 \\
10.8 \\
10.4 \\
9.9 \\
9.8 \\
10.1 \\
9.8 \\
10.3 \\
10.3\end{array}$ & $\begin{array}{l}22.1 \\
22.2 \\
23.2 \\
23.3 \\
23.4 \\
23.6 \\
22.8 \\
22.3 \\
22.0 \\
22.2 \\
21.8 \\
22.4 \\
22.6\end{array}$ & $\begin{array}{l}27.5 \\
28.4 \\
29.4 \\
29.8 \\
31.0 \\
31.8 \\
30.9 \\
30.6 \\
29.8 \\
29.4 \\
29.7 \\
30.8 \\
29.9\end{array}$ & $\begin{array}{l}3.7 \\
3.6 \\
2.6 \\
2.6 \\
3.4 \\
3.8 \\
3.0 \\
3.5 \\
3.0 \\
2.7 \\
2.6 \\
4.0 \\
3.2\end{array}$ & $\begin{array}{l}53.3 \\
54.2 \\
55.3 \\
55.7 \\
57.7 \\
59.2 \\
56.7 \\
56.4 \\
54.8 \\
54.2 \\
54.1 \\
57.2 \\
55.7\end{array}$ & $\begin{array}{l}49.4 \\
51.9 \\
52.9 \\
52.7 \\
53.2 \\
54.8 \\
53.7 \\
54.1 \\
52.8 \\
52.0 \\
52.2 \\
53.4 \\
52.8\end{array}$ & $\begin{array}{l}51.5 \\
54.3 \\
55.4 \\
55.0 \\
55.5 \\
57.2 \\
55.8 \\
56.4 \\
55.0 \\
54.2 \\
54.5 \\
55.6 \\
55.0\end{array}$ & $\begin{array}{l}78.4 \\
81.0 \\
84.1 \\
84.2 \\
84.5 \\
86.0 \\
84.2 \\
84.3 \\
82.2 \\
82.5 \\
82.2 \\
83.7 \\
83.1\end{array}$ & $\begin{array}{l}149.5 \\
155.5 \\
160.9 \\
165.1 \\
167.1 \\
173.0 \\
170.4 \\
175.4 \\
170.6 \\
165.9 \\
167.5 \\
168.9 \\
165.9\end{array}$ & $\begin{array}{l}26.5 \\
24.7 \\
24.0 \\
26.8 \\
27.8 \\
28.7 \\
24.5 \\
28.7 \\
29.2 \\
27.7 \\
26.2 \\
37.4 \\
27.7\end{array}$ & $\begin{array}{l}254.5 \\
261.2 \\
269.0 \\
276.2 \\
279.4 \\
287.7 \\
279.2 \\
288.4 \\
282.0 \\
276.1 \\
275.9 \\
290.0 \\
276.7\end{array}$ \\
\hline 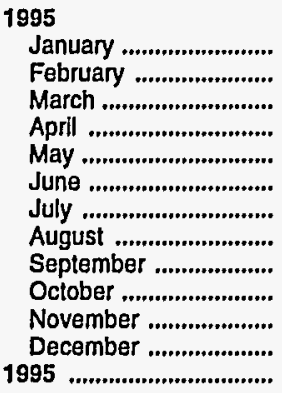 & $\begin{array}{r}9.0 \\
9.5 \\
11.2 \\
9.8 \\
9.2 \\
9.6 \\
9.7 \\
10.1 \\
9.9 \\
9.8 \\
10.0 \\
10.0 \\
9.8\end{array}$ & $\begin{array}{r}9.4 \\
9.8 \\
11.6 \\
10.1 \\
9.5 \\
10.0 \\
10.1 \\
10.4 \\
10.2 \\
10.1 \\
10.4 \\
10.4 \\
10.2\end{array}$ & $\begin{array}{c}19.9 \\
W \\
W \\
20.8 \\
W \\
20.5 \\
20.2 \\
21.0 \\
20.9 \\
21.0 \\
21.6 \\
21.4 \\
20.8\end{array}$ & $\begin{array}{l}28.9 \\
30.4 \\
31.6 \\
31.2 \\
31.1 \\
31.6 \\
31.3 \\
33.2 \\
31.3 \\
31.9 \\
32.6 \\
32.6 \\
31.5\end{array}$ & $\begin{array}{l}3.2 \\
W \\
W \\
3.3 \\
W \\
3.9 \\
3.9 \\
4.4 \\
5.3 \\
4.5 \\
5.1 \\
5.6 \\
4.1\end{array}$ & $\begin{array}{l}\mathbf{5 2 . 0} \\
54.9 \\
55.6 \\
55.3 \\
54.9 \\
56.0 \\
55.5 \\
58.6 \\
57.4 \\
57.4 \\
59.3 \\
59.5 \\
56.4\end{array}$ & $\begin{array}{l}49.1 \\
51.7 \\
53.2 \\
53.2 \\
54.1 \\
56.3 \\
54.9 \\
56.1 \\
54.5 \\
53.4 \\
53.7 \\
54.3 \\
53.7\end{array}$ & $\begin{array}{l}51.2 \\
54.1 \\
55.6 \\
55.5 \\
56.2 \\
58.5 \\
56.9 \\
58.3 \\
56.6 \\
55.6 \\
55.9 \\
56.4 \\
55.9\end{array}$ & $\begin{array}{l}73.5 \\
77.3 \\
76.7 \\
77.7 \\
78.7 \\
80.2 \\
78.1 \\
79.7 \\
77.7 \\
77.8 \\
78.6 \\
78.7 \\
77.9\end{array}$ & $\begin{array}{l}156.4 \\
166.8 \\
171.1 \\
170.9 \\
178.8 \\
185.5 \\
180.7 \\
187.1 \\
175.7 \\
176.7 \\
176.6 \\
173.2 \\
175.0\end{array}$ & $\begin{array}{l}29.8 \\
32.1 \\
34.0 \\
30.1 \\
33.2 \\
37.6 \\
35.7 \\
38.8 \\
41.4 \\
35.4 \\
38.9 \\
39.9 \\
35.6\end{array}$ & $\begin{array}{l}259.7 \\
276.2 \\
281.8 \\
278.6 \\
290.7 \\
303.3 \\
294.5 \\
305.6 \\
294.8 \\
290.0 \\
294.2 \\
291.8 \\
288.5\end{array}$ \\
\hline $\begin{array}{l}1996 \\
\text { January ......................... }\end{array}$ & 9.1 & 9.4 & 19.5 & 29.5 & 4.0 & 52.9 & 50.3 & 52.4 & 73.3 & 161.4 & 29.7 & 264.5 \\
\hline
\end{tabular}

Dash $(-)=$ No data reported.

$W=$ Withheld to avold disclosure of individual company data.

Notes: Data for the 4th quarter of 1993 were derived from two separate survey systems. The DTW, Rack, and Bulk components were derived from the revised EIA-782 survey system, while the End-Use and Average Resale categories were derived from the predecessor ElA-782 survey system. Therefore, the DTW, Rack, and Bulk components are not consistent with the Average Resale category. Beginning January 1994, all data are from the revised ElA-782 survey system and are consistent.

Noles: Motor gasoline averages and totals prior to October 1993 include leaded gasoline.

Notes: Values shown for the current month are preliminary. Values shown for previous months are revised. Data are final upon publication in the Petroleum Marketing Annual. Totals may not equal the sum of the components due to rounding.

a includes sales through retail outlets as well as all direct sales to end users that were not made through company-operated retail outlets, e.g., sales to agricultural customers, commercial sales, and industrial sales.

Source: Energy Information Administration Form ElA-782A, "Refiners'/Gas Plant Operators' Monthly Petroleum Product Sales Report." 
Table 8. U.S. Refiner Conventional Motor Gasoline Prices by Grade and Sales Type (Cents per Gallon Excluding Taxes)

\begin{tabular}{|c|c|c|c|c|c|c|c|c|c|c|c|c|}
\hline \multirow{3}{*}{$\begin{array}{c}\text { Year } \\
\text { Month }\end{array}$} & \multicolumn{6}{|c|}{ Regular } & \multicolumn{6}{|c|}{ Midgrade } \\
\hline & \multicolumn{2}{|c|}{ Sales to End Users } & \multicolumn{4}{|c|}{ Sales for Resale } & \multicolumn{2}{|c|}{ Sales to End Users } & \multicolumn{4}{|c|}{ Sales for Resale } \\
\hline & $\begin{array}{c}\text { Through } \\
\text { Retail } \\
\text { Outlets }\end{array}$ & Average $^{a}$ & DTW & Rack & Bulk & Average & $\begin{array}{c}\text { Through } \\
\text { Retail } \\
\text { Outlets }\end{array}$ & Average $^{a}$ & DTW & Rack & Bulk & Average \\
\hline \multicolumn{13}{|l|}{1994} \\
\hline January ........................... & 59.4 & 58.8 & 54.2 & 46.0 & 41.8 & 46.6 & 69.8 & 69.2 & 60.4 & 49.8 & NA & 53.6 \\
\hline February ........................ & 61.7 & 61.1 & 57.0 & 49.2 & 45.0 & 50.0 & 71.5 & 70.9 & 62.9 & 53.3 & NA & 56.9 \\
\hline 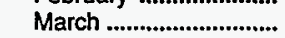 & 62.2 & 61.7 & 57.4 & 49.9 & 46.2 & 51.3 & 72.0 & 71.4 & 63.3 & 54.0 & NA & 58.0 \\
\hline 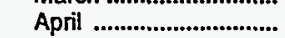 & 64.9 & 64.5 & 60.1 & 53.5 & 49.3 & 54.5 & 74.6 & 74.1 & 66.0 & 57.6 & NA & 61.2 \\
\hline 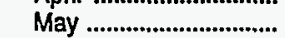 & 66.6 & 66.2 & 62.0 & 54.7 & 50.3 & 56.0 & 76.4 & 75.9 & 67.7 & 58.4 & $\mathrm{NA}$ & 62.6 \\
\hline 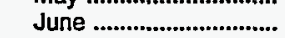 & 69.7 & 69.2 & 65.3 & 58.2 & 53.1 & 59.3 & 79.5 & 78.9 & 71.2 & 61.8 & NA & 65.9 \\
\hline 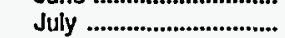 & 72.6 & 72.2 & 68.0 & 61.0 & 56.0 & 62.2 & 82.3 & 81.8 & 73.8 & 63.9 & NA & 68.3 \\
\hline 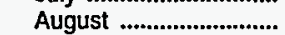 & 77.4 & 76.9 & 71.2 & 63.6 & 57.9 & 64.7 & 86.9 & 86.3 & 77.0 & 66.4 & NA & 71.0 \\
\hline September .................... & 75.5 & 74.8 & 68.6 & 54.6 & 51.6 & 57.4 & 85.2 & 84.4 & 74.7 & 57.1 & NA & 64.6 \\
\hline 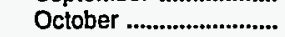 & 71.1 & 70.3 & 64.6 & 54.4 & 50.1 & 55.5 & 81.4 & 80.6 & 70.4 & 58.2 & $w$ & 62.6 \\
\hline November ..................... & 70.6 & 70.0 & 64.2 & 54.7 & 50.5 & 55.5 & 80.6 & 79.9 & 70.7 & 59.3 & $w$ & 63.0 \\
\hline December ....................... & 66.6 & 65.8 & 58.3 & 48.8 & 46.4 & 49.4 & 76.6 & 75.8 & 65.7 & 52.9 & NA & 56.3 \\
\hline 1994 & 68.7 & 68.1 & 63.6 & 54.5 & 50.0 & 55.8 & 78.4 & 77.8 & 69.4 & NA & NA & 62.7 \\
\hline \multicolumn{13}{|l|}{1995} \\
\hline 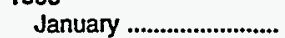 & 65.7 & 65.0 & 59.5 & 52.3 & 48.3 & 52.4 & 75.7 & 75.0 & 66.3 & 56.8 & $w$ & 59.2 \\
\hline 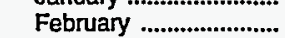 & 65.7 & 65.0 & 60.4 & 53.4 & 49.6 & 53.6 & 75.3 & 74.7 & 67.4 & 57.9 & $w$ & 60.6 \\
\hline March .............................. & 66.8 & 66.3 & 60.9 & 54.3 & 50.7 & 54.6 & 76.1 & 75.4 & 67.4 & 58.1 & $w$ & 60.7 \\
\hline 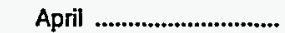 & 72.2 & 71.7 & 66.3 & 62.1 & 57.2 & 62.0 & 81.2 & 80.7 & 72.9 & 66.6 & w & 68.4 \\
\hline May .............................. & 78.8 & 78.3 & 72.5 & 67.6 & 62.1 & 67.4 & 88.0 & 87.4 & 79.2 & 72.4 & $w$ & 74.3 \\
\hline 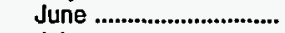 & 79.3 & 78.7 & 72.5 & 62.3 & 58.5 & 63.1 & 88.4 & 87.7 & 79.5 & 66.7 & $w$ & 70.3 \\
\hline 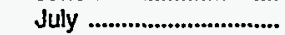 & 74.9 & 74.2 & 68.4 & 56.3 & 52.7 & 57.4 & 84.0 & 83.3 & 74.7 & 60.0 & $w$ & 64.2 \\
\hline 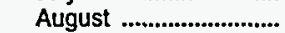 & 71.9 & 71.3 & 65.5 & 56.8 & 52.1 & 57.3 & 80.8 & 80.2 & 71.1 & 60.6 & NA & 63.4 \\
\hline September ..................... & 71.1 & 70.6 & 65.1 & 57.6 & 52.5 & 57.7 & 79.8 & 79.3 & 70.5 & 61.2 & NA & 63.9 \\
\hline 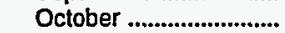 & 67.4 & 66.8 & 60.7 & 52.2 & 49.1 & 52.6 & 77.0 & 76.3 & 66.7 & 56.1 & - & 58.6 \\
\hline November ...................... & 64.6 & 64.0 & 58.3 & 52.1 & 47.8 & 51.9 & 74.0 & 73.4 & 64.0 & 55.9 & - & 57.7 \\
\hline December ....................... & 66.5 & 65.9 & 60.4 & 54.1 & 49.4 & 53.7 & 75.7 & 75.1 & 66.2 & 58.1 & - & 60.0 \\
\hline 1995 & 71.0 & 70.4 & 65.1 & 57.0 & 52.6 & 57.3 & 80.0 & 79.4 & 71.1 & 61.0 & NA & 63.7 \\
\hline \multicolumn{13}{|l|}{1996} \\
\hline 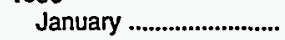 & 68.3 & 67.6 & 61.8 & 54.9 & 51.4 & 55.0 & 77.4 & 76.8 & 68.0 & 59.1 & - & 61.2 \\
\hline
\end{tabular}

See footnotes at end of table. 
Table 8. U.S. Refiner Conventional Motor Gasoline Prices by Grade and Sales Type

(Cents per Gallon Excluding Taxes) - Continued

\begin{tabular}{|c|c|c|c|c|c|c|c|c|c|c|c|c|}
\hline \multirow{3}{*}{$\begin{array}{l}\text { Year } \\
\text { Month }\end{array}$} & \multicolumn{6}{|c|}{ Premium } & \multicolumn{6}{|c|}{ All Grades } \\
\hline & \multicolumn{2}{|c|}{ Sales to End Users } & \multicolumn{4}{|c|}{ Sales for Resale } & \multicolumn{2}{|c|}{ Sales to End Users } & \multicolumn{4}{|c|}{ Sales for Resale } \\
\hline & $\begin{array}{c}\text { Through } \\
\text { Retail } \\
\text { Outlets }\end{array}$ & Average ${ }^{a}$ & DTW & Rack & Bulk & Average & $\begin{array}{c}\text { Through } \\
\text { Retail } \\
\text { Outlets }\end{array}$ & Average $^{\mathrm{a}}$ & DTW & Rack & Bulk & Average \\
\hline \multicolumn{13}{|l|}{1994} \\
\hline 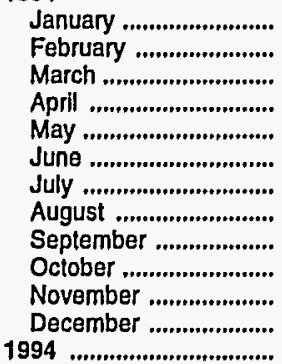 & $\begin{array}{l}78.1 \\
77.3 \\
80.4 \\
83.1 \\
84.9 \\
87.5 \\
90.4 \\
95.1 \\
93.5 \\
89.3 \\
88.6 \\
84.5 \\
86.3\end{array}$ & $\begin{array}{l}77.3 \\
76.7 \\
79.6 \\
82.3 \\
84.1 \\
86.8 \\
89.7 \\
94.3 \\
92.4 \\
88.3 \\
87.6 \\
83.4 \\
85.5\end{array}$ & $\begin{array}{l}68.5 \\
70.8 \\
71.4 \\
74.1 \\
75.8 \\
79.4 \\
82.1 \\
85.1 \\
83.1 \\
79.0 \\
78.8 \\
74.0 \\
77.8\end{array}$ & $\begin{array}{l}55.2 \\
58.4 \\
59.2 \\
62.8 \\
64.0 \\
67.6 \\
70.2 \\
72.9 \\
63.5 \\
64.2 \\
64.6 \\
58.4 \\
63.8\end{array}$ & $\begin{array}{l}46.9 \\
49.3 \\
50.6 \\
55.5 \\
56.9 \\
59.8 \\
62.8 \\
64.2 \\
55.5 \\
54.0 \\
54.7 \\
49.8 \\
55.5\end{array}$ & $\begin{array}{l}58.1 \\
61.3 \\
63.3 \\
66.7 \\
68.3 \\
71.7 \\
74.5 \\
77.1 \\
70.6 \\
68.0 \\
67.7 \\
61.1 \\
68.2\end{array}$ & $\begin{array}{l}65.0 \\
66.8 \\
67.1 \\
69.9 \\
71.6 \\
74.6 \\
77.5 \\
82.0 \\
80.2 \\
76.3 \\
75.5 \\
71.6 \\
73.6\end{array}$ & $\begin{array}{l}64.3 \\
66.2 \\
66.5 \\
69.3 \\
71.1 \\
74.0 \\
76.9 \\
81.4 \\
79.4 \\
75.4 \\
74.8 \\
70.7 \\
72.9\end{array}$ & $\begin{array}{l}59.3 \\
61.7 \\
61.9 \\
64.6 \\
66.6 \\
70.0 \\
72.7 \\
75.7 \\
73.3 \\
69.2 \\
69.0 \\
63.6 \\
68.3\end{array}$ & $\begin{array}{l}48.0 \\
51.2 \\
52.0 \\
55.5 \\
56.8 \\
60.3 \\
63.0 \\
65.5 \\
56.4 \\
56.5 \\
56.8 \\
50.9 \\
56.5\end{array}$ & $\begin{array}{l}42.5 \\
45.6 \\
46.7 \\
49.9 \\
51.2 \\
54.1 \\
56.9 \\
58.7 \\
52.0 \\
50.5 \\
50.9 \\
46.7 \\
50.7\end{array}$ & $\begin{array}{l}49.5 \\
52.9 \\
54.3 \\
57.5 \\
59.2 \\
62.6 \\
65.3 \\
67.8 \\
60.7 \\
58.5 \\
58.4 \\
52.0 \\
58.9\end{array}$ \\
\hline \multicolumn{13}{|l|}{1995} \\
\hline 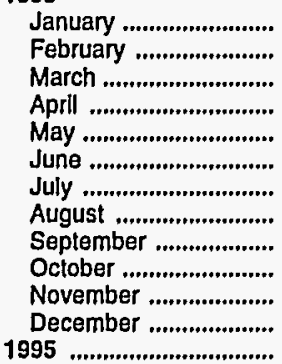 & $\begin{array}{l}84.0 \\
83.5 \\
81.7 \\
89.2 \\
96.3 \\
96.3 \\
91.9 \\
89.0 \\
88.2 \\
84.8 \\
81.7 \\
83.4 \\
87.7\end{array}$ & $\begin{array}{l}83.0 \\
82.6 \\
81.0 \\
88.5 \\
95.5 \\
95.4 \\
91.0 \\
88.2 \\
87.4 \\
84.0 \\
80.7 \\
82.6 \\
86.8\end{array}$ & $\begin{array}{l}74.5 \\
75.2 \\
75.6 \\
80.7 \\
87.2 \\
87.5 \\
83.0 \\
79.4 \\
78.7 \\
74.2 \\
71.5 \\
73.6 \\
79.0\end{array}$ & $\begin{array}{l}62.1 \\
63.2 \\
63.6 \\
71.9 \\
77.8 \\
72.4 \\
66.0 \\
66.5 \\
67.0 \\
61.7 \\
61.3 \\
63.5 \\
66.5\end{array}$ & $\begin{array}{l}51.7 \\
53.8 \\
53.7 \\
62.8 \\
66.2 \\
62.7 \\
56.9 \\
57.2 \\
56.6 \\
52.0 \\
51.9 \\
55.0 \\
56.8\end{array}$ & $\begin{array}{l}63.9 \\
65.2 \\
65.8 \\
73.4 \\
79.3 \\
75.0 \\
69.1 \\
68.7 \\
68.6 \\
63.2 \\
62.4 \\
64.3 \\
68.4\end{array}$ & $\begin{array}{l}70.7 \\
70.4 \\
71.3 \\
76.5 \\
83.0 \\
83.4 \\
79.1 \\
76.2 \\
75.4 \\
72.0 \\
69.2 \\
71.1 \\
75.3\end{array}$ & $\begin{array}{l}69.9 \\
69.7 \\
70.7 \\
76.0 \\
82.5 \\
82.7 \\
78.4 \\
75.5 \\
74.8 \\
71.3 \\
68.5 \\
70.4 \\
74.7\end{array}$ & $\begin{array}{l}64.5 \\
65.2 \\
65.5 \\
70.7 \\
76.8 \\
76.9 \\
72.7 \\
69.6 \\
69.1 \\
65.0 \\
62.6 \\
64.7 \\
69.3\end{array}$ & $\begin{array}{l}54.5 \\
55.5 \\
56.3 \\
64.3 \\
69.8 \\
64.4 \\
58.3 \\
58.8 \\
59.5 \\
54.3 \\
54.1 \\
56.2 \\
59.0\end{array}$ & $\begin{array}{l}48.6 \\
50.0 \\
50.9 \\
57.8 \\
62.5 \\
58.9 \\
53.1 \\
52.6 \\
53.0 \\
49.4 \\
48.2 \\
50.1 \\
53.0\end{array}$ & $\begin{array}{l}55.1 \\
56.4 \\
57.2 \\
64.6 \\
70.1 \\
65.7 \\
60.0 \\
59.8 \\
60.1 \\
55.0 \\
54.3 \\
56.2 \\
59.8\end{array}$ \\
\hline \multicolumn{13}{|l|}{1996} \\
\hline January .......................... & 85.3 & 84.3 & 75.3 & 64.5 & 55.9 & 65.6 & 72.7 & 72.0 & 66.2 & 57.0 & 52.0 & 57.5 \\
\hline
\end{tabular}

$\operatorname{Dash}(-)=$ No data reported.

NA $=$ Not avallable.

$W=$ Withheld to avoid disclosure of individual company data.

a Includes sales through retail outlets as well as all direct sales to end users that were not made through company-operated retail outlets, e.g., sales to agricultural customers, commercial sales, and industrial sales.

Notes: The 4th quarter of 1993 was a transitional period between the predecessor EIA-782 survey system and the revised EIA-782 survey sytem. The revised survey system contains additional product and sales categories, which may not be consistent with categories derived from the predecessor survey system. Beginning January 1994 all data are from the revised survey system and are consistent.

Notes: Values shown for the current month are preliminary. Values shown for previous months are revised. Data are final upon publication in the Petroleum Marketing Annual.

Source: Energy Information Administration Form EIA-782A, "Refiners'/Gas Plant Operators' Monthly Petroleum Product Sales Report." 
Table 9. U.S. Refiner Conventional Motor Gasoline Volumes by Grade and Sales Type (Million Gallons per Day)

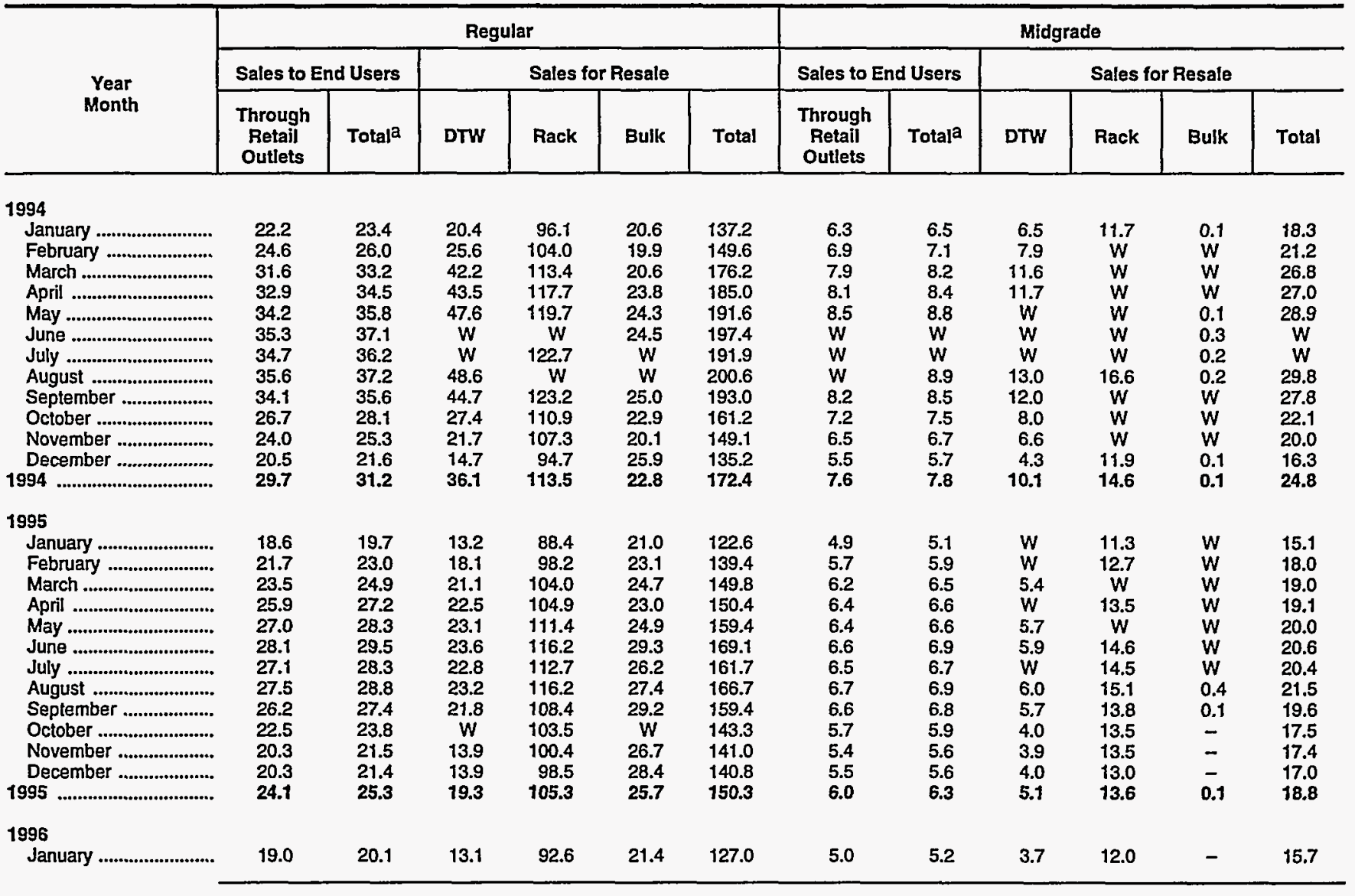

See footnotes at end of table. 
Table 9. U.S. Refiner Conventional Motor Gasoline Volumes by Grade and Sales Type (Million Gallons per Day) - Continued

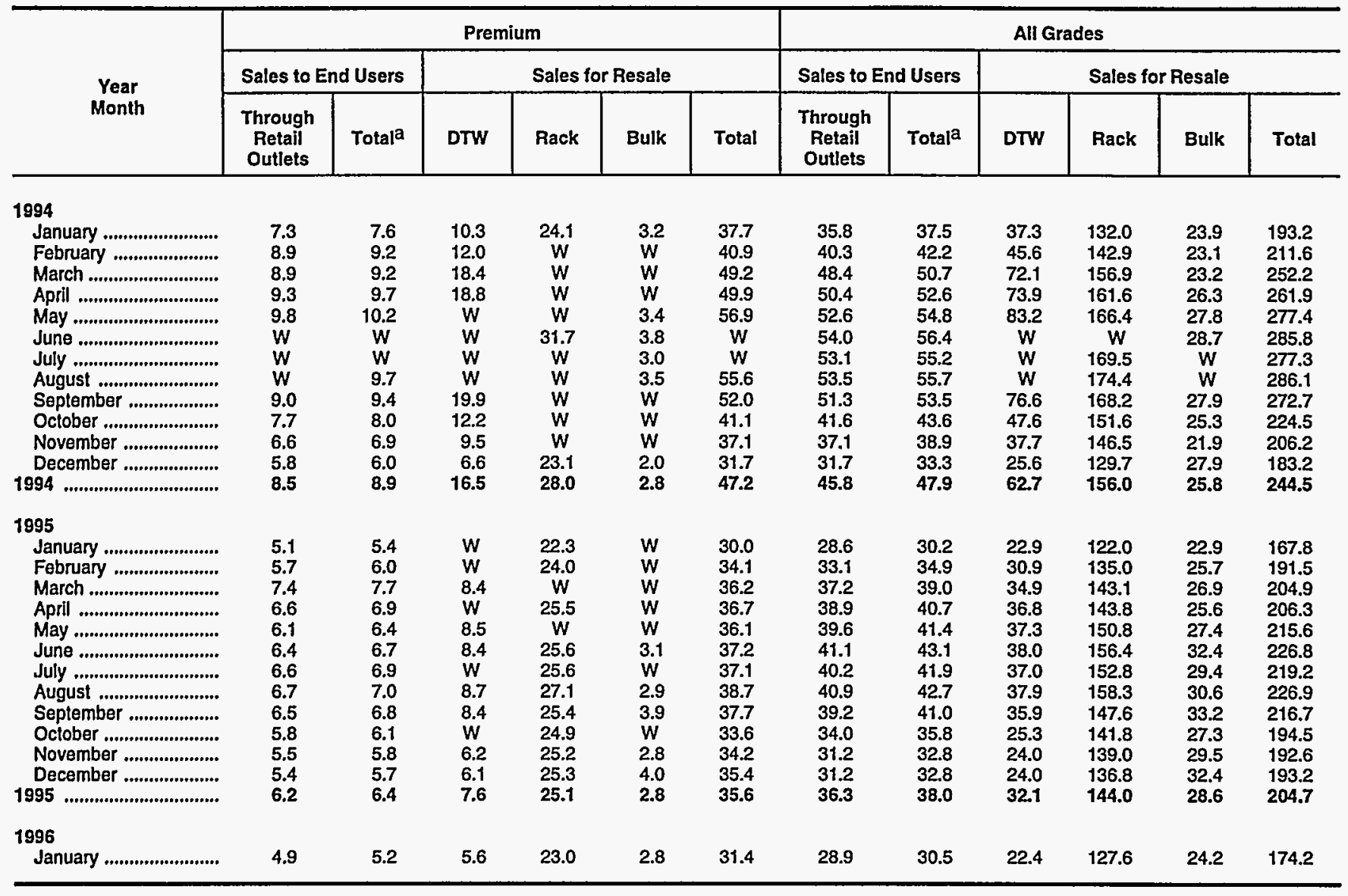

$\operatorname{Dash}(-)=$ No data reported.

$W=$ Withheld to avold disclosure of individual company data.

a Includes sales through retail outlets as well as all direct sales to end users that were not made through company-operated retail outlets, e.g., sales to agricultural customers, commercial sales, and industrial sales.

Notes: The 4th quarter of 1993 was a transitional period between the predecessor EIA-782 survey system and the revised ElA-782 survey sytem. The revised survey system contains additional product and sales calegories, which may not be consistent with categories derived from the predecessor survey system. Beginning January 1994 all data are from the revised survey system and are consistent.

Notes: Values shown for the current month are preliminary. Values shown for previous months are revised. Data are final upon publication in the Petroleum Markeling Annual. Totals may not equal the sum of the components due to rounding.

Source: Energy Information Administration Form EIA-782A, "Refiners'/Gas Plant Operators' Monthly Petroleum Product Sales Report." 
Table 10. U.S. Refiner Oxygenated Motor Gasoline Prices by Grade and Sales Type (Cents per Gallon Excluding Taxes)

\begin{tabular}{|c|c|c|c|c|c|c|c|c|c|c|c|c|}
\hline \multirow{3}{*}{$\begin{array}{c}\text { Year } \\
\text { Month }\end{array}$} & \multicolumn{6}{|c|}{ Regular } & \multicolumn{6}{|c|}{ Midgrade } \\
\hline & \multicolumn{2}{|c|}{ Sales to End Users } & \multicolumn{4}{|c|}{ Sales for Resale } & \multicolumn{2}{|c|}{ Sales to End Users } & \multicolumn{4}{|c|}{ Sales for Resale } \\
\hline & $\begin{array}{c}\text { Through } \\
\text { Retail } \\
\text { Outlets }\end{array}$ & Average ${ }^{a}$ & DTW & Rack & Bulk & Average & $\begin{array}{c}\text { Through } \\
\text { Retail } \\
\text { Outlets }\end{array}$ & Averagea & DTW & Rack & Bulk & Average \\
\hline \multicolumn{13}{|l|}{1994} \\
\hline 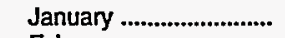 & 69.0 & 68.4 & 60.0 & 51.0 & 45.4 & 56.3 & 78.0 & 77.3 & 65.7 & 54.8 & $w$ & 62.7 \\
\hline February ......................... & 68.3 & 67.8 & 60.1 & 53.2 & 48.7 & 57.8 & 78.1 & 77.4 & 65.9 & 56.8 & W & 63.7 \\
\hline 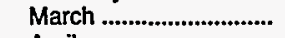 & 71.0 & 70.2 & 62.6 & 54.1 & 46.9 & 59.1 & 80.4 & 79.5 & 65.7 & 57.1 & w & 63.8 \\
\hline 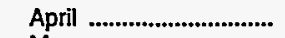 & 66.5 & 66.0 & 60.8 & 54.4 & 49.6 & 58.3 & 80.1 & 79.6 & 66.5 & 57.9 & W & 64.7 \\
\hline 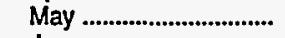 & 70.6 & 70.5 & 61.9 & 61.1 & - & 61.6 & 82.5 & 82.5 & $W$ & $w$ & - & 58.8 \\
\hline 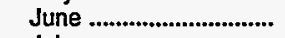 & 71.9 & 71.9 & $W$ & 64.9 & - & 63.8 & $W$ & $W$ & $W$ & $w$ & - & $w$ \\
\hline July & 75.1 & 75.1 & $W$ & 70.0 & $w$ & 67.4 & $w$ & $w$ & $w$ & $w$ & - & $w$ \\
\hline 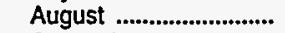 & 81.9 & 81.8 & 67.6 & 75.7 & $w$ & 70.0 & $w$ & 98.1 & - & 69.4 & - & 69.4 \\
\hline September ...................... & 80.9 & 80.5 & 74.1 & 65.4 & 54.6 & 67.8 & 94.1 & 93.5 & 77.1 & 62.1 & $w$ & 71.6 \\
\hline 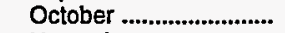 & 78.7 & 78.3 & 73.5 & 63.7 & 57.8 & 69.5 & 89.5 & 89.2 & 79.9 & 68.4 & $w$ & 77.0 \\
\hline November ....................... & 79.6 & 79.2 & 73.4 & 63.8 & 59.4 & 69.4 & 90.4 & 90.1 & 79.7 & 69.2 & $w$ & 76.6 \\
\hline December ...................... & 77.1 & 76.9 & 70.1 & 58.5 & W & 63.7 & 87.2 & 86.9 & 75.0 & 62.2 & $W$ & 69.3 \\
\hline 1994 & 74.3 & 73.9 & 66.7 & 58.6 & 52.7 & 63.2 & 84.3 & 83.8 & 72.7 & 62.2 & $w$ & 69.6 \\
\hline \multicolumn{13}{|l|}{1995} \\
\hline 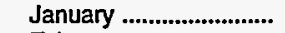 & 76.6 & 76.3 & 69.4 & 60.6 & 56.1 & 64.7 & 85.7 & 85.6 & 74.4 & 64.0 & $w$ & 70.0 \\
\hline 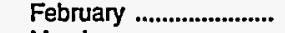 & 76.2 & 76.1 & 69.9 & 62.8 & 55.2 & 66.0 & 86.3 & 86.2 & 73.4 & 65.3 & - & 69.3 \\
\hline 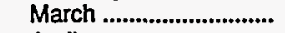 & $\pi .2$ & $\pi .1$ & 72.4 & 65.1 & - & 68.9 & 85.6 & 85.5 & 75.1 & 66.1 & - & 70.1 \\
\hline April & 83.4 & 83.4 & 76.7 & 67.9 & - & 71.5 & 91.1 & 90.9 & $w$ & 69.7 & - & 73.8 \\
\hline May & 88.9 & 88.8 & 80.4 & 71.7 & - & 75.2 & 95.3 & 95.1 & $w$ & 73.5 & - & 77.7 \\
\hline June .............................. & 90.7 & 90.7 & 80.4 & 66.5 & - & 72.0 & 97.6 & 97.4 & $W$ & 68.7 & - & 75.0 \\
\hline July & 88.1 & 88.1 & 77.9 & 62.6 & - & 68.5 & 92.4 & 92.4 & $\mathbf{w}$ & 63.9 & - & 69.7 \\
\hline 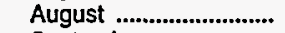 & 84.8 & 84.8 & 76.8 & 63.6 & - & 68.8 & 87.7 & 87.6 & $w$ & 65.4 & - & 70.1 \\
\hline September ........................ & 83.4 & 83.1 & 74.3 & 66.4 & 69.3 & 69.4 & 85.2 & 85.1 & 75.6 & 69.1 & $W$ & 70.7 \\
\hline 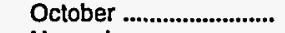 & 76.1 & 75.8 & 70.9 & 63.2 & W & 66.8 & 80.4 & 80.2 & 75.3 & 67.0 & - & 72.3 \\
\hline November ...................... & 74.9 & 74.7 & 69.9 & 62.4 & 53.6 & 65.3 & 82.8 & 82.7 & 75.0 & 65.5 & - & 71.2 \\
\hline December ......................... & 74.6 & 74.4 & 69.7 & 62.1 & 58.3 & 65.5 & 82.0 & 81.9 & 74.6 & 65.8 & - & 71.4 \\
\hline 1995 & 76.8 & 76.6 & 70.9 & 63.0 & 59.2 & 66.5 & 83.4 & 83.3 & 75.0 & 65.9 & W & 71.3 \\
\hline \multicolumn{13}{|l|}{1996} \\
\hline January ........................... & 75.8 & 75.5 & 70.4 & 61.6 & $w$ & 65.5 & 83.8 & 83.7 & 76.0 & 65.1 & - & 72.1 \\
\hline
\end{tabular}

See footnotes at end of table. 
Table 10. U.S. Refiner Oxygenated Motor Gasoline Prices by Grade and Sales Type (Cents per Gallon Excluding Taxes) - Continued

\begin{tabular}{|c|c|c|c|c|c|c|c|c|c|c|c|c|}
\hline \multirow{3}{*}{$\begin{array}{c}\text { Year } \\
\text { Month }\end{array}$} & \multicolumn{6}{|c|}{ Premium } & \multicolumn{6}{|c|}{ All Grades } \\
\hline & \multicolumn{2}{|c|}{ Sales to End Users } & \multicolumn{4}{|c|}{ Sales for Resale } & \multicolumn{2}{|c|}{ Sales to End Users } & \multicolumn{4}{|c|}{ Sales for Resale } \\
\hline & $\begin{array}{c}\text { Through } \\
\text { Retail } \\
\text { Outlets }\end{array}$ & Average $^{a}$ & DTW & Rack & Bulk & Average & $\begin{array}{c}\text { Through } \\
\text { Retail } \\
\text { Outlets }\end{array}$ & Averagea & DTW & Rack & Bulk & Average \\
\hline \multicolumn{13}{|l|}{1994} \\
\hline January ......................... & 88.4 & 87.8 & 74.0 & 60.1 & 50.6 & 70.2 & 74.0 & 73.3 & 64.7 & 53.1 & 46.4 & 60.7 \\
\hline 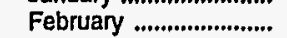 & 88.0 & 87.2 & 74.0 & 62.0 & 53.1 & 70.8 & 73.3 & 72.7 & 64.8 & 55.4 & 50.1 & 61.9 \\
\hline 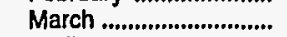 & 89.1 & 88.4 & 74.0 & 61.6 & 52.2 & 71.0 & 76.9 & 76.1 & 67.5 & 56.2 & 48.6 & 63.9 \\
\hline 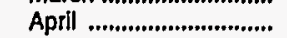 & 87.4 & 87.2 & 74.6 & 62.2 & 55.9 & 71.7 & 74.9 & 74.4 & 67.6 & 57.0 & 52.1 & 64.5 \\
\hline May ................................... & 87.9 & 87.6 & 72.9 & 63.7 & - & 71.1 & 77.0 & 76.9 & 67.8 & 61.2 & - & 65.4 \\
\hline 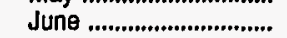 & 88.8 & 88.8 & $W$ & 68.2 & - & 73.2 & 78.8 & 78.8 & $w$ & 65.4 & - & 67.6 \\
\hline 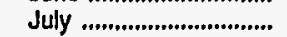 & 90.9 & 90.9 & W & 72.0 & - & 75.7 & 81.4 & 81.4 & $w$ & 70.0 & $w$ & 70.6 \\
\hline 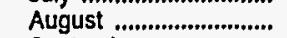 & 93.9 & 93.9 & $\ddot{W}$ & 76.1 & - & 78.7 & 86.4 & 86.3 & $w$ & 75.0 & $w$ & 72.9 \\
\hline September .................... & 97.1 & 96.8 & 85.7 & 73.0 & 60.0 & 81.3 & 86.4 & 86.0 & 78.9 & 66.3 & 55.8 & 72.3 \\
\hline 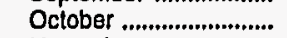 & 97.3 & 97.0 & 87.7 & 73.8 & 64.0 & 84.1 & 83.6 & 83.2 & 78.5 & 66.1 & 58.9 & 74.1 \\
\hline November ....................... & 99.2 & 98.7 & 87.8 & 74.9 & 66.1 & 84.4 & 84.5 & 84.1 & 78.2 & 66.3 & 60.0 & 73.8 \\
\hline December ...................... & 96.8 & 96.5 & 83.7 & 68.8 & $\underset{567}{W}$ & 77.8 & 81.5 & 81.2 & 73.5 & 60.4 & $W$ & 66.8 \\
\hline 1994 & 92.9 & 92.4 & 79.9 & 67.5 & 56.7 & 76.5 & 79.3 & 78.8 & 71.5 & 60.7 & 53.5 & 67.5 \\
\hline \multicolumn{13}{|l|}{1995} \\
\hline January ........................... & 95.9 & 95.7 & 83.6 & 70.6 & $w$ & 78.2 & 80.7 & 80.4 & 72.8 & 62.4 & 56.6 & 67.5 \\
\hline February ...................... & 95.1 & 95.0 & 84.0 & 71.9 & - & 78.6 & 80.3 & 80.2 & 72.7 & 64.4 & 55.2 & 68.3 \\
\hline 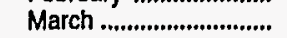 & 93,9 & 93.6 & 85.5 & 72.8 & - & 80.1 & 80.3 & 80.2 & 75.0 & 66.4 & - & 70.9 \\
\hline April ................................. & 100.7 & 100.5 & 93.0 & 74.7 & - & 81.4 & 86.3 & 86.2 & 79.1 & 69.1 & - & 73.2 \\
\hline 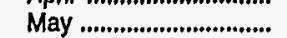 & 105.3 & 105.1 & 96.9 & 78.2 & - & 84.6 & 91.5 & 91.3 & 82.9 & 72.9 & - & 76.8 \\
\hline 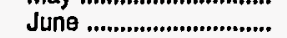 & 106.4 & 106.4 & 98.8 & 73.3 & - & 81.6 & 93.2 & 93.2 & 83.0 & 67.8 & - & 73.6 \\
\hline 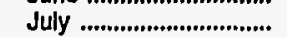 & 104.9 & 104.9 & 97.4 & 70.0 & - & 79.3 & 90.7 & 90.7 & 80.5 & 63.8 & - & 70.2 \\
\hline August ........................... & 102.2 & 102.2 & 96.2 & 70.8 & - & 79.3 & 87.5 & 87.5 & 79.5 & 64.9 & - & 70.5 \\
\hline September .................. & 100.1 & 99.6 & 87.8 & 73.9 & $w$ & 77.6 & 86.3 & 86.0 & 76.9 & 67.8 & 68.3 & 70.8 \\
\hline 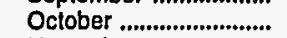 & 93.4 & 93.1 & 84.7 & 73.6 & $\ddot{w}$ & 80.3 & 79.5 & 79.2 & 74.5 & 65.2 & $W$ & 70.0 \\
\hline November .................... & 92.6 & 92.4 & 83.7 & 72.3 & 58.8 & 78.5 & 79.0 & 78.8 & 73.5 & 64.2 & 54.3 & 68.4 \\
\hline December ...................... & 93.3 & 93.0 & 83.3 & 72.2 & W & 78.7 & 78.9 & 78.6 & 73.3 & 64.0 & 59.0 & 68.6 \\
\hline 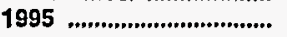 & 94.7 & 94.5 & 84.6 & 72.4 & $\mathbf{W}$ & 79.1 & 80.5 & 80.3 & 74.1 & 64.7 & 59.5 & 69.1 \\
\hline \multicolumn{13}{|l|}{1996} \\
\hline January ......................... & 94.8 & 94.5 & 84.4 & 71.7 & $w$ & 79.0 & 80.1 & 79.8 & 74.0 & 63.4 & W & 68.7 \\
\hline
\end{tabular}

Dash $(-)=$ No data reported.

$W=$ Withheld to avoid disclosure of individual company data.

a Includes sales through retail outlets as well as all direct sales to end users that were not made through company-operated retail outlets, e.g., sales to agricultural customers, commercial sales, and industrial sales.

Notes: The 4th quarter of 1993 was a transitional period between the predecessor EIA-782 survey system and the revised EIA-782 survey sytem. The revised survey system contains additlonal product and sales categories, which may not be consistent with categories derived from the predecessor survey system. Beginning January 1994 all data are from the revised survey system and are consistent.

Notes: Values shown for the current month are preliminary. Values shown for previous months are revised. Data are final upon publication in the Petroleum Markeling Annual.

Source: Energy Information Administration Form EIA-782A, "Refiners'/Gas Plant Operators' Monthly Petroleum Product Sales Report." 
Table 11. U.S. Refiner Oxygenated Motor Gasoline Volumes by Grade and Sales Type (Million Gallons per Day)

\begin{tabular}{|c|c|c|c|c|c|c|c|c|c|c|c|c|}
\hline \multirow{3}{*}{$\begin{array}{l}\text { Year } \\
\text { Month }\end{array}$} & \multicolumn{6}{|c|}{ Regular } & \multicolumn{6}{|c|}{ Midgrade } \\
\hline & \multicolumn{2}{|c|}{ Sales to End Users } & \multicolumn{4}{|c|}{ Sales for Resale } & \multicolumn{2}{|c|}{ Sales to End Users } & \multicolumn{4}{|c|}{ Sales for Resale } \\
\hline & $\begin{array}{c}\text { Through } \\
\text { Retail } \\
\text { Outlets }\end{array}$ & Totala & DTW & Rack & Bulk & Total & $\begin{array}{c}\text { Through } \\
\text { Retail } \\
\text { Outlets }\end{array}$ & Totala & DTW & Rack & Butk & Total \\
\hline \multicolumn{13}{|l|}{1994} \\
\hline January ........................... & 9.1 & 9.5 & 23.9 & 12.3 & 2.0 & 38.2 & 1.8 & 1.9 & 5.5 & 1.9 & $w$ & 7.5 \\
\hline February ......................... & 8.0 & 8.2 & 21.0 & 8.6 & 1.1 & 30.7 & 1.4 & 1.5 & 4.2 & $\dddot{w}$ & $\ddot{w}$ & 5.6 \\
\hline 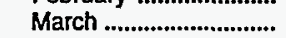 & 2.8 & 2.9 & 5.9 & 2.7 & 0.5 & 9.1 & 0.4 & 0.5 & 1.2 & $w$ & $w$ & 1.6 \\
\hline 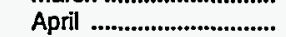 & 1.3 & 1.3 & 4.5 & 2.1 & 0.3 & 6.9 & 0.3 & 0.3 & 1.2 & $w$ & $w$ & 1.6 \\
\hline 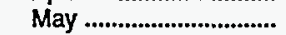 & 0.4 & 0.4 & 0.6 & 0.5 & - & 1.1 & 0.0 & 0.0 & $w$ & W & - & 0.1 \\
\hline June ................................ & 0.4 & 0.4 & $w$ & $W$ & - & 1.0 & $w$ & $w$ & W & $W$ & - & $w$ \\
\hline 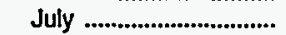 & 0.4 & 0.4 & W & 0.6 & $W$ & 1.0 & $w$ & $\ddot{W}$ & $\ddot{W}$ & $w$ & - & W \\
\hline 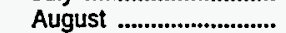 & 0.4 & 0.4 & 0.4 & $w$ & $W$ & 1.4 & $w$ & 0.0 & - & 0.1 & - & 0.1 \\
\hline 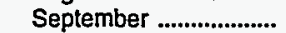 & 1.0 & 1.0 & 2.7 & 1.6 & 1.0 & 5.3 & 0.1 & 0.1 & 0.7 & $w$ & $w$ & 1.1 \\
\hline 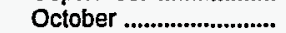 & 7.2 & 7.4 & 20.1 & 10.1 & 1.9 & 32.2 & 1.2 & 1.2 & 4.8 & $w$ & $w$ & 6.3 \\
\hline November ...................... & 9.9 & 10.2 & 24.1 & 14.2 & 2.0 & 40.2 & 1.9 & 2.0 & 5.8 & $w$ & W & 8.0 \\
\hline 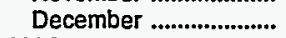 & 7.6 & 7.7 & 10.0 & $W$ & $W$ & 20.2 & 1.3 & 1.3 & 1.8 & $w$ & w & 3.1 \\
\hline 1994 & 4.0 & 4.1 & 9.4 & 5.2 & 0.9 & 15.5 & 0.7 & 0.7 & 2.1 & 0.8 & w & 2.9 \\
\hline \multicolumn{13}{|l|}{1995} \\
\hline January ......................... & 5.7 & 5.8 & 8.7 & 8.0 & 1.0 & 17.7 & 1.1 & 1.1 & $w$ & $w$ & $w$ & 2.6 \\
\hline February ......................... & 3.9 & 3.9 & 4.5 & 4.4 & 0.3 & 9.2 & 0.6 & 0.6 & $w$ & W & $\underline{-}$ & 0.9 \\
\hline March ............................. & 1.6 & 1.7 & 2.0 & 1.8 & - & 3.8 & 0.1 & 0.1 & $\ddot{w}$ & $\ddot{w}$ & - & 0.3 \\
\hline 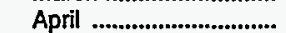 & 0.5 & 0.5 & 0.8 & 1.1 & - & 1.9 & 0.0 & 0.0 & $w$ & $\ddot{w}$ & - & 0.2 \\
\hline 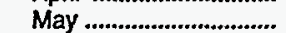 & 0.5 & 0.5 & 0.8 & 1.2 & - & 1.9 & 0.0 & 0.0 & 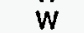 & $w$ & - & 0.2 \\
\hline June ................................. & 0.5 & 0.5 & 0.8 & 1.2 & - & 2.0 & 0.0 & 0.0 & w & 0.1 & - & 0.2 \\
\hline 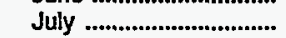 & 0.5 & 0.5 & 0.8 & 1.2 & - & 2.0 & 0.0 & 0.0 & $w$ & $w$ & - & 0.2 \\
\hline 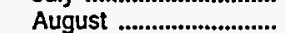 & 0.5 & 0.5 & 0.8 & 1.2 & - & 2.0 & 0.0 & 0.0 & w & 0.1 & - & 0.2 \\
\hline September .................... & 0.6 & 0.6 & 1.2 & 1.9 & 1.2 & 4.4 & 0.1 & 0.1 & 0.2 & 0.2 & $w$ & 0.6 \\
\hline October ........................... & 3.8 & 3.9 & $W$ & $w$ & $W$ & 15.4 & 0.9 & 0.9 & $w$ & $w$ & - & 3.2 \\
\hline November ..................... & 5.7 & 5.8 & 8.7 & 8.7 & 1.3 & 18.8 & 1.5 & 1.5 & 2.4 & 1.6 & - & 3.9 \\
\hline December ...................... & 5.8 & 6.0 & 8.7 & $w$ & $w$ & 18.0 & 1.7 & 1.7 & 2.4 & 1.3 & - & 3.8 \\
\hline 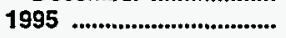 & 2.4 & 2.5 & 3.8 & 3.8 & 0.5 & 8.1 & 0.5 & 0.5 & 0.8 & $w$ & $\mathbf{w}$ & 1.3 \\
\hline \multicolumn{13}{|l|}{1996} \\
\hline 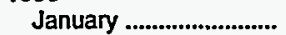 & 5.4 & 5.5 & 8.5 & $W$ & $w$ & 17.1 & 1.5 & 1.5 & $w$ & $w$ & - & 3.5 \\
\hline
\end{tabular}

See footnotes at end of table. 
Table 11. U.S. Refiner Oxygenated Motor Gasoline Volumes by Grade and Sales Type (Million Gallons per Day) - Continued

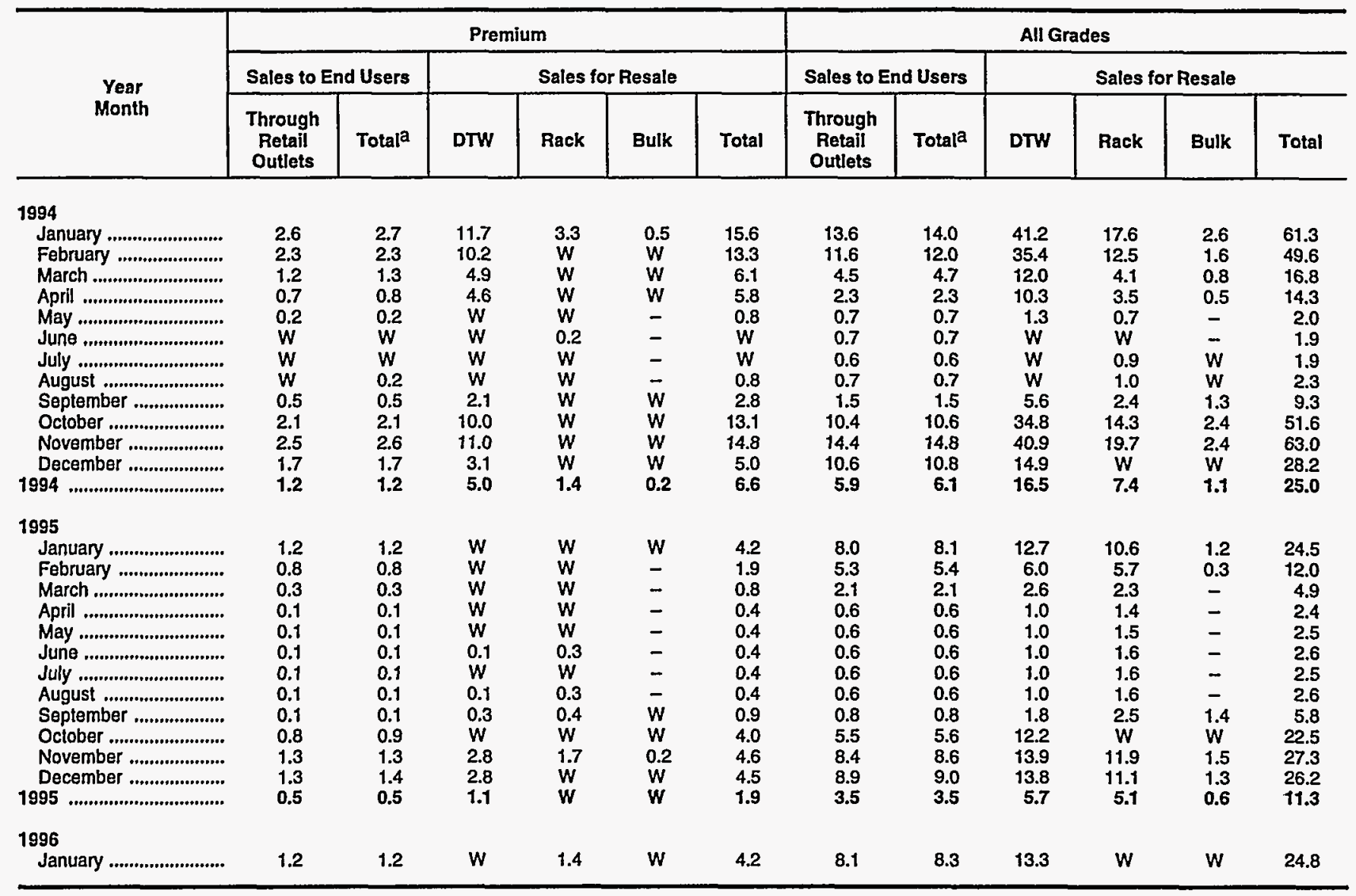

Dash $(-)=$ No data reported.

$W=$ Withheld to avold disclosure of individual company data.

a Includes sales through retall outlets as well as all direct sales to end users that were not made through company-operated retail outlets, e.g., sales to agricultural customers, commercial sales, and industrial sales.

Notes: The 4th quarter of 1993 was a transitional period between the predecessor ElA-782 survey system and the revised ElA-782 survey sytem. The revised survey system contains additional product and sales categories, which may not be consistent with categories derived from the predecessor survey system. Beginning January 1994 all data are from the revised survey system and are consistent.

Notes: Values shown for the current month are preliminary. Values shown for previous months are revised. Data are final upon publication in the Petroleum Marketing Annual. Totals may not equal the sum of the components due to rounding.

Source: Energy Information Administration Form ElA-782A, "Refiners'/Gas Plant Operators' Monthly Petroleum Product Sales Report." 
Table 12. U.S. Refiner Reformulated Motor Gasoline Prices by Grade and Sales Type (Cents per Gallon Excluding Taxes)

\begin{tabular}{|c|c|c|c|c|c|c|c|c|c|c|c|c|}
\hline \multirow{3}{*}{$\begin{array}{l}\text { Year } \\
\text { Month }\end{array}$} & \multicolumn{6}{|c|}{ Regular } & \multicolumn{6}{|c|}{ Midgrade } \\
\hline & \multicolumn{2}{|c|}{ Sales to End Users } & \multicolumn{4}{|c|}{ Sales for Resale } & \multicolumn{2}{|c|}{ Sales to End Users } & \multicolumn{4}{|c|}{ Sales for Resale } \\
\hline & $\begin{array}{c}\text { Through } \\
\text { Retail } \\
\text { Outlets }\end{array}$ & Average $^{a}$ & DTW & Rack & Bulk & Average & $\begin{array}{c}\text { Through } \\
\text { Retail } \\
\text { Outlets }\end{array}$ & Average $^{a}$ & DTW & Rack & Bulk & Average \\
\hline \multicolumn{13}{|l|}{1994} \\
\hline 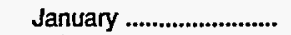 & - & - & - & - & - & - & - & - & - & - & - & - \\
\hline 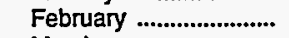 & - & - & - & - & - & - & - & - & - & - & - & - \\
\hline 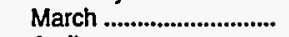 & $\rightarrow$ & - & - & - & - & - & - & - & - & - & - & - \\
\hline 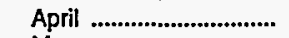 & - & - & - & - & - & - & - & - & - & - & - & - \\
\hline 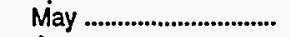 & - & - & - & - & - & - & - & - & - & - & - & - \\
\hline 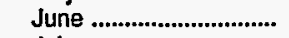 & - & - & - & - & - & - & - & - & - & - & - & - \\
\hline July & - & - & - & - & - & - & - & - & - & - & - & - \\
\hline 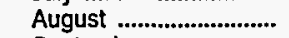 & - & - & - & - & - & - & - & - & - & - & - & - \\
\hline September ..................... & - & - & - & - & - & - & - & - & - & - & - & - \\
\hline 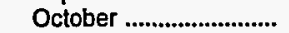 & - & - & - & - & - & - & - & - & - & - & - & - \\
\hline November ....................... & 77.9 & 77.7 & 72.6 & 60.3 & 58.7 & 65.2 & 89.8 & 89.5 & 77.9 & 69.0 & - & 75.4 \\
\hline 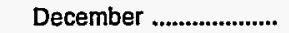 & 76.4 & 75.7 & 72.0 & 56.7 & 53.3 & 63.7 & 87.8 & 87.2 & 77.0 & 62.5 & $w$ & 72.6 \\
\hline 1994 & 76.4 & 75.8 & 72.0 & 56.9 & 54.3 & 63.8 & 87.9 & 87.3 & 77.0 & 62.8 & $w$ & 72.8 \\
\hline \multicolumn{13}{|l|}{1995} \\
\hline January ......................... & 75.8 & 75.2 & 72.2 & 59.0 & 56.0 & 65.1 & 85.3 & 84.8 & 77.1 & 63.2 & W & 73.1 \\
\hline 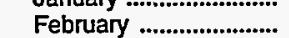 & 74.4 & 73.8 & 71.2 & 59.1 & 57.5 & 64.7 & 83.5 & 83.1 & 75.6 & 64.2 & $W$ & 72.4 \\
\hline March .............................. & 73.0 & 72.4 & 69.9 & 57.3 & 54.0 & 62.7 & 82.1 & 81.7 & 74.5 & 62.1 & - & 70.9 \\
\hline 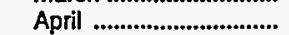 & 75.0 & 74.5 & 71.4 & 63.9 & 59.6 & 67.3 & 84.0 & 83.7 & 76.4 & 68.4 & W & 74.0 \\
\hline 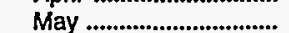 & 80.3 & 80.1 & 76.3 & 69.5 & 65.0 & 72.3 & 89.1 & 88.8 & 81.3 & 74.1 & - & 79.2 \\
\hline 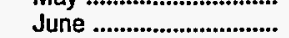 & 81.7 & 81.4 & 77.4 & 65.9 & 61.6 & 71.1 & 90.3 & 90.0 & 81.9 & 70.8 & - & 78.6 \\
\hline July & 79.2 & 78.6 & 74.2 & 59.6 & 58.1 & 66.3 & 87.7 & 87.3 & 78.2 & 64.7 & W & 74.2 \\
\hline 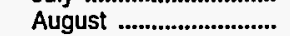 & 75.4 & 74.8 & 70.1 & 59.3 & 57.5 & 64.0 & 83.5 & 83.1 & 74.2 & 64.1 & - & 71.1 \\
\hline September ....................... & 72.6 & 72.2 & 68.3 & 60.5 & 58.0 & 63.9 & 80.5 & 80.2 & 72.4 & 64.7 & $W$ & 70.2 \\
\hline 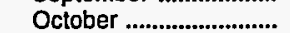 & 71.5 & 71.0 & 67.3 & 55.7 & 52.9 & - 60.9 & 80.3 & 80.0 & 71.6 & 60.2 & - & 68.3 \\
\hline November ..................... & 69.1 & 68.7 & 64.8 & 55.6 & 52.7 & 59.6 & 78.8 & 78.4 & 69.8 & 59.8 & - & 66.9 \\
\hline 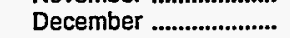 & 69.5 & 69.1 & 65.2 & 59.1 & 57.5 & 61.9 & 78.9 & 78.7 & 70.3 & 63.2 & - & 68.3 \\
\hline 1995 & 74.9 & 74.4 & 70.7 & 60.5 & 57.3 & 65.0 & 83.6 & 83.3 & 75.3 & 65.0 & NA & 72.3 \\
\hline \multicolumn{13}{|l|}{1996} \\
\hline 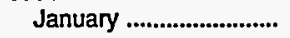 & 71.5 & 71.1 & 67.6 & 59.1 & 56.2 & 63.1 & 80.9 & 80.6 & 72.4 & 63.8 & - & 70.0 \\
\hline
\end{tabular}

See footnotes at end of table. 
Table 12. U.S. Refiner Reformulated Motor Gasoline Prices by Grade and Sales Type (Cents per Gallon Excluding Taxes) - Continued

\begin{tabular}{|c|c|c|c|c|c|c|c|c|c|c|c|c|}
\hline \multirow{3}{*}{$\begin{array}{l}\text { Year } \\
\text { Month }\end{array}$} & \multicolumn{6}{|c|}{ Premium } & \multicolumn{6}{|c|}{ All Grades } \\
\hline & \multicolumn{2}{|c|}{ Sales to End Users } & \multicolumn{4}{|c|}{ Sales for Resale } & \multicolumn{2}{|c|}{ Sales to End Users } & \multicolumn{4}{|c|}{ Sales for Resale } \\
\hline & $\begin{array}{c}\text { Through } \\
\text { Retail } \\
\text { Outlets }\end{array}$ & Averagea & DTW & Rack & Bulk & Average & $\begin{array}{c}\text { Through } \\
\text { Retail } \\
\text { Outlets }\end{array}$ & Average ${ }^{a}$ & DTW & Rack & Bulk & Average \\
\hline \multicolumn{13}{|l|}{1994} \\
\hline 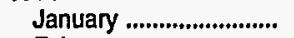 & - & - & - & - & - & - & - & $\rightarrow$ & - & - & - & - \\
\hline February ........................ & - & - & - & - & - & - & - & - & - & - & - & - \\
\hline March & - & - & - & $\rightarrow$ & - & - & - & $\rightarrow$ & - & - & - & - \\
\hline 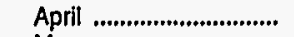 & - & - & - & - & - & - & - & - & - & - & - & - \\
\hline 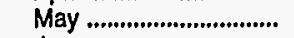 & - & - & - & - & - & - & - & - & - & - & - & - \\
\hline June ................................ & - & - & - & - & - & - & - & - & - & - & - & - \\
\hline July .................................. & - & - & - & - & - & - & - & - & - & - & - & - \\
\hline 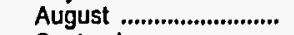 & - & - & - & - & - & - & - & - & - & - & - & - \\
\hline September ......................... & - & - & - & - & - & - & - & - & - & - & - & - \\
\hline 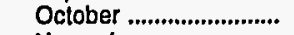 & - & - & - & $\overline{-}$ & - & - & - & - & - & - & - & - \\
\hline November .................... & 97.2 & 97.0 & 84.6 & 69.5 & 61.2 & 76.8 & 87.4 & 87.2 & 77.8 & 64.1 & 59.4 & 70.0 \\
\hline December ..................... & 96.5 & 95.7 & 86.3 & 67.5 & 54.5 & 77.8 & 82.8 & 82.1 & 77.0 & 59.7 & 53.6 & 68.6 \\
\hline 1994 & 96.6 & 95.8 & 86.1 & 67.7 & 55.8 & 77.7 & 83.1 & 82,4 & 77.1 & 59.9 & 54.7 & 68.7 \\
\hline \multicolumn{13}{|l|}{1995} \\
\hline January ........................ & 95.3 & 94.7 & 85.7 & 68.8 & 58.7 & 78.9 & 82.1 & 81.5 & 77.1 & 61.6 & 56.7 & 69.9 \\
\hline February ........................ & 93.5 & 92.9 & 84.4 & 68.9 & 58.8 & 78.5 & 80.6 & 79.9 & 76.0 & 61.8 & 57.8 & 69.4 \\
\hline March ............................ & 89.4 & 88.9 & 83.6 & 66.5 & 57.5 & 76.7 & 79.1 & 78.5 & 74.8 & 59.9 & 54.6 & 67.5 \\
\hline 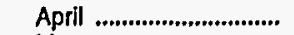 & 93.4 & 92.9 & 85.1 & 73.2 & 66.9 & 80.8 & 81.0 & 80.6 & 76.4 & 66.4 & 60.9 & 71.8 \\
\hline May ................................. & 98.5 & 98.1 & 90.2 & 80.4 & 74.0 & 86.4 & 86.0 & 85.7 & 81.3 & 72.4 & 66.5 & 76.9 \\
\hline June ................................ & 99.6 & 99.2 & 91.4 & 76.6 & 67.4 & 85.7 & 87.4 & 87.1 & 82.3 & 68.7 & 62.6 & 75.9 \\
\hline July ................................. & 97.2 & 96.7 & 87.9 & 69.9 & 62.1 & 81.2 & 84.9 & 84.4 & 78.9 & 62.4 & 58.6 & 71.2 \\
\hline August & 93.3 & 92.7 & 83.4 & 69.5 & 61.6 & 77.5 & 81.1 & 80.5 & 74.8 & 62.1 & 58.3 & 68.5 \\
\hline September .................... & 91.1 & 90.7 & 81.7 & 70.6 & 63.2 & 77.2 & 78.4 & 78.1 & 73.1 & 63.1 & 59.0 & 68.3 \\
\hline October ........................... & 89.8 & 89.3 & 80.3 & 65.4 & 57.1 & 73.9 & 77.6 & 77.1 & 72.0 & 58.4 & 54.0 & 65.5 \\
\hline November ..................... & 87.6 & 87.2 & 77.8 & 65.3 & 57.3 & 72.2 & 75.5 & 75.0 & 69.7 & 58.3 & 53.9 & 64.1 \\
\hline December ..................... & 88.0 & 87.6 & 78.7 & 69.5 & 64.1 & 74.9 & 75.8 & 75.4 & 70.3 & 61.9 & 59.0 & 66.4 \\
\hline 1995 & 92.9 & 92.4 & 84.1 & 70.4 & 61.5 & 78.5 & 80.8 & 80.3 & 75.6 & 63.1 & 58.2 & 69.6 \\
\hline \multicolumn{13}{|l|}{1996} \\
\hline 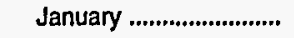 & 90.0 & 89.6 & 80.6 & 69.4 & 61.7 & 76.3 & 77.6 & 77.2 & 72.4 & 61.9 & 57.4 & 67.6 \\
\hline
\end{tabular}

Dash $(-)=$ No data reported.

NA $=$ Not available.

$W=$ Witheid to avoid disclosure of individual company data.

a Includes sales through retail outlets as well as all direct sales to end users that were not made through company-operated retail outlets, e.g., sales to agricultural customers, commercial sales, and industrial sales.

Notes: Values shown for the current month are preliminary. Values shown for previous months are revised. Data are final upon publication in the $P$ etroleum Markeling Annual.

Source: Energy Information Administration Form EIA-782A, "Refiners'/Gas Plant Operators' Monthly Petroleum Product Sales Report." 
Table 13. U.S. Refiner Reformulated Motor Gasoline Volumes by Grade and Sales Type (Million Gallons per Day)

\begin{tabular}{|c|c|c|c|c|c|c|c|c|c|c|c|c|}
\hline \multirow{3}{*}{$\begin{array}{l}\text { Year } \\
\text { Month }\end{array}$} & \multicolumn{6}{|c|}{ Regular } & \multicolumn{6}{|c|}{ Midgrade } \\
\hline & \multicolumn{2}{|c|}{ Sales to End Users } & \multicolumn{4}{|c|}{ Sales for Resale } & \multicolumn{2}{|c|}{ Sales to End Users } & \multicolumn{4}{|c|}{ Sales for Resale } \\
\hline & $\begin{array}{c}\text { Through } \\
\text { Retail } \\
\text { Outlets }\end{array}$ & Totala & DTW & Rack & Bulk & Total & $\begin{array}{c}\text { Through } \\
\text { Retail } \\
\text { Outlets }\end{array}$ & Totala & DTW & Rack & Bulk & Total \\
\hline \multicolumn{13}{|l|}{1994} \\
\hline January .......................... & - & - & - & - & - & - & - & - & - & - & - & - \\
\hline 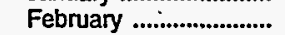 & - & - & - & - & - & - & - & - & - & - & - & - \\
\hline 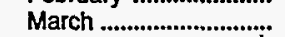 & - & - & - & - & - & - & - & - & - & - & - & - \\
\hline 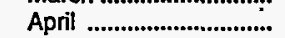 & - & - & - & - & - & - & - & - & - & - & - & - \\
\hline 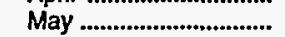 & - & - & - & - & - & - & - & - & - & - & - & - \\
\hline 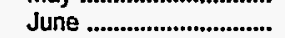 & - & - & - & - & - & - & - & - & - & - & - & - \\
\hline 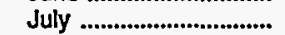 & - & - & - & - & - & - & - & - & - & - & - & - \\
\hline 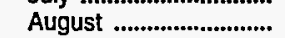 & - & - & - & - & - & - & - & - & - & - & - & - \\
\hline September ................... & - & - & - & - & - & - & - & - & - & - & - & - \\
\hline October & - & $\therefore$ & - & - & - & - & - & - & - & - & - & - \\
\hline 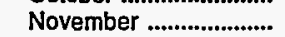 & 0.3 & 0.3 & 1.7 & 0.8 & 1.4 & 3.9 & 0.2 & 0.2 & 0.4 & 0.2 & - & 0.6 \\
\hline 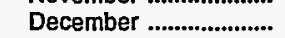 & 6.7 & 7.0 & 23.0 & $W$ & $\ddot{w}$ & 47.3 & 2.0 & 2.0 & 7.5 & $w$ & $w$ & 10.7 \\
\hline 1994 & 0.6 & 0.6 & 2.1 & 1.6 & 0.6 & 4.3 & 0.2 & 0.2 & 0.7 & 0.3 & w & 1.0 \\
\hline \multicolumn{13}{|l|}{1995} \\
\hline January .......................... & 7.0 & 7.3 & 19.5 & 15.8 & 4.4 & 39.8 & 2.8 & 2.8 & 7.0 & $w$ & $w$ & 9.8 \\
\hline February ......................... & 7.5 & 7.8 & .20 .8 & 17.8 & 5.1 & 43.7 & 2.9 & 3.0 & 7.4 & $w$ & W & 10.3 \\
\hline 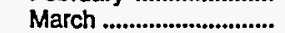 & 7.5 & 7.8 & 20.2 & 17.2 & 5.8 & 43.2 & 3.0 & 3.1 & 7.2 & 3.0 & - & 10.2 \\
\hline 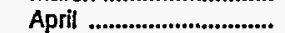 & 7.7 & 8.0 & 20.6 & $\quad 17.2$ & 3.7 & 41.5 & 3.0 & 3.0 & 7.3 & $w$ & $\mathbf{W}$ & 10.3 \\
\hline 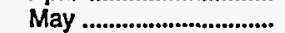 & 8.0 & 8.2 & 21.1 & 17.8 & 4.8 & 43.7 & 3.0 & 3.0 & 7.5 & 3.1 & - & 10.6 \\
\hline June ................................. & 8.3 & 8.5 & 21.6 & 18.5 & 4.3 & 44.5 & 3.1 & 3.2 & 7.7 & 3.3 & - & 11.0 \\
\hline 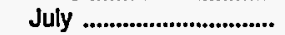 & 8.1 & 8.3 & 20.8 & 17.6 & 5.5 & 44.0 & 3.0 & 3.0 & $w$ & $w$ & w & 10.8 \\
\hline 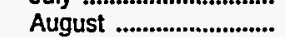 & 8.2 & 8.5 & 21.0 & 18.0 & 6.6 & 45.7 & 3.1 & 3.1 & 7.6 & 3.3 & - & 10.9 \\
\hline September ......................... & 8.0 & .8 .3 & 20.4 & 17.0 & 5.6 & 43.0 & 3.2 & 3.2 & 7.4 & 3.0 & $w$ & 10.5 \\
\hline 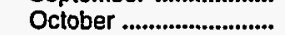 & 7.7 & 8.0 & $w$ & W & $W$ & 42.7 & 3.0 & 3.0 & $\mathbf{W}$ & $w$ & - & 10.5 \\
\hline November .................... & 7.8 & 8.1 & 20.6 & 16.9 & 5.8 & 43.3 & 3.0 & 3.1 & 7.5 & 3.0 & - & 10.5 \\
\hline December ...................... & 7.9 & 8.1 & 20.8 & $W$ & $W$ & 42.2 & 3.1 & 3.2 & 7.6 & 3.0 & - & 10.6 \\
\hline 1995 & 7.8 & 8.1 & 20.7 & 17.3 & 5.1 & 43.1 & 3.0 & 3.1 & 7.4 & $\mathbf{W}$ & $\mathbf{W}$ & 10.5 \\
\hline \multicolumn{13}{|l|}{1996} \\
\hline 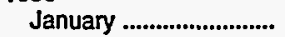 & 7.5 & 7.7 & 19.3 & $w$ & $W$ & 38.4 & 2.9 & 2.9 & $w$ & $w$ & - & 9.7 \\
\hline
\end{tabular}

See footnotes at end of table. 
Table 13. U.S. Refiner Reformulated Motor Gasoline Volumes by Grade and Sales Type (Million Gallons per Day) - Continued

\begin{tabular}{|c|c|c|c|c|c|c|c|c|c|c|c|c|}
\hline \multirow{3}{*}{$\begin{array}{l}\text { Year } \\
\text { Month }\end{array}$} & \multicolumn{6}{|c|}{ Premium } & \multicolumn{6}{|c|}{ All Grades } \\
\hline & \multicolumn{2}{|c|}{ Sales to End Users } & \multicolumn{4}{|c|}{ Sales for Resale } & \multicolumn{2}{|c|}{ Sales to End Users } & \multicolumn{4}{|c|}{ Sales for Resale } \\
\hline & $\begin{array}{c}\text { Through } \\
\text { Retail } \\
\text { Outlets }\end{array}$ & Totala & DTW & Rack & Bulk & Total & $\begin{array}{c}\text { Through } \\
\text { Retail } \\
\text { Outlets }\end{array}$ & Totala & DTW & Rack & Bulk & Total \\
\hline \multicolumn{13}{|l|}{1994} \\
\hline January ........................... & - & - & - & - & - & - & - & - & - & - & - & - \\
\hline February ............................ & - & - & - & - & - & - & - & - & - & - & - & - \\
\hline March .............................. & - & - & - & - & - & - & - & - & - & - & - & - \\
\hline 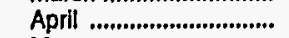 & - & - & - & - & - & - & - & - & - & - & - & - \\
\hline 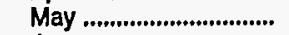 & - & - & - & - & - & - & - & - & - & - & - & - \\
\hline June ................................. & - & - & - & - & - & - & - & - & - & - & - & - \\
\hline July ................................... & - & - & - & - & - & - & - & - & - & - & - & - \\
\hline August ........................... & - & - & - & - & - & - & - & - & - & - & - & - \\
\hline September ...................... & - & - & - & - & - & - & - & - & - & - & - & - \\
\hline 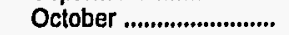 & - & - & - & - & - & - & - & - & - & - & - & - \\
\hline November ...................... & 0.3 & 0.3 & 1.4 & 0.4 & 0.5 & 2.2 & 0.7 & 0.7 & 3.5 & 1.3 & 1.9 & 6.8 \\
\hline December ..................... & 2.5 & 2.5 & 12.6 & $w$ & $w$ & 20.5 & 11.1 & 11.5 & 43.1 & $W$ & $W$ & 78.5 \\
\hline 1994 & 0.2 & 0.2 & 1.2 & 0.5 & 0.2 & 1.9 & 1.0 & 1.0 & 4.0 & 2.4 & 0.8 & 7.2 \\
\hline \multicolumn{13}{|l|}{1995} \\
\hline 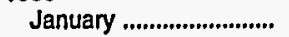 & 2.7 & 2.8 & 11.4 & $w$ & $w$ & 17.8 & 12.5 & 12.9 & 37.9 & 23.8 & 5.8 & 67.5 \\
\hline February ........................ & 2.9 & 3.0 & 12.3 & $W$ & $w$ & 18.8 & 13.4 & 13.8 & 40.5 & 26.2 & 6.1 & 72.7 \\
\hline March .............................. & 3.5 & 3.6 & 11.7 & 5.6 & 1.3 & 18.6 & 14.0 & 14.5 & 39.2 & 25.7 & 7.0 & 72.0 \\
\hline 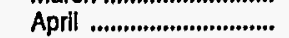 & 3.1 & 3.2 & 12.0 & $W$ & $w$ & 18.2 & 13.7 & 14.2 & 39.9 & 25.6 & 4.5 & 69.9 \\
\hline 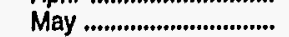 & 2.9 & 3.0 & 11.8 & 5.6 & 1.0 & 18.4 & 13.9 & 14.2 & 40.4 & 26.5 & 5.8 & 72.6 \\
\hline June .............................. & 3.1 & 3.2 & 11.9 & 5.7 & 0.8 & 18.4 & 14.6 & 14.9 & 41.2 & 27.5 & 5.2 & 73.9 \\
\hline July & 3.1 & 3.1 & $W$ & $w$ & $W$ & 18.0 & 14.1 & 14.5 & 40.1 & 26.4 & 6.3 & 72.8 \\
\hline August & 3.2 & 3.3 & 12.2 & 5.9 & 1.5 & 19.5 & 14.6 & 15.0 & 40.8 & 27.2 & 8.2 & 76.1 \\
\hline September ................... & 3.2 & 3.3 & 12.2 & 5.5 & 1.2 & 18.9 & 14.4 & 14.8 & 40.0 & 25.6 & 6.8 & 72.4 \\
\hline 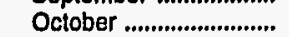 & 3.2 & 3.2 & $W$ & $W$ & 1.8 & 19.8 & 13.9 & 14.2 & 40.2 & $W$ & $w$ & 72.9 \\
\hline November .................... & 3.3 & 3.3 & 12.6 & 5.8 & 2.1 & 20.5 & 14.1 & 14.4 & 40.8 & 25.7 & 7.8 & 74.3 \\
\hline December ........................ & 3.2 & 3.3 & 12.6 & $w$ & $w$ & 19.7 & 14.2 & 14.6 & 40.9 & 25.3 & 6.2 & 72.5 \\
\hline 1995 & 3.1 & 3.2 & 12.0 & $\mathbf{W}$ & $\mathbf{W}$ & 18.9 & 14.0 & 14.3 & 40.1 & 25.9 & 6.4 & 72.5 \\
\hline \multicolumn{13}{|l|}{1996} \\
\hline January ............................ & 3.0 & 3.0 & $W$ & 5.0 & W & 17.3 & 13.3 & 13.7 & 37.6 & $w$ & $\mathbf{W}$ & 65.5 \\
\hline
\end{tabular}

Dash $(-)=$ No data reported.

$W=$ Withheld to avoid disclosure of individual company data.

a Includes sales through retail outlets as well as all direct sales to end users that were not made through company-operated retail outlets, e.g., sales to agricultural customers, commerclal sales, and industrial sales.

Notes: Values shown for the current month are preliminary. Values shown for previous months are revised. Data are final upon publication in the Petroleum Marketing Annual. Totals may not equal the sum of the components due to rounding.

Source: Energy Information Administration Form ElA-782A, "Refiners'/Gas Plant Operators' Monthly Petroleum Product Sales Report." 
Table 14. U.S. Propane (Consumer Grade) Prices by Sales Type (Cents per Gallon Excluding Taxes)

\begin{tabular}{|c|c|c|c|c|c|c|c|c|}
\hline \multirow[b]{2}{*}{$\begin{array}{l}\text { Year } \\
\text { Month }\end{array}$} & \multicolumn{7}{|c|}{ Sales to End Users } & \multirow{2}{*}{$\begin{array}{c}\text { Sales } \\
\text { for } \\
\text { Resale }\end{array}$} \\
\hline & $\begin{array}{l}\text { Residential } \\
\text { Consumers }\end{array}$ & $\begin{array}{c}\text { Commercial } \\
\text { Institutional } \\
\text { Consumers }\end{array}$ & $\begin{array}{l}\text { Industrial } \\
\text { Consumers }\end{array}$ & $\begin{array}{c}\text { Through } \\
\text { Retail } \\
\text { Outlets }\end{array}$ & $\begin{array}{l}\text { Petro- } \\
\text { Chemical }\end{array}$ & $\begin{array}{c}\text { Other } \\
\text { End Users }\end{array}$ & Average & \\
\hline \multicolumn{9}{|l|}{1994} \\
\hline 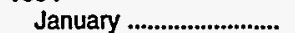 & 88.4 & 75.9 & 64.4 & 71.4 & 27.3 & 65.1 & 80.9 & 33.2 \\
\hline 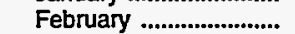 & 89.8 & 77.0 & 67.5 & 72.7 & 29.4 & 68.1 & 82.1 & 35.3 \\
\hline 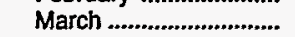 & 92.0 & 77.4 & 67.2 & 72.6 & 29.1 & 67.2 & 82.1 & 33.2 \\
\hline 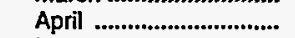 & 92.3 & 76.8 & 69.0 & 73.8 & 29.6 & 69.2 & 80.9 & 31.9 \\
\hline 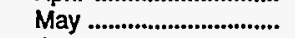 & 94.1 & 76.3 & 69.0 & 74.8 & 30.1 & 72.1 & 77.6 & 32.0 \\
\hline June ............................... & 93.2 & 77.6 & 68.8 & 75.2 & 29.5 & 70.0 & 76.9 & 31.4 \\
\hline July .................................. & 87.6 & 76.3 & 67.9 & 75.0 & 29.6 & 67.5 & 73.9 & 31.0 \\
\hline 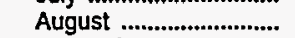 & NA & 75.2 & 63.6 & 74.6 & 31.4 & 63.8 & 71.0 & 32.0 \\
\hline September .................... & 82.5 & 75.2 & 63.0 & 73.6 & 31.4 & 62.1 & 73.0 & 32.8 \\
\hline 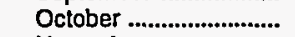 & 83.9 & 76.4 & 62.6 & 74.4 & 33.3 & 55.9 & 71.1 & 34.2 \\
\hline November ..................... & 85.1 & 77.2 & 65.0 & 74.5 & 34.7 & 60.1 & 75.7 & 36.0 \\
\hline December ....................... & 86.2 & 77.7 & 64.7 & 74.8 & 34.4 & 65.0 & 79.3 & 36.8 \\
\hline 1994 & 87.2 & 76.5 & 65.2 & 73.6 & 31.1 & 63.4 & 77.6 & 33.6 \\
\hline \multicolumn{9}{|l|}{1995} \\
\hline 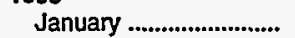 & 86.6 & 78.3 & 67.6 & 74.8 & 34.5 & 66.9 & 79.5 & 36.5 \\
\hline 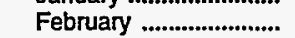 & 88.3 & 77.5 & 66.3 & 73.5 & 32.3 & 66.6 & 80.4 & 35.3 \\
\hline March ............................. & 88.0 & 76.8 & 66.5 & 73.8 & 32.6 & 68.1 & 79.3 & 35.3 \\
\hline April & 88.3 & 78,5 & 63.8 & 74.1 & 32.4 & 70.6 & 75.9 & 34.2 \\
\hline 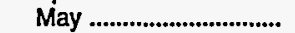 & 90.0 & 78.2 & 65.2 & 76.4 & 32.8 & 74.6 & 73.9 & 34.6 \\
\hline 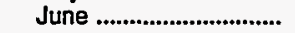 & 89.5 & 77.9 & 66.2 & 76.5 & 32.5 & 72.4 & 73.1 & 33.6 \\
\hline 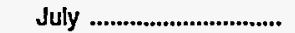 & 84.9 & 77.6 & 63.7 & 75.8 & 31.9 & 66.7 & 69.9 & 33.1 \\
\hline 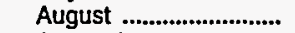 & 80.9 & 76.1 & 62.4 & 75.4 & 31.5 & 65.7 & 70.5 & 34.1 \\
\hline September ..................... & 80.9 & 76.0 & 61.2 & 74.4 & 32.2 & 62.2 & 71.1 & 34.6 \\
\hline October ............................. & 83.1 & 75.4 & 63.2 & 74.9 & 31.7 & 60.1 & 71.9 & 35.1 \\
\hline November ...................... & 84.6 & 75.2 & 64.7 & 73.6 & 31.4 & 65.3 & 76.4 & 35.5 \\
\hline December ...................... & 88.5 & 79.0 & 63.9 & 73.7 & 32.5 & 69.7 & 81.2 & 38.7 \\
\hline 1995 & 86.4 & 77.3 & 64.7 & 74.7 & 32.4 & 66.1 & 76.5 & 35.3 \\
\hline \multicolumn{9}{|l|}{1996} \\
\hline 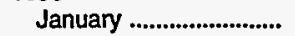 & 92.6 & 82.9 & 68.0 & NA & 34.5 & 72.7 & 86.3 & 42.3 \\
\hline
\end{tabular}

NA $=$ Not available.

Notes: The 4th quarter of 1993 was a transitional period between the predecessor ElA-782 survey system and the revised ElA-782 survey sytem. The revised survey system contains additional product and sales categories, which may not be consistent with categories derived from the predecessor survey system. Beginning January 1994 all data are from the revised survey system and are consistent.

Notes: Values shown for the current month are preliminary. Values shown for previous months are revised. Data are final upon publication in the Petroleum Marketing Annual.

Sources: Energy Information Administration Forms ElA-782A, "Refiners'/Gas Plant Operators' Monthly Petroleum Product Sales Report," and ElA-782B,

"Resellers'/Retailers' Monthly Petroleum Product Sales Report." 
Table 15. U.S. No. 2 Distillate ${ }^{\mathrm{P}}$ Prices by Sales Type (Cents per Gallon Excluding Taxes)

\begin{tabular}{|c|c|c|c|c|c|c|c|}
\hline \multirow[b]{2}{*}{$\begin{array}{l}\text { Year } \\
\text { Month }\end{array}$} & \multicolumn{6}{|c|}{ Sales to End Users } & \multirow[b]{2}{*}{$\begin{array}{c}\text { Sales } \\
\text { for } \\
\text { Resale }\end{array}$} \\
\hline & $\begin{array}{l}\text { Residential } \\
\text { Consumers }\end{array}$ & $\begin{array}{c}\text { Commercial/ } \\
\text { Institutional } \\
\text { Consumers }\end{array}$ & $\begin{array}{l}\text { Industrial } \\
\text { Consumers }\end{array}$ & $\begin{array}{l}\text { Through } \\
\text { Retail } \\
\text { Outlets }^{b}\end{array}$ & $\begin{array}{l}\text { Other } \\
\text { End Usersc }\end{array}$ & Average & \\
\hline 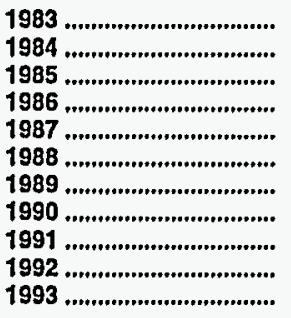 & $\begin{array}{r}107.8 \\
109.1 \\
105.3 \\
83.6 \\
80.3 \\
81.3 \\
90.0 \\
106.3 \\
101.9 \\
93.4 \\
91.1\end{array}$ & $\begin{array}{l}86.3 \\
85.7 \\
82.1 \\
51.4 \\
56.6 \\
51.9 \\
60.8 \\
75.1 \\
67.5 \\
63.7 \\
62.0\end{array}$ & $\begin{array}{l}88.3 \\
87.0 \\
83.4 \\
53.7 \\
59.7 \\
54.2 \\
63.8 \\
78.0 \\
69.7 \\
67.5 \\
66.7\end{array}$ & $\begin{array}{l}94.3 \\
92.0 \\
88.6 \\
59.8 \\
64.4 \\
61.6 \\
68.5 \\
85.2 \\
74.5 \\
72.1 \\
71.1\end{array}$ & $\begin{array}{l}89.7 \\
89.2 \\
85.3 \\
56.9 \\
63.1 \\
58.7 \\
66.7 \\
82.8 \\
73.8 \\
72.1 \\
70.5\end{array}$ & $\begin{array}{l}93.3 \\
92.6 \\
89.0 \\
61.4 \\
64.3 \\
61.2 \\
69.5 \\
84.1 \\
76.0 \\
72.6 \\
71.0\end{array}$ & $\begin{array}{l}81.8 \\
81.9 \\
78.1 \\
48.0 \\
53.5 \\
48.2 \\
57.2 \\
70.6 \\
62.7 \\
59.1 \\
56.6\end{array}$ \\
\hline 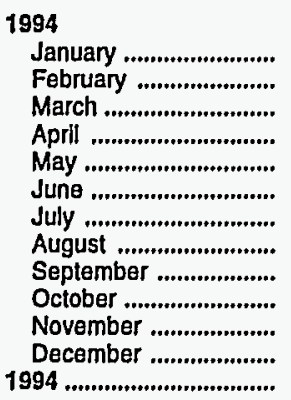 & $\begin{array}{l}89.6 \\
92.9 \\
91.4 \\
88.2 \\
86.1 \\
85.2 \\
82.7 \\
82.1 \\
83.2 \\
84.7 \\
85.7 \\
86.8 \\
88.4\end{array}$ & $\begin{array}{l}58.2 \\
60.9 \\
58.9 \\
57.7 \\
57.1 \\
57.3 \\
58.3 \\
59.2 \\
59.3 \\
59.8 \\
60.0 \\
58.0 \\
58.7\end{array}$ & $\begin{array}{l}61.6 \\
64.8 \\
63.3 \\
62.9 \\
62.4 \\
63.1 \\
64.2 \\
65.0 \\
65.0 \\
66.0 \\
65.9 \\
63.3 \\
64.1\end{array}$ & $\begin{array}{l}64.2 \\
67.5 \\
67.3 \\
67.0 \\
66.2 \\
66.6 \\
67.5 \\
68.5 \\
68.1 \\
68.2 \\
68.8 \\
66.9 \\
67.3\end{array}$ & $\begin{array}{l}61.1 \\
64.7 \\
64.7 \\
65.7 \\
64.5 \\
64.4 \\
65.4 \\
67.4 \\
67.9 \\
69.1 \\
68.1 \\
65.2 \\
65.5\end{array}$ & $\begin{array}{l}70.5 \\
72.7 \\
69.7 \\
65.8 \\
63.7 \\
63.2 \\
63.8 \\
65.3 \\
66.0 \\
67.6 \\
68.5 \\
68.9 \\
67.5\end{array}$ & $\begin{array}{l}\mathbf{5 1 . 1} \\
54.7 \\
52.6 \\
52.1 \\
51.8 \\
52.4 \\
53.8 \\
53.9 \\
53.6 \\
54.3 \\
54.1 \\
51.2 \\
52.9\end{array}$ \\
\hline 1995 & & & & & & & \\
\hline 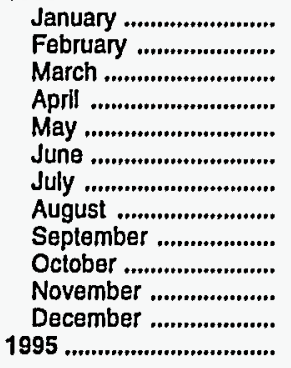 & $\begin{array}{l}87.4 \\
87.9 \\
87.4 \\
86.2 \\
86.4 \\
84.7 \\
82.0 \\
80.6 \\
82.3 \\
84.2 \\
86.6 \\
91.2 \\
87.1\end{array}$ & $\begin{array}{l}57.5 \\
57.8 \\
57.4 \\
59.7 \\
60.7 \\
58.3 \\
56.8 \\
58.8 \\
60.0 \\
59.5 \\
61.2 \\
63.6 \\
59.3\end{array}$ & $\begin{array}{l}62.0 \\
61.8 \\
62.6 \\
64.9 \\
66.0 \\
64.0 \\
62.3 \\
64.2 \\
65.2 \\
65.0 \\
66.3 \\
67.8 \\
64.4\end{array}$ & $\begin{array}{l}65.8 \\
65.1 \\
65.3 \\
67.0 \\
68.6 \\
67.6 \\
66.1 \\
66.7 \\
68.0 \\
67.5 \\
68.2 \\
69.1 \\
67.1\end{array}$ & $\begin{array}{l}64.3 \\
64.0 \\
65.4 \\
67.9 \\
69.2 \\
67.7 \\
66.3 \\
66.4 \\
69.1 \\
68.8 \\
68.9 \\
69.2 \\
67.4\end{array}$ & $\begin{array}{l}69.3 \\
69.9 \\
67.5 \\
67.5 \\
67.0 \\
64.5 \\
62.8 \\
64.2 \\
66.2 \\
66.3 \\
69.2 \\
73.5 \\
67.5\end{array}$ & $\begin{array}{l}50.7 \\
50.8 \\
50.7 \\
53.8 \\
55.4 \\
52.5 \\
51.4 \\
54.1 \\
55.2 \\
54.2 \\
55.8 \\
57.9 \\
\mathbf{5 3 . 6}\end{array}$ \\
\hline $\begin{array}{l}1996 \\
\quad \text { January ........................ }\end{array}$ & 94.7 & 63.9 & 67.7 & 69.9 & 68.8 & 75.2 & 57.3 \\
\hline
\end{tabular}

\footnotetext{
a Includes sales of No. 2 fuel oil and high- and low-sulfur diesel fuels.

b includes low-sulfur diesel fuel only.

c All end-user sales not included in the other end-user categories shown, e.g., sales to agricultural customers or utilities.

Notes: Values shown for the current month are preliminary. Values shown for previous months are revised. Data are final upon publication in the Petroleum Marketing Annual.

Sources: Energy Information Administration Forms ElA-782A, "Refiners'/Gas Plant Operators' Monthly Petroleum Product Sales Report," and ElA-782B, "Resellers'/Retailers' Monthly Petroleum Product Sales Report."
} 
Table 16. U.S. No. 2 Diesel Fuel Prices by Sales Type

(Cents per Gallon Excluding Taxes)

\begin{tabular}{|c|c|c|c|c|c|c|}
\hline \multirow[b]{2}{*}{$\begin{array}{l}\text { Year } \\
\text { Month }\end{array}$} & \multicolumn{5}{|c|}{ Sales to End Users } & \multirow[b]{2}{*}{$\begin{array}{c}\text { Sales } \\
\text { for } \\
\text { Resale }\end{array}$} \\
\hline & $\begin{array}{c}\text { Commercial/ } \\
\text { Institutional } \\
\text { Consumers }\end{array}$ & $\begin{array}{c}\text { Industrial } \\
\text { Consumers }\end{array}$ & $\begin{array}{c}\text { Through } \\
\text { Retail } \\
\text { Outlets }\end{array}$ & $\begin{array}{l}\text { Other } \\
\text { End Users } b\end{array}$ & Average & \\
\hline \multicolumn{7}{|l|}{1994} \\
\hline 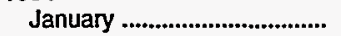 & 55.0 & 61.3 & 64.2 & 62.3 & 59.9 & 50.2 \\
\hline February .................................. & 57.9 & 64.5 & 67.5 & 64.9 & 62.6 & 53.9 \\
\hline 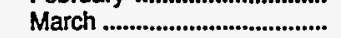 & 56.9 & 63.4 & 67.3 & 65.1 & 62.2 & 53.8 \\
\hline 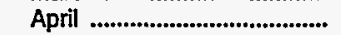 & 56.8 & 63.2 & 67.0 & 66.1 & 62.2 & 53.4 \\
\hline 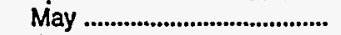 & 56.6 & 62.7 & 66.2 & 64.9 & 61.6 & 52.7 \\
\hline 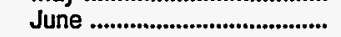 & 57.0 & 63.5 & 66.6 & 64.8 & 61.9 & 53.2 \\
\hline 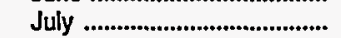 & 58.1 & 64.6 & 67.5 & 65.9 & 62.9 & 54.7 \\
\hline 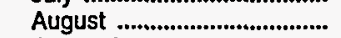 & 59.2 & 65.5 & 68.5 & 67.7 & 64.2 & 54.8 \\
\hline September .............................. & 59.4 & 65.7 & 68.1 & 68.3 & 64.4 & 54.9 \\
\hline 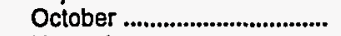 & 59.8 & 66.7 & 68.2 & 69.6 & 65.1 & 55.8 \\
\hline November .............................. & 59.6 & 66.5 & 68.8 & 68.5 & 64.9 & 55.6 \\
\hline 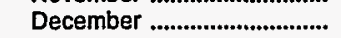 & 56.7 & 63.6 & 66.9 & . 65.8 & 62.2 & 52.0 \\
\hline 1994 & 57.7 & 64.5 & 67.3 & 66.1 & 62.8 & 53.8 \\
\hline \multicolumn{7}{|l|}{1995} \\
\hline 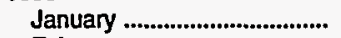 & 56.0 & 62.1 & 65.8 & 64.1 & 61.2 & 51.1 \\
\hline 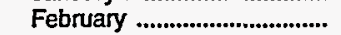 & 56.2 & 62.0 & 65.1 & 64.0 & 61.0 & 51.5 \\
\hline 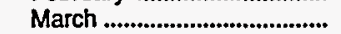 & 56.5 & 62.8 & 65.3 & 65.6 & 61.6 & 52.0 \\
\hline 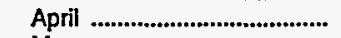 & 59.4 & 65.3 & 67.0 & 68.2 & 64.1 & 55.5 \\
\hline 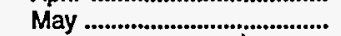 & 60.7 & 66.5 & 68.6 & 69.6 & 65.4 & 56.5 \\
\hline 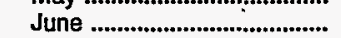 & 58.3 & 64.5 & 67.6 & 68.2 & 63.7 & 53.4 \\
\hline 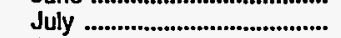 & 56.8 & 63.0 & 66.1 & 66.6 & 62.2 & 52.2 \\
\hline 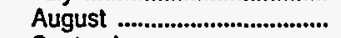 & 58.9 & 64.8 & 66.7 & 67.2 & 63.4 & 54.9 \\
\hline September .............................. & 59.9 & 66.0 & 68.0 & 69.4 & 64.8 & 56.3 \\
\hline 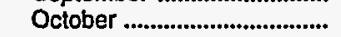 & 59.5 & 65.8 & 67.5 & 69.3 & 64.6 & 55.4 \\
\hline November .............................. & 60.7 & 67.0 & 68.2 & 69.2 & 65.3 & 57.0 \\
\hline December ................................. & 62.3 & 68.2 & 69.1 & 69.4 & 66.4 & 58.2 \\
\hline 1995 & $\mathbf{5 8 . 8}$ & 64.9 & 67.1 & 67.8 & 63.7 & 54.6 \\
\hline \multicolumn{7}{|l|}{1996} \\
\hline 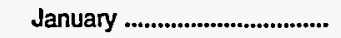 & 62.0 & 68.0 & 69.9 & 68.5 & 66.4 & 57.0 \\
\hline
\end{tabular}

a Includes low-sulfur diesel fuel only.

b All end-user sales not included in the other end-user categories shown, e.g., sales to agriculturai customers or utilities.

Notes: The 4th quarter of 1993 was a transitional period between the predecessor ElA-782 survey system and the revised ElA-782 survey sytem. The revised survey system contains additional product and sales categories, which may not be consistent with categories derived from the predecessor survey system. Beginning January 1994 all data are from the revised survey system and are consistent.

Notes: Values shown for the current month are preliminary. Values shown for previous months are revised. Data are final upon publicalion in the Petroleum Marketing Annual.

Sources: Energy Information Administration Forms ElA-782A, "Refiners'/Gas Plant Operators' Monthly Petroleum Product Sales Report," and ElA-782B,

"Resellers'/Retailers' Monthly Petroleum Product Sales Report." 
Table 17. U.S. No. 2 Diesel Fuel Prices by Sulfur Content and Sales Type (Cents per Gallon Excluding Taxes)

\begin{tabular}{|c|c|c|c|c|c|c|c|c|c|c|c|}
\hline \multirow{3}{*}{$\begin{array}{l}\text { Year } \\
\text { Month }\end{array}$} & \multicolumn{6}{|c|}{ Low-Sulfur Diesel Fuel } & \multicolumn{5}{|c|}{ High-Sulfur Diesel Fuel } \\
\hline & \multicolumn{5}{|c|}{ Sales to End Users } & \multirow{2}{*}{$\begin{array}{c}\text { Sales } \\
\text { for } \\
\text { Resale }\end{array}$} & \multicolumn{4}{|c|}{ Sales to End Users } & \multirow{2}{*}{$\begin{array}{c}\text { Sales } \\
\text { for } \\
\text { Resale }\end{array}$} \\
\hline & $\begin{array}{c}\text { Commercial } \\
\text { Instifutional } \\
\text { Consumers }\end{array}$ & $\begin{array}{l}\text { Industrial } \\
\text { Consumers }\end{array}$ & $\begin{array}{c}\text { Through } \\
\text { Retail } \\
\text { Outlets }\end{array}$ & $\begin{array}{c}\text { Other } \\
\text { End } \\
\text { Users }\end{array}$ & Average & & $\begin{array}{l}\text { Commercial/ } \\
\text { Institutional } \\
\text { Consumers }\end{array}$ & $\begin{array}{c}\text { Industrial } \\
\text { Consumers }\end{array}$ & $\begin{array}{l}\text { Other } \\
\text { End } \\
\text { Users }\end{array}$ & Average & \\
\hline \multicolumn{12}{|l|}{1994} \\
\hline 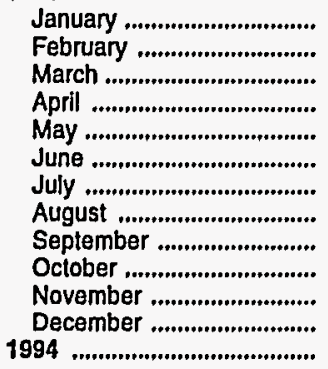 & $\begin{array}{l}56.1 \\
59.5 \\
59.1 \\
58.5 \\
57.8 \\
58.0 \\
59.3 \\
60.1 \\
60.4 \\
60.9 \\
60.8 \\
58.1 \\
59.1\end{array}$ & $\begin{array}{l}62.5 \\
66.2 \\
65.3 \\
64.9 \\
64.4 \\
64.7 \\
66.0 \\
66.8 \\
67.8 \\
68.8 \\
68.4 \\
65.2 \\
66.0\end{array}$ & $\begin{array}{l}64.2 \\
67.5 \\
67.3 \\
67.0 \\
66.2 \\
66.6 \\
67.5 \\
68.5 \\
68.1 \\
68.2 \\
68.8 \\
66.9 \\
67.3\end{array}$ & $\begin{array}{l}63.3 \\
66.2 \\
66.3 \\
66.5 \\
66.5 \\
66.8 \\
67.6 \\
68.6 \\
69.3 \\
70.2 \\
69.2 \\
66.0 \\
67.4\end{array}$ & $\begin{array}{l}60.9 \\
64.1 \\
64.0 \\
63.7 \\
62.9 \\
63.3 \\
64.3 \\
65.2 \\
65.4 \\
66.0 \\
65.9 \\
63.4 \\
64.2\end{array}$ & $\begin{array}{l}50.7 \\
54.4 \\
54.6 \\
54.0 \\
53.1 \\
53.6 \\
55.0 \\
55.1 \\
55.2 \\
56.1 \\
56.0 \\
52.2 \\
54.2\end{array}$ & $\begin{array}{l}53.3 \\
55.2 \\
53.6 \\
54.3 \\
54.7 \\
55.4 \\
56.0 \\
57.7 \\
57.7 \\
57.7 \\
57.4 \\
54.5 \\
\mathbf{5 5 . 3}\end{array}$ & $\begin{array}{l}59.8 \\
62.6 \\
61.3 \\
61.5 \\
60.9 \\
62.2 \\
63.0 \\
64.4 \\
63.7 \\
64.6 \\
64.7 \\
62.1 \\
62.9\end{array}$ & $\begin{array}{l}60.9 \\
62.9 \\
63.6 \\
65.5 \\
63.0 \\
62.5 \\
63.7 \\
66.6 \\
66.9 \\
68.8 \\
67.6 \\
65.5 \\
64.6\end{array}$ & $\begin{array}{l}57.4 \\
59.2 \\
58.1 \\
59.0 \\
58.7 \\
58.9 \\
59.7 \\
61.8 \\
61.9 \\
63.0 \\
62.3 \\
59.4 \\
59.8\end{array}$ & $\begin{array}{l}48.6 \\
51.9 \\
50.5 \\
50.9 \\
51.2 \\
51.5 \\
52.8 \\
53.7 \\
53.5 \\
54.2 \\
54.2 \\
51.0 \\
51.9\end{array}$ \\
\hline \multicolumn{12}{|l|}{1995} \\
\hline 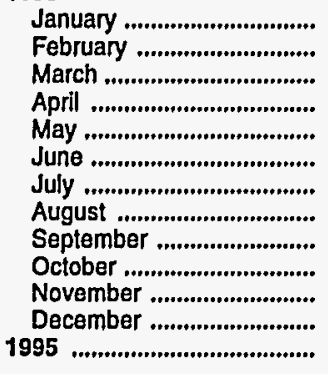 & $\begin{array}{l}57.2 \\
57.3 \\
57.9 \\
60.9 \\
61.8 \\
59.2 \\
57.8 \\
59.7 \\
61.1 \\
60.3 \\
61.8 \\
63.7 \\
59.9\end{array}$ & $\begin{array}{l}63.4 \\
63.3 \\
64.4 \\
67.3 \\
67.6 \\
65.3 \\
63.8 \\
66.4 \\
67.4 \\
67.6 \\
68.5 \\
69.5 \\
66.3\end{array}$ & $\begin{array}{l}65.8 \\
65.1 \\
65.3 \\
67.0 \\
68.6 \\
67.6 \\
66.1 \\
66.7 \\
68.0 \\
67.5 \\
68.2 \\
69.1 \\
67.1\end{array}$ & $\begin{array}{l}64.3 \\
64.3 \\
66.1 \\
68.8 \\
70.4 \\
68.7 \\
67.5 \\
68.2 \\
70.3 \\
69.9 \\
69.6 \\
70.4 \\
68.4\end{array}$ & $\begin{array}{l}62.3 \\
62.0 \\
62.6 \\
65.1 \\
66.3 \\
64.5 \\
63.1 \\
64.3 \\
65.8 \\
65.3 \\
66.0 \\
67.3 \\
64.6\end{array}$ & $\begin{array}{l}51.4 \\
51.9 \\
52.6 \\
56.1 \\
56.9 \\
53.8 \\
52.7 \\
55.4 \\
56.8 \\
55.8 \\
57.4 \\
58.8 \\
55.1\end{array}$ & $\begin{array}{l}54.0 \\
54.2 \\
53.8 \\
56.6 \\
58.4 \\
56.6 \\
54.9 \\
57.2 \\
57.8 \\
57.9 \\
58.7 \\
59.7 \\
56.7\end{array}$ & $\begin{array}{l}61.1 \\
60.8 \\
61.4 \\
63.6 \\
65.4 \\
63.7 \\
62.0 \\
63.2 \\
64.5 \\
64.1 \\
65.5 \\
67.0 \\
63.6\end{array}$ & $\begin{array}{l}63.8 \\
63.5 \\
64.9 \\
67.4 \\
68.6 \\
67.6 \\
65.6 \\
65.9 \\
68.1 \\
68.4 \\
68.7 \\
68.1 \\
66.9\end{array}$ & $\begin{array}{l}58.4 \\
58.7 \\
58.8 \\
61.5 \\
63.2 \\
61.6 \\
59.8 \\
61.3 \\
62.5 \\
62.8 \\
63.3 \\
64.1 \\
61.4\end{array}$ & $\begin{array}{l}49.7 \\
49.9 \\
49.4 \\
52.7 \\
54.4 \\
51.6 \\
50.1 \\
52.5 \\
53.8 \\
53.1 \\
54.6 \\
55.8 \\
52.4\end{array}$ \\
\hline 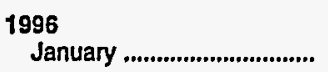 & 63.0 & 69.1 & 69.9 & 69.2 & 67.3 & 57.5 & 60.1 & 67.0 & 67.5 & 64.1 & 55.1 \\
\hline
\end{tabular}

a All end-user sales not included in the other end-user categories shown, e.g., sales to agricultural customers or utilities.

Notes: The 4th quarter of 1993 was a transitional period between the predecessor ElA-782 survey system and the revised ElA-782 survey sytem. The revised survey system contains additional product and sales categories, which may not be consistent with categories derived from the predecessor survey system. Beginning January 1994 all data are from the revised survey system and are consistent.

Notes: Values shown for the current month are preliminary. Values shown for previous months are revised. Data are final upon publication in the Petroleum Marketing Annual.

Sources: Energy Information Adminlstration Forms EIA-782A, "Refiners'/Gas Plant Operators' Monthly Petroleum Product Sales Report," and EIA-782B, "Resellers'/Retailers' Monthly Petroleum Product Sales Report." 
Table 18. Prices of No. 2 Distillate to Residences by PAD District and Selected States

(Cents per Gallon Excluding Taxes)

\begin{tabular}{|c|c|c|c|c|c|c|c|c|c|c|}
\hline \multirow{2}{*}{$\begin{array}{l}\text { Year } \\
\text { Month }\end{array}$} & \multirow{2}{*}{$\begin{array}{c}\text { U.S. } \\
\text { Average }\end{array}$} & \multicolumn{9}{|c|}{ PAD District I } \\
\hline & & Average & CT & ME & MA & NH & Rl & VT & DE & DC \\
\hline $\begin{array}{l}1978 \\
1979 \\
1980 \\
1981 \\
1982 \\
1983 \\
1984 \\
1985\end{array}$ & $\begin{array}{r}49.0 \\
70.4 \\
97.4 \\
119.4 \\
116.0 \\
107.8 \\
109.1 \\
105.3 \\
83.6 \\
80.3 \\
81.3 \\
90.0 \\
106.3 \\
101.9 \\
93.4 \\
91.1\end{array}$ & $\begin{array}{r}- \\
- \\
- \\
- \\
109.0 \\
111.3 \\
106.8 \\
86.2 \\
81.4 \\
82.7 \\
91.7 \\
108.1 \\
104.1 \\
94.9 \\
92.1\end{array}$ & $\begin{array}{r}50.1 \\
72.0 \\
98.3 \\
121.7 \\
118.3 \\
109.1 \\
112.1 \\
108.0 \\
89.0 \\
83.4 \\
85.3 \\
92.9 \\
109.8 \\
106.2 \\
94.7 \\
91.9\end{array}$ & $\begin{array}{r}48.6 \\
68.8 \\
96.3 \\
120.4 \\
115.5 \\
102.8 \\
103.9 \\
99.7 \\
74.4 \\
74.7 \\
77.7 \\
89.4 \\
98.9 \\
96.0 \\
87.1 \\
82.6\end{array}$ & $\begin{array}{r}48.8 \\
70.9 \\
97.8 \\
121.3 \\
117.6 \\
109.1 \\
111.6 \\
107.0 \\
82.1 \\
80.6 \\
82.1 \\
92.6 \\
108.4 \\
103.0 \\
92.5 \\
89.7\end{array}$ & $\begin{array}{r}50.3 \\
72.5 \\
100.4 \\
123.7 \\
117.4 \\
104.1 \\
108.4 \\
102.4 \\
75.9 \\
76.5 \\
78.2 \\
89.3 \\
102.8 \\
91.6 \\
85.6 \\
82.8\end{array}$ & $\begin{array}{r}50.7 \\
72.8 \\
101.1 \\
123.8 \\
120.1 \\
110.5 \\
111.4 \\
106.7 \\
82.8 \\
82.5 \\
83.6 \\
93.9 \\
108.6 \\
99.9 \\
91.2 \\
89.3\end{array}$ & $\begin{array}{r}50.8 \\
72.5 \\
101.5 \\
125.4 \\
120.1 \\
112.9 \\
111.9 \\
107.7 \\
86.6 \\
81.1 \\
82.6 \\
90.5 \\
107.0 \\
101.9 \\
92.1 \\
90.4\end{array}$ & $\begin{array}{r}47.8 \\
68.2 \\
95.4 \\
117.3 \\
111.3 \\
106.0 \\
109.6 \\
104.6 \\
85.0 \\
79.3 \\
80.1 \\
88.2 \\
105.8 \\
99.7 \\
92.3 \\
89.9\end{array}$ & $\begin{array}{r}50.7 \\
74.2 \\
102.6 \\
127.4 \\
124.5 \\
117.0 \\
118.7 \\
114.3 \\
93.1 \\
91.8 \\
91.6 \\
98.6 \\
107.8 \\
112.2 \\
105.7 \\
104.5\end{array}$ \\
\hline 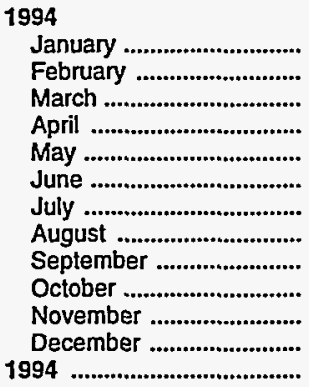 & $\begin{array}{l}89.6 \\
92.9 \\
91.4 \\
88.2 \\
86.1 \\
85.2 \\
82.7 \\
82.1 \\
83.2 \\
84.7 \\
85.7 \\
86.8 \\
88.4\end{array}$ & $\begin{array}{l}91.0 \\
94.6 \\
92.5 \\
89.0 \\
86.6 \\
85.6 \\
83.1 \\
82.4 \\
83.7 \\
85.2 \\
86.1 \\
87.3 \\
89.4\end{array}$ & $\begin{array}{l}90.2 \\
93.8 \\
92.1 \\
89.4 \\
85.4 \\
86.1 \\
84.2 \\
79.7 \\
80.5 \\
83.7 \\
84.0 \\
86.1 \\
89.0\end{array}$ & $\begin{array}{l}83.8 \\
90.4 \\
85.9 \\
80.8 \\
76.8 \\
75.6 \\
75.6 \\
78.0 \\
78.5 \\
77.5 \\
77.7 \\
77.5 \\
81.8\end{array}$ & $\begin{array}{l}88.4 \\
91.3 \\
88.3 \\
86.0 \\
85.1 \\
83.7 \\
82.1 \\
78.7 \\
81.1 \\
83.0 \\
83.6 \\
84.2 \\
87.0\end{array}$ & $\begin{array}{l}80.4 \\
86.6 \\
83.6 \\
78.2 \\
75.4 \\
73.1 \\
71.8 \\
72.8 \\
72.9 \\
74.0 \\
73.7 \\
77.3 \\
79.2\end{array}$ & $\begin{array}{l}87.3 \\
91.4 \\
89.4 \\
85.1 \\
83.3 \\
82.3 \\
81.6 \\
84.0 \\
84.7 \\
84.4 \\
85.8 \\
87.2 \\
88.5\end{array}$ & $\begin{array}{l}88.8 \\
92.3 \\
91.0 \\
88.3 \\
86.7 \\
84.6 \\
83.0 \\
83.8 \\
83.3 \\
83.9 \\
84.3 \\
85.3 \\
87.6\end{array}$ & $\begin{array}{l}92.1 \\
91.5 \\
91.2 \\
89.2 \\
84.4 \\
82.0 \\
80.5 \\
82.3 \\
83.1 \\
84.9 \\
86.0 \\
86.1 \\
89.4\end{array}$ & $\begin{array}{r}102.5 \\
105.5 \\
102.0 \\
93.7 \\
83.1 \\
W \\
W \\
81.9 \\
86.2 \\
95.5 \\
97.7 \\
101.3 \\
100.0\end{array}$ \\
\hline 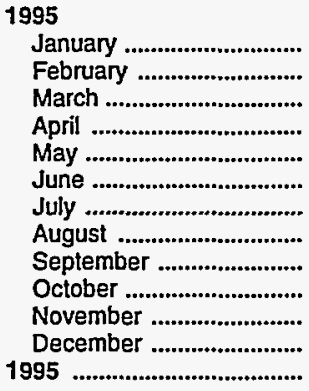 & $\begin{array}{l}87.4 \\
87.9 \\
87.4 \\
86.2 \\
86.4 \\
84.7 \\
82.0 \\
80.6 \\
82.3 \\
84.2 \\
86.6 \\
91.2 \\
87.1\end{array}$ & $\begin{array}{l}88.1 \\
88.7 \\
88.1 \\
86.7 \\
86.9 \\
85.4 \\
82.5 \\
81.1 \\
82.7 \\
84.8 \\
87.2 \\
92.1 \\
87.9\end{array}$ & $\begin{array}{l}86.7 \\
87.8 \\
87.0 \\
85.2 \\
86.5 \\
84.2 \\
79.4 \\
77.4 \\
79.2 \\
82.9 \\
85.6 \\
90.5 \\
86.3\end{array}$ & $\begin{array}{l}77.8 \\
77.4 \\
76.3 \\
76.7 \\
78.7 \\
78.0 \\
76.9 \\
76.6 \\
76.2 \\
75.8 \\
79.1 \\
87.0 \\
78.7\end{array}$ & $\begin{array}{l}84.8 \\
84.9 \\
83.7 \\
83.3 \\
85.4 \\
84.0 \\
80.6 \\
80.9 \\
81.8 \\
82.3 \\
83.8 \\
88.9 \\
84.7\end{array}$ & $\begin{array}{l}78.4 \\
78.5 \\
77.7 \\
76.6 \\
75.8 \\
74.5 \\
72.9 \\
73.1 \\
73.8 \\
73.9 \\
77.2 \\
83.8 \\
77.9\end{array}$ & $\begin{array}{l}87.3 \\
87.3 \\
87.0 \\
86.5 \\
86.1 \\
83.2 \\
81.7 \\
85.3 \\
84.5 \\
85.7 \\
87.4 \\
91.8 \\
87.3\end{array}$ & $\begin{array}{l}85.8 \\
85.9 \\
85.6 \\
84.8 \\
84.5 \\
83.7 \\
81.6 \\
81.7 \\
82.5 \\
82.5 \\
84.5 \\
88.0 \\
85.3\end{array}$ & $\begin{array}{l}88.5 \\
88.6 \\
87.6 \\
87.0 \\
85.2 \\
83.2 \\
80.0 \\
82.2 \\
82.4 \\
83.1 \\
84.5 \\
89.5 \\
87.0\end{array}$ & $\begin{array}{r}102.4 \\
103.4 \\
103.3 \\
100.0 \\
93.3 \\
\mathrm{NA} \\
85.1 \\
W \\
86.1 \\
\mathrm{NA} \\
100.2 \\
103.8 \\
101.0\end{array}$ \\
\hline 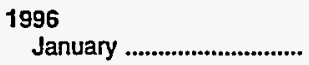 & 94.7 & 96.2 & 94.4 & 92.4 & 92.1 & 89.1 & 94.9 & 92.8 & 94.1 & 111.7 \\
\hline
\end{tabular}

See footnotes at end of table. 
Table 18. Prices of No. 2 Distillate to Residences by PAD District and Selected States

(Cents per Gallon Excluding Taxes) - Continued

\begin{tabular}{|c|c|c|c|c|c|c|c|c|c|c|}
\hline \multirow{2}{*}{$\begin{array}{l}\text { Year } \\
\text { Month }\end{array}$} & \multicolumn{6}{|c|}{ PAD District I (Continued) } & \multicolumn{4}{|c|}{ PAD District II } \\
\hline & MD & NJ & NY & PA & VA & WV & Average & IL & IN & MI \\
\hline $\begin{array}{l}1978 \\
1979 \\
1980 \\
1981 \\
1982 \\
1983 \\
1984 \\
1985 \\
1986 \ldots \ldots \ldots \ldots\end{array}$ & $\begin{array}{r}49.2 \\
70.1 \\
97.9 \\
121.4 \\
117.1 \\
110.3 \\
113.5 \\
108.8 \\
91.4 \\
86.6 \\
87.0 \\
93.8 \\
111.9 \\
108.4 \\
100.0 \\
98.1\end{array}$ & $\begin{array}{r}49.6 \\
71.0 \\
97.9 \\
121.5 \\
117.4 \\
107.9 \\
111.0 \\
105.9 \\
90.2 \\
84.3 \\
84.8 \\
91.8 \\
108.8 \\
104.0 \\
93.9 \\
92.4\end{array}$ & $\begin{array}{r}50.1 \\
71.2 \\
98.2 \\
123.2 \\
120.5 \\
112.1 \\
115.5 \\
111.3 \\
91.1 \\
85.2 \\
86.3 \\
95.8 \\
112.5 \\
111.3 \\
102.8 \\
100.1\end{array}$ & $\begin{array}{r}48.8 \\
69.8 \\
96.4 \\
118.1 \\
113.7 \\
105.8 \\
107.9 \\
102.3 \\
81.4 \\
76.9 \\
77.8 \\
85.1 \\
102.6 \\
99.7 \\
89.0 \\
86.3\end{array}$ & $\begin{array}{r}49.1 \\
70.4 \\
98.5 \\
120.5 \\
117.7 \\
108.7 \\
110.5 \\
106.3 \\
86.6 \\
79.5 \\
80.5 \\
87.0 \\
110.6 \\
101.1 \\
92.8 \\
89.3\end{array}$ & $\begin{array}{r}46.2 \\
65.1 \\
92.2 \\
115.0 \\
109.3 \\
101.0 \\
102.1 \\
98.0 \\
74.6 \\
76.4 \\
74.2 \\
83.0 \\
99.1 \\
93.4 \\
86.4 \\
85.6\end{array}$ & $\begin{array}{r}- \\
- \\
- \\
- \\
- \\
102.0 \\
101.7 \\
99.4 \\
72.7 \\
74.8 \\
74.3 \\
81.9 \\
97.8 \\
90.8 \\
82.9 \\
83.3\end{array}$ & $\begin{array}{r}46.5 \\
68.8 \\
95.8 \\
114.9 \\
110.9 \\
100.4 \\
100.1 \\
97.5 \\
\text { NA } \\
79.8 \\
77.6 \\
80.9 \\
96.1 \\
92.7 \\
87.7 \\
84.4\end{array}$ & $\begin{array}{r}48.5 \\
72.7 \\
99.6 \\
118.5 \\
114.3 \\
100.7 \\
103.1 \\
99.1 \\
74.8 \\
75.4 \\
75.4 \\
83.2 \\
99.3 \\
91.8 \\
81.2 \\
81.0\end{array}$ & $\begin{array}{r}47.9 \\
70.9 \\
97.8 \\
118.3 \\
113.9 \\
106.4 \\
105.0 \\
102.1 \\
81.0 \\
77.5 \\
77.5 \\
85.3 \\
100.9 \\
94.2 \\
87.2 \\
87.2\end{array}$ \\
\hline 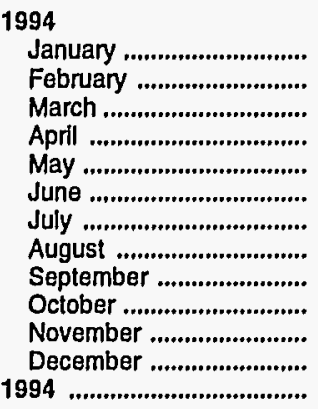 & $\begin{array}{l}98.8 \\
99.5 \\
96.3 \\
92.4 \\
86.8 \\
87.7 \\
87.8 \\
86.0 \\
87.8 \\
90.0 \\
92.4 \\
94.3 \\
95.0\end{array}$ & $\begin{array}{l}91.7 \\
94.8 \\
93.9 \\
90.7 \\
85.4 \\
83.5 \\
82.9 \\
85.9 \\
85.4 \\
86.8 \\
88.6 \\
89.6 \\
89.5\end{array}$ & $\begin{array}{r}97.2 \\
101.7 \\
100.3 \\
96.4 \\
96.3 \\
96.8 \\
93.9 \\
89.1 \\
90.8 \\
92.9 \\
93.3 \\
94.6 \\
96.6\end{array}$ & $\begin{array}{l}87.7 \\
92.5 \\
90.4 \\
86.2 \\
83.7 \\
80.1 \\
75.7 \\
77.9 \\
79.1 \\
80.2 \\
81.4 \\
82.0 \\
85.7\end{array}$ & $\begin{array}{l}88.6 \\
88.6 \\
86.6 \\
83.0 \\
82.2 \\
79.7 \\
79.6 \\
80.5 \\
80.4 \\
82.3 \\
84.1 \\
84.8 \\
85.3\end{array}$ & $\begin{array}{l}86.3 \\
86.3 \\
85.0 \\
77.8 \\
73.5 \\
72.4 \\
72.9 \\
74.8 \\
76.2 \\
79.3 \\
81.4 \\
81.3 \\
80.9\end{array}$ & $\begin{array}{l}80.4 \\
82.1 \\
82.0 \\
81.4 \\
80.6 \\
80.5 \\
80.2 \\
79.8 \\
80.0 \\
80.1 \\
80.9 \\
81.1 \\
80.8\end{array}$ & $\begin{array}{l}78.8 \\
82.2 \\
78.7 \\
76.1 \\
73.3 \\
75.5 \\
75.3 \\
77.2 \\
76.6 \\
77.6 \\
80.8 \\
80.4 \\
78.4\end{array}$ & $\begin{array}{l}79.1 \\
82.0 \\
81.0 \\
81.2 \\
79.9 \\
81.5 \\
80.0 \\
81.6 \\
82.6 \\
81.7 \\
81.2 \\
82.4 \\
81.2\end{array}$ & $\begin{array}{l}85.6 \\
88.0 \\
87.7 \\
87.7 \\
87.3 \\
86.9 \\
87.7 \\
84.3 \\
84.2 \\
85.2 \\
85.9 \\
86.1 \\
86.3\end{array}$ \\
\hline 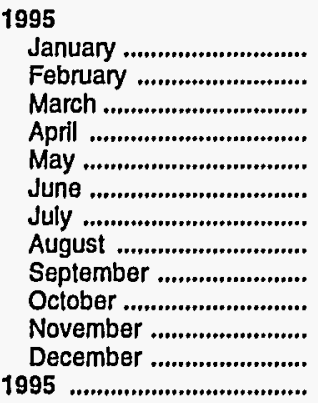 & $\begin{array}{l}94.2 \\
95.0 \\
94.2 \\
91.3 \\
89.6 \\
86.7 \\
83.2 \\
82.6 \\
85.5 \\
89.5 \\
93.1 \\
98.5 \\
93.6\end{array}$ & $\begin{array}{l}\text { NA } \\
\text { NA } \\
\text { NA } \\
\text { NA } \\
87.8 \\
87.4 \\
85.3 \\
81.9 \\
83.7 \\
85.0 \\
87.8 \\
94.1 \\
89.9\end{array}$ & $\begin{array}{l}95.6 \\
97.0 \\
97.0 \\
94.8 \\
96.0 \\
95.9 \\
92.9 \\
90.3 \\
91.1 \\
94.7 \\
96.3 \\
99.8 \\
96.3\end{array}$ & $\begin{array}{l}83.1 \\
83.4 \\
82.3 \\
80.9 \\
81.1 \\
79.5 \\
75.8 \\
75.5 \\
77.2 \\
79.5 \\
81.9 \\
87.2 \\
82.6\end{array}$ & $\begin{array}{l}84.9 \\
84.6 \\
84.0 \\
84.0 \\
83.0 \\
82.3 \\
81.2 \\
80.8 \\
81.6 \\
82.5 \\
83.8 \\
88.1 \\
84.4\end{array}$ & $\begin{array}{l}82.1 \\
82.3 \\
81.4 \\
80.2 \\
76.2 \\
77.3 \\
75.3 \\
74.3 \\
76.0 \\
77.1 \\
81.6 \\
89.4 \\
81.4\end{array}$ & $\begin{array}{l}81.0 \\
80.5 \\
80.6 \\
81.4 \\
81.5 \\
79.0 \\
77.6 \\
76.3 \\
79.4 \\
79.8 \\
81.5 \\
83.5 \\
80.9\end{array}$ & $\begin{array}{l}82.0 \\
80.8 \\
76.6 \\
81.5 \\
81.6 \\
77.0 \\
76.6 \\
72.9 \\
75.6 \\
74.6 \\
78.9 \\
82.9 \\
78.7\end{array}$ & $\begin{array}{l}81.7 \\
80.1 \\
82.3 \\
82.7 \\
83.9 \\
83.7 \\
82.0 \\
79.3 \\
81.0 \\
82.1 \\
79.3 \\
83.7 \\
81.7\end{array}$ & $\begin{array}{l}86.2 \\
85.8 \\
85.7 \\
86.3 \\
86.1 \\
83.5 \\
82.0 \\
82.1 \\
84.5 \\
83.9 \\
86.9 \\
88.7 \\
86.1\end{array}$ \\
\hline 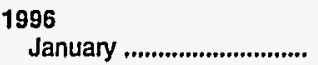 & 104.0 & 97.7 & 103.5 & 92.2 & 91.3 & 91.3 & 84.3 & 84.4 & 85.0 & 89.1 \\
\hline
\end{tabular}

See footnotes at end of table. 
Table 18. Prices of No. 2 Distillate to Residences by PAD District and Selected States

(Cents per Gallon Excluding Taxes) - Continued

\begin{tabular}{|c|c|c|c|c|c|c|c|c|c|c|}
\hline \multirow{2}{*}{$\begin{array}{l}\text { Year } \\
\text { Month }\end{array}$} & \multicolumn{3}{|c|}{ PAD District II (Continued) } & \multirow{2}{*}{$\begin{array}{c}\text { PAD } \\
\text { District III } \\
\text { Average }\end{array}$} & \multicolumn{2}{|c|}{ PAD District IV } & \multicolumn{4}{|c|}{ PAD District V } \\
\hline & MN & $\mathrm{OH}$ & WI & & Average & ID & Average & AK & OR & WA \\
\hline $\begin{array}{l}1978 \\
1979 \\
1980 \\
1981 \\
1982 \\
1983 \\
1984 \\
1985\end{array}$ & $\begin{array}{r}47.8 \\
72.4 \\
99.9 \\
118.4 \\
115.1 \\
103.1 \\
104.1 \\
101.9 \\
79.2 \\
74.6 \\
73.5 \\
82.4 \\
101.4 \\
91.1 \\
82.6 \\
83.2\end{array}$ & $\begin{array}{r}47.4 \\
68.6 \\
91.9 \\
113.2 \\
110.2 \\
101.3 \\
102.1 \\
99.7 \\
77.7 \\
74.7 \\
74.7 \\
81.6 \\
98.1 \\
91.0 \\
83.6 \\
84.0\end{array}$ & $\begin{array}{r}44.7 \\
67.3 \\
91.5 \\
109.1 \\
107.8 \\
101.2 \\
101.0 \\
98.3 \\
75.6 \\
75.1 \\
73.9 \\
81.1 \\
94.2 \\
89.5 \\
81.6 \\
82.3\end{array}$ & $\begin{array}{c}- \\
- \\
- \\
- \\
- \\
87.4 \\
97.9 \\
92.5 \\
67.4 \\
63.7 \\
62.8 \\
70.7 \\
85.5 \\
78.7 \\
71.3 \\
75.4\end{array}$ & $\begin{array}{c}- \\
- \\
- \\
- \\
- \\
94.2 \\
96.8 \\
96.1 \\
70.6 \\
69.6 \\
69.1 \\
78.6 \\
97.0 \\
92.4 \\
84.8 \\
86.0\end{array}$ & $\begin{array}{r}43.6 \\
62.1 \\
91.6 \\
110.4 \\
110.4 \\
101.8 \\
98.5 \\
97.2 \\
73.8 \\
68.8 \\
68.8 \\
77.8 \\
97.4 \\
95.1 \\
85.7 \\
86.2 \\
\end{array}$ & $\begin{array}{r}- \\
- \\
- \\
- \\
- \\
106.4 \\
101.5 \\
100.9 \\
78.1 \\
77.8 \\
76.9 \\
86.6 \\
102.6 \\
99.8 \\
92.1 \\
96.8\end{array}$ & $\begin{array}{r}53.2 \\
68.2 \\
97.8 \\
118.0 \\
117.4 \\
108.8 \\
106.9 \\
108.3 \\
94.9 \\
86.5 \\
86.9 \\
96.4 \\
110.1 \\
105.0 \\
94.1 \\
96.1\end{array}$ & $\begin{array}{r}45.8 \\
68.0 \\
97.3 \\
111.4 \\
111.6 \\
103.6 \\
99.3 \\
97.1 \\
70.4 \\
72.5 \\
70.9 \\
80.2 \\
97.0 \\
93.3 \\
87.6 \\
91.8\end{array}$ & $\begin{array}{r}48.6 \\
69.7 \\
100.8 \\
116.5 \\
117.6 \\
109.0 \\
102.6 \\
101.1 \\
77.5 \\
79.5 \\
78.5 \\
87.4 \\
102.9 \\
101.6 \\
94.0 \\
99.9\end{array}$ \\
\hline 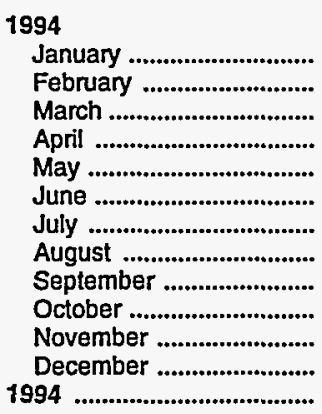 & $\begin{array}{l}80.5 \\
80.6 \\
80.0 \\
80.3 \\
79.9 \\
79.7 \\
79.8 \\
80.8 \\
81.2 \\
81.4 \\
81.2 \\
80.3 \\
80.6\end{array}$ & $\begin{array}{l}81.3 \\
84.2 \\
82.5 \\
82.7 \\
83.3 \\
82.2 \\
76.8 \\
76.0 \\
79.9 \\
79.8 \\
79.8 \\
81.1 \\
81.2\end{array}$ & $\begin{array}{l}79.9 \\
81.8 \\
82.4 \\
81.4 \\
80.8 \\
79.9 \\
81.4 \\
79.1 \\
79.8 \\
80.7 \\
80.9 \\
81.2 \\
81.1\end{array}$ & $\begin{array}{c}75.9 \\
79.3 \\
75.0 \\
\text { NA } \\
\text { NA } \\
\text { NA } \\
\text { NA } \\
71.6 \\
70.2 \\
\text { W } \\
\text { W } \\
W \\
71.4\end{array}$ & $\begin{array}{l}73.7 \\
74.0 \\
76.3 \\
75.3 \\
76.6 \\
76.8 \\
75.4 \\
82.2 \\
84.4 \\
86.2 \\
83.3 \\
85.4 \\
78.6\end{array}$ & $\begin{array}{l}73.2 \\
73.7 \\
77.4 \\
76.2 \\
76.9 \\
72.8 \\
74.6 \\
80.8 \\
83.1 \\
85.8 \\
84.8 \\
84.6 \\
78.9\end{array}$ & $\begin{array}{l}90.4 \\
92.5 \\
93.2 \\
92.7 \\
92.3 \\
88.8 \\
85.8 \\
80.6 \\
85.7 \\
90.6 \\
93.4 \\
92.2 \\
90.9\end{array}$ & $\begin{array}{l}88.8 \\
88.6 \\
89.2 \\
88.6 \\
90.0 \\
87.7 \\
88.2 \\
80.8 \\
83.4 \\
85.1 \\
86.6 \\
84.7 \\
86.5\end{array}$ & $\begin{array}{r}86.0 \\
88.3 \\
88.4 \\
88.1 \\
87.6 \\
85.1 \\
82.5 \\
\text { NA } \\
87.8 \\
91.1 \\
91.6 \\
89.4 \\
88.7\end{array}$ & $\begin{array}{r}92.8 \\
96.3 \\
97.1 \\
97.5 \\
96.2 \\
93.1 \\
\text { NA } \\
\text { NA } \\
90.2 \\
96.2 \\
99.0 \\
97.3 \\
95.0\end{array}$ \\
\hline 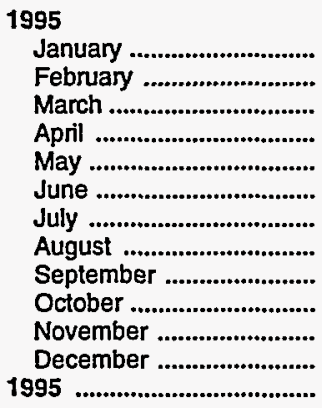 & $\begin{array}{l}80.1 \\
79.1 \\
80.4 \\
80.5 \\
80.5 \\
77.3 \\
76.5 \\
77.3 \\
79.5 \\
80.1 \\
80.5 \\
81.8 \\
80.1\end{array}$ & $\begin{array}{l}81.2 \\
80.9 \\
80.4 \\
81.9 \\
80.8 \\
78.8 \\
76.6 \\
72.6 \\
77.5 \\
79.0 \\
81.7 \\
84.0 \\
80.8\end{array}$ & $\begin{array}{l}81.1 \\
80.3 \\
80.4 \\
81.1 \\
81.5 \\
81.3 \\
81.0 \\
78.5 \\
80.7 \\
80.4 \\
81.6 \\
82.9 \\
81.2\end{array}$ & $\begin{array}{l}\text { NA } \\
W \\
76.1 \\
77.9 \\
79.2 \\
77.0 \\
W \\
69.5 \\
66.9 \\
65.7 \\
67.9 \\
69.5 \\
68.5\end{array}$ & $\begin{array}{l}80.7 \\
80.4 \\
80.7 \\
82.0 \\
84.0 \\
83.0 \\
81.7 \\
82.7 \\
87.0 \\
88.9 \\
88.9 \\
89.1 \\
84.2\end{array}$ & $\begin{array}{l}80.3 \\
79.7 \\
80.0 \\
81.0 \\
83.2 \\
82.8 \\
82.9 \\
83.5 \\
86.6 \\
88.8 \\
88.6 \\
88.8 \\
83.8\end{array}$ & $\begin{array}{l}90.6 \\
89.7 \\
90.6 \\
91.6 \\
89.1 \\
86.7 \\
85.4 \\
84.6 \\
87.4 \\
91.1 \\
92.6 \\
92.9 \\
90.5\end{array}$ & $\begin{array}{l}83.5 \\
84.0 \\
84.2 \\
82.8 \\
82.3 \\
82.7 \\
81.7 \\
81.7 \\
83.1 \\
83.5 \\
84.7 \\
84.2 \\
83.5\end{array}$ & $\begin{array}{r}88.5 \\
87.0 \\
88.8 \\
90.4 \\
91.5 \\
89.9 \\
\text { NA } \\
86.3 \\
87.1 \\
90.6 \\
92.3 \\
90.5 \\
89.4\end{array}$ & $\begin{array}{r}95.4 \\
94.8 \\
94.5 \\
\mathrm{NA} \\
\mathrm{NA} \\
\mathrm{NA} \\
94.0 \\
91.2 \\
95.5 \\
97.8 \\
99.2 \\
100.6 \\
96.0\end{array}$ \\
\hline $\begin{array}{l}1996 \\
\text { January ........................... }\end{array}$ & 82.5 & 85.5 & 83.1 & 70.9 & 88.3 & 87.6 & 92.5 & 84.1 & 90.1 & 99.7 \\
\hline
\end{tabular}

Dash $(-)=$ No data reported.

NA $=$ Not available.

$W=$ Withheld to avoid disclosure of individual company data.

Notes: Values shown for the current month are preliminary. Values shown for previous months are revised. Data are final upon publication in the

Petroleum Marketing Annual.

Sources: Energy Information Administration Forms ElA-782A, "Refiners'/Gas Plant Operators' Monthly Petroleum Product Sales Report," and ElA-782B,

"Resellers'/Retailers' Monthly Petroleum Product Sales Report," January 1983 forward; Form EIA-9A, "No. 2 Distillate Price Monitoring Report," source for backcast estimates prior to January 1983. 
Figure 6. U.S. No. 2 Distillate Prices to Residences by PAD District

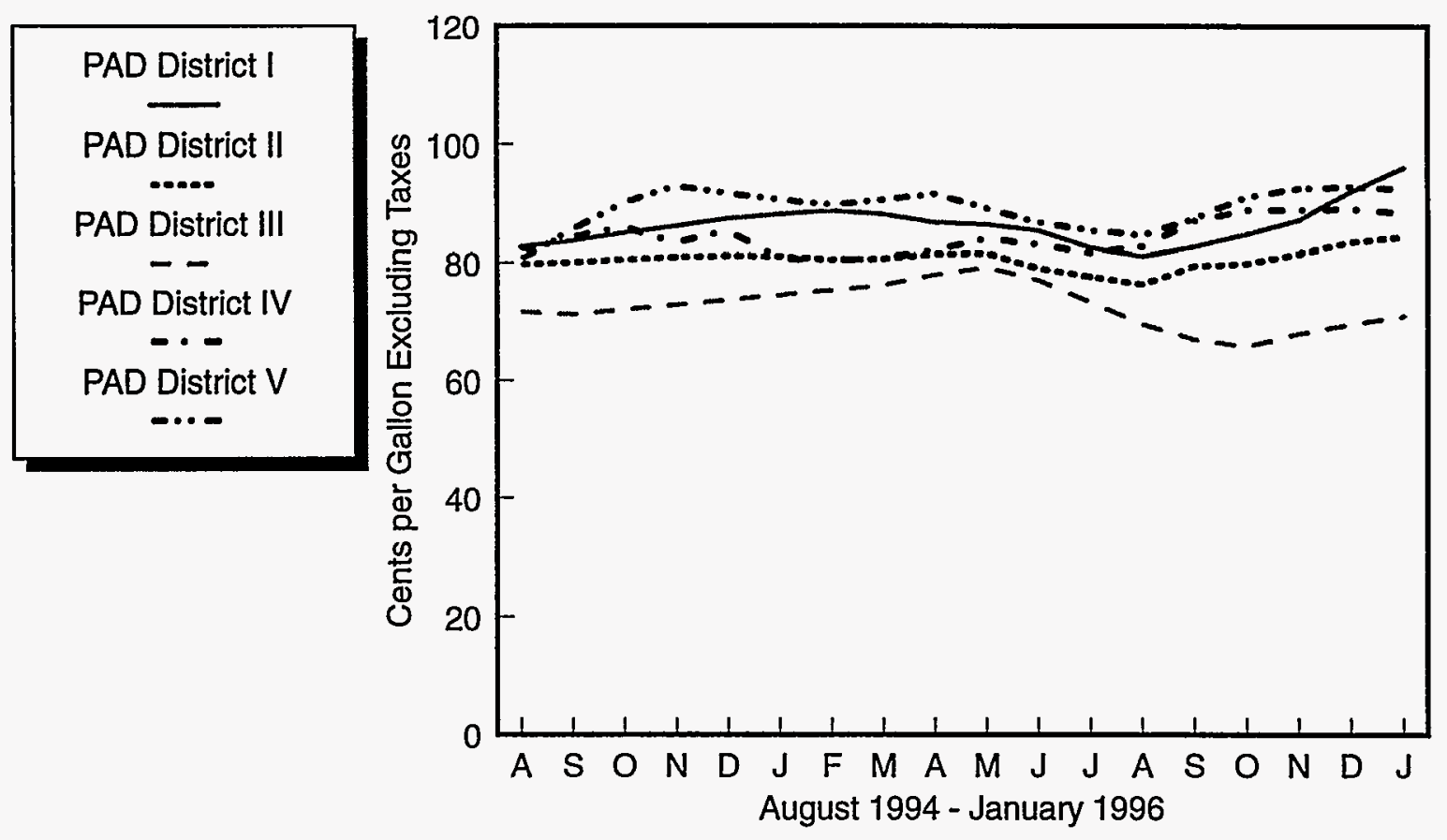

Source: Energy Information Administration, Form ElA-782A, "Refiners'/Gas Plant Operators' Monthly Petroleum Product Sales Report" and Form ElA-782B, "Resellers'/Retailers' Monthly Petroleum Product Sales Report." 
Table 19. U.S. Refiner Residual Fuel Oil Prices

(Cents per Gallon Excluding Taxes)

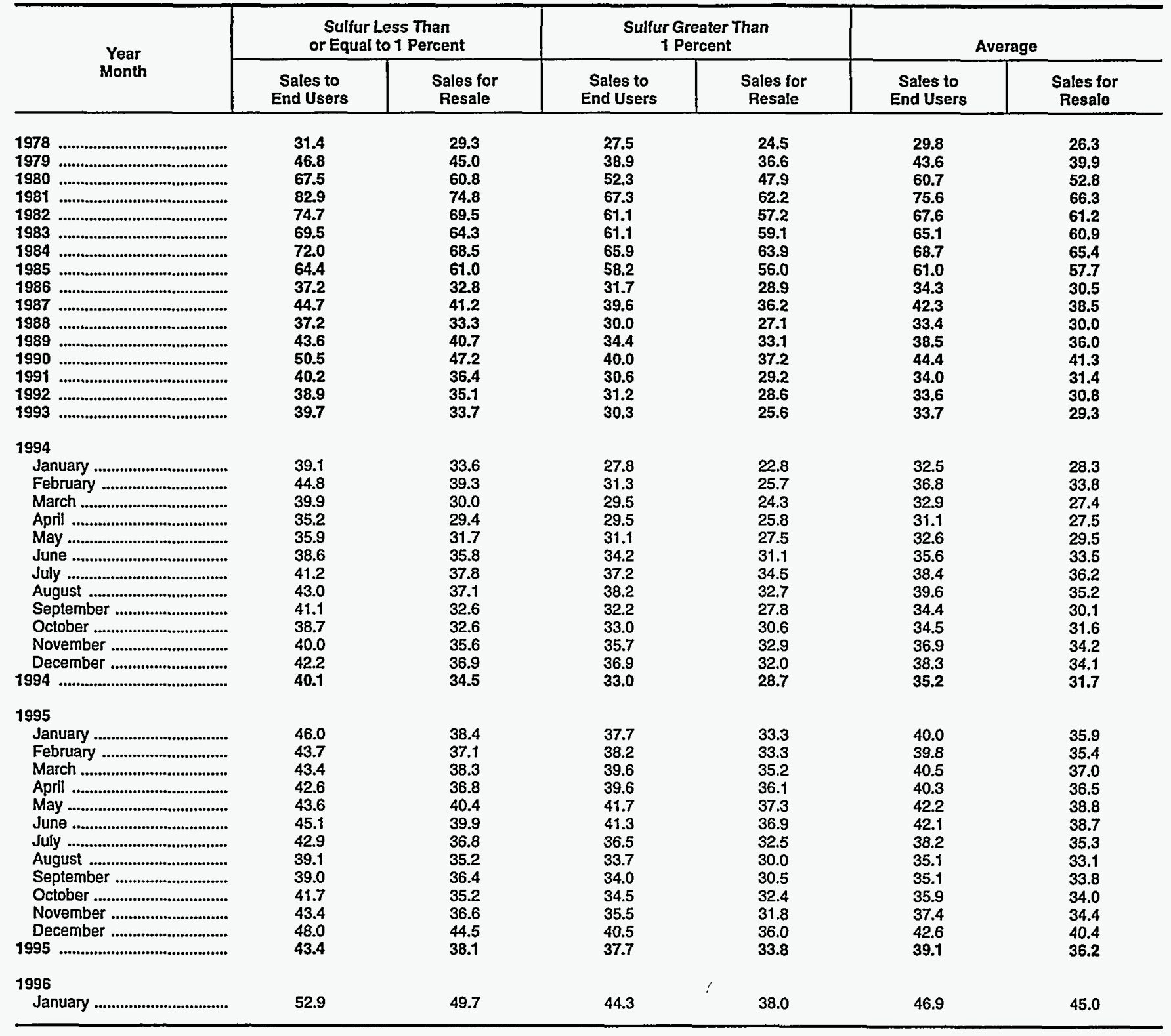

Notes: Values shown for the current month are preliminary. Values shown for previous months are revised. Data are final upon publication in the Petroleum Marketing Annual.

Sources: Energy Information Administration Form ElA-782A, "Refiners'/Gas Plant Operators' Monthly Petroleum Product Sales Report," January 1983 forward; Form ElA-460, "Petroleum Industry Monthly Report for Product Prices," source for backcast estimates prior to January 1983. 
Figure 7. U.S. Refiner Residual Fuel Oil Prices and Volumes

\section{Prices}

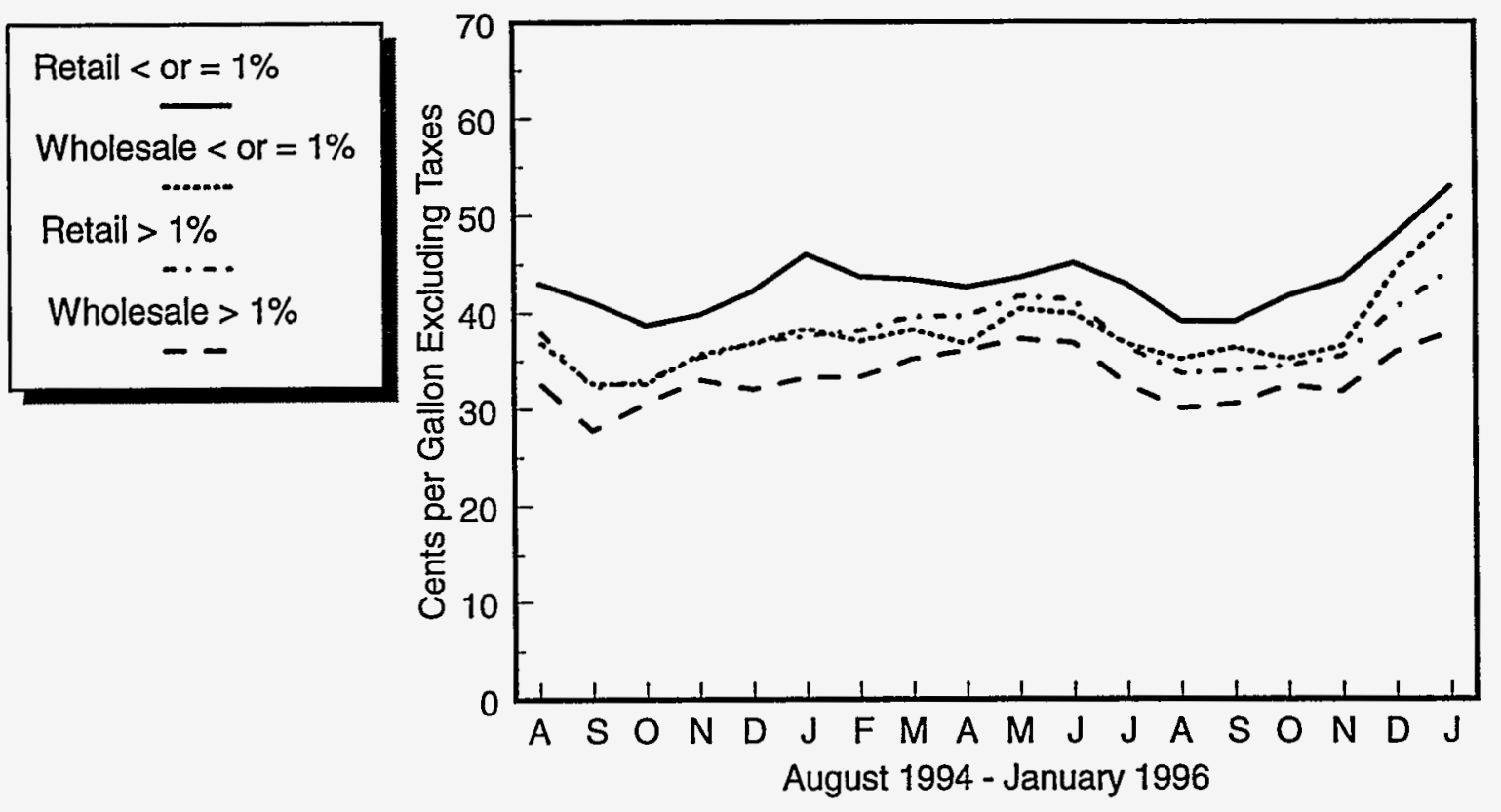

Volumes

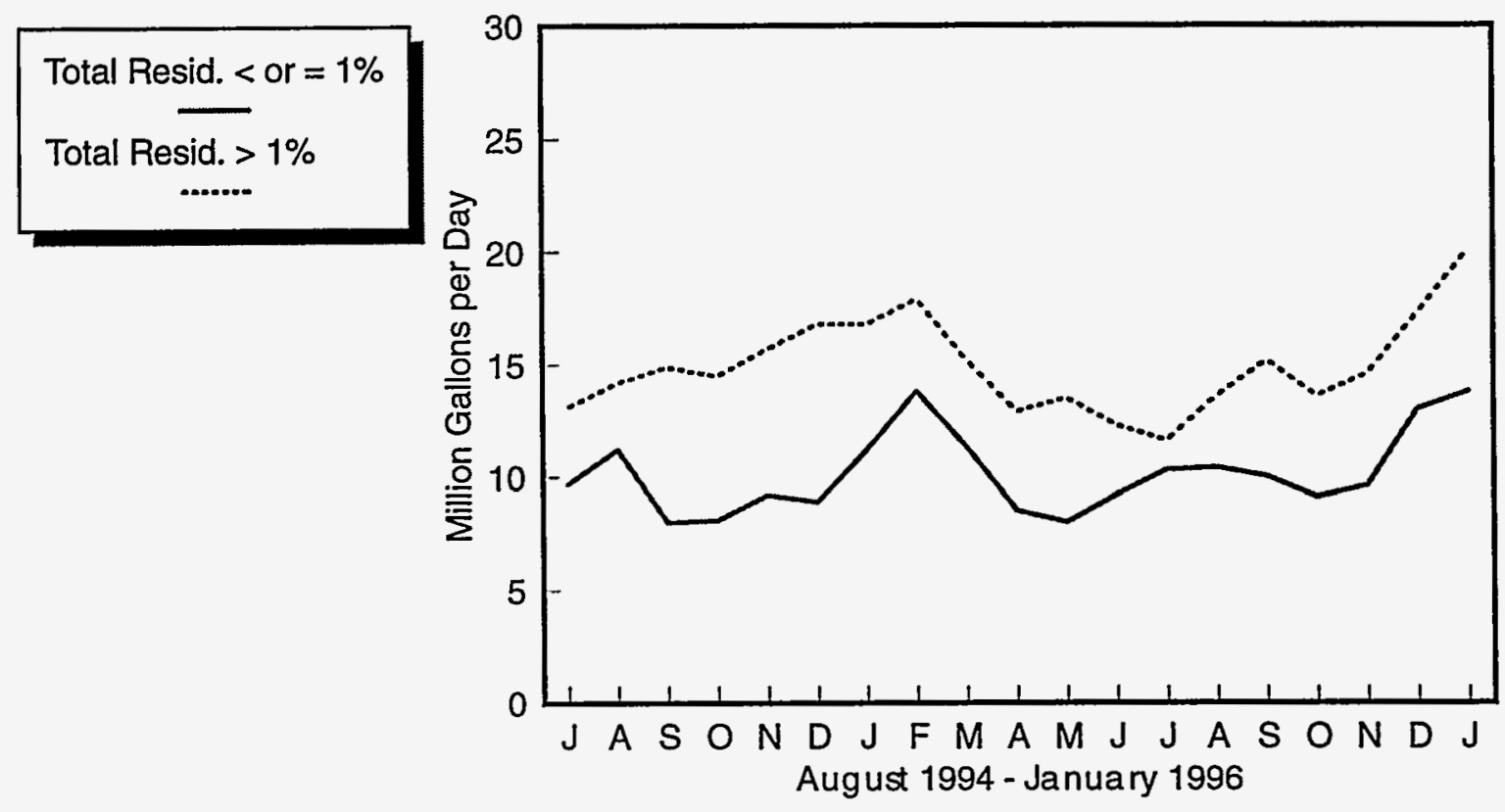

Source: Energy Information Administration, Form EIA-782A, "Refiners'/Gas Plant Operators' Monthly Petroleum Product Sales Report." 
Table 20. U.S. Refiner Residual Fuel Oil Volumes

(Million Gallons per Day)

\begin{tabular}{|c|c|c|c|c|c|c|}
\hline \multirow{2}{*}{$\begin{array}{l}\text { Year } \\
\text { Month }\end{array}$} & \multicolumn{2}{|c|}{$\begin{array}{l}\text { Sulfur Less Than } \\
\text { or Equal to } 1 \text { Percent }\end{array}$} & \multicolumn{2}{|c|}{$\begin{array}{c}\text { Sulfur Greater Than } \\
1 \text { Percent }\end{array}$} & \multicolumn{2}{|c|}{ Total } \\
\hline & $\begin{array}{l}\text { Sales to } \\
\text { End Users }\end{array}$ & $\begin{array}{l}\text { Sales for } \\
\text { Resale }\end{array}$ & $\begin{array}{l}\text { Sales to } \\
\text { End Users }\end{array}$ & $\begin{array}{l}\text { Sales for } \\
\text { Resale }\end{array}$ & $\begin{array}{l}\text { Sales to } \\
\text { End Users }\end{array}$ & $\begin{array}{l}\text { Sales for } \\
\text { Resalo }\end{array}$ \\
\hline $\begin{array}{l}1983 \\
1984 \\
1985 \\
1986 \\
1987 \\
1988 \\
1989 \\
1990 \\
1991 \\
1992\end{array}$ & $\begin{array}{r}13.4 \\
15.1 \\
11.4 \\
15.2 \\
15.1 \\
14.2 \\
13.4 \\
11.1 \\
8.6 \\
7.1 \\
6.2\end{array}$ & $\begin{array}{l}7.2 \\
6.7 \\
7.0 \\
7.9 \\
7.8 \\
8.7 \\
7.9 \\
7.4 \\
5.6 \\
5.2 \\
5.7\end{array}$ & $\begin{array}{l}14.9 \\
17.8 \\
13.8 \\
16.4 \\
13.9 \\
15.9 \\
17.0 \\
14.9 \\
15.5 \\
15.3 \\
11.1\end{array}$ & $\begin{array}{r}12.9 \\
14.6 \\
12.9 \\
10.7 \\
9.1 \\
10.3 \\
13.1 \\
10.5 \\
12.2 \\
10.0 \\
6.9\end{array}$ & $\begin{array}{l}28.3 \\
32.9 \\
25.2 \\
31.6 \\
29.0 \\
30.2 \\
30.4 \\
25.9 \\
24.0 \\
22.4 \\
17.2\end{array}$ & $\begin{array}{l}20.2 \\
21.3 \\
19.9 \\
18.6 \\
16.9 \\
18.9 \\
21.0 \\
17.9 \\
17.8 \\
15.2 \\
12.6\end{array}$ \\
\hline 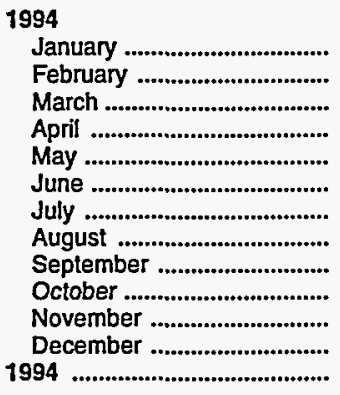 & $\begin{array}{l}7.2 \\
6.3 \\
5.0 \\
3.6 \\
3.9 \\
4.4 \\
3.4 \\
3.3 \\
3.1 \\
3.4 \\
3.5 \\
3.8 \\
4.2\end{array}$ & $\begin{array}{l}7.1 \\
9.6 \\
8.6 \\
6.1 \\
6.5 \\
6.4 \\
6.3 \\
7.9 \\
4.9 \\
4.7 \\
5.6 \\
5.1 \\
6.6\end{array}$ & $\begin{array}{r}10.3 \\
9.2 \\
10.2 \\
9.0 \\
8.3 \\
9.6 \\
8.1 \\
8.3 \\
9.3 \\
9.7 \\
9.3 \\
10.2 \\
9.3\end{array}$ & $\begin{array}{l}6.8 \\
6.4 \\
7.4 \\
6.7 \\
6.9 \\
6.3 \\
5.3 \\
5.9 \\
5.6 \\
4.8 \\
6.0 \\
6.6 \\
6.2\end{array}$ & $\begin{array}{l}17.5 \\
15.5 \\
15.2 \\
12.6 \\
12.2 \\
14.0 \\
11.5 \\
11.6 \\
12.5 \\
13.1 \\
12.9 \\
14.0 \\
13.5\end{array}$ & $\begin{array}{r}14.0 \\
16.0 \\
16.0 \\
12.8 \\
13.4 \\
12.7 \\
11.6 \\
13.8 \\
10.5 \\
9.6 \\
11.6 \\
11.7 \\
12.8\end{array}$ \\
\hline 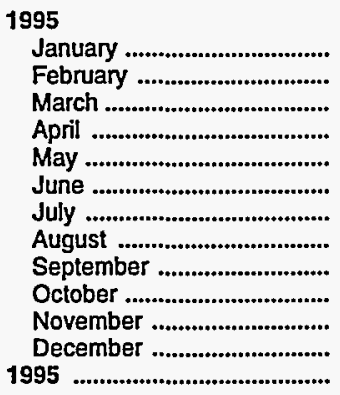 & $\begin{array}{l}3.6 \\
4.0 \\
2.9 \\
2.4 \\
2.6 \\
2.2 \\
2.7 \\
3.0 \\
2.5 \\
2.0 \\
2.8 \\
3.5 \\
2.9\end{array}$ & $\begin{array}{l}7.6 \\
9.8 \\
8.4 \\
6.1 \\
5.4 \\
7.0 \\
7.6 \\
7.4 \\
7.5 \\
7.1 \\
6.8 \\
9.5 \\
7.5\end{array}$ & $\begin{array}{r}9.3 \\
10.0 \\
9.1 \\
8.5 \\
7.9 \\
8.0 \\
7.4 \\
8.5 \\
9.3 \\
8.6 \\
8.9 \\
8.7 \\
8.7\end{array}$ & $\begin{array}{l}7.5 \\
7.9 \\
6.1 \\
4.4 \\
5.6 \\
4.3 \\
4.2 \\
5.1 \\
5.9 \\
5.0 \\
5.7 \\
8.6 \\
5.8\end{array}$ & $\begin{array}{l}13.0 \\
13.9 \\
12.0 \\
11.0 \\
10.5 \\
10.2 \\
10.1 \\
11.4 \\
11.8 \\
10.6 \\
11.8 \\
12.2 \\
11.5\end{array}$ & $\begin{array}{l}15.1 \\
17.7 \\
14.5 \\
10.5 \\
11.0 \\
11.3 \\
11.8 \\
12.5 \\
13.4 \\
12.1 \\
12.5 \\
18.2 \\
13.4\end{array}$ \\
\hline 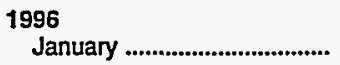 & 4.0 & 9.8 & 9.4 & 6.7 & 13.4 & 16.4 \\
\hline
\end{tabular}

Notes: Values shown for the current month are preliminary. Values shown for previous months are revised. Data are final upon publication in the Petroleum Marketing Annual. Totals may not equal the sum of the components due to rounding.

Source: Energy Information Administration Form ElA-782A, "Refiners'/Gas Plant Operators' Monthly Petroleum Product Sales Report." 
Crude Oil Prices 

Table 21. Domestic Crude Oil First Purchase Prices

(Dollars per Barrel)

\begin{tabular}{|c|c|c|c|c|c|c|c|c|c|c|c|c|c|}
\hline \multirow[b]{2}{*}{$\begin{array}{l}\text { Year } \\
\text { Month }\end{array}$} & \multicolumn{2}{|c|}{ U.S. Average } & \multicolumn{4}{|c|}{ PAD District I } & \multicolumn{7}{|c|}{ PAD District II } \\
\hline & $\begin{array}{c}\text { U.S. } \\
\text { Average }\end{array}$ & $\begin{array}{c}\text { Less } \\
\text { AK } \\
\text { North } \\
\text { Slope }\end{array}$ & Average & NY & PA & wV & Average & IL & IN & KS & $\mathbf{K Y}$ & MI & NE \\
\hline $\begin{array}{l}1978 \\
1979 \\
1980 \\
1981 \\
1982\end{array}$ & $\begin{array}{r}9.00 \\
12.64 \\
21.59 \\
31.77 \\
28.52 \\
26.19 \\
25.88 \\
24.09 \\
12.51 \\
15.40 \\
12.58 \\
15.86 \\
20.03 \\
16.54 \\
15.99 \\
14.25\end{array}$ & $\begin{array}{r}9.56 \\
13.01 \\
22.65 \\
33.71 \\
30.43 \\
28.00 \\
27.59 \\
25.74 \\
14.13 \\
16.83 \\
13.97 \\
17.13 \\
21.57 \\
18.16 \\
17.38 \\
15.31\end{array}$ & $\begin{array}{l}12.25 \\
14.30 \\
22.66 \\
35.22 \\
31.77 \\
28.32 \\
27.76 \\
25.88 \\
14.76 \\
16.52 \\
14.21 \\
17.16 \\
22.06 \\
19.01 \\
18.52 \\
17.28\end{array}$ & $\begin{array}{c}W \\
W \\
W \\
W \\
30.91 \\
28.19 \\
27.70 \\
25.19 \\
16.03 \\
17.40 \\
W \\
W \\
23.32 \\
19.67 \\
19.05 \\
17.20\end{array}$ & $\begin{array}{l}14.72 \\
24.13 \\
37.46 \\
36.03 \\
30.77 \\
28.28 \\
27.71 \\
25.05 \\
15.77 \\
17.43 \\
15.26 \\
18.15 \\
23.00 \\
19.48 \\
19.01 \\
17.53\end{array}$ & $\begin{array}{l}14.48 \\
23.35 \\
36.92 \\
35.63 \\
29.98 \\
27.27 \\
26.90 \\
24.35 \\
14.68 \\
16.56 \\
14.35 \\
17.18 \\
22.16 \\
W \\
18.09 \\
16.76\end{array}$ & $\begin{array}{l}11.91 \\
18.33 \\
30.12 \\
35.63 \\
31.65 \\
29.17 \\
28.62 \\
25.94 \\
14.30 \\
17.42 \\
14.65 \\
18.04 \\
22.88 \\
19.58 \\
18.63 \\
16.27\end{array}$ & $\begin{array}{l}13.81 \\
23.49 \\
35.78 \\
36.03 \\
31.69 \\
29.12 \\
28.76 \\
26.90 \\
14.70 \\
17.53 \\
14.79 \\
18.36 \\
23.36 \\
20.19 \\
19.26 \\
16.97\end{array}$ & $\begin{array}{l}13.70 \\
23.28 \\
35.91 \\
36.07 \\
31.66 \\
29.06 \\
28.72 \\
26.82 \\
14.55 \\
17.49 \\
14.78 \\
18.37 \\
23.46 \\
20.20 \\
19.27 \\
16.97\end{array}$ & $\begin{array}{l}13.20 \\
21.57 \\
34.20 \\
35.80 \\
30.79 \\
28.45 \\
27.99 \\
25.33 \\
14.02 \\
17.37 \\
14.55 \\
18.18 \\
23.21 \\
19.84 \\
18.50 \\
15.95\end{array}$ & $\begin{array}{l}13.73 \\
22.55 \\
34.98 \\
35.90 \\
31.19 \\
28.60 \\
28.24 \\
26.20 \\
14.20 \\
17.19 \\
14.58 \\
18.36 \\
23.20 \\
19.84 \\
18.75 \\
16.51\end{array}$ & $\begin{array}{l}11.86 \\
14.47 \\
23.54 \\
35.74 \\
31.80 \\
28.93 \\
28.54 \\
26.16 \\
14.61 \\
17.63 \\
14.92 \\
18.06 \\
22.92 \\
19.88 \\
18.99 \\
16.79\end{array}$ & $\begin{array}{l}11.40 \\
18.13 \\
30.60 \\
35.32 \\
30.98 \\
28.58 \\
27.83 \\
25.42 \\
13.70 \\
17.08 \\
14.08 \\
17.36 \\
21.94 \\
18.78 \\
17.51 \\
14.90\end{array}$ \\
\hline 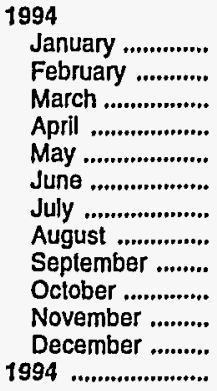 & $\begin{array}{l}10.49 \\
10.71 \\
10.94 \\
12.31 \\
14.02 \\
14.93 \\
15.34 \\
14.50 \\
13.62 \\
13.84 \\
14.14 \\
13.43 \\
13.19\end{array}$ & $\begin{array}{l}11.78 \\
11.79 \\
11.91 \\
13.40 \\
15.12 \\
16.13 \\
16.50 \\
15.47 \\
14.66 \\
14.92 \\
15.24 \\
14.43 \\
14.30\end{array}$ & $\begin{array}{l}14.13 \\
14.04 \\
13.93 \\
15.07 \\
16.58 \\
17.95 \\
18.76 \\
17.59 \\
16.45 \\
16.45 \\
16.88 \\
16.23 \\
16.23\end{array}$ & $\begin{array}{l}13.87 \\
14.13 \\
13.88 \\
14.20 \\
15.58 \\
17.34 \\
18.35 \\
17.61 \\
16.24 \\
15.90 \\
16.37 \\
16.03 \\
15.94\end{array}$ & $\begin{array}{l}14.32 \\
14.51 \\
14.31 \\
14.69 \\
16.00 \\
17.59 \\
18.50 \\
17.68 \\
16.42 \\
16.09 \\
16.52 \\
16.21 \\
16.13\end{array}$ & $\begin{array}{l}13.56 \\
13.75 \\
13.56 \\
14.31 \\
15.56 \\
17.09 \\
18.06 \\
17.16 \\
16.10 \\
15.81 \\
16.21 \\
15.90 \\
15.68\end{array}$ & $\begin{array}{l}12.75 \\
12.50 \\
12.41 \\
14.12 \\
15.93 \\
17.17 \\
17.67 \\
16.18 \\
15.33 \\
15.58 \\
15.91 \\
15.03 \\
15.05\end{array}$ & $\begin{array}{l}13.39 \\
13.11 \\
13.07 \\
14.87 \\
16.70 \\
17.95 \\
18.39 \\
16.86 \\
15.99 \\
16.28 \\
16.54 \\
15.67 \\
15.76\end{array}$ & $\begin{array}{l}13.35 \\
13.02 \\
13.04 \\
14.78 \\
16.57 \\
17.79 \\
18.24 \\
16.75 \\
15.84 \\
16.12 \\
16.40 \\
15.54 \\
15.66\end{array}$ & $\begin{array}{l}12.33 \\
12.10 \\
12.01 \\
13.83 \\
15.57 \\
16.83 \\
17.33 \\
15.85 \\
14.97 \\
15.27 \\
15.61 \\
14.73 \\
14.71\end{array}$ & $\begin{array}{l}13.05 \\
12.67 \\
12.77 \\
14.49 \\
16.17 \\
17.45 \\
17.84 \\
16.23 \\
15.42 \\
15.65 \\
15.96 \\
15.10 \\
15.33\end{array}$ & $\begin{array}{l}13.21 \\
12.88 \\
12.85 \\
14.57 \\
16.37 \\
17.61 \\
18.10 \\
16.53 \\
15.71 \\
15.95 \\
16.29 \\
15.35 \\
15.43\end{array}$ & $\begin{array}{l}11.19 \\
10.92 \\
10.88 \\
12.67 \\
14.52 \\
15.76 \\
16.32 \\
14.81 \\
13.91 \\
14.22 \\
14.55 \\
13.65 \\
13.60\end{array}$ \\
\hline 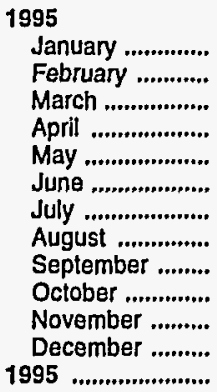 & $\begin{array}{l}14.00 \\
14.69 \\
14.68 \\
15.84 \\
15.85 \\
15.02 \\
14.01 \\
14.13 \\
14.49 \\
13.68 \\
14.03 \\
15.02 \\
14.62\end{array}$ & $\begin{array}{l}15.20 \\
15.79 \\
15.70 \\
16.95 \\
16.98 \\
15.97 \\
14.85 \\
15.30 \\
15.54 \\
14.84 \\
15.20 \\
16.29 \\
15.72\end{array}$ & $\begin{array}{l}16.69 \\
17.23 \\
17.27 \\
18.27 \\
18.36 \\
17.58 \\
16.27 \\
16.48 \\
16.75 \\
16.48 \\
16.92 \\
17.67 \\
17.18\end{array}$ & $\begin{array}{l}16.13 \\
16.61 \\
16.94 \\
17.61 \\
17.92 \\
17.58 \\
16.13 \\
16.00 \\
16.13 \\
16.43 \\
16.67 \\
16.15 \\
16.74\end{array}$ & $\begin{array}{l}16.34 \\
16.69 \\
17.05 \\
17.73 \\
18.02 \\
17.71 \\
16.23 \\
16.18 \\
16.26 \\
16.50 \\
16.88 \\
17.35 \\
16.93\end{array}$ & $\begin{array}{l}16.04 \\
16.39 \\
16.74 \\
17.35 \\
17.60 \\
17.30 \\
15.86 \\
15.81 \\
15.98 \\
16.24 \\
16.20 \\
16.49 \\
16.53\end{array}$ & $\begin{array}{l}15.95 \\
16.65 \\
16.47 \\
17.78 \\
17.52 \\
16.37 \\
15.21 \\
15.87 \\
16.17 \\
15.47 \\
15.99 \\
17.22 \\
16.39\end{array}$ & $\begin{array}{l}16.59 \\
17.25 \\
17.09 \\
18.37 \\
18.01 \\
16.80 \\
15.74 \\
16.42 \\
16.73 \\
16.12 \\
16.52 \\
17.70 \\
16.95\end{array}$ & $\begin{array}{l}16.49 \\
17.11 \\
16.99 \\
18.30 \\
18.01 \\
16.76 \\
15.64 \\
16.26 \\
16.59 \\
16.40 \\
16.46 \\
17.56 \\
16.88\end{array}$ & $\begin{array}{l}15.68 \\
16.41 \\
16.24 \\
17.58 \\
17.33 \\
16.14 \\
14.97 \\
15.72 \\
16.01 \\
15.25 \\
15.83 \\
17.17 \\
16.19\end{array}$ & $\begin{array}{l}16.03 \\
16.74 \\
16.60 \\
17.91 \\
17.52 \\
16.24 \\
15.17 \\
15.81 \\
16.17 \\
15.80 \\
16.03 \\
17.21 \\
16.43\end{array}$ & $\begin{array}{l}16.28 \\
17.02 \\
16.82 \\
18.02 \\
17.80 \\
16.60 \\
15.44 \\
16.07 \\
16.35 \\
15.59 \\
16.16 \\
17.28 \\
16.63\end{array}$ & $\begin{array}{l}14.60 \\
15.33 \\
15.15 \\
16.63 \\
16.44 \\
15.27 \\
14.08 \\
14.71 \\
14.95 \\
14.15 \\
14.77 \\
16.06 \\
15.18\end{array}$ \\
\hline $\begin{array}{l}1996 \\
\text { January .............. }\end{array}$ & 15.43 & 16.45 & 17.80 & 17.72 & 17.63 & 17.02 & 17.05 & 17.49 & 17.26 & 17.03 & 16.78 & 17.19 & 15.91 \\
\hline
\end{tabular}

See footnotes at end of table. 
Table 21. Domestic Crude Oil First Purchase Prices

(Dollars per Barrel) - Continued

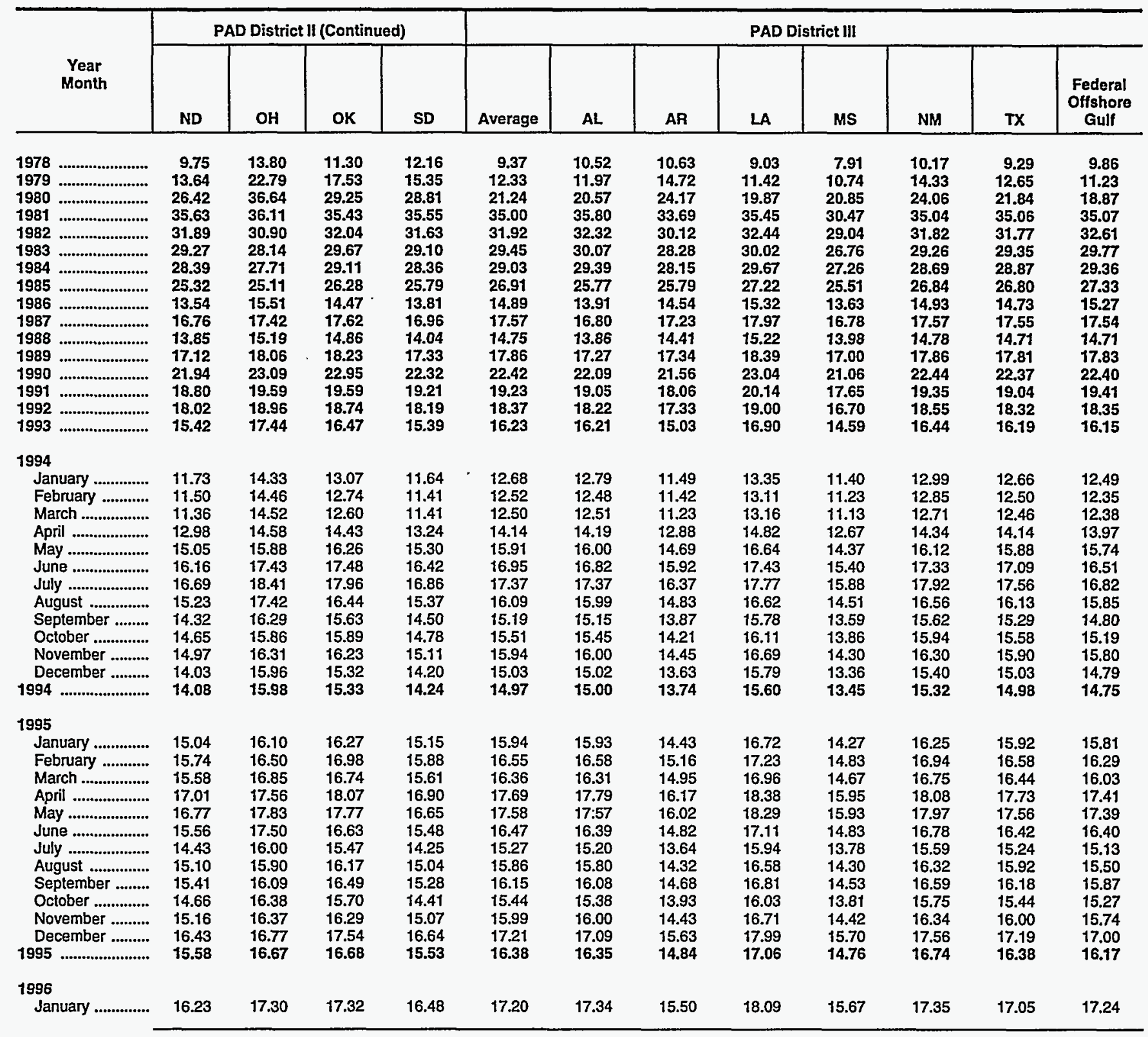

See footnotes at end of table. 
Table 21. Domestic Crude Oil First Purchase Prices

(Dollars per Barrel) - Continued

\begin{tabular}{|c|c|c|c|c|c|c|c|c|c|c|}
\hline \multirow[b]{2}{*}{$\begin{array}{l}\text { Year } \\
\text { Month }\end{array}$} & \multicolumn{5}{|c|}{ PAD District IV } & \multicolumn{5}{|c|}{ PAD District V } \\
\hline & Average & $\mathrm{Co}$ & MT & UT & WY & Average & $\begin{array}{c}\text { AK } \\
\text { North } \\
\text { Slope }\end{array}$ & $\begin{array}{c}\text { AK } \\
\text { Other }\end{array}$ & CA & $\begin{array}{c}\text { Federal } \\
\text { Offshore } \\
\text { California }\end{array}$ \\
\hline $\begin{array}{l}1978 \\
1979 \\
1980 \\
1981 \\
1982\end{array}$ & $\begin{array}{r}9.50 \\
11.98 \\
21.29 \\
33.38 \\
30.14 \\
27.81 \\
27.18 \\
24.78 \\
13.24 \\
16.77 \\
13.85 \\
17.37 \\
21.68 \\
18.21 \\
17.27 \\
15.30\end{array}$ & $\begin{array}{l}10.84 \\
13.28 \\
22.91 \\
35.69 \\
31.56 \\
28.92 \\
28.09 \\
25.64 \\
13.98 \\
17.71 \\
14.83 \\
18.86 \\
23.16 \\
19.95 \\
19.04 \\
16.59\end{array}$ & $\begin{array}{r}9.04 \\
12.03 \\
20.53 \\
34.69 \\
31.25 \\
28.80 \\
28.07 \\
25.29 \\
13.58 \\
16.57 \\
13.84 \\
17.03 \\
21.61 \\
18.17 \\
17.08 \\
14.70\end{array}$ & $\begin{array}{r}9.98 \\
11.41 \\
19.79 \\
34.14 \\
30.50 \\
28.12 \\
27.21 \\
23.98 \\
13.33 \\
17.22 \\
14.24 \\
18.63 \\
22.61 \\
19.99 \\
19.39 \\
17.48\end{array}$ & $\begin{array}{r}9.16 \\
11.73 \\
21.34 \\
32.30 \\
29.37 \\
27.19 \\
26.73 \\
24.67 \\
12.94 \\
16.45 \\
13.47 \\
16.73 \\
21.04 \\
17.33 \\
16.38 \\
14.59\end{array}$ & $\begin{array}{r}6.60 \\
11.17 \\
19.09 \\
24.79 \\
21.84 \\
19.66 \\
19.52 \\
19.11 \\
8.53 \\
11.89 \\
9.28 \\
12.71 \\
16.15 \\
12.30 \\
12.32 \\
11.23\end{array}$ & $\begin{array}{r}5.21 \\
10.57 \\
16.87 \\
23.23 \\
19.92 \\
17.69 \\
17.91 \\
16.98 \\
6.45 \\
10.83 \\
8.43 \\
12.00 \\
15.23 \\
11.57 \\
11.73 \\
10.84\end{array}$ & $\begin{array}{r}5.45 \\
6.22 \\
10.25 \\
30.15 \\
27.74 \\
23.59 \\
24.37 \\
22.46 \\
13.13 \\
15.48 \\
12.92 \\
16.17 \\
21.16 \\
15.36 \\
15.56 \\
14.11\end{array}$ & $\begin{array}{r}8.58 \\
12.78 \\
23.87 \\
26.80 \\
24.58 \\
22.61 \\
22.09 \\
22.14 \\
11.90 \\
13.92 \\
10.97 \\
14.06 \\
17.81 \\
13.72 \\
13.55 \\
12.11\end{array}$ & $\begin{array}{r}6.10 \\
8.06 \\
16.28 \\
24.56 \\
23.37 \\
20.54 \\
20.41 \\
20.08 \\
9.48 \\
11.82 \\
9.41 \\
12.64 \\
16.21 \\
11.33 \\
10.78 \\
9.20\end{array}$ \\
\hline $\begin{array}{l}1994 \\
\text { January .............. } \\
\text { February ............ } \\
\text { March ................ } \\
\text { April .................... } \\
\text { May .................. } \\
\text { June .................. } \\
\text { July ................... } \\
\text { August .............. } \\
\text { September ........ } \\
\text { Oclober .............. } \\
\text { November .......... } \\
\text { December .......... } \\
1994 \text {....................... }\end{array}$ & $\begin{array}{l}11.82 \\
11.62 \\
11.61 \\
13.21 \\
15.11 \\
16.32 \\
16.89 \\
15.47 \\
14.66 \\
15.01 \\
15.27 \\
14.31 \\
14.27\end{array}$ & $\begin{array}{l}13.12 \\
12.87 \\
12.78 \\
14.44 \\
16.23 \\
17.48 \\
18.02 \\
16.66 \\
15.77 \\
16.10 \\
16.47 \\
15.49 \\
15.44\end{array}$ & $\begin{array}{l}11.04 \\
10.80 \\
10.75 \\
12.38 \\
14.24 \\
15.49 \\
16.03 \\
14.62 \\
13.77 \\
14.07 \\
14.42 \\
13.50 \\
13.43\end{array}$ & $\begin{array}{l}14.13 \\
13.89 \\
13.73 \\
15.39 \\
17.13 \\
18.43 \\
18.95 \\
17.57 \\
16.69 \\
17.00 \\
17.29 \\
16.37 \\
16.38\end{array}$ & $\begin{array}{l}11.12 \\
10.93 \\
11.04 \\
12.59 \\
14.54 \\
15.72 \\
16.31 \\
14.89 \\
14.10 \\
14.48 \\
14.68 \\
13.71 \\
13.67\end{array}$ & $\begin{array}{r}7.31 \\
8.15 \\
8.65 \\
9.56 \\
11.26 \\
11.90 \\
12.27 \\
12.11 \\
11.26 \\
11.48 \\
11.54 \\
11.23 \\
10.53\end{array}$ & $\begin{array}{r}6.67 \\
7.38 \\
8.01 \\
8.83 \\
10.70 \\
11.19 \\
11.72 \\
11.40 \\
10.32 \\
10.63 \\
10.67 \\
10.47 \\
9.77\end{array}$ & $\begin{array}{r}9.37 \\
11.17 \\
11.66 \\
13.35 \\
15.29 \\
15.76 \\
15.15 \\
15.36 \\
14.99 \\
14.76 \\
14.76 \\
14.13 \\
13.87\end{array}$ & $\begin{array}{r}8.67 \\
9.58 \\
9.99 \\
10.88 \\
12.47 \\
13.30 \\
13.49 \\
13.61 \\
13.14 \\
13.27 \\
13.37 \\
12.95 \\
12.12\end{array}$ & $\begin{array}{r}6.43 \\
7.25 \\
7.69 \\
8.41 \\
9.58 \\
10.30 \\
10.47 \\
10.49 \\
9.82 \\
10.10 \\
9.93 \\
9.57 \\
9.32\end{array}$ \\
\hline 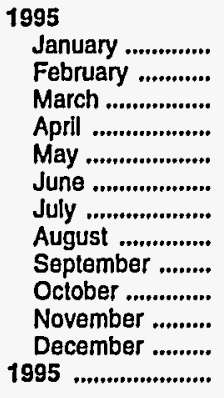 & $\begin{array}{l}15.32 \\
16.05 \\
15.80 \\
17.16 \\
17.09 \\
15.91 \\
14.81 \\
15.39 \\
15.71 \\
15.07 \\
15.70 \\
16.80 \\
15.90\end{array}$ & $\begin{array}{l}16.40 \\
17.02 \\
16.84 \\
17.81 \\
17.95 \\
16.67 \\
15.58 \\
16.17 \\
16.50 \\
15.70 \\
16.28 \\
17.30 \\
16.67\end{array}$ & $\begin{array}{l}14.50 \\
15.21 \\
14.96 \\
16.26 \\
16.11 \\
14.92 \\
13.73 \\
14.39 \\
14.71 \\
14.17 \\
14.54 \\
15.77 \\
14.93\end{array}$ & $\begin{array}{l}17.22 \\
17.87 \\
17.74 \\
19.16 \\
18.96 \\
17.77 \\
16.54 \\
16.84 \\
17.25 \\
16.82 \\
17.50 \\
18.55 \\
17.71\end{array}$ & $\begin{array}{l}14.77 \\
15.55 \\
15.26 \\
16.73 \\
16.65 \\
15.55 \\
14.50 \\
15.09 \\
15.40 \\
14.67 \\
15.32 \\
16.44 \\
15.50\end{array}$ & $\begin{array}{l}11.28 \\
12.01 \\
12.24 \\
13.17 \\
13.37 \\
12.92 \\
12.16 \\
11.61 \\
12.05 \\
11.20 \\
11.20 \\
11.85 \\
12.08\end{array}$ & $\begin{array}{l}10.43 \\
11.31 \\
11.48 \\
12.36 \\
12.32 \\
11.95 \\
11.19 \\
10.23 \\
10.89 \\
10.03 \\
10.25 \\
10.97 \\
11.12\end{array}$ & $\begin{array}{l}14.35 \\
14.80 \\
15.33 \\
16.73 \\
17.25 \\
16.55 \\
15.23 \\
15.20 \\
15.35 \\
14.33 \\
13.65 \\
14.76 \\
15.28\end{array}$ & $\begin{array}{l}13.07 \\
13.55 \\
13.91 \\
14.77 \\
15.32 \\
14.93 \\
14.08 \\
14.08 \\
14.15 \\
13.49 \\
13.10 \\
13.61 \\
14.00\end{array}$ & $\begin{array}{r}9.97 \\
10.41 \\
10.78 \\
11.95 \\
12.60 \\
11.61 \\
10.51 \\
10.71 \\
10.83 \\
10.01 \\
9.91 \\
10.57 \\
10.81\end{array}$ \\
\hline $\begin{array}{l}1996 \\
\text { January ............... }\end{array}$ & 16.74 & 17.32 & 15.73 & 18.37 & 16.38 & 12.92 & 12.13 & 15.67 & 14.53 & 11.27 \\
\hline
\end{tabular}

$W=$ Withheld to avold disclosure of individual company data.

Notes: The actual domestlc average price represents the average price at the lease (or wellhead) at which domestic crude oil is purchased.

Notes: Values shown for the current month are preliminary. Values shown for previous months are revised. Data are final upon publication in the Petroleum Marketing Annual.

Sources: Energy Information Administration, Form ERA-182, "Domestic Crude Oil First Purchaser's Report," January 1978 through December 1982 ; Form ElA-182, "Domestic Crude Oll First Purchase Report," January 1983 to present. 
Table 22. Domestic Crude Oil First Purchase Prices for Selected Crude Streams (Dollars per Barrel) .

\begin{tabular}{|c|c|c|c|c|c|c|c|c|c|}
\hline $\begin{array}{l}\text { Year } \\
\text { Month }\end{array}$ & $\begin{array}{l}\text { Alaska } \\
\text { North } \\
\text { Slope }\end{array}$ & $\begin{array}{l}\text { Californla } \\
\text { Midway- } \\
\text { Sunset }\end{array}$ & $\begin{array}{c}\text { Calffornia } \\
\text { Kem } \\
\text { River }\end{array}$ & $\begin{array}{l}\text { Califormia } \\
\text { Wilmington }\end{array}$ & $\begin{array}{l}\text { Loulsiana } \\
\text { South } \\
\text { Mix }\end{array}$ & $\begin{array}{c}\text { North } \\
\text { Dakota } \\
\text { Sweet }\end{array}$ & $\begin{array}{c}\text { West } \\
\text { Texas } \\
\text { Intermediate }\end{array}$ & $\begin{array}{l}\text { West } \\
\text { Texas } \\
\text { Sour }\end{array}$ & $\begin{array}{l}\text { Wyoming } \\
\text { Sour }\end{array}$ \\
\hline \multicolumn{10}{|l|}{1994} \\
\hline 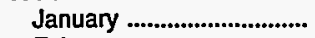 & 6.67 & 8.20 & 8.27 & 9.10 & 13.56 & 12.21 & 13.40 & 11.77 & 9.29 \\
\hline 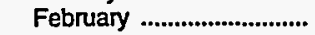 & 7.38 & 8.90 & 9.00 & 10.08 & 13.32 & 11.92 & 13.17 & 11.65 & 9.07 \\
\hline March ................................... & 8.01 & 9.29 & 9.43 & 10.38 & 13.29 & 11.82 & 13.04 & 11.61 & 9.16 \\
\hline April & 8.83 & 10.22 & 10.31 & 11.40 & $\$ 4.91$ & 13.38 & 14.76 & 13.24 & 10.87 \\
\hline May ..................................... & 10.70 & 11.50 & 11.72 & 12.82 & 16.69 & 15.52 & 16.49 & 15.01 & 12.73 \\
\hline June .................................... & 11.19 & 12.49 & 12.72 & 13.66 & 17.41 & 16.62 & 17.74 & 16.18 & 13.97 \\
\hline July ....................................... & 11.72 & 12.92 & 13.09 & 13.86 & 17.78 & 17.16 & 18.27 & 16.66 & 14.54 \\
\hline 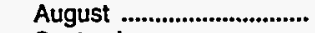 & 11.40 & 13.1 .1 & 13.25 & 14.03 & 16.72 & 15.74 & 16.85 & 15.26 & 12.99 \\
\hline September ......................... & 10.32 & 12.79 & 12.91 & 13.61 & 15.88 & 14.81 & 15.99 & 14.39 & 12.29 \\
\hline 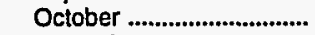 & 10.63 & 12.82 & 12.61 & 13.67 & 16.21 & 15.11 & 16.27 & 14.71 & 12.61 \\
\hline November .......................... & 10.67 & 12.79 & 12.94 & 13.63 & 16.76 & 15.46 & 16.62 & 15.08 & 12.92 \\
\hline December & $\begin{array}{r}10.47 \\
9.77\end{array}$ & $\begin{array}{r}12.33 \\
11.79\end{array}$ & $\begin{array}{l}12.47 \\
11.65\end{array}$ & $\begin{array}{l}13.55 \\
12.47\end{array}$ & 15.88 & 14.59 & 15.71 & 14.17 & 11.96 \\
\hline & & & & & & & & & \\
\hline \multicolumn{10}{|l|}{1995} \\
\hline January ............................. & 10.43 & 12.53 & 12.65 & 13.54 & 16.80 & 15.57 & 16.59 & 15.06 & 13.31 \\
\hline 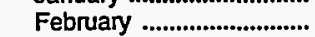 & 11.31 & 13.01 & 13.15 & 13.31 & 17.34 & 16.21 & 17.28 & 15.63 & 13.98 \\
\hline March .................................. & 11.48 & 13.19 & 13.44 & 14.34 & 17.03 & 16.06 & 17.09 & 15.56 & 13.82 \\
\hline 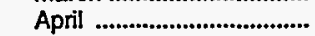 & 12.36 & 14.07 & 14.41 & 15.39 & 18.43 & 17.46 & 18.40 & 16.83 & 15.13 \\
\hline May ..................................... & 12.32 & 14.64 & 14.86 & 15.97 & 18.41 & 17.23 & 18.23 & 16.74 & 15.07 \\
\hline June .................................. & 11.95 & 1.4 .45 & 14.47 & 15.51 & 17.20 & 16.01 & 17.04 & 15.60 & 14.04 \\
\hline 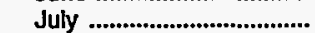 & 11.19 & 13.59 & 13.82 & 14.45 & 16.04 & 14.88 & 15.83 & 14.41 & 12.99 \\
\hline August ............................... & 10.23 & 13.59 & 13.82 & 14.54 & 16.66 & 15.50 & 16.57 & 15.03 & 13.72 \\
\hline September ......................... & 10.89 & 13.70 & 13.91 & 14.65 & 16.88 & 15.78 & 16.82 & 15.33 & 14.11 \\
\hline 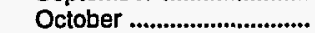 & 10.03 & 12.82 & 13.00 & 14.15 & 16.12 & 15.07 & 16.03 & 14.56 & 13.35 \\
\hline November ........................ & 10.25 & 12.35 & 12.55 & 13.55 & 16.80 & 15.62 & 16.64 & 15.14 & 13.95 \\
\hline December ........................... & 10.97 & 12.80 & 13.12 & 13.98 & 18.11 & 16.86 & 17.85 & 16.33 & 15.11 \\
\hline 1995 & 11.12 & 13.37 & 13.59 & 14.70 & 17.14 & 16.02 & 17.03 & 15.52 & 14.06 \\
\hline \multicolumn{10}{|l|}{1996} \\
\hline January .................. & 12.13 & 13.92 & 14.11 & 14.67 & 18.27 & 16.67 & 17.69 & 16.15 & 15.13 \\
\hline
\end{tabular}

Notes: Values shown for the current month are preliminary. Values shown for previous months are revised. Data are final upon publication in the Petroleum Marketing Annual.

Source: Energy Information Administration, Form ElA-182, "Domeștic Crude Oil First Purchase Report." 
Table 23. Domestic Crude Oil First Purchase Prices by API Gravity (Dollars per Barrel)

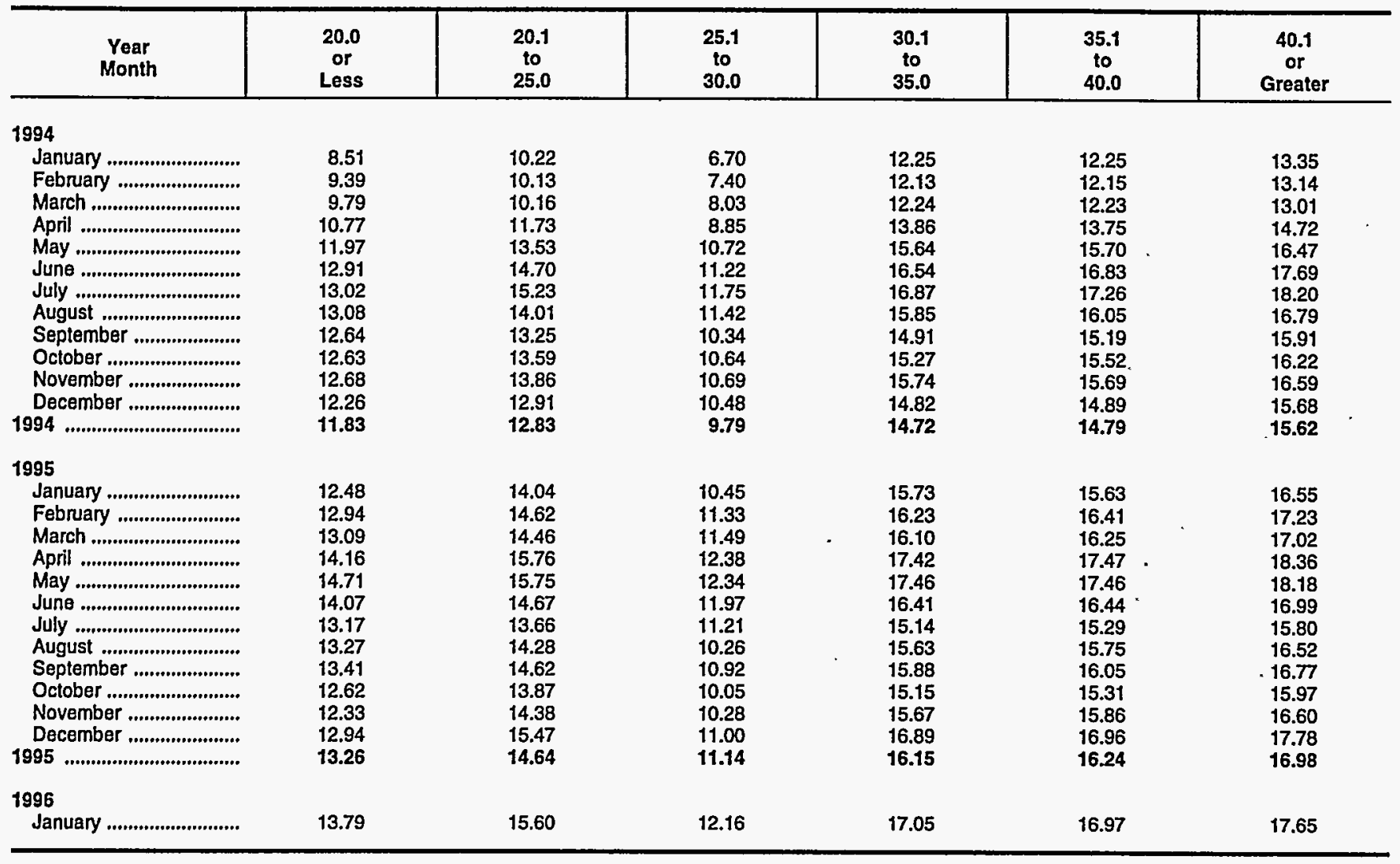

Notes: Values shown for the current month are preliminary. Values shown for previous months are revised. Data are final upon publication in the Petroleum Marketing Annual.

Source; Energy Information Administration, Form ElA-182, "Domestic Crude Oil First Purchase Report." 
Table 24. F.O.B. a Costs of Imported Crude Oil by Selected Country (Dollars per Barrel)

\begin{tabular}{|c|c|c|c|c|c|c|c|c|c|c|}
\hline \multirow{2}{*}{$\begin{array}{l}\text { Year } \\
\text { Month }\end{array}$} & \multicolumn{8}{|c|}{ Selected Countries } & \multicolumn{2}{|c|}{ OPEC } \\
\hline & Algeria & Indonesia & Mexico & Nigeria & $\begin{array}{l}\text { Saudi } \\
\text { Arabia }\end{array}$ & $\begin{array}{c}\text { United } \\
\text { Kingdom }\end{array}$ & Venezuela & $\begin{array}{l}\text { Other } \\
\text { Countries }\end{array}$ & $\begin{array}{l}\text { Arab } \\
\text { OPECb }\end{array}$ & $\begin{array}{l}\text { Total } \\
\text { OPEC }\end{array}$ \\
\hline $\begin{array}{l}1978 \\
1979 \\
1980 \\
1981 \\
1982 \\
1983 \\
1984 \\
1985 \\
1986 \\
1987 \\
1988 \\
1989 \\
1990 \\
1991\end{array}$ & $\begin{array}{l}14.12 \\
20.53 \\
36.67 \\
39.08 \\
34.20 \\
30.09 \\
28.34 \\
26.89 \\
13.62 \\
16.79 \\
W \\
W \\
W \\
W \\
W \\
W\end{array}$ & $\begin{array}{l}13.61 \\
19.03 \\
32.17 \\
35.62 \\
35.11 \\
29.92 \\
29.13 \\
27.12 \\
13.19 \\
17.40 \\
13.81 \\
17.01 \\
21.29 \\
18.69 \\
17.06 \\
17.13\end{array}$ & $\begin{array}{l}13.24 \\
20.27 \\
31.06 \\
33.01 \\
28.08 \\
25.20 \\
26.39 \\
25.33 \\
11.84 \\
16.36 \\
12.18 \\
15.96 \\
19.26 \\
15.37 \\
15.26 \\
13.74\end{array}$ & $\begin{array}{l}14.05 \\
21.69 \\
35.93 \\
38.31 \\
35.13 \\
29.81 \\
29.51 \\
28.04 \\
14.35 \\
18.47 \\
15.16 \\
18.31 \\
22.46 \\
20.29 \\
19.98 \\
17.79\end{array}$ & $\begin{array}{l}12.70 \\
17.28 \\
28.17 \\
32.60 \\
33.73 \\
27.53 \\
27.67 \\
22.04 \\
11.36 \\
15.12 \\
12.16 \\
16.29 \\
20.36 \\
14.62 \\
15.85 \\
13.77\end{array}$ & $\begin{array}{l}13.82 \\
21.70 \\
34.36 \\
36.06 \\
33.42 \\
29.91 \\
28.87 \\
27.64 \\
13.84 \\
18.28 \\
14.80 \\
17.89 \\
23.43 \\
20.81 \\
19.61 \\
16.64\end{array}$ & $\begin{array}{l}12.38 \\
16.90 \\
24.81 \\
28.95 \\
23.74 \\
21.48 \\
24.23 \\
23.64 \\
10.92 \\
15.08 \\
12.96 \\
16.09 \\
19.55 \\
14.91 \\
14.39 \\
12.46\end{array}$ & $\begin{array}{l}13.35 \\
21.10 \\
34.34 \\
36.69 \\
31.96 \\
27.96 \\
27.79 \\
26.12 \\
13.32 \\
17.11 \\
13.45 \\
17.12 \\
19.88 \\
17.79 \\
17.65 \\
15.17\end{array}$ & $\begin{array}{l}13.28 \\
19.27 \\
31.57 \\
34.79 \\
33.84 \\
28.28 \\
27.79 \\
24.34 \\
11.59 \\
15.80 \\
12.57 \\
16.72 \\
18.84 \\
15.59 \\
16.50 \\
14.25\end{array}$ & $\begin{array}{l}13.31 \\
19.88 \\
32.21 \\
35.17 \\
33.48 \\
28.46 \\
27.79 \\
25.67 \\
12.21 \\
16.43 \\
13.43 \\
17.06 \\
20.40 \\
16.99 \\
16.87 \\
14.78\end{array}$ \\
\hline 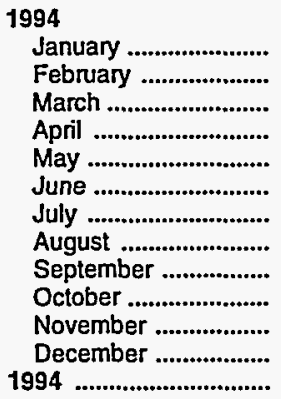 & $\begin{array}{l}w \\
\bar{w} \\
w \\
\bar{w} \\
w \\
w \\
- \\
\overline{-} \\
\bar{w} \\
w\end{array}$ & $\begin{array}{c}W \\
14.46 \\
W \\
13.52 \\
15.26 \\
15.91 \\
17.56 \\
W \\
W \\
W \\
W \\
W \\
15.57\end{array}$ & $\begin{array}{l}11.26 \\
11.44 \\
11.68 \\
12.88 \\
13.67 \\
15.02 \\
15.70 \\
14.57 \\
13.51 \\
14.42 \\
15.19 \\
14.74 \\
13.68\end{array}$ & $\begin{array}{l}15.02 \\
14.00 \\
14.27 \\
15.65 \\
16.77 \\
17.32 \\
18.02 \\
16.69 \\
16.35 \\
17.01 \\
17.13 \\
16.18 \\
16.32\end{array}$ & $\begin{array}{l}10.29 \\
12.81 \\
14.19 \\
14.91 \\
15.59 \\
14.83 \\
W \\
14.14 \\
14.80 \\
14.22 \\
W \\
W \\
14.12\end{array}$ & $\begin{array}{c}W \\
W \\
13.68 \\
W \\
15.77 \\
16.53 \\
17.29 \\
16.70 \\
15.41 \\
16.42 \\
17.01 \\
15.75 \\
15.66\end{array}$ & $\begin{array}{l}10.93 \\
10.35 \\
11.09 \\
11.81 \\
12.80 \\
13.21 \\
14.28 \\
12.31 \\
12.09 \\
12.90 \\
11.93 \\
12.38 \\
12.21\end{array}$ & $\begin{array}{l}12.16 \\
12.16 \\
12.36 \\
13.73 \\
15.23 \\
16.11 \\
16.71 \\
15.95 \\
15.44 \\
15.29 \\
15.82 \\
15.14 \\
14.68\end{array}$ & $\begin{array}{l}10.73 \\
12.19 \\
13.70 \\
14.53 \\
15.72 \\
15.21 \\
14.76 \\
14.09 \\
14.82 \\
14.20 \\
W \\
14.65 \\
14.05\end{array}$ & $\begin{array}{l}12.35 \\
11.96 \\
12.58 \\
13.75 \\
14.73 \\
15.24 \\
15.76 \\
14.29 \\
13.91 \\
14.48 \\
14.30 \\
13.94 \\
14.00\end{array}$ \\
\hline 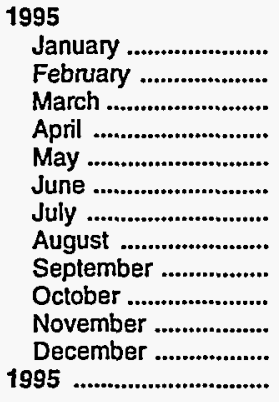 & $\begin{array}{l}- \\
\overline{-} \\
\bar{w} \\
w \\
\bar{w} \\
\bar{w} \\
w \\
- \\
\bar{w} \\
\bar{w}\end{array}$ & $\begin{array}{l}W \\
W \\
W \\
W \\
W \\
17.71 \\
W \\
W \\
W \\
W \\
W \\
W \\
17.13\end{array}$ & $\begin{array}{l}14.98 \\
15.79 \\
15.74 \\
17.16 \\
17.20 \\
16.07 \\
14.77 \\
14.54 \\
15.24 \\
15.02 \\
15.32 \\
16.41 \\
15.65\end{array}$ & $\begin{array}{l}17.13 \\
17.43 \\
17.19 \\
18.96 \\
18.66 \\
17.66 \\
15.97 \\
16.48 \\
16.91 \\
16.54 \\
17.28 \\
18.37 \\
17.40\end{array}$ & $\begin{array}{c}W \\
W \\
W \\
W \\
W \\
14.90 \\
W \\
W \\
W \\
W \\
16.19 \\
15.46 \\
15.77\end{array}$ & $\begin{array}{c}W \\
16.84 \\
W \\
W \\
18.42 \\
W \\
W \\
16.23 \\
16.47 \\
16.41 \\
W \\
W \\
16.99\end{array}$ & $\begin{array}{l}12.61 \\
13.02 \\
14.23 \\
15.97 \\
15.76 \\
13.80 \\
13.33 \\
13.73 \\
13.29 \\
12.40 \\
13.37 \\
14.70 \\
13.89\end{array}$ & $\begin{array}{l}15.57 \\
16.41 \\
16.62 \\
17.51 \\
17.96 \\
16.63 \\
15.54 \\
15.68 \\
16.06 \\
15.14 \\
15.63 \\
16.36 \\
16.27\end{array}$ & $\begin{array}{c}W \\
15.88 \\
W \\
17.33 \\
16.69 \\
14.84 \\
W \\
15.13 \\
14.97 \\
W \\
16.13 \\
15.40 \\
15.74\end{array}$ & $\begin{array}{l}14.79 \\
15.09 \\
15.47 \\
17.18 \\
16.93 \\
15.47 \\
14.43 \\
14.88 \\
14.77 \\
14.26 \\
15.10 \\
15.89 \\
15.38\end{array}$ \\
\hline $\begin{array}{l}1996 \\
\text { January }\end{array}$ & - & W & 16.32 & 18.85 & 15.35 & - & 14.33 & 16.04 & 15.17 & 15.69 \\
\hline
\end{tabular}

Dash $(-)=$ No data reported.

$W=$ Withheld to avoid disclosure of individual company data.

a Free on Board. See Glossary.

b Includes Algeria, Libya, Saudi Arabia, United Arab Emirates, Iraq, Kuwait, and Qatar.

c Includes Algeria, Gabon, Indonesia, Iran, Iraq, Kuwait, Libya, Nigeria, Qatar, Saudi Arabia, United Arab Emirates and Venezuela. Prior to January 1, 1993, data included Ecuador, which was then a member of OPEC.

Notes: Values through 1980 reflect the month of reporting; values since then reflect the month of acquisition, which can be the month of loading, the month of landing, or sometime between those events. Prices for crude oil can be determined at a time other than the acquisition date. See the Explanatory Notes section for additional detail.

Notes: Values for the current 2 months are preliminary. Values shown for previous months are revised. Data are final upon publication in the Petroleum Marketing Annual.

Sources: Energy Information Administration, Form FEA-F701-M-0, "Transfer Pricing Report," January 1978 through December 1978; Form ERA-51, "Transfer Pricing Report," January 1979 through September 1982; Form EP-51, "Monthly Foreign Crude Oil Transaction Report," October 1982 through June 1984; Form EIA-856, "Monthly Foreign Crude Oil Acquisition Report," July 1984 to present. 
Table 25. Landed Costs of Imported Crude Oil by Selected Country (Dollars per Barrel)

\begin{tabular}{|c|c|c|c|c|c|c|c|c|c|c|c|}
\hline \multirow{2}{*}{$\begin{array}{l}\text { Year } \\
\text { Month }\end{array}$} & \multicolumn{9}{|c|}{ Selected Countries } & \multicolumn{2}{|c|}{ OPEC } \\
\hline & Algeria & Canada & Indonesia & Mexico & Nigeria & $\begin{array}{l}\text { Saudi } \\
\text { Arabla }\end{array}$ & $\begin{array}{c}\text { United } \\
\text { KIngdom }\end{array}$ & Venezuela & \begin{tabular}{|c|} 
Other \\
Countries
\end{tabular} & $\begin{array}{c}\text { Arab } \\
\text { OPECa }\end{array}$ & $\begin{array}{c}\text { Total } \\
\text { OPECb }\end{array}$ \\
\hline $\begin{array}{l}1978 \\
1979 \\
1980 \\
1981 \\
1982 \\
1983 \\
1984 \\
1985\end{array}$ & $\begin{array}{r}14.93 \\
21.88 \\
37.92 \\
40.46 \\
35.35 \\
31.26 \\
29.06 \\
27.51 \\
14.82 \\
17.87 \\
W \\
19.13 \\
W \\
W \\
W \\
17.34\end{array}$ & $\begin{array}{l}14.41 \\
20.22 \\
30.11 \\
32.32 \\
27.15 \\
25.63 \\
26.56 \\
25.71 \\
13.43 \\
17.04 \\
13.50 \\
16.81 \\
20.48 \\
17.16 \\
17.04 \\
15.27\end{array}$ & $\begin{array}{l}14.65 \\
20.63 \\
33.92 \\
37.31 \\
36.70 \\
31.57 \\
30.87 \\
28.67 \\
14.63 \\
18.49 \\
15.15 \\
18.35 \\
22.50 \\
20.20 \\
18.76 \\
18.55\end{array}$ & $\begin{array}{l}13.56 \\
20.77 \\
31.77 \\
33.70 \\
28.63 \\
25.78 \\
26.85 \\
25.63 \\
12.17 \\
16.69 \\
12.58 \\
16.35 \\
19.64 \\
15.89 \\
15.60 \\
14.11\end{array}$ & $\begin{array}{l}14.88 \\
22.97 \\
37.15 \\
39.66 \\
36.16 \\
30.85 \\
30.36 \\
28.96 \\
15.29 \\
19.32 \\
15.88 \\
19.19 \\
23.33 \\
21.39 \\
20.78 \\
18.73\end{array}$ & $\begin{array}{l}13.94 \\
18.95 \\
29.80 \\
34.20 \\
34.99 \\
29.27 \\
29.20 \\
24.72 \\
12.84 \\
16.81 \\
13.37 \\
17.34 \\
21.82 \\
17.22 \\
17.48 \\
15.40\end{array}$ & $\begin{array}{l}14.53 \\
22.97 \\
35.68 \\
37.29 \\
34.25 \\
30.87 \\
29.45 \\
28.36 \\
14.63 \\
18.78 \\
15.82 \\
18.74 \\
22.65 \\
21.37 \\
20.63 \\
17.92\end{array}$ & $\begin{array}{l}12.84 \\
17.65 \\
25.92 \\
29.91 \\
24.93 \\
22.94 \\
25.19 \\
24.43 \\
11.52 \\
15.76 \\
13.66 \\
16.78 \\
20.31 \\
15.92 \\
15.13 \\
13.39\end{array}$ & $\begin{array}{l}14.58 \\
22.86 \\
36.15 \\
38.54 \\
34.03 \\
29.68 \\
29.21 \\
27.33 \\
14.25 \\
18.30 \\
14.45 \\
18.08 \\
20.52 \\
19.73 \\
19.25 \\
16.44\end{array}$ & $\begin{array}{l}14.36 \\
20.79 \\
32.97 \\
36.22 \\
35.15 \\
29.87 \\
29.10 \\
25.90 \\
13.14 \\
17.32 \\
13.60 \\
17.41 \\
20.64 \\
17.45 \\
17.63 \\
15.28\end{array}$ & $\begin{array}{l}14.34 \\
21.29 \\
33.56 \\
36.60 \\
34.81 \\
29.84 \\
29.06 \\
26.86 \\
13.46 \\
17.64 \\
14.18 \\
17.78 \\
21.23 \\
18.08 \\
17.81 \\
15.68\end{array}$ \\
\hline 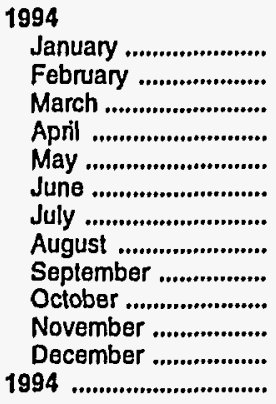 & $\begin{array}{l}W \\
\bar{W} \\
W \\
\bar{W} \\
W \\
W \\
W \\
W \\
W \\
W \\
W\end{array}$ & $\begin{array}{l}12.13 \\
12.05 \\
11.92 \\
13.43 \\
15.25 \\
16.45 \\
17.53 \\
16.51 \\
15.50 \\
15.54 \\
16.06 \\
15.41 \\
14.83\end{array}$ & $\begin{array}{c}W \\
16.17 \\
W \\
15.08 \\
16.42 \\
17.00 \\
18.41 \\
19.96 \\
W \\
W \\
W \\
16.99 \\
16.91\end{array}$ & $\begin{array}{l}11.61 \\
11.73 \\
11.97 \\
13.23 \\
14.10 \\
15.44 \\
16.17 \\
14.97 \\
14.04 \\
14.82 \\
15.61 \\
15.56 \\
14.09\end{array}$ & $\begin{array}{l}15.76 \\
14.68 \\
15.13 \\
16.46 \\
17.36 \\
18.21 \\
18.74 \\
17.78 \\
17.39 \\
17.85 \\
18.04 \\
17.24 \\
17.21\end{array}$ & $\begin{array}{l}11.66 \\
12.32 \\
13.31 \\
14.30 \\
15.81 \\
16.60 \\
16.81 \\
15.68 \\
15.62 \\
15.41 \\
15.85 \\
15.56 \\
15.11\end{array}$ & $\begin{array}{l}14.98 \\
15.40 \\
14.67 \\
15.31 \\
16.33 \\
17.40 \\
17.96 \\
17.41 \\
16.62 \\
17.06 \\
17.19 \\
16.84 \\
16.64\end{array}$ & $\begin{array}{l}11.78 \\
11.12 \\
11.87 \\
12.72 \\
13.53 \\
14.15 \\
15.02 \\
13.24 \\
13.04 \\
13.85 \\
13.03 \\
13.50 \\
13.12\end{array}$ & $\begin{array}{l}13.52 \\
13.60 \\
13.33 \\
15.09 \\
16.48 \\
17.18 \\
17.73 \\
16.92 \\
16.38 \\
16.28 \\
16.97 \\
16.45 \\
15.95\end{array}$ & $\begin{array}{l}11.86 \\
12.24 \\
12.85 \\
14.21 \\
15.72 \\
16.58 \\
16.86 \\
15.72 \\
15.46 \\
15.34 \\
15.84 \\
15.56 \\
15.02\end{array}$ & $\begin{array}{l}12.94 \\
12.59 \\
13.05 \\
14.47 \\
15.62 \\
16.48 \\
16.88 \\
15.69 \\
15.25 \\
15.51 \\
15.63 \\
15.34 \\
15.08\end{array}$ \\
\hline 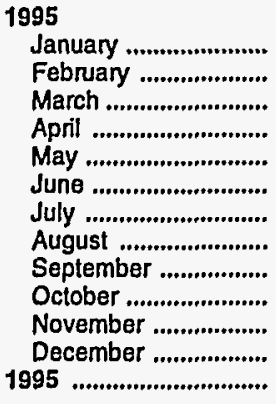 & $\begin{array}{l}W \\
W \\
W \\
W \\
W \\
\bar{W} \\
W \\
W \\
W \\
- \\
\bar{W}\end{array}$ & $\begin{array}{l}16.03 \\
16.74 \\
16.88 \\
18.27 \\
18.44 \\
17.28 \\
16.33 \\
16.35 \\
16.37 \\
15.37 \\
15.37 \\
16.07 \\
16.64\end{array}$ & $\begin{array}{c}W \\
W \\
18.78 \\
W \\
W \\
18.98 \\
17.27 \\
17.47 \\
W \\
W \\
W \\
W \\
18.43\end{array}$ & $\begin{array}{l}15.52 \\
16.23 \\
16.34 \\
17.56 \\
17.69 \\
16.58 \\
15.28 \\
15.12 \\
15.74 \\
15.61 \\
15.90 \\
17.08 \\
16.20\end{array}$ & $\begin{array}{l}17.64 \\
18.24 \\
18.13 \\
19.82 \\
19.45 \\
18.74 \\
17.29 \\
17.39 \\
17.86 \\
17.49 \\
17.98 \\
19.09 \\
18.25\end{array}$ & $\begin{array}{l}16.66 \\
17.11 \\
17.41 \\
18.45 \\
17.71 \\
16.39 \\
15.73 \\
16.16 \\
16.35 \\
16.03 \\
17.00 \\
16.92 \\
16.84\end{array}$ & $\begin{array}{l}17.35 \\
17.70 \\
18.00 \\
18.53 \\
19.16 \\
18.71 \\
17.44 \\
17.28 \\
17.44 \\
17.31 \\
17.28 \\
18.74 \\
17.95\end{array}$ & $\begin{array}{l}13.66 \\
14.01 \\
15.29 \\
16.95 \\
16.68 \\
14.85 \\
14.21 \\
14.68 \\
14.28 \\
13.33 \\
14.19 \\
15.48 \\
14.84\end{array}$ & $\begin{array}{l}16.94 \\
17.57 \\
17.78 \\
18.55 \\
18.86 \\
17.96 \\
16.72 \\
16.68 \\
17.12 \\
16.73 \\
16.96 \\
17.88 \\
17.49\end{array}$ & $\begin{array}{l}16.65 \\
17.03 \\
17.33 \\
18.41 \\
17.70 \\
16.41 \\
15.74 \\
16.12 \\
16.35 \\
15.98 \\
16.87 \\
16.83 \\
16.79\end{array}$ & $\begin{array}{l}16.14 \\
16.49 \\
16.86 \\
18.34 \\
17.90 \\
16.62 \\
15.69 \\
16.04 \\
16.22 \\
15.61 \\
16.35 \\
17.01 \\
16.62\end{array}$ \\
\hline $\begin{array}{l}1996 \\
\text { January ........................ }\end{array}$ & W & 16.07 & W & 16.81 & 19.76 & 16.88 & 18.53 & 15.31 & 18.13 & 16.87 & 17.24 \\
\hline
\end{tabular}

Dash $(-)=$ No data reported

$W=$ Withheld to avoid disclosure of individual company data.

a Includes Algeria, Libya, Saudi Arabia, United Arab Emirates, Iraq, Kuwait, and Qatar

b Includes Algeria, Gabon, Indonesia, Iran, Iraq, Kuwait, Libya, Nigeria, Qatar, Saudi Arabia, United Arab Emirates and Venezuela. Prior to January 1, 1993, data included Ecuador, which was then a member of OPEC.

Notes: Values through 1980 reflect the month of reporting; values since then reflect the month of acquisition, which can be the month of loading, the month of landing, or sometime between those events. Prices for crude oil can be determined at a time other than the acquisition date. See the Explanatory Notes section for addilional detall.

Notes: Values for the current 2 months are preliminary. Values shown for previous months are revised. Data are final upon publication in the Petroleum Marketing Annual.

Sources: Energy Information Administration, Form FEA-F701-M-0, "Transfer Pricing Report," January 1978 through December 1978; Form ERA-51, "Transler Pricing Report," January 1979 through Seplember 1982; Form EP-51, "Monthly Foreign Crude Oil Transaction Report," October 1982 through June 1984; Form ElA-856, "Monthly Foreign Crude Oil Acquisition Report," July 1984 to present. 
Table 26. F.O.B. a Costs of Imported Crude Oil by API Gravity (Dollars per Barrel)

\begin{tabular}{|c|c|c|c|c|c|c|c|}
\hline $\begin{array}{l}\text { Year } \\
\text { Month }\end{array}$ & $\begin{array}{l}20.0 \\
\text { or } \\
\text { Less }\end{array}$ & $\begin{array}{c}20.1 \\
\text { to } \\
25.0\end{array}$ & $\begin{array}{c}25.1 \\
\text { to } \\
30.0\end{array}$ & $\begin{array}{c}30.1 \\
\text { to } \\
35.0\end{array}$ & $\begin{array}{c}35.1 \\
\text { to } \\
40.0\end{array}$ & $\begin{array}{c}40.1 \\
\text { to } \\
45.0\end{array}$ & $\begin{array}{c}\mathbf{4 5 . 1} \\
\text { or } \\
\text { Greater }\end{array}$ \\
\hline $\begin{array}{l}1978 \\
1979 \\
1980 \\
1981 \\
1982 \\
1983 \\
1984 \\
1985\end{array}$ & $\begin{array}{l}11.98 \\
14.43 \\
23.09 \\
26.70 \\
23.08 \\
21.77 \\
24.10 \\
23.45 \\
10.51 \\
15.21 \\
11.92 \\
14.00 \\
15.98 \\
11.91 \\
11.83 \\
11.33\end{array}$ & $\begin{array}{l}11.74 \\
18.54 \\
28.76 \\
31.20 \\
25.82 \\
24.27 \\
25.31 \\
24.27 \\
10.96 \\
15.37 \\
11.65 \\
14.89 \\
18.00 \\
13.72 \\
13.96 \\
12.22\end{array}$ & $\begin{array}{l}12.51 \\
19.91 \\
30.72 \\
33.61 \\
31.48 \\
27.38 \\
27.22 \\
24.86 \\
12.25 \\
15.76 \\
12.48 \\
16.95 \\
20.54 \\
16.33 \\
16.74 \\
15.14\end{array}$ & $\begin{array}{l}13.06 \\
19.54 \\
30.31 \\
34.38 \\
33.56 \\
29.04 \\
28.55 \\
26.46 \\
12.83 \\
17.24 \\
13.82 \\
17.50 \\
20.77 \\
17.67 \\
18.02 \\
15.44\end{array}$ & $\begin{array}{l}13.62 \\
21.24 \\
34.33 \\
36.85 \\
34.29 \\
29.74 \\
29.18 \\
27.43 \\
13.83 \\
17.99 \\
14.38 \\
18.09 \\
22.19 \\
20.15 \\
19.50 \\
17.04\end{array}$ & $\begin{array}{l}14.00 \\
21.65 \\
35.39 \\
38.42 \\
34.82 \\
30.10 \\
29.40 \\
27.79 \\
14.19 \\
18.03 \\
14.89 \\
18.23 \\
22.78 \\
19.69 \\
19.58 \\
17.53\end{array}$ & $\begin{array}{l}14.01 \\
19.77 \\
36.13 \\
38.59 \\
34.48 \\
30.12 \\
28.02 \\
26.90 \\
13.76 \\
17.37 \\
15.25 \\
18.05 \\
22.28 \\
20.85 \\
20.05 \\
17.61\end{array}$ \\
\hline 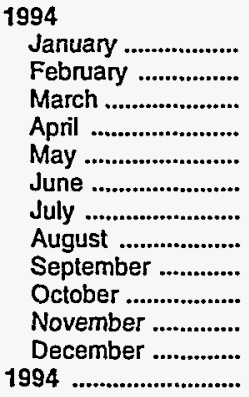 & $\begin{array}{r}10.54 \\
9.32 \\
9.60 \\
10.68 \\
11.45 \\
11.91 \\
13.01 \\
10.66 \\
11.55 \\
11.66 \\
11.93 \\
12.46 \\
11.43\end{array}$ & $\begin{array}{r}10.05 \\
9.84 \\
10.17 \\
11.51 \\
12.42 \\
13.58 \\
14.49 \\
13.12 \\
12.22 \\
13.14 \\
13.08 \\
12.67 \\
12.19\end{array}$ & $\begin{array}{l}12.65 \\
12.05 \\
12.46 \\
14.36 \\
14.99 \\
15.99 \\
16.88 \\
14.83 \\
14.73 \\
14.93 \\
14.83 \\
14.50 \\
14.45\end{array}$ & $\begin{array}{l}12.19 \\
12.95 \\
13.55 \\
14.43 \\
15.80 \\
16.09 \\
16.64 \\
15.57 \\
15.30 \\
15.12 \\
15.41 \\
14.94 \\
14.93\end{array}$ & $\begin{array}{l}14.05 \\
13.79 \\
13.82 \\
15.22 \\
16.25 \\
17.08 \\
17.75 \\
16.94 \\
15.93 \\
16.57 \\
17.02 \\
16.03 \\
15.91\end{array}$ & $\begin{array}{c}14.85 \\
14.15 \\
14.04 \\
W \\
15.69 \\
W \\
16.96 \\
W \\
16.09 \\
W \\
17.03 \\
15.87 \\
15.70\end{array}$ & $\begin{array}{c}14.24 \\
14.30 \\
13.70 \\
15.21 \\
W \\
17.47 \\
17.61 \\
17.40 \\
16.00 \\
16.10 \\
16.73 \\
16.78 \\
16.11\end{array}$ \\
\hline 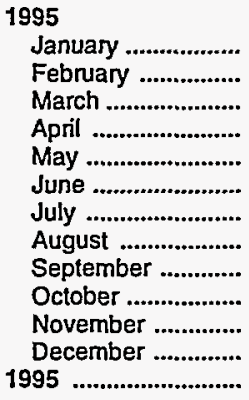 & $\begin{array}{l}12.46 \\
12.97 \\
13.46 \\
15.01 \\
15.17 \\
13.75 \\
12.98 \\
12.71 \\
12.85 \\
11.69 \\
12.76 \\
13.86 \\
13.36\end{array}$ & $\begin{array}{l}13.20 \\
13.69 \\
14.38 \\
15.89 \\
15.91 \\
14.53 \\
13.20 \\
13.46 \\
13.27 \\
12.42 \\
12.85 \\
14.23 \\
13.95\end{array}$ & $\begin{array}{l}15.27 \\
16.13 \\
16.14 \\
17.65 \\
17.62 \\
16.30 \\
15.28 \\
15.83 \\
15.90 \\
15.05 \\
15.31 \\
16.59 \\
16.14\end{array}$ & $\begin{array}{l}15.78 \\
16.26 \\
16.87 \\
17.87 \\
17.44 \\
16.35 \\
15.55 \\
15.47 \\
15.55 \\
15.31 \\
16.09 \\
16.36 \\
16.23\end{array}$ & $\begin{array}{l}16.81 \\
17.20 \\
17.32 \\
18.72 \\
18.68 \\
17.48 \\
16.18 \\
16.47 \\
16.87 \\
16.52 \\
16.99 \\
18.14 \\
17.25\end{array}$ & $\begin{array}{c}15.82 \\
17.12 \\
16.88 \\
17.87 \\
18.51 \\
18.04 \\
W \\
W \\
W \\
W \\
16.76 \\
17.79 \\
17.34\end{array}$ & $\begin{array}{c}17.02 \\
W \\
17.86 \\
18.00 \\
18.84 \\
17.72 \\
16.48 \\
16.46 \\
17.04 \\
16.66 \\
17.83 \\
W \\
17.26\end{array}$ \\
\hline $\begin{array}{l}1996 \\
\quad \text { January .................... }\end{array}$ & 13.49 & 13.72 & 16.41 & 16.45 & 18.59 & 18.65 & $w$ \\
\hline
\end{tabular}

$W=$ Witheld to avoid disclosure of individual company data.

a Free on Board. See Glossary.

Notes: Values through 1980 reflect the month of reporting; values since then reflect the month of acquisition, which can be the month of loading, the month of landing, or sometime between those events. Prices for crude oil can be determined at a time other than the acquisition date. See the Explanatory Notes section for additional detail.

Notes: Values for the current 2 months are preliminary. Values shown for previous months are revised. Data are final upon publication in the $P$ etroleum Marketing Annual.

Sources: Energy Information Administration, Form FEA-F701-M-0, "Transfer Pricing Report," January 1978 through December 1978; Form ERA-51, "Transfer Pricing Report," January 1979 through September 1982; Form EP-51, "Monthly Foreign Crude Oil Transaction Report," October 1982 through June 1984; Form ElA-856, "Monthly Foreign Crude Oil Acquisition Report," July 1984 to present. 
Table 27. Landed Costs of Imported Crude Oil by API Gravity (Dollars per Barrel)

\begin{tabular}{|c|c|c|c|c|c|c|c|}
\hline $\begin{array}{l}\text { Year } \\
\text { Month }\end{array}$ & $\begin{array}{c}20.0 \\
\text { or } \\
\text { Less }\end{array}$ & $\begin{array}{c}20.1 \\
\text { to } \\
25.0\end{array}$ & $\begin{array}{c}25.1 \\
\text { to } \\
30.0\end{array}$ & $\begin{array}{c}30.1 \\
\text { to } \\
35.0\end{array}$ & $\begin{array}{c}35.1 \\
\text { to } \\
40.0\end{array}$ & $\begin{array}{c}40.1 \\
\text { to } \\
45.0\end{array}$ & $\begin{array}{c}45.1 \\
\text { or } \\
\text { Greater }\end{array}$ \\
\hline 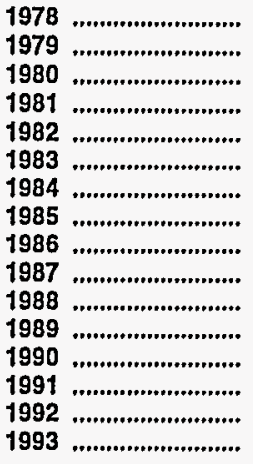 & $\begin{array}{l}12.75 \\
15.57 \\
24.54 \\
27.94 \\
24.17 \\
23.17 \\
25.08 \\
24.33 \\
11.30 \\
16.14 \\
12.75 \\
14.90 \\
16.82 \\
13.06 \\
12.89 \\
12.44\end{array}$ & $\begin{array}{l}13.11 \\
20.05 \\
29.65 \\
32.00 \\
26.46 \\
24.95 \\
25.97 \\
24.65 \\
11.49 \\
15.87 \\
12.11 \\
15.42 \\
18.54 \\
14.41 \\
14.58 \\
12.96\end{array}$ & $\begin{array}{l}13.76 \\
20.88 \\
31.83 \\
34.86 \\
32.62 \\
28.62 \\
28.38 \\
26.17 \\
13.28 \\
17.21 \\
13.54 \\
17.59 \\
21.59 \\
17.17 \\
17.39 \\
15.72\end{array}$ & $\begin{array}{l}14.10 \\
21.01 \\
31.73 \\
35.71 \\
34.76 \\
30.25 \\
29.58 \\
27.10 \\
13.59 \\
18.16 \\
14.35 \\
17.87 \\
21.18 \\
18.65 \\
18.50 \\
16.06\end{array}$ & $\begin{array}{l}14.69 \\
22.61 \\
35.81 \\
38.36 \\
35.43 \\
30.96 \\
30.16 \\
28.29 \\
14.99 \\
18.72 \\
15.21 \\
18.74 \\
22.47 \\
20.86 \\
20.11 \\
17.88\end{array}$ & $\begin{array}{l}14.87 \\
23.06 \\
36.85 \\
39.78 \\
35.78 \\
31.28 \\
30.16 \\
28.39 \\
14.80 \\
18.89 \\
15.74 \\
19.05 \\
23.47 \\
20.88 \\
20.55 \\
18.38\end{array}$ & $\begin{array}{l}15.16 \\
21.23 \\
37.57 \\
39.97 \\
35.81 \\
31.27 \\
29.05 \\
27.73 \\
15.37 \\
18.57 \\
16.24 \\
19.13 \\
23.41 \\
22.15 \\
20.95 \\
18.22\end{array}$ \\
\hline 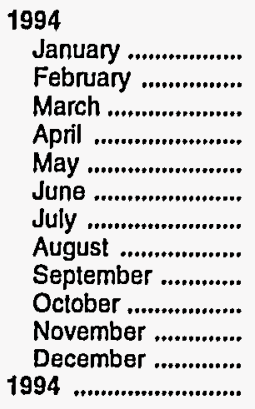 & $\begin{array}{l}11.39 \\
10.24 \\
10.61 \\
11.53 \\
12.36 \\
12.93 \\
13.87 \\
11.71 \\
12.44 \\
12.76 \\
13.22 \\
13.54 \\
12.42\end{array}$ & $\begin{array}{l}10.70 \\
10.43 \\
10.82 \\
12.24 \\
13.12 \\
14.36 \\
15.23 \\
13.83 \\
13.06 \\
13.84 \\
13.85 \\
13.83 \\
12.93\end{array}$ & $\begin{array}{l}12.93 \\
12.38 \\
12.88 \\
14.59 \\
15.56 \\
16.84 \\
17.35 \\
15.68 \\
15.36 \\
15.59 \\
15.78 \\
15.30 \\
15.10\end{array}$ & $\begin{array}{l}12.85 \\
13.21 \\
13.33 \\
14.80 \\
16.30 \\
16.99 \\
17.40 \\
16.49 \\
16.03 \\
15.84 \\
16.34 \\
15.92 \\
15.61\end{array}$ & $\begin{array}{l}14.55 \\
14.37 \\
14.59 \\
15.72 \\
16.87 \\
17.78 \\
18.32 \\
17.53 \\
16.81 \\
17.08 \\
17.41 \\
16.79 \\
16.65\end{array}$ & $\begin{array}{l}15.21 \\
14.43 \\
14.33 \\
15.73 \\
16.55 \\
17.82 \\
18.50 \\
18.06 \\
17.01 \\
17.24 \\
17.51 \\
17.28 \\
16.64\end{array}$ & $\begin{array}{l}14.63 \\
14.63 \\
14.43 \\
15.47 \\
16.50 \\
17.80 \\
18.81 \\
18.77 \\
16.65 \\
16.90 \\
17.44 \\
17.22 \\
16.91\end{array}$ \\
\hline 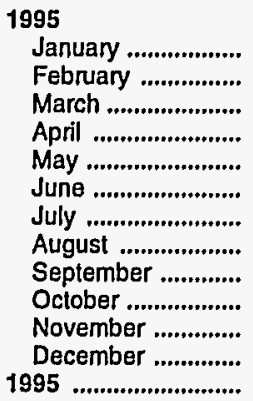 & $\begin{array}{l}13.91 \\
14.21 \\
14.72 \\
15.94 \\
16.20 \\
14.78 \\
13.96 \\
13.70 \\
14.04 \\
12.88 \\
13.56 \\
14.80 \\
14.44\end{array}$ & $\begin{array}{l}14.06 \\
14.62 \\
15.31 \\
16.74 \\
16.75 \\
15.44 \\
14.09 \\
14.30 \\
14.20 \\
13.40 \\
13.74 \\
15.03 \\
14.83\end{array}$ & $\begin{array}{l}16.26 \\
16.88 \\
16.96 \\
18.43 \\
18.26 \\
17.03 \\
15.97 \\
16.38 \\
16.49 \\
15.78 \\
16.14 \\
17.11 \\
16.84\end{array}$ & $\begin{array}{l}16.88 \\
17.23 \\
17.67 \\
18.71 \\
18.12 \\
17.20 \\
16.34 \\
16.36 \\
16.65 \\
16.24 \\
17.01 \\
17.33 \\
17.14\end{array}$ & $\begin{array}{l}17.27 \\
17.84 \\
17.94 \\
19.05 \\
19.28 \\
18.59 \\
17.15 \\
17.32 \\
17.55 \\
17.30 \\
17.54 \\
18.69 \\
17.96\end{array}$ & $\begin{array}{l}16.74 \\
17.99 \\
18.16 \\
18.83 \\
19.21 \\
18.51 \\
17.30 \\
16.95 \\
18.60 \\
17.78 \\
17.53 \\
18.48 \\
18.19\end{array}$ & $\begin{array}{l}17.41 \\
17.20 \\
18.53 \\
18.93 \\
18.92 \\
18.82 \\
17.81 \\
16.55 \\
18.02 \\
17.46 \\
18.21 \\
18.18 \\
17.85\end{array}$ \\
\hline $\begin{array}{l}1996 \\
\text { January ................... }\end{array}$ & 14.57 & 14.71 & 17.65 & 17.47 & 18.75 & 19.22 & 18.47 \\
\hline
\end{tabular}

Notes: Values through 1980 reflect the month of reporting; values since then reflect the month of acquisition, which can be the month of loading, the month of landing, or sometime between those events. Prices for cnude oil can be determined at a time other than the acquisition date. See the Explanatory Notes section for additional detail.

Notes: Values for the current 2 months are preliminary. Values shown for previous months are revised. Data are final upon publication in the Petroleum Marketing Annual.

Sources: Energy Information Administration, Form FEA-F701-M-0, "Transfer Pricing Report," January 1978 through December 1978; Form ERA-51, "Transfer Pricing Report," January 1979 through September 1982; Form EP-51, "Monthly Foreign Crude Oil Transaction Report," October 1982 through June 1984; Form EIA-856, "Monthly Foreign Crude Oil Acquisition Report," July 1984 to present. 
Table 28. Percentages of Total Imported Crude Oil by API Gravity (Percent by Interval)

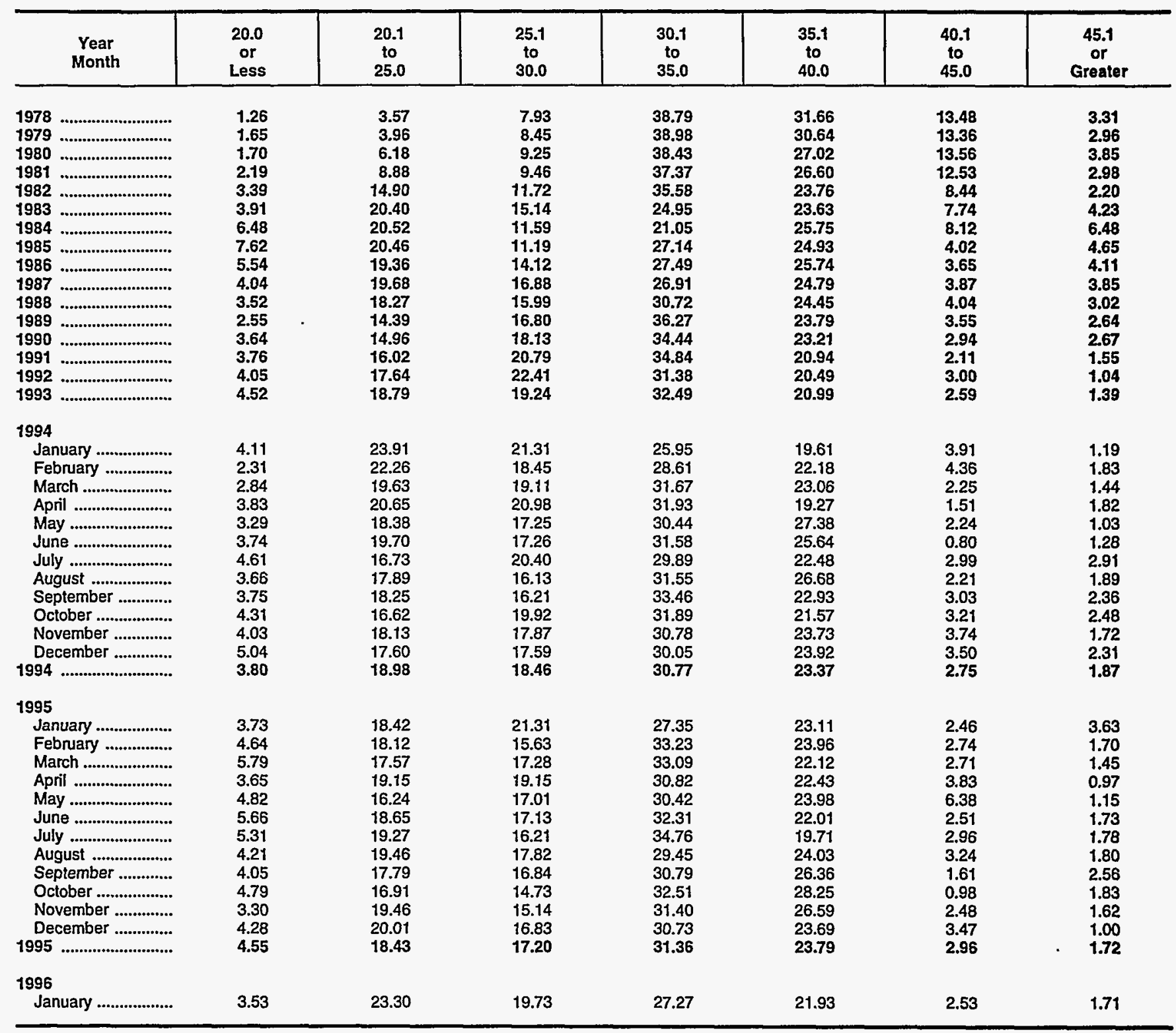

Notes: Values through 1980 reflect the month of reporting; values since then reflect the month of acquisition, which can be the month of loading, the month of landing, or sometime between those events. Prices for crude oil can be determined at a time other than the acquisition date. See the Explanatory Noles section for additional detail.

Notes: Values for the current 2 months are preliminary. Values shown for previous months are revised. Data are final upon publication in the Petroleum Marketing Annual.

Sources: Energy Information Administration, Form FEA-F701-M-0, "Transfer Pricing Report," January 1978 through December 1978; Form ERA-51, "Transfer Pricing Report," January 1979 through September 1982; Form EP-51, "Monthly Foreign Crude Oil Transaction Report," October 1982 through June 1984; Form EIA-856, "Monthly Foreign Crude Oil Acquisition Report," July 1984 to present. 
Table 29. F.O.B. ${ }^{\text {a }}$ Costs of Imported Crude Oil for Selected Crude Streams (Dollars per Barrel)

\begin{tabular}{|c|c|c|c|c|c|c|c|}
\hline $\begin{array}{l}\text { Year } \\
\text { Quarter } \\
\text { Month }\end{array}$ & $\begin{array}{c}\text { Algerian } \\
\text { Condensate }\end{array}$ & $\begin{array}{l}\text { Angolan } \\
\text { Cabinda }\end{array}$ & $\begin{array}{c}\text { Canadian } \\
\text { Lloydminster }\end{array}$ & $\begin{array}{l}\text { Cameroon } \\
\text { Kole Marine }\end{array}$ & $\begin{array}{c}\text { Ecuadorian } \\
\text { Oriente }\end{array}$ & $\begin{array}{l}\text { Mexican } \\
\text { Isthmus }\end{array}$ & $\begin{array}{l}\text { Mexican } \\
\text { Mayan }\end{array}$ \\
\hline 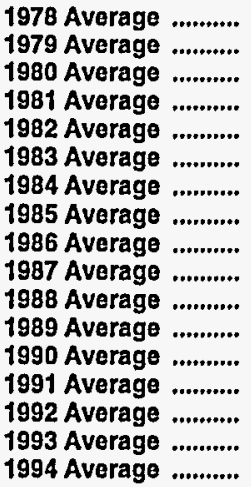 & $\begin{array}{l}W \\
W \\
35.81 \\
38.38 \\
32.58 \\
29.86 \\
27.77 \\
26.57 \\
13.25 \\
16.66 \\
W \\
W \\
W \\
W \\
W \\
W \\
W\end{array}$ & $\begin{array}{r}13.32 \\
20.35 \\
33.24 \\
35.55 \\
31.86 \\
28.14 \\
27.58 \\
26.30 \\
13.39 \\
17.31 \\
14.52 \\
17.15 \\
W \\
W \\
18.46 \\
15.65 \\
15.15\end{array}$ & $\begin{array}{c}- \\
\bar{w} \\
W \\
W \\
24.54 \\
24.70 \\
23.79 \\
12.77 \\
15.12 \\
11.28 \\
W \\
18.50 \\
13.51 \\
W \\
W \\
W\end{array}$ & $\begin{array}{c}W \\
W \\
35.92 \\
37.51 \\
33.73 \\
29.03 \\
28.56 \\
26.86 \\
13.64 \\
W \\
14.28 \\
17.46 \\
W \\
W \\
W \\
W \\
W\end{array}$ & $\begin{array}{l}12.87 \\
27.59 \\
34.51 \\
32.66 \\
32.36 \\
28.22 \\
28.16 \\
26.12 \\
13.32 \\
16.53 \\
12.96 \\
16.26 \\
W \\
16.04 \\
17.60 \\
14.57 \\
13.70\end{array}$ & $\begin{array}{l}13.24 \\
20.29 \\
32.78 \\
36.23 \\
32.50 \\
29.41 \\
29.00 \\
27.25 \\
13.88 \\
17.94 \\
14.23 \\
W \\
23.39 \\
18.55 \\
17.92 \\
15.95 \\
14.79\end{array}$ & $\begin{array}{c}- \\
21.50 \\
28.73 \\
30.82 \\
25.29 \\
23.99 \\
25.35 \\
24.23 \\
10.93 \\
15.72 \\
11.26 \\
14.71 \\
17.29 \\
13.02 \\
13.42 \\
12.03 \\
12.39\end{array}$ \\
\hline $\begin{array}{l}1995 \\
\text { January .................. } \\
\text { February ................ } \\
\text { March ..................... } \\
\text { 1st Quarter }\end{array}$ & $\begin{array}{l}- \\
-\end{array}$ & $\begin{array}{c}W \\
16.60 \\
16.54\end{array}$ & $\begin{array}{l}W \\
W \\
W\end{array}$ & $\begin{array}{l}- \\
-\end{array}$ & $\begin{array}{l}W \\
W \\
W\end{array}$ & $\begin{array}{l}W \\
W \\
W\end{array}$ & $\begin{array}{l}13.82 \\
14.60 \\
14.86\end{array}$ \\
\hline $\begin{array}{l}\text { Average .................... } \\
\text { April ......................... } \\
\text { May ......................... } \\
\text { June ....................... } \\
\text { 2nd Quarter }\end{array}$ & $\begin{array}{l}\bar{w} \\
- \\
-\end{array}$ & $\begin{array}{c}16.45 \\
17.45 \\
17.97 \\
W\end{array}$ & $\begin{array}{c}W \\
W \\
W \\
15.36\end{array}$ & $\begin{array}{l}\bar{w} \\
- \\
-\end{array}$ & $\begin{array}{c}W \\
W \\
18.42 \\
W\end{array}$ & $\begin{array}{l}w \\
w \\
w \\
w\end{array}$ & $\begin{array}{l}14.42 \\
15.99 \\
16.22 \\
14.88\end{array}$ \\
\hline $\begin{array}{l}\text { Average ................... } \\
\text { July ......................... } \\
\text { August ................ } \\
\text { Seplember ............ } \\
\text { 3rd Quarter }\end{array}$ & $\begin{array}{l}w \\
\bar{w} \\
w\end{array}$ & $\begin{array}{c}17.44 \\
W \\
W \\
W\end{array}$ & $\begin{array}{c}16.19 \\
W \\
W \\
W\end{array}$ & $\begin{array}{l}W \\
- \\
- \\
-\end{array}$ & $\begin{array}{c}17.95 \\
W \\
w\end{array}$ & $\begin{array}{c}W \\
15.85 \\
16.09 \\
16.26\end{array}$ & $\begin{array}{l}15.67 \\
13.42 \\
13.26 \\
13.62\end{array}$ \\
\hline $\begin{array}{l}\text { Average ................... } \\
\text { October .................. } \\
\text { November ............. } \\
\text { December .............. } \\
\text { 4th Quarter }\end{array}$ & $\begin{array}{l}W \\
- \\
- \\
-\end{array}$ & $\begin{array}{c}W \\
15.50 \\
W \\
W\end{array}$ & $\begin{array}{c}W \\
12.31 \\
W \\
12.55\end{array}$ & $\begin{array}{l}- \\
- \\
-\end{array}$ & $\begin{array}{c}16.00 \\
\bar{w} \\
15.62\end{array}$ & $\begin{array}{c}16.09 \\
W \\
16.02 \\
W\end{array}$ & $\begin{array}{l}13.42 \\
13.10 \\
13.47 \\
15.06\end{array}$ \\
\hline $\begin{array}{l}\text { Average ................... } \\
1995 \text { Average .......... }\end{array}$ & $\bar{w}$ & $\begin{array}{l}16.10 \\
16.39\end{array}$ & $\begin{array}{l}12.41 \\
14.44\end{array}$ & $\bar{w}$ & $\begin{array}{l}14.40 \\
16.29\end{array}$ & $\begin{array}{l}16.38 \\
16.53\end{array}$ & $\begin{array}{l}14.02 \\
14.38\end{array}$ \\
\hline $\begin{array}{l}1996 \\
\text { January ................... }\end{array}$ & - & $w$ & 12.95 & - & $w$ & $w$ & 14.68 \\
\hline
\end{tabular}

See tootnotes at end of table. 
Table 29. F.O.B. a Costs of Imported Crude Oil for Selected Crude Streams (Dollars per Barrel) - Continued

\begin{tabular}{|c|c|c|c|c|c|c|c|}
\hline $\begin{array}{l}\text { Year } \\
\text { Quarter } \\
\text { Month }\end{array}$ & $\begin{array}{l}\text { Nigerian } \\
\text { Brass River }\end{array}$ & $\begin{array}{l}\text { Nigerian } \\
\text { Bonny Light }\end{array}$ & $\begin{array}{l}\text { Norwegian } \\
\text { Ekofisk }\end{array}$ & $\begin{array}{c}\text { Saudi Arabian } \\
\text { Light }\end{array}$ & $\begin{array}{c}\text { Saudi Arabian } \\
\text { Heavy }\end{array}$ & $\begin{array}{c}\text { United Kingdom } \\
\text { Brent }\end{array}$ & $\begin{array}{c}\text { Venezuelan } \\
\text { Boscan }\end{array}$ \\
\hline 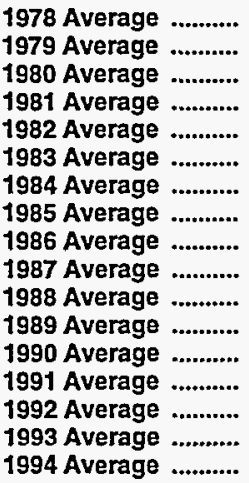 & $\begin{array}{l}14.17 \\
21.89 \\
35.57 \\
38.64 \\
35.18 \\
30.31 \\
29.82 \\
28.23 \\
14.07 \\
18.32 \\
15.39 \\
18.42 \\
23.47 \\
21.10 \\
20.26 \\
17.63 \\
15.56\end{array}$ & $\begin{array}{l}14.11 \\
25.68 \\
37.71 \\
38.19 \\
35.58 \\
30.07 \\
29.67 \\
W \\
14.02 \\
18.45 \\
15.33 \\
18.58 \\
22.67 \\
20.46 \\
20.10 \\
17.89 \\
16.06\end{array}$ & $\begin{array}{c}13.91 \\
21.36 \\
35.86 \\
37.72 \\
34.04 \\
29.73 \\
29.17 \\
W \\
16.23 \\
18.25 \\
14.58 \\
17.80 \\
W \\
20.39 \\
19.62 \\
19.03 \\
-\end{array}$ & $\begin{array}{l}12.74 \\
17.33 \\
27.79 \\
32.62 \\
34.08 \\
29.22 \\
29.11 \\
23.70 \\
11.79 \\
14.46 \\
12.98 \\
18.28 \\
21.72 \\
16.83 \\
17.34 \\
15.61 \\
14.83\end{array}$ & $\begin{array}{l}12.07 \\
15.83 \\
26.14 \\
31.42 \\
31.10 \\
26.30 \\
26.52 \\
W \\
11.00 \\
14.85 \\
11.68 \\
W \\
19.13 \\
13.25 \\
14.76 \\
12.70 \\
13.54\end{array}$ & $\begin{array}{l}-\overline{21.92} \\
33.13 \\
36.22 \\
33.55 \\
30.30 \\
29.25 \\
27.88 \\
14.07 \\
18.37 \\
14.40 \\
18.38 \\
29.33 \\
22.17 \\
19.74 \\
16.77 \\
W\end{array}$ & $\begin{array}{c}W \\
W \\
18.56 \\
W \\
19.00 \\
19.31 \\
22.24 \\
21.94 \\
9.44 \\
15.75 \\
12.29 \\
12.27 \\
12.15 \\
8.49 \\
8.25 \\
9.17 \\
8.56\end{array}$ \\
\hline $\begin{array}{l}1995 \\
\text { January ................... } \\
\text { February ................ } \\
\text { March ..................... } \\
\text { 1st Quarter }\end{array}$ & $\begin{array}{l}W \\
- \\
-\end{array}$ & $\begin{array}{l}W \\
W \\
W\end{array}$ & $\overline{-}$ & $\begin{array}{l}w \\
W \\
W\end{array}$ & $\bar{w}$ & $\begin{array}{l}W \\
W \\
W\end{array}$ & $\begin{array}{l}\vec{w} \\
w\end{array}$ \\
\hline $\begin{array}{l}\text { Average .................... } \\
\text { April ......................... } \\
\text { May ....................... } \\
\text { June ....................... } \\
\text { 2nd Quarter }\end{array}$ & $\begin{array}{l}W \\
W \\
W \\
W\end{array}$ & $\begin{array}{l}W \\
W \\
W \\
W\end{array}$ & $\begin{array}{l}W \\
- \\
- \\
-\end{array}$ & $\begin{array}{l}W \\
W \\
W \\
W\end{array}$ & $\begin{array}{l}W \\
- \\
- \\
-\end{array}$ & $\begin{array}{l}W \\
W \\
W \\
-\end{array}$ & $\begin{array}{l}W \\
\bar{w} \\
W\end{array}$ \\
\hline $\begin{array}{l}\text { Average ................. } \\
\text { July ......................... } \\
\text { August ................. } \\
\text { September ............. } \\
\text { 3rd Quarter }\end{array}$ & $\begin{array}{c}18.77 \\
- \\
- \\
-\end{array}$ & $\begin{array}{c}W \\
W \\
16.41 \\
W\end{array}$ & $\begin{array}{l}- \\
\bar{w}\end{array}$ & $\begin{array}{l}W \\
W \\
W \\
W\end{array}$ & $\begin{array}{l}- \\
- \\
-\end{array}$ & $\begin{array}{l}W \\
W \\
W \\
W\end{array}$ & $\begin{array}{l}W \\
W \\
W \\
W\end{array}$ \\
\hline $\begin{array}{l}\text { Average ..................... } \\
\text { October ................. } \\
\text { November .............. } \\
\text { December ............... } \\
\text { 4th Quarter }\end{array}$ & $\begin{array}{l}- \\
\bar{w} \\
W\end{array}$ & $\begin{array}{c}16.41 \\
W \\
W \\
W\end{array}$ & $\begin{array}{l}W \\
\overline{-} \\
\bar{W}\end{array}$ & $\begin{array}{c}W \\
W \\
16.69 \\
15.93\end{array}$ & $\begin{array}{l}- \\
- \\
-\end{array}$ & $\begin{array}{l}W \\
W \\
W \\
W\end{array}$ & $\begin{array}{l}W \\
- \\
\bar{W}\end{array}$ \\
\hline $\begin{array}{l}\text { Average ..................... } \\
1995 \text { Average .......... }\end{array}$ & $\underset{17.63}{W}$ & $\begin{array}{c}W \\
17.35\end{array}$ & $\underset{18.11}{W}$ & $\begin{array}{l}16.12 \\
16.15\end{array}$ & $\bar{w}$ & $\begin{array}{l}W \\
W\end{array}$ & $\begin{array}{l}W \\
W\end{array}$ \\
\hline $\begin{array}{l}1996 \\
\quad \text { January ..................... }\end{array}$ & $w$ & $w$ & - & $W$ & - & - & - \\
\hline
\end{tabular}

Dash $(-)=$ No data reported.

$W=$ Withheld to avoid disclosure of individual company data.

a Free on Board. See Glossary.

Notes: Values through 1980 reflect the month of reporting; values since then reflect the month of acquisition, which can be the month of loading, the month of landing, or sometime between those events. Prices for crude oil can be determined at a time other than the acquisition date. See the Explanatory Notes section for additional detail.

Notes: Values for the current 2 months are preliminary. Values shown for previous months are revised. Data are final upon publication in the Petroleum Marketing Annual.

Sources: Energy Information Administration, Form FEA-F701-M-0, "Transfer Pricing Report," January 1978 through December 1978; Form ERA-51, "Transfer Pricing Report," January 1979 through September 1982; Form EP-51, "Monthly Foreign Crude Oil Transaction Report," October 1982 through June 1984; Form EIA-856, "Monthly Foreign Crude Oil Acquisition Report," July 1984 to present. 
Table 30. Landed Costs of Imported Crude Oil for Selected Crude Streams (Dollars per Barrel)

\begin{tabular}{|c|c|c|c|c|c|c|c|}
\hline $\begin{array}{l}\text { Year } \\
\text { Quarter } \\
\text { Month }\end{array}$ & $\begin{array}{c}\text { Algerian } \\
\text { Condensate }\end{array}$ & $\begin{array}{l}\text { Angolan } \\
\text { Cabinda }\end{array}$ & $\begin{array}{c}\text { Canadian } \\
\text { Lloydminster }\end{array}$ & $\begin{array}{l}\text { Cameroon } \\
\text { Kole Marine }\end{array}$ & $\begin{array}{c}\text { Ecuadorian } \\
\text { Oriente }\end{array}$ & $\begin{array}{l}\text { Mexican } \\
\text { Isthmus }\end{array}$ & $\begin{array}{c}\text { Mexican } \\
\text { Mayan }\end{array}$ \\
\hline 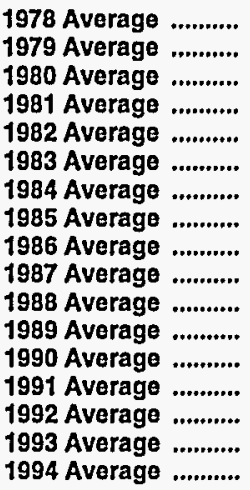 & $\begin{array}{c}W \\
W \\
37.73 \\
40.03 \\
33.71 \\
30.79 \\
28.59 \\
27.21 \\
14.54 \\
17.72 \\
W \\
W \\
W \\
W \\
W \\
W \\
W\end{array}$ & $\begin{array}{l}14.07 \\
21.51 \\
34.68 \\
36.84 \\
33.08 \\
29.31 \\
28.63 \\
27.48 \\
14.27 \\
18.43 \\
14.96 \\
18.15 \\
20.01 \\
18.31 \\
19.59 \\
16.95 \\
16.07\end{array}$ & $\begin{array}{c}- \\
\bar{w} \\
w \\
W \\
25.27 \\
25.35 \\
24.38 \\
13.52 \\
15.98 \\
12.21 \\
15.36 \\
19.55 \\
14.63 \\
W \\
13.65 \\
13.58\end{array}$ & $\begin{array}{c}W \\
25.40 \\
37.89 \\
38.95 \\
34.95 \\
30.28 \\
29.51 \\
27.94 \\
13.71 \\
18.63 \\
15.21 \\
18.71 \\
19.76 \\
W \\
W \\
W \\
W\end{array}$ & $\begin{array}{l}13.85 \\
29.17 \\
34.61 \\
33.56 \\
32.97 \\
28.90 \\
28.79 \\
26.97 \\
14.39 \\
17.60 \\
13.77 \\
17.69 \\
21.63 \\
17.52 \\
18.52 \\
15.79 \\
15.24\end{array}$ & $\begin{array}{l}13.54 \\
20.78 \\
33.42 \\
36.87 \\
33.11 \\
30.00 \\
29.46 \\
27.60 \\
14.28 \\
18.32 \\
14.69 \\
W \\
23.79 \\
19.16 \\
18.40 \\
16.50 \\
15.36\end{array}$ & $\begin{array}{c}- \\
22.23 \\
29.49 \\
31.52 \\
25.86 \\
24.56 \\
25.84 \\
24.57 \\
11.24 \\
16.03 \\
11.65 \\
15.14 \\
17.75 \\
13.62 \\
13.80 \\
12.45 \\
12.79\end{array}$ \\
\hline $\begin{array}{l}1995 \\
\text { January .................. } \\
\text { February ............... } \\
\text { March .................... } \\
\text { Ist Quarter }\end{array}$ & $\begin{array}{l}W \\
W \\
W\end{array}$ & $\begin{array}{l}16.68 \\
17.42 \\
17.67\end{array}$ & $\begin{array}{l}15.15 \\
16.00 \\
16.39\end{array}$ & $\begin{array}{l}- \\
- \\
-\end{array}$ & $\begin{array}{l}16.50 \\
17.23 \\
16.91\end{array}$ & $\begin{array}{l}W \\
W \\
W\end{array}$ & $\begin{array}{l}14.33 \\
15.00 \\
15.42\end{array}$ \\
\hline $\begin{array}{l}\text { Average .................... } \\
\text { April ......................... } \\
\text { May ........................ } \\
\text { June ........................ } \\
\text { 2nd Quarter }\end{array}$ & $\begin{array}{l}W \\
W \\
W \\
-\end{array}$ & $\begin{array}{l}17.35 \\
18.57 \\
18.73 \\
18.00\end{array}$ & $\begin{array}{l}15.81 \\
17.76 \\
17.83 \\
16.48\end{array}$ & $\begin{array}{l}\bar{w} \\
- \\
-\end{array}$ & $\begin{array}{l}16.85 \\
18.12 \\
18.85 \\
17.38\end{array}$ & $\begin{array}{l}W \\
W \\
W \\
W\end{array}$ & $\begin{array}{l}14.92 \\
16.29 \\
16.74 \\
15.43\end{array}$ \\
\hline $\begin{array}{l}\text { Average .................... } \\
\text { July ......................... } \\
\text { August ................... } \\
\text { September ............. } \\
\text { Brd Quarter }\end{array}$ & $\begin{array}{l}W \\
W \\
W \\
W\end{array}$ & $\begin{array}{l}18.43 \\
16.60 \\
16.13 \\
16.83\end{array}$ & $\begin{array}{c}17.31 \\
15.35 \\
W \\
W\end{array}$ & $\begin{array}{l}W \\
- \\
- \\
-\end{array}$ & $\begin{array}{c}18.20 \\
15.63 \\
15.67 \\
W\end{array}$ & $\begin{array}{c}W \\
16.20 \\
16.56 \\
16.82\end{array}$ & $\begin{array}{l}16.14 \\
13.92 \\
13.74 \\
14.10\end{array}$ \\
\hline $\begin{array}{l}\text { Average .................... } \\
\text { October ................... } \\
\text { November ............. } \\
\text { December .............. } \\
\text { 4th Quarter }\end{array}$ & $\begin{array}{l}W \\
W \\
- \\
-\end{array}$ & $\begin{array}{l}16.51 \\
16.59 \\
16.91 \\
17.52\end{array}$ & $\begin{array}{l}15.58 \\
13.46 \\
13.29 \\
13.66\end{array}$ & $\begin{array}{l}- \\
- \\
-\end{array}$ & $\begin{array}{c}15.80 \\
W \\
15.07 \\
16.95\end{array}$ & $\begin{array}{c}16.54 \\
W \\
16.55 \\
17.66\end{array}$ & $\begin{array}{l}13.91 \\
13.65 \\
13.98 \\
15.66\end{array}$ \\
\hline $\begin{array}{l}\text { Average .................. } \\
1995 \text { Average .......... }\end{array}$ & $\begin{array}{l}W \\
W\end{array}$ & $\begin{array}{l}16.93 \\
17.32\end{array}$ & $\begin{array}{l}13.49 \\
15.56\end{array}$ & $\bar{w}$ & $\begin{array}{l}15.69 \\
16.84\end{array}$ & $\begin{array}{l}16.87 \\
16.99\end{array}$ & $\begin{array}{l}14.59 \\
14.88\end{array}$ \\
\hline $\begin{array}{l}1996 \\
\text { January }\end{array}$ & $w$ & 17.57 & 14.07 & - & $w$ & 18.38 & 15.34 \\
\hline
\end{tabular}

See footnotes at end of table. 
Table 30. Landed Costs of Imported Crude Oil for Selected Crude Streams (Dollars per Barrel) - Continued

\begin{tabular}{|c|c|c|c|c|c|c|c|}
\hline $\begin{array}{l}\text { Year } \\
\text { Quarter } \\
\text { Month }\end{array}$ & $\begin{array}{c}\text { Nigerian } \\
\text { Brass River }\end{array}$ & $\begin{array}{c}\text { Nigerian } \\
\text { Bonny Light }\end{array}$ & $\begin{array}{l}\text { Norwegian } \\
\text { Ekofisk }\end{array}$ & $\begin{array}{c}\text { Saudi Arablan } \\
\text { Light }\end{array}$ & $\begin{array}{c}\text { Saudi Arabian } \\
\text { Heavy }\end{array}$ & $\begin{array}{c}\text { United Kingdom } \\
\text { Brent }\end{array}$ & $\begin{array}{c}\text { Venezuelan } \\
\text { Boscan }\end{array}$ \\
\hline 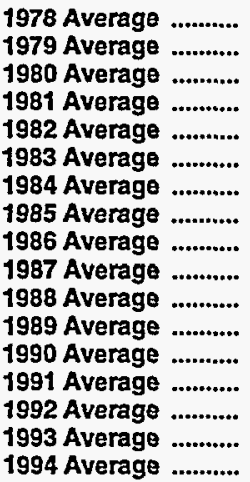 & $\begin{array}{l}14.97 \\
23.19 \\
37.02 \\
40.01 \\
36.26 \\
31.44 \\
30.71 \\
29.14 \\
14.89 \\
19.39 \\
16.06 \\
19.34 \\
24.29 \\
22.27 \\
21.25 \\
18.66 \\
16.30\end{array}$ & $\begin{array}{l}15.04 \\
27.11 \\
38.58 \\
39.25 \\
36.45 \\
31.06 \\
30.46 \\
28.98 \\
15.00 \\
19.26 \\
16.02 \\
19.38 \\
23.21 \\
21.57 \\
20.85 \\
18.75 \\
17.23\end{array}$ & $\begin{array}{l}14.68 \\
22.64 \\
36.91 \\
38.70 \\
34.70 \\
30.79 \\
29.99 \\
28.16 \\
15.07 \\
18.96 \\
15.45 \\
18.67 \\
21.53 \\
21.66 \\
20.96 \\
19.72 \\
-\end{array}$ & $\begin{array}{l}14.04 \\
19.18 \\
29.57 \\
34.32 \\
35.65 \\
30.95 \\
30.61 \\
25.35 \\
13.06 \\
17.88 \\
14.04 \\
17.96 \\
22.49 \\
18.49 \\
18.54 \\
16.62 \\
15.83\end{array}$ & $\begin{array}{l}13.32 \\
17.68 \\
28.16 \\
33.02 \\
32.64 \\
28.12 \\
28.10 \\
23.69 \\
12.17 \\
16.20 \\
12.80 \\
16.56 \\
21.46 \\
15.28 \\
16.10 \\
14.19 \\
14.27\end{array}$ & $\begin{array}{l}- \\
23.26 \\
34.57 \\
37.58 \\
34.53 \\
31.26 \\
29.89 \\
28.49 \\
14.64 \\
18.71 \\
15.84 \\
18.83 \\
24.40 \\
21.65 \\
20.68 \\
18.02 \\
16.65\end{array}$ & $\begin{array}{c}W \\
W \\
20.34 \\
W \\
20.32 \\
20.69 \\
23.20 \\
22.85 \\
10.42 \\
16.65 \\
13.23 \\
13.16 \\
13.43 \\
10.17 \\
9.65 \\
11.32 \\
10.81\end{array}$ \\
\hline $\begin{array}{l}1995 \\
\text { January ................... } \\
\text { February ............... } \\
\text { March ...................... } \\
\text { 1st Quarter }\end{array}$ & $\begin{array}{l}W \\
W \\
W\end{array}$ & $\begin{array}{l}17.85 \\
18.29 \\
18.37\end{array}$ & $\bar{w}$ & $\begin{array}{l}17.03 \\
17.63 \\
17.89\end{array}$ & $\begin{array}{c}15.97 \\
16.40 \\
W\end{array}$ & $\begin{array}{l}17.50 \\
17.75 \\
18.07\end{array}$ & $\begin{array}{l}\bar{W} \\
W\end{array}$ \\
\hline $\begin{array}{l}\text { Average ................... } \\
\text { Apil .......................... } \\
\text { May ....................... } \\
\text { June ....................... } \\
\text { 2nd Quarter }\end{array}$ & $\begin{array}{c}W \\
19.80 \\
19.53 \\
20.26\end{array}$ & $\begin{array}{c}18.15 \\
20.10 \\
W \\
W\end{array}$ & $\begin{array}{l}W \\
- \\
- \\
-\end{array}$ & $\begin{array}{l}17.52 \\
18.56 \\
17.83 \\
16.76\end{array}$ & $\begin{array}{c}16.26 \\
W \\
W \\
W\end{array}$ & $\begin{array}{l}17.89 \\
18.47 \\
19.19 \\
19.16\end{array}$ & $\begin{array}{l}w \\
w \\
W\end{array}$ \\
\hline $\begin{array}{l}\text { Average .................... } \\
\text { July ........................ } \\
\text { August ................... } \\
\text { September ............. } \\
\text { 3rd Quarter }\end{array}$ & $\begin{array}{c}19.73 \\
18.09 \\
17.49 \\
W\end{array}$ & $\begin{array}{c}19.64 \\
W \\
17.44 \\
17.81\end{array}$ & $\begin{array}{c}- \\
\overline{-} \\
18.24\end{array}$ & $\begin{array}{l}17.82 \\
16.33 \\
16.27 \\
16.65\end{array}$ & $\begin{array}{c}W \\
W \\
W \\
15.44\end{array}$ & $\begin{array}{l}18.94 \\
17.44 \\
17.41 \\
17.43\end{array}$ & $\begin{array}{l}W \\
W \\
W \\
W\end{array}$ \\
\hline $\begin{array}{l}\text { Average ................... } \\
\text { October .................. } \\
\text { November .............. } \\
\text { December ............... } \\
\text { 4th Quarter }\end{array}$ & $\begin{array}{c}18.09 \\
- \\
17.61 \\
18.63\end{array}$ & $\begin{array}{c}17.55 \\
W \\
W \\
19.22\end{array}$ & $\begin{array}{c}18.24 \\
- \\
\bar{w}\end{array}$ & $\begin{array}{l}16.45 \\
16.47 \\
17.44 \\
17.29\end{array}$ & $\begin{array}{c}15.34 \\
W \\
W \\
W\end{array}$ & $\begin{array}{l}17.43 \\
17.33 \\
17.57 \\
18.43\end{array}$ & $\begin{array}{l}w \\
\bar{w} \\
\bar{w}\end{array}$ \\
\hline $\begin{array}{l}\text { Average ..................... } \\
1995 \text { Average }\end{array}$ & $\begin{array}{l}18.20 \\
18.68\end{array}$ & $\begin{array}{l}18.21 \\
18.34\end{array}$ & $\begin{array}{c}W \\
18.75\end{array}$ & $\begin{array}{l}17.08 \\
17.22\end{array}$ & $\begin{array}{c}W \\
16.07\end{array}$ & $\begin{array}{l}17.61 \\
18.00\end{array}$ & $\begin{array}{l}W \\
W\end{array}$ \\
\hline $\begin{array}{l}1996 \\
\quad \text { January ................... }\end{array}$ & $w$ & $W$ & - & 17.26 & $w$ & 18.70 & - \\
\hline
\end{tabular}

Dash $(-)=$ No data reported.

$W=$ Withheld to avoid disclosure of individual company data.

Notes: Values through 1980 reflect the month of reporting; values since then reflect the month of acquisition, which can be the month of loading, the month of landing, or sometime between those events. Prices for crude oil can be determined at a time other than the acquisition date. See the Explanatory Notes section for additional detail.

Notes: Values for the current 2 months are preliminary. Values shown for previous months are revised. Data are final upon publication in the Petroleum Marketing Annual.

Sources: Energy Information Administration, Form FEA-F701-M-0, "Transfer Pricing Report," January 1978 through December 1978; Form ERA-51, "Transfer Pricing Report," January 1979 through September 1982; Form EP-51, "Monthly Foreign Crude Oil Transaction Report," October 1982 through June 1984; Form ElA-856, "Monthly Foreign Crude Oil Acquisition Report," July 1984 to present. 
Prices of Petroleum Products 
Table 31. Motor Gasoline Prices by Grade, Sales Type, PAD District, and State (Cents per Gallon Excluding Taxes)

\begin{tabular}{|c|c|c|c|c|c|c|c|c|c|c|c|c|}
\hline \multirow{3}{*}{$\begin{array}{c}\text { Geographic Area } \\
\text { Month }\end{array}$} & \multicolumn{6}{|c|}{ Regular } & \multicolumn{6}{|c|}{ Midgrade } \\
\hline & \multicolumn{2}{|c|}{ Sales to End Users } & \multicolumn{4}{|c|}{ Sales for Resale } & \multicolumn{2}{|c|}{ Sales to End Users } & \multicolumn{4}{|c|}{ Sales for Resale } \\
\hline & $\begin{array}{c}\text { Through } \\
\text { Retail } \\
\text { Outlets }\end{array}$ & Average ${ }^{a}$ & DTW & Rack & Bulk & Average & $\begin{array}{c}\text { Through } \\
\text { Retail } \\
\text { Outlets }\end{array}$ & Average $\mathrm{a}$ & DTW & Rack & Bulk & Averago \\
\hline \multicolumn{13}{|l|}{ United States } \\
\hline $\begin{array}{l}\text { January } 1996 \ldots . . . . . . . . . . \\
\text { December } 1995 \\
\text { January } 1995\end{array}$ & $\begin{array}{l}70.3 \\
68.9 \\
69.6\end{array}$ & $\begin{array}{l}69.9 \\
68.6 \\
69.1\end{array}$ & $\begin{array}{l}64.4 \\
63.3 \\
64.4\end{array}$ & $\begin{array}{l}56.3 \\
55.6 \\
54.2\end{array}$ & $\begin{array}{l}51.7 \\
50.8 \\
49.9\end{array}$ & $\begin{array}{l}58.2 \\
57.2 \\
56.9\end{array}$ & $\begin{array}{l}78.6 \\
77.1 \\
78.3\end{array}$ & $\begin{array}{l}78.1 \\
76.8 \\
77.7\end{array}$ & $\begin{array}{l}69.9 \\
68.6 \\
70.3\end{array}$ & $\begin{array}{l}60.8 \\
60.0 \\
58.9\end{array}$ & $\overline{\text { NA }}$ & $\begin{array}{l}65.7 \\
64.6 \\
65.2\end{array}$ \\
\hline \multicolumn{13}{|l|}{ PAD District I } \\
\hline $\begin{array}{l}\text { January } 1996 \text {............... } \\
\text { December } 1995 \text {............ } \\
\text { January } 1995 \text {.............. } \\
\text { Subdistrict IA }\end{array}$ & $\begin{array}{l}68.6 \\
66.9 \\
69.5\end{array}$ & $\begin{array}{l}68.2 \\
66.6 \\
68.8\end{array}$ & $\begin{array}{l}64.0 \\
62.6 \\
64.9\end{array}$ & $\begin{array}{l}56.4 \\
55.8 \\
55.1\end{array}$ & $\begin{array}{l}54.1 \\
55.9 \\
54.4\end{array}$ & $\begin{array}{l}58.9 \\
58.3 \\
58.6\end{array}$ & $\begin{array}{l}77.9 \\
76.3 \\
79.6\end{array}$ & $\begin{array}{l}77.2 \\
75.8 \\
78.7\end{array}$ & $\begin{array}{l}70.1 \\
68.1 \\
71.5\end{array}$ & $\begin{array}{l}60.7 \\
59.8 \\
59.5\end{array}$ & $\bar{w}$ & $\begin{array}{l}65.3 \\
64.0 \\
65.6\end{array}$ \\
\hline 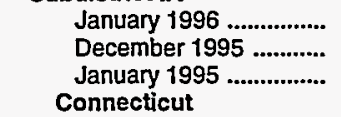 & $\begin{array}{l}75.4 \\
74.1 \\
74.2\end{array}$ & $\begin{array}{l}75.0 \\
73.8 \\
73.7\end{array}$ & $\begin{array}{l}70.2 \\
68.6 \\
69.4\end{array}$ & $\begin{array}{l}62.1 \\
62.1 \\
60.3\end{array}$ & $\begin{array}{l}55.5 \\
60.4 \\
54.6\end{array}$ & $\begin{array}{l}64.9 \\
64.7 \\
63.0\end{array}$ & $\begin{array}{l}85.1 \\
83.9 \\
84.2\end{array}$ & $\begin{array}{l}83.9 \\
82.7 \\
82.7\end{array}$ & $\begin{array}{l}77.1 \\
75.2 \\
76.2\end{array}$ & $\begin{array}{l}66.6 \\
66.3 \\
63.6\end{array}$ & $\overline{-}$ & $\begin{array}{l}72.1 \\
71.1 \\
70.3\end{array}$ \\
\hline $\begin{array}{l}\text { January } 1996 \text {................ } \\
\text { December } 1995 \text {............ } \\
\text { January } 1995 \text {................ } \\
\text { Maine }\end{array}$ & $\begin{array}{l}74.9 \\
73.3 \\
72.8\end{array}$ & $\begin{array}{l}74.3 \\
72.9 \\
72.2\end{array}$ & $\begin{array}{l}69.6 \\
68.1 \\
70.2\end{array}$ & $\begin{array}{l}62.4 \\
62.2 \\
61.3\end{array}$ & $\begin{array}{l}W \\
W \\
W\end{array}$ & $\begin{array}{l}65.5 \\
64.8 \\
64.5\end{array}$ & $\begin{array}{l}85.2 \\
83.0 \\
83.7\end{array}$ & $\begin{array}{l}82.7 \\
81.0 \\
81.6\end{array}$ & $\begin{array}{l}75.4 \\
73.6 \\
76.0\end{array}$ & $\begin{array}{l}67.0 \\
66.6 \\
65.8\end{array}$ & $\begin{array}{l}- \\
-\end{array}$ & $\begin{array}{l}71.6 \\
70.3 \\
71.3\end{array}$ \\
\hline $\begin{array}{l}\text { January } 1996 \text {................ } \\
\text { December } 1995 \text {............ } \\
\text { January } 1995 \text {................ } \\
\text { Massachusetts }\end{array}$ & $\begin{array}{l}77.0 \\
75.4 \\
75.5\end{array}$ & $\begin{array}{l}76.6 \\
75.2 \\
75.1\end{array}$ & $\begin{array}{l}69.5 \\
68.7 \\
66.1\end{array}$ & $\begin{array}{l}61.7 \\
62.3 \\
58.6\end{array}$ & $\begin{array}{l}51.5 \\
58.7 \\
52.6\end{array}$ & $\begin{array}{l}61.7 \\
63.0 \\
58.2\end{array}$ & $\begin{array}{l}87.0 \\
86.5 \\
84.6\end{array}$ & $\begin{array}{l}86.0 \\
85.7 \\
83.6\end{array}$ & $\begin{array}{l}82.1 \\
78.8 \\
76.6\end{array}$ & $\begin{array}{l}65.5 \\
66.0 \\
61.7\end{array}$ & $\begin{array}{l}- \\
-\end{array}$ & $\begin{array}{l}70.3 \\
69.9 \\
65.9\end{array}$ \\
\hline $\begin{array}{l}\text { January } 1996 \text {................ } \\
\text { December } 1995 \text {............ } \\
\text { January } 1995 \text {............... } \\
\text { New Hampshire }\end{array}$ & $\begin{array}{l}75.4 \\
74.1 \\
74.3\end{array}$ & $\begin{array}{l}75.0 \\
73.8 \\
73.8\end{array}$ & $\begin{array}{l}71.1 \\
69.1 \\
70.0\end{array}$ & $\begin{array}{l}62.2 \\
62.0 \\
60.6\end{array}$ & $\begin{array}{l}56.9 \\
62.4 \\
55.4\end{array}$ & $\begin{array}{l}65.2 \\
65.1 \\
63.9\end{array}$ & $\begin{array}{l}84.9 \\
84.1 \\
84.4\end{array}$ & $\begin{array}{l}83.9 \\
82.8 \\
82.7\end{array}$ & $\begin{array}{l}78.2 \\
76.3 \\
77.2\end{array}$ & $\begin{array}{l}67.0 \\
66.3 \\
63.4\end{array}$ & $\vec{w}$ & $\begin{array}{l}73.2 \\
72.1 \\
71.2\end{array}$ \\
\hline $\begin{array}{l}\text { January } 1996 . . . . . . . . . . . . . . \\
\text { December } 1995 \text {............ } \\
\text { January } 1995 \text {................ } \\
\text { Rhode Island }\end{array}$ & $\begin{array}{l}75.1 \\
73.7 \\
76.2\end{array}$ & $\begin{array}{l}74.9 \\
73.5 \\
75.9\end{array}$ & $\begin{array}{l}69.2 \\
68.1 \\
69.9\end{array}$ & $\begin{array}{l}64.1 \\
63.5 \\
61.4\end{array}$ & $\begin{array}{l}- \\
-\end{array}$ & $\begin{array}{l}67.6 \\
66.7 \\
67.0\end{array}$ & $\begin{array}{l}85.3 \\
83.5 \\
86.4\end{array}$ & $\begin{array}{l}84.9 \\
83.1 \\
85.8\end{array}$ & $\begin{array}{l}74.4 \\
72.5 \\
76.3\end{array}$ & $\begin{array}{l}68.4 \\
68.6 \\
60.9\end{array}$ & $\overline{-}$ & $\begin{array}{l}73.2 \\
71.7 \\
70.8\end{array}$ \\
\hline $\begin{array}{l}\text { January } 1996 \text {................ } \\
\text { December } 1995 \text {............ } \\
\text { January } 1995 \text {................ } \\
\text { Vermont }\end{array}$ & $\begin{array}{l}72.3 \\
71.5 \\
69.6\end{array}$ & $\begin{array}{l}72.1 \\
71.4 \\
69.4\end{array}$ & $\begin{array}{l}68.2 \\
66.0 \\
66.5\end{array}$ & $\begin{array}{l}61.7 \\
61.3 \\
60.1\end{array}$ & $\begin{array}{l}W \\
W \\
W\end{array}$ & $\begin{array}{l}64.2 \\
63.1 \\
61.0\end{array}$ & $\begin{array}{l}80.9 \\
80.0 \\
79.7\end{array}$ & $\begin{array}{l}80.7 \\
79.7 \\
79.1\end{array}$ & $\begin{array}{l}74.4 \\
72.3 \\
72.9\end{array}$ & $\begin{array}{l}66.1 \\
65.5 \\
63.2\end{array}$ & $\bar{z}$ & $\begin{array}{l}70.4 \\
68.9 \\
68.0\end{array}$ \\
\hline 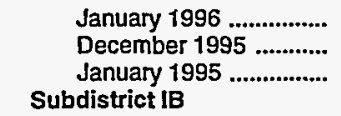 & $\begin{array}{l}78.2 \\
77.6 \\
76.1\end{array}$ & $\begin{array}{l}77.6 \\
77.0 \\
75.5\end{array}$ & $\begin{array}{l}70.4 \\
70.6 \\
68.1\end{array}$ & $\begin{array}{l}60.2 \\
61.1 \\
57.7\end{array}$ & - & $\begin{array}{l}67.7 \\
67.9 \\
65.2\end{array}$ & $\begin{array}{l}87.9 \\
86.8 \\
86.7\end{array}$ & $\begin{array}{l}86.3 \\
85.4 \\
84.6\end{array}$ & $\begin{array}{l}76.8 \\
76.9 \\
73.0\end{array}$ & $\begin{array}{l}64.2 \\
65.3 \\
62.0\end{array}$ & $\dot{-}$ & $\begin{array}{l}73.8 \\
73.7 \\
70.3\end{array}$ \\
\hline $\begin{array}{l}\text { January } 1996 \ldots . . . . . . . . . . . . \\
\text { December } 1995 \text {............ } \\
\text { January } 1995 \text {................ } \\
\text { Delaware }\end{array}$ & $\begin{array}{l}71.8 \\
70.8 \\
72.6\end{array}$ & $\begin{array}{l}71.2 \\
70.4 \\
71.9\end{array}$ & $\begin{array}{l}65.1 \\
63.9 \\
67.3\end{array}$ & $\begin{array}{l}58.5 \\
59.4 \\
57.9\end{array}$ & $\begin{array}{l}53.9 \\
56.0 \\
55.2\end{array}$ & $\begin{array}{l}60.2 \\
60.6 \\
61.6\end{array}$ & $\begin{array}{l}81.1 \\
80.0 \\
82.6\end{array}$ & $\begin{array}{l}80.1 \\
79.3 \\
81.6\end{array}$ & $\begin{array}{l}72.0 \\
70.0 \\
74.8\end{array}$ & $\begin{array}{l}63.4 \\
63.9 \\
63.3\end{array}$ & $\begin{array}{l}- \\
-\end{array}$ & $\begin{array}{l}68.6 \\
67.7 \\
70.6\end{array}$ \\
\hline $\begin{array}{l}\text { January } 1996 \ldots . . . . . . . . . . . . . \\
\text { December } 1995 . . . . . . . . . \\
\text { January } 1995 \ldots . . . . . . . . . . . . . . \\
\text { District of Columbia }\end{array}$ & $\begin{array}{l}70.2 \\
69.6 \\
72.3\end{array}$ & $\begin{array}{l}69.9 \\
69.3 \\
71.9\end{array}$ & $\begin{array}{l}63.4 \\
62.4 \\
66.8\end{array}$ & $\begin{array}{l}58.9 \\
59.3 \\
60.1\end{array}$ & $\begin{array}{l}W \\
W \\
W\end{array}$ & $\begin{array}{l}61.0 \\
60.8 \\
62.9\end{array}$ & $\begin{array}{l}80.4 \\
79.9 \\
83.0\end{array}$ & $\begin{array}{l}80.1 \\
79.8 \\
82.5\end{array}$ & $\begin{array}{l}69.1 \\
67.8 \\
73.4\end{array}$ & $\begin{array}{l}63.0 \\
63.5 \\
64.9\end{array}$ & $\begin{array}{l}- \\
-\end{array}$ & $\begin{array}{l}66.4 \\
66.0 \\
69.2\end{array}$ \\
\hline $\begin{array}{l}\text { January } 1996 \ldots . . . . . . . . . . . . . \\
\text { December } 1995 \text {........... } \\
\text { January } 1995 . . . . . . . . . . . . . .\end{array}$ & $\begin{array}{l}W \\
W \\
W\end{array}$ & $\begin{array}{l}64.8 \\
62.9 \\
69.1\end{array}$ & $\begin{array}{l}65.4 \\
62.7 \\
73.5\end{array}$ & $\overline{-}$ & $\begin{array}{l}- \\
-\end{array}$ & $\begin{array}{l}65.4 \\
62.7 \\
73.2\end{array}$ & $\begin{array}{l}W \\
W \\
W\end{array}$ & $\begin{array}{l}74.2 \\
72.2 \\
78.3\end{array}$ & $\begin{array}{l}70.0 \\
67.4 \\
79.1\end{array}$ & $\bar{w}$ & $\begin{array}{l}- \\
-\end{array}$ & $\begin{array}{l}70.0 \\
67.4 \\
79.0\end{array}$ \\
\hline $\begin{array}{l}\text { January } 1996 \text {................ } \\
\text { December } 1995 \text {............ } \\
\text { January } 1995 \text {................ } \\
\text { New Jersey }\end{array}$ & $\begin{array}{l}72.4 \\
71.4 \\
76.9\end{array}$ & $\begin{array}{l}69.6 \\
68.7 \\
73.8\end{array}$ & $\begin{array}{l}63.9 \\
61.6 \\
69.4\end{array}$ & $\begin{array}{l}58.0 \\
57.0 \\
60.0\end{array}$ & $\begin{array}{l}W \\
W \\
W\end{array}$ & $\begin{array}{l}61.8 \\
59.8 \\
66.2\end{array}$ & $\begin{array}{l}80.5 \\
79.3 \\
85.0\end{array}$ & $\begin{array}{l}77.3 \\
76.5 \\
82.0\end{array}$ & $\begin{array}{l}69.3 \\
66.6 \\
76.3\end{array}$ & $\begin{array}{l}62.7 \\
61.9 \\
65.0\end{array}$ & $\begin{array}{l}- \\
-\end{array}$ & $\begin{array}{l}67.4 \\
65.2 \\
73.3\end{array}$ \\
\hline $\begin{array}{l}\text { January } 1996 . . . . . . . . . . . . . \\
\text { December } 1995 \text {............ } \\
\text { January } 1995 \text {............... } \\
\text { New York }\end{array}$ & $\begin{array}{l}74.4 \\
72.7 \\
75.9\end{array}$ & $\begin{array}{l}73.8 \\
72.1 \\
75.1\end{array}$ & $\begin{array}{l}68.8 \\
67.0 \\
70.7\end{array}$ & $\begin{array}{l}61.5 \\
61.9 \\
61.5\end{array}$ & $\begin{array}{l}54.3 \\
57.5 \\
55.6\end{array}$ & $\begin{array}{l}59.7 \\
61.6 \\
61.8\end{array}$ & $\begin{array}{l}86.5 \\
84.8 \\
88.3\end{array}$ & $\begin{array}{l}85.3 \\
84.0 \\
87.4\end{array}$ & $\begin{array}{l}74.9 \\
72.8 \\
76.6\end{array}$ & $\begin{array}{l}67.1 \\
67.3 \\
66.8\end{array}$ & $\begin{array}{l}- \\
-\end{array}$ & $\begin{array}{l}72.3 \\
71.0 \\
73.5\end{array}$ \\
\hline $\begin{array}{l}\text { January } 1996 \text {................ } \\
\text { December } 1995 \text {............ } \\
\text { January } 1995 \text {................ } \\
\text { Pennsylvania }\end{array}$ & $\begin{array}{l}72.5 \\
72.3 \\
71.8\end{array}$ & $\begin{array}{l}72.1 \\
72.1 \\
71.3\end{array}$ & $\begin{array}{l}65.2 \\
64.6 \\
65.7\end{array}$ & $\begin{array}{l}59.2 \\
60.6 \\
58.3\end{array}$ & $\begin{array}{l}54.6 \\
53.6 \\
55.2\end{array}$ & $\begin{array}{l}61.9 \\
61.7 \\
61.9\end{array}$ & $\begin{array}{l}82.3 \\
82.2 \\
82.0\end{array}$ & $\begin{array}{l}81.5 \\
81.6 \\
80.7\end{array}$ & $\begin{array}{l}74.8 \\
72.6 \\
74.3\end{array}$ & $\begin{array}{l}65.0 \\
66.0 \\
63.6\end{array}$ & $\begin{array}{l}- \\
-\end{array}$ & $\begin{array}{l}71.3 \\
70.3 \\
71.0\end{array}$ \\
\hline $\begin{array}{l}\text { January } 1996 . . . . . . . . . . . . . . . \\
\text { December } 1995 \text {........... } \\
\text { January } 1995 . . . . . . . . . . . . . .\end{array}$ & $\begin{array}{l}70.0 \\
68.8 \\
71.6\end{array}$ & $\begin{array}{l}69.7 \\
68.6 \\
70.9\end{array}$ & $\begin{array}{l}63.0 \\
62.1 \\
65.4\end{array}$ & $\begin{array}{l}56.5 \\
57.7 \\
55.3\end{array}$ & $\begin{array}{l}51.3 \\
54.1 \\
52.1\end{array}$ & $\begin{array}{l}58.1 \\
58.6 \\
58.9\end{array}$ & $\begin{array}{l}78.8 \\
77.5 \\
80.8\end{array}$ & $\begin{array}{l}78.3 \\
77.2 \\
80.3\end{array}$ & $\begin{array}{l}69.4 \\
68.5 \\
72.0\end{array}$ & $\begin{array}{l}61.2 \\
62.2 \\
60.9\end{array}$ & $\begin{array}{l}- \\
-\end{array}$ & $\begin{array}{l}65.0 \\
65.2 \\
66.3\end{array}$ \\
\hline
\end{tabular}

See footnotes at end of table. 
Table 31. Motor Gasoline Prices by Grade, Sales Type, PAD District, and State (Cents per Gallon Excluding Taxes) - Continued

\begin{tabular}{|c|c|c|c|c|c|c|c|c|c|c|c|c|}
\hline \multirow{3}{*}{$\begin{array}{c}\text { Geographic Area } \\
\text { Month }\end{array}$} & \multicolumn{6}{|c|}{ Premium } & \multicolumn{6}{|c|}{ All Grades } \\
\hline & \multicolumn{2}{|c|}{ Sales to End Users } & \multicolumn{4}{|c|}{ Sales for Resale } & \multicolumn{2}{|c|}{ Sales to End Users } & \multicolumn{4}{|c|}{ Sales for Resale } \\
\hline & $\begin{array}{c}\text { Through } \\
\text { Retail } \\
\text { Outlets }\end{array}$ & Average ${ }^{a}$ & DTW & Rack & Bulk & Average & $\begin{array}{l}\text { Through } \\
\text { Retail } \\
\text { Outlets }\end{array}$ & Average ${ }^{a}$ & DTW & Rack & Bulk & Average \\
\hline \multicolumn{13}{|l|}{ United States } \\
\hline 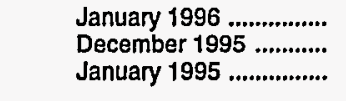 & $\begin{array}{l}87.1 \\
85.7 \\
87.2\end{array}$ & $\begin{array}{l}86.3 \\
85.0 \\
86.1\end{array}$ & $\begin{array}{l}77.2 \\
76.0 \\
78.6\end{array}$ & $\begin{array}{l}65.9 \\
65.2 \\
64.0\end{array}$ & $\begin{array}{l}58.2 \\
57.9 \\
54.9\end{array}$ & $\begin{array}{l}70.5 \\
69.4 \\
70.2\end{array}$ & $\begin{array}{l}74.5 \\
73.2 \\
74.0\end{array}$ & $\begin{array}{l}74.0 \\
72.8 \\
73.4\end{array}$ & $\begin{array}{l}68.2 \\
67.1 \\
68.7\end{array}$ & $\begin{array}{l}58.5 \\
57.9 \\
56.4\end{array}$ & $\begin{array}{l}52.5 \\
51.6 \\
50.4\end{array}$ & $\begin{array}{l}61.4 \\
60.4 \\
60.4\end{array}$ \\
\hline \multicolumn{13}{|l|}{ PAD District I } \\
\hline $\begin{array}{l}\text { January } 1996 \text {................ } \\
\text { December } 1995 \text {............. } \\
\text { January } 1995 \\
\text { Subdistrict IA }\end{array}$ & $\begin{array}{l}86.9 \\
85.3 \\
88.9\end{array}$ & $\begin{array}{l}85.9 \\
84.5 \\
87.8\end{array}$ & $\begin{array}{l}77.8 \\
76.0 \\
80.1\end{array}$ & $\begin{array}{l}66.6 \\
66.1 \\
65.5\end{array}$ & $\begin{array}{l}61.9 \\
63.9 \\
58.4\end{array}$ & $\begin{array}{l}71.6 \\
70.6 \\
72.1\end{array}$ & $\begin{array}{l}74.0 \\
72.5 \\
75.3\end{array}$ & $\begin{array}{l}73.4 \\
72.0 \\
74.4\end{array}$ & $\begin{array}{l}68.9 \\
67.4 \\
70.3\end{array}$ & $\begin{array}{l}59.2 \\
58.6 \\
57.9\end{array}$ & $\begin{array}{l}55.6 \\
57.7 \\
55.2\end{array}$ & $\begin{array}{l}62.8 \\
62.1 \\
62.9\end{array}$ \\
\hline $\begin{array}{l}\text { January } 1996 \ldots 9 . . . . . . . . . . . . \\
\text { December } 1995 \ldots . . . . . . . . \\
\text { January } 1995 \text {................ } \\
\text { Connecticut }\end{array}$ & $\begin{array}{l}93.6 \\
92.4 \\
93.7\end{array}$ & $\begin{array}{l}92.2 \\
91.3 \\
92.3\end{array}$ & $\begin{array}{l}83.4 \\
81.8 \\
83.4\end{array}$ & $\begin{array}{l}71.9 \\
72.1 \\
69.9\end{array}$ & $\begin{array}{c}\text { NA } \\
63.2 \\
W\end{array}$ & $\begin{array}{l}77.0 \\
76.1 \\
75.7\end{array}$ & $\begin{array}{l}80.2 \\
79.1 \\
79.5\end{array}$ & $\begin{array}{l}79.6 \\
78.5 \\
78.8\end{array}$ & $\begin{array}{l}74.2 \\
72.7 \\
73.8\end{array}$ & $\begin{array}{l}64.7 \\
64.6 \\
62.7\end{array}$ & $\begin{array}{l}57.7 \\
61.3 \\
55.1\end{array}$ & $\begin{array}{l}68.4 \\
68.0 \\
66.6\end{array}$ \\
\hline $\begin{array}{l}\text { January } 1996 \text {................ } \\
\text { December } 1995 \text {............ } \\
\text { January } 1995 \text {............... } \\
\text { Maine }\end{array}$ & $\begin{array}{l}94.2 \\
92.7 \\
93.4\end{array}$ & $\begin{array}{l}92.7 \\
91.4 \\
92.0\end{array}$ & $\begin{array}{l}82.0 \\
80.5 \\
83.6\end{array}$ & $\begin{array}{l}72.7 \\
72.6 \\
72.8\end{array}$ & $\begin{array}{l}\bar{w} \\
-\end{array}$ & $\begin{array}{l}77.4 \\
75.7 \\
78.1\end{array}$ & $\begin{array}{l}80.1 \\
78.6 \\
78.6\end{array}$ & $\begin{array}{l}79.1 \\
77.9 \\
77.7\end{array}$ & $\begin{array}{l}73.4 \\
71.9 \\
74.3\end{array}$ & $\begin{array}{l}65.1 \\
65.0 \\
64.5\end{array}$ & $\begin{array}{l}W \\
W \\
W\end{array}$ & $\begin{array}{l}69.0 \\
68.2 \\
68.4\end{array}$ \\
\hline $\begin{array}{l}\text { January } 1996 \text {................ } \\
\text { December } 1995 \text {............ } \\
\text { January } 1995 \text {............... } \\
\text { Massachusetts }\end{array}$ & $\begin{array}{l}94.3 \\
93.8 \\
92.9\end{array}$ & $\begin{array}{l}92.8 \\
92.5 \\
91.8\end{array}$ & $\begin{array}{l}81.5 \\
80.4 \\
78.5\end{array}$ & $\begin{array}{l}71.2 \\
72.0 \\
69.1\end{array}$ & $\begin{array}{c}W \\
61.7 \\
W\end{array}$ & $\begin{array}{l}71.9 \\
70.7 \\
70.9\end{array}$ & $\begin{array}{l}80.9 \\
79.8 \\
79.5\end{array}$ & $\begin{array}{l}80.3 \\
79.4 \\
78.9\end{array}$ & $\begin{array}{l}73.8 \\
72.4 \\
70.1\end{array}$ & $\begin{array}{l}63.8 \\
64.4 \\
60.8\end{array}$ & $\begin{array}{l}58.5 \\
59.7 \\
52.8\end{array}$ & $\begin{array}{l}64.8 \\
65.2 \\
60.9\end{array}$ \\
\hline January 1996 & 93.6 & 91.8 & 85.0 & 71.8 & $W$ & 78.1 & 80.4 & 79.8 & 75.5 & 64.8 & 57.2 & 68.9 \\
\hline December 1995 ........... & 92.5 & 91.1 & 83.1 & 71.9 & $W$ & 77.6 & 79.4 & 78.8 & 73.7 & 64.5 & 62.2 & 68.7 \\
\hline $\begin{array}{l}\text { January } 1995 \text {.................. } \\
\text { New Hampshire }\end{array}$ & 94.5 & 92.6 & 84.6 & 68.8 & W & 75.4 & 80.2 & 79.3 & 74.9 & 62.7 & 56.4 & 67.5 \\
\hline January 1996 ................ & 92.6 & 92.1 & 81.4 & 73.7 & - & 79.2 & 79.5 & 79.3 & 72.5 & 66.4 & - & 70.7 \\
\hline December 1995 ............. & 91.7 & 91.4 & 80.3 & 73.9 & - & 78.6 & 78.3 & 78.0 & 71.3 & 65.9 & - & 69.7 \\
\hline \multicolumn{6}{|l|}{ Rhode Island } & 78.0 & & & 73.2 & 63.0 & - & 69.8 \\
\hline January 1996 ................ & 88.8 & 88.2 & 81.4 & 71.7 & - & 75.9 & 77.0 & 76.7 & 72.3 & 64.5 & W & 67.7 \\
\hline December 1995 ........... & 87.9 & 87.5 & 79.4 & 71.4 & - & 74.7 & 76.2 & 76.0 & 70.3 & 64.2 & $w$ & 66.5 \\
\hline \multicolumn{12}{|l|}{ Vermont } & 64.2 \\
\hline January 1996 ................. & 96.8 & 95.5 & 83.6 & 69.6 & - & 79.8 & 83.1 & 82.2 & 73.9 & 62.6 & - & 70.9 \\
\hline December 1995 ........... & 95.7 & 94.5 & 83.3 & 70.5 & - & 79.8 & 82.3 & 81.5 & 74.0 & 63.4 & - & 71.0 \\
\hline $\begin{array}{l}\text { January } 1995 \\
\text { Subdistrlot IB }\end{array}$ & 95.6 & 94.2 & 81.7 & 67.1 & - & 77.8 & 81.4 & 80.4 & 71.7 & 60.2 & - & 68.6 \\
\hline January 1996 & 90.2 & 88.9 & 79.9 & 68.9 & 61.5 & 74.0 & 77.1 & 76.2 & 70.6 & 61.3 & 55.3 & 64.6 \\
\hline December 1995 ............ & 88.8 & 88.0 & 78.1 & 70.4 & 64.3 & 73.8 & 76.2 & 75.5 & 69.2 & 62.4 & 57.8 & 64.8 \\
\hline $\begin{array}{c}\text { January } 1995 \text {................. } \\
\text { pelaware }\end{array}$ & 91.7 & 90.5 & 83.2 & 68.2 & 58.3 & 75.5 & 78.3 & 77.3 & 73.2 & 60.8 & 55.9 & 66.3 \\
\hline \multicolumn{13}{|l|}{ Delaware } \\
\hline $\begin{array}{l}\text { January } 1996 \text {................ } \\
\text { December } 1995 \text {........... }\end{array}$ & $\begin{array}{l}89.2 \\
88.6\end{array}$ & 87.9 & 76.0 & 69.1 & - & 72.7 & 74.9 & $\begin{array}{l}75.0 \\
74.6\end{array}$ & $\begin{array}{l}67.1 \\
66.2\end{array}$ & $\begin{array}{l}61.5 \\
61.9\end{array}$ & $\begin{array}{l}W \\
W\end{array}$ & $\begin{array}{l}64.2 \\
63.9\end{array}$ \\
\hline December 1995 ............ & 92.4 & 91.7 & 80.9 & 71.1 & - & 75.7 & 77.7 & 77.1 & 70.8 & 63.0 & $\ddot{w}$ & 66.3 \\
\hline \multicolumn{13}{|l|}{ District of Columbia } \\
\hline January 1996 & $W$ & 77.5 & 77.2 & - & - & 77.2 & $W$ & 70.5 & 72.0 & - & - & 72.0 \\
\hline December 1995 ........... & $W$ & 74.0 & 74.6 & - & - & 74.6 & W & 68.1 & 69.6 & - & - & 69.6 \\
\hline $\begin{array}{l}\text { January } 1995 \text {................ } \\
\text { Maryland }\end{array}$ & \multicolumn{12}{|c|}{ Maryland } \\
\hline January 1996 & 87.5 & 84.3 & 75.5 & 68.7 & - & 73.7 & 77.1 & 74.0 & 68.2 & 61.1 & $W$ & 65.9 \\
\hline December 1995 ........... & 85.7 & 83.1 & 72.8 & 67.4 & $w$ & 71.2 & 75.9 & 73.1 & 65.8 & 60.2 & 59.9 & 63.8 \\
\hline \multicolumn{12}{|l|}{ New Jersey } & 71.1 \\
\hline January 1996 ................ & 94.7 & 93.2 & 82.9 & 70.7 & 62.3 & 74.3 & 81.9 & 81.0 & 74.8 & 64.7 & 55.7 & 64.5 \\
\hline December 1995 ............ & 93.1 & 92.2 & 81.0 & 72.2 & 64.5 & 74.4 & 80.3 & 79.6 & 73.0 & 65.4 & 59.1 & 66.2 \\
\hline $\begin{array}{l}\text { January } 1995 \text {................ } \\
\text { New York }\end{array}$ & 97.2 & 95.6 & 84.7 & 69.7 & 58.6 & 74.3 & 83.8 & 82.8 & 76.7 & 64.4 & 56.3 & 66.5 \\
\hline January 1996 & 91.0 & 90.1 & 81.6 & 69.4 & 62.6 & 76.7 & 77.4 & 76.8 & 71.4 & 61.9 & 56.6 & 66.7 \\
\hline December 1995 ............ & 90.3 & 89.7 & 80.0 & 71.9 & 65.9 & 76.5 & 77.3 & 76.9 & 70.5 & 63.4 & 56.0 & 66.4 \\
\hline $\begin{array}{l}\text { January } 1995 \text {................. } \\
\text { Pennsylvania }\end{array}$ & 90.6 & 89.6 & 82.8 & 69.1 & 58.6 & 77.3 & 77.0 & 76.2 & 72.2 & 60.9 & 56.8 & 67.1 \\
\hline January 1996 ................ & 87.0 & 86.3 & 76.2 & 67.2 & 58.3 & 69.9 & 74.9 & 74.4 & 67.1 & 59.2 & 53.0 & 61.5 \\
\hline December 1995 ............ & 85.3 & 84.9 & 75.2 & 68.6 & 62.3 & 70.5 & 73.6 & 73.3 & 66.2 & 60.5 & 55.9 & 62.0 \\
\hline January 1995 ................. & 89.1 & 88.4 & 80.7 & 65.6 & 54.5 & 71.6 & 76.7 & 76.0 & 70.0 & 58.3 & 52.5 & 62.6 \\
\hline
\end{tabular}

See footnotes at end of table. 
Table 31. Motor Gasoline Prices by Grade, Sales Type, PAD District, and State (Cents per Gallon Excluding Taxes) - Continued

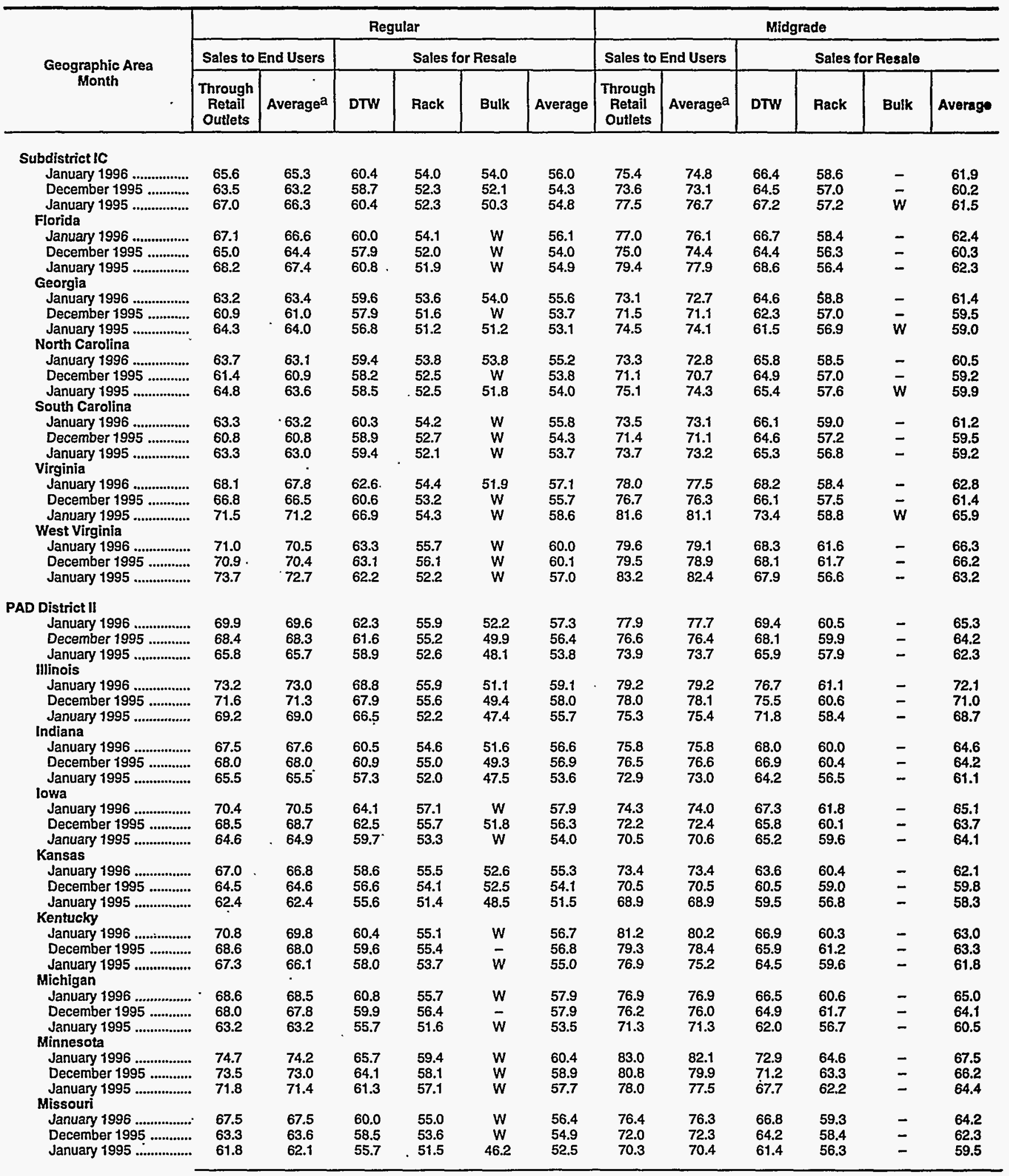

See footnotes at end of table. 
Table 31. Motor Gasoline Prices by Grade, Sales Type, PAD District, and State (Cents per Gallon Excluding Taxes) - Continued

\begin{tabular}{|c|c|c|c|c|c|c|c|c|c|c|c|c|}
\hline \multirow{3}{*}{$\begin{array}{c}\text { Geographic Area } \\
\text { Month }\end{array}$} & \multicolumn{6}{|c|}{ Premium } & \multicolumn{6}{|c|}{ All Grades } \\
\hline & \multicolumn{2}{|c|}{ Sales to End Users } & \multicolumn{4}{|c|}{ Sales for Resale } & \multicolumn{2}{|c|}{ Sales to End Users } & \multicolumn{4}{|c|}{ Sales for Resale } \\
\hline & $\begin{array}{c}\text { Through } \\
\text { Retail } \\
\text { Outlets }\end{array}$ & Average $^{\mathrm{a}}$ & DTW & Rack & Bulk & Average & $\begin{array}{c}\text { Through } \\
\text { Retail } \\
\text { Outlets }\end{array}$ & Average $^{\mathrm{a}}$ & DTW & Rack & Bulk & Average \\
\hline \multicolumn{13}{|l|}{ Subdistrict IC } \\
\hline $\begin{array}{l}\text { January } 1996 \text {............... } \\
\text { December } 1995 \text {........... } \\
\text { January } 1995 \text {.............. } \\
\text { Florida }\end{array}$ & $\begin{array}{l}84.2 \\
82.5 \\
86.7\end{array}$ & $\begin{array}{l}83.4 \\
81.8 \\
85.7\end{array}$ & $\begin{array}{l}73.8 \\
71.7 \\
75.3\end{array}$ & $\begin{array}{l}64.3 \\
62.7 \\
63.0\end{array}$ & $\begin{array}{l}60.5 \\
59.6 \\
58.7\end{array}$ & $\begin{array}{l}68.1 \\
66.3 \\
68.1\end{array}$ & $\begin{array}{l}71.3 \\
69.5 \\
73.0\end{array}$ & $\begin{array}{l}70.8 \\
69.0 \\
72.1\end{array}$ & $\begin{array}{l}65.2 \\
63.4 \\
65.7\end{array}$ & $\begin{array}{l}56.9 \\
55.3 \\
55.3\end{array}$ & $\begin{array}{l}55.4 \\
53.2 \\
51.7\end{array}$ & $\begin{array}{l}59.7 \\
58.1 \\
59.0\end{array}$ \\
\hline $\begin{array}{l}\text { January } 1996 \ldots . . . . . . . . . . . . \\
\text { December } 1995 \text {............ } \\
\text { January } 1995 \text {............... } \\
\text { Georgia }\end{array}$ & $\begin{array}{l}84.9 \\
83.1 \\
87.7\end{array}$ & $\begin{array}{l}84.1 \\
82.5 \\
86.5\end{array}$ & $\begin{array}{l}74.3 \\
72.0 \\
76.9\end{array}$ & $\begin{array}{l}64.5 \\
62.3 \\
62.7\end{array}$ & $\frac{61.6}{w}$ & $\begin{array}{l}69.5 \\
67.3 \\
70.4\end{array}$ & $\begin{array}{l}72.8 \\
70.8 \\
74.3\end{array}$ & $\begin{array}{l}72.1 \\
70.0 \\
73.2\end{array}$ & $\begin{array}{l}66.2 \\
64.0 \\
67.8\end{array}$ & $\begin{array}{l}56.9 \\
54.9 \\
54.9\end{array}$ & $\begin{array}{c}57.2 \\
W \\
49.2\end{array}$ & $\begin{array}{l}60.6 \\
58.6 \\
60.2\end{array}$ \\
\hline $\begin{array}{l}\text { January } 1996 \text {................ } \\
\text { December } 1995 \text {............ } \\
\text { January } 1995 \text {................ } \\
\text { North Carolina }\end{array}$ & $\begin{array}{l}82.9 \\
81.1 \\
84.4\end{array}$ & $\begin{array}{l}82.3 \\
80.7 \\
83.6\end{array}$ & $\begin{array}{l}71.7 \\
69.8 \\
69.6\end{array}$ & $\begin{array}{l}63.9 \\
62.1 \\
62.3\end{array}$ & $\begin{array}{l}W \\
W \\
W\end{array}$ & $\begin{array}{l}66.6 \\
65.0 \\
65.1\end{array}$ & $\begin{array}{l}68.9 \\
67.1 \\
70.2\end{array}$ & $\begin{array}{l}68.8 \\
67.0 \\
69.8\end{array}$ & $\begin{array}{l}63.4 \\
61.6 \\
60.6\end{array}$ & $\begin{array}{l}56.5 \\
54.6 \\
54.4\end{array}$ & $\begin{array}{c}55.4 \\
W \\
53.5\end{array}$ & $\begin{array}{l}58.9 \\
57.1 \\
56.7\end{array}$ \\
\hline $\begin{array}{l}\text { January } 1996 . . . . . . . . . . . . . . \\
\text { December } 1995 \text {............ } \\
\text { January } 1995 \text {................ } \\
\text { South Carolina }\end{array}$ & $\begin{array}{l}83.1 \\
80.7 \\
84.9\end{array}$ & $\begin{array}{l}82.1 \\
79.8 \\
83.5\end{array}$ & $\begin{array}{l}71.9 \\
70.8 \\
71.4\end{array}$ & $\begin{array}{l}64.2 \\
62.7 \\
63.3\end{array}$ & $\begin{array}{l}W \\
W \\
W\end{array}$ & $\begin{array}{l}66.1 \\
64.5 \\
65.4\end{array}$ & $\begin{array}{l}69.7 \\
67.6 \\
70.9\end{array}$ & $\begin{array}{l}68.9 \\
66.9 \\
69.5\end{array}$ & $\begin{array}{l}63.2 \\
62.1 \\
62.6\end{array}$ & $\begin{array}{l}56.8 \\
55.5 \\
55.6\end{array}$ & $\begin{array}{c}55.3 \\
\mathbf{W} \\
52.3\end{array}$ & $\begin{array}{l}58.4 \\
57.1 \\
57.4\end{array}$ \\
\hline $\begin{array}{l}\text { January } 1996 \text {............... } \\
\text { December } 1995 \text {............ } \\
\text { January } 1995 \text {............... } \\
\text { Virginia }\end{array}$ & $\begin{array}{l}83.7 \\
81.3 \\
84.7\end{array}$ & $\begin{array}{l}83.1 \\
80.7 \\
84.1\end{array}$ & $\begin{array}{l}73.5 \\
72.2 \\
72.9\end{array}$ & $\begin{array}{l}64.7 \\
63.2 \\
63.3\end{array}$ & $\begin{array}{l}- \\
-\end{array}$ & $\begin{array}{r}67.1 \\
65.5 \\
-65.5\end{array}$ & $\begin{array}{l}69.0 \\
66.8 \\
69.1\end{array}$ & $\begin{array}{l}68.8 \\
66.6 \\
68.7\end{array}$ & $\begin{array}{l}63.9 \\
62.7 \\
63.1\end{array}$ & $\begin{array}{l}57.0 \\
55.6 \\
55.1\end{array}$ & $\begin{array}{l}W \\
W \\
W\end{array}$ & $\begin{array}{l}58.8 \\
57.4 \\
56.9\end{array}$ \\
\hline $\begin{array}{l}\text { January } 1996 \text {............... } \\
\text { December } 1995 \text {............. } \\
\text { January } 1995 \text {.............. } \\
\text { West Virginia }\end{array}$ & $\begin{array}{l}85.1 \\
84.9 \\
89.9\end{array}$ & $\begin{array}{l}84.4 \\
84.2 \\
89.2\end{array}$ & $\begin{array}{l}75.8 \\
73.3 \\
81.3\end{array}$ & $\begin{array}{l}64.4 \\
63.5 \\
64.0\end{array}$ & $\begin{array}{l}W \\
W \\
-\end{array}$ & $\begin{array}{l}69.6 \\
67.9 \\
71.5\end{array}$ & $\begin{array}{l}73.6 \\
72.7 \\
77.5\end{array}$ & $\begin{array}{l}73.2 \\
72.3 \\
77.0\end{array}$ & $\begin{array}{l}67.5 \\
65.3 \\
72.1\end{array}$ & $\begin{array}{l}57.1 \\
56.1 \\
57.2\end{array}$ & $\begin{array}{l}53.2 \\
51.9 \\
52.2\end{array}$ & $\begin{array}{l}61.1 \\
59.7 \\
63.0\end{array}$ \\
\hline $\begin{array}{l}\text { January } 1996 \text {................ } \\
\text { December } 1995 \text {........... } \\
\text { January } 1995 \text {............... }\end{array}$ & $\begin{array}{l}88.0 \\
87.5 \\
90.8\end{array}$ & $\begin{array}{l}86.7 \\
86.5 \\
88.7\end{array}$ & $\begin{array}{l}75.4 \\
75.2 \\
74.7\end{array}$ & $\begin{array}{l}66.3 \\
66.9 \\
63.0\end{array}$ & $\overline{-}$ & $\begin{array}{l}71.9 \\
72.1 \\
69.5\end{array}$ & $\begin{array}{l}75.6 \\
75.5 \\
78.5\end{array}$ & $\begin{array}{l}75.0 \\
74.9 \\
77.4\end{array}$ & $\begin{array}{l}66.7 \\
66.5 \\
66.0\end{array}$ & $\begin{array}{l}58.7 \\
59.0 \\
55.2\end{array}$ & $\begin{array}{l}W \\
W \\
W\end{array}$ & $\begin{array}{l}63.4 \\
63.4 \\
60.7\end{array}$ \\
\hline \multicolumn{13}{|l|}{ PAD District II } \\
\hline $\begin{array}{l}\text { January } 1996 \ldots . . . . . . . . . . . \\
\text { December } 1995 \text {............. } \\
\text { January } 1995 \text {.............. } \\
\text { Illinois }\end{array}$ & $\begin{array}{l}85.3 \\
83.9 \\
81.1\end{array}$ & $\begin{array}{l}84.8 \\
83.5 \\
80.5\end{array}$ & $\begin{array}{l}74.3 \\
73.4 \\
71.1\end{array}$ & $\begin{array}{l}64.5 \\
63.8 \\
61.5\end{array}$ & $\begin{array}{l}55.7 \\
55.5 \\
52.5\end{array}$ & $\begin{array}{l}68.0 \\
67.2 \\
65.1\end{array}$ & $\begin{array}{l}73.2 \\
71.9 \\
69.2\end{array}$ & $\begin{array}{l}72.9 \\
71.7 \\
69.0\end{array}$ & $\begin{array}{l}65.5 \\
64.7 \\
62.2\end{array}$ & $\begin{array}{l}57.5 \\
56.9 \\
54.3\end{array}$ & $\begin{array}{l}52.3 \\
50.1 \\
48.2\end{array}$ & $\begin{array}{l}59.6 \\
58.8 \\
56.3\end{array}$ \\
\hline $\begin{array}{l}\text { January } 1996 \ldots . . . . . . . . . . . . \\
\text { December } 1995 \text {........... } \\
\text { January } 1995 \text {............... } \\
\text { Indiana }\end{array}$ & $\begin{array}{l}89.7 \\
87.7 \\
85.9\end{array}$ & $\begin{array}{l}89.2 \\
87.2 \\
85.3\end{array}$ & $\begin{array}{l}81.1 \\
80.3 \\
79.2\end{array}$ & $\begin{array}{l}64.8 \\
64.5 \\
62.0\end{array}$ & $\begin{array}{c}W \\
54.6 \\
W\end{array}$ & $\begin{array}{l}72.9 \\
72.7 \\
71.3\end{array}$ & $\begin{array}{l}76.9 \\
75.3 \\
72.9\end{array}$ & $\begin{array}{l}76.6 \\
75.1 \\
72.7\end{array}$ & $\begin{array}{l}73.2 \\
72.3 \\
70.6\end{array}$ & $\begin{array}{l}57.6 \\
57.3 \\
54.0\end{array}$ & $\begin{array}{l}51.1 \\
49.5 \\
47.5\end{array}$ & $\begin{array}{l}62.9 \\
61.7 \\
59.7\end{array}$ \\
\hline $\begin{array}{l}\text { January } 1996 . . . . \ldots . . . \ldots . . . \\
\text { December } 1995 \text {............ } \\
\text { January } 1995 \text {............... } \\
\text { lowa }\end{array}$ & $\begin{array}{l}82.6 \\
83.3 \\
79.0\end{array}$ & $\begin{array}{l}82.4 \\
83.2 \\
78.9\end{array}$ & $\begin{array}{l}72.0 \\
72.1 \\
69.4\end{array}$ & $\begin{array}{l}64.7 \\
65.0 \\
61.0\end{array}$ & $\overline{-}$ & $\begin{array}{l}68.2 \\
68.3 \\
64.7\end{array}$ & $\begin{array}{l}71.5 \\
72.1 \\
69.3\end{array}$ & $\begin{array}{l}71.5 \\
72.1 \\
69.2\end{array}$ & $\begin{array}{l}64.0 \\
64.1 \\
61.0\end{array}$ & $\begin{array}{l}56.7 \\
57.2 \\
54.0\end{array}$ & $\begin{array}{l}51.6 \\
49.3 \\
47.6\end{array}$ & $\begin{array}{l}59.4 \\
59.8 \\
56.5\end{array}$ \\
\hline $\begin{array}{l}\text { January } 1996 \text {................ } \\
\text { December } 1995 \text {............. } \\
\text { January } 1995 \text {.............. } \\
\text { Kansas }\end{array}$ & $\begin{array}{l}80.7 \\
79.3 \\
73.9\end{array}$ & $\begin{array}{l}80.3 \\
78.9 \\
73.7\end{array}$ & $\begin{array}{c}72.0 \\
\text { NA } \\
67.4\end{array}$ & $\begin{array}{l}64.4 \\
62.7 \\
61.2\end{array}$ & $\begin{array}{l}- \\
-\end{array}$ & $\begin{array}{l}65.6 \\
64.0 \\
62.4\end{array}$ & $\begin{array}{l}71.4 \\
69.5 \\
65.7\end{array}$ & $\begin{array}{l}71.4 \\
69.7 \\
65.9\end{array}$ & $\begin{array}{l}65.6 \\
64.2 \\
61.8\end{array}$ & $\begin{array}{l}57.9 \\
56.3 \\
54.0\end{array}$ & $\begin{array}{c}W \\
51.8 \\
W\end{array}$ & $\begin{array}{l}58.9 \\
57.4 \\
55.1\end{array}$ \\
\hline $\begin{array}{l}\text { January } 1996 \text {................ } \\
\text { December } 1995 \text {............. } \\
\text { January } 1995 \text {............... } \\
\text { Kentucky }\end{array}$ & $\begin{array}{l}80.2 \\
77.3 \\
75.5\end{array}$ & $\begin{array}{l}79.5 \\
77.0 \\
74.8\end{array}$ & $\begin{array}{l}66.5 \\
64.9 \\
64.2\end{array}$ & $\begin{array}{l}62.3 \\
61.1 \\
58.6\end{array}$ & $\begin{array}{c}W \\
54.1 \\
W\end{array}$ & $\begin{array}{l}63.4 \\
60.8 \\
59.7\end{array}$ & $\begin{array}{l}68.7 \\
66.2 \\
64.1\end{array}$ & $\begin{array}{l}68.4 \\
66.2 \\
64.1\end{array}$ & $\begin{array}{l}60.0 \\
58.1 \\
57.1\end{array}$ & $\begin{array}{l}56.3 \\
54.9 \\
52.2\end{array}$ & $\begin{array}{l}52.6 \\
52.6 \\
48.5\end{array}$ & $\begin{array}{l}56.2 \\
54.9 \\
52.3\end{array}$ \\
\hline $\begin{array}{l}\text { January } 1996 \text {............... } \\
\text { December } 1995 \text {............ } \\
\text { January } 1995 \text {................ } \\
\text { Michigan }\end{array}$ & $\begin{array}{l}86.8 \\
84.9 \\
84.0\end{array}$ & $\begin{array}{l}85.6 \\
83.9 \\
82.4\end{array}$ & $\begin{array}{l}72.1 \\
71.9 \\
70.3\end{array}$ & $\begin{array}{l}64.6 \\
64.9 \\
63.7\end{array}$ & $\begin{array}{l}\bar{w} \\
-\end{array}$ & $\begin{array}{l}66.9 \\
66.8 \\
65.8\end{array}$ & $\begin{array}{l}75.3 \\
73.4 \\
72.0\end{array}$ & $\begin{array}{l}74.3 \\
72.6 \\
70.7\end{array}$ & $\begin{array}{l}63.7 \\
62.9 \\
61.6\end{array}$ & $\begin{array}{l}57.6 \\
58.0 \\
56.6\end{array}$ & $\begin{array}{l}W \\
W \\
W\end{array}$ & $\begin{array}{l}59.5 \\
59.6 \\
58.2\end{array}$ \\
\hline $\begin{array}{l}\text { January } 1996 \text {................ } \\
\text { December } 1995 \text {........... } \\
\text { January } 1995 \text {............... } \\
\text { Minnesota }\end{array}$ & $\begin{array}{l}83.7 \\
83.5 \\
77.8\end{array}$ & $\begin{array}{l}83.2 \\
82.9 \\
77.5\end{array}$ & $\begin{array}{l}72.6 \\
71.3 \\
66.7\end{array}$ & $\begin{array}{l}65.2 \\
65.6 \\
60.8\end{array}$ & $\bar{w}$ & $\begin{array}{l}69.1 \\
68.6 \\
63.9\end{array}$ & $\begin{array}{l}71.6 \\
71.1 \\
66.5\end{array}$ & $\begin{array}{l}71.4 \\
70.8 \\
66.4\end{array}$ & $\begin{array}{l}63.7 \\
62.7 \\
58.7\end{array}$ & $\begin{array}{l}\mathbf{5 7 . 4} \\
\mathbf{5 8 . 1} \\
\mathbf{5 3 . 4}\end{array}$ & $\begin{array}{l}w \\
\bar{w}\end{array}$ & $\begin{array}{l}60.4 \\
60.4 \\
56.0\end{array}$ \\
\hline $\begin{array}{l}\text { January } 1996 \text {................ } \\
\text { December } 1995 \text {............ } \\
\text { January } 1995 \text {............... } \\
\text { Missouri }\end{array}$ & $\begin{array}{l}88.4 \\
87.1 \\
85.3\end{array}$ & $\begin{array}{l}88.2 \\
86.9 \\
85.2\end{array}$ & $\begin{array}{l}76.4 \\
74.5 \\
72.0\end{array}$ & $\begin{array}{l}67.1 \\
65.9 \\
65.1\end{array}$ & $\begin{array}{l}W \\
W \\
W\end{array}$ & $\begin{array}{l}68.8 \\
67.4 \\
66.4\end{array}$ & $\begin{array}{l}76.8 \\
75.6 \\
73.6\end{array}$ & $\begin{array}{l}76.2 \\
75.0 \\
73.1\end{array}$ & $\begin{array}{l}67.7 \\
66.1 \\
63.1\end{array}$ & $\begin{array}{l}60.5 \\
59.3 \\
58.3\end{array}$ & $\begin{array}{l}W \\
W \\
W\end{array}$ & $\begin{array}{l}61.8 \\
60.3 \\
59.1\end{array}$ \\
\hline $\begin{array}{l}\text { January } 1996 \text {................ } \\
\text { December } 1995 \text {............ } \\
\text { January } 1995 \text {.............. }\end{array}$ & $\begin{array}{l}85.3 \\
81.0 \\
78.7\end{array}$ & $\begin{array}{l}84.9 \\
80.8 \\
78.3\end{array}$ & $\begin{array}{l}71.1 \\
68.8 \\
66.5\end{array}$ & $\begin{array}{l}62.7 \\
61.3 \\
59.3\end{array}$ & $\begin{array}{l}- \\
-\end{array}$ & $\begin{array}{l}65.7 \\
63.9 \\
61.8\end{array}$ & $\begin{array}{l}70.7 \\
66.7 \\
65.0\end{array}$ & $\begin{array}{l}70.5 \\
66.8 \\
65.1\end{array}$ & $\begin{array}{l}62.8 \\
61.1 \\
58.3\end{array}$ & $\begin{array}{l}56.3 \\
55.0 \\
52.9\end{array}$ & $\begin{array}{c}W \\
W \\
46.2\end{array}$ & $\begin{array}{l}58.3 \\
56.8 \\
54.4\end{array}$ \\
\hline
\end{tabular}

See footnotes at end of table. 
Table 31. Motor Gasoline Prices by Grade, Sales Type, PAD District, and State (Cents per Gallon Excluding Taxes) - Continued

\begin{tabular}{|c|c|c|c|c|c|c|c|c|c|c|c|c|}
\hline \multirow{3}{*}{$\begin{array}{l}\text { Geographic Area } \\
\text { Month }\end{array}$} & \multicolumn{6}{|c|}{ Regular } & \multicolumn{6}{|c|}{ Midgrade } \\
\hline & \multicolumn{2}{|c|}{ Sales to End Users } & \multicolumn{4}{|c|}{ Sales for Resale } & \multicolumn{2}{|c|}{ Sales to End Users } & \multicolumn{4}{|c|}{ Sales for Resale } \\
\hline & $\begin{array}{c}\text { Through } \\
\text { Retail } \\
\text { Outlets }\end{array}$ & Average ${ }^{a}$ & DTW & Rack & Bulk & Average & $\begin{array}{c}\text { Through } \\
\text { Retail } \\
\text { Outlets }\end{array}$ & Average ${ }^{\mathrm{a}}$ & DTW & Rack & Bulk & Average \\
\hline \multicolumn{13}{|l|}{ Nebraska } \\
\hline January 1996 & 69.4 & 69.4 & 62.7 & 56.7 & $w$ & 57.9 & 73.8 & 73.6 & 63.4 & 63.1 & - & 63.2 \\
\hline December 1995 ............. & 68.7 & 68.7 & 60.8 & 55.0 & $w$ & 56.2 & 72.7 & 72.5 & 62.6 & 60.7 & - & 61.5 \\
\hline January 1995 .................. & 68.1 & 68.0 & 60.6 & 53.1 & - & 54.7 & 72.8 & 72.7 & 62.9 & 61.1 & - & 61.9 \\
\hline \multicolumn{13}{|l|}{ North Dakota } \\
\hline January 1996 ................. & 81.1 & 80.6 & 67.2 & 58.7 & $\rightarrow$ & 60.4 & 83.5 & 82.3 & 69.6 & 66.3 & - & 68.4 \\
\hline December 1995 ............ & 80.6 & 80.0 & 65.6 & 57.2 & - & 58.7 & 81.8 & 80.6 & 68.3 & 64.7 & - & 66.9 \\
\hline 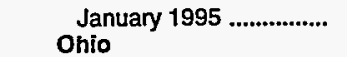 & 76.6 & & 56.8 & 85.0 & 84.4 & w & 63.4 & - & 64.3 \\
\hline January 1996 ................. & 70.0 & 69.6 & 61.2 & 54.6 & 47.8 & 57.2 & 79.4 & 78.8 & 67.8 & 59.3 & - & 64.7 \\
\hline December 1995 ............. & 69.9 & 69.5 & 61.5 & 55.4 & W & 57.5 & 79.3 & 78.8 & 68.0 & 60.3 & - & 65.0 \\
\hline January 1995 ................. & 64.2 & 63.9 & 56.2 & 51.3 & $W$ & 52.8 & 73.5 & 73.0 & 64.9 & 56.8 & - & 61.8 \\
\hline Oklahoma & & & & & & & & & & & & \\
\hline January 1996 & 64.5 & 64.4 & 59.5 & 54.2 & 52.4 & 54.0 & 70.4 & 70.4 & 64.6 & 57.5 & - & 61.3 \\
\hline December 1995 ............ & 63.0 & 62.8 & 58.0 & 52.7 & 49.1 & 51.6 & 69.0 & 69.0 & 63.2 & 55.8 & - & 59.9 \\
\hline January 1995 .................. & 61.1 & 61.0 & 58.6 & 50.6 & 48.1 & 50.0 & 68.8 & 68.7 & 62.4 & 54.0 & - & 58.9 \\
\hline South Dakota & & & & & & & & & & & & \\
\hline January 1996 & 73.1 & 72.0 & 65.5 & $57.3 \cdot$ & - & 59.4 & 80.9 & 80.6 & 68.0 & $w$ & - & 64.5 \\
\hline December 1995 & 71.8 & 71.2 & 63.6 & 55.6 & - & 57.7 & 81.1 & 80.6 & 66.7 & $W$ & - & 65.6 \\
\hline January 1995 .................. & 71.4 & 70.0 & 62.4 & 53.8 & - & 55.9 & 84.2 & 83.9 & 65.9 & $w$ & - & 62.9 \\
\hline Tennessee & & & & & & & & & & & & \\
\hline January 1996 & 64.7 & 64.4 & 61.5 & 54.0 & $w$ & 55.3 & 76.1 & 75.8 & 67.9 & 59.1 & - & 61.1 \\
\hline December 1995 ............ & 61.9 & 61.7 & 59.6 & 52.2 & $w$ & 53.4 & 73.1 & 72.8 & 65.6 & 57.3 & - & 59.1 \\
\hline January 1995 ..................... & 64.3 & 63.8 & 61.4 & 51.9 & w & 53.7 & 75.6 & 75.2 & 67.7 & 57.2 & - & 60.1 \\
\hline Wisconsin & & & & & & & & & & & & \\
\hline January 1996 & 72.8 & 72.7 & 62.7 & 57.0 & - & 58.9 & 81.1 & 81.3 & 69.2 & 62.6 & - & 65.1 \\
\hline December 1995 ............ & 71.8 & 71.7 & 62.3 & 57.0 & - & 58.8 & 79.6 & 79.5 & 67.8 & 61.8 & - & 64.0 \\
\hline January 1995 ................. & 68.7 & 68.7 & 59.5 & 53.5 & - & 55.5 & 75.8 & 75.7 & 64.7 & 58.1 & - & 60.9 \\
\hline PAD District III & & & & & & & & & & & & \\
\hline January 1996 & 68.1 & 67.5 & 61.3 & 54.3 & 49.9 & 54.2 & 77.2 & 76.4 & 65.3 & 58.9 & - & 61.5 \\
\hline December 1995 ............. & 66.2 & 65.8 & 60.3 & 53.0 & 48.3 & 52.3 & 75.4 & 74.8 & 64.0 & 57.3 & - & 60.0 \\
\hline January 1995 ................. & 69.5 & 68.3 & 62.2 & 52.3 & 47.5 & 52.6 & 79.2 & 77.9 & 66.2 & 56.8 & W & 60.8 \\
\hline Alabama & & & & & & & & & & & & \\
\hline January 1996 ................. & 68.5 & 67.9 & 64.3 & 53.9 & $W$ & 56.2 & 77.4 & 76.5 & 71.1 & 58.9 & - & 62.6 \\
\hline December 1995 ............. & 66.6 & 66.4 & 62.7 & 52.0 & $W$ & 54.3 & 75.8 & 75.0 & 69.7 & 57.0 & - & 60.9 \\
\hline January 1995 ................. & 68.1 & 67.2 & 62.4 & 51.8 & $W$ & 54.1 & 77.2 & 76.5 & 69.1 & 57.0 & - & 60.6 \\
\hline Arkansas & & & & & & & & & & & & \\
\hline January 1996 .................. & 64.9 & 63.8 & 60.7 & 54.1 & 50.2 & 54.9 & 74.3 & 73.2 & 65.7 & 58.2 & - & 60.2 \\
\hline December 1995 ............ & 62.6 & 62.7 & 59.8 & 52.7 & 48.9 & 53.6 & 72.0 & 71.3 & 64.9 & 56.7 & - & 58.8 \\
\hline January 1995 ................. & 65.0 & 64.1 & 57.6 & 51.4 & $W$ & 52.6 & 74.9 & 73.5 & 63.0 & 56.3 & - & 58.0 \\
\hline Louisiana & & & & & & & & & & & & \\
\hline January 1996 & 64.8 & 64.3 & 60.5 & 53.2 & 49.9 & 53.3 & 75.7 & 74.6 & 66.5 & 58.4 & - & 61.1 \\
\hline December 1995 ............. & 64.0 & 63.6 & 59.3 & 51.6 & 47.6 & 51.3 & 74.9 & 74.2 & 65.4 & 56.7 & - & 59.5 \\
\hline January 1995 & 67.6 & 67.3 & 61.3 & 51.2 & 46.6 & 51.3 & 78.7 & 77.8 & 66.8 & 55.5 & - & 59.5 \\
\hline Mississippi & & & & & & & & & & & & \\
\hline January 1996 & 69.2 & 68.9 & 59.9 & 53.6 & 49.5 & 54.1 & 79.0 & 78.7 & 65.5 & 58.3 & - & 60.0 \\
\hline December 1995 ............. & 67.4 & 67.1 & 58.4 & 51.9 & 48.3 & 52.5 & 77.0 & 76.6 & 63.8 & 56.4 & - & 58.2 \\
\hline January $1995 \ldots . . . . . . . . . . .$. & 69.5 & 69.1 & 60.5 & 51.4 & 47.1 & 52.3 & 79.1 & 78.6 & 67.1 & 56.2 & - & 58.9 \\
\hline New Mexico & & & & & & & & & & & & \\
\hline January 1996 ................. & 75.6 & 75.4 & 64.1 & 60.3 & - & 61.1 & 83.6 & 83.7 & 67.1 & 67.1 & - & 67.1 \\
\hline December 1995 ............ & 74.8 & 74.6 & 62.1 & 58.0 & - & 58.9 & 82.9 & 82.9 & 66.3 & 65.9 & - & 66.1 \\
\hline January 1995 & 75.8 & 75.1 & 61.8 & 54.9 & - & 56.5 & 82.6 & 82.6 & 71.6 & 62.9 & - & 65.7 \\
\hline Texas & & & & & & & & & & & & \\
\hline January 1996 & 68.1 & 67.6 & 61.0 & 54.2 & 49.9 & 53.7 & 77.1 & 76.4 & 64.3 & 58.7 & - & 61.4 \\
\hline December 1995 ............. & 65.9 & 65.5 & 60.2 & 53.2 & 48.4 & 51.8 & 75.2 & 74.5 & .63 .0 & 57.3 & - & 60.0 \\
\hline January 1995 ................... & 69.8 & 68.4 & 62.9 & 52.7 & 47.6 & 52.4 & 79.9 & 78.4 & 65.8 & 57.0 & W & 61.4 \\
\hline PAD District IV & & & & & & & & & & & & \\
\hline January 1996 ................. & 73.5 & 73.4 & 64.2 & 59.1 & $W$ & 60.3 & 83.7 & 83.2 & 69.7 & 63.5 & - & 65.9 \\
\hline December 1995 .............. & 74.5 & 74.3 & 63.3 & 58.0 & - & 59.4 & 84.4 & 83.8 & 68.8 & 62.9 & - & 65.2 \\
\hline 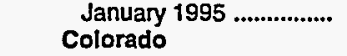 & 75.7 & 75.2 & 63.5 & 57.3 & W & 58.9 & 84.5 & 83.9 & 68.5 & 62.2 & - & 65.1 \\
\hline January 1996 & 75.2 & 75.2 & 66.4 & 59.2 & $w$ & 60.6 & 88.6 & 87.8 & 72.5 & 64.0 & - & 67.8 \\
\hline December 1995 ............. & 75.9 & 75.9 & 63.6 & 56.3 & - & 58.1 & 89.8 & 88.9 & 70.5 & 61.1 & - & 65.3 \\
\hline 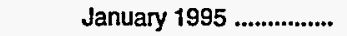 & 78.0 & 77.4 & 63.6 & 55.8 & - & 57.8 & 88.3 & 87.3 & 69.7 & 61.2 & - & 65.4 \\
\hline
\end{tabular}

See footnotes at end of table. 
Table 31. Motor Gasoline Prices by Grade, Sales Type, PAD District, and State (Cents per Gallon Excluding Taxes) - Continued

\begin{tabular}{|c|c|c|c|c|c|c|c|c|c|c|c|c|}
\hline \multirow{3}{*}{$\begin{array}{c}\text { Geographic Area } \\
\text { Month }\end{array}$} & \multicolumn{6}{|c|}{ Premium } & \multicolumn{6}{|c|}{ All Grades } \\
\hline & \multicolumn{2}{|c|}{ Sales to End Users } & \multicolumn{4}{|c|}{ Sales for Resale } & \multicolumn{2}{|c|}{ Sales to End Users } & \multicolumn{4}{|c|}{ Sales for Resale } \\
\hline & $\begin{array}{c}\text { Through } \\
\text { Retail } \\
\text { Outlets }\end{array}$ & Average $\mathrm{a}$ & DTW & Rack & Bulk & Average & $\begin{array}{c}\text { Through } \\
\text { Retail } \\
\text { Outlets } \\
\end{array}$ & Average ${ }^{a}$ & DTW & Rack & Bulk & Average \\
\hline \multicolumn{13}{|l|}{ Nebraska } \\
\hline $\begin{array}{l}\text { January } 1996 \text {................ } \\
\text { December } 1995 \text {............ } \\
\text { January } 1995 \text {............... } \\
\text { North Dakota }\end{array}$ & $\begin{array}{l}80.0 \\
80.6 \\
79.2\end{array}$ & $\begin{array}{l}83.9 \\
84.2 \\
82.5\end{array}$ & $\begin{array}{l}71.4 \\
68.6 \\
68.7\end{array}$ & $\begin{array}{l}63.8 \\
62.0 \\
60.4\end{array}$ & $\overline{-}$ & $\begin{array}{l}65.9 \\
63.7 \\
62.7\end{array}$ & $\begin{array}{l}70.6 \\
69.9 \\
69.3\end{array}$ & $\begin{array}{l}71.0 \\
70.3 \\
69.5\end{array}$ & $\begin{array}{l}63.5 \\
61.7 \\
61.4\end{array}$ & $\begin{array}{l}\mathbf{5 7 . 4} \\
55.7 \\
\mathbf{5 3 . 7}\end{array}$ & $\begin{array}{l}W \\
W \\
-\end{array}$ & $\begin{array}{l}58.7 \\
57.0 \\
55.4\end{array}$ \\
\hline $\begin{array}{l}\text { January } 1996 \text {............... } \\
\text { December } 1995 \text {............ } \\
\text { January } 1995 \text {............... } \\
\text { Ohio }\end{array}$ & $\begin{array}{l}88.7 \\
87.9 \\
85.5\end{array}$ & $\begin{array}{l}88.6 \\
88.0 \\
85.3\end{array}$ & $\begin{array}{l}77.8 \\
75.4 \\
71.5\end{array}$ & $\begin{array}{l}67.3 \\
66.0 \\
63.9\end{array}$ & $\begin{array}{l}- \\
-\end{array}$ & $\begin{array}{l}71.2 \\
69.3 \\
66.7\end{array}$ & $\begin{array}{l}81.7 \\
81.2 \\
77.7\end{array}$ & $\begin{array}{l}81.3 \\
80.5 \\
77.2\end{array}$ & $\begin{array}{l}68.8 \\
67.3 \\
63.4\end{array}$ & $\begin{array}{l}59.4 \\
58.0 \\
55.9\end{array}$ & $\vec{w}$ & $\begin{array}{l}61.6 \\
60.0 \\
57.7\end{array}$ \\
\hline $\begin{array}{l}\text { January } 1996 \text {............... } \\
\text { December } 1995 \text {........... } \\
\text { January } 1995 \text {............... } \\
\text { OkJahoma }\end{array}$ & $\begin{array}{l}86.8 \\
86.5 \\
81.0\end{array}$ & $\begin{array}{l}86.2 \\
85.9 \\
80.4\end{array}$ & $\begin{array}{l}74.2 \\
74.6 \\
70.3\end{array}$ & $\begin{array}{l}64.5 \\
64.9 \\
60.8\end{array}$ & $\begin{array}{l}W \\
W \\
-\end{array}$ & $\begin{array}{l}69.3 \\
70.0 \\
66.0\end{array}$ & $\begin{array}{l}74.4 \\
74.3 \\
68.6\end{array}$ & $\begin{array}{l}73.9 \\
73.9 \\
68.2\end{array}$ & $\begin{array}{l}64.6 \\
64.9 \\
60.3\end{array}$ & $\begin{array}{l}56.5 \\
57.3 \\
53.3\end{array}$ & $\begin{array}{l}49.0 \\
W \\
W\end{array}$ & $\begin{array}{l}60.0 \\
60.4 \\
56.0\end{array}$ \\
\hline $\begin{array}{l}\text { January } 1996 \text {............... } \\
\text { December } 1995 \text {........... } \\
\text { January } 1995 \text {............... } \\
\text { South Dakota }\end{array}$ & $\begin{array}{l}76.6 \\
75.3 \\
74.2\end{array}$ & $\begin{array}{l}76.2 \\
74.8 \\
73.5\end{array}$ & $\begin{array}{l}69.4 \\
67.7 \\
66.6\end{array}$ & $\begin{array}{l}61.3 \\
59.7 \\
57.6\end{array}$ & $\begin{array}{l}\mathbf{5 5 . 6} \\
\mathbf{5 3 . 5} \\
\mathbf{5 1 . 7}\end{array}$ & $\begin{array}{l}61.9 \\
60.5 \\
58.3\end{array}$ & $\begin{array}{l}66.9 \\
65.5 \\
63.9\end{array}$ & $\begin{array}{l}66.7 \\
65.3 \\
63.6\end{array}$ & $\begin{array}{l}61.5 \\
60.0 \\
60.2\end{array}$ & $\begin{array}{l}55.4 \\
54.0 \\
51.8\end{array}$ & $\begin{array}{l}52.5 \\
49.3 \\
48.3\end{array}$ & $\begin{array}{l}55.0 \\
52.8 \\
51.0\end{array}$ \\
\hline $\begin{array}{l}\text { January } 1996 \text {................ } \\
\text { December } 1995 \text {............ } \\
\text { January } 1995 \text {............... } \\
\text { Tennessee }\end{array}$ & $\begin{array}{l}88.8 \\
87.2 \\
83.8\end{array}$ & $\begin{array}{l}88.5 \\
86.9 \\
83.7\end{array}$ & $\begin{array}{l}72.5 \\
70.8 \\
69.8\end{array}$ & $\begin{array}{l}65.6 \\
64.2 \\
62.8\end{array}$ & $\begin{array}{l}- \\
-\end{array}$ & $\begin{array}{l}67.2 \\
65.6 \\
64.5\end{array}$ & $\begin{array}{l}75.3 \\
74.3 \\
73.7\end{array}$ & $\begin{array}{l}74.0 \\
73.3 \\
72.1\end{array}$ & $\begin{array}{l}66.1 \\
64.3 \\
63.2\end{array}$ & $\begin{array}{l}58.3 \\
56.8 \\
54.8\end{array}$ & $\overline{-}$ & $\begin{array}{l}60.3 \\
58.8 \\
56.9\end{array}$ \\
\hline $\begin{array}{l}\text { January } 1996 \text {............... } \\
\text { December } 1995 \\
\text { January } 1995 \text {................... } \\
\text { Wisconsin }\end{array}$ & $\begin{array}{l}85.0 \\
81.7 \\
84.0\end{array}$ & $\begin{array}{l}84.4 \\
81.2 \\
83.1\end{array}$ & $\begin{array}{l}74.8 \\
72.6 \\
74.1\end{array}$ & $\begin{array}{l}64.5 \\
62.8 \\
62.4\end{array}$ & $\begin{array}{l}\bar{w} \\
-\end{array}$ & $\begin{array}{l}66.4 \\
64.5 \\
64.9\end{array}$ & $\begin{array}{l}71.3 \\
68.5 \\
70.8\end{array}$ & $\begin{array}{l}70.9 \\
68.2 \\
70.2\end{array}$ & $\begin{array}{l}65.9 \\
63.9 \\
65.9\end{array}$ & $\begin{array}{l}\mathbf{5 7 . 3} \\
\mathbf{5 5 . 6} \\
\mathbf{5 5 . 3}\end{array}$ & $\begin{array}{l}W \\
W \\
W\end{array}$ & $\begin{array}{l}58.8 \\
57.0 \\
57.5\end{array}$ \\
\hline $\begin{array}{l}\text { January } 1996 \text {............... } \\
\text { December } 1995 \text {............ } \\
\text { January } 1995 \text {.............. }\end{array}$ & $\begin{array}{l}86.4 \\
85.4 \\
80.9\end{array}$ & $\begin{array}{l}85.8 \\
84.9 \\
80.5\end{array}$ & $\begin{array}{l}72.2 \\
72.0 \\
69.9\end{array}$ & $\begin{array}{l}66.2 \\
66.1 \\
62.5\end{array}$ & $\begin{array}{l}- \\
-\end{array}$ & $\begin{array}{l}68.4 \\
68.4 \\
65.3\end{array}$ & $\begin{array}{l}75.5 \\
74.4 \\
71.2\end{array}$ & $\begin{array}{r}75.3 \\
74.2 \\
71.0\end{array}$ & $\begin{array}{l}64.8 \\
64.4 \\
61.6\end{array}$ & $\begin{array}{l}58.7 \\
58.6 \\
55.1\end{array}$ & $\overline{-}$ & $\begin{array}{l}60.7 \\
60.6 \\
57.3\end{array}$ \\
\hline \multicolumn{13}{|l|}{ PAD District III } \\
\hline $\begin{array}{l}\text { January } 1996 \text {............... } \\
\text { December } 1995 \text {............ } \\
\text { January } 1995 \text {............... } \\
\text { Alabama }\end{array}$ & $\begin{array}{l}85.9 \\
84.0 \\
87.3\end{array}$ & $\begin{array}{l}84.6 \\
82.9 \\
85.2\end{array}$ & $\begin{array}{l}71.7 \\
70.7 \\
73.1\end{array}$ & $\begin{array}{l}64.0 \\
62.5 \\
61.5\end{array}$ & $\begin{array}{l}\mathbf{5 4 . 7} \\
53.2 \\
\mathbf{5 0 . 3}\end{array}$ & $\begin{array}{l}64.7 \\
62.6 \\
63.4\end{array}$ & $\begin{array}{l}72.9 \\
71.1 \\
74.3\end{array}$ & $\begin{array}{l}72.1 \\
70.5 \\
73.0\end{array}$ & $\begin{array}{l}64.3 \\
63.4 \\
65.3\end{array}$ & $\begin{array}{l}56.7 \\
55.3 \\
54.6\end{array}$ & $\begin{array}{l}50.4 \\
48.9 \\
47.8\end{array}$ & $\begin{array}{l}56.7 \\
54.8 \\
55.3\end{array}$ \\
\hline $\begin{array}{l}\text { January } 1996 \text {............... } \\
\text { December } 1995 \text {............ } \\
\text { January } 1995 \text {............... } \\
\text { Arkansas }\end{array}$ & $\begin{array}{l}87.0 \\
85.2 \\
86.1\end{array}$ & $\begin{array}{l}85.5 \\
84.0 \\
84.1\end{array}$ & $\begin{array}{l}78.1 \\
76.5 \\
76.0\end{array}$ & $\begin{array}{l}64.6 \\
62.6 \\
62.7\end{array}$ & $\begin{array}{l}- \\
-\end{array}$ & $\begin{array}{l}67.5 \\
65.7 \\
65.8\end{array}$ & $\begin{array}{l}74.0 \\
72.3 \\
73.5\end{array}$ & $\begin{array}{l}73.2 \\
71.8 \\
72.4\end{array}$ & $\begin{array}{r}68.6 \\
67.3 \\
66.8\end{array}$ & $\begin{array}{l}57.1 \\
55.3 \\
55.1\end{array}$ & $\begin{array}{l}W \\
W \\
W\end{array}$ & $\begin{array}{l}59.7 \\
58.0 \\
57.8\end{array}$ \\
\hline $\begin{array}{l}\text { January } 1996 \text {................ } \\
\text { December } 1995 \text {............. } \\
\text { January } 1995 \text {............... } \\
\text { Loulsiana }\end{array}$ & $\begin{array}{l}82.1 \\
80.5 \\
82.0\end{array}$ & $\begin{array}{l}80.3 \\
79.4 \\
79.6\end{array}$ & $\begin{array}{l}71.0 \\
70.2 \\
68.2\end{array}$ & $\begin{array}{l}63.4 \\
62.2 \\
60.5\end{array}$ & $\begin{array}{l}- \\
-\end{array}$ & $\begin{array}{l}65.1 \\
63.9 \\
61.8\end{array}$ & $\begin{array}{l}68.9 \\
66.8 \\
69.1\end{array}$ & $\begin{array}{l}67.4 \\
66.5 \\
67.9\end{array}$ & $\begin{array}{l}63.5 \\
62.7 \\
60.2\end{array}$ & $\begin{array}{l}56.3 \\
55.0 \\
53.6\end{array}$ & $\begin{array}{c}50.2 \\
48.9 \\
W\end{array}$ & $\begin{array}{l}57.3 \\
56.1 \\
54.8\end{array}$ \\
\hline $\begin{array}{l}\text { January } 1996 \text {............... } \\
\text { December } 1995 \text {............ } \\
\text { January } 1995 \text {............... } \\
\text { Mlssissippi }\end{array}$ & $\begin{array}{l}84.4 \\
82.9 \\
86.6\end{array}$ & $\begin{array}{l}82.4 \\
81.2 \\
85.1\end{array}$ & $\begin{array}{l}73.6 \\
72.3 \\
74.4\end{array}$ & $\begin{array}{l}63.0 \\
61.0 \\
59.8\end{array}$ & $\begin{array}{l}52.9 \\
54.2 \\
50.3\end{array}$ & $\begin{array}{l}64.2 \\
61.9 \\
62.8\end{array}$ & $\begin{array}{l}70.9 \\
70.1 \\
73.9\end{array}$ & $\begin{array}{l}70.2 \\
69.5 \\
73.3\end{array}$ & $\begin{array}{l}65.4 \\
64.3 \\
66.1\end{array}$ & $\begin{array}{l}56.2 \\
54.6 \\
53.8\end{array}$ & $\begin{array}{l}50.4 \\
48.9 \\
47.1\end{array}$ & $\begin{array}{l}56.6 \\
54.7 \\
54.7\end{array}$ \\
\hline $\begin{array}{l}\text { January } 1996 \text {................ } \\
\text { December } 1995 \text {........... } \\
\text { January } 1995 \text {.............. } \\
\text { New Mexico }\end{array}$ & $\begin{array}{l}88.1 \\
86.1 \\
87.5\end{array}$ & $\begin{array}{l}87.3 \\
85.6 \\
86.8\end{array}$ & $\begin{array}{l}72.2 \\
70.8 \\
72.3\end{array}$ & $\begin{array}{l}64.1 \\
62.3 \\
61.9\end{array}$ & $\begin{array}{c}56.6 \\
54.3 \\
W\end{array}$ & $\begin{array}{l}64.0 \\
62.6 \\
61.9\end{array}$ & $\begin{array}{l}74.5 \\
72.7 \\
74.6\end{array}$ & $\begin{array}{l}74.0 \\
72.4 \\
74.0\end{array}$ & $\begin{array}{l}63.3 \\
62.0 \\
64.0\end{array}$ & $\begin{array}{l}56.5 \\
\mathbf{5 4 . 8} \\
\mathbf{5 4 . 2}\end{array}$ & $\begin{array}{l}51.7 \\
50.0 \\
48.6\end{array}$ & $\begin{array}{l}57.0 \\
55.5 \\
55.2\end{array}$ \\
\hline $\begin{array}{l}\text { January } 1996 \text {................ } \\
\text { December } 1995 \text {............ } \\
\text { January } 1995 \text {............." } \\
\text { Texas }\end{array}$ & $\begin{array}{l}91.3 \\
90.3 \\
90.2\end{array}$ & $\begin{array}{l}91.2 \\
90.1 \\
89.7\end{array}$ & $\begin{array}{l}75.2 \\
73.5 \\
74.4\end{array}$ & $\begin{array}{l}69.5 \\
67.0 \\
63.3\end{array}$ & $\begin{array}{l}- \\
-\end{array}$ & $\begin{array}{l}70.4 \\
68.3 \\
65.7\end{array}$ & $\begin{array}{l}78.4 \\
77.7 \\
77.9\end{array}$ & $\begin{array}{l}78.1 \\
77.4 \\
77.3\end{array}$ & $\begin{array}{l}65.5 \\
63.8 \\
63.5\end{array}$ & $\begin{array}{l}62.0 \\
59.6 \\
56.1\end{array}$ & $\overline{-}$ & $\begin{array}{l}62.7 \\
60.5 \\
57.8\end{array}$ \\
\hline $\begin{array}{l}\text { January } 1996 \ldots . . . \ldots \ldots . . . . . . \\
\text { December } 1995 \text {........... } \\
\text { January } 1995 \text {............... }\end{array}$ & $\begin{array}{l}85.6 \\
83.5 \\
87.9\end{array}$ & $\begin{array}{l}84.6 \\
82.7 \\
85.5\end{array}$ & $\begin{array}{l}70.4 \\
69.4 \\
72.7\end{array}$ & $\begin{array}{l}63.9 \\
62.7 \\
61.6\end{array}$ & $\begin{array}{l}54.9 \\
52.9 \\
50.1\end{array}$ & $\begin{array}{l}64.0 \\
61.7 \\
63.5\end{array}$ & $\begin{array}{l}72.7 \\
70.7 \\
74.7\end{array}$ & $\begin{array}{l}72.0 \\
70.2 \\
73.0\end{array}$ & $\begin{array}{l}63.6 \\
62.8 \\
65.6\end{array}$ & $\begin{array}{l}56.2 \\
55.2 \\
54.8\end{array}$ & $\begin{array}{l}50.4 \\
48.9 \\
47.8\end{array}$ & $\begin{array}{l}55.9 \\
53.9 \\
54.9\end{array}$ \\
\hline PAD District IV & & & & & & & & & & & & \\
\hline $\begin{array}{l}\text { January } 1996 \text {............... } \\
\text { December } 1995 \text {............ } \\
\text { January } 1995 \text {............... } \\
\text { Colorado }\end{array}$ & $\begin{array}{l}88.7 \\
89.6 \\
89.7\end{array}$ & $\begin{array}{l}87.9 \\
88.8 \\
88.6\end{array}$ & $\begin{array}{l}74.1 \\
73.7 \\
72.9\end{array}$ & $\begin{array}{l}68.5 \\
67.4 \\
66.6\end{array}$ & $\overline{-}$ & $\begin{array}{l}70.1 \\
69.3 \\
68.5\end{array}$ & $\begin{array}{l}77.4 \\
78.4 \\
79.0\end{array}$ & $\begin{array}{l}77.0 \\
78.0 \\
78.4\end{array}$ & $\begin{array}{l}67.0 \\
66.3 \\
66.0\end{array}$ & $\begin{array}{l}61.2 \\
60.2 \\
59.3\end{array}$ & $\frac{w}{w}$ & $\begin{array}{l}62.7 \\
61.9 \\
61.2\end{array}$ \\
\hline $\begin{array}{l}\text { January } 1996 \text {............... } \\
\text { December } 1995 \text {............ } \\
\text { January } 1995 \text {.............. }\end{array}$ & $\begin{array}{l}92.8 \\
93.5 \\
96.3\end{array}$ & $\begin{array}{l}92.3 \\
93.1 \\
95.2\end{array}$ & $\begin{array}{l}77.5 \\
75.8 \\
75.6\end{array}$ & $\begin{array}{l}67.8 \\
64.6 \\
64.5\end{array}$ & $\begin{array}{l}- \\
-\end{array}$ & $\begin{array}{l}70.7 \\
67.9 \\
67.9\end{array}$ & $\begin{array}{l}79.7 \\
80.6 \\
82.1\end{array}$ & $\begin{array}{l}79.6 \\
80.3 \\
81.4\end{array}$ & $\begin{array}{l}69.5 \\
67.1 \\
66.6\end{array}$ & $\begin{array}{l}61.0 \\
58.0 \\
57.5\end{array}$ & $\begin{array}{l}W \\
- \\
-\end{array}$ & $\begin{array}{l}63.0 \\
60.5 \\
60.1\end{array}$ \\
\hline
\end{tabular}

See footnotes at end of table. 
Table 31. Motor Gasoline Prices by Grade, Sales Type, PAD District, and State (Cents per Gallon Excluding Taxes) - Continued

\begin{tabular}{|c|c|c|c|c|c|c|c|c|c|c|c|c|}
\hline \multirow{3}{*}{$\begin{array}{c}\text { Geographic Area } \\
\text { Month }\end{array}$} & \multicolumn{6}{|c|}{ Regular } & \multicolumn{6}{|c|}{ Midgrade } \\
\hline & \multicolumn{2}{|c|}{ Sales to End Users } & \multicolumn{4}{|c|}{ Sales for Resale } & \multicolumn{2}{|c|}{ Sales to End Users } & \multicolumn{4}{|c|}{ Sales for Resale } \\
\hline & $\begin{array}{c}\text { Through } \\
\text { Retail } \\
\text { Outlets }\end{array}$ & Average $\mathrm{a}^{\mathrm{a}}$ & DTW & Rack & Bulk & Average & $\begin{array}{c}\text { Through } \\
\text { Retail } \\
\text { Outlets }\end{array}$ & Average $\mathbf{a}$ & DTW & Rack & Bulk & Averago \\
\hline \multicolumn{13}{|l|}{ Idaho } \\
\hline January 1996 ................ & 69.9 & 70.0 & 61.9 & 55.0 & - & 57.1 & 78.1 & 77.8 & 66.6 & 58.3 & - & 61.4 \\
\hline December 1995 ............. & 72.9 & 72.5 & 62.9 & 56.8 & - & 58.8 & 81.2 & 80.2 & 68.4 & 60.8 & - & 63.7 \\
\hline January 1995 .................. & 75.0 & 74.5 & 65.0 & 59.1 & - & 61.0 & 80.0 & 79.1 & 68.9 & 62.5 & - & 64.6 \\
\hline \multicolumn{13}{|l|}{ Montana } \\
\hline January 1996 & 74.4 & 75.5 & 68.1 & 62.8 & - & 63.8 & 78.5 & 78.6 & 71.3 & 67.9 & - & 68.1 \\
\hline December 1995 .............. & 74.6 & 75.7 & 67.7 & 62.1 & - & 63.2 & 79.5 & 79.5 & 72.5 & 67.3 & - & 67.7 \\
\hline January 1995 & 75.7 & 76.0 & 66.6 & 59.5 & $w$ & 60.9 & 79.6 & 80.7 & $W$ & W & - & 66.6 \\
\hline \multicolumn{13}{|l|}{ Utah } \\
\hline January $1996 \ldots . . . . . . . . . . . . . .$. & 70.0 & 69.3 & 61.1 & 59.1 & - & 59.8 & 77.9 & 77.6 & 67.1 & 64.2 & - & 65.2 \\
\hline December 1995 ............ & 71.0 & 70.6 & 61.0 & 59.5 & - & 59.9 & 78.1 & 77.8 & 66.5 & 64.5 & - & 65.2 \\
\hline January 1995 ................ & 69.0 & 68.8 & 61.2 & 58.5 & $w$ & 59.3 & 75.9 & 75.8 & 66.5 & 63.4 & - & 64.8 \\
\hline \multicolumn{13}{|l|}{ Wyoming } \\
\hline January 1996 ................. & 77.1 & 76.6 & 64.8 & 58.0 & - & 59.3 & 83.6 & 83.5 & NA & 60.5 & - & NA \\
\hline December 1995 ............. & 77.8 & 77.2 & 63.8 & 56.0 & - & 57.5 & 83.6 & 83.4 & 65.0 & 63.6 & - & 64.7 \\
\hline January 1995 ................. & 79.2 & 78.0 & 62.3 & 54.4 & $w$ & 55.8 & 84.0 & 84.0 & 61.8 & 65.3 & - & 62.9 \\
\hline \multicolumn{13}{|l|}{ PAD District V } \\
\hline January 1996 & 77.0 & 76.5 & 68.5 & 60.5 & 54.3 & 64.4 & 82.0 & 81.8 & 72.3 & 65.3 & - & 70.8 \\
\hline December 1995 ............. & 76.3 & 76.0 & 67.2 & 62.0 & 56.0 & 64.2 & 80.7 & 80.6 & 71.5 & 66.6 & - & 70.5 \\
\hline January 1995 ................... & 79.3 & 78.7 & 70.3 & 59.7 & 54.2 & 64.9 & 85.3 & 85.0 & 75.0 & 65.5 & $w$ & 73.2 \\
\hline \multicolumn{13}{|l|}{ Alaska } \\
\hline January 1996 ................. & 112.2 & 107.8 & 86.2 & 74.9 & 63.1 & 80.5 & 103.4 & 104.2 & 97.2 & 83.7 & - & 94.7 \\
\hline December 1995 ............. & 111.3 & 107.3 & 88.5 & 71.5 & $w$ & 80.1 & 100.6 & 101.9 & 97.1 & 86.2 & - & 94.4 \\
\hline January 1995 .................. & 118.8 & 116.1 & 91.4 & 73.3 & $w$ & 83.2 & $w$ & 109.0 & 97.3 & W & $w$ & 97.1 \\
\hline $\begin{array}{l}\text { Arzzona } \\
\text { January } 1996 \ldots . . . . . . . . . . .\end{array}$ & 79.1 & 78.4 & 71.3 & 62.9 & 56.7 & 67.0 & 85.3 & 85.2 & 765 & 705 & - & 743 \\
\hline December 1995 ............... & 76.3 & 75.7 & 69.4 & 62.7 & 56.6 & 66.1 & $\begin{array}{l}05.0 \\
82.0\end{array}$ & $\begin{array}{l}00.2 \\
81.9\end{array}$ & 74.7 & 70.1 & - & $\begin{array}{l}74.3 \\
73.2\end{array}$ \\
\hline 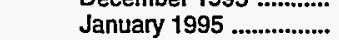 & 78.7 & 78.4 & 70.8 & 61.7 & $w$ & 66.4 & 87.9 & 87.7 & 73.3 & 67.9 & - & 70.5 \\
\hline Califomia & & & & & & & & & & & & \\
\hline January 1996 ................. & 71.5 & 71.4 & 66.5 & 59.1 & 54.6 & 63.2 & 78.4 & 78.4 & 70.6 & 63.7 & - & 69.4 \\
\hline December 1995 .............. & 70.9 & 70.9 & 64.7 & 60.8 & 56.0 & 62.3 & 77.0 & 77.1 & 69.5 & 65.2 & - & 68.8 \\
\hline January 1995 ................. & 77.0 & 76.4 & 70.2 & 59.1 & 54.5 & 64.8 & 84.6 & 84.2 & 74.8 & 65.2 & $w$ & 73.1 \\
\hline \multicolumn{13}{|l|}{ Hawaii } \\
\hline January 1996 ................. & 103.1 & 95.2 & 93.7 & 83.1 & - & 91.8 & 108.7 & 104.1 & 99.7 & $w$ & - & 99.4 \\
\hline December 1995 ............ & 105.6 & 96.9 & 94.0 & 80.7 & - & 92.1 & 111.3 & 105.3 & 99.9 & $w$ & - & 99.6 \\
\hline January 1995 ........................ & 100.0 & 92.9 & 91.4 & 80.5 & - & 89.7 & 106.6 & 99.1 & 97.4 & $w$ & - & 97.1 \\
\hline \multicolumn{13}{|c|}{ Nevada } \\
\hline January 1996 ................. & 87.6 & 86.1 & 68.6 & 63.4 & 57.3 & 64.8 & 91.3 & 91.2 & 74.7 & 68.3 & - & 73.0 \\
\hline December 1995 ............ & 86.3 & 85.1 & 67.7 & 64.6 & 58.1 & 65.2 & 90.4 & 90.3 & 73.6 & 69.5 & - & 72.5 \\
\hline January 1995 ................... & 88.1 & 86.2 & 65.3 & 62.6 & w & 63.6 & 93.1 & 92.5 & 72.3 & 66.1 & - & 70.6 \\
\hline \multicolumn{13}{|l|}{ Oregon } \\
\hline January 1996 ................. & 79.5 & 79.2 & 69.7 & 60.6 & 52.1 & 62.5 & 85.8 & 83.6 & 73.5 & 68.1 & - & 71.3 \\
\hline December 1995 ............. & 80.0 & 79.8 & 71.0 & 63.2 & 54.7 & 65.7 & 86.8 & 85.0 & 74.5 & 70.6 & - & 72.8 \\
\hline January 1995 ................. & 77.4 & 76.8 & 65.1 & 57.6 & 52.1 & 59.6 & 77.1 & 78.7 & 68.2 & 63.2 & - & 65.9 \\
\hline \multicolumn{13}{|l|}{ Washington } \\
\hline January 1996 ................. & 79.8 & 79.7 & 73.1 & 59.7 & 50.0 & 65.2 & 85.2 & 85.2 & 78.3 & 65.2 & - & 73.3 \\
\hline December 1995 .............. & 81.1 & 80.9 & 74.4 & 61.7 & 53.9 & 67.4 & 86.0 & 85.9 & 78.7 & 66.6 & - & 74.1 \\
\hline January 1995 .................. & 79.5 & 79.5 & 69.8 & 58.8 & $w$ & 64.3 & 86.0 & 85.5 & 71.9 & 67.4 & - & 70.9 \\
\hline
\end{tabular}

See footnotes at end of table. 
Table 31. Motor Gasoline Prices by Grade, Sales Type, PAD District, and State (Cents per Gallon Excluding Taxes) - Continued

\begin{tabular}{|c|c|c|c|c|c|c|c|c|c|c|c|c|}
\hline \multirow{3}{*}{$\begin{array}{l}\text { Geographic Area } \\
\text { Month }\end{array}$} & \multicolumn{6}{|c|}{ Premium } & \multicolumn{6}{|c|}{ All Grades } \\
\hline & \multicolumn{2}{|c|}{ Sales to End Users } & \multicolumn{4}{|c|}{ Sales for Resale } & \multicolumn{2}{|c|}{ Sales to End Users } & \multicolumn{4}{|c|}{ Sales for Resale } \\
\hline & $\begin{array}{c}\text { Through } \\
\text { Retail } \\
\text { Outlets }\end{array}$ & Averagea & DTW & Rack & Bulk & Average & $\begin{array}{c}\text { Through } \\
\text { Retail } \\
\text { Outlets }\end{array}$ & Averagea & DTW & Rack & Bulk & Average \\
\hline \multicolumn{13}{|l|}{ Idaho } \\
\hline $\begin{array}{l}\text { January } 1996 \text {................ } \\
\text { December } 1995 \text {............ } \\
\text { Jantuary } 1995 \ldots . . . . . . . . . . . \\
\text { Montana }\end{array}$ & $\begin{array}{l}82.5 \\
85.8 \\
86.6\end{array}$ & $\begin{array}{l}81.9 \\
84.8 \\
86.1\end{array}$ & $\begin{array}{l}71.4 \\
72.8 \\
73.9\end{array}$ & $\begin{array}{l}64.5 \\
66.1 \\
68.2\end{array}$ & $\begin{array}{l}- \\
-\end{array}$ & $\begin{array}{l}67.0 \\
68.8 \\
70.4\end{array}$ & $\begin{array}{l}72.5 \\
75.6 \\
76.8\end{array}$ & $\begin{array}{l}72.4 \\
75.0 \\
76.3\end{array}$ & $\begin{array}{l}64.4 \\
65.7 \\
67.1\end{array}$ & $\begin{array}{l}56.9 \\
58.8 \\
60.8\end{array}$ & $\begin{array}{l}- \\
-\end{array}$ & $\begin{array}{l}59.3 \\
61.2 \\
62.8\end{array}$ \\
\hline $\begin{array}{l}\text { January } 1996 . . . . . . . . . . . . . . \\
\text { December } 1995 \text {............ } \\
\text { January } 1995 \text {............... } \\
\text { Utah }\end{array}$ & $\begin{array}{l}83.6 \\
83.7 \\
84.7\end{array}$ & $\begin{array}{l}84.3 \\
84.1 \\
84.9\end{array}$ & $\begin{array}{l}76.7 \\
76.2 \\
74.4\end{array}$ & $\begin{array}{l}73.0 \\
72.1 \\
69.0\end{array}$ & $\begin{array}{l}- \\
-\end{array}$ & $\begin{array}{l}73.8 \\
73.1 \\
70.0\end{array}$ & $\begin{array}{l}76.3 \\
76.7 \\
77.4\end{array}$ & $\begin{array}{l}77.0 \\
77.3 \\
77.6\end{array}$ & $\begin{array}{l}70.1 \\
70.0 \\
68.0\end{array}$ & $\begin{array}{l}65.1 \\
64.4 \\
61.3\end{array}$ & $\bar{w}$ & $\begin{array}{l}66.1 \\
65.5 \\
62.6\end{array}$ \\
\hline $\begin{array}{l}\text { January } 1996 \text {................ } \\
\text { December } 1995 \text {............ } \\
\text { January } 1995 \text {................ } \\
\text { Wyoming }\end{array}$ & $\begin{array}{l}85.3 \\
86.1 \\
82.1\end{array}$ & $\begin{array}{l}84.1 \\
84.9 \\
81.2\end{array}$ & $\begin{array}{l}71.2 \\
71.3 \\
70.2\end{array}$ & $\begin{array}{l}68.6 \\
68.7 \\
67.7\end{array}$ & $\begin{array}{l}- \\
-\end{array}$ & $\begin{array}{l}69.4 \\
69.6 \\
68.6\end{array}$ & $\begin{array}{l}74.5 \\
75.4 \\
72.8\end{array}$ & $\begin{array}{l}73.7 \\
74.8 \\
72.5\end{array}$ & $\begin{array}{l}64.3 \\
64.5 \\
64.2\end{array}$ & $\begin{array}{l}62.1 \\
62.3 \\
61.1\end{array}$ & $\overline{-}$ & $\begin{array}{l}62.8 \\
63.0 \\
62.1\end{array}$ \\
\hline $\begin{array}{l}\text { January } 1996 . . . . . . . . . . . . . . \\
\text { December } 1995 \ldots . . . . . . . . . \\
\text { January } 1995 . . . . . . . . . . . . .\end{array}$ & $\begin{array}{l}89.0 \\
89.5 \\
90.9\end{array}$ & $\begin{array}{l}88.5 \\
89.0 \\
88.7\end{array}$ & $\begin{array}{l}74.2 \\
73.9 \\
70.6\end{array}$ & $\begin{array}{l}67.5 \\
65.4 \\
63.2\end{array}$ & $\begin{array}{l}- \\
-\end{array}$ & $\begin{array}{l}68.7 \\
66.9 \\
64.5\end{array}$ & $\begin{array}{l}79.6 \\
80.3 \\
81.4\end{array}$ & $\begin{array}{l}79.1 \\
79.6 \\
80.3\end{array}$ & $\begin{array}{l}66.5 \\
65.4 \\
63.7\end{array}$ & $\begin{array}{l}59.9 \\
57.8 \\
56.0\end{array}$ & $\overline{-}$ & $\begin{array}{l}61.1 \\
59.3 \\
57.4\end{array}$ \\
\hline \multicolumn{13}{|l|}{ PAD District V } \\
\hline $\begin{array}{l}\text { January } 1996 \ldots . . . . . . . . . . \\
\text { December } 1995 \text {............ } \\
\text { January } 1995 \text {.............. } \\
\text { Alaska }\end{array}$ & $\begin{array}{l}93.9 \\
93.0 \\
97.3\end{array}$ & $\begin{array}{l}92.9 \\
92.2 \\
96.3\end{array}$ & $\begin{array}{l}81.5 \\
80.8 \\
84.9\end{array}$ & $\begin{array}{l}72.1 \\
73.6 \\
71.5\end{array}$ & $\begin{array}{l}60.3 \\
62.5 \\
60.3\end{array}$ & $\begin{array}{l}78.4 \\
78.2 \\
81.1\end{array}$ & $\begin{array}{l}80.5 \\
79.7 \\
82.8\end{array}$ & $\begin{array}{l}80.0 \\
79.4 \\
82.2\end{array}$ & $\begin{array}{l}71.9 \\
70.8 \\
74.1\end{array}$ & $\begin{array}{l}62.7 \\
64.2 \\
61.7\end{array}$ & $\begin{array}{l}54.9 \\
56.6 \\
54.7\end{array}$ & $\begin{array}{l}67.8 \\
67.6 \\
68.7\end{array}$ \\
\hline $\begin{array}{l}\text { January } 1996 \ldots . . . . . . . . . . . . \\
\text { December } 1995 \text {............ } \\
\text { January } 1995 \text {................ } \\
\text { Arizona }\end{array}$ & $\begin{array}{l}117.1 \\
116.4 \\
116.4\end{array}$ & $\begin{array}{l}113.5 \\
113.0 \\
114.9\end{array}$ & $\begin{array}{l}100.8 \\
100.5 \\
102.1\end{array}$ & $\begin{array}{l}84.6 \\
84.1 \\
82.3\end{array}$ & $\begin{array}{l}W \\
W \\
-\end{array}$ & $\begin{array}{l}94.5 \\
93.9 \\
94.9\end{array}$ & $\begin{array}{l}111.7 \\
110.6 \\
118.5\end{array}$ & $\begin{array}{l}108.0 \\
107.2 \\
115.4\end{array}$ & $\begin{array}{l}88.6 \\
90.7 \\
93.0\end{array}$ & $\begin{array}{l}76.0 \\
73.2 \\
74.1\end{array}$ & $\begin{array}{l}63.5 \\
W \\
W\end{array}$ & $\begin{array}{l}82.6 \\
82.5 \\
84.9\end{array}$ \\
\hline $\begin{array}{l}\text { January } 1996 \text {................ } \\
\text { December } 1995 \text {............ } \\
\text { January } 1995 \text {............... } \\
\text { Callfomia }\end{array}$ & $\begin{array}{l}97.2 \\
94.2 \\
96.7\end{array}$ & $\begin{array}{l}96.7 \\
93.8 \\
96.2\end{array}$ & $\begin{array}{l}85.3 \\
83.3 \\
83.2\end{array}$ & $\begin{array}{l}74.8 \\
75.0 \\
73.0\end{array}$ & $\begin{array}{c}64.2 \\
63.5 \\
W\end{array}$ & $\begin{array}{l}81.0 \\
79.0 \\
80.0\end{array}$ & $\begin{array}{l}82.6 \\
79.8 \\
81.8\end{array}$ & $\begin{array}{l}81.9 \\
79.2 \\
81.5\end{array}$ & $\begin{array}{l}74.2 \\
72.5 \\
73.1\end{array}$ & $\begin{array}{l}65.4 \\
65.2 \\
63.5\end{array}$ & $\begin{array}{l}57.1 \\
58.3 \\
W\end{array}$ & $\begin{array}{l}69.9 \\
69.0 \\
68.6\end{array}$ \\
\hline $\begin{array}{l}\text { January } 1996 \text {................ } \\
\text { December } 1995 \text {............ } \\
\text { January } 1995 \text {.............. } \\
\text { Hawalf }\end{array}$ & $\begin{array}{l}89.4 \\
88.5 \\
96.0\end{array}$ & $\begin{array}{l}88.5 \\
87.8 \\
94.9\end{array}$ & $\begin{array}{l}79.4 \\
78.5 \\
84.4\end{array}$ & $\begin{array}{l}70.0 \\
71.5 \\
70.3\end{array}$ & $\begin{array}{l}59.8 \\
62.2 \\
59.9\end{array}$ & $\begin{array}{l}77.0 \\
76.5 \\
81.0\end{array}$ & $\begin{array}{l}76.0 \\
75.2 \\
81.5\end{array}$ & $\begin{array}{l}75.7 \\
75.0 \\
80.8\end{array}$ & $\begin{array}{l}70.2 \\
68.7 \\
74.2\end{array}$ & $\begin{array}{l}61.3 \\
63.0 \\
61.3\end{array}$ & $\begin{array}{l}55.2 \\
56.5 \\
55.0\end{array}$ & $\begin{array}{l}66.9 \\
66.0 \\
69.0\end{array}$ \\
\hline $\begin{array}{l}\text { January } 1996 \text {............... } \\
\text { December } 1995 \text {............ } \\
\text { January } 1995 \text {............... } \\
\text { Nevada }\end{array}$ & $\begin{array}{l}118.8 \\
120.5 \\
118.1\end{array}$ & $\begin{array}{l}109.8 \\
110.1 \\
108.4\end{array}$ & $\begin{array}{l}106.7 \\
106.9 \\
104.3\end{array}$ & $\begin{array}{l}97.2 \\
95.1 \\
94.8\end{array}$ & $\begin{array}{l}- \\
-\end{array}$ & $\begin{array}{l}105.6 \\
105.7 \\
103.2\end{array}$ & $\begin{array}{l}107.9 \\
110.1 \\
105.3\end{array}$ & $\begin{array}{r}100.1 \\
101.3 \\
97.6\end{array}$ & $\begin{array}{r}98.8 \\
99.2 \\
96.5\end{array}$ & $\begin{array}{l}87.3 \\
85.2 \\
85.2\end{array}$ & $\begin{array}{l}- \\
-\end{array}$ & $\begin{array}{l}97.2 \\
97.6 \\
95.1\end{array}$ \\
\hline 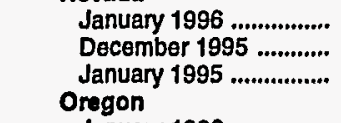 & $\begin{array}{l}101.9 \\
101.0 \\
101.9\end{array}$ & $\begin{array}{l}101.4 \\
100.6 \\
100.5\end{array}$ & $\begin{array}{l}83.1 \\
81.9 \\
81.2\end{array}$ & $\begin{array}{l}74.1 \\
75.2 \\
73.6\end{array}$ & $\begin{array}{l}W \\
64.8 \\
W\end{array}$ & $\begin{array}{l}78.9 \\
77.4 \\
77.6\end{array}$ & $\begin{array}{l}90.3 \\
89.3 \\
90.9\end{array}$ & $\begin{array}{l}89.0 \\
88.2 \\
89.1\end{array}$ & $\begin{array}{l}72.2 \\
71.3 \\
69.0\end{array}$ & $\begin{array}{l}65.3 \\
66.6 \\
64.2\end{array}$ & $\begin{array}{c}57.9 \\
59.5 \\
W\end{array}$ & $\begin{array}{l}67.9 \\
68.1 \\
66.3\end{array}$ \\
\hline $\begin{array}{l}\text { January } 1996 \text {............... } \\
\text { December } 1995 \text {........... } \\
\text { January } 1995 \text {.............. } \\
\text { Washington }\end{array}$ & $\begin{array}{l}94.9 \\
95.9 \\
93.4\end{array}$ & $\begin{array}{l}94.4 \\
95.5 \\
92.7\end{array}$ & $\begin{array}{l}84.5 \\
85.6 \\
80.7\end{array}$ & $\begin{array}{l}75.2 \\
78.1 \\
72.1\end{array}$ & $\begin{array}{c}W \\
60.6 \\
-\end{array}$ & $\begin{array}{l}76.0 \\
79.9 \\
77.0\end{array}$ & $\begin{array}{l}82.2 \\
82.7 \\
79.4\end{array}$ & $\begin{array}{l}81.6 \\
82.3 \\
78.9\end{array}$ & $\begin{array}{r}72.1 \\
73.4 \\
-67.5\end{array}$ & $\begin{array}{l}62.8 \\
65.6 \\
59.4\end{array}$ & $\begin{array}{l}53.4 \\
55.5 \\
52.1\end{array}$ & $\begin{array}{l}64.9 \\
68.1 \\
61.7\end{array}$ \\
\hline $\begin{array}{l}\text { January } 1996 \text {............... } \\
\text { December } 1995 \text {........... } \\
\text { January } 1995 \text {............... }\end{array}$ & $\begin{array}{r}98.9 \\
100.1 \\
97.6\end{array}$ & $\begin{array}{l}98.4 \\
99.8 \\
97.1\end{array}$ & $\begin{array}{l}88.3 \\
89.6 \\
84.7\end{array}$ & $\begin{array}{l}71.5 \\
73.6 \\
71.2\end{array}$ & $\begin{array}{l}- \\
-\end{array}$ & $\begin{array}{l}80.5 \\
81.9 \\
79.4\end{array}$ & $\begin{array}{l}83.2 \\
84.4 \\
82.4\end{array}$ & $\begin{array}{l}83.0 \\
84.2 \\
82.4\end{array}$ & $\begin{array}{l}76.3 \\
77.5 \\
72.7\end{array}$ & $\begin{array}{l}62.0 \\
63.9 \\
60.7\end{array}$ & $\begin{array}{l}50.0 \\
53.9 \\
W\end{array}$ & $\begin{array}{l}68.5 \\
70.5 \\
67.1\end{array}$ \\
\hline
\end{tabular}

$\operatorname{Dash}(-)=$ No data reported.

NA $=$ Not available.

$W=$ Withheld to avold disclosure of individual company data.

a Includes sales through retall outlets as well as all direct sales to end users that were not made through company-operated retail outlets, e.g., sales to agricultural customers, commercial sales, and Industrial sales.

Notes: Motor gasoline averages and totals prior to October 1993 include leaded gasoline.

Notes: Values shown for the current month are preliminary. Values shown for previous months are revised. Data are final upon publication in the Petroleum Marketing Annual.

Sources: Energy Information Administration Forms EIA-782A, "Reliners'/Gas Plant Operators' Monthly Petroleum Product Sales Report," and ElA-782B,

"Resellers'/Retallers' Monthly Petroleum Product Sales Report." 
Table 32. Conventional Motor Gasoline Prices by Grade, Sales Type, PAD District, and State

(Cents per Gallon Excluding Taxes)

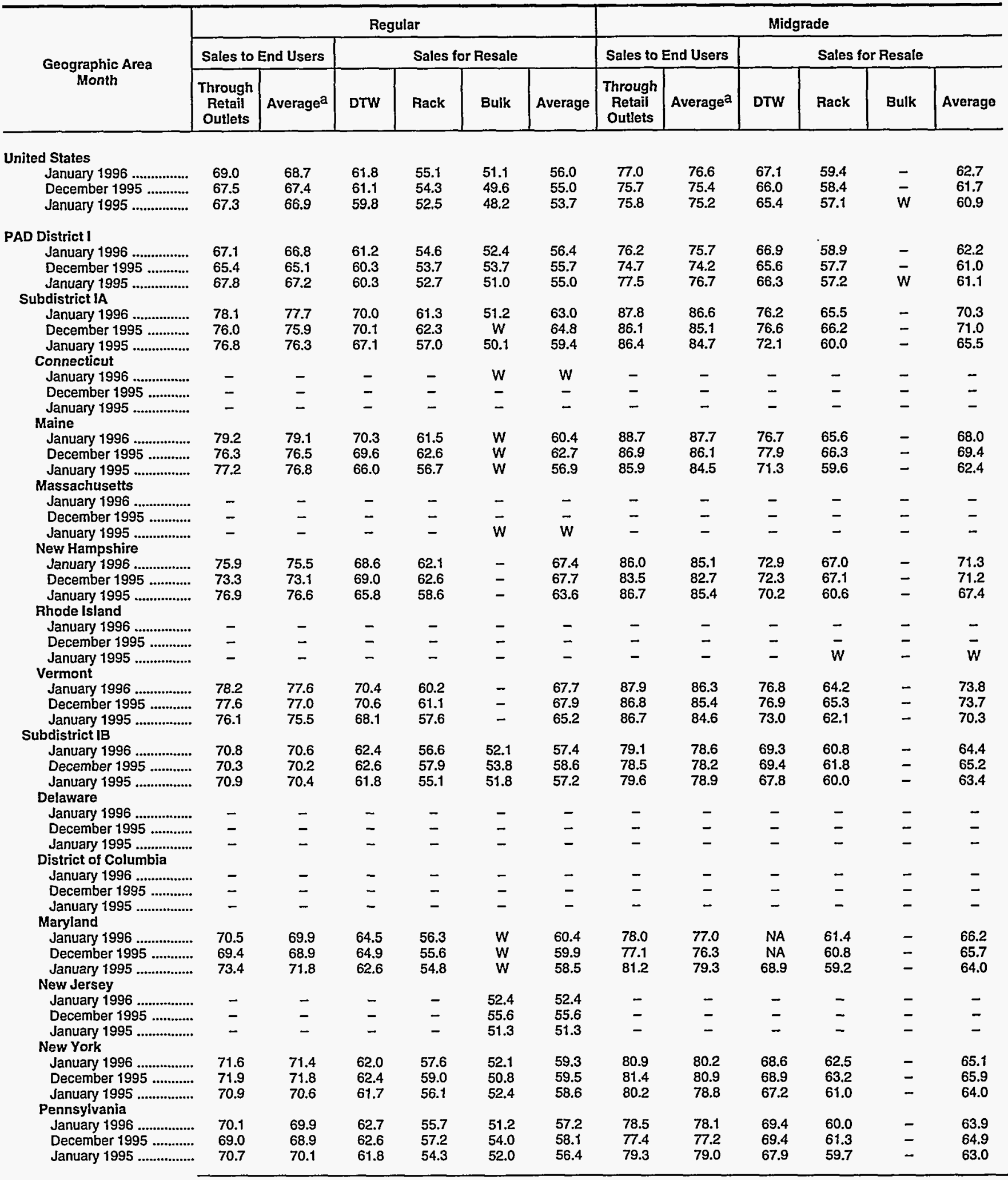

See footnotes at end of table. 
Table 32. Conventional Motor Gasoline Prices by Grade, Sales Type, PAD District, and State

(Cents per Gallon Excluding Taxes) - Continued

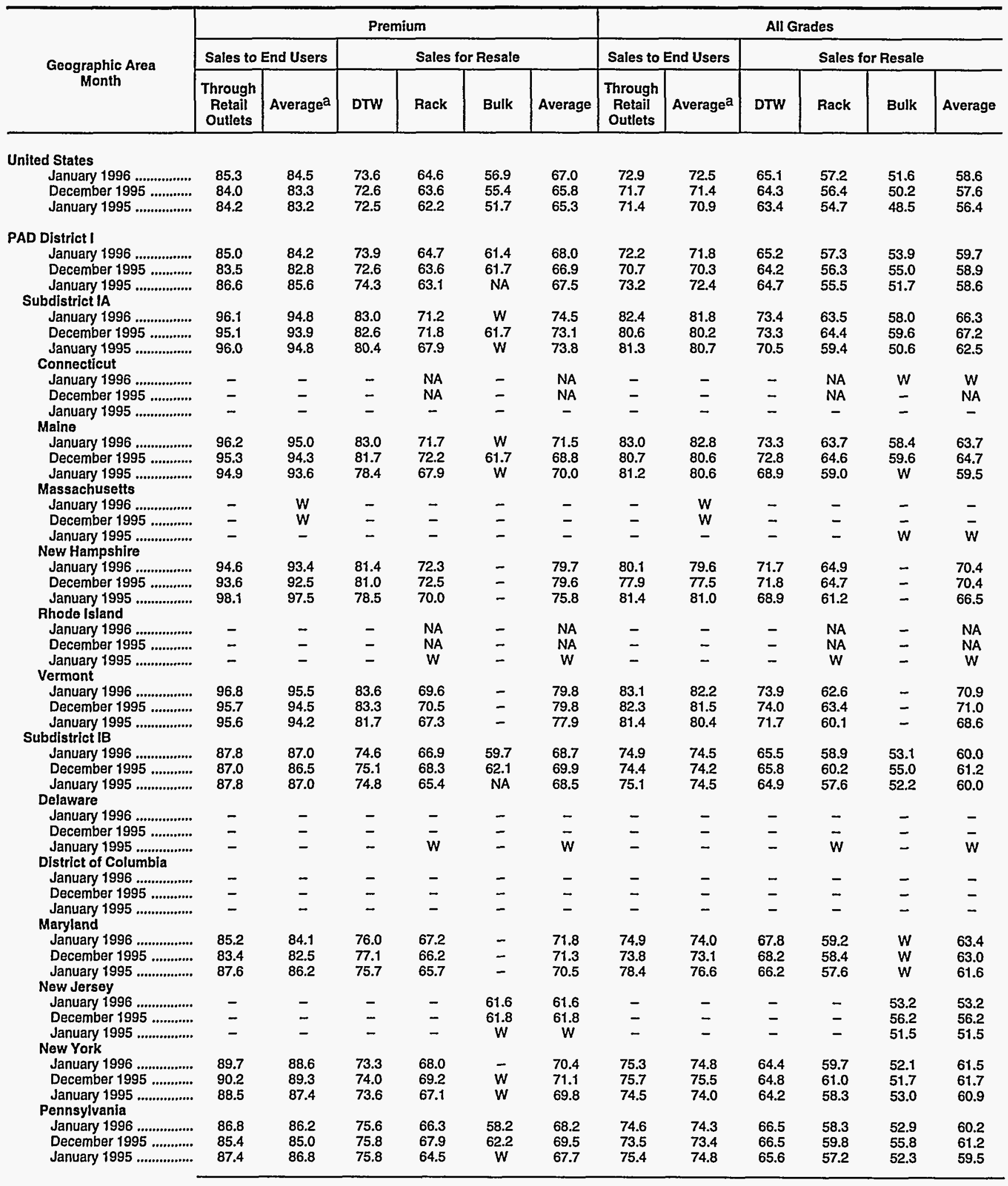

See footnotes at end of table. 
Table 32. Conventional Motor Gasoline Prices by Grade, Sales Type, PAD District, and State

(Cents per Gallon Excluding Taxes) — Continued

\begin{tabular}{|c|c|c|c|c|c|c|c|c|c|c|c|c|}
\hline \multirow{3}{*}{$\begin{array}{l}\text { Geographic Area } \\
\text { Month }\end{array}$} & \multicolumn{6}{|c|}{ Regular } & \multicolumn{6}{|c|}{ Midgrade } \\
\hline & \multicolumn{2}{|c|}{ Sales to End Users } & \multicolumn{4}{|c|}{ Sales for Resale } & \multicolumn{2}{|c|}{ Sales to End Users } & \multicolumn{4}{|c|}{ Sales for Resale } \\
\hline & $\begin{array}{c}\text { Through } \\
\text { Retail } \\
\text { Outlets }\end{array}$ & Average $^{a}$ & DTW & Rack & Bulk & Average & $\begin{array}{c}\text { Through } \\
\text { Retail } \\
\text { Outlets }\end{array}$ & Average ${ }^{a}$ & DTW & Rack & Bulk & Average \\
\hline \multicolumn{13}{|l|}{ Subdistrict IC } \\
\hline $\begin{array}{l}\text { January } 1996 \ldots . . . . . . . . . . . . . \\
\text { December } 1995 \\
\text { January } 1995 \\
\text { Florida }\end{array}$ & $\begin{array}{l}65.4 \\
63.3 \\
66.4\end{array}$ & $\begin{array}{l}65.1 \\
63.0 \\
65.7\end{array}$ & $\begin{array}{l}60.0 \\
58.5 \\
59.1\end{array}$ & $\begin{array}{l}53.8 \\
52.1 \\
51.7\end{array}$ & $\begin{array}{l}53.9 \\
52.1 \\
50.1\end{array}$ & $\begin{array}{l}55.6 \\
54.0 \\
54.0\end{array}$ & $\begin{array}{l}75.1 \\
73.3 \\
76.8\end{array}$ & $\begin{array}{l}74.6 \\
72.8 \\
75.9\end{array}$ & $\begin{array}{l}66.1 \\
64.3 \\
65.8\end{array}$ & $\begin{array}{l}58.3 \\
56.7 \\
56.6\end{array}$ & $\vec{w}$ & $\begin{array}{l}61.5 \\
59.8 \\
60.4\end{array}$ \\
\hline $\begin{array}{l}\text { January } 1996 \ldots . . . . . . . . . . . . . \\
\text { December } 1995 . . . . . . . . . . \\
\text { January } 1995 \ldots . . . . . . . . . . . . . .\end{array}$ & $\begin{array}{l}67.1 \\
65.0 \\
68.2\end{array}$ & $\begin{array}{l}66.6 \\
64.4 \\
67.4\end{array}$ & $\begin{array}{l}60.0 \\
57.9 \\
60.8\end{array}$ & $\begin{array}{l}54.1 \\
52.0 \\
51.9\end{array}$ & $\begin{array}{l}W \\
W \\
W\end{array}$ & $\begin{array}{l}56.1 \\
54.0 \\
54.9\end{array}$ & $\begin{array}{l}77.0 \\
75.0 \\
79.4\end{array}$ & $\begin{array}{l}76.1 \\
74.4 \\
77.9\end{array}$ & $\begin{array}{l}66.7 \\
64.4 \\
68.6\end{array}$ & $\begin{array}{l}58.4 \\
56.3 \\
56.4\end{array}$ & $\begin{array}{l}- \\
-\end{array}$ & $\begin{array}{l}62.4 \\
60.3 \\
62.3\end{array}$ \\
\hline $\begin{array}{l}\text { January } 1996 \text {................. } \\
\text { December } 1995 \text {............ } \\
\text { January } 1995 \text {............... } \\
\text { North Carolina }\end{array}$ & $\begin{array}{l}63.2 \\
60.9 \\
64.3\end{array}$ & $\begin{array}{l}63.4 \\
61.0 \\
64.0\end{array}$ & $\begin{array}{l}59.6 \\
57.9 \\
56.8\end{array}$ & $\begin{array}{l}53.6 \\
51.6 \\
51.2\end{array}$ & $\begin{array}{c}54.0 \\
W \\
51.2\end{array}$ & $\begin{array}{l}55.6 \\
53.7 \\
53.1\end{array}$ & $\begin{array}{l}73.1 \\
71.5 \\
74.5\end{array}$ & $\begin{array}{l}72.7 \\
71.1 \\
74.1\end{array}$ & $\begin{array}{l}64.6 \\
62.3 \\
61.5\end{array}$ & $\begin{array}{l}58.8 \\
57.0 \\
56.9\end{array}$ & $\bar{w}$ & $\begin{array}{l}61.4 \\
59.5 \\
59.0\end{array}$ \\
\hline $\begin{array}{l}\text { January } 1996 \ldots . . . . . . . . . . . \\
\text { December } 1995 \text {............ } \\
\text { January } 1995 \text {................ } \\
\text { South Carolina }\end{array}$ & $\begin{array}{l}63.7 \\
61.4 \\
64.0\end{array}$ & $\begin{array}{l}63.1 \\
60.9 \\
62.8\end{array}$ & $\begin{array}{l}59.4 \\
58.2 \\
58.2\end{array}$ & $\begin{array}{l}53.8 \\
52.5 \\
52.0\end{array}$ & $\begin{array}{c}53.8 \\
W \\
W\end{array}$ & $\begin{array}{l}55.2 \\
53.8 \\
53.6\end{array}$ & $\begin{array}{l}73.3 \\
71.1 \\
74.0\end{array}$ & $\begin{array}{l}72.8 \\
70.7 \\
73.2\end{array}$ & $\begin{array}{l}65.8 \\
64.9 \\
65.1\end{array}$ & $\begin{array}{l}58.5 \\
57.0 \\
56.8\end{array}$ & $\bar{w}$ & $\begin{array}{l}60.5 \\
59.2 \\
59.4\end{array}$ \\
\hline $\begin{array}{l}\text { January } 1996 \ldots . . . . . . . . . . . . \\
\text { December } 1995 \text {............ } \\
\text { January } 1995 \text {................ } \\
\text { Virginia }\end{array}$ & $\begin{array}{l}63.3 \\
60.8 \\
63.3\end{array}$ & $\begin{array}{l}63.2 \\
60.8 \\
63.0\end{array}$ & $\begin{array}{l}60.3 \\
58.9 \\
59.4\end{array}$ & $\begin{array}{l}54.2 \\
52.7 \\
52.1\end{array}$ & $\begin{array}{l}W \\
W \\
W\end{array}$ & $\begin{array}{l}55.8 \\
54.3 \\
53.7\end{array}$ & $\begin{array}{l}73.5 \\
71.4 \\
73.7\end{array}$ & $\begin{array}{l}73.1 \\
71.1 \\
73.2\end{array}$ & $\begin{array}{l}66.1 \\
64.6 \\
65.3\end{array}$ & $\begin{array}{l}59.0 \\
57.2 \\
56.8\end{array}$ & $\begin{array}{l}- \\
-\end{array}$ & $\begin{array}{l}61.2 \\
59.5 \\
59.2\end{array}$ \\
\hline $\begin{array}{l}\text { January } 1996 . . . . . . . . . . . . . . . \\
\text { December } 1995 \\
\text { January } 1995 \text {.................... } \\
\text { West Virginia }\end{array}$ & $\begin{array}{l}67.8 \\
66.7 \\
69.3\end{array}$ & $\begin{array}{l}67.6 \\
66.6 \\
69.2\end{array}$ & $\begin{array}{l}60.7 \\
60.3 \\
60.8\end{array}$ & $\begin{array}{l}52.1 \\
51.1 \\
51.5\end{array}$ & $\begin{array}{l}W \\
W \\
W\end{array}$ & $\begin{array}{l}54.2 \\
53.3 \\
53.7\end{array}$ & $\begin{array}{l}77.4 \\
76.4 \\
78.7\end{array}$ & $\begin{array}{l}77.0 \\
76.1 \\
78.6\end{array}$ & $\begin{array}{l}66.6 \\
66.3 \\
66.9\end{array}$ & $\begin{array}{l}55.4 \\
54.7 \\
55.6\end{array}$ & $\bar{z}$ & $\begin{array}{l}59.1 \\
58.4 \\
59.6\end{array}$ \\
\hline $\begin{array}{l}\text { January } 1996 \text {................ } \\
\text { December } 1995 \text {............ } \\
\text { January } 1995 \text {................ }\end{array}$ & $\begin{array}{l}71.0 \\
70.9 \\
73.7\end{array}$ & $\begin{array}{l}70.5 \\
70.4 \\
72.7\end{array}$ & $\begin{array}{l}63.3 \\
63.1 \\
62.2\end{array}$ & $\begin{array}{l}55.7 \\
56.1 \\
52.2\end{array}$ & $\begin{array}{l}W \\
W \\
W\end{array}$ & $\begin{array}{l}60.0 \\
60.1 \\
57.0\end{array}$ & $\begin{array}{l}79.6 \\
79.5 \\
83.2\end{array}$ & $\begin{array}{l}79.1 \\
78.9 \\
82.4\end{array}$ & $\begin{array}{l}68.3 \\
68.1 \\
67.9\end{array}$ & $\begin{array}{l}61.6 \\
61.7 \\
56.6\end{array}$ & $\overline{-}$ & $\begin{array}{l}66.3 \\
66.2 \\
63.2\end{array}$ \\
\hline \multicolumn{13}{|l|}{ PAD District II } \\
\hline $\begin{array}{l}\text { January } 1996 \ldots . . . . . . . . . . . . . . \\
\text { December } 1995 \ldots . . . . . . . \\
\text { January } 1995 . . . . . . . . . . . . . . \\
\text { Illinois }\end{array}$ & $\begin{array}{l}68.9 \\
67.6 \\
64.9\end{array}$ & $\begin{array}{l}68.8 \\
67.6 \\
64.8\end{array}$ & $\begin{array}{l}61.1 \\
60.4 \\
57.2\end{array}$ & $\begin{array}{l}55.4 \\
54.7 \\
52.0\end{array}$ & $\begin{array}{l}52.2 \\
49.9 \\
48.0\end{array}$ & $\begin{array}{l}56.5 \\
55.7 \\
52.9\end{array}$ & $\begin{array}{l}76.7 \\
75.6 \\
73.0\end{array}$ & $\begin{array}{l}76.5 \\
75.4 \\
72.7\end{array}$ & $\begin{array}{l}67.2 \\
66.0 \\
64.0\end{array}$ & $\begin{array}{l}60.1 \\
59.4 \\
57.4\end{array}$ & $\overline{-}$ & $\begin{array}{l}63.6 \\
62.7 \\
60.9\end{array}$ \\
\hline $\begin{array}{l}\text { January } 1996 \ldots . . . . . . . . . . . . . \\
\text { December } 1995 \text {............ } \\
\text { January } 1995 \ldots . . . . . . . . . . . . . .\end{array}$ & $\begin{array}{l}71.0 \\
69.8 \\
67.2\end{array}$ & $\begin{array}{l}71.0 \\
69.8 \\
67.2\end{array}$ & $\begin{array}{l}61.6 \\
61.1 \\
58.2\end{array}$ & $\begin{array}{l}54.4 \\
54.3 \\
50.7\end{array}$ & $\begin{array}{l}51.1 \\
49.4 \\
47.4\end{array}$ & $\begin{array}{l}54.9 \\
54.0 \\
51.1\end{array}$ & $\begin{array}{l}76.1 \\
75.4 \\
72.8\end{array}$ & $\begin{array}{l}76.1 \\
75.5 \\
72.8\end{array}$ & $\begin{array}{l}69.9 \\
69.6 \\
65.1\end{array}$ & $\begin{array}{l}60.3 \\
59.9 \\
56.9\end{array}$ & $\overline{-}$ & $\begin{array}{l}65.0 \\
64.4 \\
61.8\end{array}$ \\
\hline $\begin{array}{l}\text { January } 1996 \ldots . . . . . . . . . . . . . \\
\text { December } 1995 \text {............ } \\
\text { January } 1995 \text {............... } \\
\text { lowa }\end{array}$ & $\begin{array}{l}67.2 \\
67.8 \\
65.4\end{array}$ & $\begin{array}{l}67.3 \\
67.9 \\
65.4\end{array}$ & $\begin{array}{l}60.1 \\
60.5 \\
56.8\end{array}$ & $\begin{array}{l}53.9 \\
54.4 \\
51.4\end{array}$ & $\begin{array}{l}51.6 \\
49.3 \\
47.5\end{array}$ & $\begin{array}{l}56.0 \\
56.5 \\
53.1\end{array}$ & $\begin{array}{l}75.2 \\
76.1 \\
72.8\end{array}$ & $\begin{array}{l}75.3 \\
76.2 \\
72.9\end{array}$ & $\begin{array}{l}67.2 \\
66.3 \\
63.8\end{array}$ & $\begin{array}{l}58.9 \\
59.5 \\
56.4\end{array}$ & $\begin{array}{l}- \\
-\end{array}$ & $\begin{array}{l}63.9 \\
63.6 \\
60.6\end{array}$ \\
\hline $\begin{array}{l}\text { January } 1996 \ldots . . . . . . . . . . . . . \\
\text { December } 1995 \text {........... } \\
\text { January } 1995 \text {................ } \\
\text { Kansas }\end{array}$ & $\begin{array}{l}70.4 \\
68.5 \\
64.6\end{array}$ & $\begin{array}{l}70.5 \\
68.7 \\
64.9\end{array}$ & $\begin{array}{l}64.1 \\
62.5 \\
59.7\end{array}$ & $\begin{array}{l}57.1 \\
55.7 \\
53.3\end{array}$ & $\begin{array}{c}W \\
51.8 \\
W\end{array}$ & $\begin{array}{l}57.9 \\
56.3 \\
54.0\end{array}$ & $\begin{array}{l}74.3 \\
72.2 \\
70.5\end{array}$ & $\begin{array}{l}74.0 \\
72.4 \\
70.6\end{array}$ & $\begin{array}{l}67.3 \\
65.8 \\
65.2\end{array}$ & $\begin{array}{l}61.8 \\
60.1 \\
59.6\end{array}$ & $\begin{array}{l}- \\
-\end{array}$ & $\begin{array}{l}65.1 \\
63.7 \\
64.1\end{array}$ \\
\hline $\begin{array}{l}\text { January } 1996 \ldots . . . . . . . . . . . . . \\
\text { December } 1995 \text {........... } \\
\text { January } 1995 \text {................ } \\
\text { Kentucky }\end{array}$ & $\begin{array}{l}67.0 \\
64.5 \\
62.4\end{array}$ & $\begin{array}{l}66.8 \\
64.6 \\
62.4\end{array}$ & $\begin{array}{l}58.6 \\
56.6 \\
55.6\end{array}$ & $\begin{array}{l}55.5 \\
54.1 \\
51.4\end{array}$ & $\begin{array}{l}52.6 \\
52.5 \\
48.5\end{array}$ & $\begin{array}{l}55.3 \\
54.1 \\
51.5\end{array}$ & $\begin{array}{l}73.4 \\
70.5 \\
68.9\end{array}$ & $\begin{array}{l}73.4 \\
70.5 \\
68.9\end{array}$ & $\begin{array}{l}63.6 \\
60.5 \\
59.5\end{array}$ & $\begin{array}{l}60.4 \\
59.0 \\
56.8\end{array}$ & $\begin{array}{l}- \\
-\end{array}$ & $\begin{array}{l}62.1 \\
59.8 \\
58.3\end{array}$ \\
\hline $\begin{array}{l}\text { January } 1996 \text {................ } \\
\text { December } 1995 \text {............ } \\
\text { January } 1995 \text {................ } \\
\text { Michigan }\end{array}$ & $\begin{array}{l}68.9 \\
67.0 \\
66.8\end{array}$ & $\begin{array}{l}68.1 \\
66.4 \\
65.5\end{array}$ & $\begin{array}{l}59.7 \\
58.9 \\
57.5\end{array}$ & $\begin{array}{l}54.2 \\
54.5 \\
52.0\end{array}$ & $\begin{array}{l}w \\
\bar{w}\end{array}$ & $\begin{array}{l}55.9 \\
56.0 \\
53.9\end{array}$ & $\begin{array}{l}79.3 \\
77.5 \\
76.2\end{array}$ & $\begin{array}{l}78.2 \\
76.5 \\
74.2\end{array}$ & $\begin{array}{l}65.7 \\
64.8 \\
63.9\end{array}$ & $\begin{array}{l}59.2 \\
60.2 \\
57.3\end{array}$ & $\begin{array}{l}- \\
-\end{array}$ & $\begin{array}{l}62.0 \\
62.4 \\
60.6\end{array}$ \\
\hline $\begin{array}{l}\text { January } 1996 \text {................ } \\
\text { December } 1995 \\
\text { January } 1995 \text {..................... } \\
\text { Minnesota }\end{array}$ & $\begin{array}{l}68.6 \\
68.0 \\
63.2\end{array}$ & $\begin{array}{l}68.5 \\
67.8 \\
63.2\end{array}$ & $\begin{array}{l}60.8 \\
59.9 \\
55.7\end{array}$ & $\begin{array}{l}55.7 \\
56.4 \\
51.6\end{array}$ & $\begin{array}{l}w \\
\bar{w}\end{array}$ & $\begin{array}{l}57.9 \\
57.9 \\
53.5\end{array}$ & $\begin{array}{l}76.9 \\
76.2 \\
71.3\end{array}$ & $\begin{array}{l}76.9 \\
76.0 \\
71.3\end{array}$ & $\begin{array}{l}66.5 \\
64.9 \\
62.0\end{array}$ & $\begin{array}{l}60.6 \\
61.7 \\
56.7\end{array}$ & $\begin{array}{l}- \\
-\end{array}$ & $\begin{array}{l}65.0 \\
64.1 \\
60.5\end{array}$ \\
\hline $\begin{array}{l}\text { January } 1996 \text {................ } \\
\text { December } 1995 \text {............ } \\
\text { January } 1995 \text {................ } \\
\text { Missouri }\end{array}$ & $\begin{array}{l}72.6 \\
72.6 \\
71.0\end{array}$ & $\begin{array}{l}72.1 \\
72.1 \\
70.7\end{array}$ & $\begin{array}{l}65.5 \\
64.1 \\
59.5\end{array}$ & $\begin{array}{l}\mathbf{5 7 . 7} \\
\mathbf{5 6 . 6} \\
\mathbf{5 5 . 0}\end{array}$ & $\begin{array}{l}W \\
W \\
W\end{array}$ & $\begin{array}{l}58.9 \\
57.7 \\
55.6\end{array}$ & $\begin{array}{l}80.8 \\
78.7 \\
76.4\end{array}$ & $\begin{array}{l}79.7 \\
77.7 \\
75.9\end{array}$ & $\begin{array}{r}\text { NA } \\
70.0 \\
66.9\end{array}$ & $\begin{array}{l}65.6 \\
63.8 \\
63.4\end{array}$ & $\overline{-}$ & $\begin{array}{l}67.8 \\
66.8 \\
65.3\end{array}$ \\
\hline $\begin{array}{l}\text { January } 1996 . . . . . . . . . . . . . . \\
\text { December } 1995 \text {............ } \\
\text { January } 1995 \text {............... }\end{array}$ & $\begin{array}{l}67.5 \\
63.3 \\
61.8\end{array}$ & $\begin{array}{l}67.5 \\
63.6 \\
62.1\end{array}$ & $\begin{array}{l}60.0 \\
58.5 \\
55.7\end{array}$ & $\begin{array}{l}55.0 \\
53.6 \\
51.5\end{array}$ & $\begin{array}{c}W \\
W \\
46.2\end{array}$ & $\begin{array}{l}56.4 \\
54.9 \\
52.5\end{array}$ & $\begin{array}{l}76.4 \\
72.0 \\
70.3\end{array}$ & $\begin{array}{l}76.3 \\
72.3 \\
70.4\end{array}$ & $\begin{array}{l}66.8 \\
64.2 \\
61.4\end{array}$ & $\begin{array}{l}59.3 \\
58.4 \\
56.3\end{array}$ & $\begin{array}{l}- \\
-\end{array}$ & $\begin{array}{l}64.2 \\
62.3 \\
59.5\end{array}$ \\
\hline
\end{tabular}

See footnotes at end of table. 
Table 32. Conventional Motor Gasoline Prices by Grade, Sales Type, PAD District, and State

(Cents per Gallon Excluding Taxes) - Continued

\begin{tabular}{|c|c|c|c|c|c|c|c|c|c|c|c|c|}
\hline \multirow{3}{*}{$\begin{array}{c}\text { Geographic Area } \\
\text { Month }\end{array}$} & \multicolumn{6}{|c|}{ Premium } & \multicolumn{6}{|c|}{ All Grades } \\
\hline & \multicolumn{2}{|c|}{ Sales to End Users } & \multicolumn{4}{|c|}{ Sales for Resale } & \multicolumn{2}{|c|}{ Sales to End Users } & \multicolumn{4}{|c|}{ Sales for Resale } \\
\hline & $\begin{array}{c}\text { Through } \\
\text { Retail } \\
\text { Outlets }\end{array}$ & Average $^{a}$ & DTW & Rack & Bulk & Average & $\begin{array}{c}\text { Through } \\
\text { Retail } \\
\text { Outlets }\end{array}$ & Average $\mathrm{e}^{\mathrm{a}}$ & DTW & Rack & Bulk & Average \\
\hline \multicolumn{13}{|l|}{ Subdistrict IC } \\
\hline $\begin{array}{l}\text { January } 1996 \ldots . . . . . . . . . . . . \\
\text { December } 1995 \\
\text { January } 1995 \text {.................... } \\
\text { Florida }\end{array}$ & $\begin{array}{l}84.1 \\
82.3 \\
86.0\end{array}$ & $\begin{array}{l}83.3 \\
81.7 \\
85.0\end{array}$ & $\begin{array}{l}73.4 \\
71.5 \\
74.0\end{array}$ & $\begin{array}{l}64.0 \\
62.3 \\
62.4\end{array}$ & $\begin{array}{l}60.7 \\
59.6 \\
58.7\end{array}$ & $\begin{array}{l}67.6 \\
65.8 \\
67.0\end{array}$ & $\begin{array}{l}71.1 \\
69.2 \\
72.3\end{array}$ & $\begin{array}{l}70.6 \\
68.8 \\
71.4\end{array}$ & $\begin{array}{l}64.7 \\
63.1 \\
64.4\end{array}$ & $\begin{array}{l}56.6 \\
55.0 \\
54.7\end{array}$ & $\begin{array}{l}55.4 \\
53.2 \\
51.2\end{array}$ & $\begin{array}{l}59.3 \\
57.7 \\
58.0\end{array}$ \\
\hline $\begin{array}{l}\text { January } 1996 \ldots . . . . . . . . . . . . \\
\text { December } 1995 \text {............ } \\
\text { January } 1995 \text {............... } \\
\text { Georgla }\end{array}$ & $\begin{array}{l}84.9 \\
83.1 \\
87.7\end{array}$ & $\begin{array}{l}84.1 \\
82.5 \\
86.5\end{array}$ & $\begin{array}{l}74.3 \\
72.0 \\
76.9\end{array}$ & $\begin{array}{l}64.5 \\
62.3 \\
62.7\end{array}$ & $\frac{61.6}{w}$ & $\begin{array}{l}69.5 \\
67.3 \\
70.4\end{array}$ & $\begin{array}{l}72.8 \\
70.8 \\
74.3\end{array}$ & $\begin{array}{l}72.1 \\
70.0 \\
73.2\end{array}$ & $\begin{array}{l}66.2 \\
64.0 \\
67.8\end{array}$ & $\begin{array}{l}56.9 \\
54.9 \\
54.9\end{array}$ & $\begin{array}{c}57.2 \\
W \\
49.2\end{array}$ & $\begin{array}{l}60.6 \\
58.6 \\
60.2\end{array}$ \\
\hline $\begin{array}{l}\text { January } 1996 . . . . . . . . . . . . . \\
\text { December } 1995 \text {............ } \\
\text { January } 1995 \text {............... } \\
\text { North Carolina }\end{array}$ & $\begin{array}{l}82.9 \\
81.1 \\
84.4\end{array}$ & $\begin{array}{l}82.3 \\
80.7 \\
83.6\end{array}$ & $\begin{array}{l}71.7 \\
69.8 \\
69.6\end{array}$ & $\begin{array}{l}63.9 \\
62.1 \\
62.3\end{array}$ & $\begin{array}{l}W \\
W \\
W\end{array}$ & $\begin{array}{l}66.6 \\
65.0 \\
65.1\end{array}$ & $\begin{array}{l}68.9 \\
67.1 \\
70.2\end{array}$ & $\begin{array}{l}68.8 \\
67.0 \\
69.8\end{array}$ & $\begin{array}{l}63.4 \\
61.6 \\
60.6\end{array}$ & $\begin{array}{l}56.5 \\
54.6 \\
54.4\end{array}$ & $\begin{array}{c}55.4 \\
W \\
53.5\end{array}$ & $\begin{array}{l}58.9 \\
57.1 \\
56.7\end{array}$ \\
\hline $\begin{array}{l}\text { January } 1996 . . . . . . . . . . . . . \\
\text { December } 1995 \text {............ } \\
\text { January } 1995 \text {................ } \\
\text { South Carolina }\end{array}$ & $\begin{array}{l}83.1 \\
80.7 \\
83.7\end{array}$ & $\begin{array}{l}82.1 \\
79.8 \\
82.2\end{array}$ & $\begin{array}{l}71.9 \\
70.8 \\
71.2\end{array}$ & $\begin{array}{l}64.2 \\
62.7 \\
62.5\end{array}$ & $\begin{array}{l}W \\
W \\
W\end{array}$ & $\begin{array}{l}66.1 \\
64.5 \\
64.9\end{array}$ & $\begin{array}{l}69.7 \\
67.6 \\
69.8\end{array}$ & $\begin{array}{l}68.9 \\
66.9 \\
68.4\end{array}$ & $\begin{array}{l}63.2 \\
62.1 \\
62.3\end{array}$ & $\begin{array}{l}56.8 \\
55.5 \\
55.0\end{array}$ & $\begin{array}{l}55.3 \\
W \\
W\end{array}$ & $\begin{array}{l}58.4 \\
57.1 \\
56.9\end{array}$ \\
\hline $\begin{array}{l}\text { January } 1996 \text {............... } \\
\text { December } 1995 \text {............ } \\
\text { January } 1995 \text {............... } \\
\text { Virginia }\end{array}$ & $\begin{array}{l}83.7 \\
81.3 \\
84.7\end{array}$ & $\begin{array}{l}83.1 \\
80.7 \\
84.1\end{array}$ & $\begin{array}{l}73.5 \\
72.2 \\
72.9\end{array}$ & $\begin{array}{l}64.7 \\
63.2 \\
63.3\end{array}$ & $\begin{array}{l}- \\
-\end{array}$ & $\begin{array}{l}67.1 \\
65.5 \\
65.5\end{array}$ & $\begin{array}{l}69.0 \\
66.8 \\
69.1\end{array}$ & $\begin{array}{l}68.8 \\
66.6 \\
68.7\end{array}$ & $\begin{array}{l}63.9 \\
62.7 \\
63.1\end{array}$ & $\begin{array}{l}57.0 \\
55.6 \\
55.1\end{array}$ & $\begin{array}{l}W \\
W \\
W\end{array}$ & $\begin{array}{l}58.8 \\
57.4 \\
56.9\end{array}$ \\
\hline $\begin{array}{l}\text { January } 1996 \ldots . . . . . . . . . . . . \\
\text { December } 1995 \ldots . . . . . . . . \\
\text { January } 1995 \text {................. } \\
\text { West Virginia }\end{array}$ & $\begin{array}{l}84.2 \\
85.2 \\
87.1\end{array}$ & $\begin{array}{l}83.6 \\
84.5 \\
86.6\end{array}$ & $\begin{array}{l}74.7 \\
73.5 \\
73.9\end{array}$ & $\begin{array}{l}61.3 \\
60.6 \\
60.6\end{array}$ & $\begin{array}{l}- \\
-\end{array}$ & $\begin{array}{l}65.3 \\
64.2 \\
64.2\end{array}$ & $\begin{array}{l}72.9 \\
72.5 \\
75.0\end{array}$ & $\begin{array}{l}72.7 \\
72.2 \\
74.8\end{array}$ & $\begin{array}{l}65.3 \\
64.6 \\
65.1\end{array}$ & $\begin{array}{l}54.6 \\
53.7 \\
54.2\end{array}$ & $\begin{array}{l}W \\
W \\
W\end{array}$ & $\begin{array}{l}57.4 \\
56.5 \\
57.0\end{array}$ \\
\hline $\begin{array}{l}\text { January } 1996 \text {................ } \\
\text { December } 1995 \text {........... } \\
\text { January } 1995 \text {............... }\end{array}$ & $\begin{array}{l}88.0 \\
87.5 \\
90.8\end{array}$ & $\begin{array}{l}86.7 \\
86.5 \\
88.7\end{array}$ & $\begin{array}{l}75.4 \\
75.3 \\
74.7\end{array}$ & $\begin{array}{l}66.3 \\
66.9 \\
62.9\end{array}$ & $\begin{array}{l}- \\
-\end{array}$ & $\begin{array}{l}71.9 \\
72.1 \\
69.5\end{array}$ & $\begin{array}{l}75.6 \\
75.5 \\
78.5\end{array}$ & $\begin{array}{l}75.0 \\
74.9 \\
77.4\end{array}$ & $\begin{array}{l}66.7 \\
66.5 \\
66.0\end{array}$ & $\begin{array}{l}58.7 \\
59.0 \\
55.1\end{array}$ & $\begin{array}{l}W \\
W \\
W\end{array}$ & $\begin{array}{l}63.4 \\
63.4 \\
60.6\end{array}$ \\
\hline \multicolumn{13}{|l|}{ PAD District II } \\
\hline $\begin{array}{l}\text { January } 1996 \ldots . . . . \ldots \ldots . . . . . \\
\text { December } 1995 . . . . . . . . . \\
\text { January } 1995 \text {............... } \\
\text { Illinois }\end{array}$ & $\begin{array}{l}84.0 \\
82.9 \\
80.0\end{array}$ & $\begin{array}{l}83.6 \\
82.5 \\
79.5\end{array}$ & $\begin{array}{l}72.4 \\
71.6 \\
68.7\end{array}$ & $\begin{array}{l}64.0 \\
63.4 \\
60.9\end{array}$ & $\begin{array}{l}55.7 \\
55.5 \\
52.4\end{array}$ & $\begin{array}{l}66.7 \\
66.0 \\
63.6\end{array}$ & $\begin{array}{l}72.1 \\
71.0 \\
68.3\end{array}$ & $\begin{array}{l}71.9 \\
70.8 \\
68.1\end{array}$ & $\begin{array}{l}63.8 \\
63.1 \\
60.3\end{array}$ & $\begin{array}{l}\mathbf{5 7 . 0} \\
\mathbf{5 6 . 4} \\
\mathbf{5 3 . 7}\end{array}$ & $\begin{array}{l}52.3 \\
50.1 \\
48.2\end{array}$ & $\begin{array}{l}58.6 \\
57.8 \\
55.1\end{array}$ \\
\hline $\begin{array}{l}\text { January } 1996 \ldots . . . . . . . . . . . . \\
\text { December } 1995 \text {........... } \\
\text { January } 1995 \text {............... } \\
\text { Indiana }\end{array}$ & $\begin{array}{l}86.1 \\
84.8 \\
82.9\end{array}$ & $\begin{array}{l}86.0 \\
84.6 \\
82.5\end{array}$ & $\begin{array}{l}73.5 \\
72.0 \\
68.2\end{array}$ & $\begin{array}{l}62.9 \\
63.0 \\
60.1\end{array}$ & $\begin{array}{c}W \\
54.6 \\
W\end{array}$ & $\begin{array}{l}65.2 \\
65.1 \\
62.2\end{array}$ & $\begin{array}{l}73.9 \\
72.9 \\
70.4\end{array}$ & $\begin{array}{l}74.0 \\
72.9 \\
70.3\end{array}$ & $\begin{array}{l}64.9 \\
64.3 \\
61.2\end{array}$ & $\begin{array}{l}55.9 \\
55.9 \\
52.1\end{array}$ & $\begin{array}{l}51.1 \\
49.5 \\
47.4\end{array}$ & $\begin{array}{l}56.9 \\
56.0 \\
53.0\end{array}$ \\
\hline $\begin{array}{l}\text { January } 1996 \text {................ } \\
\text { December } 1995 \text {............ } \\
\text { January } 1995 \text {............... } \\
\text { Jowa }\end{array}$ & $\begin{array}{l}81.9 \\
82.9 \\
78.8\end{array}$ & $\begin{array}{l}81.8 \\
82.7 \\
78.7\end{array}$ & $\begin{array}{l}71.4 \\
71.7 \\
68.7\end{array}$ & $\begin{array}{l}63.9 \\
64.3 \\
60.2\end{array}$ & $\bar{w}$ & $\begin{array}{l}67.5 \\
67.8 \\
64.0\end{array}$ & $\begin{array}{l}71.1 \\
71.8 \\
69.1\end{array}$ & $\begin{array}{l}71.1 \\
71.8 \\
69.1\end{array}$ & $\begin{array}{l}63.3 \\
63.6 \\
60.4\end{array}$ & $\begin{array}{l}55.9 \\
56.5 \\
53.4\end{array}$ & $\begin{array}{l}51.6 \\
49.3 \\
47.6\end{array}$ & $\begin{array}{l}58.8 \\
59.3 \\
55.9\end{array}$ \\
\hline $\begin{array}{l}\text { January } 1996 \text {............... } \\
\text { December } 1995 \text {........... } \\
\text { January } 1995 \text {............... } \\
\text { Kansas }\end{array}$ & $\begin{array}{l}80.7 \\
79.3 \\
73.9\end{array}$ & $\begin{array}{l}80.3 \\
78.9 \\
73.7\end{array}$ & $\begin{array}{c}72.0 \\
\text { NA } \\
67.4\end{array}$ & $\begin{array}{l}64.4 \\
62.7 \\
61.2\end{array}$ & $\begin{array}{l}- \\
-\end{array}$ & $\begin{array}{l}65.6 \\
64.0 \\
62.4\end{array}$ & $\begin{array}{l}71.4 \\
69.5 \\
65.7\end{array}$ & $\begin{array}{l}71.4 \\
69.7 \\
65.9\end{array}$ & $\begin{array}{l}65.6 \\
64.2 \\
61.8\end{array}$ & $\begin{array}{l}57.9 \\
56.3 \\
54.0\end{array}$ & $\begin{array}{c}W \\
51.8 \\
W\end{array}$ & $\begin{array}{l}58.9 \\
57.4 \\
55.1\end{array}$ \\
\hline $\begin{array}{l}\text { January } 1996 \text {................ } \\
\text { December } 1995 \text {............ } \\
\text { January } 1995 \text {............... } \\
\text { Kentucky }\end{array}$ & $\begin{array}{l}80.2 \\
77.3 \\
75.5\end{array}$ & $\begin{array}{l}79.5 \\
77.0 \\
74.8\end{array}$ & $\begin{array}{l}66.5 \\
64.9 \\
64.2\end{array}$ & $\begin{array}{l}62.3 \\
61.1 \\
58.6\end{array}$ & $\begin{array}{c}W \\
54.1 \\
W\end{array}$ & $\begin{array}{l}63.4 \\
60.8 \\
59.7\end{array}$ & $\begin{array}{l}68.7 \\
66.2 \\
64.1\end{array}$ & $\begin{array}{l}68.4 \\
66.2 \\
64.1\end{array}$ & $\begin{array}{l}60.0 \\
58.1 \\
57.1\end{array}$ & $\begin{array}{l}56.3 \\
54.9 \\
52.2\end{array}$ & $\begin{array}{l}52.6 \\
52.6 \\
48.5\end{array}$ & $\begin{array}{l}56.2 \\
54.9 \\
52.3\end{array}$ \\
\hline 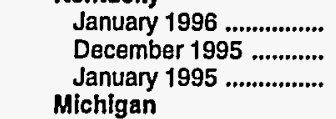 & $\begin{array}{l}84.0 \\
82.5 \\
83.0\end{array}$ & $\begin{array}{l}82.7 \\
81.4 \\
81.2\end{array}$ & $\begin{array}{l}70.9 \\
70.8 \\
69.6\end{array}$ & $\begin{array}{l}63.7 \\
64.0 \\
62.0\end{array}$ & $\vec{w}$ & $\begin{array}{l}65.9 \\
65.8 \\
64.5\end{array}$ & $\begin{array}{l}73.2 \\
71.5 \\
71.5\end{array}$ & $\begin{array}{l}72.3 \\
70.8 \\
70.0\end{array}$ & $\begin{array}{l}62.6 \\
61.9 \\
60.9\end{array}$ & $\begin{array}{l}56.7 \\
57.1 \\
54.8\end{array}$ & $\begin{array}{l}W \\
W \\
W\end{array}$ & $\begin{array}{l}58.6 \\
58.7 \\
57.0\end{array}$ \\
\hline $\begin{array}{l}\text { January } 1996 \text {................ } \\
\text { December } 1995 \text {............ } \\
\text { January } 1995 \text {................ } \\
\text { Minnesota }\end{array}$ & $\begin{array}{l}83.7 \\
83.5 \\
77.8\end{array}$ & $\begin{array}{l}83.2 \\
82.9 \\
77.5\end{array}$ & $\begin{array}{l}72.6 \\
71.3 \\
66.7\end{array}$ & $\begin{array}{l}65.2 \\
65.6 \\
60.8\end{array}$ & $\bar{w}$ & $\begin{array}{l}69.1 \\
68.6 \\
63.9\end{array}$ & $\begin{array}{l}71.6 \\
71.1 \\
66.5\end{array}$ & $\begin{array}{l}71.4 \\
70.8 \\
66.4\end{array}$ & $\begin{array}{l}63.7 \\
62.7 \\
58.7\end{array}$ & $\begin{array}{l}57.4 \\
58.1 \\
53.4\end{array}$ & $\frac{w}{w}$ & $\begin{array}{l}60.4 \\
60.4 \\
56.0\end{array}$ \\
\hline $\begin{array}{l}\text { January } 1996 \text {............... } \\
\text { December } 1995 \text {............ } \\
\text { January } 1995 \text {................ } \\
\text { Missouri }\end{array}$ & $\begin{array}{l}84.1 \\
84.7 \\
83.6\end{array}$ & $\begin{array}{l}84.1 \\
84.6 \\
83.6\end{array}$ & $\begin{array}{l}72.4 \\
71.5 \\
69.8\end{array}$ & $\begin{array}{l}65.8 \\
65.0 \\
64.0\end{array}$ & $\begin{array}{l}W \\
W \\
W\end{array}$ & $\begin{array}{l}66.9 \\
65.9 \\
65.2\end{array}$ & $\begin{array}{l}74.1 \\
74.1 \\
72.4\end{array}$ & $\begin{array}{l}73.6 \\
73.5 \\
72.0\end{array}$ & $\begin{array}{l}66.6 \\
65.4 \\
61.3\end{array}$ & $\begin{array}{l}58.7 \\
57.6 \\
56.0\end{array}$ & $\begin{array}{l}W \\
W \\
W\end{array}$ & $\begin{array}{l}60.1 \\
58.8 \\
56.9\end{array}$ \\
\hline $\begin{array}{l}\text { January } 1996 \text {............... } \\
\text { December } 1995 \text {........... } \\
\text { January } 1995 \text {............... }\end{array}$ & $\begin{array}{l}85.3 \\
81.0 \\
78.7\end{array}$ & $\begin{array}{l}84.9 \\
80.8 \\
78.3\end{array}$ & $\begin{array}{l}71.1 \\
68.8 \\
66.5\end{array}$ & $\begin{array}{l}62.7 \\
61.3 \\
59.3\end{array}$ & $\begin{array}{l}- \\
-\end{array}$ & $\begin{array}{l}65.7 \\
63.9 \\
61.8\end{array}$ & $\begin{array}{l}70.7 \\
66.7 \\
65.0\end{array}$ & $\begin{array}{l}70.5 \\
66.8 \\
65.1\end{array}$ & $\begin{array}{l}62.8 \\
61.1 \\
58.3\end{array}$ & $\begin{array}{l}56.3 \\
55.0 \\
52.9\end{array}$ & $\begin{array}{r}W \\
W \\
46.2\end{array}$ & $\begin{array}{l}58.3 \\
56.8 \\
54.4\end{array}$ \\
\hline
\end{tabular}

See footnotes at end of table. 
Table 32. Conventional Motor Gasoline Prices by Grade, Sales Type, PAD District, and State

(Cents per Gallon Excluding Taxes) - Continued

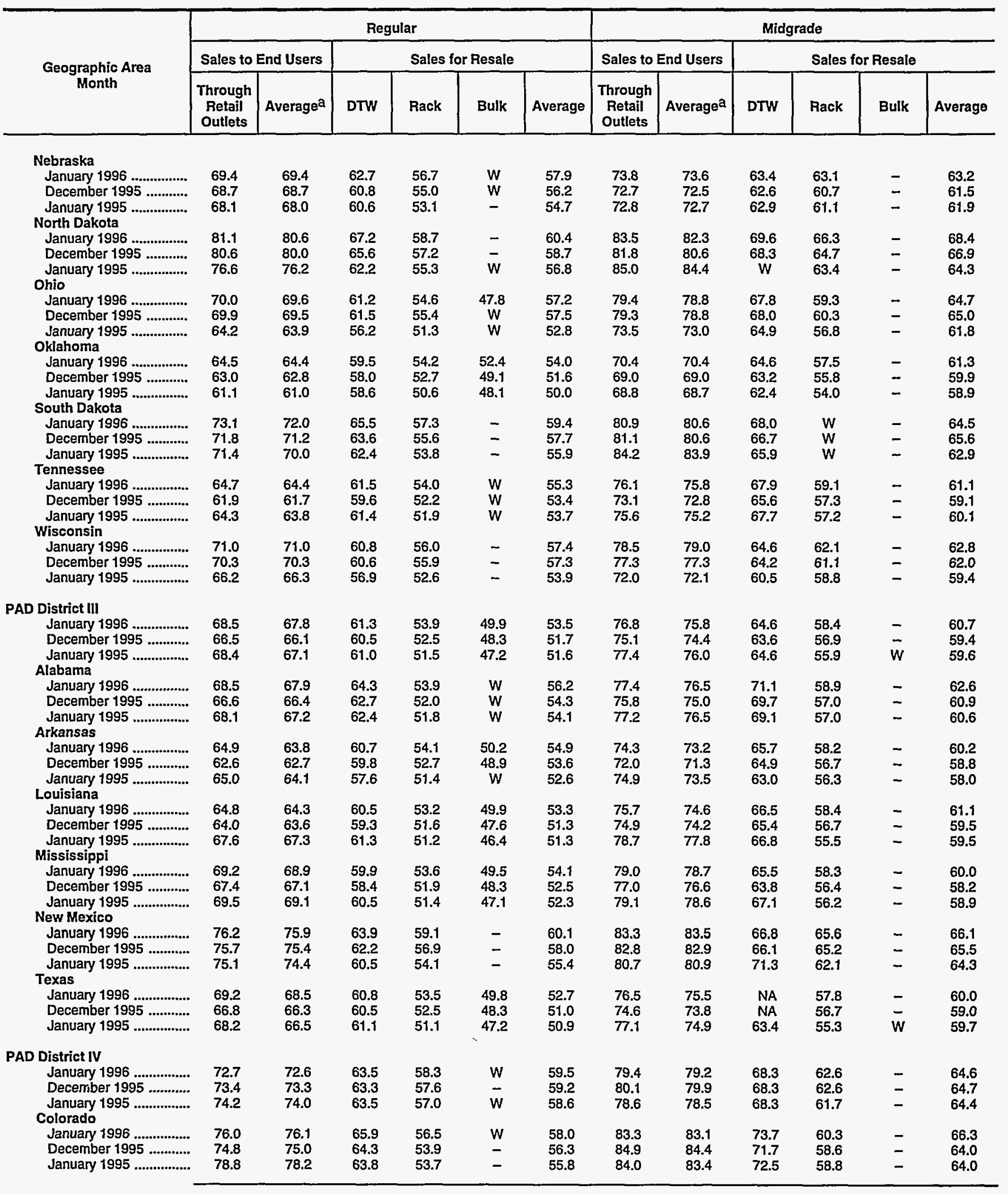

See footnotes at end of table. 
Table 32. Conventional Motor Gasoline Prices by Grade, Sales Type, PAD District, and State

(Cents per Gallon Excluding Taxes) - Continued

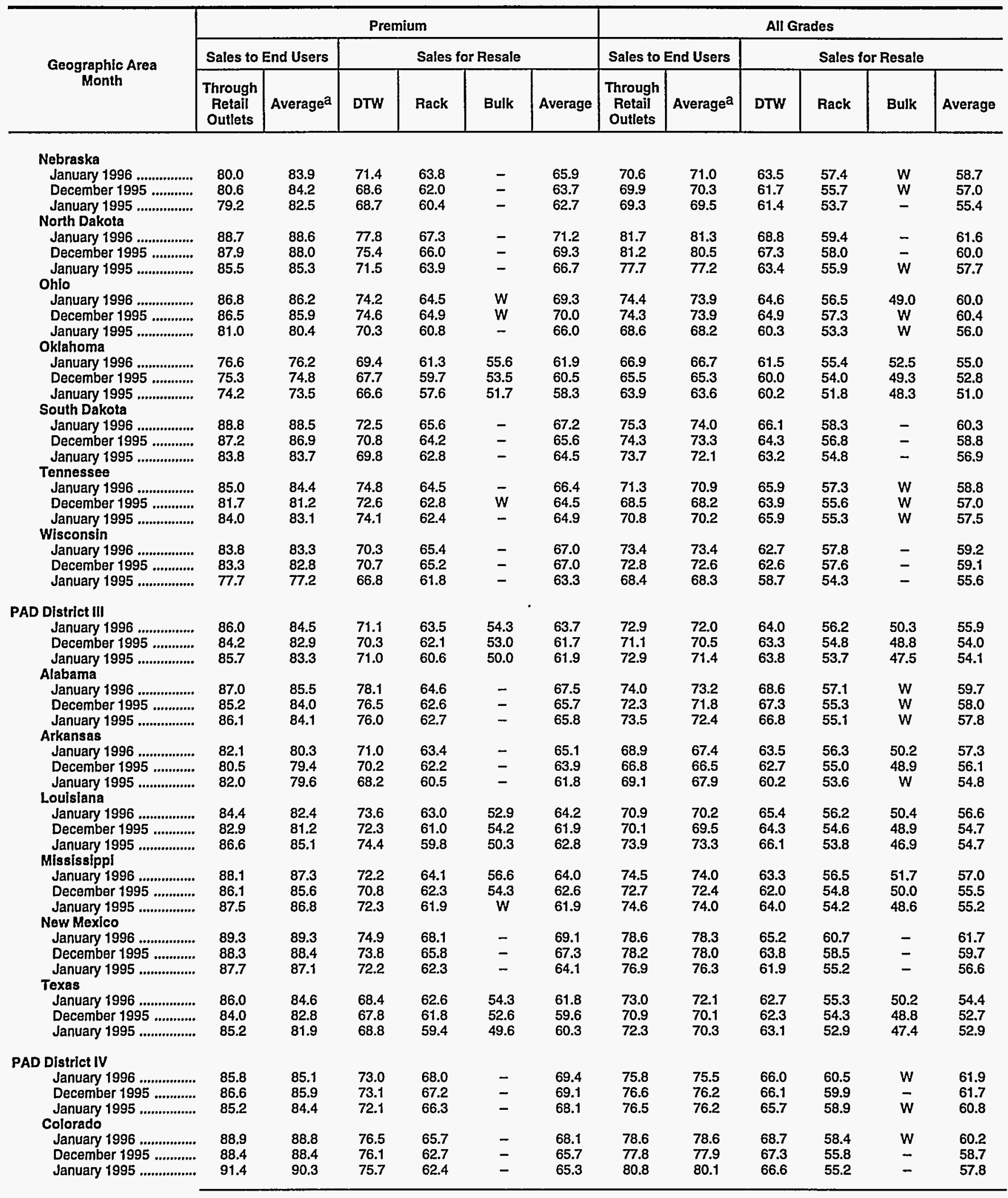

See footnotes at end of table. 
Table 32. Conventional Motor Gasoline Prices by Grade, Sales Type, PAD District, and State

(Cents per Gallon Excluding Taxes) - Continued

\begin{tabular}{|c|c|c|c|c|c|c|c|c|c|c|c|c|}
\hline \multirow{3}{*}{$\begin{array}{c}\text { Geographic Area } \\
\text { Month }\end{array}$} & \multicolumn{6}{|c|}{ Regular } & \multicolumn{6}{|c|}{ Midgrade } \\
\hline & \multicolumn{2}{|c|}{ Sales to End Users } & \multicolumn{4}{|c|}{ Sales for Resale } & \multicolumn{2}{|c|}{ Sales to End Users } & \multicolumn{4}{|c|}{ Sales for Resale } \\
\hline & $\begin{array}{c}\text { Through } \\
\text { Retail } \\
\text { Outlets }\end{array}$ & Average $^{a}$ & DTW & Rack & Bulk & Average & $\begin{array}{c}\text { Through } \\
\text { Retail } \\
\text { Outlets }\end{array}$ & Average $^{a}$ & DTW & Rack & Bulk & Average \\
\hline \multicolumn{13}{|l|}{ Idaho } \\
\hline January 1996 & 69.9 & 70.0 & 61.9 & 55.0 & - & 57.1 & 78.1 & 77.8 & 66.6 & 58.3 & - & 61.4 \\
\hline December 1995 ............... & 72.9 & 72.5 & 62.9 & 56.8 & - & 58.8 & 81.2 & 80.2 & 68.4 & 60.8 & - & 63.7 \\
\hline 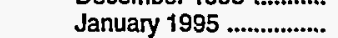 & 75.0 & 74.5 & 65.0 & 59.1 & - & 61.0 & 80.0 & 79.1 & 68.9 & 62.5 & - & 64.6 \\
\hline \multicolumn{13}{|l|}{ Montana } \\
\hline January 1996 & 74.4 & 75.6 & 68.1 & 62.5 & - & 63.6 & 78.5 & 78.6 & 71.3 & 67.3 & - & 67.7 \\
\hline December 1995 ............. & 74.6 & 75.8 & 67.8 & 61.8 & - & 63.0 & 79.4 & 79.5 & 72.7 & 66.8 & - & 67.3 \\
\hline January 1995 .................. & 75.5 & 75.9 & 66.6 & 59.0 & w & 60.5 & NA & 81,0 & $W$ & W & - & 65.9 \\
\hline \multicolumn{13}{|l|}{ Utah } \\
\hline January 1996 ................ & 69.9 & 69.2 & 61.0 & 59.0 & - & 59.7 & 77.8 & 77.5 & 67.0 & 64.1 & - & 65.1 \\
\hline December 1995 ............. & 70.7 & 70.3 & 60.9 & 59.0 & - & 59.7 & 77.9 & 77.7 & 66.4 & 63.9 & - & 64.9 \\
\hline January 1995 .................. & 68.8 & 68.6 & 61.1 & 58.1 & $W$ & 59.1 & 75.8 & 75.7 & 66.3 & 63.0 & - & 64.5 \\
\hline Wyoming & & & & & & & & & & & & \\
\hline January 1996 ................ & 77.1 & 76.6 & 64.8 & 58.0 & - & 59.3 & 83.6 & 83.5 & NA & 60.5 & - & NA \\
\hline December 1995 ............. & 77.8 & 77.2 & 63.8 & 56.0 & - & 57.5 & 83.6 & 83.4 & 65.0 & 63.6 & - & 64.7 \\
\hline January 1995 ................. & 79.2 & 78.0 & 62.3 & 54.4 & $W$ & 55.8 & 84.0 & 84.0 & 61.8 & 65.3 & - & 62.9 \\
\hline \multicolumn{13}{|l|}{ PAD District V } \\
\hline January 1996 ................. & 85.4 & 83.7 & 73.0 & 59.8 & 53.9 & 61.7 & 90.3 & 89.2 & 77.2 & 65.8 & - & 72.2 \\
\hline December 1995 ............. & 86.0 & 84.3 & 73.4 & 60.9 & 53.9 & 62.4 & 91.1 & 90.0 & 78.9 & 67.3 & - & 73.6 \\
\hline January 1995 .................. & 84.1 & 82.5 & 70.5 & 58.2 & 52.9 & 60.1 & 86.1 & 86.5 & 78.0 & 65.1 & W & 73.6 \\
\hline \multicolumn{13}{|l|}{ Alaska } \\
\hline January 1996 & 112.6 & 110.8 & 82.8 & 73.6 & $\mathbf{W}$ & 77.8 & 102.5 & 103.5 & 90.9 & 83.7 & - & 88.1 \\
\hline December 1995 ............. & 111.7 & 110.0 & 86.3 & 69.9 & $W$ & 77.0 & 99.6 & 101.3 & 92.5 & 86.2 & - & 89.5 \\
\hline January 1995 & 120.6 & 119.2 & 88.8 & 71.2 & W & 79.5 & $W$ & 108.6 & 89.8 & $W$ & $\mathbf{W}$ & 91.1 \\
\hline \multicolumn{13}{|c|}{ Arizona } \\
\hline January 1996 ................. & 81.8 & 79.7 & 67.0 & 60.3 & 56.5 & 62.4 & 89.1 & 89.0 & 70.7 & 67.5 & - & 69.3 \\
\hline December 1995 ............. & 82.4 & 80.1 & 65.6 & 60.0 & 56.6 & 61.9 & 89.6 & 89.6 & 70.6 & 67.2 & - & 69.1 \\
\hline January 1995 ................. & 81.4 & 80.0 & 66.4 & 58.4 & $w$ & 60.8 & 87.5 & 87.5 & 72.5 & $w$ & - & 69.7 \\
\hline \multicolumn{13}{|l|}{ California } \\
\hline January 1996 & $w$ & $\mathbf{W}$ & - & 57.7 & 54.6 & 54.8 & $w$ & $W$ & - & 63.4 & - & 63.4 \\
\hline December 1995 ............ & W & W & - & 58.1 & 53.2 & 53.3 & $w$ & W & - & $W$ & - & $w$ \\
\hline January 1995 .................. & - & - & - & NA & 52.9 & 53.4 & - & - & - & NA & - & 64.2 \\
\hline \multicolumn{13}{|l|}{ Hawaii } \\
\hline January 1996 ................. & 103.1 & 95.2 & 93.7 & 83.1 & - & 91.8 & 108.7 & 104.1 & 99.7 & $w$ & - & 99.4 \\
\hline December 1995 ............. & 105.6 & 96.9 & 94.0 & 80.7 & - & 92.1 & 111.3 & 105.3 & 99.9 & $W$ & - & 99.6 \\
\hline January 1995 ................. & 100.0 & 92.9 & 91.4 & 80.5 & - & 89.7 & 106.6 & 99.1 & 97.4 & $W$ & - & 97.1 \\
\hline \multicolumn{13}{|c|}{ Nevada } \\
\hline January $1996 \ldots . . . . . . . . . . . . .$. & 77.5 & 76.3 & 67.5 & 60.1 & 57.3 & 60.5 & 86.5 & 86.7 & 73.6 & 66.5 & - & 69.3 \\
\hline December 1995 ............. & 78.1 & 77.3 & 68.2 & 61.3 & 58.4 & 62.1 & 89.5 & 89.1 & 74.2 & 67.6 & - & 70.1 \\
\hline January 1995 ................. & 75.9 & 74.3 & 65.3 & 59.4 & $w$ & 60.3 & 84.1 & NA & 71.2 & 65.6 & - & 67.7 \\
\hline \multicolumn{13}{|l|}{ Oregon } \\
\hline January 1996 ................. & 79.1 & 78.8 & 67.8 & 58.5 & 52.1 & 58.8 & 85.8 & 83.6 & 69.0 & 66.8 & - & 67.8 \\
\hline December 1995 ............. & 79.9 & 79.5 & 69.0 & 61.1 & 54.7 & 62.3 & 87.2 & 85.4 & 72.8 & 69.7 & - & 70.9 \\
\hline January 1995 .................. & 76.6 & 75.8 & 63.5 & 55.7 & 52.1 & 56.8 & 76.5 & 78.5 & 66.3 & W & - & 64.3 \\
\hline Washington & & & & & & & & & & & & \\
\hline January 1996 ................ & 80.0 & 79.9 & 68.6 & 57.7 & 50.0 & 59.5 & 86.3 & 86.1 & NA & 63.9 & - & 68.7 \\
\hline December 1995 .............. & 82.0 & 81.6 & 70.6 & 59.6 & 53.9 & 62.4 & 87.8 & 87.3 & NA & 65.4 & - & 69.8 \\
\hline January 1995 .................. & 80.3 & 80.5 & 64.9 & 57.0 & $w$ & 59.0 & 83.8 & 83.6 & 67.9 & $w$ & - & 67.2 \\
\hline
\end{tabular}

See footnotes at end of table. 
Table 32. Conventional Motor Gasoline Prices by Grade, Sales Type, PAD District, and State

(Cents per Gallon Excluding Taxes) - Continued

\begin{tabular}{|c|c|c|c|c|c|c|c|c|c|c|c|c|}
\hline \multirow{3}{*}{$\begin{array}{l}\text { Geographlc Area } \\
\text { Month }\end{array}$} & \multicolumn{6}{|c|}{ Premium } & \multicolumn{6}{|c|}{ All Grades } \\
\hline & \multicolumn{2}{|c|}{ Sales to End Users } & \multicolumn{4}{|c|}{ Sales for Resale } & \multicolumn{2}{|c|}{ Sales to End Users } & \multicolumn{4}{|c|}{ Sales for Resale } \\
\hline & $\begin{array}{l}\text { Through } \\
\text { Retail } \\
\text { Outlets }\end{array}$ & Averagea & DTW & Rack & Bulk & Average & $\begin{array}{c}\text { Through } \\
\text { Retail } \\
\text { Outlets }\end{array}$ & Averagea & DTW & Rack & Bulk & Average \\
\hline \multicolumn{13}{|l|}{ Idaho } \\
\hline 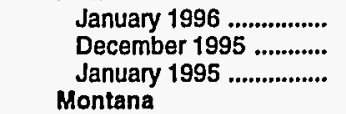 & $\begin{array}{l}82.5 \\
85.8 \\
86.6\end{array}$ & $\begin{array}{l}81.9 \\
84.8 \\
86.1\end{array}$ & $\begin{array}{l}71.4 \\
72.8 \\
73.9\end{array}$ & $\begin{array}{l}64.5 \\
66.1 \\
68.2\end{array}$ & $\begin{array}{l}- \\
-\end{array}$ & $\begin{array}{l}67.0 \\
68.8 \\
70.4\end{array}$ & $\begin{array}{l}72.5 \\
75.6 \\
76.8\end{array}$ & $\begin{array}{l}72.4 \\
75.0 \\
76.3\end{array}$ & $\begin{array}{l}64.4 \\
65.7 \\
67.1\end{array}$ & $\begin{array}{l}56.9 \\
58.8 \\
60.8\end{array}$ & $\begin{array}{l}- \\
-\end{array}$ & $\begin{array}{l}59.3 \\
61.2 \\
62.8\end{array}$ \\
\hline $\begin{array}{l}\text { January } 1996 \text {................ } \\
\text { December } 1995 \text {............. } \\
\text { January } 1995 \text {................ } \\
\text { Utah }\end{array}$ & $\begin{array}{l}83.6 \\
83.8 \\
84.2\end{array}$ & $\begin{array}{l}84.4 \\
84.2 \\
84.5\end{array}$ & $\begin{array}{l}76.7 \\
76.2 \\
74.4\end{array}$ & $\begin{array}{l}72.6 \\
71.7 \\
68.3\end{array}$ & $\begin{array}{l}- \\
-\end{array}$ & $\begin{array}{l}73.6 \\
72.9 \\
69.5\end{array}$ & $\begin{array}{l}76.3 \\
76.7 \\
77.1\end{array}$ & $\begin{array}{l}77.1 \\
77.4 \\
77.4\end{array}$ & $\begin{array}{l}70.1 \\
70.0 \\
68.0\end{array}$ & $\begin{array}{l}64.7 \\
64.0 \\
60.7\end{array}$ & $\bar{w}$ & $\begin{array}{l}65.8 \\
65.2 \\
62.2\end{array}$ \\
\hline $\begin{array}{l}\text { January } 1996 \text {................. } \\
\text { December } 1995 \text {............ } \\
\text { January } 1995 \text {................ } \\
\text { Wyoming }\end{array}$ & $\begin{array}{l}85.2 \\
85.9 \\
82.0\end{array}$ & $\begin{array}{l}84.0 \\
84.8 \\
81.1\end{array}$ & $\begin{array}{l}71.0 \\
71.2 \\
70.1\end{array}$ & $\begin{array}{l}68.6 \\
68.4 \\
67.4\end{array}$ & $\begin{array}{l}- \\
-\end{array}$ & $\begin{array}{l}69.4 \\
69.5 \\
68.4\end{array}$ & $\begin{array}{l}74.4 \\
75.2 \\
72.6\end{array}$ & $\begin{array}{l}73.6 \\
74.5 \\
72.4\end{array}$ & $\begin{array}{l}64.2 \\
64.4 \\
64.1\end{array}$ & $\begin{array}{l}62.0 \\
61.8 \\
60.7\end{array}$ & $\vec{w}$ & $\begin{array}{l}62.7 \\
62.8 \\
61.9\end{array}$ \\
\hline $\begin{array}{l}\text { January } 1996 \text {................ } \\
\text { December } 1995 \text {............ } \\
\text { January } 1995 \text {.............. }\end{array}$ & $\begin{array}{l}89.0 \\
89.5 \\
90.9\end{array}$ & $\begin{array}{l}88.5 \\
89.0 \\
88.7\end{array}$ & $\begin{array}{l}74.2 \\
73.9 \\
70.6\end{array}$ & $\begin{array}{l}67.5 \\
65.4 \\
63.2\end{array}$ & $\overline{-}$ & $\begin{array}{l}68.7 \\
66.9 \\
64.5\end{array}$ & $\begin{array}{l}79.6 \\
80.3 \\
81.4\end{array}$ & $\begin{array}{l}79.1 \\
79.6 \\
80.3\end{array}$ & $\begin{array}{l}66.5 \\
65.4 \\
63.7\end{array}$ & $\begin{array}{l}59.9 \\
57.8 \\
56.0\end{array}$ & $\bar{w}$ & $\begin{array}{l}61.1 \\
59.3 \\
57.4\end{array}$ \\
\hline \multicolumn{13}{|l|}{ PAD District $V$} \\
\hline $\begin{array}{l}\text { January } 1996 \text {............... } \\
\text { December } 1995 \\
\text { January } 1995 . . . . . . . . . . . . . . . . . \\
\text { Alaska }\end{array}$ & $\begin{array}{l}102.6 \\
103.0 \\
101.0\end{array}$ & $\begin{array}{r}100.5 \\
101.0 \\
98.6\end{array}$ & $\begin{array}{l}92.4 \\
92.7 \\
89.1\end{array}$ & $\begin{array}{l}72.3 \\
73.8 \\
71.6\end{array}$ & $\begin{array}{l}61.6 \\
62.5 \\
60.5\end{array}$ & $\begin{array}{l}77.4 \\
78.6 \\
78.8\end{array}$ & $\begin{array}{l}88.6 \\
89.1 \\
86.8\end{array}$ & $\begin{array}{l}86.8 \\
87.4 \\
85.3\end{array}$ & $\begin{array}{l}76.8 \\
77.4 \\
74.6\end{array}$ & $\begin{array}{l}62.0 \\
63.3 \\
60.1\end{array}$ & $\begin{array}{l}54.7 \\
54.8 \\
53.3\end{array}$ & $\begin{array}{l}64.7 \\
65.6 \\
63.0\end{array}$ \\
\hline $\begin{array}{l}\text { January } 1996 \text {................. } \\
\text { December } 1995 \text {............ } \\
\text { January } 1995 \text {................. } \\
\text { Arizona }\end{array}$ & $\begin{array}{l}116.8 \\
116.1 \\
116.2\end{array}$ & $\begin{array}{l}114.0 \\
113.6 \\
115.7\end{array}$ & $\begin{array}{r}94.7 \\
\text { NA } \\
95.6\end{array}$ & $\begin{array}{l}82.3 \\
82.2 \\
80.8\end{array}$ & $\begin{array}{l}W \\
W \\
-\end{array}$ & $\begin{array}{l}88.0 \\
87.6 \\
87.2\end{array}$ & $\begin{array}{l}111.9 \\
110.7 \\
120.0\end{array}$ & $\begin{array}{l}110.0 \\
109.1 \\
117.8\end{array}$ & $\begin{array}{l}84.0 \\
87.3 \\
89.4\end{array}$ & $\begin{array}{l}74.6 \\
71.6 \\
72.2\end{array}$ & $\begin{array}{l}W \\
W \\
W\end{array}$ & $\begin{array}{l}79.0 \\
78.5 \\
80.4\end{array}$ \\
\hline $\begin{array}{l}\text { January } 1996 \text {................ } \\
\text { December } 1995 \text {............ } \\
\text { January } 1995 \text {................ } \\
\text { California }\end{array}$ & $\begin{array}{l}101.4 \\
100.0 \\
100.9\end{array}$ & $\begin{array}{r}100.8 \\
99.6 \\
98.4\end{array}$ & $\begin{array}{l}80.3 \\
80.1 \\
77.5\end{array}$ & $\begin{array}{l}70.8 \\
71.2 \\
69.7\end{array}$ & $\begin{array}{c}W \\
63.5 \\
W\end{array}$ & $\begin{array}{l}73.8 \\
72.4 \\
72.6\end{array}$ & $\begin{array}{l}85.4 \\
85.9 \\
84.9\end{array}$ & $\begin{array}{l}83.6 \\
83.9 \\
83.4\end{array}$ & $\begin{array}{l}68.7 \\
67.7 \\
67.8\end{array}$ & $\begin{array}{l}62.3 \\
62.1 \\
60.0\end{array}$ & $\begin{array}{c}56.7 \\
58.3 \\
W\end{array}$ & $\begin{array}{l}64.2 \\
63.9 \\
62.2\end{array}$ \\
\hline $\begin{array}{l}\text { January } 1996 \text {............... } \\
\text { December } 1995 \text {............ } \\
\text { January } 1995 \text {................ } \\
\text { Hawail }\end{array}$ & $\begin{array}{l}- \\
-\end{array}$ & $\overline{-}$ & $\overline{-}$ & $\begin{array}{r}\text { NA } \\
\text { NA } \\
71.5\end{array}$ & $\begin{array}{c}61.8 \\
61.6 \\
W\end{array}$ & $\begin{array}{l}63.6 \\
63.7 \\
60.5\end{array}$ & $\begin{array}{l}W \\
W \\
-\end{array}$ & $\begin{array}{l}W \\
W \\
-\end{array}$ & $\begin{array}{l}- \\
-\end{array}$ & $\begin{array}{l}61.8 \\
63.7 \\
61.4\end{array}$ & $\begin{array}{l}55.4 \\
53.7 \\
53.2\end{array}$ & $\begin{array}{l}56.0 \\
54.2 \\
53.9\end{array}$ \\
\hline $\begin{array}{l}\text { January } 1996 \ldots . . . . . . . . . . . . . \\
\text { December } 1995 \text {........... } \\
\text { January } 1995 \text {............... } \\
\text { Nevada }\end{array}$ & $\begin{array}{l}118.8 \\
120.5 \\
118.1\end{array}$ & $\begin{array}{l}109.8 \\
110.1 \\
108.4\end{array}$ & $\begin{array}{l}106.7 \\
106.9 \\
104.3\end{array}$ & $\begin{array}{l}97.2 \\
95.1 \\
94.8\end{array}$ & $\begin{array}{l}- \\
-\end{array}$ & $\begin{array}{l}105.6 \\
105.7 \\
103.2\end{array}$ & $\begin{array}{l}107.9 \\
110.1 \\
105.3\end{array}$ & $\begin{array}{r}100.1 \\
101.3 \\
97.6\end{array}$ & $\begin{array}{l}98.8 \\
99.2 \\
96.5\end{array}$ & $\begin{array}{l}87.3 \\
85.2 \\
85.2\end{array}$ & $\begin{array}{l}- \\
-\end{array}$ & $\begin{array}{l}97.2 \\
97.6 \\
95.1\end{array}$ \\
\hline $\begin{array}{l}\text { January } 1996 \text {................ } \\
\text { December } 1995 \text {............ } \\
\text { January } 1995 \text {............... } \\
\text { Oregon }\end{array}$ & $\begin{array}{l}92.7 \\
94.9 \\
88.1\end{array}$ & $\begin{array}{l}91.7 \\
93.6 \\
85.4\end{array}$ & $\begin{array}{l}78.1 \\
78.5 \\
77.7\end{array}$ & $\begin{array}{l}72.1 \\
73.4 \\
71.7\end{array}$ & $\begin{array}{c}W \\
64.8 \\
W\end{array}$ & $\begin{array}{l}72.0 \\
70.9 \\
72.4\end{array}$ & $\begin{array}{l}80.4 \\
81.7 \\
78.1\end{array}$ & $\begin{array}{l}78.8 \\
80.5 \\
76.4\end{array}$ & $\begin{array}{l}69.5 \\
70.1 \\
68.0\end{array}$ & $\begin{array}{l}62.3 \\
63.5 \\
61.1\end{array}$ & $\begin{array}{c}57.9 \\
60.1 \\
W\end{array}$ & $\begin{array}{l}62.5 \\
64.1 \\
62.2\end{array}$ \\
\hline $\begin{array}{l}\text { January } 1996 \text {............... } \\
\text { December } 1995 \text {............ } \\
\text { January } 1995 \text {............... } \\
\text { Washington }\end{array}$ & $\begin{array}{l}93.0 \\
94.3 \\
91.5\end{array}$ & $\begin{array}{l}92.8 \\
94.2 \\
90.6\end{array}$ & $\begin{array}{l}81.0 \\
82.5 \\
79.6\end{array}$ & $\begin{array}{l}73.3 \\
76.4 \\
70.8\end{array}$ & $\begin{array}{c}W \\
60.6 \\
-\end{array}$ & $\begin{array}{l}71.1 \\
75.7 \\
74.5\end{array}$ & $\begin{array}{l}81.8 \\
82.6 \\
78.4\end{array}$ & $\begin{array}{l}81.2 \\
82.0 \\
77.8\end{array}$ & $\begin{array}{l}69.7 \\
71.3 \\
65.9\end{array}$ & $\begin{array}{l}60.7 \\
63.6 \\
57.7\end{array}$ & $\begin{array}{l}53.4 \\
55.5 \\
52.1\end{array}$ & $\begin{array}{l}61.0 \\
64.7 \\
58.7\end{array}$ \\
\hline $\begin{array}{l}\text { January } 1996 \text {............... } \\
\text { December } 1995 . . . . . . . . . \\
\text { January } 1995 \text {............... }\end{array}$ & $\begin{array}{r}98.0 \\
100.0 \\
97.3\end{array}$ & $\begin{array}{l}96.8 \\
99.3 \\
96.4\end{array}$ & $\begin{array}{l}83.4 \\
85.1 \\
80.1\end{array}$ & $\begin{array}{l}69.7 \\
71.8 \\
69.8\end{array}$ & $\begin{array}{l}- \\
-\end{array}$ & $\begin{array}{l}73.9 \\
75.8 \\
73.8\end{array}$ & $\begin{array}{l}83.6 \\
85.5 \\
83.3\end{array}$ & $\begin{array}{l}83.2 \\
85.1 \\
83.4\end{array}$ & $\begin{array}{l}71.6 \\
73.4 \\
68.4\end{array}$ & $\begin{array}{l}60.0 \\
61.9 \\
58.9\end{array}$ & $\begin{array}{c}50.0 \\
53.9 \\
W\end{array}$ & $\begin{array}{l}62.6 \\
65.2 \\
61.7\end{array}$ \\
\hline
\end{tabular}

Dash $(-)=$ No data reported.

$N A=$ Not avallable.

$W=$ Withheld to avoid disclosure of individual company data.

a Includes sales through retail outlets as well as all direct sales to end users that were not made through company-operated retail outlets, e.g., sales to agricultural customers, commercial sales, and Industrial sales.

Notes: Values shown for the current month are preliminary. Values shown for previous months are revised. Data are final upon publication in the Petroleum Marketing Annual.

Sources: Energy Information Administration Forms ElA-782A, "Refiners'/Gas Plant Operators' Monthly Petroleum Product Sales Report," and EIA-782B,

"Resellers'/Retallers' Monthly Petroleum Product Sales Report." 
Table 33. Oxygenated Motor Gasoline Prices by Grade, Sales Type,

PAD District, and State

(Cents per Gallon Excluding Taxes)

\begin{tabular}{|c|c|c|c|c|c|c|c|c|c|c|c|c|}
\hline \multirow{3}{*}{$\begin{array}{l}\text { Geographic Area } \\
\text { Month }\end{array}$} & \multicolumn{6}{|c|}{ Regular } & \multicolumn{6}{|c|}{ Midgrade } \\
\hline & \multicolumn{2}{|c|}{ Sales to End Users } & \multicolumn{4}{|c|}{ Sales for Resale } & \multicolumn{2}{|c|}{ Sales to End Users } & \multicolumn{4}{|c|}{ Sales for Resale } \\
\hline & $\begin{array}{c}\text { Through } \\
\text { Retail } \\
\text { Outlets }\end{array}$ & Average $^{a}$ & DTW & Rack & Bulk & Average & $\begin{array}{c}\text { Through } \\
\text { Retail } \\
\text { Outlets } \\
\end{array}$ & Average $^{a}$ & DTW & Rack & Bulk & Average \\
\hline \multicolumn{13}{|l|}{ United States } \\
\hline January 1996 & 76.7 & 76.4 & 69.7 & 61.7 & NA & 65.4 & 84.0 & 83.7 & 75.4 & 65.3 & - & 71.8 \\
\hline December 1995 ............. & 75.7 & 75.5 & 69.1 & 62.2 & 58.3 & 65.5 & 82.6 & 82.3 & 74.2 & 65.8 & - & 71.2 \\
\hline 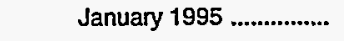 & & 76.5 & & 60.7 & & 64.4 & 85.1 & 84.6 & 73.6 & 64.3 & w & 69.9 \\
\hline \multicolumn{13}{|l|}{ PAD District I } \\
\hline January 1996 & - & - & - & - & & - & - & - & - & - & - & - \\
\hline December 1995 ............. & - & - & - & - & - & - & - & - & - & - & - & - \\
\hline January 1995 ....................... & 69.9 & 69.7 & 64.2 & 59.5 & $w$ & 59.9 & 80.7 & 80.5 & 69.5 & 63.3 & - & 64.4 \\
\hline Subdistrict IA & & & & & & & & & & & & \\
\hline January $1996 \ldots . . . . . . . . . . .$. & - & - & - & - & - & - & - & - & - & - & - & - \\
\hline December 1995 ............. & - & - & - & - & - & - & - & - & - & - & - & - \\
\hline January 1995 .................. & - & - & - & - & - & - & - & - & - & - & - & - \\
\hline Connecticut & & & & & & & & & & & & \\
\hline January 1996 & - & - & - & - & - & - & - & - & - & - & - & - \\
\hline December $1995 \ldots . . . . . . . . .$. & - & - & - & - & - & - & - & - & - & - & - & - \\
\hline January 1995 .................. & - & - & - & - & - & - & - & - & - & - & - & - \\
\hline Maine & & & & & & & & & & & & \\
\hline January $1996 . . . . . . . . . . . . . . .$. & - & - & - & - & - & - & - & - & - & - & - & - \\
\hline December 1995 ............. & - & - & - & - & - & - & - & - & - & - & - & - \\
\hline January $1995 \ldots . . . . . . . . . . . . . .$. & - & - & - & - & - & - & - & - & - & - & - & - \\
\hline Massachusetts & & & & & & & & & & & & \\
\hline January 1996 ...................... & - & - & - & - & - & - & - & - & - & - & - & - \\
\hline December 1995 ............. & - & - & - & - & - & - & - & - & - & - & - & - \\
\hline January 1995 .................. & - & - & - & - & - & - & - & - & - & - & - & - \\
\hline New Hampshire & & & & & & & & & & & & \\
\hline January 1996 .................. & - & - & - & - & - & - & - & - & - & - & - & - \\
\hline December 1995 ............. & - & - & - & - & - & - & - & - & - & - & - & - \\
\hline January 1995 ................... & - & - & - & - & - & - & - & - & - & - & - & - \\
\hline Rhode Island & & & & & & & & & & & & \\
\hline January 1996 .................. & - & - & - & - & - & - & - & - & - & - & - & - \\
\hline December 1995 ............. & - & - & - & - & - & - & - & - & - & - & - & - \\
\hline January 1995 ................... & - & - & - & - & - & - & - & - & - & - & - & - \\
\hline Vermont & & & & & & & & & & & & \\
\hline January 1996 .................. & - & - & - & - & - & - & - & - & - & - & - & - \\
\hline December 1995 ............. & - & - & - & - & - & - & - & - & - & - & - & - \\
\hline 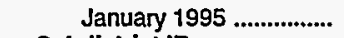 & - & - & - & - & - & - & - & - & - & - & - & - \\
\hline Subdistrict IB & & & & & & & & & & & & \\
\hline January 1996 ................. & - & - & - & - & - & - & - & - & - & - & - & - \\
\hline December 1995 ............. & - & - & - & - & - & - & - & - & - & - & - & - \\
\hline January $1995 \ldots . . . . . . . . . . . . . .$. & - & - & - & - & - & - & - & - & - & - & - & - \\
\hline Delaware & & & & & & & & & & & & \\
\hline January 1996 .................. & - & - & - & - & - & - & - & - & - & - & - & - \\
\hline December 1995 ............. & - & - & - & - & - & - & - & - & - & - & - & - \\
\hline January $1995 \ldots . . . . . . . . . . . .$. & - & - & - & - & - & - & - & - & - & - & - & - \\
\hline District of Columbia & & & & & & & & & & & & \\
\hline January 1996 .................. & - & - & - & - & - & - & - & - & - & - & - & - \\
\hline December 1995 ............. & - & - & - & - & - & - & - & - & - & - & - & - \\
\hline January 1995 ................. & - & - & - & - & - & - & - & - & - & - & - & - \\
\hline Maryland & & & & & & & & & & & & \\
\hline January 1996 ................. & - & - & - & - & - & - & - & - & - & - & - & - \\
\hline December 1995 ............. & - & - & - & - & - & - & - & - & - & - & - & - \\
\hline January $1995 \ldots . . . . . . . . . . . . . .$. & - & - & - & - & - & - & - & - & - & - & - & - \\
\hline New Jersey & & & & & & & & & & & & \\
\hline 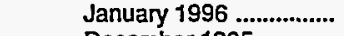 & - & - & - & - & - & - & - & - & - & - & - & - \\
\hline December 1995 ............. & - & - & - & - & - & - & - & - & - & - & - & - \\
\hline 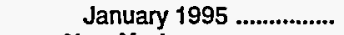 & - & - & - & - & - & - & - & - & - & - & - & - \\
\hline New York & & & & & & & & & & & & \\
\hline 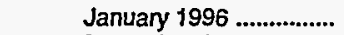 & - & - & - & - & - & - & - & - & - & - & - & - \\
\hline December 1995 .............. & - & - & - & - & - & - & - & - & - & - & - & - \\
\hline January 1995 .................. & - & - & - & - & - & - & - & - & - & - & - & - \\
\hline Pennsylvania & & & & & & & & & & & & \\
\hline January $1996 \ldots . . . . . . . . . . .$. & - & - & - & - & - & - & - & - & - & - & - & - \\
\hline December 1995 ............. & - & - & - & - & - & - & - & - & - & - & - & - \\
\hline January 1995 ................. & - & - & - & - & - & - & - & - & - & - & - & - \\
\hline
\end{tabular}

See footnotes at end of table. 
Table 33. Oxygenated Motor Gasoline Prices by Grade, Sales Type, PAD District, and State

(Cents per Gallon Excluding Taxes) - Continued

\begin{tabular}{|c|c|c|c|c|c|c|c|c|c|c|c|c|}
\hline \multirow{3}{*}{$\begin{array}{c}\text { Geographic Area } \\
\text { Month }\end{array}$} & \multicolumn{6}{|c|}{ Premium } & \multicolumn{6}{|c|}{ All Grades } \\
\hline & \multicolumn{2}{|c|}{ Sales to End Users } & \multicolumn{4}{|c|}{ Sales for Resale } & \multicolumn{2}{|c|}{ Sales to End Users } & \multicolumn{4}{|c|}{ Sales for Resale } \\
\hline & $\begin{array}{c}\text { Through } \\
\text { Retail } \\
\text { Outlets } \\
\end{array}$ & Average ${ }^{a}$ & DTW & Rack & Bulk & Average & $\begin{array}{c}\text { Through } \\
\text { Retail } \\
\text { Outlets } \\
\end{array}$ & Average ${ }^{a}$ & DTW & Rack & Bulk & Average \\
\hline \multicolumn{13}{|l|}{ United States } \\
\hline $\begin{array}{l}\text { January } 1996 \ldots . . . \ldots \ldots . . . . \\
\text { December } 1995 \\
\text { January } 1995 . . . . . . . . . . . . . . . . . .\end{array}$ & $\begin{array}{l}94.4 \\
93.3 \\
94.9\end{array}$ & $\begin{array}{l}93.8 \\
92.9 \\
94.3\end{array}$ & $\begin{array}{l}83.6 \\
82.6 \\
82.9\end{array}$ & $\begin{array}{l}71.7 \\
72.1 \\
70.5\end{array}$ & $\begin{array}{l}57.9 \\
W \\
W\end{array}$ & $\begin{array}{l}78.7 \\
78.4 \\
77.8\end{array}$ & $\begin{array}{l}80.3 \\
79.3 \\
80.4\end{array}$ & $\begin{array}{l}79.9 \\
79.0 \\
80.0\end{array}$ & $\begin{array}{l}73.2 \\
72.5 \\
71.9\end{array}$ & $\begin{array}{l}63.5 \\
64.0 \\
62.4\end{array}$ & $\begin{array}{r}\mathrm{NA} \\
59.0 \\
56.7\end{array}$ & $\begin{array}{l}68.5 \\
68.5 \\
67.2\end{array}$ \\
\hline \multicolumn{13}{|l|}{ PAD District I } \\
\hline January 1996 & - & $w$ & - & - & - & - & - & w & - & - & - & - \\
\hline December 1995 .................. & - & - & - & - & - & - & - & - & - & - & - & - \\
\hline $\begin{array}{l}\text { January } 1995 \\
\text { Subdistrict } 1 A\end{array}$ & 91.2 & 91.0 & 76.1 & 70.4 & - & 71.3 & 77.2 & 76.9 & 68.4 & 63.0 & $\mathbf{w}$ & 63.5 \\
\hline January 1996 & - & $w$ & - & - & - & - & - & W & - & - & - & - \\
\hline December 1995 .............. & - & - & - & - & - & - & - & - & - & - & - & - \\
\hline January 1995 ................... & - & - & - & - & - & - & - & - & - & - & - & - \\
\hline \multicolumn{13}{|l|}{ Connecticut } \\
\hline $\begin{array}{l}\text { January } 1996 \text {.................... } \\
\text { December } 1995 . . . . . . . . .\end{array}$ & $\overline{-}$ & W & $\overline{-}$ & $\overline{-}$ & - & $\overline{-}$ & $\overline{-}$ & $\underline{w}$ & $\overline{-}$ & - & $\overline{-}$ & - \\
\hline 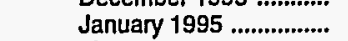 & - & - & - & - & - & - & - & - & $\overline{-}$ & 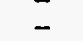 & $\overrightarrow{-}$ & - \\
\hline \multicolumn{13}{|l|}{ Maine } \\
\hline January 1996 .................. & - & - & - & - & - & - & - & - & - & - & - & - \\
\hline December 1995 ............... & - & - & - & - & - & - & - & - & - & - & - & - \\
\hline January 1995 ...................... & - & - & - & - & - & - & - & - & - & - & - & - \\
\hline \multicolumn{13}{|l|}{ Massachusetts } \\
\hline & $\overline{-}$ & $\overline{-}$ & - & $\overline{-}$ & - & $\overline{-}$ & $\overline{-}$ & $\overline{-}$ & $\overline{-}$ & - & - & - \\
\hline $\begin{array}{l}\text { December } 1995 \text {.............. } \\
\text { January } 1995 \text {................. }\end{array}$ & $\overline{-}$ & $\overline{-}$ & - & - & $\overline{-}$ & - & - & $\overline{-}$ & $\begin{array}{l}- \\
-\end{array}$ & - & - & - \\
\hline \multicolumn{10}{|l|}{ New Hampshire } & - & - & - \\
\hline January 1996 ................... & - & - & - & - & - & - & - & - & - & - & - & - \\
\hline December 1995 .............. & - & - & - & - & - & - & - & - & - & - & - & - \\
\hline January 1995 ......................... & - & - & - & - & - & - & - & - & - & - & - & - \\
\hline \multicolumn{13}{|l|}{ Rhode Island } \\
\hline & - & $\overline{-}$ & $\overline{-}$ & $\overline{-}$ & $\overline{-}$ & $\overline{-}$ & - & - & $\overline{-}$ & $\overline{-}$ & $\overline{-}$ & - \\
\hline $\begin{array}{l}\text { December } 1995 \\
\text { January } 1995\end{array}$ & - & $\overline{-}$ & - & $\overline{-}$ & - & - & $\overline{-}$ & - & - & - & - & - \\
\hline Vermont & & & & & & & & & & & & - \\
\hline January 1996 .................... & - & - & - & - & - & - & - & - & - & - & - & - \\
\hline December 1995 .............. & - & - & - & - & - & - & - & - & - & - & - & - \\
\hline $\begin{array}{l}\text { January } 1995 \text {................... } \\
\text { SubdistrictiB }\end{array}$ & - & - & - & - & - & - & - & - & - & - & - & - \\
\hline January 1996 & - & - & - & - & - & - & - & - & - & - & - & - \\
\hline December 1995 .............. & - & - & - & - & - & - & - & - & - & - & - & - \\
\hline January 1995 ................... & - & - & - & - & - & - & - & - & - & - & - & - \\
\hline Delaware & & & & & & & & & & & & \\
\hline January 1996 .................. & - & - & - & - & - & - & - & - & - & - & - & - \\
\hline December 1995 ............. & - & - & - & - & - & $\bar{z}$ & - & - & - & - & - & - \\
\hline $\begin{array}{l}\text { January } 1995 \text {..................... } \\
\text { District of Columbia }\end{array}$ & - & - & - & - & - & - & - & - & - & - & - & - \\
\hline & - & - & - & - & - & - & - & - & - & - & - & - \\
\hline December 1995 ............. & - & - & - & - & - & - & - & - & - & - & - & - \\
\hline January 1995 .................... & - & - & - & - & - & - & - & - & - & - & - & - \\
\hline Maryland & & - & - & - & - & - & - & - & - & - & _- & - \\
\hline $\begin{array}{l}\text { January } 1996 \text {.................. } \\
\text { December } 1995 . . . . . . .\end{array}$ & $\overline{-}$ & - & $\overline{-}$ & - & - & $\overline{-}$ & - & - & - & - & - & $\overline{-}$ \\
\hline & - & - & - & - & - & - & - & - & - & - & - & - \\
\hline $\begin{array}{l}\text { January } 1995 \ldots . . . . . . . . . . . . . \\
\text { New Jersey }\end{array}$ & & & & & & & & & & & & \\
\hline January 1996 & - & - & - & - & - & - & - & - & - & - & - & - \\
\hline December 1995 ............. & - & - & - & - & - & - & - & - & - & - & $-{ }^{\circ}$ & - \\
\hline January 1995 .................. & - & - & - & - & - & - & - & - & - & - & - & - \\
\hline $\begin{array}{l}\text { Now York } \\
\text { January } 1996 . . . . . . . . . . . . . . .\end{array}$ & - & & & & & & & & & - & - & \\
\hline $\begin{array}{l}\text { January } 1996 \ldots . . . . . . . . . . . . . . \\
\text { December } 1995, \ldots . . . . . .\end{array}$ & $\overline{-}$ & $\overline{-}$ & - & $\overline{-}$ & $\overline{-}$ & $\begin{array}{l}- \\
-\end{array}$ & $=$ & $\overline{-}$ & $=$ & $=$ & $=$ & $z$ \\
\hline $\begin{array}{l}\text { December } 1995, \ldots . . . . . . . . . \\
\text { January } 1995 \text {................ }\end{array}$ & - & - & - & - & - & - & - & - & - & $\overline{-}$ & - & $\overline{-}$ \\
\hline Pennsylvania & & & & & & & & & & & & - \\
\hline January 1996 ................... & - & - & - & - & - & - & - & - & - & - & - & - \\
\hline December 1995 .............. & - & - & - & - & - & - & - & - & - & - & - & - \\
\hline January 1995 .................. & - & - & - & - & - & - & - & - & - & - & - & - \\
\hline
\end{tabular}

See footnotes at end of table. 
Table 33. Oxygenated Motor Gasoline Prices by Grade, Sales Type, PAD District, and State

(Cents per Gallon Excluding Taxes) - Continued

\begin{tabular}{|c|c|c|c|c|c|c|c|c|c|c|c|c|}
\hline \multirow{3}{*}{$\begin{array}{l}\text { Geographic Area } \\
\text { Month }\end{array}$} & \multicolumn{6}{|c|}{ Regular } & \multicolumn{6}{|c|}{ Midgrade } \\
\hline & \multicolumn{2}{|c|}{ Sales to End Users } & \multicolumn{4}{|c|}{ Sales for Resale } & \multicolumn{2}{|c|}{ Sales to End Users } & \multicolumn{4}{|c|}{ Sales for Resale } \\
\hline & $\begin{array}{c}\text { Through } \\
\text { Retail } \\
\text { Outiots }\end{array}$ & Average $a$ & DTw & Rack & Bulk & Average & $\begin{array}{c}\text { Through } \\
\text { Retail } \\
\text { Outlets }\end{array}$ & Average ${ }^{a}$ & DTW & Rack & Bulk & Average \\
\hline \multicolumn{13}{|l|}{ Subdistrict IC } \\
\hline January 1996 ..................... & - & - & - & - & - & - & - & - & - & - & - & - \\
\hline December 1995 ............. & - & - & - & - & - & - & - & - & - & - & - & - \\
\hline January 1995 ................... & 69.9 & 69.7 & 64.2 & 59.5 & $w$ & 59.9 & 80.7 & 80.5 & 69.5 & 63.3 & - & 64.4 \\
\hline \multicolumn{11}{|l|}{ Florida } & & 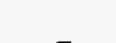 \\
\hline December 1995 .............. & $\overline{-}$ & - & - & $=$ & - & - & - & - & $\bar{z}$ & 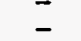 & - & $\overline{-}$ \\
\hline 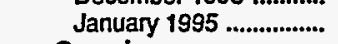 & - & - & - & - & - & - & - & - & - & - & - & - \\
\hline \multicolumn{13}{|l|}{ Georgia } \\
\hline January $1996 \ldots$ & - & - & - & - & - & - & - & - & - & - & - & - \\
\hline December 1995 ............. & - & - & - & - & - & - & - & - & - & - & - & - \\
\hline January 1995 ................... & - & - & - & - & - & - & - & - & - & - & - & - \\
\hline $\begin{array}{l}\text { North Carolina } \\
\text { January } 1996\end{array}$ & & & & & & & & & & & & \\
\hline January 1996 & - & - & - & - & - & - & - & - & - & - & - & - \\
\hline December 1995 ............. & - & - & - & - & - & - & - & - & - & - & - & - \\
\hline January 1995 & 69.9 & 69.8 & 64.2 & 59.5 & $w$ & 59.9 & 80.7 & 80.5 & 69.5 & 63.3 & - & 64.4 \\
\hline South Carolina & & & & & & & & & & & & \\
\hline January 1996 & - & - & - & - & - & - & - & - & - & - & - & - \\
\hline December 1995 .............. & - & - & - & - & - & - & - & - & - & - & - & - \\
\hline January 1995 ................... & - & - & - & - & - & - & - & - & - & - & - & - \\
\hline Virginia & & & & & & & & & & & & \\
\hline January 1996 & - & - & - & - & - & - & - & - & - & - & - & - \\
\hline December 1995 ............. & - & - & - & - & - & - & - & - & - & - & - & - \\
\hline January 1995 ................... & - & NA & - & - & - & - & - & NA & - & - & - & - \\
\hline $\begin{array}{l}\text { West Virginia } \\
\text { January } 1996\end{array}$ & & & & & & & & & & & & \\
\hline $\begin{array}{l}\text { January } 1996 \text {.................... } \\
\text { December } 1995 \text {........... }\end{array}$ & - & - & - & - & - & - & - & - & - & - & - & - \\
\hline $\begin{array}{l}\text { December } 1995 \text {.............. } \\
\text { January } 1995 \ldots . . . . . . . . . . . .\end{array}$ & - & - & - & - & - & - & - & - & - & - & - & - \\
\hline January 1995 ................... & - & - & - & - & - & - & - & - & - & - & - & - \\
\hline PAD District II & & & & & & & & & & & & \\
\hline January 1996 ................... & 77.0 & 76.5 & 66.3 & 62.4 & - & 63.2 & 84.1 & 83.6 & w & 64.1 & - & 67.3 \\
\hline December 1995 ............. & 74.5 & 74.0 & 64.2 & 60.8 & - & 61.6 & 81.9 & 81.4 & 72.4 & 63.0 & - & 65.7 \\
\hline January 1995 .................. & 72.4 & 72.0 & 64.0 & 60.2 & - & 61.1 & 78.7 & 78.3 & 68.7 & 61.7 & - & 63.8 \\
\hline Illinois & & & & & & & & & & & & \\
\hline January 1996 .................. & - & - & - & - & - & - & - & - & - & - & - & - \\
\hline December 1995 ............ & - & - & - & - & - & - & - & - & - & - & - & - \\
\hline Indiana 1995 ......................... & - & - & - & - & - & - & - & - & - & - & - & - \\
\hline January 1996 & - & - & - & - & - & - & - & - & - & - & - & - \\
\hline December 1995 ............... & - & - & - & - & - & - & - & - & - & - & - & - \\
\hline January 1995 ................. & - & - & - & - & - & - & - & - & - & - & - & - \\
\hline 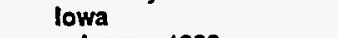 & & & & & & & & & & & & \\
\hline January 1996 & - & - & - & - & - & - & - & - & - & - & - & - \\
\hline December 1995 .............. & - & - & - & - & - & - & - & - & - & - & - & - \\
\hline January 1995 .................. & - & - & - & - & - & - & - & - & - & - & - & - \\
\hline Kansas & & & & & & & & & & & & \\
\hline January 1996 ................... & - & - & - & - & - & - & - & - & - & - & - & - \\
\hline December 1995 .............. & - & - & - & - & - & - & - & - & - & - & - & - \\
\hline January 1995 .................. & - & - & - & - & - & - & - & - & - & - & - & - \\
\hline Kentucky & & & & & & & & & & & & \\
\hline January 1996 ................... & - & -. & - & - & - & - & - & - & - & - & - & - \\
\hline December 1995 ............. & - & - & - & - & - & - & - & - & - & - & - & - \\
\hline January 1995 ................... & - & - & - & - & - & - & - & - & - & - & - & - \\
\hline Michigan & & & & & & & & & & & & \\
\hline January 1996 ................... & - & - & - & - & - & - & - & - & - & - & - & - \\
\hline December 1995 ............. & - & - & - & - & - & - & - & - & - & - & - & - \\
\hline January 1995 ................... & - & - & - & - & - & - & - & - & - & - & - & - \\
\hline Minnesota & & & & & & & & & & & & \\
\hline January 1996 ................... & & & 66.3 & 62.4 & - & 63.2 & 84.1 & 83.6 & $w$ & 64.1 & - & 67.4 \\
\hline December 1995 .............. & 74.5 & 74.0 & 64.2 & 60.8 & - & 61.6 & 81.9 & 81.4 & 72.4 & 63.0 & - & 65.7 \\
\hline January 1995 ..................... & 72.4 & 72.0 & 64.0 & 60.2 & - & 61.1 & 78.7 & 78.3 & 68.7 & 61.7 & - & 63.8 \\
\hline Missourí & & & & & & & & & & & & \\
\hline January 1996 .................. & - & - & - & - & - & - & - & - & - & - & - & - \\
\hline December 1995 ............. & - & - & - & - & - & - & - & - & - & - & - & - \\
\hline January 1995 ................... & - & - & - & - & - & - & - & - & - & - & - & - \\
\hline
\end{tabular}

See footnoles at end of table. 
Table 33. Oxygenated Motor Gasoline Prices by Grade, Sales Type,

PAD District, and State

(Cents per Gallon Excluding Taxes) - Continued

\begin{tabular}{|c|c|c|c|c|c|c|c|c|c|c|c|c|}
\hline \multirow{3}{*}{$\begin{array}{l}\text { Geographic Area } \\
\text { Month }\end{array}$} & \multicolumn{6}{|c|}{ Premium } & \multicolumn{6}{|c|}{ All Grades } \\
\hline & \multicolumn{2}{|c|}{ Sales to End Users } & \multicolumn{4}{|c|}{ Sales for Resale } & \multicolumn{2}{|c|}{ Sales to End Users } & \multicolumn{4}{|c|}{ Sales for Resale } \\
\hline & $\begin{array}{c}\text { Through } \\
\text { Retail } \\
\text { Outlets } \\
\end{array}$ & Average ${ }^{a}$ & DTW & Rack & Bulk & Average & $\begin{array}{c}\text { Through } \\
\text { Retail } \\
\text { Outlets }\end{array}$ & Averagea & DTW & Rack & Bulk & Average \\
\hline \multicolumn{13}{|l|}{ Subdistrict IC } \\
\hline January 1996 .................. & - & - & - & - & - & - & - & - & - & - & - & - \\
\hline December 1995 ............. & - & - & - & - & - & - & - & - & - & - & - & - \\
\hline January 1995 ................... & 91.2 & 91.0 & 76.1 & 70.4 & - & 71.3 & 77.2 & 76.9 & 68.4 & 63.0 & $w$ & 63.5 \\
\hline \multicolumn{13}{|l|}{ Florida } \\
\hline Jantary 1996 .................. & $=$ & $\overline{-}$ & $\overline{-}$ & - & $=$ & - & - & - & - & - & - & - \\
\hline & $\overline{-}$ & - & - & - & - & $=$ & $\overline{-}$ & - & - & - & - & - \\
\hline \multicolumn{13}{|l|}{ Georgia } \\
\hline January 1996 ................... & - & - & - & - & - & - & - & - & - & - & - & - \\
\hline December 1995 ............. & - & - & - & - & - & - & - & - & - & - & - & - \\
\hline $\begin{array}{l}\text { January } 1995 \text {................. } \\
\text { North Carolina }\end{array}$ & - & - & - & - & \multicolumn{2}{|c|}{ North Carolina } & - & - & - & - & & - \\
\hline January 1996 .................. & - & - & - & - & - & - & - & - & - & - & - & _- \\
\hline December 1995 .............. & - & - & - & - & - & - & - & - & - & - & - & - \\
\hline January 1995 .................. & 91.2 & 91.0 & 76.1 & 70.4 & - & 71.3 & 77.2 & 77.0 & 68.4 & 63.0 & $w$ & 63.5 \\
\hline \multicolumn{13}{|l|}{ South Carolina } \\
\hline January 1996 ................... & - & - & - & - & - & - & - & - . & - & - & - & - \\
\hline December 1995 ............. & - & - & - & - & - & - & - & - & - & - & - & - \\
\hline January $1995 \ldots . . . . . . . . . . . . .$. & - & - & - & - & - & - & - & - & - & - & - & - \\
\hline Virginia & & & & & & & & & & & & \\
\hline January 1996 ................. & - & - & - & - & - & - & - & - & - & - & - & - \\
\hline December 1995 .............. & - & - & - & - & - & - & - & - & - & - & - & - \\
\hline $\begin{array}{c}\text { January } 1995 \text {................. } \\
\text { West Virginia }\end{array}$ & - & NA & - & - & - & - & - & NA & - & - & - & - \\
\hline $\begin{array}{l}\text { West Virginia } \\
\text { January } 1996 \text {................. }\end{array}$ & - & - & - & - & - & - & - & - & - & - & - & - \\
\hline December 1995 ........................ & - & - & - & - & - & - & - & - & - & - & - & - \\
\hline January 1995 ................... & - & - & - & - & - & - & - & - & - & - & - & - \\
\hline PAD District II & & & & & & & & & & & & \\
\hline January 1996 & 91.5 & 91.1 & $w$ & 68.5 & - & 71.0 & 79.5 & 78.9 & 69.6 & 63.4 & - & 64.6 \\
\hline December 1995 .............. & 89.0 & 88.7 & 77.4 & 66.9 & - & 69.1 & 77.1 & 76.6 & 67.1 & 61.9 & - & 63.1 \\
\hline January 1995 ................... & 86.5 & 86.3 & 74.4 & 65.9 & - & 67.5 & 74.6 & 74.2 & 65.9 & 61.2 & - & 62.2 \\
\hline Illinois & & & & & & & & & & & & \\
\hline January 1996 .................. & - & - & - & - & - & - & - & - & - & - & - & - \\
\hline December 1995 .............. & - & - & - & - & - & - & - & - & - & - & - & - \\
\hline January 1995 ................... & - & - & - & - & - & - & - & - & - & - & - & - \\
\hline 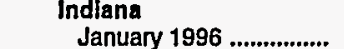 & & & & & & & & & & & & \\
\hline $\begin{array}{l}\text { January } 1996 \ldots . . . . . . . . . . . . . . \\
\text { December } 1995 . . . . . . . . .\end{array}$ & $\overline{-}$ & $\overline{-}$ & $\overline{-}$ & $\overline{-}$ & - & $\overline{-}$ & $\overline{-}$ & $\overline{-}$ & $\bar{z}$ & $\overline{-}$ & $\overline{-}$ & $\overline{-}$ \\
\hline January 1995 ................... & - & - & - & - & - & - & - & - & - & - & - & - \\
\hline lowa & & & & & & & & & & & & \\
\hline January 1996 ................... & - & - & - & - & - & - & - & - & - & - & - & - \\
\hline December 1995 ............ & - & - & - & - & - & - & - & - & - & - & - & - \\
\hline $\begin{array}{c}\text { January } 1995 \text {..................... } \\
\text { Kansas }\end{array}$ & - & - & - & - & - & - & - & - & - & - & - & - \\
\hline 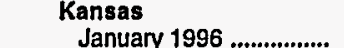 & - & - & - & - & - & - & - & - & - & - & - & - \\
\hline December 1995 .............. & - & - & - & - & - & - & - & - & - & - & - & - \\
\hline January $1995 \ldots . . . . . . . . . . . . . . .$. & - & - & - & - & - & - & - & - & - & - & - & - \\
\hline Kentucky & & & & & & & & & & & & \\
\hline January 1996 .................... & - & - & - & - & - & - & - & - & - & - & - & - \\
\hline December 1995 .............. & - & - & - & - & - & - & - & - & - & - & - & - \\
\hline January 1995 .................. & - & - & - & - & - & - & - & - & - & - & - & - \\
\hline Michigan & & & & & & & & & & & & \\
\hline January 1996 ................... & - & - & - & - & - & - & - & - & - & - & - & - \\
\hline December 1995 ............. & - & - & - & - & - & - & - & - & - & - & - & - \\
\hline January 1995 ................... & - & - & - & - & - & - & - & - & - & - & - & - \\
\hline Minnesota & & & & & & & & & & & & \\
\hline January 1996 ................... & 91.5 & 91.1 & W & 68.5 & - & 71.0 & 79.5 & 78.9 & 69.6 & 63.4 & - & 64.6 \\
\hline December 1995 .............. & 89.0 & 88.7 & 77.4 & 66.9 & - & 69.1 & 77.1 & 76.6 & 67.1 & 61.9 & - & 63.1 \\
\hline January 1995 ................... & 86.5 & 86.3 & 74.4 & 65.9 & - & 67.5 & 74.6 & 74.2 & 65.9 & 61.2 & - & 62.2 \\
\hline Missouri & & & & & & & & & & & & \\
\hline January 1996 ................... & - & - & - & - & - & - & - & - & - & - & - & - \\
\hline December 1995 .............. & - & - & - & - & - & - & - & - & - & - & - & - \\
\hline January 1995 .................. & - & - & - & - & - & - & - & - & - & - & - & - \\
\hline
\end{tabular}

See footnotes at end of table. 
Table 33. Oxygenated Motor Gasoline Prices by Grade, Sales Type, PAD District, and State

(Cents per Gallon Excluding Taxes) - Continued

\begin{tabular}{|c|c|c|c|c|c|c|c|c|c|c|c|c|}
\hline \multirow{3}{*}{$\begin{array}{c}\text { Geographic Area } \\
\text { Month }\end{array}$} & \multicolumn{6}{|c|}{ Regular } & \multicolumn{6}{|c|}{ Midgrade } \\
\hline & \multicolumn{2}{|c|}{ Sales to End Users } & \multicolumn{4}{|c|}{ Sales for Resale } & \multicolumn{2}{|c|}{ Sales to End Users } & \multicolumn{4}{|c|}{ Sales for Resale } \\
\hline & $\begin{array}{c}\text { Through } \\
\text { Retail } \\
\text { Outlets }\end{array}$ & Averagea & DTW & Rack & Bulk & Average & $\begin{array}{c}\text { Through } \\
\text { Retail } \\
\text { Outlets }\end{array}$ & Average ${ }^{a}$ & DTW & Rack & Bulk & Average \\
\hline \multicolumn{13}{|l|}{ Nebraska } \\
\hline January 1996 & - & - & - & - & - & - & - & - & - & - & - & - \\
\hline December 1995 ............. & - & - & - & $\rightarrow$ & - & $\rightarrow$ & - & - & - & - & - & - \\
\hline January 1995 .................. & - & - & - & - & - & - & - & - & - & - & - & - \\
\hline \multicolumn{13}{|l|}{ North Dakota } \\
\hline January 1996 & - & - & - & - & - & - & - & - & - & - & - & - \\
\hline December 1995 ............. & - & - & - & - & - & - & - & - & - & - & - & - \\
\hline January 1995 ................. & - & - & - & - & - & - & - & - & - & - & - & - \\
\hline \multicolumn{13}{|l|}{ Ohio } \\
\hline January 1996 ................. & - & - & - & - & - & - & - & - & - & - & - & - \\
\hline December 1995 .............. & - & - & - & - & - & - & - & - & - & - & - & - \\
\hline 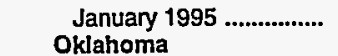 & - & - & - & $\cdot \quad-$ & - & - & - & - & - & - & - & - \\
\hline January 1996 ................. & - & - & - & - & - & - & - & - & - & - & - & - \\
\hline December 1995 ............ & - & - & - & - & - & - & - & - & - & - & - & - \\
\hline January 1995 ................. & - & - & - & - & - & - & - & - & - & - & - & - \\
\hline South Dakota & & & & & & & & & & & & \\
\hline January 1996 ................... & - & - & - & - & - & - & - & - & - & - & - & - \\
\hline December 1995 ............. & - & - & - & - & - & - & - & - & - & - & - & - \\
\hline $\begin{array}{l}\text { January } 1995 \text {................ } \\
\text { Tennessee }\end{array}$ & - & - & - & - & - & - & - & - & - & - & - & - \\
\hline $\begin{array}{l}\text { Tennessee } \\
\text { January } 1996 . . . . . . . . . . . . . . . .\end{array}$ & - & - & - & - & - & - & - & - & - & - & - & - \\
\hline December 1995 ............. & - & - & - & - & - & - & - & - & - & - & - & - \\
\hline January 1995 .................. & - & - & - & - & - & - & - & - & - & - & - & - \\
\hline Wisconsin & & & & & & & & & & & & \\
\hline January 1996 & - & - & - & $W$ & - & $w$ & - & - & - & $w$ & - & $W$ \\
\hline December 1995 ............ & - & - & - & W & - & W & - & - & - & W & - & $W$ \\
\hline January 1995 ................. & - & - & - & - & - & - & - & - & - & - & - & - \\
\hline PAD District III & & & & & & & & & & & & \\
\hline January 1996 & 75.7 & 75.2 & 66.8 & 65.3 & - & 65.5 & 84.9 & 84.8 & 72.1 & 70.9 & - & 71.2 \\
\hline December 1995 ............ & 74.2 & 73.7 & 63.4 & 64.0 & - & 63.9 & 83.2 & 83.1 & 69.1 & 69.0 & - & 69.0 \\
\hline January 1995 ................. & 80.2 & 79.3 & 68.4 & 61.0 & $w$ & 59.9 & 89.3 & 89.2 & 76.6 & 66.7 & - & 71.0 \\
\hline Alabama & & & & & & & & & & & & \\
\hline January 1996 .................. & - & - & - & - & - & - & - & - & - & - & - & - \\
\hline December 1995 ............. & - & - & - & - & - & - & - & - & - & - & - & - \\
\hline January 1995 & - & - & - & $\cdot-$ & - & - & - & - & - & - & - & - \\
\hline Arkansas & & & & & & & & & & & & \\
\hline January 1996 & - & - & - & - & - & - & - & - & - & - & - & - \\
\hline December 1995 ............. & - & - & - & - & - & - & - & - & - & - & - & - \\
\hline January 1995 .................. & - & - & - & - & - & - & - & - & - & - & - & - \\
\hline Louisiana & & & & & & & & & & & & \\
\hline January 1996 & - & - & - & - & - & - & - & - & - & - & - & - \\
\hline December 1995 ............ & - & - & - & - & - & - & - & - & - & - & - & - \\
\hline January 1995 .................. & - & - & - & - & - & - & - & - & - & - & - & - \\
\hline Mississippi & & & & & & & & & & & & \\
\hline January 1996 & - & - & - & - & - & - & - & - & - & - & - & - \\
\hline December 1995 ............ & - & - & - & - & - & - & - & - & - & - & - & - \\
\hline January 1995 ................. & - & - & - & - & - & - & - & - & - & - & - & - \\
\hline New Mexico & & & & & & & & & & & & \\
\hline January 1996 .................. & 74.8 & 74.6 & 65.1 & 64.9 & - & 64.9 & 84.0 & 83.9 & 68.5 & 70.4 & - & 70.0 \\
\hline December 1995 ............. & 73.6 & 73.3 & 62.1 & 62.1 & - & 62.1 & 83.0 & 82.8 & 67.0 & 67.7 & - & 67.5 \\
\hline January 1995 ................... & 76.9 & 76.3 & 65.2 & 58.9 & - & 61.0 & 85.2 & 84.9 & 71.9 & 64.6 & - & 67.9 \\
\hline Texas & & & & & & & & & & & & \\
\hline January 1996 .................. & 79.5 & 77.4 & W & 65.6 & - & 66.1 & $\mathbf{W}$ & $\mathbf{W}$ & W & 72.1 & - & 73.3 \\
\hline December 1995 ............. & 76.9 & 75.1 & $W$ & 66.0 & - & 66.0 & $w$ & $W$ & W & 72.3 & - & 72.1 \\
\hline January 1995 .................. & 85.3 & 84.2 & W & 62.7 & $w$ & 59.2 & 92.0 & 92.0 & W & 69.4 & - & 75.2 \\
\hline PAD District IV & & & & & & & & & & & & \\
\hline January 1996 & 74.8 & 74.7 & 66.7 & 61.7 & - & 62.9 & 89.9 & 89.0 & 72.1 & 65.8 & - & 68.5 \\
\hline December 1995 ............ & 76.5 & 76.3 & 63.2 & 59.1 & - & 60.0 & 90.9 & 90.0 & 70.0 & 63.8 & - & 66.4 \\
\hline January 1995 ................. & 77.7 & 77.1 & 63.6 & 58.6 & - & 60.0 & 88.8 & 87.8 & 68.8 & 63.3 & - & 66.3 \\
\hline Colorado & & & & & & & & & & & & \\
\hline January 1996 ................. & 74.9 & 74.8 & 66.7 & 61.4 & - & 62.8 & 90.2 & 89.3 & 72.1 & 65.4 & - & 68.5 \\
\hline December 1995 ............. & 76.4 & 76.3 & 63.1 & 58.2 & - & 59.5 & 91.2 & 90.3 & 69.9 & 62.5 & - & 66.0 \\
\hline January 1995 .................. & 77.7 & 77.2 & 63.6 & 57.8 & - & 59.5 & 88.9 & 87.9 & 68.8 & 62.8 & - & 66.1 \\
\hline
\end{tabular}

See footnotes at end of table. 
Table 33. Oxygenated Motor Gasoline Prices by Grade, Sales Type, PAD District, and State

(Cents per Gallon Excluding Taxes) - Continued

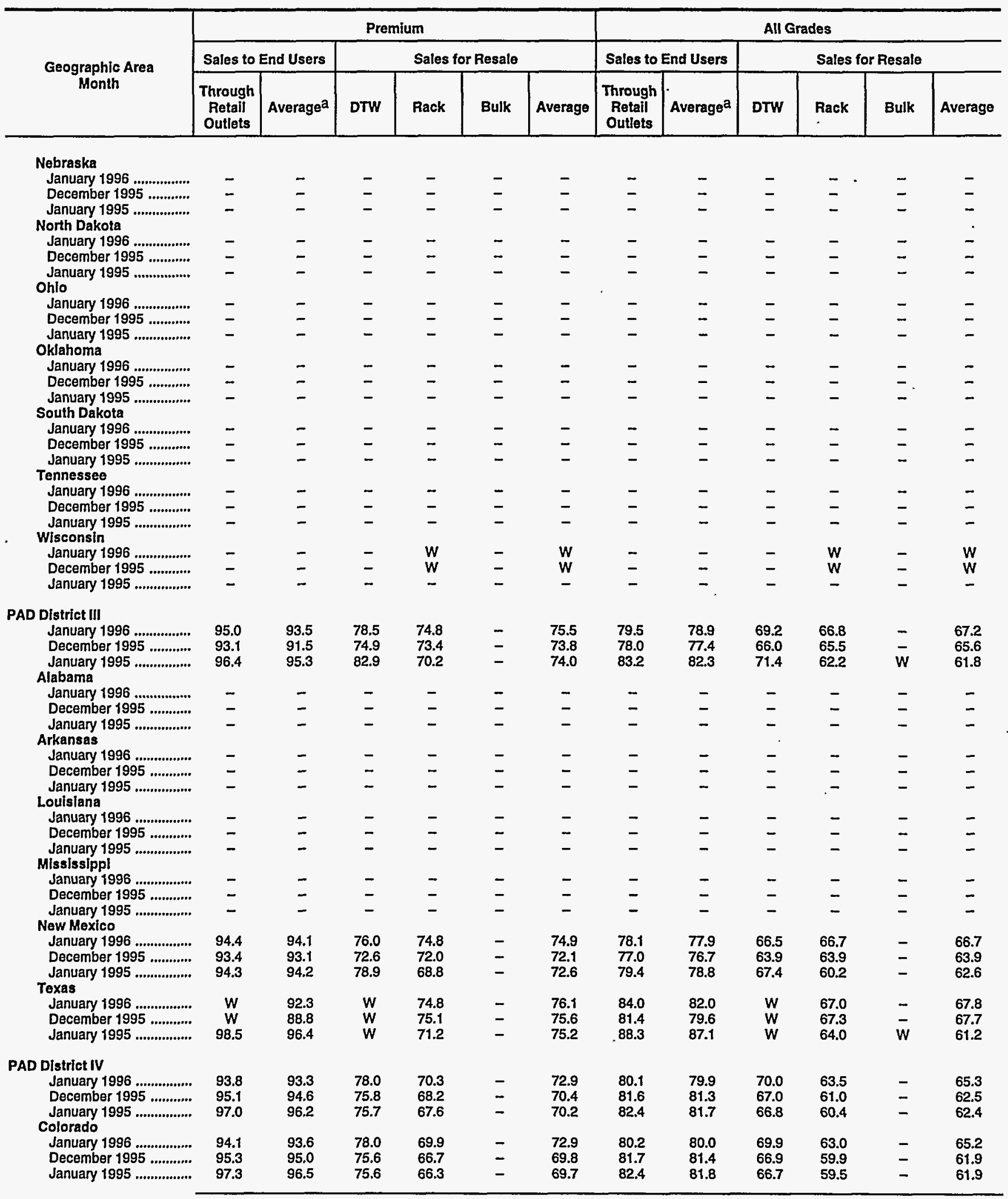

See footnotes at end of table. 
Table 33. Oxygenated Motor Gasoline Prices by Grade, Sales Type, PAD District, and State

(Cents per Gallon Excluding Taxes) - Continued

\begin{tabular}{|c|c|c|c|c|c|c|c|c|c|c|c|c|}
\hline \multirow{3}{*}{$\begin{array}{l}\text { Geographic Area } \\
\text { Month }\end{array}$} & \multicolumn{6}{|c|}{ Regular } & \multicolumn{6}{|c|}{ Midgrade } \\
\hline & \multicolumn{2}{|c|}{ Sales to End Users } & \multicolumn{4}{|c|}{ Sales for Resale } & \multicolumn{2}{|c|}{ Sales to End Users } & \multicolumn{4}{|c|}{ Sales for Resale } \\
\hline & $\begin{array}{c}\text { Through } \\
\text { Retail } \\
\text { Outlets }\end{array}$ & Average ${ }^{a}$ & DTW & Rack & Bulk & Average & $\begin{array}{c}\text { Through } \\
\text { Retail } \\
\text { Outlets }\end{array}$ & Average ${ }^{a}$ & DTW & Rack & Bulk & Average \\
\hline \multicolumn{13}{|l|}{ Idaho } \\
\hline January 1996 & - & - & - & - & - & - & - & - & - & - & - & - \\
\hline December 1995 ............. & - & - & - & - & - & - & - & - & - & - & - & - \\
\hline $\begin{array}{l}\text { January } 1995 \\
\text { Montana }\end{array}$ & - & - & - & - & - & - & - & - & - & - & - & - \\
\hline January 1996 & 74.1 & 72.5 & $w$ & 68.0 & - & 67.9 & 78.7 & 78.4 & W & 72.3 & - & 72.3 \\
\hline December 1995 ............ & 74.5 & 73.6 & w & 66.9 & - & 66.9 & 79.8 & 79.5 & $W$ & 71.3 & - & 71.1 \\
\hline January 1995 .................. & 81.6 & 79.9 & $\ddot{w}$ & 67.2 & - & 67.2 & $w$ & $W$ & - & W & - & W \\
\hline \multicolumn{13}{|l|}{ Utah } \\
\hline January 1996 & NA & 73.3 & 73.0 & 61.4 & - & 61.8 & 81.8 & 80.8 & W & 66.9 & - & 67.9 \\
\hline December 1995 ............. & 78.9 & 78.2 & 72.2 & 61.8 & - & 62.0 & 83.3 & 82.1 & W & 67.8 & - & 68.2 \\
\hline January 1995 .................. & 74.5 & 74.4 & 67.1 & 62.5 & - & 62.9 & 79.9 & 79.9 & $w$ & 68.3 & - & 69.4 \\
\hline Wyoming & & & & & & & & & & & & \\
\hline January 1996 & - & - & - & - & - & - & - & - & - & - & - & - \\
\hline December 1995 ............. & - & - & - & - & - & - & - & - & - & - & - & - \\
\hline January 1995 ................... & - & - & - & - & - & - & - & - & - & - & - & - \\
\hline \multicolumn{13}{|l|}{ PAD District V } \\
\hline January $1996 \ldots$ & 77.1 & 76.8 & 69.9 & 61.1 & NA & 65.9 & 82.7 & 82.5 & 75.6 & 65.1 & - & 72.5 \\
\hline December 1995 .............. & 75.9 & 75.7 & 69.6 & 63.2 & 58.3 & 66.6 & 81.0 & 80.9 & 74.5 & 66.6 & - & 72.2 \\
\hline January 1995 ................ & 77.9 & 77.5 & 69.0 & 61.2 & 56.9 & 65.6 & 85.5 & 85.0 & 74.4 & 65.3 & $w$ & 71.4 \\
\hline \multicolumn{13}{|l|}{ Alaska } \\
\hline January 1996 ................... & $W$ & 91.1 & 94.0 & 82.3 & W & 89.0 & $W$ & $w$ & $w$ & - & - & $w$ \\
\hline December 1995 .............. & $W$ & 90.4 & 92.8 & 81.4 & - & 89.6 & $W$ & $W$ & $W$ & - & - & $W$ \\
\hline January 1995 & 101.7 & 97.2 & 96.6 & 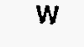 & - & 95.4 & W & $w$ & W & - & - & $w$ \\
\hline $\begin{array}{l}\text { Arizona } \\
\text { January } 1996 . . . . . . . . . . . . . . .\end{array}$ & & & & & & & 83.4 & & 78.7 & 723 & & 76.6 \\
\hline $\begin{array}{l}\text { January } 1996 \text {.................. } \\
\text { December } 1995 \text {............ }\end{array}$ & $\begin{array}{l}78.3 \\
74.5\end{array}$ & 74.2 & 71.5 & 66.8 & - & 70.0 & 78.6 & 78.6 & 76.2 & 72.6 & $\overline{-}$ & $\begin{array}{l}76.6 \\
75.2\end{array}$ \\
\hline January 1995 .................. & 78.1 & 78.0 & 72.9 & 68.3 & - & 71.8 & 89.0 & 88.2 & 73.8 & $w$ & - & 70.9 \\
\hline \multicolumn{13}{|l|}{ California } \\
\hline January 1996 & 72.6 & 72.6 & 67.5 & 58.4 & $W$ & 63.0 & 79.8 & 80.0 & 73.6 & 63.0 & - & 70.5 \\
\hline December 1995 ............. & 71.8 & 72.0 & 66.9 & 60.7 & 58.4 & 63.9 & 78.4 & 78.6 & 72.5 & 65.0 & - & 70.2 \\
\hline January 1995 ................. & 75.0 & 74.6 & 67.9 & 59.5 & 56.9 & 63.7 & 84.2 & 83.8 & 74.2 & 64.8 & $w$ & 71.1 \\
\hline \multicolumn{13}{|l|}{ Hawail } \\
\hline January 1996 ................. & - & - & - & - & - & - & - & - & - &.- & - & - \\
\hline December 1995 ............. & - & - & - & - & - & - & - & - & - & - & - & - \\
\hline January 1995 & - & - & - & - & - & - & - & - & - & - & - & - \\
\hline \multicolumn{13}{|l|}{ Nevada } \\
\hline January 1996 .................. & 89.8 & 89.2 & 68.8 & 67.8 & - & 68.4 & 91.9 & 91.8 & 74.9 & 70.7 & - & 74.2 \\
\hline December 1995 & 88.2 & 87.6 & 67.6 & 68.9 & $w$ & 67.4 & 90.5 & 90.4 & 73.5 & 71.7 & - & 73.2 \\
\hline January 1995 & 90.4 & 89.4 & 65.3 & 66.0 & $w$ & 65.5 & 94.2 & 93.8 & 72.4 & 66.9 & - & 71.5 \\
\hline \multicolumn{13}{|l|}{ Oregon } \\
\hline 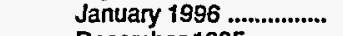 & 80.0 & 79.6 & 70.8 & 64.9 & - & 68.5 & 86.0 & 83.6 & 77.1 & 71.3 & - & 75.6 \\
\hline December 1995 .............. & 80.2 & 80.2 & 72.3 & 67.2 & - & 70.3 & 86.1 & 84,2 & 75.5 & 72.8 & - & 74.8 \\
\hline January 1995 & 78.7 & 78.4 & 66.2 & 61.9 & - & 64.7 & 79.2 & 79.6 & 74.5 & W & - & 72.7 \\
\hline \multicolumn{13}{|l|}{ Washington } \\
\hline January 1996 & 79.8 & 79.7 & 74.9 & 63.8 & - & 71.3 & 84.7 & 84.7 & 81.7 & 66.6 & - & 77.0 \\
\hline December 1995 ............. & 80.8 & 80.6 & 75.9 & 65.9 & - & 72.5 & 85.2 & 85.2 & 81.4 & 68.2 & - & 77.3 \\
\hline 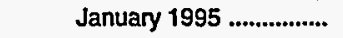 & 79.2 & 79.1 & 71.5 & 63.3 & - & 69.3 & 87.4 & 86.9 & $w$ & 71.2 & - & 76.7 \\
\hline
\end{tabular}

See footnotes at end of table. 
Table 33. Oxygenated Motor Gasoline Prices by Grade, Sales Type, PAD District, and State

(Cents per Gallon Excluding Taxes) - Continued

\begin{tabular}{|c|c|c|c|c|c|c|c|c|c|c|c|c|}
\hline \multirow{3}{*}{$\begin{array}{c}\text { Geographic Area } \\
\text { Month }\end{array}$} & \multicolumn{6}{|c|}{ Premium } & \multicolumn{6}{|c|}{ All Grades } \\
\hline & \multicolumn{2}{|c|}{ Sales to End Users } & \multicolumn{4}{|c|}{ Sales for Resale } & \multicolumn{2}{|c|}{ Sales to End Users } & \multicolumn{4}{|c|}{ Sales for Resale } \\
\hline & $\begin{array}{c}\text { Through } \\
\text { Retail } \\
\text { Outlets }\end{array}$ & Average $\mathrm{a}$ & DTW & Rack & Bulk & Average & $\begin{array}{c}\text { Through } \\
\text { Retall } \\
\text { Outlats }\end{array}$ & Averngea & DTW & Rack & Bulk & Average : \\
\hline \multicolumn{13}{|l|}{ Idaho } \\
\hline January 1996 ................ & - & - & - & - & - & - & - & - & - & - & - & - \\
\hline December 1995 .............. & - & - & - & - & - & - & - & - & - & - & - & - \\
\hline \multicolumn{13}{|l|}{ Montana } \\
\hline January 1996 ................ & 83.1 & 82.7 & $w$ & 77.5 & - & $\pi 7.5$ & $\pi 7.0$ & 75.6 & $w$ & 70.6 & - & 70.6 \\
\hline December 1995 ............. & 83.2 & 83.0 & $w$ & 76.6 & - & 76.6 & 77.3 & 76.5 & $w$ & 69.5 & - & 69.4 \\
\hline \multicolumn{12}{|l|}{ Utah } & 69.4 \\
\hline January 1996 ................ & 87.5 & 86.9 & 83.1 & 69.0 & - & 69.8 & 77.8 & 77.4 & 77.4 & 64.1 & - & 64.8 \\
\hline December 1995 ............. & 90.9 & 89.5 & 84.8 & 70.7 & - & 71.1 & 82.2 & 81.3 & 76.8 & 64.6 & - & 64.9 \\
\hline January $1995 \ldots . . . . . . . . . .$. & 85.5 & 85.5 & 77.9 & 71.3 & - & 72.0 & 77.3 & 77.2 & 71.1 & 65.1 & - & 65.7 \\
\hline \multicolumn{13}{|l|}{ Wyoming } \\
\hline January 1996 ............... & - & - & - & - & - & - & - & - & - & - & - & - \\
\hline December 1995 ............ & - & - & - & - & - & - & - & - & - & - & - & - \\
\hline January 1995 ................. & - & - & - & - & - & - & - & - & - & - & - & - \\
\hline \multicolumn{13}{|l|}{ PAD District V } \\
\hline January 1996 & 94.9 & 94.2 & 84.0 & 72.5 & NA & 79.9 & 80.6 & 80.2 & 73.5 & 63.2 & NA & 69.3 \\
\hline December 1995 ............. & 93.5 & 93.1 & 83.1 & 74.3 & $w$ & 80.1 & 79.3 & 79.1 & 73.1 & 65.2 & 59.0 & 69.8 \\
\hline January 1995 ................ & 95.8 & 95.1 & 83.4 & 72.6 & $w$ & 79.8 & 81.2 & 80.7 & 72.4 & 63.2 & 57.5 & 68.6 \\
\hline \multicolumn{13}{|l|}{ Alaska } \\
\hline January 1996 & $w$ & 110.1 & 105.9 & 95.0 & - & 104.0 & $w$ & 94.8 & 96.9 & 83.8 & $w$ & 92.3 \\
\hline December 1995 ............. & w & 108.9 & 104.7 & 94.5 & - & 103.2 & $w$ & 93.9 & 95.9 & 82.8 & - & 92.8 \\
\hline January 1995 ................. & $w$ & 109.2 & 106.7 & $W$ & - & 106.3 & 104.6 & 99.4 & 98.9 & $w$ & - & 97.7 \\
\hline \multicolumn{13}{|l|}{ Arizona } \\
\hline January 1996 .................. & 96.2 & 95.8 & 86.5 & 79.7 & $W$ & 84.7 & 81.8 & 81.3 & 76.7 & 69,5 & $w$ & 74.5 \\
\hline December 1995 ........... & 92.8 & 92.5 & 84.1 & 79.6 & - & 83.0 & 78.0 & 77.7 & 74.7 & 69.3 & - & 73.1 \\
\hline January $1995 \ldots . . . . . . . . . . .$. & 95.7 & 95.6 & 84.4 & 77.8 & - & 83.2 & 81.0 & 80.9 & 75.4 & 69.7 & - & 74.0 \\
\hline January 1996 ................. & 90.8 & 90.2 & 82.0 & 69.5 & $w$ & 77.3 & 76.6 & 76.5 & 71.5 & 60.6 & $w$ & 66.7 \\
\hline December 1995 ............. & 89.4 & 89.2 & 80.8 & 71.7 & $W$ & 77.5 & 75.7 & 75.8 & 70.8 & 62.9 & 59.0 & 67.3 \\
\hline 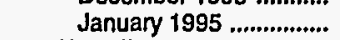 & 93.6 & 92.7 & 82.5 & 71.1 & $w$ & 78.2 & 79.0 & 78.5 & 71.8 & 61.7 & 57.5 & 67.1 \\
\hline \multicolumn{13}{|l|}{ Hawail } \\
\hline January 1996 ................. & - & - & - & - & - & - & - & - & - & - & - & - \\
\hline December 1995 ............ & - & - & - & - & - & - & - & - & - & - & - & - \\
\hline January 1995 & - & - & - & - & - & - & - & - & - & - & - & - \\
\hline \multicolumn{13}{|l|}{ Nevada } \\
\hline January 1996 ................. & 103.3 & 103.3 & 83.5 & 76.8 & - & 82.0 & 92.2 & 91.7 & 72.7 & 69.3 & - & 71.7 \\
\hline December 1995 ............ & 102.0 & 102.0 & 82.2 & 77.5 & - & 81.1 & 90.8 & 90.4 & 71.5 & 70.4 & $W$ & 70.6 \\
\hline $\begin{array}{l}\text { January } 1995 \ldots . . . . . . . . . . . \\
\text { Oregon }\end{array}$ & 103.5 & 102.9 & 81.6 & 75.0 & - & 79.6 & 93.0 & 92.2 & 69.1 & 67.4 & $\ddot{w}$ & 68.5 \\
\hline January 1996 ................ & 97.8 & 96.6 & 86.5 & 78.9 & - & 83.9 & 82.7 & 82.1 & 73.5 & 67.0 & - & 71.1 \\
\hline December 1995 ............ & 98.5 & 97.5 & 87.6 & 81.2 & - & 85.4 & 82.9 & 82.7 & 74.7 & 69.3 & - & 72.7 \\
\hline \multirow{2}{*}{\multicolumn{13}{|c|}{ Washington }} \\
\hline & & & & & & & & & & & & \\
\hline December 1995 ............... & $\begin{array}{r}59.3 \\
100.1\end{array}$ & $\begin{array}{r}99.2 \\
100.1\end{array}$ & 91.3 & $\begin{array}{l}75.8 \\
77.5\end{array}$ & $\overline{-}$ & $\begin{array}{l}86.4 \\
87.4\end{array}$ & $\begin{array}{l}83.0 \\
84.0\end{array}$ & $\begin{array}{l}82.8 \\
83.8\end{array}$ & $\begin{array}{l}78.3 \\
79.2\end{array}$ & $\begin{array}{l}65.7 \\
67.7\end{array}$ & $\overline{-}$ & $\begin{array}{l}74.3 \\
75.5\end{array}$ \\
\hline January 1995 .................. & 97.8 & 97.5 & 86.8 & 74.9 & - & 84.4 & 82.1 & 81.9 & 74.4 & 65.0 & - & 72.0 \\
\hline
\end{tabular}

Dash $(-)=$ No data reported.

NA $=$ Not available.

$W=$ Withheld to avoid disclosure of individual company data.

a Includes sales through retall outlets as well as all direct sales to end users that were not made through company-operated retall outlets, e.g., sales to agricultural customers, commerclal sales, and Industrial sales.

Notes: Values shown for the current month are preliminary. Values shown for previous months are revised. Data are final upon publication in the Petroleum Markoting Annual.

Sources: Energy Information Administration Forms ElA-782A, "Refiners'Gas Plant Operators' Monthly Petroleum Product Sales Report," and EIA-782B,

"Resellers'/Retailers' Monthly Petroleum Product Sales Report." 
Table 34. Reformulated Motor Gasoline Prices by Grade, Sales Type, PAD District, and State

(Cents per Gallon Excluding Taxes)

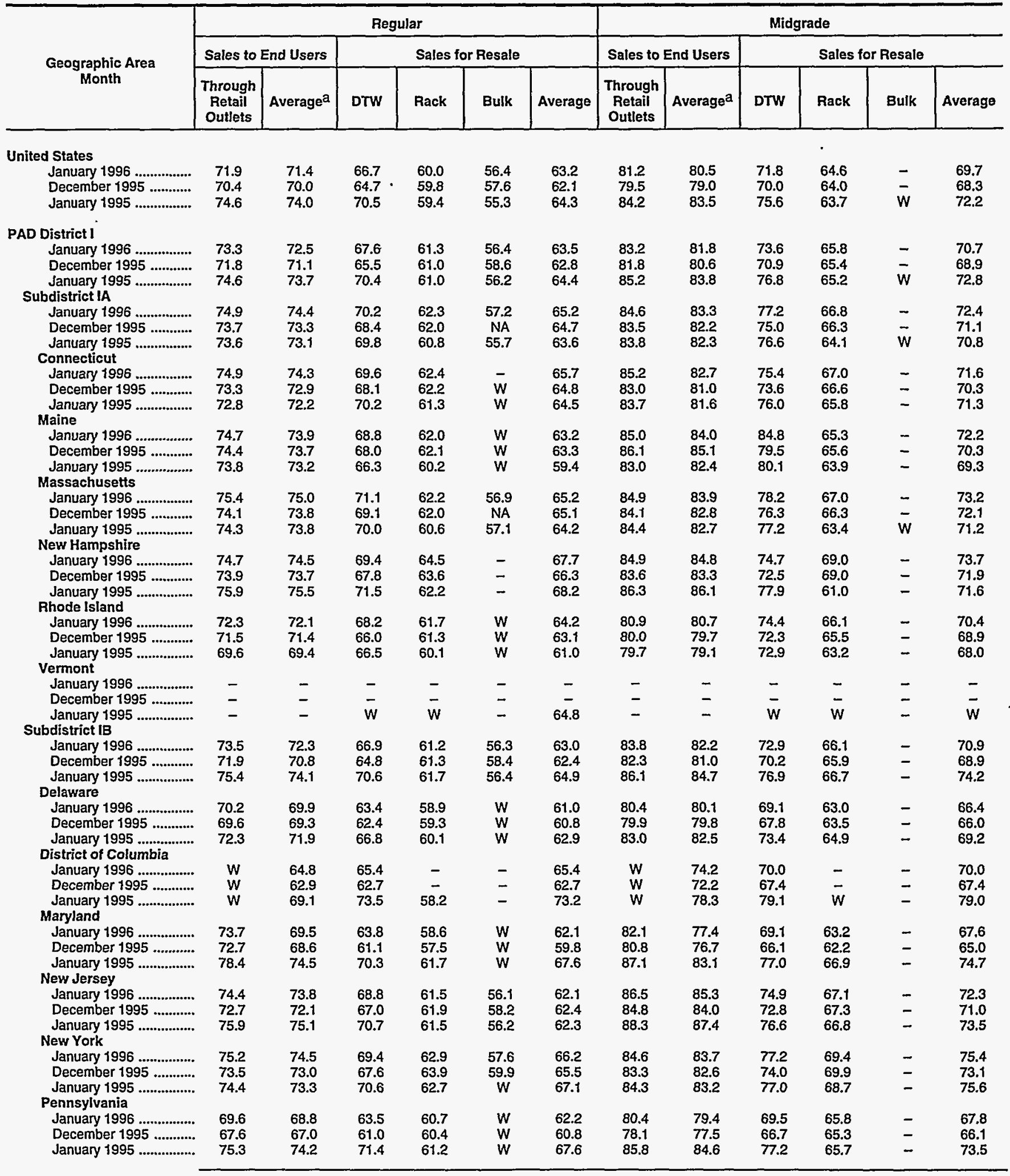

See footnotes at end of table. 
Table 34. Reformulated Motor Gasoline Prices by Grade, Sales Type, PAD District, and State

(Cents per Gallon Excluding Taxes) - Continued

\begin{tabular}{|c|c|c|c|c|c|c|c|c|c|c|c|c|}
\hline \multirow{3}{*}{$\begin{array}{l}\text { Geographic Area } \\
\text { Month }\end{array}$} & \multicolumn{6}{|c|}{ Premium } & \multicolumn{6}{|c|}{ All Grades } \\
\hline & \multicolumn{2}{|c|}{ Sales to End Users } & \multicolumn{4}{|c|}{ Sales for Resale } & \multicolumn{2}{|c|}{ Sales to End Users } & \multicolumn{4}{|c|}{ Sales for Resale } \\
\hline & $\begin{array}{l}\text { Through } \\
\text { Retail } \\
\text { Outlets }\end{array}$ & Average $\mathrm{a}^{\mathrm{a}}$ & DTW & Rack & Bulk & Average & $\begin{array}{c}\text { Through } \\
\text { Retail } \\
\text { Outlets }\end{array}$ & Average $\mathrm{a}^{\mathrm{a}}$ & DTW & Rack & Bulk & Average \\
\hline \multicolumn{13}{|l|}{ United States } \\
\hline $\begin{array}{l}\text { January } 1996 \ldots . . . . . . . . . . . . \\
\text { December } 1995 \text {........... } \\
\text { January } 1995 \ldots . . . . . . . . . .\end{array}$ & $\begin{array}{l}89.9 \\
88.3 \\
93.5\end{array}$ & $\begin{array}{l}88.9 \\
87.5 \\
92.4\end{array}$ & $\begin{array}{l}79.8 \\
78.1 \\
84.3\end{array}$ & $\begin{array}{l}69.8 \\
70.1 \\
69.1\end{array}$ & $\begin{array}{l}62.3 \\
64.4 \\
58.5\end{array}$ & $\begin{array}{l}75.8 \\
74.7 \\
78.0\end{array}$ & $\begin{array}{l}77.4 \\
75.9 \\
80.3\end{array}$ & $\begin{array}{l}76.7 \\
75.4 \\
79.5\end{array}$ & $\begin{array}{l}71.2 \\
69.4 \\
75.3\end{array}$ & $\begin{array}{l}62.7 \\
62.6 \\
62.0\end{array}$ & $\begin{array}{l}57.6 \\
59.1 \\
55.9\end{array}$ & $\begin{array}{l}67.3 \\
66.2 \\
68.8\end{array}$ \\
\hline \multicolumn{13}{|l|}{ PAD District I } \\
\hline 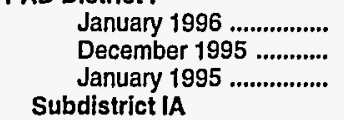 & $\begin{array}{l}91.6 \\
90.0 \\
94.3\end{array}$ & $\begin{array}{l}90.1 \\
88.9 \\
92.8\end{array}$ & $\begin{array}{l}81.2 \\
78.8 \\
84.8\end{array}$ & $\begin{array}{l}70.9 \\
71.5 \\
70.4\end{array}$ & $\begin{array}{l}62.3 \\
65.2 \\
58.6\end{array}$ & $\begin{array}{l}76.3 \\
75.2 \\
77.7\end{array}$ & $\begin{array}{l}79.2 \\
77.9 \\
81.0\end{array}$ & $\begin{array}{l}78.1 \\
76.9 \\
79.9\end{array}$ & $\begin{array}{l}72.9 \\
70.7 \\
76.0\end{array}$ & $\begin{array}{l}64.1 \\
64.1 \\
63.8\end{array}$ & $\begin{array}{l}57.7 \\
60.5 \\
56.8\end{array}$ & $\begin{array}{l}68.0 \\
67.1 \\
69.2\end{array}$ \\
\hline 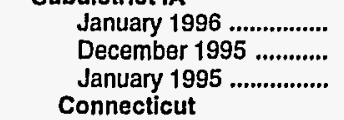 & $\begin{array}{l}93.1 \\
92.0 \\
93.4\end{array}$ & $\begin{array}{l}91.7 \\
90.8 \\
91.9\end{array}$ & $\begin{array}{l}83.4 \\
81.7 \\
83.7\end{array}$ & $\begin{array}{l}72.0 \\
72.1 \\
70.1\end{array}$ & $\begin{array}{l}W \\
W \\
W\end{array}$ & $\begin{array}{l}77.4 \\
76.5 \\
75.9\end{array}$ & $\begin{array}{l}79.8 \\
78.8 \\
79.2\end{array}$ & $\begin{array}{l}79.1 \\
78.2 \\
78.4\end{array}$ & $\begin{array}{l}74.4 \\
72.6 \\
74.3\end{array}$ & $\begin{array}{l}64.8 \\
64.6 \\
63.1\end{array}$ & $\begin{array}{l}57.5 \\
63.0 \\
56.2\end{array}$ & $\begin{array}{l}68.7 \\
68.1 \\
67.2\end{array}$ \\
\hline $\begin{array}{l}\text { January } 1996 \ldots . . . . . . . . . . . . \\
\text { December } 1995 \text {............ } \\
\text { January } 1995 \text {................ } \\
\text { Maine }\end{array}$ & $\begin{array}{l}94.2 \\
92.7 \\
93.4\end{array}$ & $\begin{array}{l}92.7 \\
91.4 \\
92.0\end{array}$ & $\begin{array}{l}82.0 \\
80.5 \\
83.6\end{array}$ & $\begin{array}{l}72.7 \\
72.6 \\
72.8\end{array}$ & $\bar{w}$ & $\begin{array}{l}77.4 \\
75.7 \\
78.1\end{array}$ & $\begin{array}{l}80.1 \\
78.6 \\
78.6\end{array}$ & $\begin{array}{l}79.1 \\
77.9 \\
77.7\end{array}$ & $\begin{array}{l}73.4 \\
71.9 \\
74.3\end{array}$ & $\begin{array}{l}65.1 \\
65.0 \\
64.5\end{array}$ & $\begin{array}{l}\bar{w} \\
W\end{array}$ & $\begin{array}{l}69.1 \\
68.2 \\
68.4\end{array}$ \\
\hline $\begin{array}{l}\text { January } 1996 \text {............... } \\
\text { December } 1995 \text {........... } \\
\text { January } 1995 \text {................ } \\
\text { Massachusetts }\end{array}$ & $\begin{array}{l}92.2 \\
92.1 \\
90.8\end{array}$ & $\begin{array}{l}90.4 \\
90.6 \\
89.7\end{array}$ & $\begin{array}{l}80.2 \\
79.4 \\
78.7\end{array}$ & $\begin{array}{l}70.8 \\
71.8 \\
70.2\end{array}$ & $\overline{-}$ & $\begin{array}{l}72.6 \\
73.4 \\
71.7\end{array}$ & $\begin{array}{l}78.7 \\
78.8 \\
77.6\end{array}$ & $\begin{array}{l}77.7 \\
78.0 \\
76.9\end{array}$ & $\begin{array}{l}74.1 \\
72.0 \\
71.4\end{array}$ & $\begin{array}{l}63.9 \\
64.2 \\
62.3\end{array}$ & $\begin{array}{l}W \\
W \\
W\end{array}$ & $\begin{array}{l}66.0 \\
65.9 \\
62.1\end{array}$ \\
\hline $\begin{array}{l}\text { January } 1996 \text {............... } \\
\text { December } 1995 \text {.......... } \\
\text { January } 1995 \text {................ } \\
\text { New Hampshire }\end{array}$ & $\begin{array}{l}93.6 \\
92.5 \\
94.5\end{array}$ & $\begin{array}{l}91.9 \\
91.1 \\
92.6\end{array}$ & $\begin{array}{l}85.0 \\
83.1 \\
84.6\end{array}$ & $\begin{array}{l}71.8 \\
71.9 \\
68.8\end{array}$ & $\begin{array}{l}W \\
W \\
W\end{array}$ & $\begin{array}{l}78.1 \\
77.6 \\
75.4\end{array}$ & $\begin{array}{l}80.4 \\
79.4 \\
80.2\end{array}$ & $\begin{array}{l}79.8 \\
78.8 \\
79.3\end{array}$ & $\begin{array}{l}75.5 \\
73.7 \\
74.9\end{array}$ & $\begin{array}{l}64.8 \\
64.5 \\
62.7\end{array}$ & $\begin{array}{l}57.2 \\
62.2 \\
57.8\end{array}$ & $\begin{array}{l}68.9 \\
68.7 \\
67.7\end{array}$ \\
\hline $\begin{array}{l}\text { January } 1996 \text {................ } \\
\text { December } 1995 \text {........... } \\
\text { January } 1995 \text {................ } \\
\text { Rhode Island }\end{array}$ & $\begin{array}{l}91.9 \\
91.1 \\
94.9\end{array}$ & $\begin{array}{l}91.7 \\
91.0 \\
94.7\end{array}$ & $\begin{array}{l}81.4 \\
80.1 \\
83.0\end{array}$ & $\begin{array}{l}74.0 \\
74.2 \\
70.5\end{array}$ & 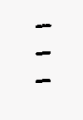 & $\begin{array}{l}79.1 \\
78.3 \\
78.8\end{array}$ & $\begin{array}{l}79.3 \\
78.4 \\
81.0\end{array}$ & $\begin{array}{l}79.1 \\
78.2 \\
80.7\end{array}$ & $\begin{array}{l}72.8 \\
71.1 \\
74.8\end{array}$ & $\begin{array}{l}66.7 \\
66.1 \\
63.6\end{array}$ & $\begin{array}{l}- \\
-\end{array}$ & $\begin{array}{l}70.8 \\
69.5 \\
70.8\end{array}$ \\
\hline $\begin{array}{l}\text { January } 1996 \text {................ } \\
\text { December } 1995 \text {........... } \\
\text { January } 1995 \text {................ } \\
\text { Vermont }\end{array}$ & $\begin{array}{l}88.8 \\
87.9 \\
86.5\end{array}$ & $\begin{array}{l}88.2 \\
87.5 \\
85.9\end{array}$ & $\begin{array}{l}81.4 \\
79.4 \\
80.3\end{array}$ & $\begin{array}{l}71.8 \\
71.4 \\
68.5\end{array}$ & $\overline{-}$ & $\begin{array}{l}75.9 \\
74.8 \\
73.4\end{array}$ & $\begin{array}{l}77.0 \\
76.2 \\
75.0\end{array}$ & $\begin{array}{l}76.7 \\
76.0 \\
74.6\end{array}$ & $\begin{array}{l}72.3 \\
70.3 \\
71.1\end{array}$ & $\begin{array}{l}64.5 \\
64.2 \\
62.4\end{array}$ & $\begin{array}{l}W \\
W \\
W\end{array}$ & $\begin{array}{l}67.7 \\
66.5 \\
64.2\end{array}$ \\
\hline $\begin{array}{l}\text { January } 1996 \text {............... } \\
\text { December } 1995 \ldots . . . \ldots \ldots . . . \\
\text { January } 1995 \ldots . . . . . . . . . \\
\text { Subdistrict 1B }\end{array}$ & $\begin{array}{l}- \\
-\end{array}$ & $\overline{-}$ & $\bar{w}$ & $\overline{-}$ & $\overline{-}$ & $\overline{-}$ & $\begin{array}{l}- \\
-\end{array}$ & $\begin{array}{l}- \\
-\end{array}$ & $\overline{-}$ & $\bar{w}$ & $\begin{array}{l}- \\
-\end{array}$ & $\begin{array}{c}- \\
\overline{-} \\
65.2\end{array}$ \\
\hline $\begin{array}{l}\text { January } 1996 \ldots . . . . . . . . . . . . . \\
\text { December } 1995 \\
\text { January } 1995 \text {.................... } \\
\text { Delaware }\end{array}$ & $\begin{array}{l}92.2 \\
90.5 \\
94.9\end{array}$ & $\begin{array}{l}90.6 \\
89.3 \\
93.3\end{array}$ & $\begin{array}{l}81.2 \\
78.8 \\
85.0\end{array}$ & $\begin{array}{l}70.5 \\
71.9 \\
70.8\end{array}$ & $\begin{array}{l}62.5 \\
65.3 \\
58.6\end{array}$ & $\begin{array}{l}76.4 \\
75.3 \\
78.2\end{array}$ & $\begin{array}{l}80.3 \\
78.8 \\
82.4\end{array}$ & $\begin{array}{l}78.7 \\
77.5 \\
80.8\end{array}$ & $\begin{array}{l}72.9 \\
70.7 \\
76.7\end{array}$ & $\begin{array}{l}64.3 \\
64.8 \\
64.7\end{array}$ & $\begin{array}{l}57.8 \\
60.3 \\
57.0\end{array}$ & $\begin{array}{l}68.2 \\
67.4 \\
70.2\end{array}$ \\
\hline 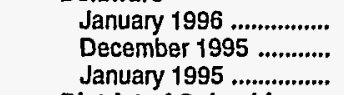 & $\begin{array}{l}89.2 \\
88.6 \\
92.4\end{array}$ & $\begin{array}{l}88.4 \\
87.9 \\
91.7\end{array}$ & $\begin{array}{l}77.1 \\
76.0 \\
80.9\end{array}$ & $\begin{array}{l}68.4 \\
69.1 \\
71.1\end{array}$ & - & $\begin{array}{l}72.8 \\
72.7 \\
75.7\end{array}$ & $\begin{array}{l}75.4 \\
74.9 \\
77.7\end{array}$ & $\begin{array}{l}75.0 \\
74.6 \\
77.1\end{array}$ & $\begin{array}{l}67.1 \\
66.2 \\
70.8\end{array}$ & $\begin{array}{l}61.5 \\
61.9 \\
63.0\end{array}$ & $\begin{array}{l}W \\
W \\
W\end{array}$ & $\begin{array}{l}64.2 \\
63.9 \\
66.3\end{array}$ \\
\hline $\begin{array}{l}\text { District of Columbia } \\
\text { January } 1996 \text {............... } \\
\text { December } 1995 \text {........... } \\
\text { January } 1995 . . . . . . . . . . . . . \\
\text { Maryland }\end{array}$ & $\begin{array}{l}W \\
W \\
W\end{array}$ & $\begin{array}{l}77.5 \\
74.0 \\
83.9\end{array}$ & $\begin{array}{l}77.2 \\
74.6 \\
87.1\end{array}$ & $\overline{-}$ & $\begin{array}{l}- \\
-\end{array}$ & $\begin{array}{l}77.2 \\
74.6 \\
87.0\end{array}$ & $\begin{array}{l}W \\
W \\
W\end{array}$ & $\begin{array}{l}70.5 \\
68.1 \\
74.5\end{array}$ & $\begin{array}{l}72.0 \\
69.6 \\
80.9\end{array}$ & $\overline{-}$ & $\begin{array}{l}- \\
-\end{array}$ & $\begin{array}{l}72.0 \\
69.6 \\
80.8\end{array}$ \\
\hline $\begin{array}{l}\text { January } 1996 . . . . . . . . . . . . . . \\
\text { December } 1995 \text {............ } \\
\text { January } 1995 \text {................ } \\
\text { New Jersey }\end{array}$ & $\begin{array}{l}88.9 \\
87.1 \\
94.4\end{array}$ & $\begin{array}{l}84.4 \\
83.5 \\
90.3\end{array}$ & $\begin{array}{l}75.5 \\
72.5 \\
84.1\end{array}$ & $\begin{array}{l}69.1 \\
67.7 \\
72.7\end{array}$ & $\bar{w}$ & $\begin{array}{l}73.9 \\
71.2 \\
81.6\end{array}$ & $\begin{array}{l}78.5 \\
77.3 \\
83.2\end{array}$ & $\begin{array}{l}74.0 \\
73.0 \\
79.0\end{array}$ & $\begin{array}{l}68.2 \\
65.5 \\
75.5\end{array}$ & $\begin{array}{l}61.8 \\
60.7 \\
65.1\end{array}$ & $\begin{array}{l}W \\
60.3 \\
W\end{array}$ & $\begin{array}{l}66.4 \\
64.0 \\
72.6\end{array}$ \\
\hline $\begin{array}{l}\text { January } 1996 \text {................ } \\
\text { December } 1995 \text {............ } \\
\text { January } 1995 \text {................ } \\
\text { New York }\end{array}$ & $\begin{array}{l}94.7 \\
93.1 \\
97.2\end{array}$ & $\begin{array}{l}93.2 \\
92.2 \\
95.6\end{array}$ & $\begin{array}{l}82.9 \\
81.0 \\
84.7\end{array}$ & $\begin{array}{l}70.7 \\
72.2 \\
69.7\end{array}$ & $\begin{array}{l}62.5 \\
64.8 \\
58.7\end{array}$ & $\begin{array}{l}75.1 \\
74.8 \\
74.4\end{array}$ & $\begin{array}{l}81.9 \\
80.3 \\
83.8\end{array}$ & $\begin{array}{l}81.0 \\
79.6 \\
82.8\end{array}$ & $\begin{array}{l}74.8 \\
73.0 \\
76.7\end{array}$ & $\begin{array}{l}64.7 \\
65.4 \\
64.4\end{array}$ & $\begin{array}{l}57.6 \\
60.0 \\
56.8\end{array}$ & $\begin{array}{l}66.9 \\
67.0 \\
67.0\end{array}$ \\
\hline $\begin{array}{l}\text { January } 1996 \text {................ } \\
\text { December } 1995 \text {............ } \\
\text { January } 1995 \text {............... } \\
\text { Pennsylvania }\end{array}$ & $\begin{array}{l}92.3 \\
90.5 \\
92.7\end{array}$ & $\begin{array}{l}91.5 \\
90.0 \\
91.7\end{array}$ & $\begin{array}{l}84.2 \\
81.8 \\
85.5\end{array}$ & $\begin{array}{l}70.9 \\
74.1 \\
71.2\end{array}$ & $\begin{array}{l}62.6 \\
67.0 \\
W\end{array}$ & $\begin{array}{l}79.7 \\
78.9 \\
80.6\end{array}$ & $\begin{array}{l}82.1 \\
80.6 \\
81.7\end{array}$ & $\begin{array}{l}81.1 \\
79.9 \\
80.4\end{array}$ & $\begin{array}{l}76.8 \\
74.7 \\
78.0\end{array}$ & $\begin{array}{l}65.8 \\
67.6 \\
65.6\end{array}$ & $\begin{array}{c}59.7 \\
62.6 \\
W\end{array}$ & $\begin{array}{l}72.5 \\
71.6 \\
73.4\end{array}$ \\
\hline $\begin{array}{l}\text { January } 1996 \text {............... } \\
\text { December } 1995 \text {........... } \\
\text { January } 1995 \text {............... }\end{array}$ & $\begin{array}{l}87.5 \\
85.0 \\
94.8\end{array}$ & $\begin{array}{l}86.3 \\
84.2 \\
93.2\end{array}$ & $\begin{array}{l}76.9 \\
74.3 \\
86.3\end{array}$ & $\begin{array}{l}71.6 \\
71.6 \\
71.9\end{array}$ & $\begin{array}{l}W \\
W \\
W\end{array}$ & $\begin{array}{l}75.0 \\
73.3 \\
81.1\end{array}$ & $\begin{array}{l}76.2 \\
74.1 \\
82.0\end{array}$ & $\begin{array}{l}75.0 \\
73.2 \\
80.6\end{array}$ & $\begin{array}{l}68.3 \\
65.9 \\
76.4\end{array}$ & $\begin{array}{l}63.8 \\
63.6 \\
64.1\end{array}$ & $\begin{array}{c}W \\
62.9 \\
W\end{array}$ & $\begin{array}{l}66.3 \\
65.0 \\
72.0\end{array}$ \\
\hline
\end{tabular}

See foolnotes at end of table. 
Table 34. Reformulated Motor Gasoline Prices by Grade, Sales Type, PAD District, and State

(Cents per Gallon Excluding Taxes) — Continued

\begin{tabular}{|c|c|c|c|c|c|c|c|c|c|c|c|c|}
\hline \multirow{3}{*}{$\begin{array}{c}\text { Geographic Area } \\
\text { Month }\end{array}$} & \multicolumn{6}{|c|}{ Regular } & \multicolumn{6}{|c|}{ Midgrade } \\
\hline & \multicolumn{2}{|c|}{ Sales to End Users } & \multicolumn{4}{|c|}{ Sales for Resale } & \multicolumn{2}{|c|}{ Sales to End Users } & \multicolumn{4}{|c|}{ Sales for Resale } \\
\hline & $\begin{array}{l}\text { Through } \\
\text { Retail } \\
\text { Outlets }\end{array}$ & Averagea & DTW & Rack & Bulk & Average & $\begin{array}{c}\text { Through } \\
\text { Retail } \\
\text { Outlets }\end{array}$ & Average $\mathrm{a}^{\mathrm{a}}$ & DTW & Rack & Bulk & Average \\
\hline \multicolumn{13}{|l|}{ Subdistrict IC } \\
\hline $\begin{array}{l}\text { January } 1996 \ldots \ldots . . . . . . . . . \\
\text { December } 1995\end{array}$ & 68.5 & $\begin{array}{l}68.1 \\
66.5\end{array}$ & 63.8 & $\begin{array}{l}57.9 \\
56.5\end{array}$ & W & 60.5 & 78.7 & $\begin{array}{l}78.0 \\
765\end{array}$ & 69.0 & 62.6 & - & 66.2 \\
\hline $\begin{array}{l}\text { December } 1995 \text {.............. } \\
\text { January } 1995 \text {................ }\end{array}$ & $\begin{array}{l}66.9 \\
74.6\end{array}$ & $\begin{array}{l}66.5 \\
73.9\end{array}$ & $\begin{array}{l}60.8 \\
70.7\end{array}$ & $\begin{array}{l}56.5 \\
59.1\end{array}$ & $\bar{w}$ & $\begin{array}{l}58.4 \\
64.6\end{array}$ & $\begin{array}{l}77.2 \\
85.5\end{array}$ & $\begin{array}{l}76.5 \\
84.5\end{array}$ & $\begin{array}{l}65.9 \\
76.7\end{array}$ & $\begin{array}{l}61.5 \\
63.6\end{array}$ & $\bar{w}$ & $\begin{array}{l}64.7 \\
71.5\end{array}$ \\
\hline \multicolumn{13}{|l|}{ Florida } \\
\hline $\begin{array}{l}\text { January } 1996 \text {................. } \\
\text { December } 1995 \text {............ }\end{array}$ & - & - & - & - & - & - & - & - & - & - & - & - \\
\hline & $\overline{-}$ & $\bar{z}$ & $\bar{z}$ & $z$ & $\begin{array}{l}- \\
-\end{array}$ & $z$ & $\overline{-}$ & $\overline{-}$ & $\overline{-}$ & $\overline{-}$ & $\overline{-}$ & $\overline{-}$ \\
\hline \multicolumn{13}{|l|}{$\begin{array}{l}\text { January } 1995 \\
\text { Georgia }\end{array}$} \\
\hline $\begin{array}{l}\text { January } 1996 . . . . . . . . . . . . . . \\
\text { December } 1995 . . . . . . . . .\end{array}$ & - & - & - & - & - & - & - & - & - & - & - & - \\
\hline December 1995 .............. & - & - & - & - & - & - & - & - & - & - & - & - \\
\hline $\begin{array}{l}\text { January } 1995 \text {........................... } \\
\text { North Carolina }\end{array}$ & - & - & \multicolumn{3}{|c|}{ North Carolina } & - & - & - & - & - & - & - \\
\hline January 1996 ..................... & - & - & - & - & _ & - & - & - & _- & - & - & - \\
\hline December 1995 ............. & - & - & - & - & - & - & - & - & - & - & - & - \\
\hline January 1995 & - & - & - & w & - & $w$ & - & - & - & - & - & - \\
\hline $\begin{array}{l}\text { South Carolina } \\
\text { January } 1996\end{array}$ & - & - & - & - & - & - & _ & _- & - & - & - & 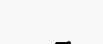 \\
\hline December 1995 ................. & - & - & - & - & - & - & - & - & $=$ & $\overline{-}$ & $\overline{-}$ & $\overline{-}$ \\
\hline 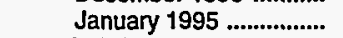 & - & - & - & - & - & - & - & - & - & - & - & - \\
\hline Virginia & & & & & & & & & & & & \\
\hline January 1996 .................. & 68.5 & 68.1 & 63.8 & 57.9 & $w$ & 60.5 & 78.7 & 78.0 & 69.0 & 62.6 & - & 66.2 \\
\hline December 1995 .............. & 66.9 & 66.5 & 60.8 & 56.5 & $\overrightarrow{-}$ & 58.5 & 77.2 & 76.5 & 65.9 & 61.5 & - & 64.1 \\
\hline January 1995 ..................... & 74.6 & 73.9 & 70.7 & 59.1 & w & 64.6 & 85.5 & 84.5 & 76.7 & 63.6 & W & 71.5 \\
\hline West Virginia & & & & & & & & & & & & \\
\hline 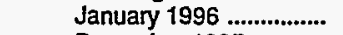 & - & - & NA & - & - & NA & - & - & - & - & - & - \\
\hline December 1995 .............. & - & - & NA & - & - & NA & - & - & - & - & - & - \\
\hline January 1995 ................... & - & W & - & - & - & - & - & - & - & - & - & - \\
\hline PAD District II & & & & & & & & & & & & \\
\hline 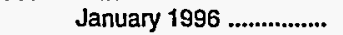 & 77.2 & 76.4 & 70.5 & 59.9 & - & 64.9 & 86.3 & 86.1 & 77.9 & 63.4 & - & 74.0 \\
\hline December 1995 ............. & 74.9 & 74.2 & 69.5 & 59.7 & - & 64.3 & 84.3 & 84.1 & 76.2 & 63.3 & - & 72.7 \\
\hline 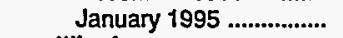 & 73.4 & 72.8 & 68.7 & 57.3 & w & 62.9 & 81.6 & 81.4 & 73.4 & 60.9 & - & 70.2 \\
\hline Illinois & & & & & & & & & & & & \\
\hline January 1996 ................... & 78.1 & 77.0 & 72.8 & 59.7 & - & 66.9 & 87.1 & 87.0 & 79.2 & 62.7 & - & 76.7 \\
\hline December 1995 .............. & 75.5 & 74.6 & 71.9 & 58.9 & - & 66.1 & 85.0 & 84.7 & 77.6 & 62.0 & - & 75.2 \\
\hline January 1995 .................. & 74.1 & 73.4 & 71.2 & 56.6 & - & 65.1 & 82.8 & 82.5 & 74.6 & 61.1 & - & 72.8 \\
\hline Indiana & & & & & & & & & & & & \\
\hline 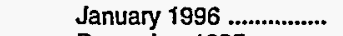 & 72.1 & 71.6 & 67.1 & 60.5 & - & 62.3 & 83.0 & 82.7 & 73.4 & 64.6 & - & 68.8 \\
\hline December 1995 ............. & 71.0 & 70.5 & 66.3 & 59.8 & - & 61.6 & 81.8 & 81.6 & 72.0 & 64.0 & - & 67.7 \\
\hline January 1995 ....................... & 68.8 & 67.8 & 64.4 & 56.9 & - & 59.1 & 75.2 & 74.5 & 67.4 & 60.2 & - & 66.2 \\
\hline Jowa & & & & & & & & & & & & \\
\hline 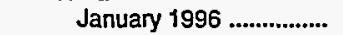 & - & - & - & - & - & - & - & - & - & - & - & - \\
\hline December 1995 .............. & - & - & - & - & - & - & - & - & - & - & - & - \\
\hline January 1995 ................... & - & - & - & - & - & - & - & - & - & - & - & - \\
\hline Kansas & & & & & & & & & & & & \\
\hline January 1996 .................. & - & - & - & - & - & - & - & - & - & - & - & - \\
\hline December 1995 .............. & - & - & - & - & - & - & - & - & - & - & - & - \\
\hline January 1995 ..................... & - & - & - & - & - & - & - & - & - & - & - & - \\
\hline Kentucky & & & & & & & & & & & & \\
\hline 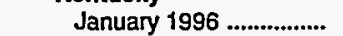 & 75.7 & 74.8 & 64.8 & 58.8 & - & 60.4 & 84.9 & 84.7 & 70.8 & 62.8 & - & 65.6 \\
\hline December 1995 ............. & 73.3 & 72.5 & 63.5 & 59.0 & - & 60.1 & 82.9 & 82.7 & 69.6 & 63.3 & - & 65.7 \\
\hline 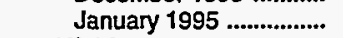 & 69.0 & 68.5 & 62.1 & 60.0 & $w$ & 60.4 & 78.9 & 78.7 & 67.2 & 64.0 & - & 65.0 \\
\hline Michigan & & & & & & & & & & & & \\
\hline January 1996 .................. & - & - & - & - & - & - & - & - & - & - & - & - \\
\hline December 1995 ............ & - & - & - & - & - & - & - & - & - & - & - & - \\
\hline January 1995 ................. & - & - & - & - & - & - & - & - & - & - & - & - \\
\hline Minnesota & & & & & & & & & & & & \\
\hline 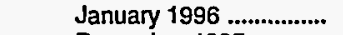 & - & - & - & - & - & - & - & - & - & - & - & - \\
\hline December 1995 .............. & - & - & - & - & - & - & - & - & - & - & - & - \\
\hline 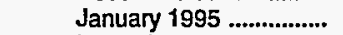 & - & - & - & - & - & - & - & - & - & - & - & - \\
\hline Missouri & & & & & & & & & & & & \\
\hline 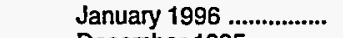 & - & - & - & - & - & - & - & - & - & - & - & - \\
\hline December 1995 .............. & - & - & - & - & - & - & - & - & - & - & - & - \\
\hline January 1995 ....................... & - & - & - & - & - & - & - & - & - & - & - & - \\
\hline
\end{tabular}

See footnotes at end of table. 
Table 34. Reformulated Motor Gasoline Prices by Grade, Sales Type, PAD District, and State

(Cents per Gallon Excluding Taxes) - Continued

\begin{tabular}{|c|c|c|c|c|c|c|c|c|c|c|c|c|}
\hline \multirow{3}{*}{$\begin{array}{c}\text { Geographic Area } \\
\text { Month }\end{array}$} & \multicolumn{6}{|c|}{ Premium } & \multicolumn{6}{|c|}{ All Grades } \\
\hline & \multicolumn{2}{|c|}{ Sales to End Users } & \multicolumn{4}{|c|}{ Sales for Resale } & \multicolumn{2}{|c|}{ Sales to End Users } & \multicolumn{4}{|c|}{ Sales for Resale } \\
\hline & $\begin{array}{c}\text { Through } \\
\text { Retall } \\
\text { Outlets }\end{array}$ & Average $^{\mathrm{a}}$ & DTW & Rack & Bulk & Average & \begin{tabular}{|c|} 
Through \\
Retail \\
Outlets
\end{tabular} & Average ${ }^{a}$ & DTW & Rack & Bulk & Average \\
\hline \multicolumn{13}{|l|}{ Subdistrict IC } \\
\hline $\begin{array}{l}\text { January } 1996 . . . \ldots . . . . . . . . \\
\text { December } 1995 \text {............ } \\
\text { January } 1995 \\
\text { Florida }\end{array}$ & $\begin{array}{l}86.2 \\
84.5 \\
93.7\end{array}$ & $\begin{array}{l}85.4 \\
83.8 \\
92.6\end{array}$ & $\begin{array}{l}76.3 \\
73.2 \\
84.5\end{array}$ & $\begin{array}{l}69.0 \\
67.7 \\
69.9\end{array}$ & $\begin{array}{l}W \\
W \\
-\end{array}$ & $\begin{array}{l}73.2 \\
70.9 \\
78.6\end{array}$ & $\begin{array}{l}74.5 \\
73.0 \\
80.9\end{array}$ & $\begin{array}{l}73.9 \\
72.4 \\
80.0\end{array}$ & $\begin{array}{l}68.7 \\
65.8 \\
76.1\end{array}$ & $\begin{array}{l}61.1 \\
59.9 \\
62.3\end{array}$ & $\begin{array}{l}W \\
W \\
W\end{array}$ & $\begin{array}{l}64.9 \\
62.9 \\
69.6\end{array}$ \\
\hline January 1996 & - & - & - & - & - & - & - & - & - & - & - & - \\
\hline December 1995 ............ & - & - & - & - & - & - & - & - & - & - & - & - \\
\hline $\begin{array}{l}\text { January } 1995 \ldots . . . . . . . . . . . . . \\
\text { Georgia }\end{array}$ & - & - & - & - & - & - & - & - & - & - & - & - \\
\hline January 1996 ................ & - & - & - & - & - & - & - & - & - & - & - & - \\
\hline December 1995 ............ & - & - & - & - & - & - & - & - & - & - & - & - \\
\hline \multicolumn{13}{|l|}{ North Carolina } \\
\hline January 1996 & - & - & - & - & - & - & - & - & - & - & - & - \\
\hline December 1995 ............ & - & - & - & - & - & - & - & - & - & - & - & - \\
\hline $\begin{array}{l}\text { January } 1995 \text {................. } \\
\text { South Carolina }\end{array}$ & - & - & - & $w$ & - & $W$ & - & - & - & $w$ & - & $W$ \\
\hline January 1996 & - & - & - & - & - & - & - & - & - & - & - & - \\
\hline December 1995 ............. & - & - & - & - & - & - & - & - & - & - & - & - \\
\hline 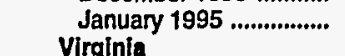 & - & - & \multicolumn{9}{|c|}{ Virginia } & - \\
\hline $\begin{array}{l}\text { January } 1996 \ldots . . . . . . . . . . . . . . . \\
\text { December } 1995 \text {........... } \\
\text { January } 1995 \text {............... } \\
\text { West Virginia }\end{array}$ & $\begin{array}{l}86.2 \\
84.5 \\
93.7\end{array}$ & $\begin{array}{l}85.4 \\
83.8 \\
92.6\end{array}$ & $\begin{array}{l}76.3 \\
73.2 \\
84.5\end{array}$ & $\begin{array}{l}69.0 \\
67.7 \\
69.9\end{array}$ & $\begin{array}{l}W \\
W \\
-\end{array}$ & $\begin{array}{l}73.2 \\
70.9 \\
78.7\end{array}$ & $\begin{array}{l}74.5 \\
73.0 \\
80.9\end{array}$ & $\begin{array}{l}73.9 \\
72.4 \\
80.0\end{array}$ & $\begin{array}{l}68.7 \\
65.8 \\
76.1\end{array}$ & $\begin{array}{l}61.1 \\
59.9 \\
62.3\end{array}$ & $\begin{array}{l}W \\
W \\
W\end{array}$ & $\begin{array}{l}64.9 \\
62.9 \\
69.6\end{array}$ \\
\hline January 1996 & - & - & NA & - & - & NA & - & - & NA & - & - & NA \\
\hline December 1995 ............ & - & - & NA & - & - & NA & - & - & NA & - & - & NA \\
\hline January 1995 ................... & - & $w$ & - & $w$ & - & $w$ & - & $w$ & - & $w$ & - & $w$ \\
\hline \multicolumn{13}{|l|}{ PAD Dlstrict II } \\
\hline January 1996 & 93.9 & 93.1 & 81.6 & 68.5 & - & 76.4 & 81.7 & 80.9 & 74.5 & 61.8 & - & 68.6 \\
\hline December 1995 ........... & 91.2 & 90.6 & 80.9 & 68.3 & - & 75.9 & 79.5 & 78.7 & 73.5 & 61.6 & - & 68.0 \\
\hline 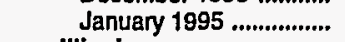 & 89.4 & 88.6 & 80.5 & 66.3 & $w$ & 74.8 & 77.6 & 76.9 & 72.5 & 59.4 & $w$ & 66.6 \\
\hline \multicolumn{13}{|l|}{ Illinois } \\
\hline January 1996 ................ & 94.1 & 93.4 & 83.1 & 68.1 & - & 78.6 & 82.9 & 82.0 & 76.9 & 61.5 & - & 71.2 \\
\hline December 1995 ........... & 91.2 & 90.5 & 82.6 & 67.3 & - & 78.2 & 80.4 & 79.5 & 76.0 & 60.7 & - & 70.4 \\
\hline January 1995 ................. & 90.3 & 89.4 & 82.2 & 65.0 & $w$ & 77.2 & 78.8 & 78.1 & 74.8 & 58.6 & $W$ & 69.3 \\
\hline \multicolumn{13}{|l|}{ Indlana } \\
\hline January 1996 ................ & 90.7 & 89.8 & 78.2 & 69.9 & - & 73.0 & 77.6 & 76.8 & 71.1 & 62.8 & - & 65.5 \\
\hline December 1995 ............. & 89.2 & 88.4 & 78.0 & 69.1 & - & 72.1 & 76.4 & 75.7 & 70.1 & 62.1 & - & 64.6 \\
\hline \multicolumn{13}{|l|}{$\begin{array}{l}\text { January } 1995 \text {................. } \\
\text { lowa }\end{array}$} \\
\hline January 1996 ................. & - & - & - & - & - & - & - & - & - & - & - & - \\
\hline December 1995 ........... & - & - & - & - & - & - & - & - & - & - & - & - \\
\hline January 1995 ................. & - & - & - & - & - & - & - & - & - & - & - & - \\
\hline $\begin{array}{l}\text { Kansas } \\
\text { January } 1996\end{array}$ & & & & & & & & & & & & \\
\hline January 1996 ................. & - & - & - & - & - & - & - & - & - & - & - & - \\
\hline December 1995 ............ & - & - & - & - & - & - & - & - & - & - & - & - \\
\hline $\begin{array}{l}\text { January } 1995 \text {................. } \\
\text { Kentucky }\end{array}$ & - & - & - & - & - & - & - & - & - & - & - & - \\
\hline January 1996 ................ & 94.2 & 94.0 & 77.0 & 68.1 & - & 70.9 & 80.7 & 79.9 & 68.8 & 61.3 & - & 63.4 \\
\hline December 1995 ............ & 91.8 & 91.7 & 76.9 & 68.2 & - & 70.6 & 78.4 & 77.7 & 67.5 & 61.5 & - & 63.2 \\
\hline 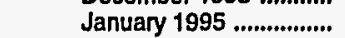 & 88.0 & 87.7 & 74.0 & 70.0 & - & 71.0 & 73.9 & 73.3 & 66.3 & 62.8 & $W$ & 63.5 \\
\hline Michigan & & & & & & & & & & & & \\
\hline January 1996 & - & - & - & - & - & - & - & - & - & - & - & - \\
\hline December 1995 ............ & - & - & - & - & - & - & - & - & - & - & - & - \\
\hline January 1995 & - & - & - & - & - & - & - & - & - & - & - & - \\
\hline Minnesota & & & & & & & & & & & & \\
\hline January 1996 ................ & - & - & - & - & - & - & - & - & - & - & - & - \\
\hline December 1995 ............. & - & - & - & - & - & - & - & - & - & - & - & - \\
\hline January 1995 ................ & - & - & - & - & - & - & - & - & - & - & - & - \\
\hline Missouri & & & & & & & & & & & & \\
\hline January 1996 ................. & - & - & - & - & - & - & - & - & - & - & - & - \\
\hline December 1995 ............ & - & - & - & - & - & - & - & - & - & - & - & - \\
\hline January 1995 ................ & - & - & - & - & - & - & - & - & - & - & - & - \\
\hline
\end{tabular}

See footnotes at end of table. 
Table 34. Reformulated Motor Gasoline Prices by Grade, Sales Type, PAD District, and State

(Cents per Gallon Excluding Taxes) - Continued

\begin{tabular}{|c|c|c|c|c|c|c|c|c|c|c|c|c|}
\hline \multirow{3}{*}{$\begin{array}{c}\text { Geographic Area } \\
\text { Month }\end{array}$} & \multicolumn{6}{|c|}{ Regular } & \multicolumn{6}{|c|}{ Midgrade } \\
\hline & \multicolumn{2}{|c|}{ Sales to End Users } & \multicolumn{4}{|c|}{ Sales for Resale } & \multicolumn{2}{|c|}{ Sales to End Users } & \multicolumn{4}{|c|}{ Sales for Resale } \\
\hline & $\begin{array}{c}\text { Through } \\
\text { Retail } \\
\text { Outlets }\end{array}$ & Averagea & DTW & Rack & Bulk & Average & $\begin{array}{c}\text { Through } \\
\text { Retail } \\
\text { Outlets }\end{array}$ & Average $^{a}$ & DTW & Rack & Bulk & Averago \\
\hline \multicolumn{13}{|l|}{ Nebraska } \\
\hline January 1996 & - & - & - & - & - & - & - & - & - & - & - & - \\
\hline December 1995 ............... & - & - & - & - & - & - & - & - & - & - & - & - \\
\hline January 1995 .................. & - & - & - & - & - & - & - & - & - & - & - & - \\
\hline \multicolumn{13}{|l|}{ North Dakota } \\
\hline January 1996 & - & - & - & - & - & - & - & - & - & - & - & - \\
\hline December 1995 & - & - & - & $\cdot-$ & - & - & - & - & - & - & - & - \\
\hline 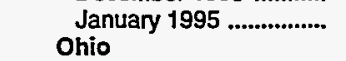 & - & - & - & - & - & - & - & - & - & - & - & - \\
\hline January 1996 ................ & - & - & - & - & - & - & - & - & - & - & - & - \\
\hline December 1995 ............ & - & - & - & - & - & - & - & - & - & - & - & - \\
\hline & \multicolumn{12}{|c|}{ Oklahoma } \\
\hline January 1996 ................. & - & - & - & - & - & - & - & - & - & - & - & - \\
\hline December 1995 ............ & - & - & - & - & - & - & - & - & - & - & - & - \\
\hline $\begin{array}{l}\text { January } 1995 \ldots . . . . . . . . . . . . . . \\
\text { South Dakota }\end{array}$ & - & - & - & - & - & - & - & - & - & - & - & - \\
\hline January 1996 & - & - & - & - & - & - & - & - & - & - & - & - \\
\hline December 1995 ............. & - & - & - & - & - & - & - & - & - & - & - & - \\
\hline January 1995 .................. & - & - & - & - & - & - & - & - & - & - & - & - \\
\hline \multicolumn{13}{|l|}{ Tennessee } \\
\hline January 1996 ................ & - & - & - & - & - & - & - & - & - & - & - & - \\
\hline December 1995 ............. & - & - & - & - & - & - & - & - & - & - & - & - \\
\hline $\begin{array}{l}\text { January } 1995 \text {................. } \\
\text { Wisconsin }\end{array}$ & - & - & - & - & - & - & - & - & - & - & - & - \\
\hline $\begin{array}{l}\text { Wisconsin } \\
\text { January } 1996 . . . . . . . . . . . . . . .\end{array}$ & 77.9 & 77.4 & 66.5 & 60.8 & - & 63.4 & 86.6 & 86.5 & 74.2 & 64.8 & - & Wisconsin \\
\hline 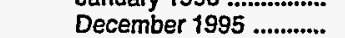 & 76.0 & 75.5 & 65.6 & 61.6 & - & 63.4 & 84.9 & 84.8 & 72.1 & 65.6 & - & $\begin{array}{l}70.9 \\
69.8\end{array}$ \\
\hline January 1995 .................. & 75.0 & 74.5 & 64.4 & 57.2 & - & 60.6 & 82.7 & 82.2 & 70.5 & 56.2 & - & 64.0 \\
\hline PAD District III & & & & & & & & & & & & \\
\hline January 1996 & 65.9 & 65.7 & 61.2 & 55.6 & 54.6 & 57.9 & 77.7 & 77.3 & 66.9 & 60.7 & - & 64.2 \\
\hline December 1995 .............. & 64.0 & 63.8 & 59.7 & 54.2 & 50.6 & 56.0 & 75.7 & 75.4 & 65.2 & 58.5 & - & 61.9 \\
\hline January 1995 .................. & 71.5 & 71.3 & 67.7 & 56.3 & 52.7 & 58.8 & 83.2 & 83.0 & 73.6 & 60.1 & - & 65.6 \\
\hline Alabama & & & & & & & & & & & & \\
\hline January 1996 ................. & - & - & - & - & - & - & - & - & - & - & - & - \\
\hline December 1995 ............. & - & - & - & - & - & - & - & - & - & - & - & - \\
\hline January 1995 & - & - & - & - & - & - & - & - & - & - & - & - \\
\hline Arkansas & & & & & & & & & & & & \\
\hline January 1996 ................. & - & - & - & - & $\rightarrow$ & - & - & - & - & - & - & - \\
\hline December 1995 ............ & - & - & - & - & - & - & - & - & - & - & - & - \\
\hline $\begin{array}{l}\text { January } 1995 \text {.................. } \\
\text { Louisiana }\end{array}$ & - & - & - & - & - & - & - & - & - & - & - & - \\
\hline January 1996 & - & $w$ & - & - & - & - & - & - & - & - & - & - \\
\hline December 1995 ............. & - & $\dddot{w}$ & - & - & $w$ & $w$ & - & - & - & - & - & - \\
\hline January 1995 ................. & - & - & - & - & $w$ & $w$ & - & - & - & - & - & - \\
\hline Mississippi & & & & & & & & & & & & \\
\hline January 1996 ............... & - & - & - & - & - & - & - & - & - & - & - & - \\
\hline December 1995 ............ & - & - & - & - & - & - & - & - & - & - & - & - \\
\hline January 1995 ................. & - & - & - & - & - & - & - & - & - & - & - & - \\
\hline New Mexico & & & & & & & & & & & & \\
\hline January 1996 ................... & - & - & - & - & - & - & - & - & - & - & - & - \\
\hline December 1995 ............ & - & - & - & - & - & - & - & - & - & - & - & - \\
\hline January 1995 & - & - & - & - & - & - & - & - & - & - & - & - \\
\hline Texas & & & & & & & & & & & & \\
\hline January 1996 ................. & 65.9 & 65.7 & 61.2 & 55.6 & 54.6 & 57.9 & 77.7 & 77.3 & 66.9 & 60.7 & - & 64.2 \\
\hline December 1995 ............ & 64.0 & 63.8 & 59.7 & 54.2 & 50.6 & 56.0 & 75.7 & 75.4 & 65.2 & 58.5 & - & 61.9 \\
\hline January 1995 .................. & 71.5 & 71.3 & 67.7 & 56.3 & 52.6 & 58.8 & 83.2 & 83.0 & 73.6 & 60.1 & - & 65.6 \\
\hline PAD District IV & & & & & & & & & & & & \\
\hline January 1996 & - & - & - & - & - & - & - & - & - & - & - & - \\
\hline December 1995 ............ & - & - & - & - & - & - & - & - & - & - & - & - \\
\hline $\begin{array}{l}\text { January } 1995 \text {................... } \\
\text { Colorado }\end{array}$ & - & - & - & - & - & - & - & - & - & - & - & - \\
\hline January 1996 ................ & - & - & - & - & - & - & - & - & - & - & - & - \\
\hline December 1995 & - & - & - & - & - & - & - & - & - & - & - & - \\
\hline January 1995 ................ & - & - & - & - & - & - & - & - & - & - & - & - \\
\hline
\end{tabular}

See footnotes at end of table. 
Table 34. Reformulated Motor Gasoline Prices by Grade, Sales Type,

PAD District, and State

(Cents per Gallon Excluding Taxes) - Continued

\begin{tabular}{|c|c|c|c|c|c|c|c|c|c|c|c|c|}
\hline \multirow{3}{*}{$\begin{array}{l}\text { Geographic Area } \\
\text { Month }\end{array}$} & \multicolumn{6}{|c|}{ Premium } & \multicolumn{6}{|c|}{ All Grades } \\
\hline & \multicolumn{2}{|c|}{ Sales to End Users } & \multicolumn{4}{|c|}{ Sales for Resale } & \multicolumn{2}{|c|}{ Sales to End Users } & \multicolumn{4}{|c|}{ Sales for Resale } \\
\hline & $\begin{array}{c}\text { Through } \\
\text { Retail } \\
\text { Outlets }\end{array}$ & Average $^{a}$ & DTW & Rack & Bulk & Average & $\begin{array}{c}\text { Through } \\
\text { Retail } \\
\text { Outlets }\end{array}$ & Average ${ }^{a}$ & DTW & Rack & Bulk & Average \\
\hline
\end{tabular}

Nebraska

January 1996 .................

December 1995

January 1995

North Dakota

January 1996

December 1995

January 1995 .................

Ohio

January 1996

December 1995 .............

January 1995

Oklahoma

January 1996

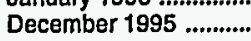

January 1995

South Dakota

January 1996 .

December 1995

Tennesseo

January 1996

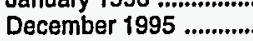

January 1995

Wisconsin

January 1996 ................

December 1995 ............

January 1995

\begin{tabular}{l}
\hline \\
\hline \\
\hline \\
\hline \\
9 \\
90 \\
9
\end{tabular}

PAD District III

January 1996 ................

December 1995

January 1995 .................

Alabama

January 1996 .................

December 1995

January 1995 ................

Arkansas

January 1996 ................

December 1995

January 1995 ................

Loulsiana

January 1996 ...............

December 1995

January 1995 .................

Mississippi

January 1996 ................

December 1995

January 1995 ................

New Mexico

January 1996 ................

December 1995

January 1995 .................

Texas

January 1996

December 1995 .............

January 1995 .................

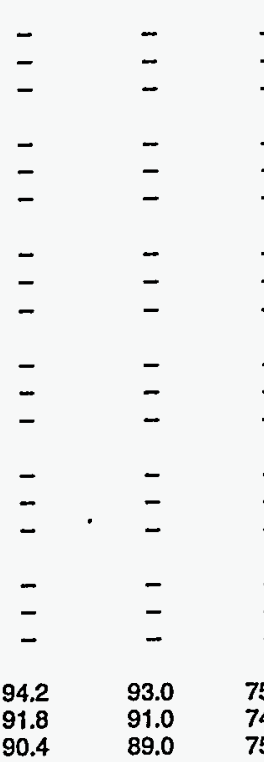

-
-
-
-
-
-
-
-
-
-
-
-

$\begin{array}{lll}- & - & - \\ - & - & -\end{array}$

\section{$=\quad-$}

$\overline{-} \quad \overline{-} \quad \overline{-}$

$\overline{-}=\overline{-} \quad \bar{z}$

$-$

\section{-}

$\begin{array}{lll}- & - & -\end{array}$

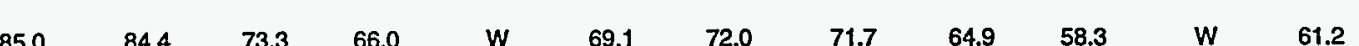

$\begin{array}{lllllllllll}85.0 & 84.4 & 73.3 & 66.0 & W & 69.1 & 72.0 & 71.7 & 64.9 & 58.3 & 52.4 \\ 82.8 & 82.4 & 71.8 & 64.2 & 58.8 & 67.3 & 70.2 & 70.0 & 63.6 & 56.9 & 59.4\end{array}$

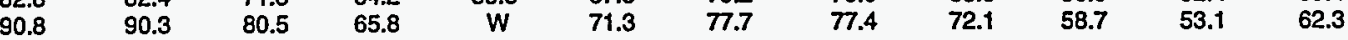

PAD District IV

January 1996

December 1995 ............

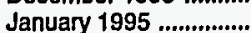

Colorado

January 1996 ...............

December 1995 ...........

January 1995

-
-
-
-
-
-
-
-
-
-
-
-
85.
82
90

$\overline{-}$

$\pm \quad=$

$\bar{z} \quad \bar{z}=$

$=$

$=$

$\overline{-}= \pm \overline{ }=\overline{-}$

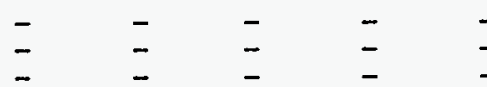

$\overline{-}$

$=\quad-\quad=$

$\overline{-}=\bar{z}$

-

$\begin{array}{rrrrrrrrrrrr}5.0 & 84.4 & 73.3 & 66.0 & W & 69.1 & 72.0 & 71.7 & 64.9 & \mathbf{5 8 . 3} & W & 61.2 \\ 2.8 & 82.4 & 71.8 & 64.2 & \mathbf{5 8 . 8} & 67.3 & 70.2 & 70.0 & 63.6 & \mathbf{5 6 . 9} & \mathbf{5 2 . 5} & \mathbf{5 9 . 4} \\ 0.8 & 90.3 & 80.5 & 65.8 & W & 71.3 & 77.7 & 77.4 & \mathbf{7 2 . 1} & \mathbf{5 8 . 7} & \mathbf{5 3 . 1} & \mathbf{6 2 . 4}\end{array}$

See tootnotes at end of table. 
Table 34. Reformulated Motor Gasoline Prices by Grade, Sales Type, PAD District, and State

(Cents per Gallon Excluding Taxes) - Continued

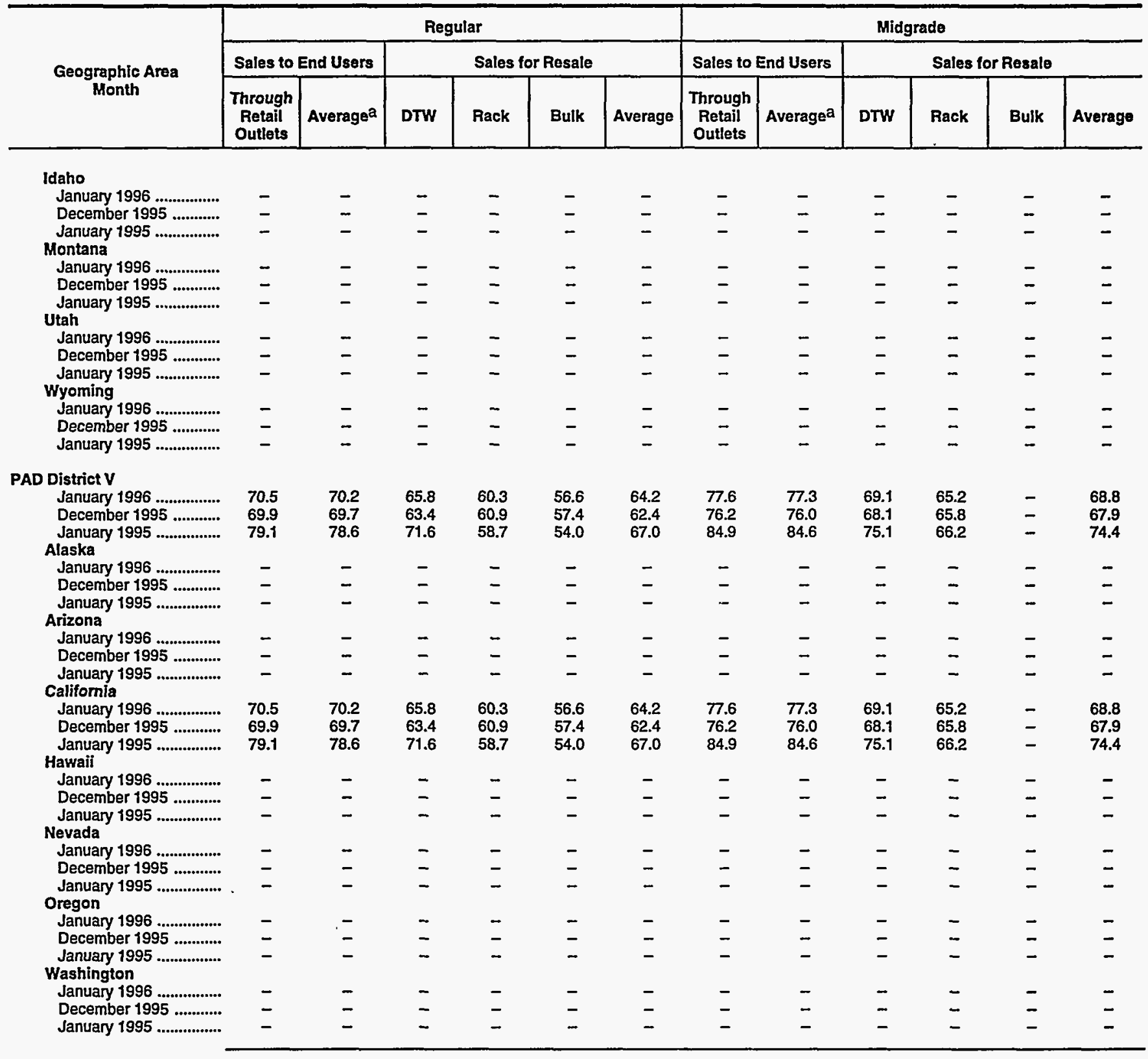

See footnotes at end of table. 
Table 34. Reformulated Motor Gasoline Prices by Grade, Sales Type, PAD District, and State

(Cents per Gallon Excluding Taxes) — Continued

\begin{tabular}{|c|c|c|c|c|c|c|c|c|c|c|c|c|}
\hline \multirow{3}{*}{$\begin{array}{l}\text { Geographic Area } \\
\text { Month }\end{array}$} & \multicolumn{6}{|c|}{ Premium } & \multicolumn{6}{|c|}{ All Grades } \\
\hline & \multicolumn{2}{|c|}{ Sales to End Users } & \multicolumn{4}{|c|}{ Sales for Resale } & \multicolumn{2}{|c|}{ Sales to End Users } & \multicolumn{4}{|c|}{ Sales for Resale } \\
\hline & $\begin{array}{c}\text { Through } \\
\text { Retail } \\
\text { Outlets }\end{array}$ & Average ${ }^{a}$ & DTW & Rack & Bulk & Average & $\begin{array}{c}\text { Through } \\
\text { Retail } \\
\text { Outlets }\end{array}$ & Averagea & DTW & Rack & Bulk & Average \\
\hline \multicolumn{13}{|l|}{ Idaho } \\
\hline January 1996 ................... & - & - & - & - & - & - & - & - & - & - & - & - \\
\hline December 1995 ............. & - & - & - & - & - & - & - & - & - & - & - & - \\
\hline January 1995 .................... & - & - & - & - & - & - & - & - & - & - & - & - \\
\hline $\begin{array}{l}\text { Montana } \\
\text { January } 1996 \ldots . . . . . . . . . . . . . .\end{array}$ & - & - & - & - & - & - & - & - & - & - & - & - \\
\hline $\begin{array}{l}\text { January } 1996 \text {.................. } \\
\text { December } 1995 \text {........... }\end{array}$ & $\overline{-}$ & $\overline{-}$ & $\overline{-}$ & $\overline{-}$ & - & $\overline{-}$ & $\overline{-}$ & - & 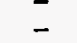 & - & - & - \\
\hline January 1995 ........................ & - & - & - & - & - & - & - & - & - & - & - & - \\
\hline $\begin{array}{l}\text { Utah } \\
\text { January } 1996 \ldots . . . . . . . . . . .\end{array}$ & - & - & . & - & - & - & - & - & - & - & - & - \\
\hline $\begin{array}{l}\text { January } 1996 \text {.................. } \\
\text { December } 1995 \text {............ }\end{array}$ & $\overline{-}$ & $\overline{-}$ & $\overline{-}$ & - & $\overline{-}$ & - & - & - & - & - & - & - \\
\hline 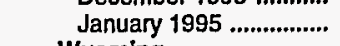 & - & - & - & - & - & - & - & - & - & - & - & - \\
\hline $\begin{array}{l}\text { Wyoming } \\
\text { January } 1996 \text {................. }\end{array}$ & - & - & - & - & - & - & - & - & - & - & - & - \\
\hline December 1995 .............. & - & - & - & - & - & - & - & - & - & - & - & - \\
\hline January 1995 ................... & - & - & - & - & - & - & - & - & - & - & - & - \\
\hline \multicolumn{13}{|l|}{ PAD District V } \\
\hline January 1996 ................ & 88.5 & 87.4 & 78.2 & 70.7 & $\begin{array}{l}W \\
W\end{array}$ & 77.3 & 75.5 & 75.0 & $\begin{array}{l}69.4 \\
675\end{array}$ & 62.4 & $\begin{array}{l}56.8 \\
577\end{array}$ & $\begin{array}{l}67.8 \\
66.2\end{array}$ \\
\hline $\begin{array}{l}\text { December } 1995 \text {.............. } \\
\text { January } 1995 \text {................ }\end{array}$ & 87.9 & $\begin{array}{l}86.9 \\
96.7\end{array}$ & $\begin{array}{l}77.3 \\
85.3\end{array}$ & $\begin{array}{l}71.4 \\
69.1\end{array}$ & $\begin{array}{l}W \\
W\end{array}$ & $\begin{array}{l}76.4 \\
83.0\end{array}$ & $\begin{array}{l}74.8 \\
83.7\end{array}$ & $\begin{array}{l}74.4 \\
83.1\end{array}$ & $\begin{array}{l}67.5 \\
75.5\end{array}$ & $\begin{array}{l}63.0 \\
60.8\end{array}$ & $\begin{array}{l}5 \% .7 \\
54.3\end{array}$ & $\begin{array}{l}66.2 \\
71.5\end{array}$ \\
\hline 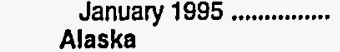 & 97.6 & 96.7 & \multicolumn{10}{|c|}{ Alaska } \\
\hline 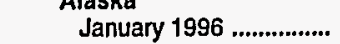 & - & - & - & - & - & - & - & - & - & - & - & - \\
\hline December 1995 .............. & - & - & - & - & - & - & - & - & - & - & - & - \\
\hline January 1995 ..................... & - & - & - & - & - & - & - & - & - & - & - & - \\
\hline Arjzona & & & & & & & - & - & & - & - & - \\
\hline $\begin{array}{l}\text { January } 1996 \text {................... } \\
\text { December } 1995 \ldots . . . . . .\end{array}$ & $\overline{-}$ & $\overline{-}$ & $\overline{-}$ & $\overline{-}$ & $\overline{-}$ & $\overline{-}$ & $\overline{-}$ & - & $\overline{-}$ & - & - & - \\
\hline January 1995 .................... & - & - & - & - & - & - & - & - & - & - & - & - \\
\hline Californla & & & & & & & & & & & & \\
\hline January 1996 .................. & 88.5 & 87.4 & 78.2 & 70.7 & $w$ & $\begin{array}{l}77.3 \\
76.4\end{array}$ & $\begin{array}{l}75.5 \\
74.8\end{array}$ & $\begin{array}{l}75.0 \\
74.4\end{array}$ & $\begin{array}{l}69.4 \\
675\end{array}$ & 62.4 & $\begin{array}{l}56.8 \\
577\end{array}$ & $\begin{array}{l}67.8 \\
66.2\end{array}$ \\
\hline December 1995 ............ & 87.9 & 86.9 & 77.3 & $\begin{array}{l}71.4 \\
69.1\end{array}$ & $\begin{array}{l}W \\
W\end{array}$ & $\begin{array}{l}76.4 \\
83.0\end{array}$ & $\begin{array}{l}74.8 \\
83.7\end{array}$ & $\begin{array}{l}74.4 \\
83.1\end{array}$ & $\begin{array}{l}67.5 \\
75.5\end{array}$ & $\begin{array}{l}63.0 \\
60.8\end{array}$ & $\begin{array}{l}57.7 \\
54.3\end{array}$ & $\begin{array}{l}66.2 \\
71.5\end{array}$ \\
\hline $\begin{array}{l}\text { January } 1995 \ldots . . . . . . . . . . . . . . . \\
\text { Hawail }\end{array}$ & 97.6 & 96.7 & 85.3 & \\
\hline January $1996 \ldots . . . . . . . . . . . . . .$. & - & - & - & - & - & - & - & - & - & - & - & - \\
\hline December 1995 ............. & - & - & - & - & - & - & - & - & - & - & - & - \\
\hline January $1995 \ldots . . . . . . . . . . . . . .$. & - & - & - & - & - & - & - & - & - & - & - & - \\
\hline \multicolumn{13}{|l|}{ Nevada } \\
\hline January 1996 .................. & $\bar{z}$ & $\overline{-}$ & z- & $\overline{-}$ & $\begin{array}{c}- \\
-\end{array}$ & - & $=$ & $=$ & $=$ & $=$ & $\bar{z}$ & $=$ \\
\hline $\begin{array}{l}\text { December } 1995 \text {.............. } \\
\text { January } 1995\end{array}$ & $\bar{z}$ & $\overline{-}$ & $\overline{-}$ & $\overline{-}$ & $\overline{-}$ & $\overline{-}$ & $\bar{z}-$ & $=$ & - & $=$ & $\overline{-}$ & z \\
\hline \multicolumn{13}{|l|}{ Oregon } \\
\hline January $1996 \ldots . . . . . . . . . . . . .$. & - & - & - & - & - & - & - & - & - & - & - & - \\
\hline December 1995 ............. & - & - & - & - & - & - & - & - & - & - & - & - \\
\hline January 1995 & - & - & - & - & - & - & - & - & - & - & - & - \\
\hline \multicolumn{13}{|l|}{ Washington } \\
\hline January $1996 . . . . . . . . . . . . . . .$. & $=$ & $\bar{z}$ - & $=$ & $\bar{z}$ - & $\overline{-}$ & $\overline{-}$ & $z$ & $\ddot{-}$ & $\overline{-}$ & - & - & $=$ \\
\hline $\begin{array}{l}\text { December } 1995 \ldots . . . . . . . . . \\
\text { January } 1995\end{array}$ & $\overline{-}$ & $=$ & $=$ & - & - & - & - & - & - & - & $\overline{-}$ & $\overline{-}$ \\
\hline & & & & & & & & & & & & \\
\hline
\end{tabular}

Dash $(-)=$ No data reported.

NA $=$ Not available.

$W=$ Withheld to avoid disclosure of individual company data.

a Includes sales through retall outlets as well as all direct sales to end users that were not made through company-operated retail outlets, e.g., sales to agricultural customers, commercial sales, and industrial sales.

Notes: Values shown for the current month are preliminary. Values shown for previous months are revised. Data are final upon publication in the Petroleum Marketing Annual.

Sources: Energy Information Administration Forms ElA-782A, "Refiners'/Gas Plant Operators' Monthly Petroleum Product Sales Report," and ElA-782B,

"Resellers'/Retallers' Monthly Petroleum Product Sales Report." 
Table 35. Refiner Motor Gasoline Prices by Grade, Sales Type, PAD District, and State (Cents per Gallon Excluding Taxes)

\begin{tabular}{|c|c|c|c|c|c|c|c|c|c|c|c|c|}
\hline \multirow{3}{*}{$\begin{array}{l}\text { Geographic Area } \\
\text { Month }\end{array}$} & \multicolumn{3}{|c|}{ Regular } & \multicolumn{3}{|c|}{ Midgrade } & \multicolumn{3}{|c|}{ Premium } & \multicolumn{3}{|c|}{ All Grades } \\
\hline & \multicolumn{2}{|c|}{ Sales to End Users } & \multirow{2}{*}{$\begin{array}{l}\text { Sales } \\
\text { for } \\
\text { Resale }\end{array}$} & \multicolumn{2}{|c|}{ Sales to End Users } & \multirow{2}{*}{$\begin{array}{l}\text { Sales } \\
\text { for } \\
\text { Resale }\end{array}$} & \multicolumn{2}{|c|}{ Sales to End Users } & \multirow[b]{2}{*}{$\begin{array}{l}\text { Sales } \\
\text { for } \\
\text { Resale }\end{array}$} & \multicolumn{2}{|c|}{ Sales to End Users } & \multirow[b]{2}{*}{$\begin{array}{l}\text { Sales } \\
\text { for } \\
\text { Resale }\end{array}$} \\
\hline & $\begin{array}{l}\text { Through } \\
\text { Retail } \\
\text { Outlets }\end{array}$ & $\begin{array}{l}\text { Other } \\
\text { End } \\
\text { Usersa }\end{array}$ & & $\begin{array}{c}\text { Through } \\
\text { Retail } \\
\text { Outlets }\end{array}$ & $\begin{array}{l}\text { Other } \\
\text { End } \\
\text { Usersa }\end{array}$ & & $\begin{array}{l}\text { Through } \\
\text { Retail } \\
\text { Outlets }\end{array}$ & $\begin{array}{c}\text { Other } \\
\text { End } \\
\text { Users }\end{array}$ & & $\begin{array}{c}\text { Through } \\
\text { Retail } \\
\text { Outlets }\end{array}$ & $\begin{array}{l}\text { Other } \\
\text { End } \\
\text { Users }\end{array}$ & \\
\hline \multicolumn{13}{|l|}{ United States } \\
\hline $\begin{array}{l}\text { January } 1996 \ldots . . . . . . . . . . \\
\text { December } 1995 \\
\text { January } 1995\end{array}$ & $\begin{array}{l}70.3 \\
68.6 \\
69.9\end{array}$ & $\begin{array}{l}58.3 \\
56.5 \\
56.0\end{array}$ & $\begin{array}{l}57.7 \\
56.5 \\
56.4\end{array}$ & $\begin{array}{l}79.5 \\
77.7 \\
80.0\end{array}$ & $\begin{array}{l}61.2 \\
60.0 \\
58.7\end{array}$ & $\begin{array}{l}65.5 \\
64.2 \\
65.2\end{array}$ & $\begin{array}{l}88.1 \\
86.2 \\
88.9\end{array}$ & $\begin{array}{l}67.1 \\
65.6 \\
64.6\end{array}$ & $\begin{array}{l}70.2 \\
68.9 \\
70.2\end{array}$ & $\begin{array}{l}75.2 \\
73.6 \\
75.2\end{array}$ & $\begin{array}{l}60.0 \\
58.3 \\
57.7\end{array}$ & $\begin{array}{l}61.1 \\
59.9 \\
60.1\end{array}$ \\
\hline \multicolumn{13}{|l|}{ PAD District I } \\
\hline 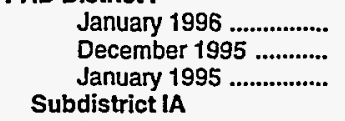 & $\begin{array}{l}66.8 \\
64.9 \\
68.5\end{array}$ & $\begin{array}{l}57.2 \\
54.3 \\
55.8\end{array}$ & $\begin{array}{l}58.1 \\
57.3 \\
58.2\end{array}$ & $\begin{array}{l}77.4 \\
75.6 \\
80.4\end{array}$ & $\begin{array}{l}60.2 \\
58.4 \\
58.0\end{array}$ & $\begin{array}{l}64.7 \\
63.2 \\
65.7\end{array}$ & $\begin{array}{l}86.2 \\
84.2 \\
89.4\end{array}$ & $\begin{array}{l}65.5 \\
63.2 \\
63.8\end{array}$ & $\begin{array}{l}71.3 \\
70.2 \\
72.3\end{array}$ & $\begin{array}{l}73.2 \\
71.5 \\
75.6\end{array}$ & $\begin{array}{l}59.0 \\
56.2 \\
57.4\end{array}$ & $\begin{array}{l}62.4 \\
61.5 \\
62.8\end{array}$ \\
\hline $\begin{array}{l}\text { January } 1996 \ldots \ldots . . . . . . . . . \\
\text { December } 1995 \ldots . . . . . . . \\
\text { January } 1995 \ldots . . . . . . . . . . . \\
\text { Connecticut }\end{array}$ & $\begin{array}{l}76.7 \\
74.9 \\
79.0\end{array}$ & $\begin{array}{l}61.2 \\
59.6 \\
59.9\end{array}$ & $\begin{array}{l}64.8 \\
64.2 \\
63.0\end{array}$ & $\begin{array}{l}87.4 \\
86.2 \\
88.8\end{array}$ & $\begin{array}{l}64.8 \\
63.9 \\
62.3\end{array}$ & $\begin{array}{l}72.0 \\
70.9 \\
70.8\end{array}$ & $\begin{array}{r}95.6 \\
94.5 \\
100.7\end{array}$ & $\begin{array}{l}W \\
W \\
W\end{array}$ & $\begin{array}{l}77.2 \\
76.4 \\
76.1\end{array}$ & $\begin{array}{l}83.3 \\
81.9 \\
86.5\end{array}$ & $\begin{array}{l}63.2 \\
61.8 \\
61.4\end{array}$ & $\begin{array}{l}68.6 \\
68.0 \\
67.1\end{array}$ \\
\hline $\begin{array}{l}\text { January } 1996 \ldots . . . . . . . . . . . . . . . \\
\text { December } 1995 \text {............ } \\
\text { January } 1995 \text {................. } \\
\text { Maine }\end{array}$ & $\begin{array}{l}W \\
W \\
W\end{array}$ & $\begin{array}{l}W \\
W \\
W\end{array}$ & $\begin{array}{l}64.6 \\
64.1 \\
64.6\end{array}$ & $\begin{array}{l}W \\
W \\
W\end{array}$ & $\begin{array}{c}W \\
64.0 \\
W\end{array}$ & $\begin{array}{l}71.6 \\
70.3 \\
71.7\end{array}$ & $\begin{array}{l}W \\
W \\
W\end{array}$ & $\begin{array}{l}W \\
W \\
W\end{array}$ & $\begin{array}{l}77.2 \\
75.4 \\
78.2\end{array}$ & $\begin{array}{l}w \\
w \\
w\end{array}$ & $\begin{array}{c}W \\
61.1 \\
W\end{array}$ & $\begin{array}{l}68.4 \\
67.9 \\
68.8\end{array}$ \\
\hline $\begin{array}{l}\text { January } 1996 \ldots . . . . . . . . . . . . . \\
\text { December } 1995 . . . . . . . . . \\
\text { January } 1995 \ldots . . . . . . . . . . . . .\end{array}$ & $\begin{array}{l}- \\
-\end{array}$ & $\begin{array}{l}W \\
W \\
W\end{array}$ & $\begin{array}{l}60.7 \\
61.5 \\
57.1\end{array}$ & $\begin{array}{l}- \\
-\end{array}$ & $\begin{array}{l}W \\
W \\
W\end{array}$ & $\begin{array}{l}65.3 \\
65.7 \\
61.9\end{array}$ & $\overline{-}$ & $\begin{array}{l}W \\
W \\
W\end{array}$ & $\begin{array}{l}70.6 \\
71.7 \\
69.0\end{array}$ & $\begin{array}{l}- \\
-\end{array}$ & $\begin{array}{l}W \\
W \\
W\end{array}$ & $\begin{array}{l}63.0 \\
63.7 \\
59.5\end{array}$ \\
\hline $\begin{array}{l}\text { January } 1996 \text {................ } \\
\text { December } 1995 \text {............. } \\
\text { January } 1995 \text {............... }\end{array}$ & $\begin{array}{l}77.8 \\
76.3 \\
79.6\end{array}$ & $\begin{array}{l}61.3 \\
59.8 \\
60.4\end{array}$ & $\begin{array}{l}66.2 \\
65.2 \\
64.3\end{array}$ & $\begin{array}{l}88.1 \\
87.2 \\
87.6\end{array}$ & $\begin{array}{c}W \\
W \\
62.5\end{array}$ & $\begin{array}{l}74.3 \\
72.9 \\
73.1\end{array}$ & $\begin{array}{r}97.5 \\
96.0 \\
100.9\end{array}$ & $\begin{array}{l}W \\
W \\
W\end{array}$ & $\begin{array}{l}78.8 \\
78.3 \\
76.4\end{array}$ & $\begin{array}{l}84.5 \\
83.3 \\
86.8\end{array}$ & $\begin{array}{l}63.6 \\
62.5 \\
61.8\end{array}$ & $\begin{array}{l}70.4 \\
69.4 \\
68.5\end{array}$ \\
\hline $\begin{array}{l}\text { New Hampshire } \\
\text { January } 1996 \ldots . . . . . . . . . . . \\
\text { December } 1995 \text {........... } \\
\text { January } 1995 \text {................ } \\
\text { Rhode Island }\end{array}$ & $\begin{array}{l}W \\
W \\
W\end{array}$ & $\begin{array}{l}W \\
W \\
W\end{array}$ & $\begin{array}{l}67.4 \\
65.7 \\
67.8\end{array}$ & $\begin{array}{l}W \\
W \\
W\end{array}$ & $\begin{array}{l}\bar{w} \\
w\end{array}$ & $\begin{array}{l}73.5 \\
71.4 \\
70.5\end{array}$ & $\begin{array}{l}W \\
W \\
W\end{array}$ & $\begin{array}{l}W \\
W \\
W\end{array}$ & $\begin{array}{l}80.5 \\
78.7 \\
80.3\end{array}$ & $\begin{array}{l}w \\
W \\
w\end{array}$ & $\begin{array}{l}W \\
W \\
W\end{array}$ & $\begin{array}{l}71.0 \\
69.3 \\
71.0\end{array}$ \\
\hline $\begin{array}{l}\text { January } 1996 \ldots . . . . . . . . . . . . . \\
\text { December } 1995 \text {............ } \\
\text { January } 1995 \text {................. } \\
\text { Vermont }\end{array}$ & $\begin{array}{l}74.2 \\
71.4 \\
74.9\end{array}$ & $\begin{array}{l}61.5 \\
61.5 \\
59.6\end{array}$ & $\begin{array}{l}64.0 \\
63.3 \\
61.1\end{array}$ & $\begin{array}{l}82.9 \\
81.7 \\
85.7\end{array}$ & $\begin{array}{c}W \\
W \\
61.8\end{array}$ & $\begin{array}{l}70.4 \\
69.4 \\
69.0\end{array}$ & $\begin{array}{l}89.8 \\
88.4 \\
92.9\end{array}$ & $\begin{array}{l}W \\
W \\
W\end{array}$ & $\begin{array}{l}76.2 \\
75.3 \\
75.1\end{array}$ & $\begin{array}{l}79.8 \\
77.7 \\
81.5\end{array}$ & $\begin{array}{l}63.5 \\
62.3 \\
61.1\end{array}$ & $\begin{array}{l}67.7 \\
67.0 \\
64.9\end{array}$ \\
\hline $\begin{array}{l}\text { January } 1996 \ldots . . . . . . . . . . . \\
\text { December } 1995 \text {............ } \\
\text { January } 1995 \text {................ } \\
\text { Subdistrict IB }\end{array}$ & $\overline{-}$ & $\begin{array}{l}- \\
-\end{array}$ & $\begin{array}{l}64.2 \\
64.0 \\
62.3\end{array}$ & $\overline{-}$ & $\begin{array}{l}- \\
-\end{array}$ & $\begin{array}{l}70.4 \\
69.7 \\
69.1\end{array}$ & $\begin{array}{l}- \\
-\end{array}$ & $\begin{array}{l}- \\
-\end{array}$ & $\begin{array}{l}74.9 \\
75.3 \\
73.6\end{array}$ & $\begin{array}{l}- \\
-\end{array}$ & $\begin{array}{l}- \\
-\end{array}$ & $\begin{array}{l}67.3 \\
67.0 \\
65.7\end{array}$ \\
\hline $\begin{array}{l}\text { January } 1996 \ldots . . . . . . . . . . . . . \\
\text { December } 1995 \text {............ } \\
\text { January } 1995 \text {............... } \\
\text { Delaware }\end{array}$ & $\begin{array}{l}70.8 \\
69.6 \\
72.3\end{array}$ & $\begin{array}{l}60.5 \\
59.3 \\
60.1\end{array}$ & $\begin{array}{l}60.2 \\
60.3 \\
61.7\end{array}$ & $\begin{array}{l}82.2 \\
80.6 \\
84.3\end{array}$ & $\begin{array}{l}63.7 \\
63.2 \\
61.9\end{array}$ & $\begin{array}{l}68.4 \\
67.2 \\
71.1\end{array}$ & $\begin{array}{l}91.6 \\
90.0 \\
93.9\end{array}$ & $\begin{array}{l}69.9 \\
69.0 \\
68.7\end{array}$ & $\begin{array}{l}74.4 \\
74.0 \\
76.3\end{array}$ & $\begin{array}{l}77.5 \\
76.3 \\
79.3\end{array}$ & $\begin{array}{l}62.3 \\
61.1 \\
61.4\end{array}$ & $\begin{array}{l}65.1 \\
65.0 \\
67.0\end{array}$ \\
\hline $\begin{array}{l}\text { January } 1996 . . . . . . . . . . . . . . \\
\text { December } 1995 \text {........... } \\
\text { January } 1995 \text {............... } \\
\text { District of Columbia }\end{array}$ & $\begin{array}{l}72.4 \\
69.2 \\
74.7\end{array}$ & $\begin{array}{l}W \\
W \\
W\end{array}$ & $\begin{array}{l}60.4 \\
59.9 \\
63.0\end{array}$ & $\begin{array}{l}85.2 \\
81.6 \\
87.1\end{array}$ & $\begin{array}{l}W \\
- \\
-\end{array}$ & $\begin{array}{l}65.8 \\
65.1 \\
69.3\end{array}$ & $\begin{array}{l}95.3 \\
92.5 \\
97.3\end{array}$ & $\begin{array}{l}W \\
W \\
W\end{array}$ & $\begin{array}{l}72.4 \\
72.4 \\
76.4\end{array}$ & $\begin{array}{l}80.1 \\
77.1 \\
82.4\end{array}$ & $\begin{array}{l}W \\
W \\
W\end{array}$ & $\begin{array}{l}63.6 \\
63.3 \\
66.7\end{array}$ \\
\hline 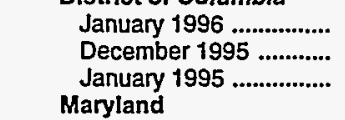 & $\overline{-}$ & $\begin{array}{l}56.9 \\
54.5 \\
59.7\end{array}$ & $\begin{array}{l}65.5 \\
62.7 \\
73.8\end{array}$ & $\begin{array}{l}- \\
-\end{array}$ & $\begin{array}{l}W \\
W \\
W\end{array}$ & $\begin{array}{l}70.1 \\
67.4 \\
79.3\end{array}$ & $\begin{array}{l}- \\
-\end{array}$ & $\begin{array}{l}64.9 \\
62.3 \\
67.6\end{array}$ & $\begin{array}{l}77.2 \\
74.7 \\
87.2\end{array}$ & $\begin{array}{l}- \\
-\end{array}$ & $\begin{array}{l}59.6 \\
57.3 \\
61.7\end{array}$ & $\begin{array}{l}72.1 \\
69.7 \\
81.2\end{array}$ \\
\hline $\begin{array}{l}\text { January } 1996 \text {................ } \\
\text { December } 1995 \text {............ } \\
\text { January } 1995 \text {............... } \\
\text { New Jersey }\end{array}$ & $\begin{array}{l}W \\
W \\
-\end{array}$ & $\begin{array}{l}59.4 \\
57.3 \\
60.5\end{array}$ & $\begin{array}{l}61.5 \\
59.3 \\
67.1\end{array}$ & $\begin{array}{l}W \\
W \\
-\end{array}$ & $\begin{array}{l}60.4 \\
58.5 \\
63.7\end{array}$ & $\begin{array}{l}66.9 \\
64.6 \\
74.3\end{array}$ & $\begin{array}{l}W \\
W \\
-\end{array}$ & $\begin{array}{l}65.8 \\
64.0 \\
69.5\end{array}$ & $\begin{array}{l}73.6 \\
71.0 \\
81.6\end{array}$ & $\begin{array}{l}W \\
W \\
-\end{array}$ & $\begin{array}{l}60.1 \\
58.1 \\
61.9\end{array}$ & $\begin{array}{l}65.8 \\
63.5 \\
72.3\end{array}$ \\
\hline $\begin{array}{l}\text { January } 1996 \text {................ } \\
\text { December } 1995 \text {............ } \\
\text { January } 1995 \text {................. } \\
\text { New York }\end{array}$ & $\begin{array}{l}74.8 \\
72.4 \\
77.0\end{array}$ & $\begin{array}{l}60.7 \\
60.0 \\
59.7\end{array}$ & $\begin{array}{l}60.9 \\
61.9 \\
62.6\end{array}$ & $\begin{array}{l}86.9 \\
84.5 \\
90.2\end{array}$ & $\begin{array}{l}62.7 \\
63.3 \\
63.3\end{array}$ & $\begin{array}{l}72.3 \\
71.0 \\
73.7\end{array}$ & $\begin{array}{l}95.6 \\
93.5 \\
99.2\end{array}$ & $\begin{array}{l}69.6 \\
70.3 \\
68.4\end{array}$ & $\begin{array}{l}74.4 \\
74.5 \\
74.8\end{array}$ & $\begin{array}{l}82.8 \\
80.7 \\
85.6\end{array}$ & $\begin{array}{l}63.6 \\
63.1 \\
62.3\end{array}$ & $\begin{array}{l}66.0 \\
66.8 \\
67.5\end{array}$ \\
\hline $\begin{array}{l}\text { January } 1996 \\
\text { December } 1995 \ldots . . . . . . . . . . . . . . \\
\text { January } 1995 \text {................. } \\
\text { Pennsylvania }\end{array}$ & $\begin{array}{l}68.9 \\
68.6 \\
69.1\end{array}$ & $\begin{array}{l}63.3 \\
62.1 \\
61.8\end{array}$ & $\begin{array}{l}62.3 \\
62.5 \\
62.0\end{array}$ & $\begin{array}{l}80.8 \\
80.3 \\
81.5\end{array}$ & $\begin{array}{l}66.5 \\
65.7 \\
60.8\end{array}$ & $\begin{array}{l}71.7 \\
70.6 \\
71.7\end{array}$ & $\begin{array}{l}90.6 \\
89.6 \\
91.3\end{array}$ & $\begin{array}{l}72.1 \\
71.3 \\
68.0\end{array}$ & $\begin{array}{l}78.5 \\
77.9 \\
78.5\end{array}$ & $\begin{array}{l}75.2 \\
74.9 \\
75.7\end{array}$ & $\begin{array}{l}64.7 \\
63.7 \\
62.2\end{array}$ & $\begin{array}{l}68.2 \\
68.1 \\
68.2\end{array}$ \\
\hline $\begin{array}{l}\text { January } 1996 . . . . . . . . . . . . . \\
\text { December } 1995 . . . . . . . . . \\
\text { January } 1995 \text {.............. }\end{array}$ & $\begin{array}{l}70.7 \\
69.1 \\
72.8\end{array}$ & $\begin{array}{l}59.3 \\
58.8 \\
57.2\end{array}$ & $\begin{array}{l}57.0 \\
57.4 \\
58.0\end{array}$ & $\begin{array}{l}80.6 \\
78.7 \\
82.9\end{array}$ & $\begin{array}{l}64.8 \\
65.0 \\
61.0\end{array}$ & $\begin{array}{l}63.5 \\
63.6 \\
65.6\end{array}$ & $\begin{array}{l}89.0 \\
87.3 \\
91.9\end{array}$ & $\begin{array}{l}75.0 \\
74.2 \\
69.9\end{array}$ & $\begin{array}{l}69.0 \\
69.5 \\
71.5\end{array}$ & $\begin{array}{l}76.4 \\
74.8 \\
78.8\end{array}$ & $\begin{array}{l}61.2 \\
60.5 \\
58.7\end{array}$ & $\begin{array}{l}60.4 \\
60.9 \\
62.0\end{array}$ \\
\hline
\end{tabular}

See footnotes at end of table. 
Table 35. Refiner Motor Gasoline Prices by Grade, Sales Type, PAD District, and State (Cents per Gallon Excluding Taxes) - Continued

\begin{tabular}{|c|c|c|c|c|c|c|c|c|c|c|c|c|}
\hline \multirow{3}{*}{$\begin{array}{l}\text { Geographic Area } \\
\text { Month }\end{array}$} & \multicolumn{3}{|c|}{ Regular } & \multicolumn{3}{|c|}{ Midgrade } & \multicolumn{3}{|c|}{ Premium } & \multicolumn{3}{|c|}{ All Grades } \\
\hline & \multicolumn{2}{|c|}{ Sales to End Users } & \multirow{2}{*}{$\begin{array}{l}\text { Sales } \\
\text { for } \\
\text { Resale }\end{array}$} & \multicolumn{2}{|c|}{ Sales to End Users } & \multirow{2}{*}{$\begin{array}{l}\text { Sales } \\
\text { for } \\
\text { Resale }\end{array}$} & \multicolumn{2}{|c|}{ Sales to End Users } & \multirow{2}{*}{$\begin{array}{l}\text { Sales } \\
\text { for } \\
\text { Resale }\end{array}$} & \multicolumn{2}{|c|}{ Sales to End Users } & \multirow[b]{2}{*}{$\begin{array}{l}\text { Sales } \\
\text { for } \\
\text { Resale }\end{array}$} \\
\hline & $\begin{array}{c}\text { Through } \\
\text { Retail } \\
\text { Outlets }\end{array}$ & $\begin{array}{l}\text { Other } \\
\text { End } \\
\text { Usersa }\end{array}$ & & $\begin{array}{c}\text { Through } \\
\text { Retail } \\
\text { Outlets }\end{array}$ & $\begin{array}{l}\text { Other } \\
\text { End } \\
\text { Usersa }\end{array}$ & & $\begin{array}{c}\text { Through } \\
\text { Retail } \\
\text { Outlets }\end{array}$ & $\begin{array}{c}\text { Other } \\
\text { End } \\
\text { Usersa }\end{array}$ & & $\begin{array}{l}\text { Through } \\
\text { Retail } \\
\text { Outlets }\end{array}$ & $\begin{array}{l}\text { Other } \\
\text { End } \\
\text { Usersa }\end{array}$ & \\
\hline \multicolumn{13}{|l|}{ Subdistrict IC } \\
\hline $\begin{array}{l}\text { January } 1996 \ldots \ldots \ldots . . . \ldots . \\
\text { December } 1995 \ldots . . . \ldots \ldots . . . \\
\text { January } 1995 \text {.............. } \\
\text { Florida }\end{array}$ & $\begin{array}{l}63.5 \\
61.0 \\
65.5\end{array}$ & $\begin{array}{l}55.3 \\
51.7 \\
52.5\end{array}$ & $\begin{array}{l}54.8 \\
52.9 \\
53.8\end{array}$ & $\begin{array}{l}74.0 \\
71.9 \\
77.6\end{array}$ & $\begin{array}{l}58.5 \\
55.9 \\
56.1\end{array}$ & $\begin{array}{l}60.7 \\
58.7 \\
60.8\end{array}$ & $\begin{array}{l}82.1 \\
79.6 \\
85.9\end{array}$ & $\begin{array}{l}63.4 \\
60.4 \\
61.5\end{array}$ & $\begin{array}{l}67.0 \\
65.0 \\
67.4\end{array}$ & $\begin{array}{l}69.8 \\
67.5 \\
72.5\end{array}$ & $\begin{array}{l}57.2 \\
53.6 \\
54.7\end{array}$ & $\begin{array}{l}58.6 \\
56.8 \\
58.2\end{array}$ \\
\hline $\begin{array}{l}\text { January } 1996 \ldots . . . . . . . . . . . . . \\
\text { December } 1995 \ldots . . . . . . . . \\
\text { January } 1995 \ldots . . . . . . . . . . . .\end{array}$ & $\begin{array}{l}63.6 \\
60.8 \\
64.8\end{array}$ & $\begin{array}{l}55.1 \\
50.9 \\
51.9\end{array}$ & $\begin{array}{l}55.3 \\
53.0 \\
54.2\end{array}$ & $\begin{array}{l}74.5 \\
72.2 \\
78.0\end{array}$ & $\begin{array}{l}57.6 \\
55.1 \\
54.8\end{array}$ & $\begin{array}{l}61.8 \\
59.4 \\
62.0\end{array}$ & $\begin{array}{l}82.7 \\
80.1 \\
85.9\end{array}$ & $\begin{array}{l}62.5 \\
59.3 \\
59.9\end{array}$ & $\begin{array}{l}69.0 \\
66.7 \\
70.3\end{array}$ & $\begin{array}{l}70.7 \\
68.3 \\
73.0\end{array}$ & $\begin{array}{l}56.6 \\
52.3 \\
53.9\end{array}$ & $\begin{array}{l}60.1 \\
57.9 \\
59.9\end{array}$ \\
\hline $\begin{array}{l}\text { January } 1996 \text {............... } \\
\text { December } 1995 \text {............ } \\
\text { January } 1995 \text {................ } \\
\text { North Carolina }\end{array}$ & $\begin{array}{l}61.7 \\
58.6 \\
62.8\end{array}$ & $\begin{array}{l}55.0 \\
51.8 \\
51.9\end{array}$ & $\begin{array}{l}54.0 \\
51.7 \\
51.8\end{array}$ & $\begin{array}{l}71.9 \\
69.1 \\
74.0\end{array}$ & $\begin{array}{l}58.6 \\
55.5 \\
55.6\end{array}$ & $\begin{array}{l}59.9 \\
57.7 \\
58.3\end{array}$ & $\begin{array}{l}80.1 \\
77.1 \\
83.4\end{array}$ & $\begin{array}{l}62.5 \\
59.5 \\
59.8\end{array}$ & $\begin{array}{l}64.7 \\
63.0 \\
63.8\end{array}$ & $\begin{array}{l}67.7 \\
64.9 \\
69.4\end{array}$ & $\begin{array}{l}57.4 \\
54.2 \\
54.0\end{array}$ & $\begin{array}{l}57.3 \\
55.2 \\
55.4\end{array}$ \\
\hline $\begin{array}{l}\text { January } 1996 \ldots 9 . . . . . . . . . . . . \\
\text { December } 1995 \text {........... } \\
\text { January } 1995 \text {................ } \\
\text { South Carolina }\end{array}$ & $\begin{array}{l}61.3 \\
59.0 \\
63.0\end{array}$ & $\begin{array}{l}54.7 \\
51.3 \\
51.0\end{array}$ & $\begin{array}{l}53.7 \\
52.3 \\
52.7\end{array}$ & $\begin{array}{l}71.8 \\
69.2 \\
73.5\end{array}$ & $\begin{array}{c}W \\
W \\
55.7\end{array}$ & $\begin{array}{l}58.6 \\
57.1 \\
57.9\end{array}$ & $\begin{array}{l}79.4 \\
76.7 \\
81.3\end{array}$ & $\begin{array}{c}W \\
60.3 \\
60.7\end{array}$ & $\begin{array}{l}64.5 \\
62.8 \\
63.7\end{array}$ & $\begin{array}{l}66.8 \\
64.6 \\
68.3\end{array}$ & $\begin{array}{l}56.3 \\
53.3 \\
52.8\end{array}$ & $\begin{array}{l}56.9 \\
55.4 \\
55.9\end{array}$ \\
\hline $\begin{array}{l}\text { January } 1996 \text {............... } \\
\text { December } 1995 \text {............ } \\
\text { January } 1995 \text {................ } \\
\text { Virginia }\end{array}$ & $\begin{array}{l}61.8 \\
59.0 \\
61.8\end{array}$ & $\begin{array}{l}55.1 \\
51.8 \\
51.7\end{array}$ & $\begin{array}{l}54.0 \\
52.3 \\
52.0\end{array}$ & $\begin{array}{l}72.1 \\
69.8 \\
72.1\end{array}$ & $\begin{array}{c}W \\
55.0 \\
55.7\end{array}$ & $\begin{array}{l}59.2 \\
57.3 \\
57.2\end{array}$ & $\begin{array}{l}80.8 \\
77.8 \\
81.6\end{array}$ & $\begin{array}{c}64.1 \\
W \\
60.2\end{array}$ & $\begin{array}{l}65.0 \\
63.2 \\
63.3\end{array}$ & $\begin{array}{l}67.0 \\
64.6 \\
67.1\end{array}$ & $\begin{array}{l}57.4 \\
54.2 \\
53.9\end{array}$ & $\begin{array}{l}57.0 \\
55.4 \\
55.0\end{array}$ \\
\hline $\begin{array}{l}\text { January } 1996 \text {................ } \\
\text { December } 1995 \text {............ } \\
\text { January } 1995 \text {............... } \\
\text { West Virginia }\end{array}$ & $\begin{array}{l}65.7 \\
63.6 \\
74.0\end{array}$ & $\begin{array}{l}57.5 \\
54.7 \\
58.5\end{array}$ & $\begin{array}{l}56.4 \\
54.8 \\
58.0\end{array}$ & $\begin{array}{l}76.7 \\
75.0 \\
86.6\end{array}$ & $\begin{array}{l}60.3 \\
57.7 \\
62.0\end{array}$ & $\begin{array}{l}61.9 \\
60.2 \\
65.5\end{array}$ & $\begin{array}{l}84.0 \\
82.0 \\
\text { NA }\end{array}$ & $\begin{array}{l}66.2 \\
63.3 \\
68.8\end{array}$ & $\begin{array}{l}68.6 \\
66.9 \\
70.9\end{array}$ & $\begin{array}{l}71.9 \\
70.1 \\
81.0\end{array}$ & $\begin{array}{l}59.8 \\
57.0 \\
61.0\end{array}$ & $\begin{array}{l}60.4 \\
58.8 \\
62.5\end{array}$ \\
\hline $\begin{array}{l}\text { January } 1996 \text {............... } \\
\text { December } 1995 \\
\text { January } 1995 \text {..................... }\end{array}$ & $\begin{array}{l}69.8 \\
70.1 \\
74.5\end{array}$ & $\begin{array}{r}\text { NA } \\
59.0 \\
56.4\end{array}$ & $\begin{array}{l}55.9 \\
56.6 \\
53.1\end{array}$ & $\begin{array}{l}78.7 \\
79.5 \\
85.7\end{array}$ & $\begin{array}{c}W \\
W \\
63.3\end{array}$ & $\begin{array}{l}62.6 \\
62.8 \\
59.0\end{array}$ & $\begin{array}{l}87.4 \\
87.5 \\
92.8\end{array}$ & $\begin{array}{l}\text { NA } \\
W \\
69.3\end{array}$ & $\begin{array}{l}67.7 \\
68.3 \\
65.3\end{array}$ & $\begin{array}{l}74.0 \\
74.4 \\
79.3\end{array}$ & $\begin{array}{l}\text { NA } \\
61.6 \\
60.0\end{array}$ & $\begin{array}{l}59.2 \\
59.6 \\
56.5\end{array}$ \\
\hline 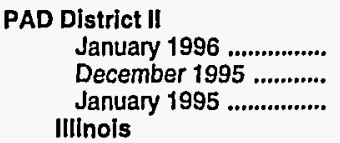 & $\begin{array}{l}70.9 \\
69.2 \\
65.8\end{array}$ & $\begin{array}{l}59.5 \\
58.4 \\
55.6\end{array}$ & $\begin{array}{l}56.6 \\
55.6 \\
53.0\end{array}$ & $\begin{array}{l}80.3 \\
78.6 \\
75.4\end{array}$ & $\begin{array}{l}65.9 \\
65.8 \\
60.8\end{array}$ & $\begin{array}{l}65.0 \\
63.7 \\
61.4\end{array}$ & $\begin{array}{l}87.6 \\
85.8 \\
82.5\end{array}$ & $\begin{array}{l}70.2 \\
68.8 \\
64.7\end{array}$ & $\begin{array}{l}67.4 \\
66.5 \\
64.4\end{array}$ & $\begin{array}{l}74.9 \\
73.3 \\
70.0\end{array}$ & $\begin{array}{l}61.2 \\
60.2 \\
57.4\end{array}$ & $\begin{array}{l}58.9 \\
58.0 \\
55.5\end{array}$ \\
\hline $\begin{array}{l}\text { January } 1996 \ldots . . . . . . . . . . . . \\
\text { December } 1995 \text {............ } \\
\text { January } 1995 \text {............... } \\
\text { Indlana }\end{array}$ & $\begin{array}{l}76.6 \\
74.2 \\
72.8\end{array}$ & $\begin{array}{l}57.6 \\
57.0 \\
55.1\end{array}$ & $\begin{array}{l}59.4 \\
58.2 \\
55.6\end{array}$ & $\begin{array}{l}85.9 \\
84.1 \\
81.4\end{array}$ & $\begin{array}{l}W \\
W \\
W\end{array}$ & $\begin{array}{l}72.7 \\
71.2 \\
68.8\end{array}$ & $\begin{array}{l}93.2 \\
90.6 \\
89.4\end{array}$ & $\begin{array}{l}W \\
W \\
W\end{array}$ & $\begin{array}{l}73.0 \\
72.8 \\
71.8\end{array}$ & $\begin{array}{l}81.1 \\
78.9 \\
77.3\end{array}$ & $\begin{array}{l}58.1 \\
57.3 \\
56.1\end{array}$ & $\begin{array}{l}63.4 \\
62.2 \\
59.8\end{array}$ \\
\hline $\begin{array}{l}\text { January } 1996 \text {................ } \\
\text { December } 1995 \text {............ } \\
\text { January } 1995 \ldots . . . . . . . . . . . \\
\text { lowa }\end{array}$ & $\begin{array}{l}67.7 \\
68.4 \\
65.3\end{array}$ & $\begin{array}{l}55.4 \\
59.9 \\
54.4\end{array}$ & $\begin{array}{l}55.7 \\
55.9 \\
52.8\end{array}$ & $\begin{array}{l}75.6 \\
76.1 \\
72.1\end{array}$ & $\begin{array}{l}W \\
W \\
W\end{array}$ & $\begin{array}{l}63.6 \\
63.3 \\
59.7\end{array}$ & $\begin{array}{l}81.4 \\
82.1 \\
77.6\end{array}$ & $\begin{array}{l}W \\
W \\
60.3\end{array}$ & $\begin{array}{l}67.2 \\
67.2 \\
63.6\end{array}$ & $\begin{array}{l}71.2 \\
71.9 \\
68.7\end{array}$ & $\begin{array}{l}55.7 \\
60.9 \\
55.2\end{array}$ & $\begin{array}{l}58.5 \\
58.7 \\
55.6\end{array}$ \\
\hline $\begin{array}{l}\text { January } 1996 \text {................ } \\
\text { December } 1995 \text {............ } \\
\text { January } 1995 \text {............... } \\
\text { Kansas }\end{array}$ & $\begin{array}{l}\text { NA } \\
68.2 \\
62.5\end{array}$ & $\begin{array}{c}W \\
W \\
55.3\end{array}$ & $\begin{array}{l}57.1 \\
55.4 \\
53.5\end{array}$ & $\begin{array}{l}W \\
W \\
W\end{array}$ & $\begin{array}{l}- \\
-\end{array}$ & $\begin{array}{l}61.0 \\
58.8 \\
56.8\end{array}$ & $\begin{array}{r}\text { NA } \\
78.1 \\
71.8\end{array}$ & $\begin{array}{l}- \\
-\end{array}$ & $\begin{array}{l}65.2 \\
63.4 \\
62.1\end{array}$ & $\begin{array}{l}\text { NA } \\
69.1 \\
63.8\end{array}$ & $\begin{array}{c}W \\
W \\
55.3\end{array}$ & $\begin{array}{l}57.9 \\
56.2 \\
54.2\end{array}$ \\
\hline $\begin{array}{l}\text { January } 1996 \text {................ } \\
\text { December } 1995 \text {............ } \\
\text { January } 1995 \text {............... } \\
\text { Kentucky }\end{array}$ & $\begin{array}{l}66.7 \\
62.9 \\
60.3\end{array}$ & $\begin{array}{l}57.0 \\
55.4 \\
52.6\end{array}$ & $\begin{array}{l}54.8 \\
53.7 \\
50.9\end{array}$ & $\begin{array}{l}75.6 \\
70.7 \\
70.2\end{array}$ & $\vec{w}$ & $\begin{array}{l}62.2 \\
59.7 \\
58.4\end{array}$ & $\begin{array}{l}82.2 \\
77.8 \\
76.4\end{array}$ & $\begin{array}{l}w \\
\vec{w}\end{array}$ & $\begin{array}{l}62.9 \\
60.2 \\
58.9\end{array}$ & $\begin{array}{l}69.3 \\
65.4 \\
62.9\end{array}$ & $\begin{array}{l}57.2 \\
55.4 \\
53.4\end{array}$ & $\begin{array}{l}55.6 \\
54.5 \\
51.7\end{array}$ \\
\hline $\begin{array}{l}\text { January } 1996 \text {............... } \\
\text { Decembet } 1995 \text {............ } \\
\text { January } 1995 \text {............... } \\
\text { Michigan }\end{array}$ & $\begin{array}{l}74.8 \\
73.0 \\
69.8\end{array}$ & $\begin{array}{l}59.1 \\
58.6 \\
58.2\end{array}$ & $\begin{array}{l}55.7 \\
56.0 \\
54.4\end{array}$ & $\begin{array}{l}83.6 \\
83.0 \\
80.6\end{array}$ & $\begin{array}{c}W \\
W \\
68.6\end{array}$ & $\begin{array}{l}61.5 \\
62.4 \\
61.0\end{array}$ & $\begin{array}{l}91.5 \\
90.3 \\
87.2\end{array}$ & $\begin{array}{l}72.5 \\
73.4 \\
70.9\end{array}$ & $\begin{array}{l}65.5 \\
65.6 \\
65.0\end{array}$ & $\begin{array}{l}79.2 \\
77.8 \\
75.1\end{array}$ & $\begin{array}{l}61.3 \\
60.9 \\
61.0\end{array}$ & $\begin{array}{l}58.4 \\
58.7 \\
57.6\end{array}$ \\
\hline $\begin{array}{l}\text { January } 1996 \text {................ } \\
\text { December } 1995 \text {............ } \\
\text { January } 1995 \text {............... } \\
\text { Minnesota }\end{array}$ & $\begin{array}{l}69.3 \\
67.2 \\
61.9\end{array}$ & $\begin{array}{l}60.7 \\
59.4 \\
55.9\end{array}$ & $\begin{array}{l}57.3 \\
56.8 \\
52.5\end{array}$ & $\begin{array}{l}78.1 \\
76.1 \\
70.7\end{array}$ & $\begin{array}{c}63.2 \\
W \\
W\end{array}$ & $\begin{array}{l}65.0 \\
63.3 \\
59.6\end{array}$ & $\begin{array}{l}85.1 \\
83.8 \\
76.6\end{array}$ & $\begin{array}{l}69.8 \\
66.9 \\
64.9\end{array}$ & $\begin{array}{l}68.8 \\
67.8 \\
63.5\end{array}$ & $\begin{array}{l}72.4 \\
70.4 \\
65.4\end{array}$ & $\begin{array}{l}62.1 \\
60.5 \\
57.5\end{array}$ & $\begin{array}{l}59.9 \\
59.3 \\
55.0\end{array}$ \\
\hline $\begin{array}{l}\text { January } 1996 \text {................ } \\
\text { December } 1995 \text {............ } \\
\text { January } 1995 \text {................. } \\
\text { Missouri }\end{array}$ & $\begin{array}{l}76.5 \\
74.2 \\
71.9\end{array}$ & $\begin{array}{l}61.7 \\
59.7 \\
59.6\end{array}$ & $\begin{array}{l}59.2 \\
57.7 \\
57.1\end{array}$ & $\begin{array}{l}84.5 \\
82.1 \\
79.8\end{array}$ & $\begin{array}{l}W \\
W \\
W\end{array}$ & $\begin{array}{l}67.6 \\
66.0 \\
63.9\end{array}$ & $\begin{array}{l}92.3 \\
90.0 \\
88.1\end{array}$ & $\vec{w}$ & $\begin{array}{l}68.2 \\
66.8 \\
66.1\end{array}$ & $\begin{array}{l}79.1 \\
76.8 \\
74.5\end{array}$ & $\begin{array}{l}62.0 \\
59.7 \\
59.9\end{array}$ & $\begin{array}{l}60.6 \\
59.1 \\
58.5\end{array}$ \\
\hline $\begin{array}{l}\text { January } 1996 \text {............... } \\
\text { December } 1995 \text {............ } \\
\text { January } 1995 \text {............... }\end{array}$ & $\begin{array}{l}66.5 \\
60.9 \\
60.6\end{array}$ & $\begin{array}{l}57.3 \\
55.6 \\
51.9\end{array}$ & $\begin{array}{l}55.5 \\
53.7 \\
51.8\end{array}$ & $\begin{array}{l}77.8 \\
71.6 \\
71.5\end{array}$ & $\overline{-}$ & $\begin{array}{l}63.9 \\
60.2 \\
59.0\end{array}$ & $\begin{array}{l}87.0 \\
81.2 \\
80.5\end{array}$ & $\begin{array}{c}W \\
67.2 \\
60.1\end{array}$ & $\begin{array}{l}64.9 \\
62.5 \\
61.1\end{array}$ & $\begin{array}{l}71.1 \\
65.5 \\
64.8\end{array}$ & $\begin{array}{l}58.3 \\
57.0 \\
53.6\end{array}$ & $\begin{array}{l}57.4 \\
55.5 \\
53.8\end{array}$ \\
\hline
\end{tabular}

Seo footnotes at end of table. 
Table 35. Refiner Motor Gasoline Prices by Grade, Sales Type, PAD District, and State (Cents per Gallon Excluding Taxes) - Continued

\begin{tabular}{|c|c|c|c|c|c|c|c|c|c|c|c|c|}
\hline \multirow{3}{*}{$\begin{array}{l}\text { Geographic Area } \\
\text { Month }\end{array}$} & \multicolumn{3}{|c|}{ Regular } & \multicolumn{3}{|c|}{ Midgrade } & \multicolumn{3}{|c|}{ Premium } & \multicolumn{3}{|c|}{ All Grades } \\
\hline & \multicolumn{2}{|c|}{ Sales to End Users } & \multirow{2}{*}{$\begin{array}{l}\text { Sales } \\
\text { for } \\
\text { Resale }\end{array}$} & \multicolumn{2}{|c|}{ Sales to End Users } & \multirow{2}{*}{$\begin{array}{l}\text { Sales } \\
\text { for } \\
\text { Resale }\end{array}$} & \multicolumn{2}{|c|}{ Sales to End Users } & \multirow{2}{*}{$\begin{array}{l}\text { Sales } \\
\text { for } \\
\text { Resale }\end{array}$} & \multicolumn{2}{|c|}{ Sales to End Users } & \multirow[b]{2}{*}{$\begin{array}{c}\text { Sales } \\
\text { for } \\
\text { Resale }\end{array}$} \\
\hline & $\begin{array}{c}\text { Through } \\
\text { Retail } \\
\text { Outlets }\end{array}$ & $\begin{array}{l}\text { Other } \\
\text { End } \\
\text { Usersa }\end{array}$ & & $\begin{array}{c}\text { Through } \\
\text { Retail } \\
\text { Outlets }\end{array}$ & $\begin{array}{l}\text { Other } \\
\text { End } \\
\text { Usersa }\end{array}$ & & $\begin{array}{c}\text { Through } \\
\text { Retail } \\
\text { Outlets }\end{array}$ & $\begin{array}{l}\text { Other } \\
\text { End } \\
\text { Usersa }\end{array}$ & & $\begin{array}{c}\text { Through } \\
\text { Retail } \\
\text { Outlets }\end{array}$ & $\begin{array}{l}\text { Other } \\
\text { End } \\
\text { Usersa }\end{array}$ & \\
\hline Nebraska & & & & & & & & & & & & \\
\hline $\begin{array}{l}\text { January } 1996 \ldots . . . . . . . . . . . . . \\
\text { December } 1995 \text {............ } \\
\text { January } 1995 \text {................ } \\
\text { North Dakota }\end{array}$ & $\begin{array}{l}69.7 \\
68.0 \\
65.6\end{array}$ & $\begin{array}{c}W \\
W \\
53.6\end{array}$ & $\begin{array}{l}57.0 \\
55.2 \\
53.5\end{array}$ & $\begin{array}{c}76.6 \\
70.0 \\
W\end{array}$ & $\begin{array}{l}- \\
-\end{array}$ & $\begin{array}{c}62.1 \\
59.7 \\
W\end{array}$ & $\begin{array}{l}82.8 \\
81.3 \\
80.2\end{array}$ & $\begin{array}{l}- \\
-\end{array}$ & $\begin{array}{l}65.5 \\
63.2 \\
62.3\end{array}$ & $\begin{array}{l}71.1 \\
69.4 \\
67.4\end{array}$ & $\begin{array}{c}W \\
W \\
53.6\end{array}$ & $\begin{array}{l}57.7 \\
55.8 \\
54.1\end{array}$ \\
\hline January 1996 & $w$ & $w$ & 58.9 & $w$ & - & W & $w$ & - & 69.8 & $w$ & $w$ & 60.1 \\
\hline December 1995 ............. & $w$ & $W$ & 57.4 & $W$ & - & $W$ & $w$ & - & 68.2 & $W$ & $W$ & 58.7 \\
\hline 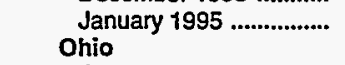 & 69.4 & $w$ & 55.6 & $w$ & - & $w$ & 86.4 & - & 65.8 & 72.2 & $w$ & 56.6 \\
\hline 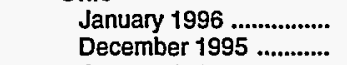 & $\begin{array}{l}70.9 \\
71.0\end{array}$ & $\begin{array}{c}61.4 \\
W\end{array}$ & $\begin{array}{l}56.8 \\
57.3\end{array}$ & $\begin{array}{l}80.4 \\
80.5\end{array}$ & $\begin{array}{l}W \\
W\end{array}$ & $\begin{array}{l}65.1 \\
65.3\end{array}$ & $\begin{array}{l}88.3 \\
88.4\end{array}$ & $\begin{array}{c}73.0 \\
W\end{array}$ & $\begin{array}{l}69.3 \\
70.0\end{array}$ & $\begin{array}{l}75.3 \\
75.5\end{array}$ & $\begin{array}{c}63.7 \\
W\end{array}$ & $\begin{array}{l}59.8 \\
60.3\end{array}$ \\
\hline $\begin{array}{l}\text { January } 1995 \text {.................. } \\
\text { Oklahoma }\end{array}$ & 64.7 & 56.2 & 52.3 & 74.5 & $w$ & 60.8 & 82.6 & 67.6 & 65.7 & 69.6 & 58.5 & 55.5 \\
\hline January 1996 ................ & 63.3 & 56.6 & 53.2 & 71.3 & 58.3 & 57.6 & 78.7 & 64.5 & 60.1 & 66.9 & 58.0 & 54.0 \\
\hline December 1995 ............. & 60.4 & 52.9 & 50.9 & 68.4 & $W$ & 55.9 & 75.7 & 60.6 & 58.7 & 64.1 & 54.4 & 51.9 \\
\hline $\begin{array}{l}\text { January } 1995 \text {.................. } \\
\text { South Dakota }\end{array}$ & 59.7 & 51.1 & 49.1 & 68.1 & 54.2 & 54.2 & 75.9 & 57.4 & 56.4 & 63.5 & 52.4 & 49.9 \\
\hline January 1996 & $w$ & - & 57.7 & W & - & $w$ & 84.5 & - & 66.5 & 76.1 & - & 58.4 \\
\hline December 1995 ............. & $W$ & - & 55.8 & $w$ & - & W & 83.7 & - & 64.7 & 75.5 & - & 56.6 \\
\hline January 1995 & 68.8 & - & 54.1 & $w$ & - & $w$ & 82.1 & - & 63.3 & 69.5 & - & 54.8 \\
\hline Tennessee & & & & & & & & & & & & \\
\hline January 1996 .................. & 65.5 & 54.3 & 54.3 & 77.8 & 56.8 & 59.8 & 86.0 & 63.4 & 65.3 & 72.6 & 56.1 & 57.8 \\
\hline December 1995 .............. & 60.9 & 53.4 & 52.4 & 72.6 & 56.2 & 57.7 & 80.8 & 61.1 & 63.3 & 67.9 & 55.0 & 55.9 \\
\hline January $1995 \ldots . . . . . . . . . . . .$. & 64.4 & $54: 3$ & 52.4 & 77.7 & 55.9 & 58.2 & 85.0 & 58.9 & 63.3 & 71.6 & 55.3 & 56.0 \\
\hline Wisconsin & & & & & & & & & & & & \\
\hline January 1996 .................. & 74.2 & 56.9 & 57.4 & 82.9 & $W$ & 64.9 & 89.4 & $W$ & 67.2 & 77.1 & 58.0 & 59.4 \\
\hline December 1995 & 73.7 & 57.4 & 57.3 & 82.7 & $W$ & 63.9 & 89.0 & $w$ & 67.0 & 76.8 & 57.7 & 59.3 \\
\hline January 1995 .................. & 69.9 & 57.7 & 54.2 & 77.6 & - & 60.2 & 84.0 & 68.2 & 63.9 & 72.7 & 58.6 & 56.0 \\
\hline PAD District III & & & & & & & & & & & & \\
\hline January 1996 & 67.0 & 54.0 & 53.5 & 78.0 & 56.1 & 60.1 & 86.0 & 61.8 & 63.3 & 73.1 & 55.7 & 55.8 \\
\hline December 1995 ............. & 64.1 & 52.2 & 51.2 & 75.5 & 53.9 & 58.3 & 83.3 & 60.3 & 60.8 & 70.4 & 53.8 & 53.5 \\
\hline January 1995 ................... & 70.2 & 51.0 & 51.4 & 81.7 & 53.0 & 59.0 & 90.2 & 59.7 & 61.7 & 76.5 & 52.8 & 53.9 \\
\hline Alabama & & & & & & & & & & & & \\
\hline January 1996 .................. & 64.3 & 54.4 & 54.1 & 75.0 & $W$ & 59.4 & 82.5 & 62.2 & 65.0 & 70.6 & 56.5 & 57.4 \\
\hline December 1995 ............. & 61.6 & 51.3 & 52.2 & 72.0 & $w$ & 57.5 & 79.4 & 59.6 & 63.0 & 68.0 & 53.5 & 55.5 \\
\hline January 1995 .................. & 65.4 & 50.9 & 52.0 & 75.8 & 54.6 & 57.6 & 83.5 & 59.6 & 63.2 & 71.6 & 53.6 & 55.4 \\
\hline Arkansas & & & & & & & & & & & & \\
\hline January 1996 & 64.1 & 56.0 & 53.6 & 73.1 & - & 58.5 & 82.7 & $\mathbf{W}$ & 63.7 & 68.4 & 57.0 & 55.8 \\
\hline December 1995 ............. & 60.6 & 53.3 & 52.2 & 69.9 & $w$ & 57.0 & 79.3 & $w$ & 62.4 & 65.1 & 54.4 & 54.5 \\
\hline January 1995 ................. & 62.8 & 52.0 & 51.5 & 73.0 & W & 56.5 & 81.8 & W & 60.8 & 67.3 & 52.8 & 53.8 \\
\hline Louisiana & & & & & & & & & & & & \\
\hline January 1996 & 66.1 & 54.6 & 52.7 & 78.0 & $w$ & 60.3 & 85.9 & $w$ & 62.9 & 73.6 & 56.5 & 55.7 \\
\hline December 1995 ............. & 64.7 & $W$ & 50.5 & 76.5 & $w$ & 58.5 & 84.2 & $W$ & 60.7 & 72.2 & $W$ & 53.8 \\
\hline January 1995 ................. & 69.1 & 49.7 & 50.3 & 81.0 & W & 58.4 & 88.5 & 57.6 & 61.2 & 76.4 & 51.8 & 53.4 \\
\hline Mississippi & & & & & & & & & & & & \\
\hline January 1996 ................. & 65.2 & 53.4 & 53.2 & 76.5 & 57.2 & 58.5 & 84.9 & 60.6 & 62.6 & 70.3 & 55.2 & 56.0 \\
\hline December 1995 ............ & 61.8 & 50.7 & 51.6 & 72.6 & 54.4 & 56.6 & 80.8 & 59.4 & 60.9 & 67.0 & 52.5 & 54.4 \\
\hline January 1995 ................ & 65.1 & 49.7 & 50.7 & 76.0 & 53.1 & 56.5 & 85.0 & 57.9 & 60.0 & 70.2 & 51.7 & 53.5 \\
\hline New Mexico & & & & & & & & & & & & \\
\hline January 1996 & 77.1 & 60.3 & 60.0 & 86.3 & - & 65.6 & 94.8 & $W$ & 69.6 & 80.4 & 61.6 & 61.7 \\
\hline December 1995 ............ & 75.9 & 61.7 & 57.6 & 85.3 & - & 63.5 & 93.8 & $\ddot{w}$ & 67.1 & 79.4 & 62.7 & 59.2 \\
\hline January 1995 .................. & 78.1 & 57.2 & 54.9 & 89.8 & - & 64.4 & 94.2 & W & 64.5 & 80.7 & 58.2 & 56.3 \\
\hline Texas & & & & & & & & & & & & \\
\hline January 1996 .................. & 66.6 & 53.5 & 53.2 & 78.2 & 55.3 & 60.5 & 86.0 & 61.4 & 62.7 & 72.9 & 55.1 & 55.2 \\
\hline December 1995 ............. & 63.3 & - 52.3 & 50.8 & 75.6 & 53.9 & 58.7 & 83.1 & 60.4 & 59.9 & 70.0 & 53.9 & 52.7 \\
\hline January 1995 ................. & 70.6 & 50.9 & 51.4. & 82.6 & 52.6 & 60.0 & 91.1 & 60.0 & 61.7 & 77.2 & 52.6 & 53.7 \\
\hline PAD District IV & & & & & & & & & & & & \\
\hline January 1996 & 71.2 & 56.7 & 58.8 & 86.5 & 61.0 & 64.2 & 90.9 & W & 68.7 & 77.4 & 59.2 & 61.1 \\
\hline December 1995 ............. & 71.1 & 58.2 & 57.9 & 86.5 & 62.0 & 63.7 & 90.3 & 67.2 & 67.8 & 77.3 & 60.6 & 60.3 \\
\hline $\begin{array}{l}\text { January } 1995 \text {.................. } \\
\text { Colorado }\end{array}$ & 75.1 & 59.0 & 57.5 & 86.3 & $w$ & 63.9 & 94.1 & 65.7 & 67.2 & 80.4 & 60.5 & 59.7 \\
\hline January 1996 & 71.9 & 62.1 & 59.1 & 89.7 & - & 66.2 & 92.3 & $w$ & 69.2 & 78.1 & 62.5 & 61.4 \\
\hline December 1995 ............. & 72.0 & 56.4 & 56.6 & 90.5 & - & 63.6 & 92.2 & $w$ & 66.2 & 78.4 & 59.5 & 58.9 \\
\hline January 1995 ................... & 76.8 & $w$ & 56.3 & 89.1 & - & 64.4 & 98.9 & 64.1 & 66.2 & 82.5 & $W$ & 58.6 \\
\hline
\end{tabular}

See footnotes at end of table. 
Table 35. Refiner Motor Gasoline Prices by Grade, Sales Type, PAD District, and State (Cents per Gallon Excluding Taxes) - Continued

\begin{tabular}{|c|c|c|c|c|c|c|c|c|c|c|c|c|}
\hline \multirow{3}{*}{$\begin{array}{l}\text { Geographic Area } \\
\text { Month }\end{array}$} & \multicolumn{3}{|c|}{ Regular } & \multicolumn{3}{|c|}{ Midgrade } & \multicolumn{3}{|c|}{ Premium } & \multicolumn{3}{|c|}{ All Grades } \\
\hline & \multicolumn{2}{|c|}{ Sales to End Users } & \multirow{2}{*}{$\begin{array}{c}\text { Sales } \\
\text { for } \\
\text { Resale }\end{array}$} & \multicolumn{2}{|c|}{ Sales to End Users } & \multirow{2}{*}{$\begin{array}{l}\text { Sales } \\
\text { for } \\
\text { Resale }\end{array}$} & \multicolumn{2}{|c|}{ Sales to End Users } & \multirow{2}{*}{$\begin{array}{l}\text { Sales } \\
\text { for } \\
\text { Resale }\end{array}$} & \multicolumn{2}{|c|}{ Sales to End Users } & \multirow[b]{2}{*}{$\begin{array}{l}\text { Sales } \\
\text { for } \\
\text { Resale }\end{array}$} \\
\hline & $\begin{array}{c}\text { Through } \\
\text { Retail } \\
\text { Outlets }\end{array}$ & $\begin{array}{l}\text { Other } \\
\text { End } \\
\text { Usersa }\end{array}$ & & $\begin{array}{c}\text { Through } \\
\text { Retail } \\
\text { Outlets }\end{array}$ & $\begin{array}{l}\text { Other } \\
\text { End } \\
\text { Usersa }\end{array}$ & & $\begin{array}{c}\text { Through } \\
\text { Retail } \\
\text { Outlets }\end{array}$ & $\begin{array}{c}\text { Other } \\
\text { End } \\
\text { Usersa }\end{array}$ & & $\begin{array}{c}\text { Through } \\
\text { Retail } \\
\text { Outlets }\end{array}$ & $\begin{array}{l}\text { Other } \\
\text { End } \\
\text { Usersa }\end{array}$ & \\
\hline \multicolumn{13}{|l|}{ Idaho } \\
\hline $\begin{array}{l}\text { January } 1996 \ldots . . . . . . . . . . . . . \\
\text { December } 1995 \text {............ } \\
\text { January } 1995 \text {............... }\end{array}$ & $\begin{array}{l}66.3 \\
69.5 \\
73.3\end{array}$ & $\begin{array}{l}54.1 \\
55.3 \\
61.3\end{array}$ & $\begin{array}{l}55.0 \\
56.7 \\
59.3\end{array}$ & $\begin{array}{c}74.7 \\
76.7 \\
W\end{array}$ & $\begin{array}{l}W \\
W \\
W\end{array}$ & $\begin{array}{l}58.9 \\
61.3 \\
63.2\end{array}$ & $\begin{array}{l}82.4 \\
84.0 \\
84.5\end{array}$ & $\begin{array}{l}W \\
W \\
W\end{array}$ & $\begin{array}{l}64.8 \\
66.6 \\
69.0\end{array}$ & $\begin{array}{l}69.5 \\
72.3 \\
75.0\end{array}$ & $\begin{array}{c}W \\
57.0 \\
61.5\end{array}$ & $\begin{array}{l}57.2 \\
59.0 \\
61.2\end{array}$ \\
\hline \multicolumn{13}{|l|}{ Montana } \\
\hline January 1996 & W & 61.3 & 62.6 & $W$ & - & 67.4 & $w$ & $w$ & 72.8 & $w$ & 61.7 & 64.8 \\
\hline 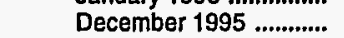 & $w$ & $W$ & 61.9 & $w$ & - & 66.9 & w & W & 72.0 & $w$ & 66.5 & 64.2 \\
\hline $\begin{array}{l}\text { January } 1995 \text {................. } \\
\text { Utah }\end{array}$ & w & 59.1 & 59.3 & $w$ & - & $w$ & $w$ & $w$ & 68.8 & w & 60.5 & 61.1 \\
\hline January 1996 ................ & 68.6 & 57.0 & 58.7 & 78.4 & 62.3 & 64.0 & 87.9 & 66.4 & 68.6 & 75.6 & 59.7 & 61.7 \\
\hline December 1995 ............ & 67.9 & 58.9 & 59.1 & 77.9 & 63.8 & 64.3 & 86.6 & 67.7 & 68.8 & 74.8 & 61.4 & 61.9 \\
\hline 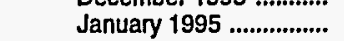 & 65.4 & 57.8 & 58.3 & 74.5 & $W$ & 63.6 & 82.7 & 66.0 & 67.8 & 71.7 & 60.2 & 61.2 \\
\hline Wyoming & & & & & & & & & & & & \\
\hline January 1996 ............... & 68.4 & 62.0 & 57.7 & 82.2 & - & 60.6 & 88.1 & w & 67.1 & 73.2 & 63.7 & 59.5 \\
\hline December 1995 ........... & 66.6 & 59.4 & 55.5 & 79.2 & - & 60.1 & 86.2 & w & 65.0 & 71.6 & 62.0 & 57.3 \\
\hline January 1995 ................ & 73.6 & 57.1 & 53.9 & 84.2 & $\rightarrow$ & 60.9 & 90.3 & $\ddot{w}$ & 62.9 & 77.1 & 60.5 & 55.5 \\
\hline \multicolumn{13}{|l|}{ PAD District V } \\
\hline January 1996 & 75.3 & 64.8 & 64.3 & 80.3 & 67.8 & 71.1 & 93.2 & 76.5 & 78.7 & 79.4 & 67.0 & 68.0 \\
\hline December 1995 ........... & 74.2 & 65.2 & 64.1 & 78.5 & 68.4 & 70.6 & 92.0 & 76.1 & 78.5 & 78.2 & 67.3 & 67.8 \\
\hline January 1995 ............... & 78.8 & 64.5 & 65.6 & 84.9 & 70.2 & 74.9 & 98.3 & 78.5 & 82.3 & 83.1 & 67.1 & 69.7 \\
\hline \multicolumn{13}{|l|}{ Alaska } \\
\hline January 1996 ................ & 108.9 & $w$ & 79.2 & W & - & 95.4 & 122.5 & $W$ & 94.2 & 110.3 & $w$ & 81.8 \\
\hline December 1995 ........... & 107.3 & W & 77.0 & $W$ & - & 94.1 & 120.6 & W & 93.3 & 108.3 & $W$ & 80.1 \\
\hline 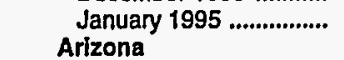 & 110.6 & $\ddot{w}$ & 79.9 & w & - & $W$ & 116.3 & $W$ & 94.3 & $\$ 11.7$ & W & 82.3 \\
\hline January 1996 ................. & 78.7 & 65.4 & 66.9 & 83.3 & $w$ & 74.8 & 96.8 & $w$ & 81.5 & 82.6 & 67.5 & 70.0 \\
\hline December 1995 ........... & 74.6 & 66.1 & 66.1 & 78.0 & $w$ & 73.4 & 93.1 & $w$ & 79.2 & 78.4 & 67.8 & 69.2 \\
\hline January 1995 ................. & 80.0 & 64.0 & 66.4 & 88.6 & - & 70.4 & 98.1 & 81.6 & 80.4 & 83.5 & 65.9 & 68.8 \\
\hline \multicolumn{13}{|l|}{ California } \\
\hline $\begin{array}{l}\text { January } 1996 \text {.................. } \\
\text { December } 1995 \text {........... }\end{array}$ & $\begin{array}{l}70.5 \\
69.7\end{array}$ & 59.4 & 62.3 & 76.5 & 67.2 & 68.9 & 88.2 & 71.2 & 76.8 & 74.7 & 62.5 & $\begin{array}{l}67.2 \\
66.3\end{array}$ \\
\hline January 1995 ................. & 76.9 & 60.1 & 66.1 & 84.5 & $w$ & 74.7 & 97.1 & 77.6 & 82.5 & 82.0 & 64.0 & 70.6 \\
\hline \multicolumn{13}{|l|}{ Hawail } \\
\hline January 1996 ................ & 102.7 & $W$ & 91.7 & 106.9 & $w$ & 99.2 & 118.0 & $W$ & 105.4 & 107.5 & $W$ & 97.1 \\
\hline December 1995 ............ & 103.6 & $W$ & 91.8 & 108.2 & $w$ & 99.4 & 118.8 & $W$ & 105.4 & 108.4 & $W$ & 97.4 \\
\hline $\begin{array}{l}\text { January } 1995 \text {................ } \\
\text { Nevada }\end{array}$ & 103.4 & W & 89.4 & 107.3 & W & 97.1 & 119.1 & W & 103.0 & 108.2 & W & 94.9 \\
\hline \multicolumn{4}{|l|}{ Nevada } & 76.4 & $w$ & 73.0 & 89.3 & - & 79.2 & & & \\
\hline December 1995 ............. & 70.6 & $w$ & 64.9 & 74.3 & $w$ & 72.3 & 87.9 & $w$ & 77.5 & 74.4 & 72.5 & 68.1 \\
\hline January 1995 ................. & 72.6 & 67.3 & 63.8 & 77.4 & $w$ & 70.6 & 89.1 & - & 77.8 & 75.8 & 67.5 & 66.6 \\
\hline Oregon & & & & & & & & & & & & \\
\hline January 1996 ................. & 82.3 & 62.2 & $62.3 \cdot$ & 88.4 & - & 72.5 & 102.7 & W & 75.7 & 85.5 & 62.3 & 64.7 \\
\hline December 1995 ........... & 82.4 & 67.6 & 65.4 & 87.8 & - & 73.4 & 103.0 & $w$ & 79.9 & 85.6 & 67.6 & 67.9 \\
\hline January 1995 ................. & 79.4 & 58.0 & 59.0 & $W$ & - & $w$ & 97.3 & $w$ & 76.4 & 82.3 & 58.3 & 61.0 \\
\hline $\begin{array}{l}\text { Washington } \\
\text { January } 1996\end{array}$ & & & & & & & & & & & & \\
\hline January 1996 .................. & 79.8 & 61.1 & $\begin{array}{l}65.1 \\
67 ?\end{array}$ & 84.9 & $\begin{array}{l}W \\
W\end{array}$ & $\begin{array}{l}73.9 \\
74.5\end{array}$ & 101.1 & $\begin{array}{l}W \\
W\end{array}$ & $\begin{array}{l}80.3 \\
81.9\end{array}$ & $\begin{array}{r}83.3 \\
83.8\end{array}$ & $\begin{array}{l}61.4 \\
65.8\end{array}$ & $\begin{array}{l}68.4 \\
70.4\end{array}$ \\
\hline $\begin{array}{l}\text { December } 1995 \text {............ } \\
\text { January } 1995 \text {............... }\end{array}$ & $\begin{array}{l}80.4 \\
78.7\end{array}$ & $\begin{array}{l}64.9 \\
58.7\end{array}$ & $\begin{array}{l}67.2 \\
64.5\end{array}$ & $\begin{array}{c}84.9 \\
W\end{array}$ & $\frac{w}{-}$ & $\begin{array}{c}7.5 \\
W\end{array}$ & $\begin{array}{r}101.3 \\
98.0\end{array}$ & - & 79.6 & 81.8 & $\begin{array}{l}5.8 \\
58.7\end{array}$ & $\begin{array}{l}70.4 \\
67.1\end{array}$ \\
\hline
\end{tabular}

$\operatorname{Dash}(-)=$ No data reported

NA $=$ Not avallable.

$W=$ Withheld to avoid disciosure of individual company data.

a Sales to "other end users" are all end-user sales that were not made through company-operated relail outlets, e.g., sales to agricultural customers or utilities. Notes: Motor gasoline averages and totals prior to October 1993 include leaded gasoline.

Notes: Values shown for the current month are preliminary. Values shown for previous months are revised. Data are final upon publication in the Petroleum Markeling Annual.

Source: Energy Information Administration Form EIA-782A, "Refiners'/Gas Plant Operators' Monthly Petroleum Product Sales Report." 
Table 36. Refiner Prices of Aviation Fuels and Kerosene by PAD District and State (Cents per Gallon Excluding Taxes)

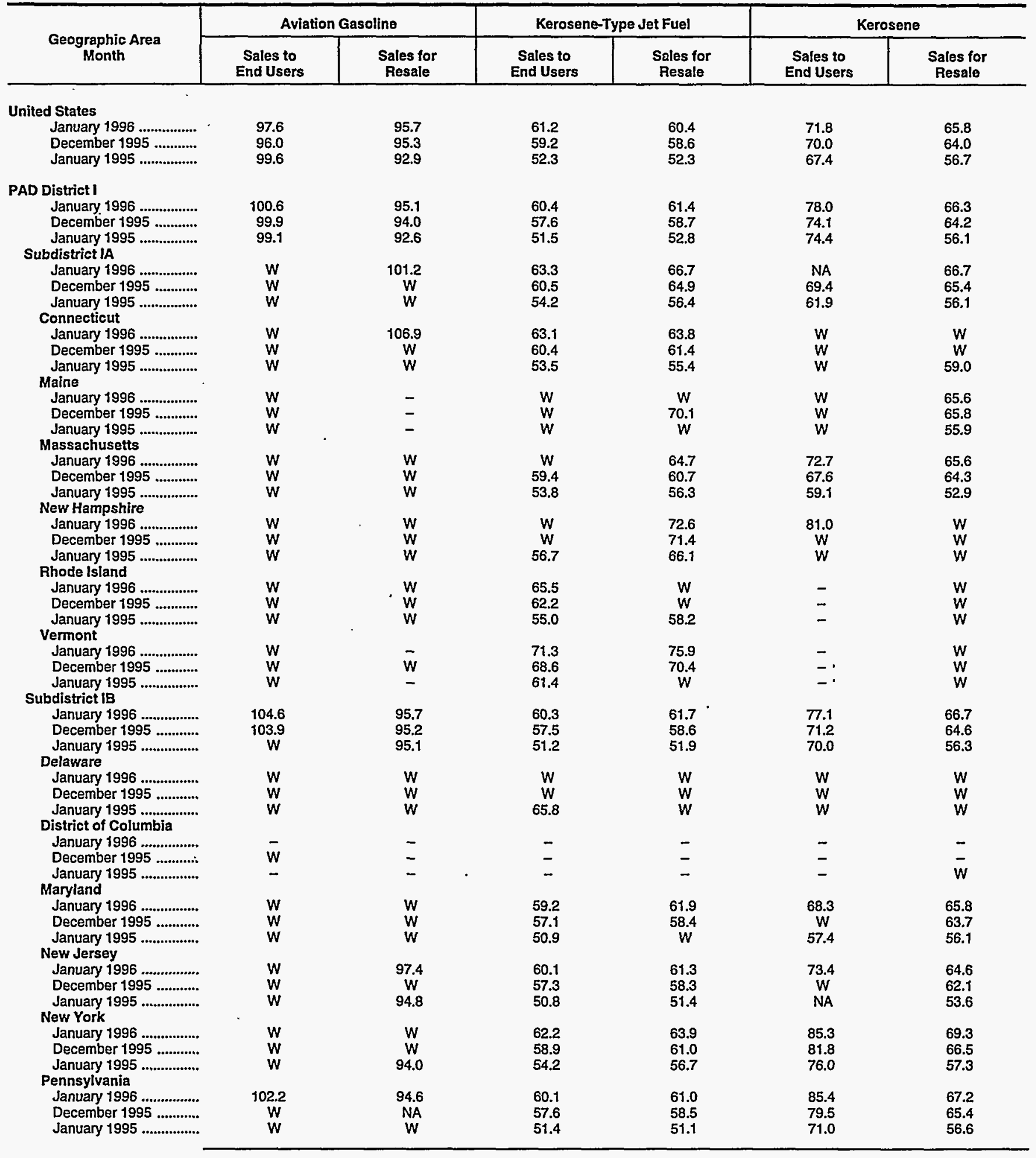

See footnotes at end of table. 
Table 36. Refiner Prices of Aviation Fuels and Kerosene by PAD District and State (Cents per Gallon Excluding Taxes) - Continued

\begin{tabular}{|c|c|c|c|c|c|c|}
\hline \multirow{2}{*}{$\begin{array}{l}\text { Geographic Area } \\
\text { Month }\end{array}$} & \multicolumn{2}{|c|}{ Aviation Gasoline } & \multicolumn{2}{|c|}{ Kerosene-Type Jet Fuel } & \multicolumn{2}{|c|}{ Kerosene } \\
\hline & $\begin{array}{l}\text { Sales to } \\
\text { End Users }\end{array}$ & $\begin{array}{l}\text { Sales for } \\
\text { Resale }\end{array}$ & $\begin{array}{l}\text { Sales to } \\
\text { End Users }\end{array}$ & $\begin{array}{l}\text { Sales for } \\
\text { Resale }\end{array}$ & $\begin{array}{l}\text { Sales to } \\
\text { End Users }\end{array}$ & $\begin{array}{l}\text { Sales for } \\
\text { Resale }\end{array}$ \\
\hline \multicolumn{7}{|l|}{ Subdistrict IC } \\
\hline January 1996 & 98.4 & 94.3 & 59.9 & 60.1 & 91.0 & 65.8 \\
\hline December 1995 ............ & 97.4 & 93.0 & 57.1 & 58.1 & 93.1 & 63.4 \\
\hline January 1995 .................... & 96.7 & 91.7 & 51.3 & 53.1 & 88.1 & 55.9 \\
\hline \multicolumn{7}{|l|}{ Florida } \\
\hline January 1996 ................... & 95.6 & 91.6 & 60.6 & 62.8 & 115.5 & 67.9 \\
\hline December 1995 ............. & 94.9 & 92.3 & 57.6 & 61.5 & 115.1 & 64.5 \\
\hline January 1995 ........................ & & 90.3 & 51.6 & 56.7 & NA & 57.1 \\
\hline \multicolumn{7}{|l|}{ Georgla } \\
\hline January 1996 ......................... & 100.0 & 96.6 & 58.5 & 57.7 & 114.0 & 66.0 \\
\hline December 1995 ............ & 97.8 & 93.2 & 56.1 & 56.0 & 116.7 & 63.7 \\
\hline January 1995 ................... & 97.6 & 92.2 & 50.4 & 49.1 & NA & 55.5 \\
\hline \multicolumn{7}{|l|}{ North Carolina } \\
\hline January 1996 .................. & w & 98.7 & 59.8 & 60.7 & 74.3 & 65.3 \\
\hline December 1995 ............. & $\ddot{w}$ & 94.2 & 56.9 & 58.2 & 76.6 & 63.0 \\
\hline January 1995 ................... & $w$ & $w$ & 50.9 & 52.2 & 80.2 & 55.2 \\
\hline \multicolumn{7}{|l|}{ South Carolina } \\
\hline January 1996 .................. & 101.6 & 98.4 & 63.9 & 65.7 & 96.5 & 65.5 \\
\hline December 1995 ............ & 100.3 & 94.0 & 61.9 & 64.4 & 99.8 & 63.0 \\
\hline January 1995 .................. & 98.9 & $w$ & 55.4 & 60.5 & 99.8 & 55.9 \\
\hline \multicolumn{7}{|l|}{ Virginia } \\
\hline January 1996 ................... & $w$ & w & 59.6 & 62.3 & 67.8 & 66.3 \\
\hline December 1995 ............ & $\ddot{w}$ & $\ddot{w}$ & 57.2 & 59.0 & 67.2 & 63.8 \\
\hline January 1995 ......................... & $\dddot{w}$ & 91.0 & 50.9 & 52.2 & 73.6 & 56.3 \\
\hline West Virginja & & & & & & \\
\hline January 1996 .................. & w & w & w & w & 73.0 & 66.9 \\
\hline December 1995 .............. & $\mathbf{w}$ & - & $w$ & $w$ & 71.7 & 65.7 \\
\hline January 1995 .................... & $w$ & 96.3 & W & $\ddot{w}$ & 72.3 & 60.0 \\
\hline \multicolumn{7}{|l|}{ PAD District II } \\
\hline January 1996 ................... & 86.8 & 94.1 & 60.3 & 62.5 & 76.4 & 65.4 \\
\hline December 1995 ............. & 84.4 & 92.6 & 57.9 & 60.3 & NA & 64.8 \\
\hline January 1995 ..................... & 93.8 & 93.2 & 52.6 & 53.5 & 76.9 & 58.4 \\
\hline \multicolumn{7}{|l|}{ Illinols } \\
\hline 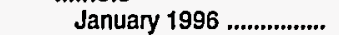 & w & w & 58.8 & 60.4 & 97.7 & 63.5 \\
\hline December 1995 ............. & W & W & 56.5 & 60.3 & NA & 62.6 \\
\hline January 1995 ................... & $w$ & $\ddot{w}$ & 50.7 & 52.5 & 93.5 & 55.8 \\
\hline \multicolumn{7}{|l|}{ Indlana } \\
\hline January 1996 ................... & w & 95.7 & 59.3 & 62.2 & NA & 64.2 \\
\hline December 1995 ............. & $\ddot{w}$ & 92.7 & 56.7 & 58.0 & 103.5 & 62.0 \\
\hline January 1995 ................. & w & 93.4 & 51.4 & 52.9 & 118.0 & 54.9 \\
\hline lowa & & & & & & \\
\hline January 1996 ................... & w & w & 63.1 & 65.4 & - & 66.5 \\
\hline December 1995 ............. & - & $\ddot{w}$ & 62.2 & 65.8 & - & 71.4 \\
\hline January 1995 .................. & W & 94.7 & 57.1 & 60.1 & - & 64.1 \\
\hline Kansas & & & & & & \\
\hline January 1996 ................... & w & 91.6 & w & w & - & 65.9 \\
\hline December 1995 ............. & $w$ & 90.3 & $w$ & $w$ & - & 69.4 \\
\hline January 1995 .................. & w & 91.3 & $w$ & W & - & 63.9 \\
\hline Kentucky & & & & & & \\
\hline January 1996 ................... & W & $\mathbf{w}$ & 62.9 & 61.3 & 82.6 & 67.1 \\
\hline December 1995 ............. & 100.1 & 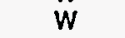 & 60.4 & 57.2 & 81.1 & 66.6 \\
\hline January 1995 .................. & $w$ & 90.4 & 54.5 & 50.0 & 77.9 & 59.8 \\
\hline Michigan & & & & & & \\
\hline January 1996 ................... & w & $w$ & 60.5 & 62.2 & 116.1 & 63.9 \\
\hline December 1995 ............. & $\ddot{w}$ & $\ddot{w}$ & 57.3 & 59.7 & 115.4 & 65.5 \\
\hline January 1995 .................... & $w$ & $w$ & 52.7 & 53.3 & 110.6 & 60.7 \\
\hline Minnesota & & & & & & \\
\hline January 1996 ................... & w & $w$ & 63.2 & 62.3 & $w$ & 69.5 \\
\hline December 1995 .............. & $\mathbf{w}$ & w & 57.7 & 61.0 & $w$ & 71.8 \\
\hline January 1995 ................. & $\ddot{w}$ & 92.9 & 53.3 & 54.4 & $\ddot{w}$ & 62.9 \\
\hline Missouri & & & & & & \\
\hline January 1996 ................... & 90.3 & 93.6 & 61.3 & 65.4 & - & 64.9 \\
\hline December 1995 ............ & $w$ & 91.5 & 58.7 & 63.7 & - & 65.7 \\
\hline January 1995 ................. & w & 92.4 & 52.8 & 57.8 & w & 58.4 \\
\hline
\end{tabular}

See footnotes at end of table. 
Table 36. Refiner Prices of Aviation Fuels and Kerosene by PAD District and State (Cents per Gallon Excluding Taxes) - Continued

\begin{tabular}{|c|c|c|c|c|c|c|}
\hline \multirow{2}{*}{$\begin{array}{c}\text { Geographic Area } \\
\text { Month }\end{array}$} & \multicolumn{2}{|c|}{ Aviation Gasoline } & \multicolumn{2}{|c|}{ Kerosene-Type Jet Fuel } & \multicolumn{2}{|c|}{ Kerosene } \\
\hline & $\begin{array}{l}\text { Sales to } \\
\text { End Users }\end{array}$ & $\begin{array}{l}\text { Sales for } \\
\text { Resale }\end{array}$ & $\begin{array}{l}\text { Sales to } \\
\text { End Users }\end{array}$ & $\begin{array}{l}\text { Sales for } \\
\text { Resale }\end{array}$ & $\begin{array}{l}\text { Sales to } \\
\text { End Users }\end{array}$ & $\begin{array}{c}\text { Sales for } \\
\text { Resale }\end{array}$ \\
\hline \multicolumn{7}{|l|}{ Nebraska } \\
\hline January 1996 & $w$ & $w$ & 61.3 & 65.2 & - & 68.4 \\
\hline December 1995 ............. & $W$ & $\ddot{W}$ & 59.4 & 63.7 & - & 75.2 \\
\hline January 1995 .................. & W & 91.8 & 52.8 & 60.8 & - & NA \\
\hline \multicolumn{7}{|l|}{ North Dakota } \\
\hline January 1996 & $w$ & $w$ & 65.8 & 68.7 & - & $w$ \\
\hline December 1995 ............. & $w$ & $\ddot{W}$ & 65.1 & 71.1 & - & 77.9 \\
\hline January 1995 ................... & 93.0 & $w$ & 61.7 & $\dddot{w}$ & - & $w$ \\
\hline \multicolumn{7}{|l|}{ Ohio } \\
\hline January 1996 & $w$ & 93.0 & 61.3 & 63.0 & 85.9 & 64.9 \\
\hline December 1995 ................. & 96.8 & 92.1 & 58.8 & 59.9 & 86.6 & 65.8 \\
\hline January 1995 ................... & $w$ & 98.0 & 53.6 & 56.0 & 89.0 & 60.3 \\
\hline \multicolumn{7}{|l|}{ Oklahoma } \\
\hline January 1996 ................. & $w$ & 91.8 & 60.8 & 62.2 & - & 63.2 \\
\hline December 1995 ............. & $w$ & 91.6 & 60.3 & 60.5 & - & 68.9 \\
\hline January 1995 .................. & $w$ & 87.8 & 53.7 & 51.0 & $w$ & $w$ \\
\hline \multicolumn{7}{|l|}{ South Dakota } \\
\hline January 1996 & $w$ & $w$ & 63.2 & $w$ & - & 70.6 \\
\hline December 1995 ............. & w & $W$ & 66.2 & $w$ & - & 73.5 \\
\hline January 1995 ................. & $w$ & $W$ & 60.8 & w & - & $w$ \\
\hline \multicolumn{7}{|l|}{ Tennessee } \\
\hline January 1996 & 99.9 & 95.3 & 58.4 & 63.0 & 84.9 & 66.6 \\
\hline December 1995 .............. & 99.8 & 94.6 & 57.7 & 61.8 & 84.7 & 63.4 \\
\hline January 1995 ................. & W & 93.8 & 53.2 & 56.4 & 83.3 & 56.5 \\
\hline \multicolumn{7}{|l|}{ Wisconsin } \\
\hline January 1996 ................. & $W$ & $W$ & 60.8 & 63.4 & $w$ & 65.8 \\
\hline December 1995 ............. & $W$ & $W$ & 58.8 & 62.0 & $W$ & 68.3 \\
\hline January $1995 \ldots . . . . . . . . . . . . .$. & $W$ & 94.8 & 53.0 & 53.7 & $w$ & 61.0 \\
\hline \multicolumn{7}{|l|}{ PAD District III } \\
\hline January 1996 & 93.7 & 88.4 & 57.2 & 56.7 & $w$ & 60.3 \\
\hline December 1995 ............. & 93.1 & 88.7 & 55.3 & 56.3 & $\ddot{w}$ & 58.8 \\
\hline January 1995 ................. & 96.4 & 83.3 & 49.5 & 49.8 & $W$ & 52.7 \\
\hline \multicolumn{7}{|l|}{ Alabama } \\
\hline January 1996 & 97.3 & 95.1 & 61.3 & NA & $w$ & 69.2 \\
\hline December 1995 ............. & 97.6 & 93.2 & 58.1 & 61.5 & W & 66.6 \\
\hline January 1995 ................ & 96.4 & 92.7 & 53.8 & NA & $W$ & 58.0 \\
\hline Arkansas & & & & & & \\
\hline January 1996 ................ & $w$ & $w$ & 65.0 & 64.8 & $w$ & 62.9 \\
\hline December 1995 .............. & $w$ & W & 62.5 & 59.6 & - & 61.7 \\
\hline January 1995 ................. & $\ddot{w}$ & 90.6 & 57.7 & 57.9 & $w$ & 48.6 \\
\hline Louisiana & & & & & & \\
\hline January 1996 ................. & 96.4 & 95.3 & 57.7 & 56.3 & $W$ & 54.2 \\
\hline December 1995 ............. & 94.9 & 91.1 & 55.3 & 56.2 & $W$ & 56.1 \\
\hline 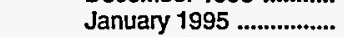 & 95.4 & 90.1 & 49.3 & 51.1 & W & 50.5 \\
\hline Mississippi & & & & & & \\
\hline January 1996 & W & W & 56.6 & $w$ & $W$ & $w$ \\
\hline December 1995 ............. & $\ddot{w}$ & $\ddot{W}$ & 54.3 & 55.2 & $\ddot{w}$ & 58.7 \\
\hline January 1995 ................. & $\ddot{W}$ & 92.4 & 49.3 & $W$ & $w$ & $w$ \\
\hline New Mexico & & & & & & \\
\hline January 1996 & $w$ & $w$ & 63.2 & 63.1 & - & $w$ \\
\hline December 1995 ............ & W & W & 61.4 & 59.4 & - & $\ddot{w}$ \\
\hline January 1995 ................ & $W$ & $w$ & 54.4 & 52.9 & $w$ & 84.9 \\
\hline Texas & & & & & & \\
\hline January 1996 ................. & 92.2 & 86.1 & 56.7 & 56.7 & $w$ & 62.2 \\
\hline December 1995 ............. & 91.0 & 87.5 & 55.1 & 56.3 & w & 61.4 \\
\hline January 1995 ................. & 97.1 & 79.9 & 49.2 & 49.3 & $\ddot{w}$ & 53.0 \\
\hline PAD District IV & & & & & & \\
\hline January 1996 & 101.5 & 97.1 & 68.9 & 72.0 & $w$ & 70.9 \\
\hline December 1995 .............. & 101.6 & 95.0 & 69.0 & 69.9 & $w$ & 71.8 \\
\hline 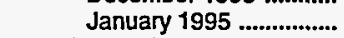 & 101.7 & 95.8 & 57.2 & 60.0 & $W$ & 65.0 \\
\hline Colorado & & & & & & \\
\hline January 1996 .................. & $W$ & 95.7 & 61.4 & 67.1 & - & 67.5 \\
\hline December 1995 ............ & 99.2 & 92.3 & 59.9 & 62.6 & - & 66.8 \\
\hline January 1995 .................. & 96.2 & 95.3 & 52.9 & 56.8 & - & 61.8 \\
\hline
\end{tabular}

See footnotes at end of table. 
Table 36. Refiner Prices of Aviation Fuels and Kerosene by PAD District and State (Cents per Gallon Excluding Taxes) - Continued

\begin{tabular}{|c|c|c|c|c|c|c|}
\hline \multirow{2}{*}{$\begin{array}{l}\text { Geographic Area } \\
\text { Month }\end{array}$} & \multicolumn{2}{|c|}{ Aviation Gasoline } & \multicolumn{2}{|c|}{ Kerosene-Type Jet Fuel } & \multicolumn{2}{|c|}{ Kerosene } \\
\hline & $\begin{array}{c}\text { Sales to } \\
\text { End Users }\end{array}$ & $\begin{array}{c}\text { Sales for } \\
\text { Resale }\end{array}$ & $\begin{array}{c}\text { Sales to } \\
\text { End Users }\end{array}$ & $\begin{array}{l}\text { Sales for } \\
\text { Resale }\end{array}$ & $\begin{array}{c}\text { Sales to } \\
\text { End Users }\end{array}$ & $\begin{array}{l}\text { Sales for } \\
\text { Resale }\end{array}$ \\
\hline \multicolumn{7}{|l|}{ Idaho } \\
\hline January 1996 & - & $w$ & 78.3 & w & - & $w$ \\
\hline December 1995 ............ & $W$ & W & 76.6 & 78.3 & - & $w$ \\
\hline January 1995 ................ & $W$ & $W$ & 67.1 & 71.7 & - & w \\
\hline \multicolumn{7}{|l|}{ Montana } \\
\hline January 1996 ................. & W & $W$ & 75.8 & 78.6 & - & 78.8 \\
\hline December 1995 ............. & W & $W$ & 74.3 & 79.1 & - & 79.6 \\
\hline January 1995 ................ & $W$ & W & 72.3 & 76.5 & - & 64.5 \\
\hline \multicolumn{7}{|l|}{ Utah } \\
\hline January 1996 & W & 98.2 & 76.4 & 77.6 & - & $w$ \\
\hline December 1995 ............ & $W$ & 93.5 & 74.7 & 76.5 & - & $w$ \\
\hline January 1995 ................ & $W$ & 95.7 & 61.7 & 66.7 & - & w \\
\hline Wyoming & & & & & & \\
\hline January 1996 ................. & W & W & 75.6 & 79.9 & $w$ & - \\
\hline December 1995 ............ & $W$ & $W$ & 74.5 & $W$ & $w$ & - \\
\hline January 1995 .................. & $W$ & $W$ & 72.5 & $W$ & $W$ & $w$ \\
\hline \multicolumn{7}{|l|}{ PAD District V } \\
\hline January 1996 ................. & 112.4 & 103.5 & 64.4 & 63.8 & W & 70.0 \\
\hline December 1995 ............. & 112.4 & 103.7 & 62.9 & 63.1 & $w$ & 72.0 \\
\hline $\begin{array}{l}\text { January } 1995 \text {.................. } \\
\text { Alaska }\end{array}$ & 109.8 & 100.7 & 54.8 & 55.5 & w & 62.2 \\
\hline January 1996 ................. & $w$ & 123.8 & 67.5 & 66.2 & - & NA \\
\hline December 1995 ............ & $W$ & 122.4 & 66.6 & 63.6 & - & $w$ \\
\hline January 1995 ................... & W & 118.5 & 59.3 & 53.7 & - & $\ddot{w}$ \\
\hline \multicolumn{7}{|l|}{ Arizona } \\
\hline January 1996 .................. & 104.7 & 99.9 & 65.8 & 68.1 & $W$ & W \\
\hline December 1995 ............ & 102.4 & 100.0 & 64.7 & 67.2 & $w$ & $w$ \\
\hline $\begin{array}{l}\text { January } 1995 \ldots . . . . . . . . . . . . . \\
\text { Calffornia }\end{array}$ & 106.8 & 92.8 & 56.6 & 59.0 & $w$ & $w$ \\
\hline January 1996 ................. & 108.2 & 100.6 & 63.5 & 62.5 & $w$ & 66.6 \\
\hline December 1995 ............. & 107.2 & 98.4 & 61.7 & 62.4 & $w$ & 66.4 \\
\hline \multirow{2}{*}{\multicolumn{7}{|c|}{ Hawall }} \\
\hline & & & & & & \\
\hline January 1996 ................. & $W$ & $W$ & 68.1 & $W$ & - & - \\
\hline December 1995 ............ & W & $W$ & 66.2 & $w$ & - & - \\
\hline January 1995 & W & W & 58.1 & $W$ & - & - \\
\hline \multicolumn{7}{|l|}{ Nevada } \\
\hline January 1996 .................. & $W$ & 103.0 & 66.2 & 67.4 & - & - \\
\hline December 1995 ............. & $W$ & 105.9 & 64.8 & 65.3 & - & - \\
\hline January 1995 & - & $w$ & 57.0 & 57.5 & - & $w$ \\
\hline \multicolumn{7}{|l|}{ Oregon } \\
\hline January 1996 ................... & $\mathbf{w}$ & $w$ & 65.3 & 69.7 & - & 70.4 \\
\hline December 1995 ............ & - & $w$ & 64.3 & 68.0 & - & 73.3 \\
\hline January 1995 .................. & W & 97.2 & 55.0 & 61.6 & - & 61.0 \\
\hline \multicolumn{7}{|l|}{ Washington } \\
\hline January 1996 ................... & $\mathbf{w}$ & $w$ & 63.3 & 66.2 & - & 71.3 \\
\hline December 1995 ............ & $w$ & $w$ & 62.6 & 65.0 & - & 73.6 \\
\hline January 1995 ................. & $w$ & $w$ & 53.8 & 56.0 & - & 60.6 \\
\hline
\end{tabular}

Dash $(-)=$ No data reported.

NA $=$ Not available

$W=$ Withheld to avoid disclosure of Individual company data.

Notes: PAD District and U.S. averages represent data for all States. In certain PAD Districts, however, prices are not shown for every State.

Notes: Values shown for the current month are preliminary. Values shown for previous months are revised. Data are final upon publication in the Petroleum Markoting Annual.

Source: Energy Information Administration Form ElA-782A, "Refiners'/Gas Plant Operators' Monthly Petroleum Product Sales Report." 
Table 37. Refiner Prices of Distillate Fuels by PAD District and State

(Cents per Gallon Excluding Taxes)

\begin{tabular}{|c|c|c|c|c|c|c|}
\hline \multirow{2}{*}{$\begin{array}{c}\text { Geographic Area } \\
\text { Month }\end{array}$} & \multicolumn{2}{|c|}{ No. 1 Distillate } & \multicolumn{2}{|c|}{ No. 2 Distillatea } & \multicolumn{2}{|c|}{ No. 4 Fuel $^{b}$} \\
\hline & $\begin{array}{l}\text { Sales to } \\
\text { End Users }\end{array}$ & $\begin{array}{l}\text { Sales for } \\
\text { Resale }\end{array}$ & $\begin{array}{l}\text { Sales to } \\
\text { End Users }\end{array}$ & $\begin{array}{l}\text { Sales for } \\
\text { Resale }\end{array}$ & $\begin{array}{l}\text { Sales to } \\
\text { End Users }\end{array}$ & $\begin{array}{l}\text { Sales for } \\
\text { Resale }\end{array}$ \\
\hline \multicolumn{7}{|l|}{ United States } \\
\hline January 1996 & 65.1 & 65.0 & 59.7 & 56.4 & 60.4 & 60.3 \\
\hline December 1995 ............. & 66.2 & 66.9 & 59.8 & 57.5 & 57.3 & 51.6 \\
\hline January $1995 \ldots . . . . . . . . . . . . . .$. & 59.3 & 58.9 & 53.9 & 49.8 & 50.5 & 45.9 \\
\hline \multicolumn{7}{|l|}{ PAD District I } \\
\hline Januany 1996 ................. & 67.9 & 67.9 & 61.9 & 57.7 & 64.8 & 61.5 \\
\hline December 1995 ............. & 67.0 & 66.3 & 61.0 & 58.1 & 59.6 & 55.2 \\
\hline January 1995 .................. & 60.4 & 58.0 & 55.6 & 50.2 & 52.4 & 48.3 \\
\hline \multicolumn{7}{|l|}{ Subdistrict lA } \\
\hline January 1996 & $w$ & 70.3 & 64.0 & 58.6 & $w$ & $w$ \\
\hline December 1995 ............. & W & 69.2 & 64.8 & 59.1 & $\ddot{w}$ & $\dddot{w}$ \\
\hline January 1995 .................. & 64.5 & 61.1 & 61.6 & 51.0 & $\ddot{w}$ & w \\
\hline \multicolumn{7}{|l|}{ Connecticut } \\
\hline January 1996 & $w$ & 68.8 & 62.0 & 58.9 & $w$ & $w$ \\
\hline December 1995 ............. & $w$ & 66.2 & 61.3 & 59.6 & $w$ & $W$ \\
\hline 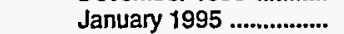 & 62.5 & $w$ & 55.2 & 51.4 & $w$ & W \\
\hline \multicolumn{7}{|l|}{ Maine } \\
\hline January 1996 & $w$ & $W$ & 60.0 & 59.3 & - & - \\
\hline December 1995 .............. & 68.3 & 70.5 & 61.3 & 59.6 & - & - \\
\hline January 1995 .................. & $w$ & 61.3 & 54.5 & 50.6 & - & $w$ \\
\hline \multicolumn{7}{|l|}{ Massachusetts } \\
\hline January 1996 & $w$ & $w$ & 64.2 & 58.2 & $w$ & $w$ \\
\hline December 1995 ............ & $w$ & $\ddot{w}$ & 65.2 & 58.7 & w & W \\
\hline January $1995 \ldots . . . . . . . . . . . . . . . .$. & NA & W & 63.1 & 50.9 & $\ddot{W}$ & $w$ \\
\hline \multicolumn{7}{|l|}{ New Hampshire } \\
\hline January 1996 & $w$ & W & 71.8 & 59.3 & $w$ & - \\
\hline December 1995 ............. & $W$ & $W$ & $w$ & 59.6 & $w$ & - \\
\hline 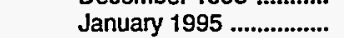 & $w$ & $w$ & $w$ & 52.2 & $w$ & - \\
\hline \multicolumn{7}{|l|}{ Rhode Island } \\
\hline January 1996 ................. & $W$ & $W$ & 61.9 & 58.1 & $w$ & - \\
\hline 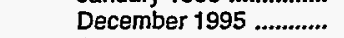 & w & 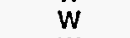 & 70.6 & 58.8 & w & - \\
\hline 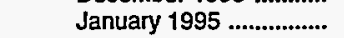 & - & $\ddot{W}$ & 62.2 & 50.5 & w & - \\
\hline \multicolumn{7}{|l|}{ Vermont } \\
\hline January 1996 & $w$ & $w$ & 59.7 & $W$ & $w$ & - \\
\hline December 1995 .............. & $w$ & $\ddot{W}$ & 59.3 & 62.6 & $\ddot{w}$ & - \\
\hline January 1995 & W & W & 57.1 & 53.2 & $\ddot{w}$ & - \\
\hline Subdistrict iB & & & & & & \\
\hline January 1996 & 67.4 & 67.8 & 66.3 & 58.6 & 65.2 & 62.2 \\
\hline December 1995 ............ & 66.7 & 66.3 & 64.6 & 58.6 & 60.1 & 56.7 \\
\hline 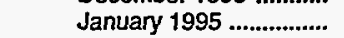 & 60.0 & 57.3 & 58.4 & 50.2 & 52.5 & 48.5 \\
\hline Delaware & & & & & & \\
\hline January 1996 ................. & W & $W$ & 60.3 & $w$ & $w$ & - \\
\hline December 1995 ............. & - & - & 61.3 & $\ddot{W}$ & $\ddot{W}$ & - \\
\hline January 1995 ................. & - & - & 52.9 & 49.1 & $w$ & - \\
\hline District of Columbia & & & & & & \\
\hline January 1996 & - & - & 59.5 & 59.8 & $w$ & - \\
\hline December 1995 ............. & - & $\rightarrow$ & 58.9 & 66.0 & w & - \\
\hline January 1995 & - & - & 52.7 & 51.7 & W & - \\
\hline Maryland & & & & & & \\
\hline January 1996 & 64.1 & 66.7 & 59.2 & 57.4 & $W$ & $w$ \\
\hline December 1995 .............. & 64.9 & $w$ & 58.0 & 57.6 & $\ddot{w}$ & $w$ \\
\hline January 1995 ................... & 59.8 & 55.1 & 50.7 & 50.6 & $w$ & $w$ \\
\hline New Jersey & & & & & & \\
\hline January 1996 .................. & W & 63.5 & 63.2 & 58.1 & 65.3 & 61.9 \\
\hline December 1995 ............. & $\ddot{W}$ & 65.7 & 61.3 & 57.6 & 59.3 & 55.9 \\
\hline January 1995 ......................... & $w$ & $w$ & 53.9 & 49.6 & 52.1 & 49.1 \\
\hline New York & & & & & & \\
\hline January 1996 ................. & $W$ & 69.0 & 69.5 & 60.5 & $w$ & 62.4 \\
\hline December 1995 ............. & W. & 66.8 & 68.4 & 60.9 & w & 57.0 \\
\hline 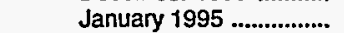 & W & 58.5 & 62.5 & 51.3 & $w$ & $w$ \\
\hline Pennsylvania & & & & & & \\
\hline January 1996 ................. & 70.1 & 67.4 & 70.1 & 57.9 & $w$ & - \\
\hline December 1995 ............. & 69.3 & 65.9 & 67.1 & 58.7 & w & - \\
\hline January 1995 .................. & 63.0 & 56.7 & 61.5 & 50.2 & w & - \\
\hline
\end{tabular}

See footnotes at end of table. 
Table 37. Refiner Prices of Distillate Fuels by PAD District and State

(Cents per Gallon Excluding Taxes) - Continued

\begin{tabular}{|c|c|c|c|c|c|c|}
\hline \multirow{2}{*}{$\begin{array}{c}\text { Geographic Area } \\
\text { Month }\end{array}$} & \multicolumn{2}{|c|}{ No.1 Distillate } & \multicolumn{2}{|c|}{ No. 2 Distillate ${ }^{a}$} & \multicolumn{2}{|c|}{ No. 4 Fuelb $^{b}$} \\
\hline & $\begin{array}{l}\text { Sales to } \\
\text { End Users }\end{array}$ & $\begin{array}{l}\text { Sales for } \\
\text { Resale }\end{array}$ & $\begin{array}{l}\text { Sales to } \\
\text { End Users }\end{array}$ & $\begin{array}{l}\text { Sales for } \\
\text { Resale }\end{array}$ & $\begin{array}{l}\text { Sales to } \\
\text { End Users }\end{array}$ & $\begin{array}{c}\text { Sales for } \\
\text { Resale }\end{array}$ \\
\hline \multicolumn{7}{|l|}{ Subdistrict IC } \\
\hline January 1996 & 65.2 & 65.3 & 58.3 & 55.6 & 61.4 & w \\
\hline December 1995 ................... & 66.1 & 62.9 & 57.8 & 56.5 & 54.3 & $\ddot{w}$ \\
\hline January 1995 .................... & 59.0 & $w$ & 52.9 & 49.7 & $w$ & w \\
\hline \multicolumn{7}{|l|}{ Florida } \\
\hline January 1996 ................... & - & - & 58.5 & 56.0 & $\mathbf{w}$ & - \\
\hline December 1995 ............. & - & - & 57.7 & 56.7 & $w$ & - \\
\hline January 1995 ................... & - & - & 53.1 & 50.2 & $w$ & - \\
\hline \multicolumn{7}{|l|}{ Georgia } \\
\hline January 1996 .................. & $w$ & - & 58.2 & 55.2 & w & - \\
\hline December 1995 .............. & w & - & 57.2 & 56.0 & - & - \\
\hline January 1995 .................... & - & - & 53.8 & 49.4 & w & - \\
\hline \multicolumn{7}{|l|}{ North Carolina } \\
\hline January 1996 ................... & 63.9 & w & 57.5 & 55.3 & w & - \\
\hline December 1995 ............. & $w$ & W & 57.4 & 56.2 & w & w \\
\hline January 1995 .................. & $w$ & - & 51.3 & 49.6 & $w$ & - \\
\hline \multicolumn{7}{|l|}{ South Carolina } \\
\hline January 1996 .................. & - & NA & 60.3 & 55.7 & $\mathbf{w}$ & - \\
\hline December 1995 ............. & - & w & 59.4 & 56.5 & W & - \\
\hline January 1995 ................... & - & - & 53.6 & 49.6 & w & - \\
\hline \multicolumn{7}{|l|}{ Virginia } \\
\hline January 1996 ................... & w & 65.3 & 56.8 & 55.6 & $w$ & w \\
\hline December 1995 ............ & 65.3 & 62.1 & 57.5 & 56.9 & w & $w$ \\
\hline January 1995 ........................ & NA & $w$ & 51.0 & 49.4 & $\dddot{w}$ & $w$ \\
\hline \multicolumn{7}{|l|}{ West Virginia } \\
\hline January 1996 ................... & 70.6 & w & 60.1 & 56.9 & $w$ & - \\
\hline December 1995 .............. & 68.3 & W & 59.5 & 57.4 & w & - \\
\hline January 1995 ..................... & $w$ & - & 55.6 & 49.8 & $\ddot{w}$ & - \\
\hline \multicolumn{7}{|l|}{ PAD District II } \\
\hline January 1996 & 63.0 & 63.3 & 56.8 & 54.7 & w & w \\
\hline December 1995 .............. & 64.3 & 65.8 & 57.3 & 56.4 & 55.9 & $w$ \\
\hline January 1995 ................... & 57.3 & 57.5 & 51.9 & 49.4 & $w$ & $w$ \\
\hline \multicolumn{7}{|l|}{ Illinois } \\
\hline January 1996 ................... & 62.0 & 62.3 & 56.0 & 53.5 & - & - \\
\hline December 1995 ............. & $w$ & 63.0 & 57.1 & 54.9 & - & - \\
\hline January 1995 ................... & w & 55.0 & 50.0 & 47.2 & w & - \\
\hline Indlana & & & & & & \\
\hline January 1996 ................... & 61.9 & 63.1 & 56.4 & 54.4 & w & w \\
\hline December 1995 ............. & 63.9 & 63.3 & 57.3 & 55.5 & - & $\ddot{w}$ \\
\hline January 1995 ....................... & 56.5 & 56.5 & 50.7 & 48.1 & w & - \\
\hline lowa & & & & & & \\
\hline January 1996 ................... & 63.7 & 63.5 & 57.0 & 56.2 & - & - \\
\hline December 1995 .................. & $w$ & 67.2 & 58.2 & 58.9 & - & - \\
\hline January 1995 ................... & $w$ & 58.5 & 51.3 & 50.7 & - & - \\
\hline Kansas & & & & & & \\
\hline January 1996 & 64.0 & 60.2 & 57.5 & 53.8 & - & - \\
\hline December 1995 .............. & 66.0 & 63.8 & 53.4 & 56.4 & - & - \\
\hline January 1995 ...................... & 57.4 & 55.7 & 51.6 & 49.5 & - & - \\
\hline Kentucky & & & & & & \\
\hline January 1996 & 67.3 & 65.1 & 57.6 & 55.2 & $w$ & - \\
\hline December 1995 & 73.1 & 64.4 & 58.1 & 56.5 & $w$ & - \\
\hline January 1995 ........................ & 64.1 & 56.6 & 52.5 & 49.8 & w & - \\
\hline Michigan & & & & & & \\
\hline January 1996 .................... & 63.3 & 63.9 & 56.7 & 54.5 & - & - \\
\hline December 1995 ................. & 63.3 & 65.5 & 57.6 & 56.7 & - & - \\
\hline January 1995 .................... & 56.3 & 56.9 & 51.5 & 49.0 & - & - \\
\hline Minnesota & & & & & & \\
\hline January 1996 ....................... & 63.4 & 64.4 & 54.9 & 55.8 & w & - \\
\hline December 1995 .............. & 64.3 & 67.8 & 56.9 & 58.9 & W & - \\
\hline 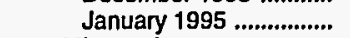 & 58.4 & 59.9 & 50.6 & 52.1 & - & - \\
\hline Missouri & & & & & & \\
\hline January 1996 ....................... & $w$ & 63.2 & 55.4 & 54.5 & - & - \\
\hline December 1995 ............. & $w$ & 66.0 & 57.8 & 56.4 & - & - \\
\hline January 1995 .................... & $w$ & 57.8 & 51.4 & 49.7 & - & - \\
\hline
\end{tabular}

See footnotes at end of table. 
Table 37. Refiner Prices of Distillate Fuels by PAD District and State (Cents per Gallon Excluding Taxes) - Continued

\begin{tabular}{|c|c|c|c|c|c|c|}
\hline \multirow{2}{*}{$\begin{array}{l}\text { Geographic Area } \\
\text { Month }\end{array}$} & \multicolumn{2}{|c|}{ No. 1 Distillate } & \multicolumn{2}{|c|}{ No. 2 Distillatea } & \multicolumn{2}{|c|}{ No. 4 Fuelb } \\
\hline & $\begin{array}{l}\text { Sales to } \\
\text { End Users }\end{array}$ & $\begin{array}{c}\text { Sales for } \\
\text { Resale }\end{array}$ & $\begin{array}{l}\text { Sales to } \\
\text { End Users }\end{array}$ & $\begin{array}{c}\text { Sales for } \\
\text { Resale }\end{array}$ & $\begin{array}{l}\text { Sales to } \\
\text { End Users }\end{array}$ & $\begin{array}{c}\text { Sales for } \\
\text { Resale }\end{array}$ \\
\hline \multicolumn{7}{|l|}{ Nebraska } \\
\hline January 1996 .................. & 63.1 & 63.4 & 54.9 & 56.0 & - & - \\
\hline December 1995 ............. & $w$ & 66.9 & 59.9 & 58.5 & - & - \\
\hline January 1995 ................. & w & 59.2 & 52.5 & 51.5 & - & - \\
\hline \multicolumn{7}{|l|}{ North Dakota } \\
\hline January 1996 ................. & 65.7 & 65.6 & $W$ & 57.1 & - & - \\
\hline December 1995 ............. & 67.0 & 69.2 & $W$ & 60.6 & - & - \\
\hline January 1995 .................. & W & 62.0 & $w$ & 53.6 & - & - \\
\hline \multicolumn{7}{|l|}{ Ohio } \\
\hline January 1996 & 65.1 & 62.4 & 60.2 & 56.0 & $W$ & - \\
\hline December 1995 .............. & 68.3 & 64.0 & 60.7 & 57.2 & W & - \\
\hline January 1995 .................. & 60.8 & $\mathbf{5 7 . 8}$ & 55.2 & 50.3 & W & - \\
\hline \multicolumn{7}{|l|}{ Oklahoma } \\
\hline January 1996 & W & 57.4 & 51.5 & 53.5 & - & - \\
\hline December 1995 ............. & W & 60.3 & 52.5 & 54.8 & - & - \\
\hline January 1995 .................. & 61.3 & 52.9 & 49.5 & 48.5 & - & - \\
\hline \multicolumn{7}{|l|}{ South Dakota } \\
\hline January 1996 & W & 66.3 & $W$ & 58.1 & - & - \\
\hline December 1995 .............. & $\mathbf{W}$ & 68.9 & W & 60.3 & - & - \\
\hline January 1995 ................... & $\mathbf{W}$ & 61.5 & W & 53.0 & - & - \\
\hline \multicolumn{7}{|l|}{ Tennessee } \\
\hline January 1996 .................. & 63.2 & 62.9 & 57.6 & 54.4 & $W$ & - \\
\hline December 1995 .............. & - & $w$ & 56.7 & 55.4 & W & - \\
\hline January 1995 .................. & W & 56.4 & 51.4 & 48.9 & W & $W$ \\
\hline \multicolumn{7}{|l|}{ Wisconsin } \\
\hline January 1996 ................... & 62.2 & 62.9 & 56.5 & 54.0 & $W$ & - \\
\hline December 1995 .............. & 63.3 & 65.3 & 57.7 & 56.5 & $W$ & - \\
\hline January 1995 ................... & 55.5 & 57.1 & 50.4 & 48.6 & W & - \\
\hline \multicolumn{7}{|l|}{ PAD District IIl } \\
\hline January 1996 & W & 65.2 & 56.8 & 53.5 & - & - \\
\hline December 1995 ............. & $\mathbf{W}$ & 61.7 & 56.8 & 54.2 & $W$ & 43.0 \\
\hline January 1995 .................. & $W$ & 57.3 & 52.1 & 48.0 & $W$ & $W$ \\
\hline \multicolumn{7}{|l|}{ Alabama } \\
\hline January 1996 ................. & - & - & 56.8 & 54.5 & - & - \\
\hline December 1995 ............. & - & - & 57.3 & 55.7 & - & - \\
\hline January 1995 & - & - & 50.5 & 48.9 & - & - \\
\hline Arkanses & & & & & & \\
\hline January 1996 & - & $w$ & 60.2 & 52.9 & - & - \\
\hline December 1995 ............. & - & - & 57.2 & 54.4 & - & - \\
\hline January 1995 .................. & - & $w$ & 53.1 & 48.9 & - & - \\
\hline Loulsiana & & & & & & \\
\hline January 1996 ................... & - & - & 56.2 & 52.8 & - & - \\
\hline December 1995 ............. & - & W & 55.6 & 52.9 & - & W \\
\hline January 1995 & - & W & 50.1 & 47.3 & - & - \\
\hline Mississippi & & & & & & \\
\hline January 1996 .................... & - & - & 56.3 & 54.4 & - & - \\
\hline December 1995 ............. & - & - & 57.1 & 55.0 & W & $W$ \\
\hline January 1995 & - & - & 50.3 & 47.9 & W & $W$ \\
\hline New Mexico & & & & & & \\
\hline January 1996 ................... & - & 74.3 & 64.8 & 60.0 & - & - \\
\hline December 1995 ............. & $\mathbf{W}$ & 74.4 & 65.8 & 60.9 & - & - \\
\hline January 1995 ................. & $\mathbf{W}$ & 70.3 & 61.4 & 54.5 & - & - \\
\hline Texas & & & & & & \\
\hline January 1996 & $\mathbf{W}$ & 62.6 & 56.3 & 53.5 & - & - \\
\hline December 1995 ............. & $W$ & 63.5 & 56.5 & 54.3 & - & - \\
\hline January 1995 .................. & W & 53.5 & 52.6 & 47.6 & - & W \\
\hline PAD District IV & & & & & & \\
\hline January 1996 & 69.9 & 71.2 & 62.4 & 60.1 & - & - \\
\hline December 1995 ............. & 72.8 & 73.4 & 65.6 & 62.6 & - & - \\
\hline January 1995 ................. & 64.9 & 64.1 & 56.3 & 54.7 & - & - \\
\hline Colorado & & & & & & \\
\hline January 1996 & 63.0 & 65.3 & 60.7 & 57.0 & - & - \\
\hline December 1995 ............. & W & 65.1 & 61.4 & 57.6 & - & - \\
\hline January 1995 ................. & W & 59.7 & 55.3 & 52.5 & - & - \\
\hline
\end{tabular}

See footnotes at end of table. 
Table 37. Refiner Prices of Distillate Fuels by PAD District and State

(Cents per Gallon Excluding Taxes) - Continued

\begin{tabular}{|c|c|c|c|c|c|c|}
\hline \multirow{2}{*}{$\begin{array}{l}\text { Geographic Area } \\
\text { Month }\end{array}$} & \multicolumn{2}{|c|}{ No. 1 Distillate } & \multicolumn{2}{|c|}{ No. 2 Distillatea } & \multicolumn{2}{|c|}{ No. 4 Fuel $^{b}$} \\
\hline & $\begin{array}{l}\text { Sales to } \\
\text { End Users }\end{array}$ & $\begin{array}{c}\text { Sales for } \\
\text { Resale }\end{array}$ & $\begin{array}{l}\text { Sales to } \\
\text { End Users }\end{array}$ & $\begin{array}{l}\text { Sales for } \\
\text { Resale }\end{array}$ & $\begin{array}{l}\text { Sales to } \\
\text { End Users }\end{array}$ & $\begin{array}{c}\text { Sales for } \\
\text { Resale }\end{array}$ \\
\hline \multicolumn{7}{|l|}{ Idaho } \\
\hline January 1996 ..................... & 76.2 & 73.5 & 61.8 & 63.8 & - & - \\
\hline December 1995 ................ & 82.1 & 78.8 & 65.3 & 69.1 & - & - \\
\hline January 1995 ........................ & 68.6 & 66.5 & 57.4 & 56.4 & $=$ & - \\
\hline \multicolumn{7}{|l|}{ Montana } \\
\hline January 1996 ................. & 73.9 & 74.8 & 62.3 & 61.5 & - & - \\
\hline December 1995 ............ & 76.0 & 76.8 & 63.5 & 64.3 & - & - \\
\hline January 1995 & 65.8 & 65.0 & 54.3 & 54.5 & - & $=$ \\
\hline \multicolumn{7}{|l|}{ Utah } \\
\hline January 1996 & 68.0 & 70.5 & 63.3 & 61.0 & _ & - \\
\hline December 1995 ............. & 75.0 & 74.5 & 67.9 & 65.6 & - & - \\
\hline January 1995 .................... & 65.9 & 66.1 & 57.9 & 56.3 & - & - \\
\hline \multicolumn{7}{|l|}{ Wyoming } \\
\hline January 1996 & 72.1 & 70.7 & 63.3 & 59.4 & - & - \\
\hline December 1995 ............. & 75.0 & 72.4 & 68.1 & 60.2 & - & - \\
\hline 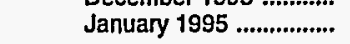 & 66.0 & 65.2 & 56.7 & 53.8 & - & - \\
\hline \multicolumn{7}{|l|}{ PAD District V } \\
\hline January 1996 & 74.5 & 68.7 & 64.2 & 59.7 & $w$ & w \\
\hline December 1995 ................. & 75.5 & 70.3 & 65.5 & 62.2 & $w$ & $w$ \\
\hline January 1995 .................. & 71.1 & 63.3 & 57.3 & 51.8 & $w$ & $w$ \\
\hline \multicolumn{7}{|l|}{ Alaska } \\
\hline January 1996 & 74.3 & 67.5 & 82.4 & 57.2 & $w$ & $w$ \\
\hline December 1995 ............. & 73.6 & 68.4 & 75.6 & 55.9 & $\ddot{w}$ & $w$ \\
\hline January 1995 ........................ & 70.5 & 65.2 & 77.5 & 51.7 & $\dddot{w}$ & $w$ \\
\hline \multicolumn{7}{|l|}{ Arizona } \\
\hline January 1996 ....................... & $w$ & $w$ & 65.5 & 61.5 & - & - \\
\hline December 1995 ............. & $w$ & W & 65.7 & 62.0 & - & - \\
\hline 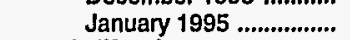 & w & 61.9 & 56.8 & 50.6 & - & - \\
\hline \multicolumn{7}{|l|}{ California } \\
\hline 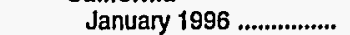 & $w$ & $w$ & 64.3 & 61.3 & - & w \\
\hline December 1995 .............. & $\ddot{w}$ & $w$ & 65.1 & 64.6 & - & w \\
\hline January 1995 ........................ & $w$ & $\ddot{w}$ & 57.2 & 54.1 & - & $W$ \\
\hline \multicolumn{7}{|l|}{ Hawail } \\
\hline 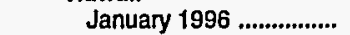 & - & w & 64.0 & 75.2 & - & - \\
\hline December 1995 ................ & - & $w$ & $w$ & 70.1 & - & - \\
\hline January 1995 ................... & - & $\ddot{w}$ & $w$ & 68.8 & - & - \\
\hline \multicolumn{7}{|l|}{ Nevada } \\
\hline 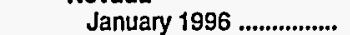 & $w$ & $w$ & 62.5 & 61.9 & - & - \\
\hline December 1995 ................. & $w$ & 75.9 & 65.3 & 63.5 & - & - \\
\hline January 1995 .................... & $\ddot{w}$ & 70.5 & 53.9 & 54.1 & - & - \\
\hline \multicolumn{7}{|l|}{ Oregon } \\
\hline January 1996 & w & 67.8 & 59.6 & 56.9 & - & - \\
\hline December 1995 ............. & $\ddot{w}$ & 73.2 & 61.0 & 59.4 & - & $\therefore$ \\
\hline \multirow{2}{*}{\multicolumn{7}{|c|}{$\begin{array}{l}\text { January ig95 ................ } \\
\text { Washington }\end{array}$}} \\
\hline & & & & & & \\
\hline January 1996 & 85.5 & 71.6 & 61.3 & 55.8 & - & - \\
\hline December 1995 ............. & 82.3 & 74.2 & 66.1 & 57.5 & - & - \\
\hline January 1995 ........................ & 86.7 & 60.2 & 55.9 & 48.3 & - & - \\
\hline
\end{tabular}

Dash $(-)=$ No data reported.

$\mathrm{NA}=$ Not avallable.

$W=$ Withheld to avoid disclosure of individual company data.

a Includes sales of No, 2 fuel oll and high- and low-sulfur diesel fuels.

b Includes No. 4 fuel oil and No. 4 diesel fuel.

Notes: Values shown for the current month are preliminary. Values shown for previous months are revised. Data are final upon publication in the Petroleum Marketing Annual.

Source: Energy Information Administration Form ElA-782A, "Refiners'/Gas Plant Operators' Monthly Petroleum Product Sales Report."

Notes: PAD District and U.S. averages represent data for all States. In certain PAD Districts, however, prices are not shown for every State. 
Table 38. Propane (Consumer Grade) Prices by Sales Type and PAD District (Cents per Gallon Excluding Taxes)

\begin{tabular}{|c|c|c|c|c|c|c|c|c|}
\hline \multirow[b]{2}{*}{$\begin{array}{c}\text { Geographic Area } \\
\text { Month }\end{array}$} & \multicolumn{7}{|c|}{ Sales to End Users } & \multirow[b]{2}{*}{$\begin{array}{c}\text { Sales } \\
\text { for } \\
\text { Resale }\end{array}$} \\
\hline & $\begin{array}{l}\text { Residential } \\
\text { Consumers }\end{array}$ & $\begin{array}{l}\text { Commercial/ } \\
\text { Institutional } \\
\text { Consumers }\end{array}$ & $\begin{array}{l}\text { Industrial } \\
\text { Consumers }\end{array}$ & $\begin{array}{l}\text { Through } \\
\text { Retail } \\
\text { Outlets }\end{array}$ & $\begin{array}{l}\text { Petro- } \\
\text { Chemical }\end{array}$ & $\begin{array}{c}\text { Other } \\
\text { End Users }\end{array}$ & Average & \\
\hline 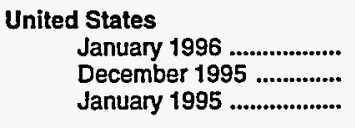 & $\begin{array}{l}92.6 \\
88.5 \\
86.6\end{array}$ & $\begin{array}{l}82.9 \\
79.0 \\
78.3\end{array}$ & $\begin{array}{l}68.0 \\
63.9 \\
67.6\end{array}$ & $\begin{array}{l}\text { NA } \\
73.7 \\
74.8\end{array}$ & $\begin{array}{l}34.5 \\
32.5 \\
34.5\end{array}$ & $\begin{array}{l}72.7 \\
69.7 \\
66.9\end{array}$ & $\begin{array}{l}86.3 \\
81.2 \\
79.5\end{array}$ & $\begin{array}{l}42.3 \\
38.7 \\
36.5\end{array}$ \\
\hline 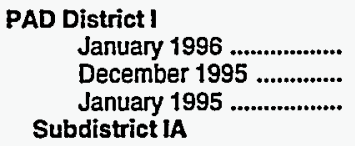 & $\begin{array}{l}113.3 \\
107.8 \\
107.5\end{array}$ & $\begin{array}{l}89.4 \\
84.6 \\
84.8\end{array}$ & $\begin{array}{l}69.0 \\
64.2 \\
65.5\end{array}$ & $\begin{array}{r}\text { NA } \\
70.6 \\
69.8\end{array}$ & $\begin{array}{l}W \\
W \\
W\end{array}$ & $\begin{array}{l}81.6 \\
75.8 \\
72.8\end{array}$ & $\begin{array}{r}102.0 \\
96.5 \\
96.3\end{array}$ & $\begin{array}{l}52.6 \\
44.8 \\
42.0\end{array}$ \\
\hline $\begin{array}{l}\text { January } 1996 \ldots . . . . . . . . . . . . . . \\
\text { December } 1995 \\
\text { January } 1995 \\
\text { Subdistrict IB.................... }\end{array}$ & $\begin{array}{l}117.5 \\
111.7 \\
112.4\end{array}$ & $\begin{array}{l}91.8 \\
87.1 \\
87.6\end{array}$ & $\begin{array}{r}58.9 \\
\text { NA } \\
56.7\end{array}$ & $\begin{array}{l}\text { NA } \\
68.1 \\
66.1\end{array}$ & $\begin{array}{l}- \\
- \\
-\end{array}$ & $\begin{array}{l}98.5 \\
99.0 \\
94.5\end{array}$ & $\begin{array}{r}104.7 \\
98.4 \\
99.2\end{array}$ & $\begin{array}{r}\text { NA } \\
48.7 \\
46.0\end{array}$ \\
\hline $\begin{array}{l}\text { January } 1996 \ldots . . . . . . . . . . . . . \\
\text { December } 1995 \\
\text { January } 1995 \\
\text { Subdistrict IC................... }\end{array}$ & $\begin{array}{l}118.7 \\
112.8 \\
115.6\end{array}$ & $\begin{array}{l}94.0 \\
89.8 \\
90.0\end{array}$ & $\begin{array}{r}64.9 \\
\text { NA } \\
62.0\end{array}$ & $\begin{array}{r}\text { NA } \\
73.4 \\
73.9\end{array}$ & $\begin{array}{l}- \\
-\end{array}$ & $\begin{array}{l}90.5 \\
86.2 \\
88.4\end{array}$ & $\begin{array}{l}106.4 \\
101.0 \\
103.5\end{array}$ & $\begin{array}{l}53.9 \\
45.0 \\
43.1\end{array}$ \\
\hline 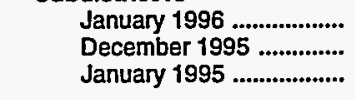 & $\begin{array}{l}109.5 \\
104.0 \\
102.1\end{array}$ & $\begin{array}{l}85.9 \\
80.3 \\
79.9\end{array}$ & $\begin{array}{l}74.8 \\
70.4 \\
71.1\end{array}$ & $\begin{array}{c}W \\
W \\
68.1\end{array}$ & $\begin{array}{l}W \\
W \\
W\end{array}$ & $\begin{array}{l}77.3 \\
70.3 \\
68.0\end{array}$ & $\begin{array}{l}99.1 \\
93.6 \\
92.0\end{array}$ & $\begin{array}{l}51.7 \\
44.1 \\
40.9\end{array}$ \\
\hline $\begin{array}{l}\text { PAD District II } \\
\text { January } 1996 \ldots . . . . . . . . . . . . . . \\
\text { December } 1995 \ldots . . . . . . . . . . \\
\text { January } 1995 \ldots . . . . . . . . . . . .\end{array}$ & $\begin{array}{l}80.4 \\
76.7 \\
73.9\end{array}$ & $\begin{array}{l}73.2 \\
69.8 \\
67.7\end{array}$ & $\begin{array}{l}75.1 \\
71.6 \\
70.8\end{array}$ & $\begin{array}{l}\text { NA } \\
75.7 \\
76.0\end{array}$ & $\begin{array}{l}W \\
W \\
W\end{array}$ & $\begin{array}{l}65.0 \\
61.7 \\
59.0\end{array}$ & $\begin{array}{l}77.9 \\
74.4 \\
71.7\end{array}$ & $\begin{array}{l}41.3 \\
38.9 \\
34.7\end{array}$ \\
\hline 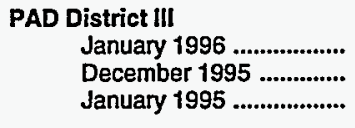 & $\begin{array}{l}93.9 \\
89.3 \\
86.8\end{array}$ & $\begin{array}{l}79.7 \\
75.7 \\
74.7\end{array}$ & $\begin{array}{l}57.0 \\
\text { NA } \\
65.6\end{array}$ & $\begin{array}{r}\text { NA } \\
73.6 \\
72.1\end{array}$ & $\begin{array}{l}33.3 \\
32.1 \\
34.3\end{array}$ & $\begin{array}{l}70.5 \\
66.0 \\
64.9\end{array}$ & $\begin{array}{l}79.3 \\
69.0 \\
68.2\end{array}$ & $\begin{array}{l}38.9 \\
35.5 \\
34.4\end{array}$ \\
\hline 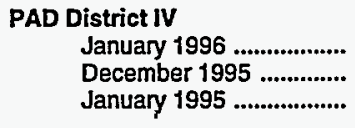 & $\begin{array}{l}82.9 \\
78.8 \\
75.7\end{array}$ & $\begin{array}{l}\text { NA } \\
71.2 \\
72.5\end{array}$ & $\begin{array}{l}64.9 \\
56.1 \\
56.2\end{array}$ & $\begin{array}{l}\text { NA } \\
62.6 \\
69.3\end{array}$ & $\begin{array}{l}- \\
-\end{array}$ & $\begin{array}{l}73.1 \\
71.0 \\
68.4\end{array}$ & $\begin{array}{l}79.3 \\
74.8 \\
72.7\end{array}$ & $\begin{array}{l}41.2 \\
38.6 \\
36.8\end{array}$ \\
\hline 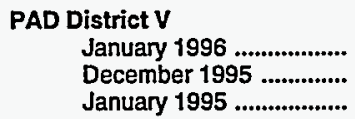 & $\begin{array}{r}103.5 \\
99.8 \\
106.7\end{array}$ & $\begin{array}{l}\text { NA } \\
86.9 \\
90.4\end{array}$ & $\begin{array}{l}67.9 \\
65.4 \\
72.2\end{array}$ & $\begin{array}{c}W \\
W \\
86.5\end{array}$ & $\begin{array}{l}- \\
-\end{array}$ & $\begin{array}{l}106.3 \\
106.5 \\
110.3\end{array}$ & $\begin{array}{l}95.0 \\
93.0 \\
99.4\end{array}$ & $\begin{array}{l}45.5 \\
41.6 \\
45.4\end{array}$ \\
\hline
\end{tabular}

Dash $(-)=$ No data reported.

NA $=$ Not available.

$W=$ Withheld to avoid disclosure of individual company data.

Notes: Values shown for the current month are preliminary. Values shown for previous months are revised. Data are final upon publication in the Petroleum Marketing Annual.

Sources: Energy Information Administration Forms ElA-782A, "Refiners'/Gas Plant Operators' Monthly Petroleum Product Sales Report," and ElA-782B,

"Resellers'/Retailers' Monthly Petroleum Product Sales Report." 
Table 39. No. 2 Distillate ${ }^{a}$ Prices by Sales Type, PAD District, and Selected States ${ }^{b}$ (Cents per Gallon Excluding Taxes)

\begin{tabular}{|c|c|c|c|c|c|c|c|}
\hline \multirow[b]{2}{*}{$\begin{array}{l}\text { Geographic Area } \\
\text { Month }\end{array}$} & \multicolumn{6}{|c|}{ Sales to End Users } & \multirow[b]{2}{*}{$\begin{array}{l}\text { Sales } \\
\text { for } \\
\text { Resale }\end{array}$} \\
\hline & $\begin{array}{l}\text { Residential } \\
\text { Consumers }\end{array}$ & $\begin{array}{l}\text { Commercial/ } \\
\text { Institutional } \\
\text { Consumers }\end{array}$ & $\begin{array}{l}\text { Industrial } \\
\text { Consumers }\end{array}$ & $\begin{array}{l}\text { Through } \\
\text { Retail } \\
\text { Outletsc }\end{array}$ & $\begin{array}{l}\text { Other } \\
\text { End Users d }\end{array}$ & Average & \\
\hline \multicolumn{8}{|l|}{ United States } \\
\hline 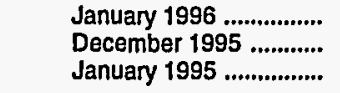 & $\begin{array}{l}94.7 \\
91.2 \\
87.4\end{array}$ & $\begin{array}{l}63.9 \\
63.6 \\
57.5\end{array}$ & $\begin{array}{l}67.7 \\
67.8 \\
62.0\end{array}$ & $\begin{array}{l}69.9 \\
69.1 \\
65.8\end{array}$ & $\begin{array}{l}68.8 \\
69.2 \\
64.3\end{array}$ & $\begin{array}{l}75.2 \\
73.5 \\
69.3\end{array}$ & $\begin{array}{l}57.3 \\
57.9 \\
50.7\end{array}$ \\
\hline PAD District I & & & & a & & & \\
\hline 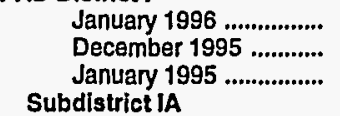 & $\begin{array}{l}96.2 \\
92.1 \\
88.1\end{array}$ & $\begin{array}{l}67.6 \\
66.2 \\
61.2\end{array}$ & $\begin{array}{l}68.0 \\
66.8 \\
62.6\end{array}$ & $\begin{array}{l}71.0 \\
68.1 \\
67.2\end{array}$ & $\begin{array}{l}68.6 \\
66.9 \\
63.5\end{array}$ & $\begin{array}{l}82.1 \\
79.2 \\
76.1\end{array}$ & $\begin{array}{l}58.5 \\
58.3 \\
51.2\end{array}$ \\
\hline $\begin{array}{l}\text { January } 1996 \ldots . . . . . . . . . . . . . \\
\text { December } 1995 \text {............ } \\
\text { January } 1995 \text {.............. } \\
\text { Connecticut }\end{array}$ & $\begin{array}{l}92.9 \\
88.9 \\
84.1\end{array}$ & $\begin{array}{l}72.1 \\
70.6 \\
66.7\end{array}$ & $\begin{array}{l}75.5 \\
74.2 \\
68.7\end{array}$ & $\begin{array}{l}79.1 \\
75.9 \\
73.4\end{array}$ & $\begin{array}{l}72.1 \\
69.5 \\
65.8\end{array}$ & $\begin{array}{l}86.4 \\
83.1 \\
78.7\end{array}$ & $\begin{array}{l}59.1 \\
58.4 \\
51.8\end{array}$ \\
\hline $\begin{array}{l}\text { January } 1996 \text {................. } \\
\text { December } 1995 \text {............ } \\
\text { January } 1995 \text {............... } \\
\text { Maino }\end{array}$ & $\begin{array}{l}94.4 \\
90.5 \\
86.7\end{array}$ & $\begin{array}{l}71.4 \\
69.0 \\
65.7\end{array}$ & $\begin{array}{l}73.1 \\
70.8 \\
67.1\end{array}$ & $\begin{array}{l}80.6 \\
78.4 \\
77.8\end{array}$ & $\begin{array}{l}77.8 \\
75.2 \\
70.3\end{array}$ & $\begin{array}{l}88.4 \\
84.9 \\
81.0\end{array}$ & $\begin{array}{l}57.9 \\
57.0 \\
51.7\end{array}$ \\
\hline $\begin{array}{l}\text { January } 1996 \text {............... } \\
\text { December } 1995 \text {............ } \\
\text { January } 1995 \text {................ } \\
\text { Massachusetts }\end{array}$ & $\begin{array}{l}92.4 \\
87.0 \\
77.8\end{array}$ & $\begin{array}{l}75.9 \\
74.2 \\
66.9\end{array}$ & $\begin{array}{l}73.7 \\
72.5 \\
65.4\end{array}$ & $\begin{array}{l}79.4 \\
75.1 \\
72.0\end{array}$ & $\begin{array}{l}72.8 \\
72.9 \\
65.9\end{array}$ & $\begin{array}{l}85.0 \\
81.6 \\
73.9\end{array}$ & $\begin{array}{l}61.3 \\
61.4 \\
53.5\end{array}$ \\
\hline $\begin{array}{l}\text { January } 1996 \text {................ } \\
\text { December } 1995 \text {............ } \\
\text { January } 1995 \text {............... } \\
\text { New Hampshire }\end{array}$ & $\begin{array}{l}92.1 \\
88.9 \\
84.8\end{array}$ & $\begin{array}{l}70.6 \\
70.0 \\
65.6\end{array}$ & $\begin{array}{l}\text { NA } \\
78.5 \\
74.5\end{array}$ & $\begin{array}{l}79.0 \\
76.9 \\
72.3\end{array}$ & $\begin{array}{l}64.6 \\
62.4 \\
61.1\end{array}$ & $\begin{array}{l}85.3 \\
82.6 \\
78.8\end{array}$ & $\begin{array}{l}59.2 \\
58.9 \\
51.2\end{array}$ \\
\hline $\begin{array}{l}\text { January } 1996 \ldots . . . . . . . . . . . . . . \\
\text { December } 1995 \text {........... } \\
\text { January } 1995 \text {................ } \\
\text { Rhode Island }\end{array}$ & $\begin{array}{l}89.1 \\
83.8 \\
78.4\end{array}$ & $\begin{array}{l}72.1 \\
69.4 \\
64.6\end{array}$ & $\begin{array}{l}67.9 \\
65.9 \\
66.2\end{array}$ & $\begin{array}{l}78.5 \\
75.2 \\
74.5\end{array}$ & $\begin{array}{l}74.2 \\
73.4 \\
64.5\end{array}$ & $\begin{array}{l}83.5 \\
79.1 \\
74.4\end{array}$ & $\begin{array}{l}59.0 \\
55.1 \\
52.0\end{array}$ \\
\hline $\begin{array}{l}\text { January } 1996 . . . . . . . . . . . . . . \\
\text { December } 1995 \text {............ } \\
\text { January } 1995 \text {................ } \\
\text { Vermont }\end{array}$ & $\begin{array}{l}94.9 \\
91.8 \\
87.3\end{array}$ & $\begin{array}{l}73.0 \\
71.7 \\
76.5\end{array}$ & $\begin{array}{l}74.5 \\
72.1 \\
65.2\end{array}$ & $\begin{array}{l}74.1 \\
73.1 \\
65.0\end{array}$ & $\begin{array}{l}\text { NA } \\
68.4 \\
74.5\end{array}$ & $\begin{array}{l}89.5 \\
85.6 \\
83.2\end{array}$ & $\begin{array}{l}59.6 \\
59.4 \\
52.1\end{array}$ \\
\hline 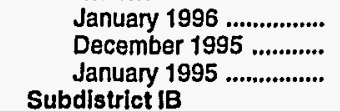 & $\begin{array}{l}92.8 \\
88.0 \\
85.8\end{array}$ & $\begin{array}{l}75.6 \\
72.7 \\
68.8\end{array}$ & $\begin{array}{r}\text { NA } \\
73.1 \\
69.7\end{array}$ & $\begin{array}{l}80.3 \\
75.9 \\
76.0\end{array}$ & $\begin{array}{l}86.2 \\
81.1 \\
77.3\end{array}$ & $\begin{array}{l}88.0 \\
83.3 \\
80.7\end{array}$ & $\begin{array}{l}64.9 \\
64.5 \\
56.3\end{array}$ \\
\hline $\begin{array}{l}\text { January } 1996 \text {............... } \\
\text { December } 1995 \text {........... } \\
\text { January } 1995 \text {............... } \\
\text { Delaware }\end{array}$ & $\begin{array}{l}98.4 \\
94.2 \\
90.1\end{array}$ & $\begin{array}{l}69.5 \\
67.5 \\
62.8\end{array}$ & $\begin{array}{l}71.4 \\
70.1 \\
65.8\end{array}$ & $\begin{array}{l}73.9 \\
70.3 \\
69.6\end{array}$ & $\begin{array}{l}70.8 \\
69.2 \\
63.9\end{array}$ & $\begin{array}{l}86.9 \\
83.6 \\
80.9\end{array}$ & $\begin{array}{l}59.0 \\
58.7 \\
51.1\end{array}$ \\
\hline $\begin{array}{l}\text { January } 1996 \text {................ } \\
\text { December } 1995 \text {............ } \\
\text { January } 1995 \text {................ } \\
\text { District of Columbia }\end{array}$ & $\begin{array}{l}94.1 \\
89.5 \\
88.5\end{array}$ & $\begin{array}{l}64.5 \\
63.4 \\
56.8\end{array}$ & $\begin{array}{r}\text { NA } \\
73.1 \\
68.7\end{array}$ & $\begin{array}{l}69.6 \\
65.9 \\
65.9\end{array}$ & $\begin{array}{l}64.7 \\
68.0 \\
58.3\end{array}$ & $\begin{array}{l}83.2 \\
80.0 \\
76.2\end{array}$ & $\begin{array}{l}58.1 \\
57.8 \\
49.9\end{array}$ \\
\hline $\begin{array}{l}\text { January } 1996 \text {................ } \\
\text { December } 1995 \text {............ } \\
\text { January } 1995 \text {............... } \\
\text { Maryland }\end{array}$ & $\begin{array}{l}111.7 \\
103.8 \\
102.4\end{array}$ & $\begin{array}{l}66.0 \\
65.3 \\
61.3\end{array}$ & $\begin{array}{l}\text { W } \\
69.3 \\
\text { NA }\end{array}$ & $\begin{array}{l}W \\
W \\
W\end{array}$ & $\begin{array}{l}62.6 \\
70.1 \\
W\end{array}$ & $\begin{array}{l}70.9 \\
70.9 \\
69.6\end{array}$ & $\begin{array}{l}\text { NA } \\
\text { NA } \\
50.2\end{array}$ \\
\hline $\begin{array}{l}\text { January } 1996 \text {................ } \\
\text { December } 1995 \text {............ } \\
\text { January } 1995 \text {.............. }\end{array}$ & $\begin{array}{r}104.0 \\
98.5 \\
94.2\end{array}$ & $\begin{array}{l}65.0 \\
63.2 \\
57.3\end{array}$ & $\begin{array}{l}67.3 \\
64.4 \\
60.1\end{array}$ & $\begin{array}{l}70.3 \\
67.4 \\
64.2\end{array}$ & $\begin{array}{l}68.3 \\
67.4 \\
61.4\end{array}$ & $\begin{array}{l}81.6 \\
78.4 \\
74.3\end{array}$ & $\begin{array}{l}58.7 \\
58.2 \\
51.3\end{array}$ \\
\hline $\begin{array}{l}\text { New Jersey } \\
\text { January } 1996 \text {............... } \\
\text { December } 1995 \text {........... } \\
\text { January } 1995 \text {............... } \\
\text { New York }\end{array}$ & $\begin{array}{r}97.7 \\
94.1 \\
\text { NA }\end{array}$ & $\begin{array}{l}64.3 \\
63.2 \\
57.9\end{array}$ & $\begin{array}{r}\text { NA } \\
74.7 \\
69.9\end{array}$ & $\begin{array}{l}73.3 \\
69.2 \\
69.6\end{array}$ & $\begin{array}{l}65.9 \\
64.3 \\
57.5\end{array}$ & $\begin{array}{r}85.5 \\
82.1 \\
\text { NA }\end{array}$ & $\begin{array}{l}57.8 \\
57.2 \\
50.0\end{array}$ \\
\hline $\begin{array}{l}\text { January } 1996 \text {................ } \\
\text { December } 1995 \text {............ } \\
\text { January } 1995 \text {............... }\end{array}$ & $\begin{array}{r}103.5 \\
99.8 \\
95.6\end{array}$ & $\begin{array}{l}76.6 \\
74.2 \\
69.5\end{array}$ & $\begin{array}{l}68.8 \\
68.0 \\
65.5\end{array}$ & $\begin{array}{l}81.0 \\
77.6 \\
80.0\end{array}$ & $\begin{array}{l}73.5 \\
70.9 \\
65.7\end{array}$ & $\begin{array}{l}94.1 \\
91.4 \\
87.2\end{array}$ & $\begin{array}{l}60.9 \\
60.8 \\
52.3\end{array}$ \\
\hline
\end{tabular}

See footnotes at end of table. 
Table 39. No. 2 Distillate ${ }^{a}$ Prices by Sales Type, PAD District, and Selected States ${ }^{b}$ (Cents per Gallon Excluding Taxes) - Continued

\begin{tabular}{|c|c|c|c|c|c|c|c|}
\hline \multirow[b]{2}{*}{$\begin{array}{c}\text { Geographic Area } \\
\text { Month }\end{array}$} & \multicolumn{6}{|c|}{ Sales to End Users } & \multirow[b]{2}{*}{$\begin{array}{l}\text { Sales } \\
\text { for } \\
\text { Resale }\end{array}$} \\
\hline & $\begin{array}{l}\text { Residential } \\
\text { Consumers }\end{array}$ & $\begin{array}{l}\text { Commercial } \\
\text { Institutional } \\
\text { Consumers }\end{array}$ & $\begin{array}{l}\text { Industrial } \\
\text { Consumers }\end{array}$ & $\begin{array}{l}\text { Through } \\
\text { Retail } \\
\text { Outletsc }\end{array}$ & $\begin{array}{l}\text { Other } \\
\text { End Users } d\end{array}$ & Average & \\
\hline \multicolumn{8}{|l|}{ Pennsylvania } \\
\hline $\begin{array}{l}\text { January } 1996 \ldots . . . . . . . . . . . . \\
\text { December } 1995 \ldots . . . . . . . . \\
\text { January } 1995 \ldots . . . . . . . . . . . . \\
\text { Subdistrict IC }\end{array}$ & $\begin{array}{l}92.2 \\
87.2 \\
83.1\end{array}$ & $\begin{array}{l}68.3 \\
66.4 \\
61.7\end{array}$ & $\begin{array}{l}71.2 \\
69.9 \\
65.2\end{array}$ & $\begin{array}{l}72.1 \\
68.3 \\
66.6\end{array}$ & $\begin{array}{l}71.8 \\
69.8 \\
64.8\end{array}$ & $\begin{array}{l}82.7 \\
78.6 \\
74.5\end{array}$ & $\begin{array}{l}59.2 \\
59.6 \\
51.5\end{array}$ \\
\hline $\begin{array}{l}\text { January } 1996 \ldots . . . . . . . . . . . . . \\
\text { December } 1995 \text {............ } \\
\text { January } 1995 \text {............... } \\
\text { Virginia }\end{array}$ & $\begin{array}{l}92.4 \\
89.7 \\
86.3\end{array}$ & $\begin{array}{l}61.9 \\
61.5 \\
56.0\end{array}$ & $\begin{array}{l}64.0 \\
63.2 \\
59.6\end{array}$ & $\begin{array}{l}68.9 \\
66.4 \\
65.4\end{array}$ & $\begin{array}{l}66.3 \\
65.0 \\
62.6\end{array}$ & $\begin{array}{l}69.4 \\
67.4 \\
64.2\end{array}$ & $\begin{array}{l}56.5 \\
57.1 \\
50.7\end{array}$ \\
\hline $\begin{array}{l}\text { January } 1996 \text {................ } \\
\text { December } 1995 \text {............ } \\
\text { January } 1995 \text {................ } \\
\text { West Virginia }\end{array}$ & $\begin{array}{l}91.3 \\
88.1 \\
84.9\end{array}$ & $\begin{array}{l}64.8 \\
64.4 \\
59.5\end{array}$ & $\begin{array}{l}66.7 \\
65.2 \\
62.9\end{array}$ & $\begin{array}{l}67.7 \\
66.0 \\
65.3\end{array}$ & $\begin{array}{l}66.8 \\
65.4 \\
63.3\end{array}$ & $\begin{array}{l}74.1 \\
71.9 \\
69.0\end{array}$ & $\begin{array}{l}57.1 \\
57.6 \\
50.4\end{array}$ \\
\hline $\begin{array}{l}\text { January } 1996 \ldots . . . . . . . . . . . . . \\
\text { December } 1995 \text {........... } \\
\text { January } 1995 . . . . . . . . . . . . . .\end{array}$ & $\begin{array}{l}91.3 \\
89.4 \\
82.1\end{array}$ & $\begin{array}{l}63.1 \\
62.9 \\
57.1\end{array}$ & $\begin{array}{l}66.2 \\
66.1 \\
59.3\end{array}$ & $\begin{array}{l}72.8 \\
69.3 \\
70.7\end{array}$ & $\begin{array}{l}68.3 \\
64.8 \\
60.8\end{array}$ & $\begin{array}{l}70.1 \\
68.2 \\
62.4\end{array}$ & $\begin{array}{l}58.0 \\
58.4 \\
51.2\end{array}$ \\
\hline \multicolumn{8}{|l|}{ PAD District I] } \\
\hline $\begin{array}{l}\text { January } 1996 \text {................ } \\
\text { December } 1995 \text {............ } \\
\text { January } 1995 \text {.............. } \\
\text { lllinois }\end{array}$ & $\begin{array}{l}84.3 \\
83.5 \\
81.0\end{array}$ & $\begin{array}{l}59.0 \\
59.6 \\
54.2\end{array}$ & $\begin{array}{l}67.1 \\
68.1 \\
61.5\end{array}$ & $\begin{array}{l}67.9 \\
67.4 \\
63.7\end{array}$ & $\begin{array}{l}70.6 \\
71.5 \\
67.3\end{array}$ & $\begin{array}{l}67.3 \\
67.1 \\
62.6\end{array}$ & $\begin{array}{l}55.5 \\
57.2 \\
50.3\end{array}$ \\
\hline $\begin{array}{l}\text { January } 1996 \ldots . . . . . . . . . . . . \\
\text { December } 1995 \text {............ } \\
\text { January } 1995 \text {................ } \\
\text { Indiana }\end{array}$ & $\begin{array}{l}84.4 \\
82.9 \\
82.0\end{array}$ & $\begin{array}{l}62.3 \\
62.9 \\
56.1\end{array}$ & $\begin{array}{l}71.4 \\
74.4 \\
64.4\end{array}$ & $\begin{array}{l}69.8 \\
69.2 \\
67.8\end{array}$ & $\begin{array}{l}71.1 \\
72.9 \\
68.6\end{array}$ & $\begin{array}{l}67.7 \\
68.7 \\
62.7\end{array}$ & $\begin{array}{l}54.1 \\
55.5 \\
48.1\end{array}$ \\
\hline $\begin{array}{l}\text { January } 1996 \text {................ } \\
\text { December } 1995 \text {............ } \\
\text { January } 1995 \text {................ } \\
\text { Michigan }\end{array}$ & $\begin{array}{l}85.0 \\
83.7 \\
81.7\end{array}$ & $\begin{array}{l}56.2 \\
57.8 \\
53.2\end{array}$ & $\begin{array}{l}67.9 \\
67.5 \\
59.8\end{array}$ & $\begin{array}{l}68.3 \\
67.4 \\
61.9\end{array}$ & $\begin{array}{l}73.4 \\
75.4 \\
69.9\end{array}$ & $\begin{array}{l}65.9 \\
66.2 \\
61.1\end{array}$ & $\begin{array}{l}54.7 \\
55.9 \\
48.8\end{array}$ \\
\hline $\begin{array}{l}\text { January } 1996 \text {................ } \\
\text { December } 1995 \text {........... } \\
\text { January } 1995 \text {................. } \\
\text { Minnesota }\end{array}$ & $\begin{array}{l}89.1 \\
88.7 \\
86.2\end{array}$ & $\begin{array}{l}62.2 \\
62.3 \\
54.8\end{array}$ & $\begin{array}{l}64.0 \\
66.0 \\
61.0\end{array}$ & $\begin{array}{l}67.8 \\
69.4 \\
64.9\end{array}$ & $\begin{array}{l}68.9 \\
69.2 \\
68.3\end{array}$ & $\begin{array}{l}71.1 \\
70.9 \\
67.2\end{array}$ & $\begin{array}{l}56.0 \\
57.9 \\
51.1\end{array}$ \\
\hline $\begin{array}{l}\text { January } 1996 \text {................. } \\
\text { December } 1995 \text {............. } \\
\text { January } 1995 \text {................ } \\
\text { Ohio }\end{array}$ & $\begin{array}{l}82.5 \\
81.8 \\
80.1\end{array}$ & $\begin{array}{l}60.8 \\
61.6 \\
52.4\end{array}$ & $\begin{array}{l}\text { NA } \\
\text { NA } \\
\text { NA }\end{array}$ & $\begin{array}{l}71.1 \\
70.9 \\
72.2\end{array}$ & $\begin{array}{l}73.7 \\
77.8 \\
74.0\end{array}$ & $\begin{array}{l}72.7 \\
72.7 \\
68.1\end{array}$ & $\begin{array}{l}56.8 \\
59.9 \\
53.2\end{array}$ \\
\hline $\begin{array}{l}\text { January } 1996 \text {................. } \\
\text { December } 1995 \text {............ } \\
\text { January } 1995 \text {............... }\end{array}$ & $\begin{array}{l}85.5 \\
84.0 \\
81.2\end{array}$ & $\begin{array}{l}59.6 \\
60.6 \\
54.2\end{array}$ & $\begin{array}{l}66.1 \\
66.5 \\
60.4\end{array}$ & $\begin{array}{l}67.8 \\
67.8 \\
63.1\end{array}$ & $\begin{array}{l}72.5 \\
70.2 \\
65.8\end{array}$ & $\begin{array}{l}68.2 \\
67.8 \\
62.4\end{array}$ & $\begin{array}{l}56.8 \\
57.8 \\
50.9\end{array}$ \\
\hline $\begin{array}{l}\text { Wisconsin } \\
\text { January } 1996 \text {................ } \\
\text { December } 1995 \text {............ } \\
\text { January } 1995 \text {............... }\end{array}$ & $\begin{array}{l}83.1 \\
82.9 \\
81.1\end{array}$ & $\begin{array}{l}62.5 \\
63.7 \\
57.9\end{array}$ & $\begin{array}{l}62.4 \\
63.1 \\
60.0\end{array}$ & $\begin{array}{l}68.8 \\
68.9 \\
64.9\end{array}$ & $\begin{array}{l}70.4 \\
72.3 \\
66.6\end{array}$ & $\begin{array}{l}71.4 \\
71.4 \\
67.1\end{array}$ & $\begin{array}{l}55.5 \\
57.9 \\
50.2\end{array}$ \\
\hline $\begin{array}{l}\text { PAD District III } \\
\text { January } 1996 \ldots . . . . . . . . . . . . \\
\text { December } 1995 \\
\text { January } 1995 . . . . . . . . . . . . . . . . . . . .\end{array}$ & $\begin{array}{r}70.9 \\
69.5 \\
\text { NA }\end{array}$ & $\begin{array}{l}57.2 \\
57.7 \\
52.4\end{array}$ & $\begin{array}{l}61.8 \\
61.9 \\
57.8\end{array}$ & $\begin{array}{l}66.5 \\
66.3 \\
63.8\end{array}$ & $\begin{array}{l}62.1 \\
63.5 \\
59.1\end{array}$ & $\begin{array}{l}61.6 \\
61.9 \\
57.4\end{array}$ & $\begin{array}{l}54.4 \\
54.8 \\
48.7\end{array}$ \\
\hline $\begin{array}{l}\text { PAD District IV } \\
\text { January } 1996 . . . . . . . . . . . . . \\
\text { December } 1995 \text {........... } \\
\text { January } 1995 \text {................ } \\
\text { Idaho }\end{array}$ & $\begin{array}{l}88.3 \\
89.1 \\
80.7\end{array}$ & $\begin{array}{l}63.5 \\
66.5 \\
57.6\end{array}$ & $\begin{array}{l}74.7 \\
75.9 \\
66.4\end{array}$ & $\begin{array}{l}75.6 \\
77.1 \\
68.3\end{array}$ & $\begin{array}{l}78.8 \\
81.3 \\
71.9\end{array}$ & $\begin{array}{l}71.6 \\
73.7 \\
64.5\end{array}$ & $\begin{array}{l}61.7 \\
64.2 \\
56.1\end{array}$ \\
\hline 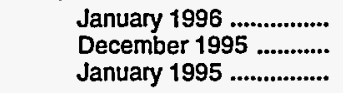 & $\begin{array}{l}87.6 \\
88.8 \\
80.3\end{array}$ & $\begin{array}{l}67.5 \\
69.8 \\
60.8\end{array}$ & $\begin{array}{l}\text { NA } \\
81.6 \\
71.2\end{array}$ & $\begin{array}{l}80.8 \\
84.5 \\
74.6\end{array}$ & $\begin{array}{l}83.3 \\
86.4 \\
72.0\end{array}$ & $\begin{array}{l}78.4 \\
80.6 \\
70.6\end{array}$ & $\begin{array}{r}65.1 \\
70.3 \\
+\quad 57.6\end{array}$ \\
\hline
\end{tabular}

See footnotes at end of table. 
Table 39. No. 2 Distillate ${ }^{a}$ Prices by Sales Type, PAD District, and Selected States ${ }^{b}$ (Cents per Gallon Excluding Taxes) - Continued

\begin{tabular}{|c|c|c|c|c|c|c|c|}
\hline \multirow[b]{2}{*}{$\begin{array}{l}\text { Geographic Area } \\
\text { Month }\end{array}$} & \multicolumn{6}{|c|}{ Sales to End Users } & \multirow{2}{*}{$\begin{array}{c}\text { Sales } \\
\text { for } \\
\text { Resale }\end{array}$} \\
\hline & $\begin{array}{l}\text { Residential } \\
\text { Consumers }\end{array}$ & $\begin{array}{c}\text { Commercial } \\
\text { Institutional } \\
\text { Consumers }\end{array}$ & $\begin{array}{l}\text { Industrial } \\
\text { Consumers }\end{array}$ & $\begin{array}{c}\text { Through } \\
\text { Retail } \\
\text { Outletsc }\end{array}$ & $\begin{array}{l}\text { Other } \\
\text { End Users }\end{array}$ & Average & \\
\hline \multicolumn{8}{|l|}{ PAD District V } \\
\hline January 1996 & 92.5 & 68.4 & 72.1 & 78.2 & 73.4 & 73.3 & 60.8 \\
\hline December 1995 ............. & 92.9 & 69.6 & 72.8 & 79.5 & 75.4 & 74.4 & 62.9 \\
\hline January 1995 ................. & 90.6 & 60.6 & 65.6 & 72.8 & 65.9 & 67.1 & 52.6 \\
\hline \multicolumn{8}{|l|}{ Alaska } \\
\hline $\begin{array}{l}\text { January } 1996 \text {................ } \\
\text { December } 1995 \text {............ }\end{array}$ & $\begin{array}{l}84.1 \\
84.2\end{array}$ & $\begin{array}{l}\text { NA } \\
\text { NA }\end{array}$ & 64.4 & 95.1 & 81.7 & $\begin{array}{l}83.4 \\
82.5\end{array}$ & 58.2 \\
\hline January 1995 ................. & 83.5 & 81.3 & 81.5 & 80.9 & 78.2 & 81.7 & 55.4 \\
\hline \multicolumn{8}{|l|}{ Oregon } \\
\hline January 1996 .................. & 90.1 & 64.7 & 69.8 & 75.7 & 68.9 & 71.1 & 57.5 \\
\hline December 1995 ............. & 90.5 & 65.9 & 71.0 & 78.0 & 70.0 & 72.0 & 60.0 \\
\hline January 1995 ................ & 88.5 & 55.9 & 63.5 & 71.4 & 63.2 & 65.1 & 48.4 \\
\hline \multicolumn{8}{|l|}{ Washington } \\
\hline January 1996 & 99.7 & 64.0 & 70.1 & 76.2 & 77.7 & 73.0 & 56.5 \\
\hline December 1995 ............ & 100.6 & 65.6 & 68.4 & 75.7 & 78.7 & 73.9 & 58.2 \\
\hline January 1995 .................. & 95.4 & 57.3 & 64.7 & 68.9 & 66.7 & 67.8 & 48.8 \\
\hline
\end{tabular}

NA $=$ Not avallable.

$W=$ Withheld to avoid disclosure of individual company data.

a includes sales of No. 2 fuel oil and high- and low-sulfur diesel fuels.

b Some State data are not sufficient for publication individually, but are used in calculating the PAD District average.

c Includes low-sulfur diesel fuel only with the exception of Alaska, which currently is exempt from the Clean Air Act's diesel fuel sulfur content requirement.

d All end-user sales not included in the other end-user categories shown, e.g., sales to agricultural customers or utilities.

Notes: Values shown for the current month are preliminary. Values shown for previous months are revised. Data are final upon publication in the Petroleum Marketing Annual.

Sources: Energy Information Administration Forms EIA-782A, "Refiners'/Gas Plant Operators' Monthly Petroleum Product Sales Report," and ElA-782B, "Resellers'/Retailers' Monthly Petroleum Product Sales Report." 
Table 40. No. 2 Diesel Fuel Prices by Sales Type, PAD District, and Selected States (Cents per Gallon Excluding Taxes)

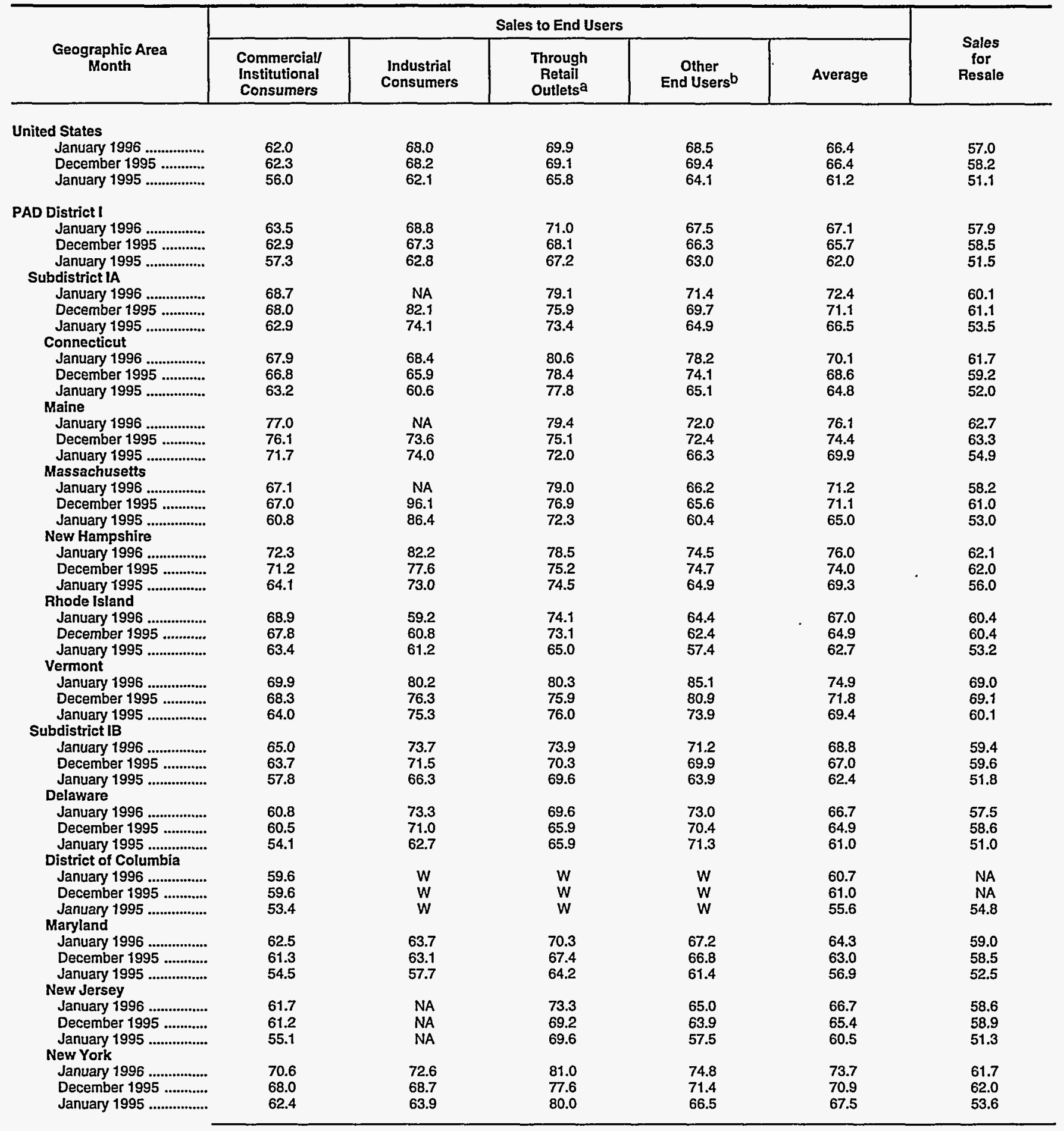

See footnotes at end of table. 
Table 40. No. 2 Diesel Fuel Prices by Sales Type, PAD District, and Selected States (Cents per Gallon Excluding Taxes) - Continued

\begin{tabular}{|c|c|c|c|c|c|c|}
\hline \multirow[b]{2}{*}{$\begin{array}{c}\text { Geographic Area } \\
\text { Month }\end{array}$} & \multicolumn{5}{|c|}{ Sales to End Users } & \multirow[b]{2}{*}{$\begin{array}{l}\text { Sales } \\
\text { for } \\
\text { Resale }\end{array}$} \\
\hline & $\begin{array}{l}\text { Commercialy } \\
\text { Institutional } \\
\text { Consumers }\end{array}$ & $\begin{array}{l}\text { Industrial } \\
\text { Consumers }\end{array}$ & $\begin{array}{l}\text { Through } \\
\text { Retail } \\
\text { Outletsa }\end{array}$ & $\begin{array}{l}\text { Other } \\
\text { End Users b }\end{array}$ & Average & \\
\hline \multicolumn{7}{|l|}{ Pennsylvania } \\
\hline 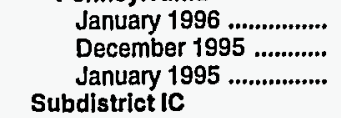 & $\begin{array}{l}65.4 \\
64.4 \\
59.0\end{array}$ & $\begin{array}{l}71.9 \\
70.2 \\
65.5\end{array}$ & $\begin{array}{l}72.1 \\
68.3 \\
66.6\end{array}$ & $\begin{array}{l}71.5 \\
70.9 \\
64.0\end{array}$ & $\begin{array}{l}69.2 \\
67.2 \\
62.8\end{array}$ & $\begin{array}{l}59.5 \\
60.0 \\
51.5\end{array}$ \\
\hline $\begin{array}{l}\text { January } 1996 \ldots . . . . . . . . . . . . \\
\text { December } 1995 \text {............ } \\
\text { January } 1995 \text {............... }\end{array}$ & $\begin{array}{l}61.1 \\
60.9 \\
55.6\end{array}$ & $\begin{array}{l}65.2 \\
63.7 \\
60.1\end{array}$ & $\begin{array}{l}68.9 \\
66.4 \\
65.4\end{array}$ & $\begin{array}{l}65.7 \\
64.7 \\
62.4\end{array}$ & $\begin{array}{l}65.4 \\
64.1 \\
61.0\end{array}$ & $\begin{array}{l}56.3 \\
57.1 \\
50.9\end{array}$ \\
\hline $\begin{array}{l}\text { Virginia } \\
\text { January } 1996 \ldots . . . . . . . . . . . \\
\text { December } 1995 \text {............ } \\
\text { January } 1995 \text {............... }\end{array}$ & $\begin{array}{l}63.1 \\
62.8 \\
58.5\end{array}$ & $\begin{array}{l}66.5 \\
64.8 \\
63.0\end{array}$ & $\begin{array}{l}67.7 \\
66.0 \\
65.3\end{array}$ & $\begin{array}{l}65.9 \\
64.7 \\
64.0\end{array}$ & $\begin{array}{l}65.9 \\
64.9 \\
63.0\end{array}$ & $\begin{array}{l}56.4 \\
57.4 \\
51.1\end{array}$ \\
\hline $\begin{array}{l}\text { West Virginia } \\
\text { January } 1996 \text {................ } \\
\text { December } 1995 \text {............ } \\
\text { January } 1995 \text {.............. }\end{array}$ & $\begin{array}{l}62.4 \\
62.6 \\
56.7\end{array}$ & $\begin{array}{l}67.0 \\
66.8 \\
60.2\end{array}$ & $\begin{array}{l}72.8 \\
69.3 \\
70.7\end{array}$ & $\begin{array}{l}68.8 \\
69.7 \\
62.5\end{array}$ & $\begin{array}{l}66.9 \\
66.1 \\
60.5\end{array}$ & $\begin{array}{l}58.6 \\
58.7 \\
51.6\end{array}$ \\
\hline $\begin{array}{l}\text { PAD District It } \\
\text { January } 1996 \ldots . . . . . . . . . . . \\
\text { December } 1995 \text {............ } \\
\text { January } 1995 \ldots . . . . . . . . . . \\
\text { lllinols }\end{array}$ & $\begin{array}{l}59.4 \\
60.0 \\
54.8\end{array}$ & $\begin{array}{l}67.5 \\
68.7 \\
62.1\end{array}$ & $\begin{array}{l}67.9 \\
67.4 \\
63.7\end{array}$ & $\begin{array}{l}70.9 \\
72.2 \\
67.4\end{array}$ & $\begin{array}{l}65.1 \\
65.4 \\
60.7\end{array}$ & $\begin{array}{l}55.8 \\
57.6 \\
50.7\end{array}$ \\
\hline 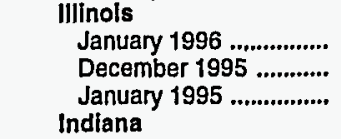 & $\begin{array}{l}62.4 \\
63.2 \\
57.5\end{array}$ & $\begin{array}{l}72.0 \\
74.7 \\
64.7\end{array}$ & $\begin{array}{l}69.8 \\
69.2 \\
67.8\end{array}$ & $\begin{array}{l}71.3 \\
72.7 \\
68.3\end{array}$ & $\begin{array}{l}67.3 \\
68.4 \\
63.0\end{array}$ & $\begin{array}{l}54.8 \\
55.7 \\
49.0\end{array}$ \\
\hline $\begin{array}{l}\text { January } 1996 \ldots . . . . . . . . . . . . . \\
\text { December } 1995 \ldots . . . . . . . . . \\
\text { January } 1995 \text {................ } \\
\text { Mlchigan }\end{array}$ & $\begin{array}{l}56.6 \\
58.6 \\
54.9\end{array}$ & $\begin{array}{l}70.2 \\
69.7 \\
64.0\end{array}$ & $\begin{array}{l}68.3 \\
67.4 \\
61.9\end{array}$ & $\begin{array}{l}73.2 \\
77.0 \\
71.3\end{array}$ & $\begin{array}{l}65.0 \\
65.8 \\
60.8\end{array}$ & $\begin{array}{l}54.7 \\
56.1 \\
49.3\end{array}$ \\
\hline $\begin{array}{l}\text { January } 1996 \text {................ } \\
\text { December } 1995 \text {............ } \\
\text { January } 1995 \text {............... } \\
\text { MInnesota }\end{array}$ & $\begin{array}{l}61.9 \\
62.3 \\
54.8\end{array}$ & $\begin{array}{l}63.8 \\
66.4 \\
61.1\end{array}$ & $\begin{array}{l}67.8 \\
69.4 \\
64.9\end{array}$ & $\begin{array}{l}66.7 \\
69.6 \\
64.5\end{array}$ & $\begin{array}{l}64.8 \\
65.8 \\
60.6\end{array}$ & $\begin{array}{l}56.2 \\
58.4 \\
51.5\end{array}$ \\
\hline $\begin{array}{l}\text { January } 1996 \text {................ } \\
\text { December } 1995 \text {............ } \\
\text { January } 1995 \text {............... } \\
\text { Ohio }\end{array}$ & $\begin{array}{l}63.6 \\
65.0 \\
54.9\end{array}$ & $\begin{array}{l}69.8 \\
69.9 \\
66.3\end{array}$ & $\begin{array}{l}71.1 \\
70.9 \\
72.2\end{array}$ & $\begin{array}{l}78.0 \\
78.0 \\
73.4\end{array}$ & $\begin{array}{l}71.0 \\
71.2 \\
67.2\end{array}$ & $\begin{array}{l}57.7 \\
60.7 \\
53.8\end{array}$ \\
\hline $\begin{array}{l}\text { January } 1996 \text {................ } \\
\text { December } 1995 \text {............. } \\
\text { January } 1995 \text {.............. } \\
\text { Wisconsin }\end{array}$ & $\begin{array}{l}59.3 \\
60.3 \\
54.5\end{array}$ & $\begin{array}{l}65.8 \\
66.4 \\
59.3\end{array}$ & $\begin{array}{l}67.8 \\
67.8 \\
63.1\end{array}$ & $\begin{array}{l}74.4 \\
75.6 \\
71.0\end{array}$ & $\begin{array}{l}64.0 \\
64.9 \\
59.1\end{array}$ & $\begin{array}{l}57.5 \\
58.5 \\
51.6\end{array}$ \\
\hline $\begin{array}{l}\text { January } 1996 . . . . . . . . . . . . . . \\
\text { December } 1995 \text {........... } \\
\text { January } 1995 . . . . . . . . . . . .\end{array}$ & $\begin{array}{l}61.8 \\
63.3 \\
57.1\end{array}$ & $\begin{array}{l}61.4 \\
62.9 \\
59.9\end{array}$ & $\begin{array}{l}68.8 \\
68.9 \\
64.9\end{array}$ & $\begin{array}{l}70.4 \\
72.1 \\
65.2\end{array}$ & $\begin{array}{l}65.2 \\
66.4 \\
61.1\end{array}$ & $\begin{array}{l}55.8 \\
58.2 \\
50.4\end{array}$ \\
\hline 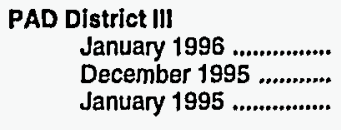 & $\begin{array}{l}57.1 \\
57.7 \\
52.4\end{array}$ & $\begin{array}{l}61.8 \\
62.0 \\
57.9\end{array}$ & $\begin{array}{l}66.5 \\
66.3 \\
63.8\end{array}$ & $\begin{array}{l}62.1 \\
63.5 \\
59.1\end{array}$ & $\begin{array}{l}61.7 \\
62.0 \\
57.4\end{array}$ & $\begin{array}{l}54.8 \\
55.2 \\
49.5\end{array}$ \\
\hline $\begin{array}{l}\text { PAD District IV } \\
\text { January } 1996 \ldots . . . \ldots \ldots \ldots . . . . \\
\text { December } 1995 \ldots . . . . . . . . \\
\text { January } 1995 \\
\text { Idaho }\end{array}$ & $\begin{array}{l}63.4 \\
66.3 \\
57.6\end{array}$ & $\begin{array}{l}74.8 \\
75.9 \\
66.5\end{array}$ & $\begin{array}{l}75.6 \\
77.1 \\
68.3\end{array}$ & $\begin{array}{l}78.8 \\
81.3 \\
71.9\end{array}$ & $\begin{array}{l}71.5 \\
73.6 \\
64.4\end{array}$ & $\begin{array}{l}61.7 \\
64.3 \\
56.2\end{array}$ \\
\hline $\begin{array}{l}\text { January } 1996 \text {................ } \\
\text { December } 1995 . . . . . . . . . \\
\text { January } 1995 \text {............... }\end{array}$ & $\begin{array}{l}67.1 \\
69.6 \\
60.7\end{array}$ & $\begin{array}{l}\text { NA } \\
81.6 \\
71.3\end{array}$ & $\begin{array}{l}80.8 \\
84.5 \\
74.6\end{array}$ & $\begin{array}{l}83.4 \\
86.4 \\
72.0\end{array}$ & $\begin{array}{l}77.7 \\
80.1 \\
69.9\end{array}$ & $\begin{array}{l}65.0 \\
70.2 \\
57.5\end{array}$ \\
\hline
\end{tabular}

See lootnotes at end of table. 
Table 40. No. 2 Diesel Fuel Prices by Sales Type, PAD District, and Selected States (Cents per Gallon Excluding Taxes) - Continued

\begin{tabular}{|c|c|c|c|c|c|c|}
\hline \multirow[b]{2}{*}{$\begin{array}{l}\text { Geographic Area } \\
\text { Month }\end{array}$} & \multicolumn{5}{|c|}{ Sales to End Users } & \multirow[b]{2}{*}{$\begin{array}{c}\text { Sales } \\
\text { for } \\
\text { Resale }\end{array}$} \\
\hline & $\begin{array}{l}\text { Commercial/ } \\
\text { Institutional } \\
\text { Consumers }\end{array}$ & $\begin{array}{l}\text { Industrial } \\
\text { Consumers }\end{array}$ & $\begin{array}{l}\text { Through } \\
\text { Retail } \\
\text { Outlets }\end{array}$ & $\begin{array}{c}\text { Other } \\
\text { End Users }\end{array}$ & Average & \\
\hline \multicolumn{7}{|l|}{ PAD District V } \\
\hline January 1996 & 68.0 & 72.0 & 78.2 & 73.4 & 71.9 & 60.7 \\
\hline December 1995 ............. & 69.2 & 72.7 & 79.5 & 75.5 & 73.2 & 63.1 \\
\hline 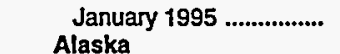 & 60.5 & 65.4 & 72.8 & 65.8 & 65.4 & 52.4 \\
\hline January 1996 .................. & NA & NA & 95.1 & 84.1 & \multicolumn{2}{|c|}{ Alaska } \\
\hline December 1995 ............ & NA & 63.1 & 95.1 & $\begin{array}{l}84.1 \\
85.3\end{array}$ & $\begin{array}{l}82.9 \\
80.8\end{array}$ & $\begin{array}{l}39.1 \\
61.5\end{array}$ \\
\hline January 1995 ................. & 81.7 & 81.0 & 80.9 & 83.5 & 82.1 & $\begin{array}{l}61.5 \\
53.7\end{array}$ \\
\hline \multicolumn{7}{|l|}{ Oregon } \\
\hline January 1996 ................. & 64.5 & 69.8 & 75.7 & 68.6 & 69.2 & 57.5 \\
\hline December 1995 ........... & 65.6 & 71.0 & 78.0 & 69.9 & 70.4 & 60.0 \\
\hline January 1995 ................. & 55.5 & 63.5 & 71.4 & 63.0 & 62.9 & 48.3 \\
\hline \multicolumn{7}{|l|}{ Washington } \\
\hline January 1996 ................ & 64.0 & 69.8 & 76.2 & 77.8 & 68.7 & 56.4 \\
\hline December 1995 ............. & 65.5 & 68.2 & 75.7 & 78.9 & 69.6 & 58.1 \\
\hline January $1995 \ldots . . . . . . . . . . . . .$. & 57.3 & 64.3 & 68.9 & 66.7 & 62.7 & 48.6 \\
\hline
\end{tabular}

NA $=$ Not available.

$W=$ Withheld to avoid disclosure of individual company data

a Includes low-sulfur diesel fuel only with the exception of Alaska, which currently is exempt from the Clean Air Act's diesel fuel sulfur content requirement.

b All end-user sales not included in the other end-user categories shown, e.g., sales to agricultural customers or utilities.

Notes: Values shown for the current month are preliminary. Values shown for previous months are revised. Data are final upon publication in the Petroleum Marketing Annual.

Sources: Energy Information Administration Forms ElA-782A, "Refiners'/Gas Plant Operators' Monthly Petroleum Product Sales Report," and EIA-782B, 'Resellers'/Retailers' Monthly Petroleum Product Sales Report." 
Table 41. No. 2 Diesel Fuel Prices by Sulfur Content, Sales Type, and PAD District (Cents per Gallon Excluding Taxes)

\begin{tabular}{|c|c|c|c|c|c|c|c|c|c|c|c|}
\hline \multirow{3}{*}{$\begin{array}{c}\text { Geographic Area } \\
\text { Month }\end{array}$} & \multicolumn{6}{|c|}{ Low-Sulfur Diesel Fuel } & \multicolumn{5}{|c|}{ High-Sulfur Diesel Fuel } \\
\hline & \multicolumn{5}{|c|}{ Sales to End Users } & \multirow{2}{*}{$\begin{array}{l}\text { Sales } \\
\text { for } \\
\text { Resale }\end{array}$} & \multicolumn{4}{|c|}{ Sales to End Users } & \multirow{2}{*}{$\begin{array}{l}\text { Sales } \\
\text { for } \\
\text { Resale }\end{array}$} \\
\hline & $\begin{array}{c}\text { Commercialy } \\
\text { Institutional } \\
\text { Consumers }\end{array}$ & $\begin{array}{c}\text { Industrial } \\
\text { Consumers }\end{array}$ & $\begin{array}{c}\text { Through } \\
\text { Retail } \\
\text { Outlets }\end{array}$ & $\begin{array}{l}\text { Other } \\
\text { End } \\
\text { Users b }\end{array}$ & Average & & $\begin{array}{l}\text { Commercial } \\
\text { Institutional } \\
\text { Consumers }\end{array}$ & $\begin{array}{c}\text { Industrial } \\
\text { Consumers }\end{array}$ & $\begin{array}{l}\text { Other } \\
\text { End } \\
\text { Users b }\end{array}$ & Average & \\
\hline $\begin{array}{l}\text { United States } \\
\text { January } 1996 \ldots . . . . . . . . . . \\
\text { December } 1995 \ldots . . . . . . . \\
\text { January } 1995 \ldots . . . . . . . . .\end{array}$ & $\begin{array}{l}63.0 \\
63.7 \\
57.2\end{array}$ & $\begin{array}{l}69.1 \\
69.5 \\
63.4\end{array}$ & $\begin{array}{l}69.9 \\
69.1 \\
65.8\end{array}$ & $\begin{array}{l}69.2 \\
70.4 \\
64.3\end{array}$ & $\begin{array}{l}67.3 \\
67.3 \\
62.3\end{array}$ & $\begin{array}{l}57.5 \\
58.8 \\
51.4\end{array}$ & $\begin{array}{l}60.1 \\
59.7 \\
54.0\end{array}$ & $\begin{array}{l}67.0 \\
67.0 \\
61.1\end{array}$ & $\begin{array}{l}67.5 \\
68.1 \\
63.8\end{array}$ & $\begin{array}{l}64.1 \\
64.1 \\
58.4\end{array}$ & $\begin{array}{l}55.1 \\
55.8 \\
49.7\end{array}$ \\
\hline $\begin{array}{l}\text { PAD District I } \\
\text { January } 1996 \ldots . . . . . . . . . . \\
\text { December } 1995 \ldots . . . . . . \\
\text { January } 1995 \text {............. } \\
\text { Subdistrict IA }\end{array}$ & $\begin{array}{l}63.2 \\
62.7 \\
57.4\end{array}$ & $\begin{array}{l}69.8 \\
68.1 \\
63.8\end{array}$ & $\begin{array}{l}71.0 \\
68.1 \\
67.2\end{array}$ & $\begin{array}{l}66.4 \\
65.8 \\
61.7\end{array}$ & $\begin{array}{l}67.1 \\
65.6 \\
62.2\end{array}$ & $\begin{array}{l}58.1 \\
58.7 \\
51.7\end{array}$ & $\begin{array}{l}64.1 \\
63.2 \\
56.9\end{array}$ & $\begin{array}{l}67.9 \\
66.5 \\
61.9\end{array}$ & $\begin{array}{l}69.3 \\
67.1 \\
64.8\end{array}$ & $\begin{array}{l}67.4 \\
66.1 \\
61.3\end{array}$ & $\begin{array}{l}57.0 \\
57.1 \\
50.1\end{array}$ \\
\hline $\begin{array}{l}\text { January } 1996 \ldots . . . . . . . . . \\
\text { December } 1995 \text {......... } \\
\text { January } 1995 \text {............. } \\
\text { Subdistrict IB }\end{array}$ & $\begin{array}{l}68.0 \\
67.5 \\
62.4\end{array}$ & $\begin{array}{l}86.0 \\
84.5 \\
75.2\end{array}$ & $\begin{array}{l}79.1 \\
75.9 \\
73.4\end{array}$ & $\begin{array}{l}71.0 \\
69.5 \\
64.3\end{array}$ & $\begin{array}{l}72.2 \\
70.9 \\
66.4\end{array}$ & $\begin{array}{l}60.1 \\
61.0 \\
53.7\end{array}$ & $\begin{array}{l}75.0 \\
72.0 \\
65.9\end{array}$ & $\begin{array}{l}\text { NA } \\
\text { NA } \\
68.3\end{array}$ & $\begin{array}{l}74.4 \\
72.4 \\
74.5\end{array}$ & $\begin{array}{l}75.3 \\
73.1 \\
67.6\end{array}$ & $\begin{array}{l}60.0 \\
62.7 \\
50.5\end{array}$ \\
\hline $\begin{array}{l}\text { January } 1996 \ldots . . . . . . . . . . \\
\text { December } 1995 \text {......... } \\
\text { January } 1995 \ldots . . . . . . . . . . \\
\text { Subdistrict 1C }\end{array}$ & $\begin{array}{l}64.9 \\
63.7 \\
58.0\end{array}$ & $\begin{array}{l}77.5 \\
74.8 \\
68.6\end{array}$ & $\begin{array}{l}73.9 \\
70.3 \\
69.6\end{array}$ & $\begin{array}{l}68.3 \\
67.8 \\
62.5\end{array}$ & $\begin{array}{l}68.8 \\
66.9 \\
62.9\end{array}$ & $\begin{array}{l}59.6 \\
59.8 \\
52.0\end{array}$ & $\begin{array}{l}65.2 \\
63.8 \\
57.4\end{array}$ & $\begin{array}{l}70.5 \\
68.2 \\
64.1\end{array}$ & $\begin{array}{l}80.7 \\
75.9 \\
68.6\end{array}$ & $\begin{array}{l}68.7 \\
67.1 \\
60.7\end{array}$ & $\begin{array}{l}58.4 \\
58.2 \\
50.2\end{array}$ \\
\hline $\begin{array}{l}\text { January } 1996 \ldots . . . . . . . . . \\
\text { December } 1995 . . . . . . . \\
\text { January } 1995 . . . . . . . . . .\end{array}$ & $\begin{array}{l}60.0 \\
60.2 \\
55.6\end{array}$ & $\begin{array}{l}63.8 \\
62.3 \\
59.4\end{array}$ & $\begin{array}{l}68.9 \\
66.4 \\
65.4\end{array}$ & $\begin{array}{l}64.4 \\
63.7 \\
60.6\end{array}$ & $\begin{array}{l}64.9 \\
63.6 \\
61.0\end{array}$ & $\begin{array}{l}56.4 \\
57.3 \\
51.1\end{array}$ & $\begin{array}{l}62.8 \\
62.3 \\
55.7\end{array}$ & $\begin{array}{l}66.4 \\
65.2 \\
60.7\end{array}$ & $\begin{array}{l}67.2 \\
65.6 \\
64.1\end{array}$ & $\begin{array}{l}66.5 \\
65.4 \\
61.2\end{array}$ & $\begin{array}{l}55.8 \\
56.3 \\
50.0\end{array}$ \\
\hline $\begin{array}{l}\text { PAD District If } \\
\text { January } 1996 \ldots \ldots \ldots \ldots . . . . \\
\text { December } 1995 \ldots . . . \ldots . . \\
\text { January } 1995 \ldots \ldots . . . . . .\end{array}$ & $\begin{array}{l}60.1 \\
61.2 \\
55.7\end{array}$ & $\begin{array}{l}65.6 \\
66.7 \\
60.4\end{array}$ & $\begin{array}{l}67.9 \\
67.4 \\
63.7\end{array}$ & $\begin{array}{l}70.4 \\
72.2 \\
67.5\end{array}$ & $\begin{array}{l}65.4 \\
65.7 \\
61.2\end{array}$ & $\begin{array}{l}55.8 \\
57.7 \\
50.6\end{array}$ & $\begin{array}{l}57.6 \\
57.5 \\
53.0\end{array}$ & $\begin{array}{l}69.3 \\
70.4 \\
63.5\end{array}$ & $\begin{array}{l}71.6 \\
72.3 \\
67.3\end{array}$ & $\begin{array}{l}64.1 \\
64.4 \\
59.1\end{array}$ & $\begin{array}{l}56.0 \\
56.9 \\
50.8\end{array}$ \\
\hline $\begin{array}{l}\text { PAD District III } \\
\text { January } 1996 \ldots . . . . . . . . . . \\
\text { December } 1995 \text {......... } \\
\text { January } 1995 \ldots . . . . . . . . .\end{array}$ & $\begin{array}{l}59.0 \\
59.8 \\
54.2\end{array}$ & $\begin{array}{l}63.8 \\
64.2 \\
61.6\end{array}$ & $\begin{array}{l}66.5 \\
66.3 \\
63.8\end{array}$ & $\begin{array}{l}62.3 \\
64.5 \\
59.3\end{array}$ & $\begin{array}{l}63.6 \\
64.0 \\
60.1\end{array}$ & $\begin{array}{l}55.7 \\
56.0 \\
50.0\end{array}$ & $\begin{array}{l}55.2 \\
56.0 \\
51.0\end{array}$ & $\begin{array}{l}61.0 \\
61.2 \\
56.1\end{array}$ & $\begin{array}{l}61.9 \\
62.8 \\
58.8\end{array}$ & $\begin{array}{l}58.5 \\
59.1 \\
53.5\end{array}$ & $\begin{array}{l}52.5 \\
53.6 \\
47.9\end{array}$ \\
\hline $\begin{array}{l}\text { PAD District IV } \\
\text { January } 1996 \ldots . . . . . . . . . . \\
\text { December } 1995 \ldots . . . . . . \\
\text { January } 1995 \ldots . . . . . . . .\end{array}$ & $\begin{array}{l}66.4 \\
68.2 \\
59.3\end{array}$ & $\begin{array}{l}73.4 \\
74.9 \\
66.1\end{array}$ & $\begin{array}{l}75.6 \\
77.1 \\
68.3\end{array}$ & $\begin{array}{l}79.3 \\
81.1 \\
72.4\end{array}$ & $\begin{array}{l}73.5 \\
75.0 \\
65.9\end{array}$ & $\begin{array}{l}61.6 \\
64.0 \\
56.2\end{array}$ & $\begin{array}{l}60.2 \\
64.4 \\
55.6\end{array}$ & $\begin{array}{r}\text { NA } \\
79.7 \\
68.7\end{array}$ & $\begin{array}{l}76.3 \\
81.7 \\
70.6\end{array}$ & $\begin{array}{l}65.7 \\
69.9 \\
59.8\end{array}$ & $\begin{array}{l}62.2 \\
66.8 \\
55.9\end{array}$ \\
\hline $\begin{array}{l}\text { PAD DistrictV } \\
\text { January } 1996 . . . . . . . . . . \\
\text { December } 1995 \text {......... } \\
\text { January } 1995 \text {........... }\end{array}$ & $\begin{array}{l}71.0 \\
72.7 \\
62.1\end{array}$ & $\begin{array}{l}72.5 \\
73.6 \\
66.5\end{array}$ & $\begin{array}{l}78.2 \\
79.5 \\
72.8\end{array}$ & $\begin{array}{l}75.4 \\
77.3 \\
67.6\end{array}$ & $\begin{array}{l}74.1 \\
75.5 \\
67.0\end{array}$ & $\begin{array}{l}61.6 \\
64.1 \\
53.1\end{array}$ & $\begin{array}{l}63.5 \\
62.9 \\
57.8\end{array}$ & $\begin{array}{l}71.1 \\
71.2 \\
64.3\end{array}$ & $\begin{array}{l}69.2 \\
71.8 \\
63.5\end{array}$ & $\begin{array}{l}67.1 \\
67.4 \\
61.9\end{array}$ & $\begin{array}{l}57.3 \\
58.8 \\
50.2\end{array}$ \\
\hline
\end{tabular}

NA $=$ Not avallable.

a Includes low-sulfur diesel fuel only with the exception of Alaska, which currently is exempt from the Clean Air Act's diesel fuel sulfur content requirement.

b All end-user sales not included in the other end-user categories shown, e.g., sales to agricultural customers or utilities.

Notes: Some State data are not sufficient for publication individually, but are used in calculating the PAD District average.

Notes: Values shown for the current month are preliminary. Values shown for previous months are revised. Data are final upon publication in the Petroleum Marketing Annual.

Sources: Energy Iniormation Administration Forms ElA-782A, "Refiners'/Gas Plant Operators' Monthly Petroleum Product Sales Report," and ElA-782B,

"Resellers'/Retailers' Monthly Petroleum Product Sales Report." 
Table 42. Residual Fuel Oil Prices by PAD District and State (Cents per Gallon Excluding Taxes)

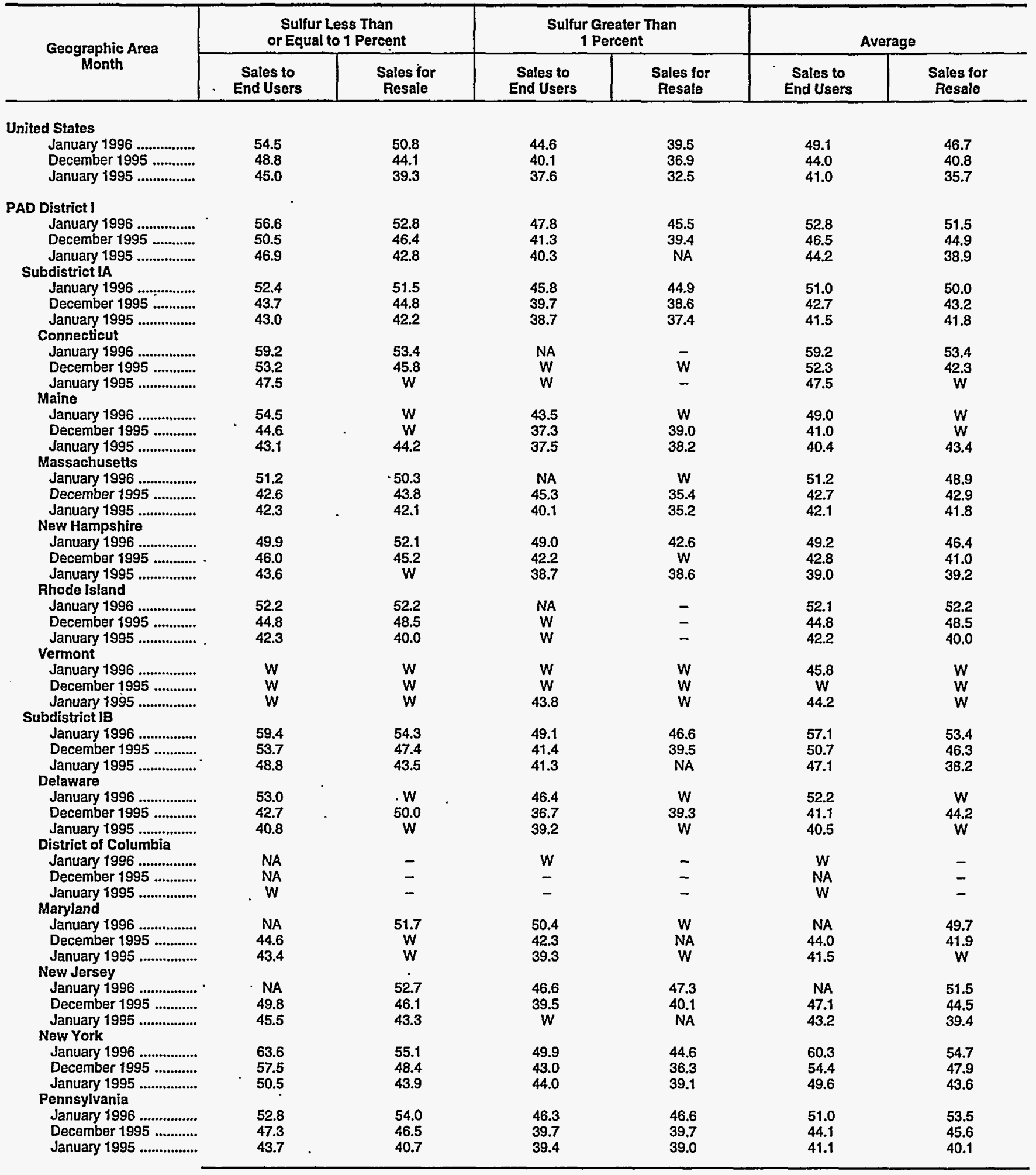

See footnotes at end of table. 
Table 42. Residual Fuel Oil Prices by PAD District and State (Cents per Gallon Excluding Taxes) —Continued

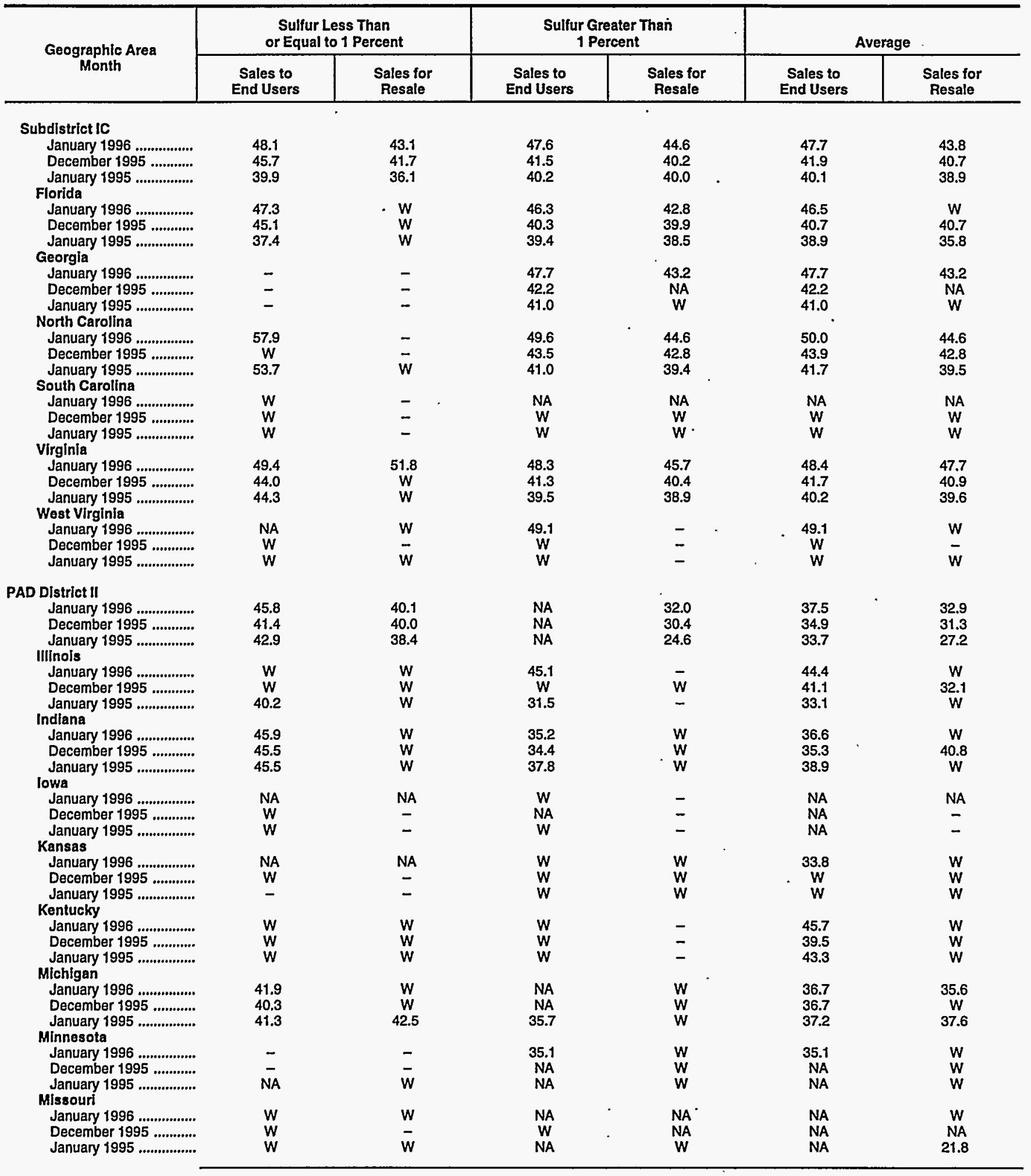

See footnotes at end of table. 
Table 42. Residual Fuel Oil Prices by PAD District and State (Cents per Gallon Excluding Taxes) - Continued

\begin{tabular}{|c|c|c|c|c|c|c|}
\hline \multirow{2}{*}{$\begin{array}{l}\text { Geographic Area } \\
\text { Month }\end{array}$} & \multicolumn{2}{|c|}{$\begin{array}{l}\text { Sulfur Less Than } \\
\text { or Equal to I Percent }\end{array}$} & \multicolumn{2}{|c|}{$\begin{array}{c}\text { Sulfur Greater Than } \\
\text { I Percent }\end{array}$} & \multicolumn{2}{|c|}{ Average } \\
\hline & $\begin{array}{l}\text { Sales to } \\
\text { End Users }\end{array}$ & $\begin{array}{l}\text { Sales for } \\
\text { Resale }\end{array}$ & $\begin{array}{l}\text { Sales to } \\
\text { End Users }\end{array}$ & $\begin{array}{l}\text { Sales for } \\
\text { Resale }\end{array}$ & $\begin{array}{c}\text { Sales to } \\
\text { End Users }\end{array}$ & $\begin{array}{c}\text { Sales for } \\
\text { Resale }\end{array}$ \\
\hline \multicolumn{7}{|l|}{ Nebraska } \\
\hline January 1996 & NA & - & $W$ & - & NA & - \\
\hline December 1995 ............. & $w$ & - & NA & - & $w$ & - \\
\hline January 1995 .................. & W & - & NA & - & $W$ & - \\
\hline \multicolumn{7}{|l|}{ North Dakota } \\
\hline January 1996 .................. & - & - & W & - & $w$ & - \\
\hline December 1995 ............ & - & - & W & - & $w$ & - \\
\hline January 1995 ................. & - & - & $w$ & - & $W$ & - \\
\hline \multicolumn{7}{|l|}{ Ohio } \\
\hline January 1996 & $W$ & - & 47.0 & $W$ & 49.3 & $W$ \\
\hline December 1995 ............. & 47.2 & - & 39.7 & $\ddot{w}$ & 41.2 & $W$ \\
\hline January 1995 .................. & 45.5 & $W$ & 36.3 & $W$ & 37.5 & 26.4 \\
\hline \multicolumn{7}{|l|}{ OkJahoma } \\
\hline January 1996 & NA & - & W & $W$ & $W$ & $W$ \\
\hline December 1995 ............. & NA & - & W & - & $W$ & - \\
\hline January 1995 .................. & NA & W & $W$ & - & W & $w$ \\
\hline \multicolumn{7}{|l|}{ South Dakota } \\
\hline January 1996 & - & NA & NA & - & NA & NA \\
\hline December 1995 ............... & - & - & NA & - & NA & - \\
\hline January 1995 ................... & - & - & NA & - & NA & - \\
\hline \multicolumn{7}{|l|}{ Tennessee } \\
\hline January 1996 ............... & $W$ & $W$ & $w$ & W & $W$ & 43.6 \\
\hline December 1995 ............. & $W$ & $W$ & $w$ & $W$ & $W$ & $w$ \\
\hline January 1995 .................... & W & W & $W$ & $W$ & $w$ & 37.1 \\
\hline \multicolumn{7}{|l|}{ Wisconsin } \\
\hline January 1996 & $W$ & $W$ & NA & 32.3 & NA & 32.4 \\
\hline December 1995 ............. & $W$ & $W$ & NA & 26.4 & NA & 26.5 \\
\hline January 1995 .................. & $W$ & W & $w$ & W & W & W \\
\hline \multicolumn{7}{|l|}{ PAD District III } \\
\hline January 1996 & 36.7 & 37.8 & 38.9 & 34.3 & 38.5 & 35.4 \\
\hline December 1995 ............. & 35.4 & 36.0 & 36.9 & 34.7 & 36.6 & 35.0 \\
\hline January 1995 ................. & W & 33.3 & 33.8 & 32.7 & 34.0 & 32.9 \\
\hline \multicolumn{7}{|l|}{ Alabama } \\
\hline January 1996 .................. & $W$ & $W$ & 42.7 & NA & 42.7 & NA \\
\hline December 1995 ............. & $W$ & $W$ & NA & NA & NA & NA \\
\hline January 1995 ................. & $\mathbf{W}$ & $W$ & 38.3 & NA & 38.3 & NA \\
\hline Arkansas & & & & & & \\
\hline January 1996 & - & - & 41.4 & - & 41.4 & - \\
\hline December 1995 ............. & - & - & 36.6 & - & 36.6 & - \\
\hline January 1995 & - & - & 30.2 & - & 30.2 & - \\
\hline Louisiana & & & & & & \\
\hline January 1996 & W & 35.3 & 37.9 & 33.9 & 38.1 & 34.0 \\
\hline December 1995 ............. & $W$ & 37.9 & 35.2 & 34.4 & 36.1 & 34.6 \\
\hline January 1995 & W & W & 32.8 & 31.4 & NA & 31.5 \\
\hline Mississippi & & & & & & \\
\hline January 1996 ................. & - & - & NA & NA & NA & NA \\
\hline December 1995 .............. & - & - & NA & $W$ & NA & $w$ \\
\hline January 1995 .................. & - & - & NA & NA & NA & NA \\
\hline New Mexico & & & & & & \\
\hline January 1996 ................. & - & W & - & - & - & $w$ \\
\hline December 1995 ............. & - & W & - & - & - & $w$ \\
\hline January 1995 .................. & - & W & - & - & - & $w$ \\
\hline Texas & & & & & & \\
\hline January 1996 .................. & NA & 38.0 & 38.2 & 34.3 & 37.3 & 36.3 \\
\hline December 1995 ............. & $w$ & 35.9 & 37.5 & 34.8 & 36.3 & 35.3 \\
\hline January 1995 .................. & $W$ & 33.3 & 33.7 & 33.7 & 33.8 & 33.5 \\
\hline PAD District IV & & & & & & \\
\hline January 1996 & $W$ & $w$ & NA & $W$ & NA & 23.7 \\
\hline December 1995 .............. & W & W & 33.0 & $w$ & 33.2 & 24.4 \\
\hline January 1995 .................. & 32.8 & $w$ & 30.9 & W & 31.1 & 24.6 \\
\hline Colorado & & & & & & \\
\hline January 1996 & - & - & - & - & - & - \\
\hline December 1995 ............... & - & $\rightarrow$ & - & - & - & - \\
\hline January 1995 .................. & - & - & - & - & - & - \\
\hline
\end{tabular}

See footnotes at end of table. 
Table 42. Residual Fuel Oil Prices by PAD District and State (Cents per Gallon Excluding Taxes) - Continued

\begin{tabular}{|c|c|c|c|c|c|c|}
\hline \multirow{2}{*}{$\begin{array}{c}\text { Geographic Area } \\
\text { Month }\end{array}$} & \multicolumn{2}{|c|}{$\begin{array}{l}\text { Sulfur Less Than } \\
\text { or Equal to } 1 \text { Percent }\end{array}$} & \multicolumn{2}{|c|}{$\begin{array}{c}\text { Sulfur Greater Than } \\
1 \text { Percent }\end{array}$} & \multicolumn{2}{|c|}{ Average } \\
\hline & $\begin{array}{l}\text { Sales to } \\
\text { End Users }\end{array}$ & $\begin{array}{l}\text { Sales for } \\
\text { Resale }\end{array}$ & $\begin{array}{l}\text { Sales to } \\
\text { End Users }\end{array}$ & $\begin{array}{c}\text { Sales for } \\
\text { Resale }\end{array}$ & $\begin{array}{l}\text { Sales to } \\
\text { End Users }\end{array}$ & $\begin{array}{c}\text { Sales for } \\
\text { Resale }\end{array}$ \\
\hline \multicolumn{7}{|l|}{ Idaho } \\
\hline January 1996 & - & $\rightarrow$ & - & - & - & - \\
\hline December 1995 ........... & - & - & - & - & - & - \\
\hline January 1995 ................. & $w$ & - & - & - & $\mathbf{W}$ & - \\
\hline \multicolumn{7}{|l|}{ Montana } \\
\hline January 1996 ................ & - & - & $W$ & $w$ & $\mathbf{w}$ & W \\
\hline December 1995 ............ & - & - & $W$ & w & W & $w$ \\
\hline January 1995 ................. & $w$ & - & $w$ & $\ddot{w}$ & $w$ & $w$ \\
\hline \multicolumn{7}{|l|}{ Utah } \\
\hline January 1996 & $W$ & NA & $w$ & - & $w$ & NA \\
\hline December 1995 ............ & $\ddot{W}$ & $W$ & w & - & $w$ & $w$ \\
\hline January 1995 ................ & W & $w$ & 26.5 & - & 26.8 & $w$ \\
\hline \multicolumn{7}{|l|}{ Wyoming } \\
\hline January 1996 & - & W & - & $\mathbf{W}$ & - & $w$ \\
\hline December 1995 ............. & - & W & - & $w$ & - & $w$ \\
\hline January 1995 ................... & - & $w$ & $W$ & $w$ & $\mathbf{W}$ & $w$ \\
\hline \multicolumn{7}{|l|}{ PAD District V } \\
\hline January 1996 & 47.5 & 32.8 & 43.9 & 42.3 & 44.9 & 40.8 \\
\hline December 1995 ............ & 44.8 & 33.5 & 41.8 & 41.3 & 42.4 & 39.4 \\
\hline January 1995 ................. & 40.6 & 32.9 & 37.8 & 35.8 & 38.4 & 34.9 \\
\hline \multicolumn{7}{|l|}{ Alaska } \\
\hline January 1996 .................. & - & - & NA & - & NA & - \\
\hline December 1995 ............ & - & - & NA & - & NA & - \\
\hline January 1995 ................ & - & - & - & $w$ & - & $w$ \\
\hline \multicolumn{7}{|l|}{ Arizona } \\
\hline January 1996 ................. & $W$ & $W$ & W & - & $w$ & $w$ \\
\hline December 1995 ............ & $W$ & $\mathbf{W}$ & $W$ & - & $w$ & $W$ \\
\hline January 1995 ................. & $W$ & $W$ & W & - & $\mathbf{w}$ & $w$ \\
\hline \multicolumn{7}{|l|}{ Califomia } \\
\hline January 1996 .................. & $W$ & 32.6 & 43.4 & 41.4 & 43.4 & 39.3 \\
\hline December 1995 ............ & $w$ & 33.2 & 42.1 & 40.5 & 42.2 & 37.6 \\
\hline January 1995 ................ & 41.7 & 32.9 & 37.6 & 37.2 & 37.9 & 35.2 \\
\hline \multicolumn{7}{|l|}{ Hawall } \\
\hline January 1996 ................. & $W$ & - & 43.6 & 54.1 & 46.9 & 54.1 \\
\hline December 1995 ............ & w & - & 37.4 & $\dddot{w}$ & 43.3 & $W$ \\
\hline January 1995 ................ & $W$ & - & 39.7 & $W$ & 40.1 & $W$ \\
\hline \multicolumn{7}{|l|}{ Nevada } \\
\hline January 1996 ................. & $W$ & - & - & - & $w$ & - \\
\hline December 1995 ........... & W & $w$ & - & - & $\mathrm{w}$ & $w$ \\
\hline January 1995 ................. & $W$ & $W$ & - & - & w & $\ddot{w}$ \\
\hline \multicolumn{7}{|l|}{ Oregon } \\
\hline January 1996 ................ & NA & $W$ & NA & 43.0 & NA & 42.0 \\
\hline December 1995 ............ & NA & $\ddot{w}$ & 42.5 & 43.6 & 42.6 & 39.9 \\
\hline January 1995 ................ & NA & - & 38.4 & 36.7 & 38.5 & 36.7 \\
\hline \multicolumn{7}{|l|}{ Washington } \\
\hline Januany 1996 ................. & - & - & 44.7 & 43.8 & 44.7 & 43.8 \\
\hline December 1995 ........... & - & - & 41.8 & 41.8 & 41.8 & 41.8 \\
\hline January 1995 ................. & NA & - & 37.2 & 35.4 & 37.2 & 35.4 \\
\hline
\end{tabular}

Dash $(-)=$ No data reported.

NA $=$ Not avallablo.

$W=$ Withheld to avoid disclosure of individual company data.

Notes: Some State data are not sufficient for publication individually, but are used in calculating the PAD District average.

Notes: Values shown for the current month are preliminary. Values shown for previous months are revised. Data are final upon publication in the Petroleum Markeling Annual.

Sources: Energy Information Administration Forms EIA-782A, "Refiners'/Gas Plant Operators' Monthly Petroleum Product Sales Report," and ElA-782B, "Resellers'/Retallers' Monthly Petroleum Product Sales Report." 


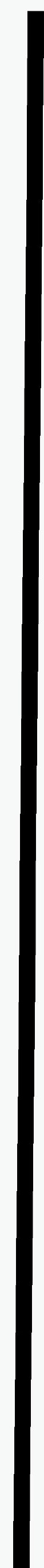




\section{Volumes of Petroleum Products}


Table 43. Refiner Motor Gasoline Volumes by Grade, Sales Type, PAD District, and State (Thousand Gallons per Day)

\begin{tabular}{|c|c|c|c|c|c|c|c|c|c|c|c|c|}
\hline \multirow{3}{*}{$\begin{array}{c}\text { Geographic Area } \\
\text { Month }\end{array}$} & \multicolumn{6}{|c|}{ Regular } & \multicolumn{6}{|c|}{ Midgrade } \\
\hline & \multicolumn{2}{|c|}{ Sales to End Users } & \multicolumn{4}{|c|}{ Sales for Resale } & \multicolumn{2}{|c|}{ Sales to End Users } & \multicolumn{4}{|c|}{ Sales for Resale } \\
\hline & $\begin{array}{l}\text { Through } \\
\text { Retail } \\
\text { Outlets }\end{array}$ & Totala & DTW & Rack & Bulk & Total & $\begin{array}{c}\text { Through } \\
\text { Retail } \\
\text { Outlets }\end{array}$ & Totala & DTW & Rack & Bulk & Total \\
\hline \multicolumn{13}{|l|}{ United States } \\
\hline $\begin{array}{l}\text { January } 1996 \\
\text { December } 1995 . . . . . . . . . . . . . . . . . \\
\text { January } 1995\end{array}$ & $\begin{array}{l}31,832.5 \\
33,957.2 \\
31,302.4\end{array}$ & $\begin{array}{l}33,354.9 \\
35,519.5 \\
32,812.0\end{array}$ & $\begin{array}{l}40,886.2 \\
43,391.6 \\
41,409.8\end{array}$ & $\begin{array}{l}115,969.4 \\
123,295.7 \\
112,247.8\end{array}$ & $\begin{array}{l}25,714.3 \\
34,282.5 \\
26,480.6\end{array}$ & $\begin{array}{l}182,569.8 \\
200,969.8 \\
180,138.2\end{array}$ & $\begin{array}{r}9,403.1 \\
10,297.7 \\
8,732.9\end{array}$ & $\begin{array}{r}9,661.1 \\
10,549.1 \\
9,007.9\end{array}$ & $\begin{array}{l}12,924.1 \\
13,940.9 \\
12,193.4\end{array}$ & $\begin{array}{l}16,015.6 \\
17,372.4 \\
15,235.0\end{array}$ & $\overline{-}$ & $\begin{array}{l}28,939.7 \\
31,313.2 \\
27,540.2\end{array}$ \\
\hline \multicolumn{13}{|l|}{ PAD District I } \\
\hline $\begin{array}{l}\text { January } 1996 \ldots . . . . . . . . . . . \\
\text { December } 1995 \\
\text { January } 1995 \text {................... } \\
\text { Subdistrict IA }\end{array}$ & $\begin{array}{l}7,288.4 \\
7,862.8 \\
7,174.9\end{array}$ & $\begin{array}{l}7,904.5 \\
8,551.1 \\
7,799.9\end{array}$ & $\begin{array}{l}13,649.6 \\
14,989.5 \\
14,403.5\end{array}$ & $\begin{array}{l}34,159.4 \\
37,075.6 \\
33,708.7\end{array}$ & $\begin{array}{l}4,739.3 \\
5,541.9 \\
5,072.1\end{array}$ & $\begin{array}{l}52,548.4 \\
57,607.0 \\
53,184.3\end{array}$ & $\begin{array}{l}2,360.1 \\
2,588.4 \\
2,380.2\end{array}$ & $\begin{array}{l}2,499.5 \\
2,717.6 \\
2,537.8\end{array}$ & $\begin{array}{l}4,686.1 \\
5,136.6 \\
5,192.9\end{array}$ & $\begin{array}{r}6,639.5 \\
7,271.2 \\
W\end{array}$ & $\bar{w}$ & $\begin{array}{l}11,325.6 \\
12,407.9 \\
11,954.8\end{array}$ \\
\hline $\begin{array}{l}\text { January } 1996 \\
\text { December } 1995 \\
\text { January } 1995 \\
\text { Connecticut }\end{array}$ & $\begin{array}{l}328.8 \\
356.0 \\
278.3\end{array}$ & $\begin{array}{l}367.9 \\
397.5 \\
326.8\end{array}$ & $\begin{array}{r}W \\
W \\
2,385.0\end{array}$ & $\begin{array}{l}3,622,7 \\
4,049.8 \\
3,708.2\end{array}$ & $\begin{array}{c}W \\
W \\
656.9\end{array}$ & $\begin{array}{l}6,274.1 \\
6,925.3 \\
6,750.1\end{array}$ & $\begin{array}{l}116.9 \\
127.6 \\
130.5\end{array}$ & $\begin{array}{l}129.4 \\
139.2 \\
147.7\end{array}$ & $\begin{array}{c}738.8 \\
803.1 \\
W\end{array}$ & $\begin{array}{l}588.0 \\
674.0 \\
W\end{array}$ & $\vec{w}$ & $\begin{array}{l}1,326.8 \\
1,477.1 \\
1,424.4\end{array}$ \\
\hline $\begin{array}{l}\text { January } 1996 \text {................. } \\
\text { December } 1995 \text {............ } \\
\text { January } 1995 \text {................ } \\
\text { Maine }\end{array}$ & $\begin{array}{l}W \\
W \\
W\end{array}$ & $\begin{array}{l}70.8 \\
78.1 \\
72.9\end{array}$ & $\begin{array}{c}711.0 \\
703.0\end{array}$ & $\begin{array}{r}W \\
1,202.7 \\
W\end{array}$ & $\begin{array}{l}w \\
w \\
w\end{array}$ & $\begin{array}{l}1,783.1 \\
1,973.3 \\
1,737.9\end{array}$ & $\begin{array}{l}W \\
W \\
W\end{array}$ & $\begin{array}{l}W \\
W \\
W\end{array}$ & $\begin{array}{l}w \\
W \\
w\end{array}$ & $\begin{array}{l}W \\
W \\
W\end{array}$ & $\overline{-}$ & $\begin{array}{l}390.5 \\
456.8 \\
424.7\end{array}$ \\
\hline $\begin{array}{l}\text { January } 1996 . . . . . . . . . . . . . . \\
\text { December } 1995 \text {............. } \\
\text { January } 1995 \text {................ } \\
\text { Massachusetts }\end{array}$ & $\begin{array}{l}- \\
- \\
-\end{array}$ & $\begin{array}{l}W \\
W \\
W\end{array}$ & $\begin{array}{l}w \\
w \\
w\end{array}$ & $\begin{array}{l}777.5 \\
828.6 \\
794.0\end{array}$ & $\begin{array}{l}W \\
W \\
W\end{array}$ & $\begin{array}{r}905.4 \\
969.1 \\
1,011.7\end{array}$ & $\overline{-}$ & $\begin{array}{l}W \\
W \\
W\end{array}$ & $\begin{array}{l}w \\
W \\
W\end{array}$ & $\begin{array}{l}W \\
W \\
W\end{array}$ & $\begin{array}{l}- \\
-\end{array}$ & $\begin{array}{l}149.8 \\
157.7 \\
161.2\end{array}$ \\
\hline $\begin{array}{l}\text { January } 1996 \ldots . . . . . . . . . . . . . \\
\text { December } 1995 \text {............ } \\
\text { January } 1995 \text {............... } \\
\text { New Hampshire }\end{array}$ & $\begin{array}{l}179.6 \\
191.2 \\
145.1\end{array}$ & $\begin{array}{l}202.3 \\
216.2 \\
170.4\end{array}$ & $\begin{array}{c}W \\
1,413.6 \\
1,245.7\end{array}$ & $\begin{array}{c}1,283.6 \\
W \\
1,389.4\end{array}$ & $\begin{array}{c}W \\
W \\
279.6\end{array}$ & $\begin{array}{l}2,619.5 \\
2,918.3 \\
2,914.7\end{array}$ & $\begin{array}{l}66.8 \\
71.4 \\
80.6\end{array}$ & $\begin{array}{l}76.3 \\
80.0 \\
94.5\end{array}$ & $\begin{array}{c}W \\
406.6 \\
388.6\end{array}$ & $\begin{array}{c}W \\
224.8 \\
W\end{array}$ & $\overline{-}$ & $\begin{array}{l}573.6 \\
631.4 \\
611.1\end{array}$ \\
\hline $\begin{array}{l}\text { January } 1996 \ldots . . . . . . . . . . \\
\text { December } 1995 \text {............ } \\
\text { January } 1995 \text {................ } \\
\text { Rhode Island }\end{array}$ & $\begin{array}{l}w \\
w \\
w\end{array}$ & $\begin{array}{c}52.2 \\
W \\
44.9\end{array}$ & $\begin{array}{l}161.7 \\
167.2 \\
137.9\end{array}$ & $\begin{array}{l}41.7 \\
42.2 \\
75.3\end{array}$ & $\begin{array}{l}- \\
-\end{array}$ & $\begin{array}{l}203.4 \\
209.4 \\
213.3\end{array}$ & $\begin{array}{l}w \\
w \\
w\end{array}$ & $\begin{array}{c}W \\
17.8 \\
W\end{array}$ & $\begin{array}{l}48.9 \\
51.5 \\
47.5\end{array}$ & $\begin{array}{r}7.0 \\
7.4 \\
33.8\end{array}$ & $\begin{array}{l}- \\
-\end{array}$ & $\begin{array}{l}55.8 \\
58.9 \\
81.4\end{array}$ \\
\hline $\begin{array}{l}\text { January } 1996 \ldots . . . . . . . . . . . . \\
\text { December } 1995 \ldots . . . . . . . . . \\
\text { January } 1995 \text {................. } \\
\text { Vermont }\end{array}$ & $\begin{array}{l}35.2 \\
39.1 \\
32.5\end{array}$ & $\begin{array}{c}W \\
41.4 \\
W\end{array}$ & $\begin{array}{l}227.5 \\
246.3 \\
228.5\end{array}$ & $\begin{array}{l}W \\
W \\
W\end{array}$ & $\begin{array}{l}W \\
W \\
W\end{array}$ & $\begin{array}{l}629.6 \\
697.4 \\
759.6\end{array}$ & $\begin{array}{l}14.7 \\
15.9 \\
14.8\end{array}$ & $\begin{array}{c}15.6 \\
W \\
16.3\end{array}$ & $\begin{array}{l}W \\
W \\
W\end{array}$ & $\begin{array}{l}W \\
W \\
W\end{array}$ & $\begin{array}{l}- \\
-\end{array}$ & $\begin{array}{l}130.7 \\
140.1 \\
121.9\end{array}$ \\
\hline $\begin{array}{l}\text { January } 1996 \text {................ } \\
\text { December } 1995 \text {............. } \\
\text { January } 1995 \text {................ } \\
\text { Subdistrict IB }\end{array}$ & $\begin{array}{l}- \\
-\end{array}$ & $\begin{array}{l}- \\
-\end{array}$ & $\begin{array}{l}W \\
W \\
W\end{array}$ & $\begin{array}{l}W \\
W \\
w\end{array}$ & $\begin{array}{l}- \\
-\end{array}$ & $\begin{array}{l}133.1 \\
157.8 \\
113.0\end{array}$ & $\begin{array}{l}- \\
-\end{array}$ & $\begin{array}{l}- \\
-\end{array}$ & $\begin{array}{l}W \\
W \\
W\end{array}$ & $\begin{array}{l}W \\
W \\
W\end{array}$ & $\begin{array}{l}- \\
-\end{array}$ & $\begin{array}{l}26.4 \\
32.3 \\
24.1\end{array}$ \\
\hline $\begin{array}{l}\text { January } 1996 . . . . . . . . . . . . . . \\
\text { December } 1995 \text {............. } \\
\text { January } 1995 \text {............... } \\
\text { Delaware }\end{array}$ & $\begin{array}{l}2,685.2 \\
3,014.5 \\
2,634.4\end{array}$ & $\begin{array}{l}2,866.5 \\
3,210.8 \\
2,859.1\end{array}$ & $\begin{array}{r}W \\
W \\
8,284.5\end{array}$ & $\begin{array}{r}9,647.1 \\
10,482.1 \\
9,604.8\end{array}$ & $\begin{array}{r}W \\
W \\
3,533.3\end{array}$ & $\begin{array}{l}20,936.4 \\
23,686.3 \\
21,422.6\end{array}$ & $\begin{array}{l}781.4 \\
895.5 \\
774.0\end{array}$ & $\begin{array}{l}811.8 \\
926.7 \\
809.1\end{array}$ & $\begin{array}{l}2,315.5 \\
2,584.8 \\
2,689.2\end{array}$ & $\begin{array}{l}1,603.3 \\
1,775.3 \\
1,541.5\end{array}$ & $\overline{-}$ & $\begin{array}{l}3,918.8 \\
4,360.1 \\
4,230.7\end{array}$ \\
\hline $\begin{array}{l}\text { January } 1996 . . . . . . . . . . . . . . . \\
\text { December } 1995 \text {............. } \\
\text { January } 1995 \text {............... } \\
\text { District of Columbia. }\end{array}$ & $\begin{array}{c}W \\
W \\
15.0\end{array}$ & $\begin{array}{l}20.8 \\
25.7 \\
21.4\end{array}$ & $\begin{array}{c}170.3 \\
W \\
192.3\end{array}$ & $\begin{array}{l}W \\
W \\
W\end{array}$ & $\begin{array}{l}W \\
W \\
W\end{array}$ & $\begin{array}{l}396.1 \\
430.6 \\
505.5\end{array}$ & $\begin{array}{l}W \\
W \\
6.1\end{array}$ & $\begin{array}{l}W \\
W \\
W\end{array}$ & $\begin{array}{l}56.6 \\
W \\
W\end{array}$ & $\begin{array}{l}47.9 \\
W \\
W\end{array}$ & $\begin{array}{l}- \\
-\end{array}$ & $\begin{array}{l}104.5 \\
111.3 \\
137.0\end{array}$ \\
\hline $\begin{array}{l}\text { January } 1996 \text {................. } \\
\text { December } 1995 \text {............. } \\
\text { January } 1995 \text {............... } \\
\text { Maryland }\end{array}$ & $\begin{array}{l}- \\
-\end{array}$ & $\begin{array}{l}5.0 \\
6.4 \\
7.3\end{array}$ & $\begin{array}{l}91.9 \\
W \\
W\end{array}$ & $\bar{w}$ & $\begin{array}{l}- \\
-\end{array}$ & $\begin{array}{r}91.9 \\
109.4 \\
114.1\end{array}$ & $\overline{-}$ & $\begin{array}{l}W \\
W \\
W\end{array}$ & $\begin{array}{l}64.4 \\
78.8 \\
W\end{array}$ & $\bar{w}$ & $\begin{array}{l}- \\
-\end{array}$ & $\begin{array}{l}64.4 \\
78.8 \\
80.4\end{array}$ \\
\hline $\begin{array}{l}\text { January } 1996 . . . . . . . . . . . . . . \\
\text { December } 1995 \text {........... } \\
\text { January } 1995 \text {............... }\end{array}$ & $\begin{array}{l}W \\
W \\
-\end{array}$ & $\begin{array}{l}43.7 \\
47.7 \\
46.2\end{array}$ & $\begin{array}{c}W \\
1,572.9 \\
W\end{array}$ & $\begin{array}{c}905.6 \\
W \\
895.6\end{array}$ & $\begin{array}{l}W \\
W \\
W\end{array}$ & $\begin{array}{l}2,310.2 \\
2,852.8 \\
2,580.5\end{array}$ & $\begin{array}{l}W \\
W \\
-\end{array}$ & $\begin{array}{l}6.5 \\
7.2 \\
7.4\end{array}$ & $\stackrel{551.7}{W}$ & $\begin{array}{c}260.6 \\
W \\
W\end{array}$ & $\begin{array}{l}- \\
-\end{array}$ & $\begin{array}{l}812.3 \\
960.4 \\
911.5\end{array}$ \\
\hline $\begin{array}{l}\text { January } 1996 \text {................ } \\
\text { December } 1995 \text {........... } \\
\text { January } 1995 \text {................ } \\
\text { New York }\end{array}$ & $\begin{array}{l}577.3 \\
658.5 \\
583.4\end{array}$ & $\begin{array}{l}610.0 \\
693.6 \\
621.6\end{array}$ & $\begin{array}{l}2,008.7 \\
2,253.1 \\
2,161.5\end{array}$ & $\begin{array}{l}1,521.0 \\
1,671.7 \\
1,510.7\end{array}$ & $\begin{array}{l}2,534.7 \\
2,552.5 \\
2,495.6\end{array}$ & $\begin{array}{l}6,064.4 \\
6,477.3 \\
6,167.9\end{array}$ & $\begin{array}{l}184.6 \\
208.6 \\
188.4\end{array}$ & $\begin{array}{l}192.3 \\
214.9 \\
194.5\end{array}$ & $\begin{array}{l}600.2 \\
654.5 \\
675.4\end{array}$ & $\begin{array}{l}262.8 \\
299.2 \\
270.1\end{array}$ & $\begin{array}{l}- \\
-\end{array}$ & $\begin{array}{l}863.0 \\
953.7 \\
945.5\end{array}$ \\
\hline $\begin{array}{l}\text { January } 1996 \text {................ } \\
\text { December } 1995 \text {............ } \\
\text { January } 1995 \text {............... } \\
\text { Pennsylyania }\end{array}$ & $\begin{array}{l}1,065.1 \\
1,168.5 \\
1,072.8\end{array}$ & $\begin{array}{l}1,109.3 \\
1,215.9 \\
1.151 .7\end{array}$ & $\begin{array}{l}2,342.9 \\
2,561.1 \\
2,401.2\end{array}$ & $\begin{array}{c}W \\
3,575.6 \\
W\end{array}$ & $\begin{array}{c}W \\
464.8 \\
W\end{array}$ & $\begin{array}{l}5,917.6 \\
6,601.6 \\
5,873.4\end{array}$ & $\begin{array}{l}243.3 \\
272.7 \\
246.4\end{array}$ & $\begin{array}{l}253.2 \\
283.5 \\
260.3\end{array}$ & $\begin{array}{l}650.6 \\
704.9 \\
732.0\end{array}$ & $\begin{array}{l}340.9 \\
352.2 \\
287.6\end{array}$ & $\begin{array}{l}- \\
-\end{array}$ & $\begin{array}{r}991.5 \\
1,057.1 \\
1,019.6\end{array}$ \\
\hline $\begin{array}{l}\text { January } 1996 \text {............... } \\
\text { December } 1995 \text {........... } \\
\text { January } 1995 \text {............... }\end{array}$ & $\begin{array}{r}1,025.7 \\
1,165.0 \\
963.2\end{array}$ & $\begin{array}{l}1,077.6 \\
1,221.5 \\
1,010.9\end{array}$ & $\begin{array}{r}1,624.0 \\
1,889.7 \\
W\end{array}$ & $\begin{array}{l}3,678.9 \\
3,851.7 \\
3,737.0\end{array}$ & $\begin{array}{r}853.2 \\
1,473.1 \\
W\end{array}$ & $\begin{array}{l}6,156.1 \\
7,214.5 \\
6,181.3\end{array}$ & $\begin{array}{l}346.6 \\
405.2 \\
333.0\end{array}$ & $\begin{array}{l}352.2 \\
411.5 \\
339.0\end{array}$ & $\begin{array}{l}392.1 \\
452.6 \\
482.2\end{array}$ & $\begin{array}{l}691.1 \\
746.2 \\
654.6\end{array}$ & $\begin{array}{l}- \\
-\end{array}$ & $\begin{array}{l}1,083.2 \\
1,198.8 \\
1,136.8\end{array}$ \\
\hline
\end{tabular}


Table 43. Refiner Motor Gasoline Volumes by Grade, Sales Type, PAD District, and State

(Thousand Gallons per Day) - Continued

\begin{tabular}{|c|c|c|c|c|c|c|c|c|c|c|c|c|}
\hline \multirow{3}{*}{$\begin{array}{l}\text { Geographic Area } \\
\text { Month }\end{array}$} & \multicolumn{6}{|c|}{ Premium } & \multicolumn{6}{|c|}{ All Grades } \\
\hline & \multicolumn{2}{|c|}{ Sales to End Users } & \multicolumn{4}{|c|}{ Sales for Resale } & \multicolumn{2}{|c|}{ Sales to End Users } & \multicolumn{4}{|c|}{ Sales for Resale } \\
\hline & $\begin{array}{c}\text { Through } \\
\text { Retail } \\
\text { Outlets }\end{array}$ & Totala & DTW & Rack & Bulk & Total & $\begin{array}{l}\text { Through } \\
\text { Retail } \\
\text { Outlets }\end{array}$ & Totala & DTW & Rack & Buik & Total \\
\hline \multicolumn{13}{|l|}{ United States } \\
\hline $\begin{array}{l}\text { January } 1996 \\
\text { December } 1995 \\
\text { January } 1995\end{array}$ & $\begin{array}{r}9,095.0 \\
10,037.4 \\
9,028.6\end{array}$ & $\begin{array}{r}9,422.4 \\
10,360.3 \\
9,358.9\end{array}$ & $\begin{array}{l}19,472.5 \\
21,415.3 \\
19,867.9\end{array}$ & $\begin{array}{l}29,462.3 \\
32,561.6 \\
28,933.1\end{array}$ & $\begin{array}{l}4,008.7 \\
5,568.0 \\
3,239.0\end{array}$ & $\begin{array}{l}52,943.5 \\
59,544.9 \\
52,040.1\end{array}$ & $\begin{array}{l}50,330.6 \\
54,292.3 \\
49,063.9\end{array}$ & $\begin{array}{l}52,438.4 \\
56,428.9 \\
51,178.8\end{array}$ & $\begin{array}{l}73,282.8 \\
78,747.7 \\
73,471.1\end{array}$ & $\begin{array}{l}161,447.3 \\
173,229.7 \\
156,415.9\end{array}$ & $\begin{array}{l}29,722.9 \\
39,850.5 \\
29,831.5\end{array}$ & $\begin{array}{l}264,453.0 \\
291,827.9 \\
259,718.5\end{array}$ \\
\hline \multicolumn{13}{|l|}{ PAD District I } \\
\hline $\begin{array}{l}\text { January } 1996 \ldots . . . . . . . . . . . . . \\
\text { December } 1995 . . . . . . . . . \\
\text { January } 1995 \text {................ } \\
\text { Subdistrict IA }\end{array}$ & $\begin{array}{l}2,828.6 \\
3,191.7 \\
2,806.9\end{array}$ & $\begin{array}{l}2,977.7 \\
3,339.4 \\
2,953.6\end{array}$ & $\begin{array}{r}9,152.4 \\
10,485.3 \\
9,728.9\end{array}$ & $\begin{array}{c}11,519.5 \\
13,035.3 \\
W\end{array}$ & $\begin{array}{c}1,473.4 \\
1,825.4 \\
W\end{array}$ & $\begin{array}{l}22,145.4 \\
25,346.0 \\
22,682.8\end{array}$ & $\begin{array}{l}12,477.1 \\
13,642.9 \\
12,362.0\end{array}$ & $\begin{array}{l}13,381.7 \\
14,608.0 \\
13,291.4\end{array}$ & $\begin{array}{l}27,488.2 \\
30,611.5 \\
29,325.2\end{array}$ & $\begin{array}{l}52,318.4 \\
57,382.1 \\
51,973.4\end{array}$ & $\begin{array}{l}6,212.7 \\
7,367.3 \\
6,523.3\end{array}$ & $\begin{array}{l}86,019.3 \\
95,360.9 \\
87,821.9\end{array}$ \\
\hline January 1996 & 139.6 & 149.5 & $w$ & $1,115.4$ & $w$ & $2,236.5$ & 585.3 & 646.8 & $4,283.7$ & $5,326.1$ & 227.6 & $9,837.4$ \\
\hline & $\begin{array}{l}157.6 \\
125.2\end{array}$ & 167.6 & $w$ & $1,300.4$ & $\underset{w}{w}$ & $\begin{array}{l}2,571.4 \\
2,3831\end{array}$ & $\begin{array}{l}641.3 \\
5341\end{array}$ & $\begin{array}{l}704.3 \\
615.0\end{array}$ & $4,642.3$ & $6,024.2$ & $\begin{array}{l}307.2 \\
804.4\end{array}$ & $\begin{array}{l}10,973.7 \\
10.5575\end{array}$ \\
\hline \\
\hline January 1996 .................. & $w$ & w & 310.7 & 334.0 & - & 644.7 & w & 123.2 & $w$ & $1,533.9$ & W & $2,818.3$ \\
\hline December 1995 ............ & $\ddot{w}$ & $w$ & 345.2 & $w$ & w & 830.3 & 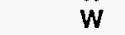 & 139.8 & $w$ & $\uparrow, 827.0$ & $\ddot{w}$ & $3,260.4$ \\
\hline January 1995 ................... & $\ddot{w}$ & 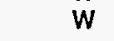 & 315.3 & 336.7 & - & 652.0 & $w$ & 124.2 & $w$ & $1,500.9$ & $w$ & $2,814.5$ \\
\hline \multicolumn{13}{|l|}{ Malne } \\
\hline $\begin{array}{l}\text { January } 1996 \text {.................... } \\
\text { December } 1995 . . . . . . . .\end{array}$ & $\overline{-}$ & $\underset{w}{w}$ & $\begin{array}{l}W \\
w\end{array}$ & $\begin{array}{l}W \\
W\end{array}$ & $\begin{array}{l}W \\
w\end{array}$ & $\begin{array}{l}220.3 \\
223.3\end{array}$ & - & $\begin{array}{l}W \\
W\end{array}$ & $\begin{array}{l}W \\
W\end{array}$ & $\begin{array}{l}1,110.3 \\
1,176.6\end{array}$ & $\underset{W}{W}$ & $\begin{array}{l}1,275.4 \\
1,350.0\end{array}$ \\
\hline January 1995 .................... & - & w & $w$ & $\ddot{w}$ & $\ddot{w}$ & 220.5 & - & $w$ & $\ddot{w}$ & $1,144.5$ & $\ddot{w}$ & $1,393.4$ \\
\hline \multicolumn{13}{|l|}{ Massachusetts } \\
\hline $\begin{array}{l}\text { January } 1996 \text {................... } \\
\text { December } 1995 \ldots . . . . . . .\end{array}$ & $\begin{array}{l}73.9 \\
83.0\end{array}$ & $\begin{array}{l}82.0 \\
91.4\end{array}$ & $\begin{array}{l}573.5 \\
637.5\end{array}$ & $\begin{array}{l}W \\
w\end{array}$ & $\begin{array}{l}W \\
w\end{array}$ & $\begin{array}{l}1,035.3 \\
1,135.5\end{array}$ & $\begin{array}{l}320.3 \\
3455\end{array}$ & $\begin{array}{l}360.5 \\
387.6\end{array}$ & $\begin{array}{l}2,257.8 \\
2,457.7\end{array}$ & $\begin{array}{c}W \\
1484\end{array}$ & $\begin{array}{c}W \\
790\end{array}$ & $\begin{array}{l}4,228.4 \\
4,685,2\end{array}$ \\
\hline 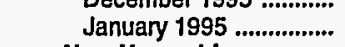 & 69.1 & 82.0 & 578.2 & $w$ & $w$ & $1,159.8$ & 294.8 & 346.8 & $2,212.5$ & $2,054.8$ & 418.4 & $4,685.7$ \\
\hline \multicolumn{13}{|l|}{ New Hampshire } \\
\hline January 1996 ................... & $w$ & w & 53.7 & 9.2 & - & 63.0 & $\mathbf{w}$ & 88.7 & 264.3 & 57.9 & - & 322.2 \\
\hline December 1995 .............. & $w$ & $w$ & 58.3 & 9.9 & - & 68.2 & $w$ & 95.7 & 276.9 & 59.5 & - & 336.4 \\
\hline January 1995 ................... & $w$ & $w$ & 51.9 & 24.0 & - & 75.9 & $w$ & $\mathbf{7 4 . 8}$ & 237.4 & 133.1 & - & 370.5 \\
\hline \multicolumn{13}{|l|}{ Rhode Island } \\
\hline January 1996 ................... & 15.1 & 15.3 & $\mathbf{w}$ & w & - & 229.5 & 65.0 & w & 388.9 & w & w & 989.8 \\
\hline December 1995 ............. & 16.8 & 16.9 & w & w & - & 266.3 & 71.8 & w & 424.5 & w & $w$ & $1,103.8$ \\
\hline January 1995 ................... & 13.0 & 13.5 & w & w & - & 237.3 & 60.3 & w & 401.4 & $w$ & $w$ & $1,118.8$ \\
\hline Vermont & & & & & & & & & & & & \\
\hline January 1996 & - & - & $w$ & w & - & 43.8 & - & - & w & $w$ & - & 203.3 \\
\hline December 1995 ............. & - & - & w & W & - & 47.8 & - & - & W & w & - & 237.9 \\
\hline January 1995 .................... & - & - & W & W & - & 37.5 & - & - & W & $w$ & - & 174.6 \\
\hline Subdistrict IB & & & & & & & & & & & & \\
\hline January 1996 ................... & $1,008.3$ & $1,046.9$ & w & $3,173.4$ & w & $9,634.8$ & $4,474.9$ & $4,725.2$ & $15,203.9$ & $14,423.8$ & $4,862.4$ & $34,490.0$ \\
\hline December 1995 .............. & $1,177.4$ & $1,215.2$ & $w$ & $3,563.1$ & $w$ & $11,416.0$ & $5,087.5$ & $5,352.7$ & 17.415 .6 & $15,820.5$ & $6,226.3$ & 39.462 .4 \\
\hline January 1995 . & $1,006.0$ & $1,045.1$ & $5,750.7$ & $3,284.8$ & $1,152.8$ & $10,188.3$ & 4,414.4 & $4,713.3$ & $16,724.5$ & $14,431.1$ & $4,686.1$ & $35,841.6$ \\
\hline Delaware & & & & & & & & & & & & \\
\hline January 1996 .................... & $w$ & w & w & w & - & 123.3 & w & 33.8 & $w$ & 309.1 & w & 623.9 \\
\hline December 1995 ........... & $w$ & $w$ & 69.9 & 63.7 & - & 133.6 & $w$ & 42.2 & 323.4 & $w$ & $\ddot{w}$ & 675.5 \\
\hline $\begin{array}{l}\text { January } 1995 \text {.................... } \\
\text { District of Columbia }\end{array}$ & 5.7 & W & w & $w$ & - & 155.5 & 26.8 & 34.2 & 323.2 & $w$ & $w$ & 797.9 \\
\hline January 1996 .................. & - & $w$ & 145.5 & - & - & 145.5 & - & 8.9 & 301.7 & - & - & 301.7 \\
\hline December 1995 ............ & - & $w$ & $w$ & - & - & 185.9 & - & 11.9 & 374.1 & - & - & 374.1 \\
\hline January 1995 .................. & - & w & $\mathbf{w}$ & w & - & 164.0 & - & 11.6 & $w$ & $w$ & - & 358.5 \\
\hline Maryland & & & & & & & & & & & & \\
\hline January 1996 ................... & w & 5.9 & 816.7 & 338.2 & - & $1,154.9$ & $\mathbf{w}$ & 56.1 & & $1,504.4$ & $w$ & $4,277.4$ \\
\hline December 1995 ............. & $\underline{w}$ & 6.6 & $\begin{array}{l}W \\
w\end{array}$ & $\begin{array}{l}444.9 \\
w\end{array}$ & $\underline{w}$ & $1,469.2$ & w & 61.5 & $3,197.2$ & $\begin{array}{c}W \\
14809\end{array}$ & $w$ & $5,282.5$ \\
\hline New & & & & & & & & 60 & & $1,480.9$ & & .5 \\
\hline & 306.2 & 323.6 & $1,558.5$ & 728.1 & 779.6 & $3,066.3$ & $1,068.1$ & $1,125.8$ & $4,167.4$ & $2,511.9$ & $3,314.4$ & $9,993.7$ \\
\hline December 1995 .............. & 357.4 & 372.3 & $1,808.9$ & 808.0 & 978.3 & $3,595.3$ & $1,224.5$ & $1,280.8$ & $4,716.5$ & $2,779.0$ & $3,530.8$ & $11,026.3$ \\
\hline January 1995 .. & 304.3 & 319.9 & $1,745.8$ & 735.7 & 840.3 & $3,321.9$ & $1,076.1$ & $1,135.9$ & $4,582.8$ & $2,516.5$ & $3,335.9$ & $10,435.3$ \\
\hline New York & & & & & & & & & & & & \\
\hline January 1996 ................. & 348.1 & 354.6 & $1,957.9$ & w & $w$ & $3,012.3$ & $1,656.5$ & $1,717.1$ & $4,951.4$ & $4,634.2$ & 335.8 & $9,921.4$ \\
\hline December 1995 .............. & 403.6 & 410.7 & $2,278.2$ & $1,040.7$ & 175.1 & $3,494.1$ & $1,844.8$ & $1,910.2$ & $5,544.3$ & $4,968.6$ & 639.9 & $11,152.8$ \\
\hline January $1995 . . . .$. & 364.1 & 372.7 & $2,054.0$ & w & w & $3,150.4$ & $1,683.4$ & $1,784.7$ & $5,187.2$ & $4,335.7$ & 520.4 & $10,043.3$ \\
\hline Pennsylvanja & & & & & & & & & & & & \\
\hline January 1996 .................. & 347.9 & 353.6 & 758.2 & $1,094.2$ & 280.3 & $2,132.7$ & $1,720.3$ & $1,783.5$ & $2,774.3$ & $5,464.2$ & $1,133.5$ & $9,372.0$ \\
\hline December 1995 ............ & 408.2 & 413.1 & 917.8 & $1,205.7$ & 414.4 & $2,537.9$ & $1,978.4$ & $2,046.2$ & $3,260.1$ & $5,803.6$ & $1,887.5$ & $10,951.2$ \\
\hline January 1995 & 331.8 & 336.8 & W & $1,271.4$ & $w$ & $2,170.0$ & $1,628.0$ & $1,686.7$ & $3,120.6$ & $5,663.0$ & 704.4 & $9,488.1$ \\
\hline
\end{tabular}

See footnotes at end of table. 
Table 43. Refiner Motor Gasoline Volumes by Grade, Sales Type, PAD District, and State (Thousand Gallons per Day) - Continued

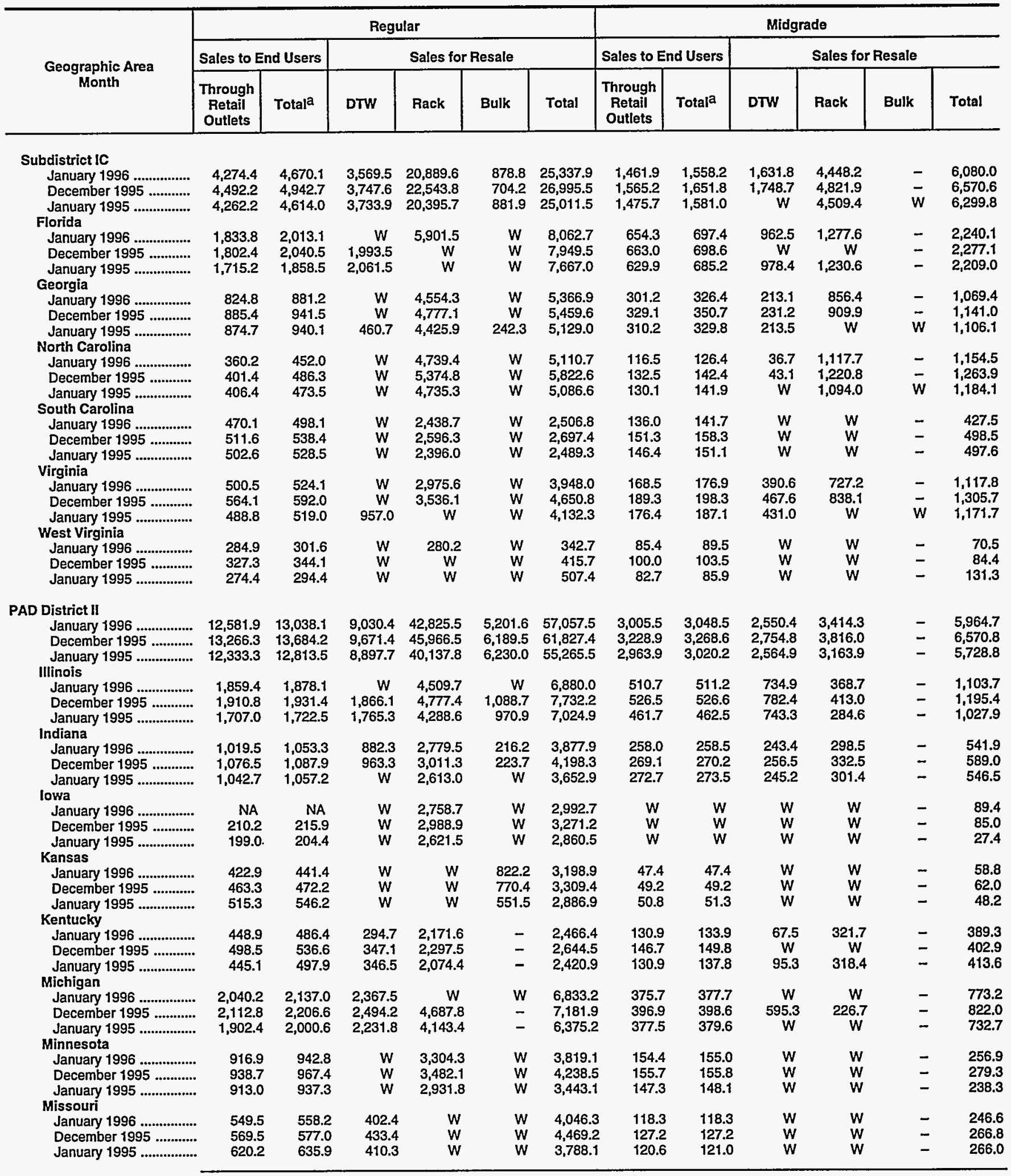

See footnotes at end of table. 
Table 43. Refiner Motor Gasoline Volumes by Grade, Sales Type, PAD District, and State (Thousand Gallons per Day) - Continued

\begin{tabular}{|c|c|c|c|c|c|c|c|c|c|c|c|c|}
\hline \multirow{3}{*}{$\begin{array}{l}\text { Geographic Area } \\
\text { Month }\end{array}$} & \multicolumn{6}{|c|}{ Premium } & \multicolumn{6}{|c|}{ All Grades } \\
\hline & \multicolumn{2}{|c|}{ Sales to End Users } & \multicolumn{4}{|c|}{ Sales for Resale } & \multicolumn{2}{|c|}{ Sales to End Users } & \multicolumn{4}{|c|}{ Sales for Resale } \\
\hline & $\begin{array}{l}\text { Through } \\
\text { Retail } \\
\text { Outlets }\end{array}$ & Totala & DTW & Rack & Bulk & Total & $\begin{array}{l}\text { Through } \\
\text { Retail } \\
\text { Outlets }\end{array}$ & Totala & DTW & Rack & Bulk & Total \\
\hline \multicolumn{13}{|l|}{ Subdistrict IC } \\
\hline 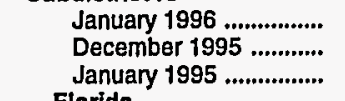 & $\begin{array}{l}1,680.7 \\
1,856.7 \\
1,675.7\end{array}$ & $\begin{array}{l}1,781.4 \\
1,956.5 \\
1,768.0\end{array}$ & $\begin{array}{r}2,799.3 \\
3,057.3 \\
W\end{array}$ & $\begin{array}{l}7,230.8 \\
8,171.8 \\
7,113.5\end{array}$ & $\begin{array}{r}244.0 \\
129.5 \\
W\end{array}$ & $\begin{array}{l}10,274.1 \\
11,358.6 \\
10,111.4\end{array}$ & $\begin{array}{l}7,417.0 \\
7,914.2 \\
7,413.6\end{array}$ & $\begin{array}{l}8,009.7 \\
8,551.0 \\
7,963.1\end{array}$ & $\begin{array}{l}8,000.6 \\
8,553.6 \\
8,371.3\end{array}$ & $\begin{array}{l}32,568.6 \\
35,537.5 \\
32,018.6\end{array}$ & $\begin{array}{r}1,122.8 \\
833.7 \\
1,032.8\end{array}$ & $\begin{array}{l}41,691.9 \\
44,924.8 \\
41,422.7\end{array}$ \\
\hline \multicolumn{13}{|l|}{ Florida } \\
\hline $\begin{array}{l}\text { January } 1996 \text {................. } \\
\text { December } 1995 \text {........... }\end{array}$ & $\begin{array}{l}889.4 \\
921.9\end{array}$ & $\begin{array}{l}929.5 \\
958.1\end{array}$ & $\begin{array}{c}W \\
1,869.4\end{array}$ & $\begin{array}{l}1,990.9 \\
2,085.0\end{array}$ & W & $\begin{array}{l}3,830.7 \\
3,954.5\end{array}$ & $\begin{array}{l}3,377.5 \\
3,387.4\end{array}$ & $\begin{array}{l}3,640.0 \\
3,697.2\end{array}$ & $\begin{array}{c}4,804.4 \\
W\end{array}$ & $\begin{array}{l}9,170.0 \\
9,305.6\end{array}$ & 159.2 & $\begin{array}{l}14,133.6 \\
14,181.0\end{array}$ \\
\hline $\begin{array}{l}\text { January } 1995 \text {................ } \\
\text { Georgla }\end{array}$ & 851.5 & 891.5 & $1,849.9$ & W & $W$ & $3,680.5$ & $3,196.7$ & $3,435.1$ & $4,889.7$ & $8,384.9$ & 281.9 & $13,556.6$ \\
\hline $\begin{array}{l}\text { January } 1996 \text {............... } \\
\text { December } 1995 \text {............ } \\
\text { January } 1995 \text {............... } \\
\text { North Carollna }\end{array}$ & $\begin{array}{l}301.6 \\
348.4 \\
305.9\end{array}$ & $\begin{array}{l}322.8 \\
368.7 \\
324.5\end{array}$ & $\begin{array}{c}W \\
W \\
299.2\end{array}$ & $\begin{array}{c}1,540.2 \\
1,677.6 \\
W\end{array}$ & $\begin{array}{l}W \\
W \\
W\end{array}$ & $\begin{array}{l}1,978.1 \\
2,070.8 \\
1,889.2\end{array}$ & $\begin{array}{l}1,427.7 \\
1,562.9 \\
1,490.8\end{array}$ & $\begin{array}{l}1,530.5 \\
1,660.9 \\
1,594.5\end{array}$ & $\begin{array}{c}950.8 \\
W \\
973.4\end{array}$ & $\begin{array}{l}6,950.8 \\
7,364.6 \\
6,812.3\end{array}$ & $\begin{array}{c}512.9 \\
W \\
338.5\end{array}$ & $\begin{array}{l}8,414.5 \\
8,671.4 \\
8,124.3\end{array}$ \\
\hline $\begin{array}{l}\text { January } 1996 \text {................ } \\
\text { December } 1995 \text {............ } \\
\text { January } 1995 \text {................ } \\
\text { South Carolina }\end{array}$ & $\begin{array}{l}111.7 \\
134.0 \\
115.9\end{array}$ & $\begin{array}{l}129.9 \\
155.2 \\
127.4\end{array}$ & $\begin{array}{c}W \\
W \\
87.8\end{array}$ & $\begin{array}{l}1,745.5 \\
2,079.7 \\
1,684.7\end{array}$ & $\begin{array}{l}W \\
W \\
-\end{array}$ & $\begin{array}{l}1,832.5 \\
2,197.2 \\
1,772.5\end{array}$ & $\begin{array}{l}588.4 \\
667.8 \\
652.5\end{array}$ & $\begin{array}{l}708.4 \\
783.8 \\
742.8\end{array}$ & $\begin{array}{c}166.8 \\
W \\
W\end{array}$ & $\begin{array}{l}7,602.7 \\
8,675.3 \\
7,514.0\end{array}$ & $\begin{array}{l}328.2 \\
W \\
W\end{array}$ & $\begin{array}{l}8,097.7 \\
9,283.7 \\
8,043.2\end{array}$ \\
\hline January 1996 ................. & 127.2 & 136.4 & $w$ & $w$ & - & 812.9 & 733.3 & 776.1 & $w$ & $3,650.1$ & $w$ & $3,747.3$ \\
\hline December 1995 ............ & 156.2 & 165.4 & $w$ & W & - & 922.8 & 819.2 & 862.1 & $w$ & $3,987.0$ & $w$ & $4,118.7$ \\
\hline January 1995 & 134.5 & 141.9 & $w$ & $W$ & - & 786.2 & 783.5 & 821.5 & $w$ & $3,637.6$ & $W$ & $3,773.1$ \\
\hline \multicolumn{13}{|l|}{ Virginia } \\
\hline January 1996 .................. & 191.5 & 199.1 & $W$ & $1,069.9$ & $W$ & $1,715.8$ & 860.5 & 900.2 & $1,938.5$ & $4,772.7$ & 70.5 & $6,781.7$ \\
\hline December 1995 ............ & 225.6 & 234.5 & $W$ & $1,328.1$ & W & $2,097.3$ & 978.9 & $1,024.8$ & $2,326.8$ & $5,702.3$ & 24.7 & $8,053.8$ \\
\hline January 1995 .................. & NA & NA & 622.9 & $1,201.6$ & - & $1,824.5$ & 872.7 & 922.3 & $2,010.9$ & $5,011.4$ & 106.2 & $7,128.5$ \\
\hline $\begin{array}{l}\text { West Virginia } \\
\text { January } 1996\end{array}$ & 59.3 & 63.6 & W & W & - & 103.9 & 429.6 & 454.6 & $\mathbf{W}$ & 422.4 & $w$ & 517.2 \\
\hline $\begin{array}{l}\text { December } 1995 \text {.............. } \\
\text { January } 1995 \text {............... }\end{array}$ & $\begin{array}{l}70.6 \\
60.2\end{array}$ & $\begin{array}{l}74.6 \\
66.6\end{array}$ & $\begin{array}{c}W \\
21.9\end{array}$ & $\underset{136.5}{W}$ & - & $\begin{array}{l}116.0 \\
158.4\end{array}$ & $\begin{array}{l}497.9 \\
417.3\end{array}$ & $\begin{array}{l}522.2 \\
447.0\end{array}$ & $\begin{array}{l}W \\
W\end{array}$ & $\begin{array}{l}502.7 \\
658.2\end{array}$ & $\begin{array}{l}W \\
W\end{array}$ & $\begin{array}{l}616.1 \\
797.0\end{array}$ \\
\hline \multirow{2}{*}{\multicolumn{13}{|c|}{ PAD District II }} \\
\hline & & & & & & & & & & & & \\
\hline January 1996 & $2,722.1$ & $2,785.5$ & $3,105.8$ & $8,398.6$ & 258.1 & $11,762.5$ & $18,309.5$ & $18,872.0$ & $14,686.6$ & $54,638.5$ & $5,459.7$ & $74,784.7$ \\
\hline December 1995 ............ & $2,955.8$ & $3,015.6$ & $3,379.5$ & 9.418 .1 & 296.6 & $13,094.2$ & $19,451.0$ & $19,968.5$ & $15,805.7$ & $59,200.6$ & $6,486.1$ & $81,492.4$ \\
\hline January 1995 .................. & $2,922.6$ & $3,010.8$ & $3,270.2$ & $8,051.7$ & 200.7 & $11,522.6$ & $18,219.8$ & $18,844.5$ & $14,732.8$ & $51,353.4$ & $6,430.6$ & $72,516.9$ \\
\hline \multicolumn{13}{|l|}{ Illinois } \\
\hline January 1996 ................. & 499.4 & 499.9 & W & 924.4 & $W$ & $1,828.0$ & $2,869.6$ & $2,889.2$ & $3,382.9$ & $5,802.8$ & 625.9 & $9,811.7$ \\
\hline December 1995 ............ & 523.8 & 524.2 & 918.9 & 935.6 & 30.8 & $1,885.3$ & $2,961.1$ & 2,982.2 & $3,567.4$ & $6,125.9$ & $1,119.5$ & $10,812.8$ \\
\hline $\begin{array}{l}\text { January } 1995 \text {................. } \\
\text { Indlana }\end{array}$ & 465.1 & 465.6 & 866.5 & 812.6 & - & $1,679.1$ & $2,633.8$ & $2,650.5$ & $3,375.1$ & $5,385.8$ & 970.9 & $9,731.8$ \\
\hline January 1996 & 245.2 & 245.5 & 300.5 & 617.7 & - & 918.2 & $1,522.7$ & $1,557.4$ & $1,426.1$ & $3,695.7$ & 216.2 & $5,338.0$ \\
\hline December 1995 ............ & 262.7 & 263.1 & 328.4 & 736.8 & - & $1,065.2$ & $1,608.3$ & $1,621.3$ & $1,548.1$ & $4,080.6$ & 223.7 & $5,852.5$ \\
\hline January 1995 .................. & 290.2 & 291.0 & $W$ & 637.8 & W & 960.6 & $1,605.6$ & $1,621.7$ & $1,404.5$ & $3,552.2$ & 203.4 & $5,160.0$ \\
\hline $\begin{array}{l}\text { lowa } \\
\text { January } 1996, \ldots . . . . . . . . . . .\end{array}$ & NA & W & $W$ & $w$ & - & 282.6 & $w$ & NA & $w$ & $3,093.0$ & W & $3,364.7$ \\
\hline $\begin{array}{l}\text { January } 1996 \text {................. } \\
\text { December } 1995 \text {............ }\end{array}$ & W & W & W & $w$ & - & 321.6 & 243.7 & 249.4 & w & $3,350.9$ & $W$ & $3,677.9$ \\
\hline January 1995 ................. & w & 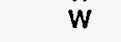 & $W$ & W & - & 248.4 & 235.8 & 241.2 & W & $2,855.5$ & $w$ & $3,136.2$ \\
\hline \multicolumn{13}{|l|}{ Kansas } \\
\hline January 1996 ................. & 61.5 & $\begin{array}{l}62.7 \\
71.3\end{array}$ & $\begin{array}{l}W \\
W\end{array}$ & $\begin{array}{l}262.0 \\
297.8\end{array}$ & $\begin{array}{l}w \\
W\end{array}$ & $\begin{array}{l}295.3 \\
394.9\end{array}$ & $\begin{array}{l}531.7 \\
583.7\end{array}$ & $\begin{array}{l}551.4 \\
592.7\end{array}$ & $\begin{array}{l}W \\
W\end{array}$ & $\begin{array}{l}2,562.4 \\
2,756.8\end{array}$ & $\begin{array}{l}W \\
W\end{array}$ & $\begin{array}{l}3,553.1 \\
3,766.3\end{array}$ \\
\hline $\begin{array}{l}\text { December } 1995 \text {............ } \\
\text { January } 1995 \text {............... } \\
\text { Kentucky }\end{array}$ & $\begin{array}{l}71.3 \\
71.1\end{array}$ & $\begin{array}{l}71.3 \\
76.0\end{array}$ & $\mathbf{W}$ & 238.8 & $\dddot{w}$ & $\begin{array}{l}394.9 \\
272.4\end{array}$ & 637.2 & 673.5 & w & $2,507.3$ & $W$ & \\
\hline $\begin{array}{l}\text { Kentucky } \\
\text { January } 1996 . . . . . . . . . . . . . . .\end{array}$ & 116.5 & 120.3 & 100.4 & 644.4 & - & 744.7 & 696.3 & 740.5 & 462.6 & $3,137.7$ & - & $3,600.4$ \\
\hline January 1996 ................. & 132.4 & 136.1 & $W$ & $\begin{array}{c}W \\
7128\end{array}$ & $W$ & 836.9 & 777.6 & 822.5 & $\begin{array}{c}W \\
58 n\end{array}$ & $3,335.6$ & W & $3,884.4$ \\
\hline \multicolumn{3}{|l|}{ Michlgan } & 652.8 & 845.8 & - & $1,498.6$ & $2,743.2$ & $2,860.0$ & $w$ & $5,502.3$ & W & \\
\hline $\begin{array}{l}\text { January } 1996 \text {.................. } \\
\text { December } 1995 \text {........... }\end{array}$ & 348.3 & 364.5 & 726.0 & 937.8 & - & $1,663.8$ & $2,858.0$ & $2,969.8$ & $3,815.5$ & $5,852.3$ & - & $9,667.7$ \\
\hline January 1995 ................. & 401.1 & 421.6 & 694.2 & $W$ & $W$ & $1,566.3$ & $2,680.9$ & $2,801.9$ & $W$ & $5,225.7$ & $W$ & $8,674.2$ \\
\hline \multicolumn{13}{|l|}{ Minnesota } \\
\hline January 1996 ................. & 119.9 & 119.9 & $W$ & $W$ & $W$ & 495.4 & $1,191.2$ & $1,217.7$ & W & $3,892.2$ & $W$ & $4,571.4$ \\
\hline December 1995 ............ & 125.7 & 125.7 & $W$ & $W$ & $W$ & 540.5 & $1,220.2$ & $1,248.9$ & W & $4,127.9$ & $W$ & $5,058.4$ \\
\hline January 1995 ................. & 117.9 & 118.0 & W & $W$ & $W$ & 465.4 & 1.178 .2 & $1,203.4$ & W & $3,488.0$ & $W$ & $4,146.8$ \\
\hline Mlssourí & & & & & & & & & & & & \\
\hline January 1996 ................ & 109.1 & 109.8 & 148.1 & 663.9 & - & 812.0 & 776.9 & 786.3 & $W$ & $4,318.3$ & $W$ & $5,104.9$ \\
\hline December 1995 ............. & 120.2 & 121.2 & 167.7 & 779.0 & - & 946.6 & 816.9 & 825.4 & $W$ & $4,833.8$ & W & $5,682.6$ \\
\hline January 1995 ................. & 117.1 & 120.9 & 161.4 & 657.3 & - & 818.6 & 857.9 & 877.9 & W & $4,065.9$ & $\mathbf{W}$ & $4,872.7$ \\
\hline
\end{tabular}

See footnotes at end of table. 
Table 43. Refiner Motor Gasoline Volumes by Grade, Sales Type, PAD District, and State (Thousand Gallons per Day) - Continued

\begin{tabular}{|c|c|c|c|c|c|c|c|c|c|c|c|c|}
\hline \multirow{3}{*}{$\begin{array}{c}\text { Geographic Area } \\
\text { Month }\end{array}$} & \multicolumn{6}{|c|}{ Regular } & \multicolumn{6}{|c|}{ Midgrade } \\
\hline & \multicolumn{2}{|c|}{ Sales to End Users } & \multicolumn{4}{|c|}{ Sales for Resale } & \multicolumn{2}{|c|}{ Sales to End Users } & \multicolumn{4}{|c|}{ Sales for Resale } \\
\hline & $\begin{array}{c}\text { Through } \\
\text { Retail } \\
\text { Outlets }\end{array}$ & Total $^{a}$ & DTW & Rack & Bulk & Total & $\begin{array}{c}\text { Through } \\
\text { Retail } \\
\text { Outlets }\end{array}$ & Totala & DTW & Rack & Bulk & Total \\
\hline \multicolumn{13}{|l|}{ Nebraska } \\
\hline January 1996 . & 141.5 & 143.2 & 136.5 & w & $\mathbf{w}$ & $1,734.2$ & 2.4 & 2.4 & $w$ & $w$ & - & 49.9 \\
\hline December 1995 ............. & 146.9 & 149.4 & 137.1 & W & w & $1,767.7$ & 4.0 & 4.0 & $w$ & $w$ & - & 41.1 \\
\hline January 1995 .................. & 124.8 & 126.6 & 122.5 & $1,462.3$ & - & $1,584.8$ & $\dddot{w}$ & $\dddot{w}$ & $w$ & $\ddot{W}$ & - & $w$ \\
\hline \multicolumn{13}{|l|}{ North Dakota } \\
\hline January 1996 & $w$ & $W$ & $w$ & $w$ & - & 744.3 & $w$ & $w$ & $w$ & $w$ & - & $w$ \\
\hline December 1995 ............ & $w$ & w & w & W & - & 831.1 & W & W & w & $W$ & - & $W$ \\
\hline January 1995 ................... & 9.7 & 10.2 & $w$ & 645.0 & $w$ & 704.8 & w & $\ddot{w}$ & $\ddot{w}$ & $\dddot{w}$ & - & $\dddot{W}$ \\
\hline \multicolumn{13}{|l|}{ Ohio } \\
\hline January 1996 & $3,105.5$ & $3,258.4$ & $w$ & $3,623.5$ & $w$ & $5,663.4$ & 899.7 & 931.9 & $w$ & $w$ & - & 807.2 \\
\hline December 1995 ............ & $3,343.3$ & $3,485.0$ & $2,042.1$ & $W$ & W & $6,217.3$ & 991.8 & $1,021.5$ & $w$ & $w$ & - & 941.9 \\
\hline $\begin{array}{l}\text { January } 1995 \text {.................. } \\
\text { Oklahoma }\end{array}$ & $2,966.8$ & $3,116.3$ & $1,829.0$ & $w$ & $w$ & $5,872.1$ & 883.5 & 923.8 & $w$ & $w$ & - & 872.6 \\
\hline January 1996 & 521.7 & 551.6 & $w$ & $W$ & $2,682.6$ & $5,507.1$ & 118.7 & 119.6 & $w$ & $w$ & - & 76.9 \\
\hline 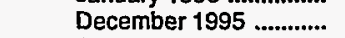 & 549.2 & 581.7 & w & $\dddot{W}$ & $2,898.8$ & $5,682.6$ & 126.6 & 127.1 & $w$ & $w$ & - & 84.3 \\
\hline January 1995 ................. & 515.4 & 546.7 & $w$ & $2,585.7$ & W & $5,832.3$ & 114.6 & 115.4 & W & $w$ & - & 57.0 \\
\hline South Dakota & & & & & & & & & & & & \\
\hline January 1996 ................. & $w$ & $w$ & 30.5 & 840.5 & - & 870.9 & $w$ & $w$ & $w$ & $w$ & - & $w$ \\
\hline December 1995 ............ & $W$ & $w$ & 33.3 & 914.1 & - & 947.4 & $W$ & $W$ & $w$ & $w$ & - & $W$ \\
\hline January 1995 .................. & 18.4 & 18.4 & 30.1 & 779.2 & - & 809.3 & $\ddot{w}$ & $w$ & $w$ & $\dddot{w}$ & - & $w$ \\
\hline Tennessee & & & & & & & & & & & & \\
\hline January 1996 ................. & 756.3 & 776.6 & $w$ & $3,917.1$ & W & $4,404.5$ & 281.6 & 284.6 & $w$ & $w$ & - & $1,162.5$ \\
\hline December 1995 ............ & 836.5 & 857.8 & $w$ & $4,623.7$ & $w$ & $5,143.0$ & 318.5 & 321.6 & $w$ & $w$ & - & $1,354.2$ \\
\hline January 1995 .................. & 785.4 & 815.8 & $w$ & $3,811.6$ & w & $4,230.0$ & 283.6 & 286.5 & $w$ & $\ddot{w}$ & - & $1,118.6$ \\
\hline Wisconsin & & & & & & & & & & & & \\
\hline January 1996 .................. & 575.3 & 581.0 & 373.3 & $3,645.4$ & - & $4,018.7$ & 102.5 & 102.6 & 97.2 & 272.6 & - & 369.8 \\
\hline December 1995 ............. & 583.9 & 589.1 & 395.4 & $3,797.6$ & - & $4,193.1$ & 106.3 & 106.4 & 107.9 & 297.9 & - & 405.8 \\
\hline January 1995 .................. & 568.2 & 577.5 & 376.6 & $3,403.3$ & - & $3,779.9$ & 99.5 & 99.5 & 96.6 & 245.8 & - & 342.5 \\
\hline PAD District III & & & & & & & & & & & & \\
\hline January 1996 & $4,407.4$ & $4,620.8$ & $1,601.5$ & $23,948.5$ & $11,851.8$ & $37,401.8$ & $1,442.8$ & $1,486.9$ & 600.3 & $3,815.6$ & - & $4,415.9$ \\
\hline December 1995 ............. & $4,562.5$ & $4,775.9$ & $1,681.5$ & $24,983.1$ & $17,927.3$ & $44,591.8$ & $1,504.8$ & $1,551.3$ & 643.5 & $4,057.7$ & - & $4,701.2$ \\
\hline January 1995 ................. & $4,388.2$ & $4,614.4$ & $1,677.1$ & W & W & $36,620.3$ & $1,458.3$ & $1,499.1$ & 650.7 & $W$ & $W$ & $4,656.6$ \\
\hline Alabama & & & & & & & & & & & & \\
\hline January 1996 & 192.1 & 217.5 & $w$ & $2,920.5$ & $w$ & $3,076.0$ & 73.0 & 83.1 & $w$ & $w$ & - & 606.5 \\
\hline December 1995 ............ & 205.4 & 230.7 & $w$ & $3,057.0$ & $w$ & $3,218.2$ & 79.3 & 88.8 & $W$ & W & - & 639.6 \\
\hline January 1995 ................. & 204.5 & 228.3 & $W$ & $2,831.4$ & $w$ & $2,991.0$ & 79.5 & 88.8 & 36.3 & 615.0 & - & 651.3 \\
\hline Arkansas & & & & & & & & & & & & \\
\hline January 1996 ................ & 166.8 & 174.7 & $w$ & $2,186.9$ & $w$ & $2,547.3$ & 46.2 & 46.2 & $w$ & $w$ & - & 306.4 \\
\hline December 1995 ............. & 192.6 & 200.4 & $w$ & $2,278.9$ & $\ddot{W}$ & $2,632.5$ & 52.5 & 52.7 & $\ddot{w}$ & $\ddot{w}$ & - & 310.1 \\
\hline January 1995 & 189.5 & 196.0 & w & $W$ & - & $2,450.4$ & 51.6 & 51.7 & w & w & - & 317.5 \\
\hline Louisiana & & & & & & & & & & & & \\
\hline January 1996 & 347.9 & 370.1 & $w$ & $2,530.0$ & $W$ & $4,234.3$ & 146.9 & 153.4 & $w$ & $w$ & - & 628.1 \\
\hline December 1995 ............. & 363.5 & 386.0 & $w$ & $2,710.5$ & $W$ & $4,705.5$ & 158.5 & 165.4 & W & W & - & 695.2 \\
\hline January 1995 ................ & 354.4 & 368.3 & 246.2 & $2,539.9$ & $1,689.1$ & $4,475.2$ & 146.8 & 150.5 & 112.0 & 535.8 & - & 647.8 \\
\hline Mississippi & & & & & & & & & & & & \\
\hline January 1996 & 63.5 & 78.4 & 9.8 & $w$ & W & $2,279.7$ & 16.8 & 20.2 & $w$ & $w$ & - & 377.6 \\
\hline December 1995 ............. & 69.5 & 84.4 & 10.1 & W & W & $2,299.4$ & 19.2 & 22.0 & $w$ & w & - & 412.7 \\
\hline January 1995 ................ & 66.1 & 82.1 & 12.3 & $1,928.8$ & 386.2 & $2,327.3$ & 19.0 & 22.1 & 5.3 & 377.5 & - & 382.8 \\
\hline New Mexico & & & & & & & & & & & & \\
\hline January 1996 & 293.7 & 298.7 & $w$ & w & - & $1,416.2$ & 32.3 & 32.3 & $w$ & $w$ & - & 124.1 \\
\hline December 1995 ............. & 303.6 & 309.0 & W & $W$ & - & $1,529.0$ & 34.0 & 34.0 & w & $w$ & - & 124.7 \\
\hline January 1995 ................ & 262.7 & 272,6 & 209.6 & $1,279.2$ & - & $1,488.8$ & 14.2 & 14.2 & $W$ & $w$ & - & 59.3 \\
\hline Texas & & & & & & & & & & & & \\
\hline January 1996 & $3,343.4$ & $3,481.4$ & $1,141.4$ & $13,139.6$ & $9,567.4$ & $23,848.3$ & $1,127.6$ & $1,151.8$ & 434.7 & $1,938.5$ & - & $2,373.2$ \\
\hline December 1995 ............. & $3,427.9$ & $3,565,4$ & $1,176.3$ & $13,564.3$ & $15,466.7$ & $30,207.3$ & $1,161.4$ & $1,188.4$ & 458.8 & $2,060.1$ & - & $2,518.9$ \\
\hline January 1995 ................... & $3,310.8$ & $3,467.0$ & $1,112.3$ & $12,419.8$ & $9,355.6$ & $22,887.6$ & $1,147.1$ & $1,171.7$ & 471.5 & W & $w$ & $2,598.1$ \\
\hline PAD District IV & & & & & & & & & & & & \\
\hline January 1996 & $1,445.9$ & $1,508.9$ & 580.5 & W & W & $5,358.5$ & 421.7 & 429.0 & $W$ & $W$ & - & 752.4 \\
\hline December 1995 ............ & $1,555.5$ & $1,618.6$ & 594.1 & $4,862.2$ & - & $5,456.3$ & 459.0 & 466.5 & 176.4 & 619.8 & - & 796.2 \\
\hline January 1995 .................. & $1,403.7$ & $1,436.0$ & 526.4 & $W$ & $\mathbf{W}$ & $5,171.2$ & 446.5 & 447.4 & $w$ & W & - & 527.3 \\
\hline $\begin{array}{l}\text { Colorado } \\
\text { January } 1996\end{array}$ & & & & & & & & & & & & \\
\hline $\begin{array}{l}\text { January } 1996 \text {................. } \\
\text { December } 1995 \text {............ }\end{array}$ & $\begin{array}{l}1,153.9 \\
1,205.0\end{array}$ & $\begin{array}{l}1,154.6 \\
1,205.9\end{array}$ & $\begin{array}{c}W \\
183.4\end{array}$ & $\begin{array}{l}1,781.9 \\
1,889.8\end{array}$ & $\begin{array}{l}W \\
-\end{array}$ & $\begin{array}{l}2,096.2 \\
2,073.2\end{array}$ & $\begin{array}{l}299.5 \\
313.7\end{array}$ & $\begin{array}{l}299.5 \\
313.7\end{array}$ & $\begin{array}{c}W \\
78.5\end{array}$ & $\begin{array}{c}W \\
233.5\end{array}$ & $\overline{-}$ & $\begin{array}{l}291.2 \\
312.1\end{array}$ \\
\hline January $1995 \ldots . . . . . . . . . . . . .$. & $1,111.6$ & $1,119.4$ & 179.6 & $1,773.5$ & - & $1,953.1$ & 355.5 & 355.5 & 72.2 & 184.5 & - & 256.6 \\
\hline
\end{tabular}

See footnotes at end of table. 
Table 43. Refiner Motor Gasoline Volumes by Grade, Sales Type, PAD District, and State (Thousand Gallons per Day) — Continued

\begin{tabular}{|c|c|c|c|c|c|c|c|c|c|c|c|c|}
\hline \multirow{3}{*}{$\begin{array}{l}\text { Geographic Area } \\
\text { Month }\end{array}$} & \multicolumn{6}{|c|}{ Premium } & \multicolumn{6}{|c|}{ All Grades } \\
\hline & \multicolumn{2}{|c|}{ Sales to End Users } & \multicolumn{4}{|c|}{ Sales for Resale } & \multicolumn{2}{|c|}{ Sales to End Users } & \multicolumn{4}{|c|}{ Sales for Resale } \\
\hline & $\begin{array}{l}\text { Through } \\
\text { Retail } \\
\text { Outlets }\end{array}$ & Totala & DTW & Rack & Bulk & Total & $\begin{array}{l}\text { Through } \\
\text { Retail } \\
\text { Outlets }\end{array}$ & Totala & DTW & Rack & Bulk & Total \\
\hline \multicolumn{13}{|l|}{ Nebraska } \\
\hline January 1996 ................. & 16.1 & 16.1 & $w$ & $w$ & - & 127.3 & 160.0 & 161.7 & 165.3 & $w$ & $w$ & $1,911,4$ \\
\hline December 1995 .............. & 17.0 & 17.0 & $w$ & $w$ & - & 139.5 & 167.8 & 170.4 & 166.5 & $w$ & $w$ & $1,948.3$ \\
\hline $\begin{array}{l}\text { January } 1995 \text {................. } \\
\text { North Dakota }\end{array}$ & $w$ & $W$ & W & $W$ & - & $w$ & 147.4 & 149.3 & 146.9 & $1,550.8$ & - & $1,697.7$ \\
\hline January 1996 ................ & $w$ & $w$ & $w$ & 46.6 & - & $w$ & $w$ & 8.4 & $w$ & $w$ & - & 836.5 \\
\hline December 1995 .............. & $w$ & $w$ & $w$ & 57.1 & - & $w$ & w & 8.5 & $w$ & $w$ & - & 936.1 \\
\hline $\begin{array}{l}\text { January } 1995 \ldots . . . . . . . . . . . . . . \\
\text { Ohio }\end{array}$ & $w$ & $W$ & W & $w$ & - & $w$ & 11.9 & 12.4 & $\mathbf{W}$ & 695.8 & $w$ & 784.8 \\
\hline $\begin{array}{l}\text { January } 1996 \ldots . . . . . . . . . . . . . \\
\text { December } 1995 \text {............ } \\
\text { January } 1995 \text {................ } \\
\text { Oklahoma }\end{array}$ & $\begin{array}{l}706.1 \\
763.1 \\
775.0\end{array}$ & $\begin{array}{l}733.8 \\
788.5 \\
805.3\end{array}$ & $\begin{array}{l}603.8 \\
677.3 \\
653.2\end{array}$ & $\begin{array}{c}W \\
804.2 \\
687.6\end{array}$ & $\begin{array}{l}W \\
- \\
-\end{array}$ & $\begin{array}{l}1,339.0 \\
1,481.5 \\
1,340.8\end{array}$ & $\begin{array}{l}4,711.3 \\
5,098.2 \\
4,625.3\end{array}$ & $\begin{array}{l}4,924.1 \\
5,294.9 \\
4,845.4\end{array}$ & $\begin{array}{l}W \\
W \\
W\end{array}$ & $\begin{array}{l}4,685.1 \\
5,169.2 \\
4,352.9\end{array}$ & $\begin{array}{l}W \\
W \\
W\end{array}$ & $\begin{array}{l}7,809.5 \\
8,640.7 \\
8,085.6\end{array}$ \\
\hline January 1996 ................. & 116.3 & 122.7 & $w$ & 579.0 & $w$ & 763.6 & 756.7 & 794.0 & $w$ & $3,415.7$ & $w$ & $6,347.6$ \\
\hline December 1995 .............. & 129.9 & 137.6 & $w$ & 600.9 & $w$ & 746.9 & 805.7 & 846.5 & $w$ & $3,403.3$ & $w$ & $6,513.8$ \\
\hline January 1995 ................. & 114.9 & 122.8 & $w$ & 524.9 & W & 680.0 & 744.9 & 784.8 & $W$ & $W$ & $3,338.7$ & $6,569.3$ \\
\hline South Dakota & & & & & & & & & & & & \\
\hline January 1996 ................. & $w$ & 1.4 & $w$ & $w$ & - & $w$ & 19.3 & 19.3 & 41.9 & 909.5 & - & 951.4 \\
\hline December 1995 ............ & 1.1 & 1.1 & w & $\ddot{w}$ & - & $W$ & $w$ & 20.1 & 45.2 & 986.3 & - & $1,031.5$ \\
\hline January 1995 .................. & 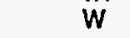 & $w$ & W & $w$ & - & $w$ & 19.5 & 19.5 & 41.0 & 834.6 & - & 875.6 \\
\hline Tonnessee & & & & & & & & & & & & \\
\hline January 1996 & 287.8 & 292.4 & w & $w$ & - & $1,748.0$ & $1,325.7$ & $1,353.6$ & $w$ & $6,572.3$ & W & $7,315.1$ \\
\hline December 1995 ............. & 340.0 & 344.9 & $w$ & $w$ & $w$ & $2,088.4$ & $1,495.0$ & $1,524.3$ & $w$ & $7,774.3$ & $w$ & $8,585.5$ \\
\hline January 1995 .................. & 292.2 & 301.0 & 174.6 & $1,533.8$ & - & $1,708.5$ & $1,361.2$ & $1,403.3$ & 609.5 & $W$ & $\ddot{W}$ & $7,057.1$ \\
\hline Wlsconsin & & & & & & & & & & & & \\
\hline January 1996 & 91.2 & 91.5 & 106.6 & 669.0 & - & 775.5 & 769.0 & 775.1 & 577.1 & $4,587.0$ & - & $5,164.1$ \\
\hline $\begin{array}{l}\text { December } 1995 \text {.............. } \\
\text { January } 1995 \text {............... }\end{array}$ & $\begin{array}{r}96.1 \\
102.7\end{array}$ & $\begin{array}{r}96.2 \\
103.5\end{array}$ & $\begin{array}{l}117.8 \\
105.6\end{array}$ & $\begin{array}{l}717.1 \\
603.7\end{array}$ & - & $\begin{array}{l}835.0 \\
709.3\end{array}$ & $\begin{array}{l}786.4 \\
770.4\end{array}$ & $\begin{array}{l}791.7 \\
780.5\end{array}$ & $\begin{array}{l}621.2 \\
578.8\end{array}$ & $\begin{array}{l}4,812.6 \\
4,252.8\end{array}$ & 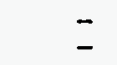 & $\begin{array}{l}5,433.8 \\
4,831.6\end{array}$ \\
\hline \multicolumn{13}{|l|}{ PAD District III } \\
\hline $\begin{array}{l}\text { January } 1996 \text {............... } \\
\text { December } 1995 \text {........... } \\
\text { January } 1995 \text {.............. }\end{array}$ & $\begin{array}{l}1,492.9 \\
1,619.9 \\
1,449.1\end{array}$ & $\begin{array}{l}1,549.3 \\
1,674.0 \\
1,507.3\end{array}$ & $\begin{array}{l}838.5 \\
916.7 \\
907.8\end{array}$ & $\begin{array}{l}6,439.3 \\
6,861.5 \\
6,442.3\end{array}$ & $\begin{array}{l}1,828.5 \\
2,948.3 \\
1,375.6\end{array}$ & $\begin{array}{r}9,106.3 \\
10,726.5 \\
8,725.8\end{array}$ & $\begin{array}{l}7,343.1 \\
7,687.3 \\
7,295.5\end{array}$ & $\begin{array}{l}7,657.0 \\
8,001.2 \\
7,620.8\end{array}$ & $\begin{array}{l}3,040.4 \\
3,241.8 \\
3,235.7\end{array}$ & $\begin{array}{l}34,203.3 \\
35,902.2 \\
33,858.7\end{array}$ & $\begin{array}{l}13,680.3 \\
20,875.5 \\
12,908.3\end{array}$ & $\begin{array}{l}50,924.0 \\
60,019.5 \\
50,002.7\end{array}$ \\
\hline \multicolumn{13}{|l|}{ Alabama } \\
\hline $\begin{array}{l}\text { January } 1996 \text {................. } \\
\text { December } 1995 \text {........... }\end{array}$ & $\begin{array}{l}75.6 \\
87.6\end{array}$ & $\begin{array}{l}83.0 \\
95.6\end{array}$ & $\begin{array}{l}W \\
W\end{array}$ & $\begin{array}{l}W \\
W\end{array}$ & - & $\begin{array}{l}1,165.7 \\
1,268.0\end{array}$ & $\begin{array}{l}340.8 \\
372.3\end{array}$ & $\begin{array}{l}383.6 \\
415.1\end{array}$ & $\begin{array}{l}W \\
W\end{array}$ & $\begin{array}{l}4,619.6 \\
4,882.6\end{array}$ & $\begin{array}{l}W \\
W\end{array}$ & $\begin{array}{l}4,848.3 \\
5,125.7\end{array}$ \\
\hline $\begin{array}{l}\text { January } 1995 \text {.................. } \\
\text { Arkansas }\end{array}$ & 78.4 & 87.7 & 43.5 & $1,063.4$ & - & $1,106.9$ & 362.4 & 404.8 & $W$ & $\begin{array}{l}4,00<.0 \\
4,509.7\end{array}$ & $W$ & $4,749.2$ \\
\hline January 1996 ................. & 35.0 & 36.4 & $w$ & $w$ & - & 620.6 & 247.9 & 257.3 & $w$ & $3,098.1$ & $w$ & $3,474.3$ \\
\hline December 1995 ............. & 42.1 & 43.6 & $\ddot{w}$ & $w$ & - & 671.6 & 287.2 & 296.7 & $w$ & $3,243.4$ & $w$ & $3,614.2$ \\
\hline January 1995 .................. & 39.5 & 40.6 & $W$ & W & - & 678.4 & 280.7 & 288.3 & W & w & - & $3,446.2$ \\
\hline $\begin{array}{l}\text { Loulstana } \\
\text { January } 1996 . . . . . . . . . . . . . .\end{array}$ & 157.4 & 165.1 & w & $w$ & $w$ & $1,420.8$ & 652.2 & 688.6 & 469.8 & 40075 & 18060 & 6.283 .2 \\
\hline December 1995 .............. & 173.9 & 182.2 & $\ddot{w}$ & $\ddot{w}$ & $w$ & $1,820.5$ & 695.9 & 733.5 & 505.5 & $4,373.3$ & $2,342.5$ & $7,221.3$ \\
\hline January 1996 ................ & 15.3 & 18.9 & $w$ & 642.1 & $w$ & 808.8 & 95.7 & 117.5 & 17.9 & $\mathbf{w}$ & $w$ & 34661 \\
\hline December 1995 ............. & 18.7 & 21.9 & $w$ & 703.3 & $w$ & 842.9 & 107.4 & 128.3 & 18.5 & $W$ & $\begin{array}{l}w \\
W\end{array}$ & $\begin{array}{l}3,466.1 \\
3,555.0\end{array}$ \\
\hline $\begin{array}{l}\text { January } 1995 \ldots \ldots . . . . . . . . \\
\text { New Mexico }\end{array}$ & 15.3 & 19.7 & $W$ & 625.8 & W & 799.6 & 100.4 & 123.9 & $w$ & $2,932.1$ & $w$ & $3,509.7$ \\
\hline January 1996 ................. & 55.3 & 56.3 & $w$ & $w$ & - & 240.6 & 381.3 & 387.2 & $w$ & $w$ & - & $1,780.8$ \\
\hline December 1995 ............. & 60.3 & 61.2 & $w$ & $w$ & - & 258.0 & 397.9 & 404.1 & $w$ & $w$ & - & $1,911.6$ \\
\hline January 1995 ................... & 42.4 & 43.8 & 22.0 & 179.9 & - & 201.8 & 319.4 & 330.6 & $\ddot{w}$ & $\dddot{w}$ & - & $1,749.9$ \\
\hline Texas & & & & & & & & & & & & \\
\hline January 1996 ................. & $1,154.2$ & $1,189.6$ & 594.2 & $2,888.5$ & $1,367.1$ & $4,849.8$ & $5,625.2$ & $5,822.8$ & $2,170.3$ & $17,966.5$ & $10,934.5$ & $31,071.3$ \\
\hline December 1995 ............ & $1,237.3$ & $1,269.5$ & 646.6 & $2,963.8$ & $2,255.2$ & $5,865.5$ & $5,826.5$ & $6,023.4$ & $2,281.7$ & $18,588.1$ & $17,721.9$ & $38,591.7$ \\
\hline January 1995 ................. & $1,111.7$ & $1,148.8$ & 619.6 & $w$ & $w$ & $4,546.1$ & $5,569.6$ & $5,787.6$ & $2,203.4$ & $17,485.7$ & $10,342.8$ & $30,031.8$ \\
\hline \multicolumn{13}{|l|}{ PAD District IV } \\
\hline January 1996 ................. & 376.2 & 396.9 & 179.2 & $1,185.1$ & - & $1,364.3$ & $2,243.8$ & $2,334.8$ & w & $6,407.9$ & $w$ & $7,475.2$ \\
\hline December 1995 ............. & 418.7 & 440.1 & 188.7 & $1,225.1$ & - & $1,413.8$ & $2,433.2$ & $2,525.3$ & 959.2 & $6,707.1$ & - & $7,666.3$ \\
\hline January 1995 ................. & 349.5 & 358.7 & 209.1 & $1,073.1$ & - & $1,282.1$ & $2,199.7$ & $2,242.1$ & $W$ & $6,093.9$ & $w$ & $6,980.6$ \\
\hline January 1996 ................ & 265.5 & 265.7 & $w$ & $w$ & - & 433.1 & $1,718.9$ & $1,719.8$ & $w$ & $2,368.5$ & $w$ & $2,820.5$ \\
\hline December 1995 ............ & 282.9 & 283.4 & 63.2 & 397.4 & - & 460.6 & $1,801.7$ & $1,803.0$ & 325.2 & $2,520.7$ & - & $2,845.9$ \\
\hline January 1995 ................. & 240.5 & 241.2 & 54.5 & 326.7 & - & 381.3 & $1,707.5$ & $1,716.0$ & 306.3 & $2,284.7$ & - & $2,591.0$ \\
\hline
\end{tabular}

See footnotes at end of table. 
Table 43. Refiner Motor Gasoline Volumes by Grade, Sales Type, PAD District, and State (Thousand Gallons per Day) - Continued

\begin{tabular}{|c|c|c|c|c|c|c|c|c|c|c|c|c|}
\hline \multirow{3}{*}{$\begin{array}{l}\text { Geographic Area } \\
\text { Month }\end{array}$} & \multicolumn{6}{|c|}{ Regular } & \multicolumn{6}{|c|}{ Midgrade } \\
\hline & \multicolumn{2}{|c|}{ Sales to End Users } & \multicolumn{4}{|c|}{ Sales for Resale } & \multicolumn{2}{|c|}{ Sales to End Users } & \multicolumn{4}{|c|}{ Sales for Resale } \\
\hline & $\begin{array}{c}\text { Through } \\
\text { Retail } \\
\text { Outlets }\end{array}$ & Totala & DTW & Rack & Bulk & Total & $\begin{array}{c}\text { Through } \\
\text { Retail } \\
\text { Outlets }\end{array}$ & Totala & DTW & Rack & Bulk & Total \\
\hline \multicolumn{13}{|l|}{ Idaho } \\
\hline January 1996 & W & 47.0 & 115.8 & 754.6 & - & 870.5 & $w$ & 8.4 & 26.4 & 113.2 & - & 139.5 \\
\hline December 1995 ............... & $\dddot{w}$ & 51.6 & 121.5 & 717.6 & - & 839.1 & w & $W$ & 24.2 & 118.2 & - & 142.4 \\
\hline 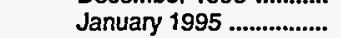 & $\ddot{w}$ & 42.0 & 84.1 & 702.6 & - & 786.7 & w & $\ddot{w}$ & $w$ & W & - & 96.6 \\
\hline \multicolumn{13}{|l|}{ Montana } \\
\hline January 1996 ................. & $w$ & 12.4 & $w$ & $W$ & - & 796.8 & $w$ & W & $W$ & $W$ & - & 67.5 \\
\hline December 1995 ............. & $w$ & 11.5 & $\dddot{w}$ & $w$ & - & 844.9 & $w$ & $w$ & $w$ & $w$ & - & 72.9 \\
\hline January 1995 .................. & $w$ & 17.5 & - & $W$ & $w$ & 786.0 & W & W & - & $w$ & - & W \\
\hline \multicolumn{13}{|l|}{ Utah } \\
\hline January 1996 ................. & 204.3 & 250.0 & 268.7 & 874.9 & - & $1,143.6$ & 106.5 & 112.1 & 66.4 & 185.8 & - & 252.2 \\
\hline December 1995 .............. & 252.9 & 300.3 & 267.6 & 958.2 & - & $1,225.8$ & 127.0 & 132.5 & 70.8 & 195.3 & - & 266.1 \\
\hline 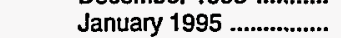 & 198.4 & 213.5 & $w$ & $w$ & $w$ & $1,182.3$ & 81.2 & 82.0 & W & $W$ & - & 168.9 \\
\hline Wyoming & & & & & & & & & & & & \\
\hline January 1996 .................. & 43.2 & 44.9 & $w$ & $w$ & - & 451.4 & 7.4 & $w$ & $w$ & $w$ & - & 1.8 \\
\hline December 1995 ............... & 47.8 & 49.4 & $\ddot{w}$ & W & - & 473.2 & 8.9 & 8.9 & $\ddot{W}$ & w & - & 2.7 \\
\hline January 1995 ................... & 41.7 & 43.7 & $\ddot{w}$ & 455.0 & $w$ & 463.1 & 5.8 & 5.8 & - & W & - & $w$ \\
\hline \multicolumn{13}{|l|}{ PAD District $V$} \\
\hline January 1996 & $6,108.9$ & $6,282.6$ & $16,024.1$ & $w$ & $w$ & $30,203.6$ & $2,172.9$ & $2,197.2$ & $\mathbf{W}$ & $w$ & - & $6,481.1$ \\
\hline December 1995 ............. & $6,710.1$ & $6,889.7$ & $16,455.1$ & $10,408.4$ & $4,623.9$ & $31,487.3$ & $2,516.5$ & $2,545.0$ & $5,229.5$ & $1,607.6$ & - & $6,837.1$ \\
\hline January 1995 ................. & $6,002.3$ & $6,148.1$ & $15,905.2$ & $10,336.5$ & $3,655.3$ & $29,896.9$ & $1,484.1$ & $1,503.4$ & $W$ & W & $w$ & $4,672.7$ \\
\hline \multicolumn{13}{|l|}{ Alaska } \\
\hline 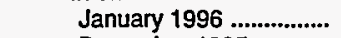 & 161.3 & 181.2 & W & 206.5 & W & 307.3 & $w$ & $w$ & W & $w$ & - & 17.4 \\
\hline December 1995 ............. & 163.9 & 183.8 & W & $w$ & $\mathbf{W}$ & 281.3 & $w$ & $w$ & $\mathbf{W}$ & $w$ & - & 18.0 \\
\hline January 1995 ................. & 129.1 & 139.7 & 86.5 & 170.4 & - & 256.8 & $w$ & $w$ & w & $w$ & - & $W$ \\
\hline \multicolumn{13}{|l|}{ Arizona } \\
\hline January 1996 ................ & 844.1 & 859.6 & W & $1,118.9$ & w & $2,499.9$ & 207.9 & 209.0 & 217.7 & 135.6 & - & 353.4 \\
\hline December 1995 ............ & 931.8 & 944.9 & $1,203.0$ & $w$ & $w$ & $2,470.3$ & 248.9 & 249.5 & 231.7 & 129.2 & - & 360.9 \\
\hline January 1995 ................... & 804.3 & 821.2 & $1,298.0$ & W & $\ddot{w}$ & $2,532.2$ & 26.7 & 26.7 & W & $W$ & - & 98.8 \\
\hline \multicolumn{13}{|l|}{ California } \\
\hline January 1996 ................. & $3,715.1$ & $3,781.9$ & $11,499.6$ & $4,855.9$ & $2,751.7$ & $19,107.2$ & $1,569.6$ & $1,586.5$ & $3,978.2$ & 921.1 & - & $4,899.3$ \\
\hline December 1995 ............. & $4,028.7$ & $4,098.3$ & $11,757.1$ & $4,610.7$ & $4,005.5$ & $20,373.3$ & $1,822.7$ & $1,842.8$ & $4,189.8$ & 918.4 & - & $5,108.2$ \\
\hline January 1995 ................. & $3,702.9$ & $3,753.1$ & $11,079.7$ & $5,070.4$ & $2,842.4$ & $18,992.5$ & $1,390.6$ & $1,400.9$ & $3,336.2$ & $W$ & $w$ & $4,200.8$ \\
\hline \multicolumn{13}{|l|}{ Hawaii } \\
\hline January 1996 .................. & 117.8 & 163.5 & $W$ & $w$ & - & 387.5 & 27.9 & 33.8 & $w$ & $w$ & - & 92.8 \\
\hline December 1995 ............ & 113.9 & 159.9 & 316.7 & 59.6 & - & 376.3 & 25.4 & 32.3 & W & $\ddot{w}$ & - & 101.0 \\
\hline January 1995 .................. & 102.9 & 144.3 & 312.7 & 58.8 & - & 371.5 & 20.7 & 29.6 & W & $\ddot{w}$ & - & 85.8 \\
\hline \multicolumn{13}{|l|}{ Nevada } \\
\hline January 1996 ................. & 75.5 & 82.9 & 591.4 & $\mathbf{W}$ & $w$ & $1,348.9$ & $w$ & $w$ & 145.3 & 53.2 & - & 198.5 \\
\hline December 1995 .............. & 92.7 & 100.6 & 591.0 & 541.5 & 189.4 & $1,321.8$ & $W$ & $\ddot{w}$ & $W$ & $W$ & - & 216.8 \\
\hline January 1995 & 86.3 & 97.0 & W & 610.1 & W & $1,290.5$ & 17.5 & 17.6 & $\ddot{W}$ & $\ddot{w}$ & - & 155.3 \\
\hline \multicolumn{13}{|l|}{ Oregon } \\
\hline January 1996 ................. & 347.5 & 356.5 & $w$ & $1,388.3$ & $w$ & $2,618.9$ & 51.5 & 51.5 & 95.2 & 111.5 & - & 206.6 \\
\hline December 1995 ............. & 380.7 & 390.8 & $W$ & $1,406.5$ & $w$ & $2,497.3$ & 58.9 & 58.9 & 115.5 & 115.6 & - & 231.1 \\
\hline January 1995 .................... & 313.3 & 323.8 & W & $1,276.5$ & W & $2,637.5$ & $W$ & $W$ & $W$ & $W$ & - & $W$ \\
\hline Washington & & & & & & & & & & & & \\
\hline January 1996 ................. & 847.6 & 857.1 & $1,538.3$ & $2,200.1$ & 195.5 & $3,933.9$ & 250.7 & 251.1 & 375.8 & 337.3 & - & 713.1 \\
\hline December 1995 ............. & 998.4 & $1,011.4$ & $1,640.9$ & $2,477.4$ & 48.8 & $4,167.0$ & 283.7 & 284.5 & 424.5 & 376.6 & - & 801.2 \\
\hline January 1995 .................. & 863.5 & 869.0 & $1,723.5$ & $w$ & $w$ & $3,815.9$ & $W$ & $w$ & $w$ & 34.1 & - & $w$ \\
\hline
\end{tabular}

See footnotes at end of table. 
Table 43. Refiner Motor Gasoline Volumes by Grade, Sales Type, PAD District, and State (Thousand Gallons per Day) - Continued

\begin{tabular}{|c|c|c|c|c|c|c|c|c|c|c|c|c|}
\hline \multirow{3}{*}{$\begin{array}{c}\text { Geographic Area } \\
\text { Month }\end{array}$} & \multicolumn{6}{|c|}{ Premium } & \multicolumn{6}{|c|}{ All Grades } \\
\hline & \multicolumn{2}{|c|}{ Sales to End Users } & \multicolumn{4}{|c|}{ Sales for Resale } & \multicolumn{2}{|c|}{ Sales to End Users } & \multicolumn{4}{|c|}{ Sales for Resale } \\
\hline & $\begin{array}{c}\text { Through } \\
\text { Retail } \\
\text { Outlets }\end{array}$ & Totala & DTW & Rack & Bulk & Total & $\begin{array}{c}\text { Through } \\
\text { Retail } \\
\text { Outlets }\end{array}$ & Totala & DTW & Rack & Bulk & Total \\
\hline \multicolumn{13}{|l|}{ Idaho } \\
\hline January 1996 & $w$ & 8.3 & 41.6 & 170.2 & - & 211.8 & $w$ & 63.6 & 183.8 & $1,038.0$ & - & $1,221.8$ \\
\hline December 1995 ............. & w & $W$ & 46.5 & 162.1 & - & 208.5 & w & 70.8 & 192.1 & 997.9 & - & $1,190.1$ \\
\hline January 1995 ................... & $w$ & $w$ & $w$ & W & - & 171.6 & $w$ & 52.2 & 132.5 & 922.5 & - & $1,054.9$ \\
\hline \multicolumn{13}{|l|}{ Montana } \\
\hline January 1996 & $W$ & $w$ & $w$ & $w$ & - & 206.0 & $w$ & 16.3 & $w$ & W & - & $1,070.4$ \\
\hline December 1995 ........... & $W$ & $w$ & $w$ & $W$ & - & 216.2 & $W$ & 15.6 & $W$ & $W$ & - & $1,134.0$ \\
\hline January 1995 ................. & $w$ & $\dddot{w}$ & - & $\ddot{w}$ & - & $W$ & w & 21.1 & - & $w$ & $w$ & 975.8 \\
\hline \multicolumn{13}{|l|}{ Utah } \\
\hline January 1996 & 93.3 & 110.2 & 73.8 & 330.5 & - & 404.3 & 404.2 & 472.3 & 408.9 & $1,391.2$ & - & $1,800.1$ \\
\hline December 1995 ........... & 115.1 & 132.2 & 74.9 & 343.4 & - & 418.3 & 495.1 & 565.0 & 413.4 & $1,496.8$ & - & $1,910.3$ \\
\hline January 1995 ................ & 92.7 & 98.5 & 117.9 & 322.4 & - & 440.3 & 372.2 & 393.9 & 429.3 & $W$ & $w$ & $1,791.5$ \\
\hline \multicolumn{13}{|l|}{ Wyoming } \\
\hline January 1996 ................. & 9.6 & $W$ & $w$ & $W$ & - & 109.1 & 60.2 & 62.8 & $w$ & $w$ & - & 562.4 \\
\hline December 1995 ............. & 11.7 & 12.7 & $\ddot{w}$ & $\ddot{W}$ & - & 110.2 & 68.4 & 70.9 & w & $w$ & - & 586.1 \\
\hline January 1995 ................... & 7.7 & 9.4 & $w$ & $W$ & - & $w$ & 55.3 & 58.9 & w & 556.1 & $W$ & 567.4 \\
\hline \multicolumn{13}{|l|}{ PAD District V } \\
\hline January 1996 ................. & $1,675.2$ & $1,713.0$ & $6,196.6$ & $1,919.8$ & 448.7 & $8,565.1$ & $9,957.0$ & $10,192.8$ & $W$ & $13,879.1$ & $\mathbf{W}$ & $45,249.7$ \\
\hline December 1995 ............ & $1,851.3$ & $1,891.3$ & $6,445.0$ & $2,021.7$ & 497.7 & $8,964.4$ & $11,077.9$ & $11,326.0$ & $28,129.5$ & $14,037.7$ & $5,121.6$ & $47,288.8$ \\
\hline January 1995 & $1,500.5$ & $1,528.5$ & $5,752.0$ & W & W & $7,826.8$ & $8,986.8$ & $9,180.0$ & W & $13,136.5$ & W & $42,396.4$ \\
\hline \multicolumn{13}{|l|}{ Alaska } \\
\hline January 1996 ................. & $W$ & $W$ & 23.1 & $W$ & W & 46.1 & 222.0 & 246.4 & $w$ & 232.6 & W & 370.8 \\
\hline December 1995 ............. & $w$ & $W$ & 22.5 & W & $W$ & 45.6 & 228.2 & 252.3 & $w$ & 221.6 & $W$ & 344.9 \\
\hline January 1995 & $w$ & W & W & 18.1 & - & $w$ & 161.9 & 175.1 & w & W & - & 304.5 \\
\hline \multicolumn{13}{|l|}{ Arizona } \\
\hline January $1996 \ldots$ & 219.8 & 221.5 & $W$ & 213.6 & W & 531.7 & $1,271.8$ & $1,290.1$ & $1,717.9$ & $1,468.2$ & 198.9 & $3,385.0$ \\
\hline December 1995 ............ & 243.3 & 244.8 & 340.4 & $W$ & $\mathbf{W}$ & 610.0 & $1,424.0$ & $1,439.2$ & $1,775.1$ & $1,473.8$ & 192.3 & $3,441.2$ \\
\hline $\begin{array}{l}\text { January } 1995 \text {.................. } \\
\text { California }\end{array}$ & 182.9 & 184.8 & $W$ & 167.9 & $W$ & 505.3 & $1,013.9$ & $1,032.7$ & $1,670.8$ & $W$ & $W$ & $3,136.3$ \\
\hline January 1996 ................. & $1,149.3$ & $1,162.6$ & $4,991.2$ & 949.6 & 338.3 & $6,279.2$ & $6,434.0$ & $6,531.0$ & $20,469.0$ & $6,726.7$ & \\
\hline December 1995 ............ & $1,260.9$ & $1,274.6$ & $5,143.2$ & 966.1 & 368.5 & $6,477.7$ & $7,112.4$ & $7,215.8$ & $21,090.1$ & $\begin{array}{l}0,720.1 \\
6,495.2\end{array}$ & $4,373.9$ & $31,959.2$ \\
\hline January 1995 .................... & $1,015.5$ & $1,023.7$ & $4,476.8$ & W & W & $5,703.1$ & $6,108.9$ & $6,177.8$ & $18,892.7$ & $6,875.1$ & $3,128.7$ & $28,896.4$ \\
\hline \multicolumn{13}{|l|}{ Hawaii } \\
\hline January 1996 ................. & 54.7 & 71.7 & 202.1 & 28.0 & - & 230.1 & 200.4 & 269.0 & 606.2 & 104.2 & - & 710.4 \\
\hline December 1995 ............. & 53.5 & 72.4 & $W$ & $W$ & - & 239.2 & 192.8 & 264.6 & 627.9 & 88.5 & - & 716.5 \\
\hline January 1995 ................. & 46.3 & 61.1 & $w$ & $W$ & - & 228.8 & 169.9 & 235.0 & 597.4 & 88.7 & - & 686.1 \\
\hline \multicolumn{13}{|l|}{ Nevada } \\
\hline January 1996 ................... & $w$ & $w$ & 180.0 & $W$ & $W$ & 310.2 & 130.0 & 137.5 & 916.7 & 718.8 & 222.0 & $1,857.5$ \\
\hline December 1995 ............. & $w$ & $\dddot{W}$ & 185.4 & W & $\ddot{w}$ & 349.5 & 161.1 & 169.3 & $W$ & 717.5 & $w$ & $1,888.1$ \\
\hline January 1995 .................. & 18.9 & 18.9 & 153.5 & W & $w$ & 275.4 & 122.6 & 133.4 & 830.2 & $w$ & $w$ & $1,721.2$ \\
\hline \multicolumn{13}{|l|}{ Oregon } \\
\hline January 1996 ................ & 55.8 & 56.1 & $w$ & 196.6 & $w$ & 423.3 & 454.9 & 464.1 & $1,035.7$ & $1,696.3$ & 516.8 & $3,248.8$ \\
\hline December 1995 ............ & 62.8 & 63.0 & $w$ & 214.3 & $W$ & 405.9 & 502.4 & 512.7 & $1,132.3$ & $1,736.5$ & 265.5 & $3,134.3$ \\
\hline January 1995 ................ & $w$ & $w$ & 154.2 & $W$ & - & $W$ & 377.5 & 388.3 & $w$ & $1,468.0$ & W & $2,995.6$ \\
\hline Washington & & & & & & & & & & & & \\
\hline January 1996 & 145.6 & 146.6 & 349.7 & 394.9 & $\rightarrow$ & 744.6 & $1,243.9$ & $1,254.8$ & $2,263.8$ & $2,932.3$ & 195.5 & $5,391.6$ \\
\hline December 1995 ............. & 174.9 & 176.3 & 385.9 & 450.5 & - & 836.4 & $1,457.0$ & $1,472.2$ & $2,451.3$ & $3,304.5$ & 48.8 & $5,804.6$ \\
\hline January 1995 .................. & w & $W$ & 408.8 & $W$ & - & $W$ & $1,032.1$ & $1,037.6$ & $W$ & $2,435.6$ & $W$ & $4,656.3$ \\
\hline
\end{tabular}

Dash $(-)=$ No data reported.

NA $=$ Not available.

$W=$ Withheld to avoid disclosure of individual company data.

a Includes sales through retail outlets as well as all direct sales to end users that were not made through company-operated retail outlets, e.g., sales to agricultural customers, commercial sales, and industrial sales.

Notes: Motor gasoline averages and totals prior to October 1993 include leaded gasoline.

Notes: Values shown for the current month are preliminary. Values shown for previous months are revised. Data are final upon publication in the Petroleum Markeling Annual.

Source: Energy Information Administration Form EIA-782A, "Refiners'/Gas Plant Operators' Monthly Petroleum Product Sales Report." 
Table 44. Refiner Motor Gasoline Volumes by Formulation, Sales Type, PAD District, and State

(Thousand Gallons per Day)

\begin{tabular}{|c|c|c|c|c|c|c|c|c|c|c|c|c|}
\hline \multirow{3}{*}{$\begin{array}{c}\text { Geographic Area } \\
\text { Month }\end{array}$} & \multicolumn{6}{|c|}{ Conventional } & \multicolumn{6}{|c|}{ Oxygenated } \\
\hline & \multicolumn{2}{|c|}{ Sales to End Users } & \multicolumn{4}{|c|}{ Sales for Resale } & \multicolumn{2}{|c|}{ Sales to End Users } & \multicolumn{4}{|c|}{ Sales for Resale } \\
\hline & \begin{tabular}{|c|} 
Through \\
Retail \\
Outlets
\end{tabular} & Totala & DTW & Rack & Bulk & Total & $\begin{array}{c}\text { Through } \\
\text { Retail } \\
\text { Outlets }\end{array}$ & Totala & DTW & Rack & Bulk & Total \\
\hline \multicolumn{13}{|l|}{ United States } \\
\hline 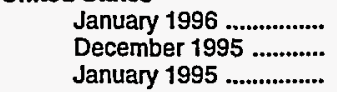 & $\begin{array}{l}28,883.0 \\
31,155.8 \\
28,595.6\end{array}$ & $\begin{array}{l}30,492.3 \\
32,760.5 \\
30,183.6\end{array}$ & $\begin{array}{l}22,368.7 \\
23,997.1 \\
22,863.4\end{array}$ & $\begin{array}{l}127,631.5 \\
136,791.6 \\
122,012.4\end{array}$ & $\begin{array}{l}24,219.8 \\
32,365.0 \\
22,890.7\end{array}$ & $\begin{array}{l}174,220.1 \\
193,153.8 \\
167,766.5\end{array}$ & $\begin{array}{l}8,145.0 \\
8,892.1 \\
7,952.1\end{array}$ & $\begin{array}{l}8,286.5 \\
9,044.3 \\
8,077.9\end{array}$ & $\begin{array}{l}13,310.0 \\
13,834.4 \\
12,718.0\end{array}$ & $\begin{array}{r}W \\
11,116.9 \\
10,597.1\end{array}$ & $\begin{array}{r}W \\
1,271.0 \\
1,180.4\end{array}$ & $\begin{array}{l}24,764.0 \\
26,222.3 \\
24,495.5\end{array}$ \\
\hline \multicolumn{13}{|l|}{ PAD District I } \\
\hline $\begin{array}{l}\text { January } 1996 \ldots . . . . . . . . . . \\
\text { December } 1995 \ldots . . . . . . . . . . \\
\text { January } 1995\end{array}$ & $\begin{array}{l}9,065.5 \\
9,803.5 \\
8,860.7\end{array}$ & $\begin{array}{r}9,702.6 \\
10,484.1 \\
9,469.5\end{array}$ & $\begin{array}{c}8,765.1 \\
9,311.5 \\
W\end{array}$ & $\begin{array}{l}39,822.4 \\
42,873.0 \\
38,549.0\end{array}$ & $\begin{array}{c}2,987.9 \\
3,278.2 \\
W\end{array}$ & $\begin{array}{l}51,575.4 \\
55,462.6 \\
49,944.6\end{array}$ & $\frac{-}{70.8}$ & $\overline{-}$ & $\overline{\bar{w}}$ & $\frac{-}{684.7}$ & $\overline{\bar{w}}$ & $\overline{-}$ \\
\hline \multicolumn{13}{|c|}{$\begin{array}{lllll} & 0,04.0 & \text { of } & 49,944.0 \\
\text { Subdistrict IA }\end{array}$} \\
\hline January 1996 .................. & - & $w$ & $w$ & 727.4 & w & 910.7 & - & - & - & - & - & - \\
\hline December 1995 ............. & - & $w$ & $\ddot{w}$ & 763.6 & W & 919.9 & - & - & - & - & - & $\overline{-}$ \\
\hline \multicolumn{13}{|l|}{ Connecticut } \\
\hline January 1996 ...................... & - & - & - & - & $w$ & w & - & - & - & - & - & - \\
\hline December 1995 ............. & - & - & - & - & - & - & - & - & - & - & - & - \\
\hline January 1995 ................... & - & - & - & - & - & - & - & - & - & - & - & - \\
\hline \multicolumn{13}{|l|}{ Maine } \\
\hline Jecember 1995 ............... & $\overline{-}$ & $\overline{-}$ & $\overline{-}$ & $w$ & $\begin{array}{l}w \\
w\end{array}$ & $\begin{array}{l}651.8 \\
660.6\end{array}$ & $\overline{-}$ & $\overline{-}$ & $\overline{-}$ & $\bar{z}$ & $\overline{-}$ & $\overline{-}$ \\
\hline 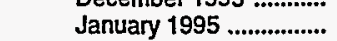 & - & - & - & $\ddot{w}$ & $w$ & 708.9 & - & - & - & - & - & - \\
\hline \multicolumn{13}{|l|}{ Massachusetts } \\
\hline January $1996 \ldots . . . . . . . . . . . . .$. & - & - & - & - & - & - & - & - & - & - & - & - \\
\hline December 1995 ............. & - & - & - & - & - & - & - & - & - & - & - & - \\
\hline January 1995 .................. & - & - & - & - & w & $w$ & - & - & - & - & - & - \\
\hline New Hampshire & & & & & & & & & & & & \\
\hline January 1996 .................. & - & W & W & w & - & w & - & - & - & - & - & - \\
\hline December 1995 ............ & - & $w$ & $w$ & $w$ & - & 21.4 & - & - & - & - & - & - \\
\hline January 1995 .................. & - & w & w & w & - & $w$ & - & - & - & - & - & - \\
\hline $\begin{array}{l}\text { Rhode Island } \\
\text { January } 1996\end{array}$ & - & - & - & - & - & - & - & - & - & - & & \\
\hline $\begin{array}{l}\text { January } 1996 \ldots . . . . . . . . . . . . \\
\text { December } 1995 \ldots . . . . . . .\end{array}$ & $\overline{-}$ & $\overline{-}$ & - & - & $\overline{-}$ & $\overline{-}$ & $z$ & $\overline{-}$ & $\overline{-}$ & $\overline{-}$ & $\bar{z}$ & $\overline{-}$ \\
\hline 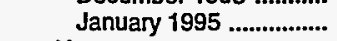 & - & - & - & $w$ & - & $w$ & - & - & - & - & - & - \\
\hline Vermont & & & & & & & & & & & & \\
\hline January 199 & - & - & w & w & - & 203.3 & - & - & - & - & - & - \\
\hline December 1995 ............. & - & - & $w$ & $w$ & - & 237.9 & - & - & - & - & - & - \\
\hline January 1995 .................. & - & - & $w$ & $w$ & - & $W$ & - & - & - & - & - & - \\
\hline $\begin{array}{l}\text { Subdistrict IB } \\
\text { January } 1996\end{array}$ & & & & 8.430 .0 & & & - & - & - & - & - & - \\
\hline 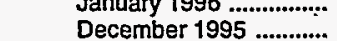 & $\begin{array}{l}2,315.8 \\
2,712.2\end{array}$ & $w$ & $w$ & $\begin{array}{l}8,430.0 \\
8,847.5\end{array}$ & $w$ & $\begin{array}{l}12,154.6 \\
14,098.5\end{array}$ & $=$ & - & 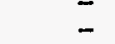 & - & $\overline{-}$ & $\overline{-}$ \\
\hline 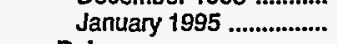 & $2,259.1$ & $\dddot{w}$ & $\ddot{w}$ & $8,436.1$ & $\ddot{w}$ & $12,113.6$ & - & - & - & - & - & - \\
\hline Delaware & & & & & & & & & & & & \\
\hline January 1996 ................... & - & - & - & - & - & - & - & - & - & - & - & - \\
\hline December 1995 ............... & - & - & - & - & - & - & - & - & - & - & - & - \\
\hline 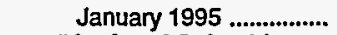 & - & - & - & $w$ & - & $w$ & - & - & - & - & - & - \\
\hline District of Columbia & & & & & & & & & & & & \\
\hline 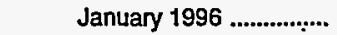 & - & - & - & - & - & - & - & - & - & - & - & - \\
\hline December $1995 \ldots . . . . . . . . . .$. & - & - & - & - & - & - & - & - & - & - & - & - \\
\hline January 1995 ................... & - & - & - & - & - & - & - & - & - & - & - & - \\
\hline Maryland. & & & & & & & & & & & & \\
\hline January 1996 & $w$ & w & w & $w$ & w & 404.7 & - & - & - & - & - & - \\
\hline December 1995 .............. & w & $w$ & w & w & w & 484.8 & - & - & - & - & - & - \\
\hline January 1995 ................. & - & $w$ & w & $w$ & w & $w$ & - & - & - & - & - & - \\
\hline New Jersey & & & & & & & & & & & & \\
\hline January 1996 .................. & - & - & - & - & 551.2 & 551.2 & - & - & - & - & - & - \\
\hline December 1995 ............. & - & - & - & - & 305.0 & 305.0 & - & - & - & - & - & - \\
\hline January $1995 \ldots . . . . . . . . . . . . . . . .$. & - & - & - & - & 189.0 & 189.0 & - & - & - & - & - & - \\
\hline New York & & & & & & & & & & & & \\
\hline January 1996 & w & $1,129.4$ & $1,231.6^{*}$ & $3,462.3$ & 94.6 & $4,788.5$ & - & - & - & - & - & - \\
\hline December 1995 ............. & $W$ & $1,256.3$ & $1,370.2$ & $3,585.9$ & 162.4 & $5,118.4$ & - & - & - & - & - & - \\
\hline January 1995 ................... & $1,091.0$ & $1,148.4$ & $1,164.6$ & $w$ & $w$ & $4,526.7$ & - & - & - & - & - & - \\
\hline Pennsylvania & & & & & & & & & & & & \\
\hline January 1996 .................. & $1,271.3$ & $1,320.5$ & $1,288.6$ & w & w & $7,010.2$ & - & - & - & - & - & - \\
\hline December 1995 ............. & 1.480 .9 & $1,532.8$ & $1,495.0$ & $\ddot{w}$ & $\ddot{w}$ & $8,190.3$ & - & - & - & - & - & - \\
\hline January 1995 ................... & $1,168.1$ & $\{, 214.3$ & $1,420.8$ & w & w & $7,024.0$ & - & - & - & - & - & - \\
\hline
\end{tabular}

See footnotes at end of table. 
Table 44. Refiner Motor Gasoline Volumes by Formulation, Sales Type, PAD District, and State

(Thousand Gallons per Day) - Continued

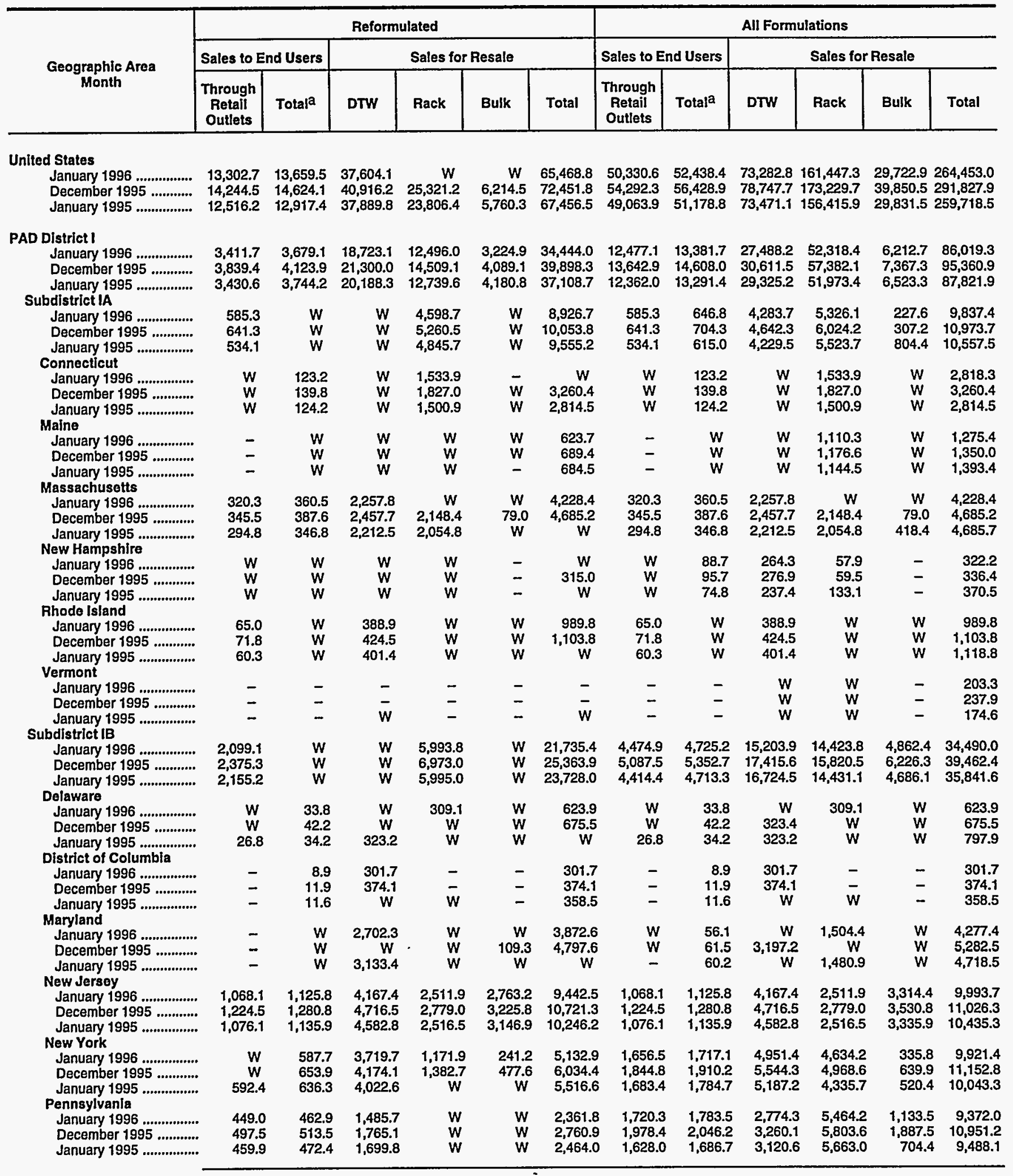

See footnotes at end of table. 
Table 44. Refiner Motor Gasoline Volumes by Formulation, Sales Type, PAD District, and State

(Thousand Gallons per Day) - Continued

\begin{tabular}{|c|c|c|c|c|c|c|c|c|c|c|c|c|}
\hline \multirow{3}{*}{$\begin{array}{c}\text { Geographic Area } \\
\text { Month }\end{array}$} & \multicolumn{6}{|c|}{ Conventional } & \multicolumn{6}{|c|}{ Oxygenated } \\
\hline & \multicolumn{2}{|c|}{ Sales to End Users } & \multicolumn{4}{|c|}{ Sales for Resale } & \multicolumn{2}{|c|}{ Sales to End Users } & \multicolumn{4}{|c|}{ Sales for Resale } \\
\hline & $\begin{array}{c}\text { Through } \\
\text { Retail } \\
\text { Outlets }\end{array}$ & Totala & DTW & Rack & Bulk & Total & $\begin{array}{c}\text { Through } \\
\text { Retail } \\
\text { Outlets }\end{array}$ & Totala & DTW & Rack & Bulk & Total \\
\hline \multicolumn{13}{|l|}{ Subdistrict IC } \\
\hline January 1996 & $6,689.6$ & $7,244.1$ & W & $30,665.0$ & $w$ & $37,910.1$ & - & - & - & - & - & - \\
\hline December 1995 ............. & $7,091.4$ & $7,683.3$ & W & $33,261.9$ & $w$ & $40,444.2$ & - & - & - & - & - & - \\
\hline $\begin{array}{l}\text { January } 1995 \\
\text { Florida }\end{array}$ & $6,601.5$ & $7,098.2$ & $6,423.0$ & $29,434.9$ & 970.7 & $36,828.6$ & 70.8 & 77.7 & $w$ & 684.7 & W & 768.6 \\
\hline January 1996 & $3,377.5$ & $3,640.0$ & $4,804.4$ & $9,170.0$ & 159.2 & $14,133.6$ & - & - & - & - & - & - \\
\hline December 1995 ............. & $3,387.4$ & $3,697.2$ & W & $9,305.6$ & $w$ & $14,181.0$ & - & - & - & - & - & - \\
\hline \multirow{2}{*}{\multicolumn{13}{|c|}{ Georgia }} \\
\hline & & & & & & & & & & & & \\
\hline January 1996 ................. & $1,427.7$ & $1,530.5$ & 950.8 & $6,950.8$ & 512.9 & $8,414.5$ & - & - & - & - & - & - \\
\hline December 1995 ............. & $1,562.9$ & $1,660.9$ & $W$ & $7,364.6$ & $W$ & $8,671.4$ & - & - & - & - & - & - \\
\hline January 1995 ................. & $1,490.8$ & $1,594.5$ & 973.4 & $6,812.3$ & 338.5 & $8,124.3$ & - & - & - & - & - & - \\
\hline North Carolina & & & & & & & & & & & & \\
\hline January 1996 & 588.4 & 708.4 & 166.8 & $7,602.7$ & 328.2 & $8,097.7$ & - & - & - & - & - & - \\
\hline December 1995 ............. & 667.8 & 783.8 & W & $8,675.3$ & W & $9,283.7$ & - & - & - & - & - & - \\
\hline 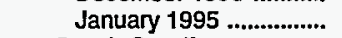 & 581.7 & $W$ & 261.6 & W & $\ddot{w}$ & W & 70.8 & w & $w$ & 684.7 & $w$ & 768.6 \\
\hline South Carolina & & & & & & & & & & & & \\
\hline January 1996 ................. & 733.3 & 776.1 & $w$ & $3,650.1$ & w & $3,747.3$ & - & - & - & - & - & - \\
\hline December 1995 ............. & 819.2 & 862.1 & $w$ & $3,987.0$ & $w$ & $4,118.7$ & - & - & - & - & - & - \\
\hline 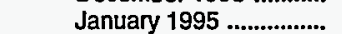 & 783.5 & 821.5 & $w$ & $3,637,6$ & $w$ & $3,773.1$ & - & - & - & - & - & - \\
\hline Virginia & & & & & & & & & & & & \\
\hline January 1996 .................. & 133.2 & 134.5 & $w$ & $2,869.1$ & $w$ & $2,999.8$ & - & - & - & - & - & - \\
\hline 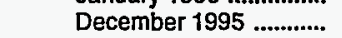 & 156.1 & 157.1 & $\dddot{w}$ & $3,426.6$ & $\ddot{w}$ & $3,573.2$ & - & - & - & - & - & - \\
\hline 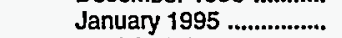 & 131.5 & $w$ & $w$ & $3,116.0$ & $\ddot{w}$ & $3,306.6$ & - & - & - & - & - & - \\
\hline West Virginia & & & & & & & & & & & & \\
\hline January 1996 ................. & 429.6 & 454.6 & $w$ & 422.4 & W & 517.2 & - & - & - & - & - & - \\
\hline December 1995 & 497.9 & 522.2 & $w$ & 502.7 & $\mathbf{W}$ & 616.1 & - & - & - & - & - & - \\
\hline January 1995 .................. & 417.3 & $W$ & W & W & W & $W$ & - & - & - & - & - & - \\
\hline PAD District II & & & & & & & & & & & & \\
\hline January 1996 ................. & $14,859.9$ & $15,361.4$ & $w$ & $w$ & $5,459.7$ & $65,259.3$ & $1,012.6$ & $1,036.3$ & $W$ & $w$ & - & $1,865.5$ \\
\hline December 1995 ............. & $15,947.3$ & $16,398.1$ & W & W & $6,486.1$ & $71,381.5$ & $1,016.1$ & $1,041.2$ & $W$ & $w$ & - & $1,951.3$ \\
\hline January 1995 ................. & $14,960.1$ & $15,523.3$ & $W$ & $w$ & $6,430.6$ & $63,348.2$ & 969.8 & 992.1 & $W$ & $w$ & - & $1,831.9$ \\
\hline Illinois & & & & & & & & & & & & \\
\hline January 1996 ................ & $1,157.1$ & $1,166.5$ & $W$ & $w$ & $W$ & $5,012.1$ & - & - & - & - & - & - \\
\hline December 1995 ............. & $1,204.6$ & $1,213.6$ & W & W & $W$ & $5,777.1$ & - & - & - & - & - & - \\
\hline January 1995 .................. & $1,079.8$ & $1,087.9$ & W & W & $\dddot{w}$ & $5,234.8$ & - & - & - & - & - & - \\
\hline Indiana & & & & & & & & & & & & \\
\hline January 1996 .................. & $1,431.5$ & $1,464.3$ & $1,204.8$ & $3,241.7$ & 216.2 & $4,662.7$ & - & - & - & - & - & - \\
\hline December 1995 ............. & $1,516.0$ & $1,526.3$ & $1,313.6$ & $3,563.9$ & 223.7 & $5,101.1$ & - & - & - & - & - & - \\
\hline January 1995 ................. & $1,501.6$ & $1,516.2$ & $1,179.6$ & $3,181.9$ & 203.4 & $4,564.9$ & - & - & - & - & - & - \\
\hline lowa & & & & & & & & & & & & \\
\hline January 1996 ................. & W & NA & $w$ & $3,093.0$ & $W$ & $3,364.7$ & - & - & - & - & - & - \\
\hline December 1995 .............. & 243.7 & 249.4 & $w$ & $3,350.9$ & $w$ & $3,677.9$ & - & - & - & - & - & - \\
\hline January 1995 ................... & 235.8 & 241.2 & $w$ & $2,855.5$ & $w$ & $3,136.2$ & - & - & - & - & - & - \\
\hline Kansas & & & & & & & & & & & & \\
\hline January 1996 .................. & 531.7 & 551.4 & $W$ & $2,562.4$ & $W$ & $3,553.1$ & - & - & - & - & - & - \\
\hline December 1995 ............. & 583.7 & 592.7 & W & $2,756.8$ & W & $3,766.3$ & - & - & - & - & - & - \\
\hline January 1995 ,.................. & 637.2 & 673.5 & $\ddot{W}$ & $2,507.3$ & $\ddot{W}$ & $3,207.5$ & - & - & - & - & - & - \\
\hline Kentucky & & & & & & & & & & & & \\
\hline 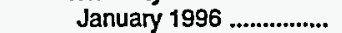 & 456.1 & 477.8 & W & w & - & $2,778.5$ & - & - & - & - & - & - \\
\hline December 1995 ............. & 531.8 & 552.6 & 319.8 & $w$ & $W$ & $2,945.9$ & - & - & - & - & $\leftrightarrow$ & - \\
\hline January 1995 ................. & 472.6 & 520.6 & W & W & - & $W$ & - & - & - & - & - & - \\
\hline Michigan & & & & & & & & & & & & \\
\hline January 1996 ................ & $2,743.2$ & $2,860.0$ & W & $5,502.3$ & $W$ & $9,105.0$ & - & - & - & - & - & - \\
\hline December 1995 ............. & $2,858.0$ & $2,969.8$ & $3,815.5$ & $5,852.3$ & - & $9,667.7$ & - & - & - & - & - & - \\
\hline January 1995 .................. & $2,680.9$ & $2,801.9$ & $\mathbf{W}$ & $5,225.7$ & $W$ & $8,674.2$ & - & - & - & - & - & - \\
\hline Minnesóta & & & & & & & & & & & & \\
\hline January 1996 ................. & 178.5 & 181.4 & 59.5 & $2,415.2$ & $W$ & w & $1,012.6$ & $1,036.3$ & $w$ & $1,477.0$ & - & w \\
\hline December 1995 ............ & 204.1 & 207.8 & 60.2 & $2,576.9$ & $W$ & $W$ & $1,016.1$ & $1,041.2$ & $w$ & $1,551.0$ & - & $w$ \\
\hline January 1995 ................. & 208.4 & 211.3 & 68.1 & $W$ & $\ddot{w}$ & $2,314.9$ & 969.8 & 992.1 & $w$ & W & - & $1,831.9$ \\
\hline Missouri & & & & & & & & & & & & \\
\hline January 1996 ................. & 776.9 & 786.3 & $w$ & $4,318.3$ & $w$ & $5,104.9$ & - & - & - & - & - & - \\
\hline December 1995 ............ & 816.9 & 825.4 & $\ddot{w}$ & $4,833.8$ & w & $5,682.6$ & - & - & - & - & - & - \\
\hline 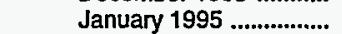 & 857.9 & 877.9 & w & $4,065.9$ & $\ddot{W}$ & $4,872.7$ & - & - & - & - & - & - \\
\hline
\end{tabular}

See footnotes at end of table. 
Table 44. Refiner Motor Gasoline Volumes by Formulation, Sales Type, PAD District, and State

(Thousand Gallons per Day) - Continued

\begin{tabular}{|c|c|c|c|c|c|c|c|c|c|c|c|c|}
\hline \multirow{3}{*}{$\begin{array}{l}\text { Geographic Area } \\
\text { Month }\end{array}$} & \multicolumn{6}{|c|}{ Reformulated } & \multicolumn{6}{|c|}{ All Formulations } \\
\hline & \multicolumn{2}{|c|}{ Sales to End Users } & \multicolumn{4}{|c|}{ Sales for Resale } & \multicolumn{2}{|c|}{ Sales to End Users } & \multicolumn{4}{|c|}{ Sales for Resale } \\
\hline & $\begin{array}{c}\text { Through } \\
\text { Retail } \\
\text { Outlets }\end{array}$ & Total ${ }^{\mathrm{a}}$. & DTW & Rack & Bulk & Total & $\begin{array}{c}\text { Through } \\
\text { Retail } \\
\text { Outlets }\end{array}$ & Total ${ }^{\mathrm{a}}$ & DTW & Rack & Bulk & Total \\
\hline \multicolumn{13}{|l|}{ Subdistrict IC } \\
\hline January 1996 & $\begin{array}{l}727.3 \\
822.8\end{array}$ & $\begin{array}{l}765.6 \\
867.7\end{array}$ & $\underset{w}{w}$ & $\begin{array}{l}1,903.6 \\
2,275.6\end{array}$ & $\begin{array}{l}w \\
W\end{array}$ & $\begin{array}{l}3,781.9 \\
4,480.6\end{array}$ & $\begin{array}{l}7,417.0 \\
7,914.2\end{array}$ & $\begin{array}{l}8,009.7 \\
8,551.0\end{array}$ & $\begin{array}{l}8,000.6 \\
8,553.6\end{array}$ & $\begin{array}{l}32,568.6 \\
35.537 .5\end{array}$ & $\begin{array}{r}1,122.8 \\
833.7\end{array}$ & $\begin{array}{l}41,691.9 \\
44,924.8\end{array}$ \\
\hline \\
\hline January 1996 & _ & _ & _ & - & _- & _ & $3,377.5$ & $3,640.0$ & $4,804.4$ & $9,170.0$ & 159.2 & $14,133.6$ \\
\hline December 1995 .............. & - & - & - & - & - & - & $3,387.4$ & $3,697.2$ & & $9,305.6$ & W & $14,181.0$ \\
\hline \multicolumn{12}{|l|}{ Georgla } & $13,556.6$ \\
\hline January 1996 ................... & - & - & - & - & - & - & $1,427.7$ & $1,530.5$ & 950.8 & $6,950.8$ & 512.9 & $8,414.5$ \\
\hline December 1995 ............ & - & - & - & - & - & - & $1,562.9$ & $1,660.9$ & $w$ & $7,364.6$ & $w$ & $8,671.4$ \\
\hline January 1995 .................... & - & - & - & - & - & - & $1,490.8$ & $1,594.5$ & 973.4 & $6,812.3$ & 338.5 & $8,124.3$ \\
\hline \multicolumn{13}{|l|}{ North Carolina } \\
\hline 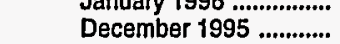 & - & $\overline{-}$ & $=$ & $\overline{-}$ & $\overline{-}$ & $\overline{-}$ & $\begin{array}{l}588.4 \\
667.8\end{array}$ & $\begin{array}{l}708.4 \\
783.8\end{array}$ & $\stackrel{165.8}{W}$ & $\begin{array}{l}7,602.7 \\
8,675.3\end{array}$ & $\begin{array}{l}328.2 \\
W\end{array}$ & $\begin{array}{l}8,097.7 \\
9,283.7\end{array}$ \\
\hline January 1995 ................... & - & - & - & $w$ & - & $w$ & 652.5 & 742.8 & $w$ & $7,514.0$ & $w$ & $8,043.2$ \\
\hline South Carolina & & & & & & & & & & & & \\
\hline January 1996 .................. & - & - & - & - & - & - & 733.3 & 776.1 & w & $3,650.1$ & $w$ & $3,747.3$ \\
\hline December 1995 ............ & - & - & - & - & - & - & 819.2 & 862.1 & w & $3,987.0$ & $w$ & $4,118.7$ \\
\hline January 1995 .......................... & - & - & - & - & - & - & 783.5 & 821.5 & W & $3,637.6$ & w & $3,773.1$ \\
\hline Virginia & & & & & & & & & & & & \\
\hline January 1996 & 727.3 & 765.6 & $w$ & $1,903.6$ & $w$ & $3,781.9$ & 860.5 & 900.2 & $1,938.5$ & $4,772.7$ & 70.5 & $6,781.7$ \\
\hline December 1995 ............. & 822.8 & 867.7 & $\ddot{w}$ & $2,275.6$ & $w$ & $4,480.6$ & 978.9 & $1,024.8$ & $2,326.8$ & $5,702.3$ & 24.7 & $8,053.8$ \\
\hline January 1995 ................. & 741.3 & $w$ & $\ddot{w}$ & $1,895.4$ & $\ddot{w}$ & $3,822.0$ & 872.7 & 922.3 & $2,010.9$ & $5,011.4$ & 106.2 & $7,128.5$ \\
\hline West Virginia & & & & & & & & & & & & \\
\hline January 1996 ................... & - & - & - & - & - & - & 429.6 & 454.6 & w & 422.4 & $w$ & 517.2 \\
\hline December 1995 .............. & - & - & - & - & - & - & 497.9 & 522.2 & w & 502.7 & w & 616.1 \\
\hline January 1995 .................. & - & $w$ & - & $w$ & - & $w$ & 417.3 & 447.0 & $\ddot{w}$ & 658.2 & $\ddot{w}$ & 797.0 \\
\hline PAD Distr & & & & & & & & & & & & \\
\hline January 1996 ................... & $2,437.0$ & $2,474.4$ & $3,779.5$ & $3,880.5$ & - & $7,660.0$ & $18,309.5$ & $18,872.0$ & $14,686.6$ & $54,638.5$ & $5,459.7$ & $74,784.7$ \\
\hline December 1995 ............. & $2,487.6$ & $2,529.2$ & $4,005.4$ & $4,154.3$ & - & $8,159.7$ & $19,451.0$ & $19,968.5$ & $15,805.7$ & $59,200.6$ & $6,486.1$ & $81,492.4$ \\
\hline January 1995 .................... & $2,289.9$ & $2,329.2$ & $3,706.6$ & $3,630.2$ & - & $7,336.9$ & $18,219.8$ & $18,844.5$ & $14,732.8$ & $51,353.4$ & $6,430.6$ & $72,516.9$ \\
\hline Illinois & & & & & & & & & & & & \\
\hline 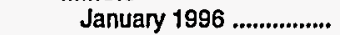 & $1,712.5$ & $1,722.7$ & w & $w$ & - & $4,799.6$ & $2,869.6$ & $2,889.2$ & $3,382.9$ & $5,802.8$ & 625.9 & $9,811.7$ \\
\hline December 1995 ........... & $1,756.5$ & $1,768.6$ & $w$ & $w$ & - & $5,035.7$ & $2,961.1$ & $2,982.2$ & $3,567.4$ & $6,125.9$ & $1,119.5$ & $10,812.8$ \\
\hline January 1995 .................. & $1,553.9$ & $1,562.6$ & $w$ & $w$ & - & $4,497.0$ & $2,633.8$ & $2,650.5$ & $3,375.1$ & $5,385.8$ & 970.9 & $9,731.8$ \\
\hline Indiana & & & & & & & & & & & & \\
\hline January 1996 ................... & 91.2 & 93.1 & 221.3 & 454.0 & - & 675.3 & $1,522.7$ & $1,557.4$ & $1,426.1$ & $3,695.7$ & 216.2 & $5,338.0$ \\
\hline December 1995 ............. & 92.3 & 94.9 & 234.5 & 516.8 & - & 751.3 & $1,608.3$ & 1.621 .3 & $1,548.1$ & $4,080.6$ & 223.7 & $5,852.5$ \\
\hline January 1995 & & 105.6 & 224.9 & 370.2 & - & 595.1 & $1,605.6$ & $1,621.7$ & $1,404.5$ & $3,552.2$ & 203.4 & $5,160.0$ \\
\hline lowa & & & & & & & & & & & & \\
\hline January 1996 .................. & - & - & - & - & - & - & w & NA & $w$ & $3,093.0$ & $w$ & $3,364.7$ \\
\hline December 1995 .............. & - & - & - & - & - & - & 243.7 & 249.4 & W & 0.9 & $w$ & $3,677.9$ \\
\hline January 1995 ................ & - & - & - & - & - & - & 235.8 & 241.2 & $w$ & $2,855.5$ & 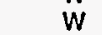 & $3,136.2$ \\
\hline Kansas & & & & & & & & & & & & \\
\hline January 1996 ................... & - & - & - & - & - & - & 531.7 & 551.4 & w & $2,562.4$ & w & $3,553.1$ \\
\hline December 1995 ................... & - & - & - & - & - & - & 583.7 & 592.7 & $\ddot{w}$ & $2,756.8$ & $w$ & $3,766.3$ \\
\hline January 1995 .................. & - & - & - & 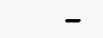 & - & - & 637.2 & 673.5 & $w$ & $2,507.3$ & $\ddot{w}$ & $3,207.5$ \\
\hline Kentucky & & & & & & & & & & & & \\
\hline January 1996 ................... & 240.3 & 262.7 & w & w & - & 821.9 & 696.3 & 740.5 & 462.6 & $3,137.7$ & - & $3,600,4$ \\
\hline December 1995 ............ & 245.8 & 269.9 & $w$ & $w$ & - & 938.5 & 777.6 & 822.5 & $w$ & $3,335.6$ & $w$ & $3,884.4$ \\
\hline January 1995 .................. & 237.2 & 258.7 & $w$ & $w$ & - & w & 709.9 & 779.3 & 580.6 & $3,105.5$ & - & $3,686.1$ \\
\hline Michlgan & & & & & & & & & & & & \\
\hline January 1996 .................. & - & - & - & - & - & - & $2,743.2$ & $2,860.0$ & w & $5,502.3$ & $w$ & $9,105.0$ \\
\hline December 1995 ............ & - & - & - & - & - & - & $2,858.0$ & $2,969.8$ & $3,815.5$ & & - & $9,667.7$ \\
\hline January 1995 ................... & - & - & - & - & - & - & $2,680.9$ & $2,801.9$ & $w$ & $5,225.7$ & $w$ & $8,674.2$ \\
\hline Minn & & & & & & & & & & & & \\
\hline January 1996 ................... & - & - & - & - & - & - & $1,191.2$ & $1,217.7$ & $w$ & $3,892.2$ & w & $4,571.4$ \\
\hline December 1995 ............ & - & - & - & - & - & - & $1,220.2$ & $1,248.9$ & $\ddot{w}$ & $4,127.9$ & $w$ & $5,058.4$ \\
\hline January 1995 .................. & - & - & - & - & - & - & $1,178.2$ & $1,203.4$ & $w$ & $3,488.0$ & $\ddot{w}$ & $4,146.8$ \\
\hline MIssouri & & & & & & & & & & & & \\
\hline January 1996 .................. & - & - & - & - & - & - & 776.9 & 786.3 & w & $4,318.3$ & $w$ & $5,104.9$ \\
\hline December 1995 .............. & - & - & - & - & - & - & 816.9 & 825.4 & $w$ & $4,833.8$ & $\ddot{w}$ & $5,682.6$ \\
\hline January 1995 ................... & - & - & - & - & - & - & 857.9 & 877.9 & $w$ & $4,065.9$ & $w$ & $4,872.7$ \\
\hline
\end{tabular}

See footnotes at end of table. 
Table 44. Refiner Motor Gasoline Volumes by Formulation, Sales Type, PAD District, and State

(Thousand Gallons per Day) - Continued

\begin{tabular}{|c|c|c|c|c|c|c|c|c|c|c|c|c|}
\hline \multirow{3}{*}{$\begin{array}{l}\text { Geographic Area } \\
\text { Month }\end{array}$} & \multicolumn{6}{|c|}{ Conventional } & \multicolumn{6}{|c|}{ Oxygenated } \\
\hline & \multicolumn{2}{|c|}{ Sales to End Users } & \multicolumn{4}{|c|}{ Sales for Resale } & \multicolumn{2}{|c|}{ Sales to End Users } & \multicolumn{4}{|c|}{ Sales for Resale } \\
\hline & $\begin{array}{c}\text { Through } \\
\text { Retail } \\
\text { Outlets }\end{array}$ & Totala & DTW & Rack & Bulk & Total & $\begin{array}{c}\text { Through } \\
\text { Retail } \\
\text { Outlets }\end{array}$ & Totala & DTW & Rack & Bulk & Total \\
\hline \multicolumn{13}{|l|}{ Nebraska } \\
\hline January 1996 & 160.0 & 161.7 & 165.3 & $w$ & $w$ & $1,911.4$ & - & $\rightarrow$ & - & - & - & - \\
\hline December 1995 ............ & 167.8 & 170.4 & 166.5 & W & W & $1,948.3$ & - & - & - & - & - & - \\
\hline \multicolumn{13}{|l|}{ North Dakota } \\
\hline January 1996 & $W$ & 8.4 & W & $w$ & - & 836.5 & - & - & - & - & - & - \\
\hline December 1995 ............. & $W$ & 8.5 & $W$ & W & - & 936.1 & - & - & - & - & - & - \\
\hline 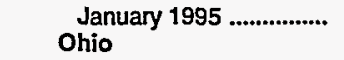 & 11.9 & 12.4 & $w$ & 695.8 & W & 784.8 & - & - & - & - & - & - \\
\hline January 1996 & $4,711.3$ & $4,924.1$ & $w$ & $4,685.1$ & $w$ & $7,809.5$ & - & - & - & - & - & - \\
\hline December 1995 .............. & $5,098.2$ & $5,294.9$ & W & $5,169.2$ & $w$ & $8,640.7$ & - & - & - & - & - & - \\
\hline \multicolumn{12}{|l|}{ Oklahoma } & - \\
\hline January 1996 ................. & 756.7 & 794.0 & $w$ & $3,415.7$ & $w$ & $6,347.6$ & - & - & - & - & - & - \\
\hline December 1995 .............. & 805.7 & 846.5 & W & $3,403.3$ & $w$ & $6,513.8$ & - & - & - & - & - & - \\
\hline $\begin{array}{l}\text { January } 1995 \\
\text { South Dakota }\end{array}$ & \multicolumn{7}{|c|}{ South Dakota } & & & - & - & - \\
\hline January 1996 & 19.3 & 19.3 & 41.9 & 909.5 & - & 951.4 & - & - & - & - & - & - \\
\hline December 1995 ............. & $W$ & 20.1 & 45.2 & 986.3 & - & $1,031.5$ & - & - & - & - & - & - \\
\hline 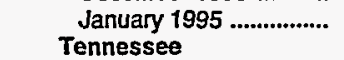 & 19.5 & \multicolumn{8}{|c|}{ Tennessee } & - & & - \\
\hline January 1996 & $1,325.7$ & $1,353.6$ & $w$ & $6,572.3$ & $w$ & $7,315.1$ & - & - & - & - & - & - \\
\hline December 1995 ............. & $1,495.0$ & $1,524.3$ & $W$ & $7,774.3$ & $w$ & $8,585.5$ & - & - & - & - & - & - \\
\hline January 1995 .................. & $1,361.2$ & $1,403.3$ & 609.5 & W & W & $7,057.1$ & - & - & - & - & - & - \\
\hline Wisconsin & & & & & & & & & & & & \\
\hline January 1996 .................. & 376.0 & 379.3 & $W$ & $3,618.0$ & - & W & - & - & - & $w$ & - & $\mathbf{W}$ \\
\hline December 1995 ............. & 393.4 & 396.0 & $W$ & $3,798.3$ & - & $w$ & - & - & - & $w$ & - & $w$ \\
\hline PAD District lil & & & & & & & & & & & & \\
\hline January 1996 & $3,464.3$ & $3,744.8$ & $W$ & $28,865.1$ & $\mathbf{W}$ & $43,262.5$ & 414.7 & 429.3 & 117.0 & 719.3 & - & 836.4 \\
\hline December 1995 ............. & $3,738.1$ & $4,019.1$ & $1,105.4$ & $30,543.3$ & $20,275.3$ & $51,924.0$ & 439.0 & 455.3 & 144.6 & 726.4 & - & 871.1 \\
\hline January 1995 .................. & $3,585.5$ & $3,878.7$ & $w$ & $28,204.1$ & $w$ & $41,549.1$ & 568.8 & 585.0 & $W$ & 620.0 & $W$ & 969.0 \\
\hline Alabama & & & & & & & & & & & & \\
\hline January 1996 & 340.8 & 383.6 & $w$ & $4,619.6$ & $w$ & $4,848.3$ & - & - & - & - & - & - \\
\hline December 1995 ............. & 372.3 & 415.1 & $\mathbf{W}$ & $4,882.6$ & $W$ & $5,125.7$ & - & - & - & - & - & - \\
\hline January 1995 .................. & 362.4 & 404.8 & $W$ & $4,509.7$ & $w$ & $4,749.2$ & - & - & - & - & - & - \\
\hline Arkansas & & & & & & & & & & & & \\
\hline January 1996 .................. & 247.9 & 257.3 & $W$ & $3,098.1$ & $w$ & $3,474.3$ & - & - & - & - & - & - \\
\hline December 1995 ............. & 287.2 & 296.7 & $w$ & $3,243,4$ & $w$ & $3,614.2$ & - & $\rightarrow$ & - & - & - & - \\
\hline January 1995 ................. & 280.7 & 288.3 & $W$ & W & - & $3,446.2$ & - & - & - & - & - & - \\
\hline $\begin{array}{l}\text { Louisiana } \\
\text { January } 1996 . . . . . . . . . . . . . . . .\end{array}$ & 652.2 & W & 469.8 & $4,007.5$ & $1,806.0$ & $6,283,2$ & - & - & - & - & - & - \\
\hline December 1995 ............. & 695.9 & $\ddot{w}$ & 505.5 & $4,373.3$ & W & $W$ & - & - & $\overline{-}$ & $\overline{-}$ & $\overline{-}$ & $\bar{z}$ \\
\hline 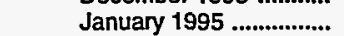 & 663.1 & 685.5 & 567.8 & $4,021.9$ & w & $w$ & - & - & - & - & $=$ & $\overline{-}$ \\
\hline Mississippi & & & & & & & & & & & & \\
\hline January 1996 & 95.7 & 117.5 & 17.9 & $w$ & $w$ & $3,466.1$ & - & - & - & - & - & - \\
\hline December 1995 ............ & 107.4 & 128.3 & 18.5 & $w$ & $w$ & $3,555.0$ & - & - & - & - & - & - \\
\hline January 1995 ................. & 100.4 & 123.9 & $w$ & $2,932.1$ & $\ddot{W}$ & $3,509.7$ & - & - & - & - & - & - \\
\hline New Mexico & & & & & & & & & & & & \\
\hline January 1996 ................ & NA & $w$ & $W$ & $w$ & - & $1,340.5$ & 286.0 & $W$ & $w$ & $w$ & - & 440.3 \\
\hline December 1995 ............ & 90.6 & W & 155.5 & $w$ & - & W & 307.3 & $w$ & $w$ & $w$ & - & $w$ \\
\hline January 1995 .................. & $w$ & 54.6 & 126.5 & $\ddot{w}$ & - & $w$ & $w$ & 276.0 & $w$ & $w$ & - & $\ddot{W}$ \\
\hline Texas & & & & & & & & & & & & \\
\hline January 1996 ................ & $2,032.4$ & $2,199.9$ & $w$ & $13,005.6$ & $w$ & $23,850.1$ & 128.8 & $w$ & $W$ & $w$ & - & 396.1 \\
\hline December 1995 ............ & $2,184.6$ & $2,351,1$ & W & $13,609.0$ & W & $30,994.9$ & 131.6 & $w$ & $\mathrm{~W}$ & $w$ & - & $W$ \\
\hline January 1995 .................. & $W$ & $2,321.5$ & W & $12,118.0$ & W & $22,056.4$ & $w$ & 309.0 & $W$ & $w$ & $w$ & $W$ \\
\hline PAD District IV & & & & & & & & & & & & \\
\hline January 1996 & 713.2 & 802.3 & 671.5 & $w$ & $w$ & $5,728.5$ & $1,530.6$ & $1,532.5$ & $w$ & $w$ & - & $1,746.7$ \\
\hline December 1995 ............ & 851.1 & 939.6 & W & $w$ & - & $5,695.8$ & $1,582.1$ & $1,585.6$ & $w$ & $w$ & - & $1,970.5$ \\
\hline January 1995 & 561.0 & 595.7 & $\ddot{w}$ & $\ddot{w}$ & $w$ & $5,319.9$ & $1,638.7$ & $1,646.4$ & $\ddot{w}$ & $\ddot{w}$ & - & $1,660.7$ \\
\hline Colorado & & & & & & & & & & & & \\
\hline January 1996 .................. & $W$ & 190.4 & $w$ & $w$ & $w$ & $1,237.0$ & $W$ & $1,529.4$ & $w$ & $w$ & - & $1,583.5$ \\
\hline December 1995 ............ & $w$ & 226.6 & $w$ & $w$ & - & $1,192.7$ & $w$ & $1,576.4$ & $w$ & $w$ & - & $1,653.1$ \\
\hline January 1995 .................. & $W$ & 72.8 & $w$ & $w$ & - & $1,105.7$ & W & $1,643.2$ & w & $w$ & - & $1,485.3$ \\
\hline
\end{tabular}

See footnotes at end of table. 
Table 44. Refiner Motor Gasoline Volumes by Formulation, Sales Type, PAD District, and State

(Thousand Gallons per Day) - Continued

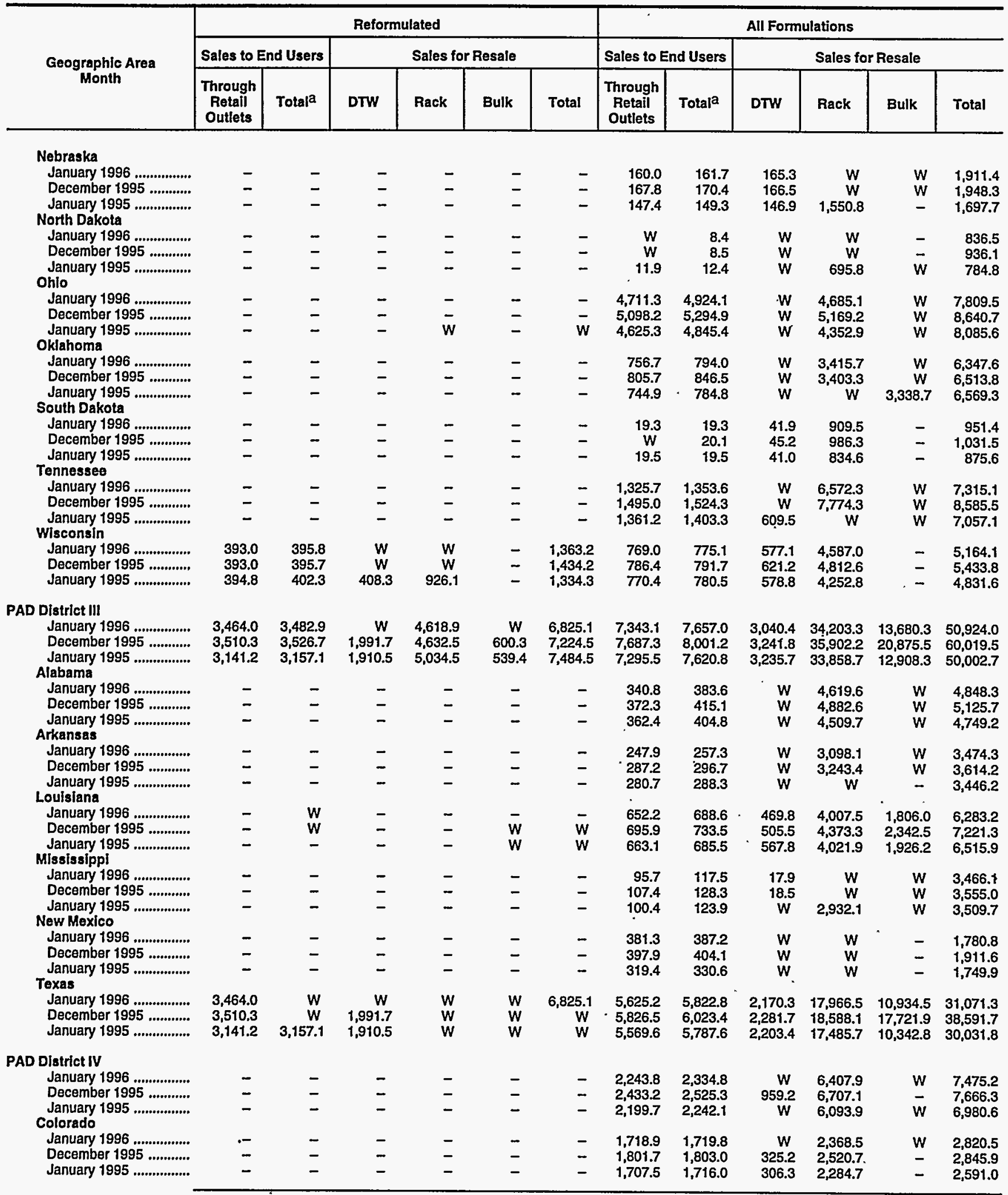

See footnotes at end of table. 
Table 44. Refiner Motor Gasoline Volumes by Formulation, Sales Type, PAD District, and State

(Thousand Gallons per Day) - Continued

\begin{tabular}{|c|c|c|c|c|c|c|c|c|c|c|c|c|}
\hline \multirow{3}{*}{$\begin{array}{l}\text { Geographic Area } \\
\text { Month }\end{array}$} & \multicolumn{6}{|c|}{ Conventional } & \multicolumn{6}{|c|}{ Oxygenated } \\
\hline & \multicolumn{2}{|c|}{ Sales to End Users } & \multicolumn{4}{|c|}{ Sales for Resale } & \multicolumn{2}{|c|}{ Sales to End Users } & \multicolumn{4}{|c|}{ Sales for Resale } \\
\hline & $\begin{array}{c}\text { Through } \\
\text { Retail } \\
\text { Outlets }\end{array}$ & Totala & DTW & Rack & Bulk & Total & $\begin{array}{c}\text { Through } \\
\text { Retail } \\
\text { Outlets }\end{array}$ & Totala & DTW & Rack & Bulk & Total \\
\hline \multicolumn{13}{|l|}{ Idaho } \\
\hline January 1996 ................. & $w$ & 63.6 & 183.8 & $1,038.0$ & - & $1,221.8$ & - & - & - & - & - & - \\
\hline December 1995 ............. & $\ddot{w}$ & 70.8 & 192.1 & 997.9 & - & $1,190.1$ & - & - & - & - & - & - \\
\hline January 1995 .................. & w & 52.2 & 132.5 & 922.5 & - & $1,054.9$ & - & - & - & - & - & - \\
\hline January 1996 ................. & $w$ & $w$ & $w$ & $w$ & - & $1,006.2$ & - & w & $w$ & $w$ & - & 64.2 \\
\hline December 1995 ............. & $w$ & $\ddot{w}$ & $\ddot{w}$ & $\ddot{w}$ & - & $1,059.1$ & - & $W$ & $\ddot{w}$ & W & - & 74.9 \\
\hline January 1995 ................... & $w$ & W & - & $\ddot{w}$ & $w$ & 916.0 & - & $w$ & - & W & - & 59.9 \\
\hline \multicolumn{13}{|l|}{ Utah } \\
\hline January 1996 & $w$ & $w$ & $W$ & $w$ & - & $1,701.1$ & $w$ & W & $w$ & W & - & 99.0 \\
\hline December 1995 ............. & w & $w$ & $w$ & $w$ & - & $1,667.9$ & $w$ & w & $\ddot{w}$ & $\ddot{W}$ & - & 242.4 \\
\hline 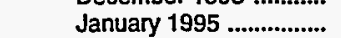 & $w$ & w & $W$ & $w$ & $w$ & $1,675.9$ & $w$ & w & w & $W$ & - & 115.5 \\
\hline \multicolumn{13}{|l|}{ wyoming } \\
\hline January 1996 ................. & 60.2 & 62.8 & $w$ & $w$ & - & 562.4 & - & - & - & - & - & - \\
\hline December 1995 ............. & 68.4 & 70.9 & $W$ & $\ddot{w}$ & - & 586.1 & - & - & - & - & - & - \\
\hline January $1995 \ldots . . . . . . . . . . . . .$. & 55.3 & 58.9 & $W$ & 556.1 & W & 567.4 & - & - & - & - & - & - \\
\hline \multicolumn{13}{|l|}{ PAD District V } \\
\hline January 1996 & 780.1 & 881.2 & $w$ & $w$ & $2,261.9$ & $8,394.5$ & $5,186.9$ & $5,288.4$ & $12,567.7$ & $\mathbf{w}$ & $w$ & $20,315.5$ \\
\hline December 1995 .............. & 815.8 & 919.5 & $1,462.5$ & $4,901.8$ & $2,325.5$ & $8,689.9$ & $5,854.9$ & $5,962.3$ & $13,047.9$ & $7,110.6$ & $1,271.0$ & $21,429.5$ \\
\hline January 1995 .................. & 628.3 & 716.4 & W & W & $1,941.1$ & $7,604.7$ & $4,704.0$ & $4,776.7$ & $11,892.6$ & W & $W$ & $19,265.4$ \\
\hline \multicolumn{13}{|l|}{ Alaska } \\
\hline January 1996 ................. & $w$ & 218.5 & $W$ & $w$ & $w$ & 225.8 & W & 27.9 & $w$ & $\mathbf{w}$ & $w$ & 145.0 \\
\hline December 1995 ............ & $W$ & 224.8 & $W$ & W & $W$ & 211.2 & $W$ & 27.5 & $\ddot{W}$ & $w$ & - & 133.7 \\
\hline January 1995 .................. & W & $w$ & 20.5 & 169.8 & - & 190.3 & w & $w$ & $\ddot{w}$ & w & - & 114.3 \\
\hline \multicolumn{13}{|l|}{ Arizona } \\
\hline January 1996 ................ & 93.0 & 103.4 & $W$ & 870.7 & $w$ & $1,268.9$ & $1,178.8$ & $1,186.7$ & $w$ & 597.4 & $w$ & $2,116.0$ \\
\hline December 1995 ............. & 76.4 & 84.6 & 222.5 & 857.6 & 192.3 & $1,272.4$ & $1,347.6$ & $1,354.6$ & $1,552.5$ & 616.3 & - & $2,168.8$ \\
\hline January 1995 & 74.5 & 85.7 & $W$ & 836.0 & $W$ & $1,185.2$ & 939.4 & 947.0 & $w$ & $w$ & - & $1,951.0$ \\
\hline \multicolumn{13}{|l|}{ California } \\
\hline January 1996 ................. & - & - & - & $w$ & $W$ & $1,233.9$ & $2,444.0$ & $2,507.8$ & $W$ & $4,290.2$ & $w$ & $12,512.1$ \\
\hline December 1995 ............. & - & - & - & $w$ & $W$ & $1,687.5$ & $2,705.2$ & $2,771.5$ & $7,471.0$ & $w$ & $w$ & $13,102.3$ \\
\hline January 1995 ................. & - & - & - & 125.5 & $1,113.2$ & $1,238.6$ & $2,454.4$ & $2,491.0$ & $6,808.4$ & $W$ & $w$ & $12,131.4$ \\
\hline \multicolumn{13}{|l|}{ Hawaii } \\
\hline January 1996 ................. & 200.4 & 269.0 & 606.2 & 104.2 & - & 710.4 & - & - & - & - & - & - \\
\hline December 1995 ............ & 192.8 & 264.6 & $W$ & $W$ & - & 716.5 & - & - & - & - & - & - \\
\hline January 1995 ................... & 169.9 & 235.0 & 597.4 & 88.7 & - & 686.1 & - & - & - & - & - & - \\
\hline \multicolumn{13}{|l|}{ Nevada } \\
\hline January 1996 ................. & $w$ & 8.5 & $w$ & $w$ & $w$ & 702.9 & $w$ & 129.0 & $w$ & $w$ & - & $1,154.6$ \\
\hline December 1995 .............. & $w$ & 17.5 & 84.9 & 376.4 & 184.1 & 645.4 & $W$ & 151.8 & $W$ & 341.1 & $w$ & $1,242.7$ \\
\hline January 1995 .................. & $w$ & $w$ & 41.3 & W & W & 519.2 & W & W & 788.9 & $W$ & $\ddot{w}$ & $1,202.0$ \\
\hline \multicolumn{13}{|l|}{ Oregon } \\
\hline January 1996 & 88.0 & 91.0 & 200.6 & $1,139.2$ & 516.8 & $1,856.6$ & 366.9 & 373.1 & 835.1 & 557.1 & - & $1,392.2$ \\
\hline December 1995 ............. & 112.7 & 116.3 & 228.1 & $1,140.4$ & 265.5 & $1,633.9$ & 389.7 & 396.4 & 904.2 & 596.2 & - & $1,500.4$ \\
\hline January 1995 .................. & 89.2 & 94.3 & $W$ & $1,033.7$ & $W$ & $1,783.6$ & 288.2 & 294.0 & 777.7 & 434.3 & - & $1,212.0$ \\
\hline Washington & & & & & & 23050 & & & & & & \\
\hline $\begin{array}{l}\text { January } 1996 \\
\text { December } 1995\end{array}$ & $\begin{array}{l}185.5 \\
204.5\end{array}$ & $\begin{array}{l}190.9 \\
211.7\end{array}$ & $\begin{array}{l}248.4 \\
280.3\end{array}$ & $\begin{array}{l}1,952.2 \\
2,193.9\end{array}$ & $\begin{array}{r}195.5 \\
48.8\end{array}$ & $\begin{array}{l}2,396.0 \\
2,523.0\end{array}$ & $\begin{array}{l}1,058.4 \\
1,252.5\end{array}$ & $\begin{array}{l}1,063.8 \\
1,260.5\end{array}$ & $\begin{array}{l}2,015.5 \\
2,171.0\end{array}$ & $\begin{array}{r}980.1 \\
1110.6\end{array}$ & $\overline{-}$ & $\begin{array}{l}2,995.6 \\
3,281.6\end{array}$ \\
\hline 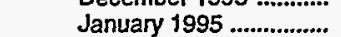 & 134.6 & 136.3 & $W$ & $W$ & $W$ & $2,001.6$ & 897.5 & 901.3 & $W$ & W & - & $2,654.6$ \\
\hline
\end{tabular}

See footnotes at end of table. 
Table 44. Refiner Motor Gasoline Volumes by Formulation, Sales Type,

PAD District, and State

(Thousand Gallons per Day) - Continued

\begin{tabular}{|c|c|c|c|c|c|c|c|c|c|c|c|c|}
\hline \multirow{3}{*}{$\begin{array}{l}\text { Geographic Area } \\
\text { Month }\end{array}$} & \multicolumn{6}{|c|}{ Reformulated } & \multicolumn{6}{|c|}{ All Formulations } \\
\hline & \multicolumn{2}{|c|}{ Sales to End Users } & \multicolumn{4}{|c|}{ Sales for Resale } & \multicolumn{2}{|c|}{ Sales to End Users } & \multicolumn{4}{|c|}{ Sales for Resale } \\
\hline & $\begin{array}{c}\text { Through } \\
\text { Retail } \\
\text { Outlets }\end{array}$ & Totafa & DTW & Rack & Bulk & Total & $\begin{array}{c}\text { Through } \\
\text { Retail } \\
\text { Outlets }\end{array}$ & Totala & DTW & Rack & Bulk & Total \\
\hline \multicolumn{13}{|l|}{ Idaho } \\
\hline January 1996 ................... & - & - & - & - & - & - & $w$ & 63.6 & 183.8 & $1,038.0$ & - & $1,221.8$ \\
\hline December 1995 .............. & - & - & - & - & - & - & w & 70.8 & 192.1 & 997.9 & - & $1,190.1$ \\
\hline January 1995 .................... & - & - & - & - & - & - & w & 52.2 & 132.5 & 922.5 & - & $1,054.9$ \\
\hline \multicolumn{13}{|l|}{ Montana } \\
\hline January 1996 ................... & - & - & - & - & - & - & W & 16.3 & W & w & - & $1,070.4$ \\
\hline December 1995 .............. & - & - & - & - & - & - & $w$ & 15.6 & w & w & - & $1,134.0$ \\
\hline January 1995 .................. & - & - & - & - & - & - & W & 21.1 & - & w & w & 975.8 \\
\hline \multicolumn{13}{|l|}{ Utah } \\
\hline January 1996 .................. & - & - & - & - & - & - & 404.2 & 472.3 & 408.9 & $1,391.2$ & - & $1,800.1$ \\
\hline December 1995 .............. & - & - & - & - & - & - & 495.1 & 565.0 & 413.4 & $1,496.8$ & - & $1,910.3$ \\
\hline $\begin{array}{c}\text { January } 1995 \text {.................... } \\
\text { Wyoming }\end{array}$ & - & - & - & - & - & - & 372.2 & 393.9 & 429.3 & W & $w$ & $1,791.5$ \\
\hline January 1996 ................... & - & - & - & - & - & - & 60.2 & 62.8 & w & w & - & 562.4 \\
\hline December 1995 .............. & - & - & - & - & - & - & 68.4 & 70.9 & $w$ & w & - & 586.1 \\
\hline January 1995 .................. & - & - & - & - & - & - & 55.3 & 58.9 & $w$ & 556.1 & w & 567.4 \\
\hline \multicolumn{13}{|l|}{ PAD District V } \\
\hline January 1996 ................... & $3,990.0$ & $4,023.2$ & w & W & $w$ & $16,539.7$ & $9,957.0$ & $10,192.8$ & $w$ & $13,879.1$ & w & $45,249.7$ \\
\hline December 1995 ............. & $4,407.2$ & $4,444.2$ & $13,619.0$ & $2,025.3$ & $1,525.1$ & $17,169.4$ & $11,077.9$ & $11,326.0$ & $28,129.5$ & $14,037.7$ & $5,121.6$ & $47,288.8$ \\
\hline January $1995 \ldots . . . . . . . . . . . . . . .$. & $3,654.5$ & $3,686.8$ & $12,084.3$ & $2,402.0$ & $1,040.1$ & $15,526.4$ & $8,986.8$ & $9,180.0$ & $w$ & $13,136.5$ & $w$ & $42,396.4$ \\
\hline \multicolumn{13}{|l|}{ Alaska } \\
\hline January 1996 ................... & - & - & - & - & - & - & 222.0 & 246.4 & w & 232.6 & $w$ & 370.8 \\
\hline December 1995 ............. & - & - & - & - & - & - & 228.2 & 252.3 & $w$ & 221.6 & w & 344.9 \\
\hline January 1995 ................... & - & - & - & - & - & - & 161.9 & 175.1 & $w$ & $w$ & - & 304.5 \\
\hline \multicolumn{13}{|l|}{ Arizona } \\
\hline January 1996 ................... & - & - & - & - & - & - & $1,271.8$ & $1,290.1$ & $1,717.9$ & $1,468.2$ & 198.9 & $3,385.0$ \\
\hline December 1995 .............. & - & - & - & - & - & - & $1,424.0$ & $1,439.2$ & $1,775.1$ & $1,473.8$ & 192.3 & $3,441.2$ \\
\hline \multirow{2}{*}{\multicolumn{13}{|c|}{ California }} \\
\hline & & & & & & & & & & & & \\
\hline January 1996 .................. & $3,990.0$ & $4,023.2$ & w & $w$ & $w$ & $16,539.7$ & $6,434.0$ & $6,531.0$ & $20,469.0$ & $6,726.7$ & $3,090.0$ & $30,285.7$ \\
\hline December 1995 ............. & $4,407.2$ & $4,444.2$ & $13,619.0$ & $2,025.3$ & $1,525.1$ & $17,169.4$ & $7,112.4$ & $7,215.8$ & $21,090.1$ & $6,495.2$ & $4,373.9$ & $31,959.2$ \\
\hline \multirow{2}{*}{\multicolumn{13}{|c|}{ Hawail }} \\
\hline & & & & & & & & & & & & \\
\hline January 1996 .................... & - & - & - & - & - & - & 200.4 & 269.0 & 606.2 & 104.2 & - & 710.4 \\
\hline December 1995 .............. & - & - & - & - & - & - & 192.8 & 264.6 & 627.9 & 88.5 & - & 716.5 \\
\hline January 1995 ................... & - & - & - & - & - & - & 169.9 & 235.0 & 597.4 & 88.7 & - & 686.1 \\
\hline \multicolumn{13}{|l|}{ Nevada } \\
\hline January 1996 ................... & - & - & - & - & - & - & 130.0 & 137.5 & 916.7 & 718.8 & 222.0 & $1,857.5$ \\
\hline December 1995 ........... & - & - & - & - & - & - & 161.1 & 169.3 & $w$ & 717.5 & $w$ & $1,888.1$ \\
\hline January 1995 . & - & - & - & - & 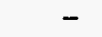 & - & 122.6 & 133.4 & 830.2 & W & W & $1,721.2$ \\
\hline \multicolumn{13}{|l|}{ Oregon } \\
\hline January 1996 ................... & - & - & - & - & - & - & 454.9 & 464.1 & $1,035.7$ & $1,696.3$ & 516.8 & $3,248.8$ \\
\hline December 1995 ............. & - & - & - & - & - & - & 502.4 & 512.7 & $1,132.3$ & $1,736.5$ & 265.5 & $3,134.3$ \\
\hline \multirow{2}{*}{\multicolumn{13}{|c|}{ Washington }} \\
\hline & & & & & & & & & & & & \\
\hline January $1996 \ldots . . . . . . . . . . . .$. & - & - & - & - & - & - & $1,243.9$ & $1,254.8$ & $2,263.8$ & $2,932.3$ & 195.5 & $5,391,6$ \\
\hline December 1995 ............. & - & - & - & - & - & - & $1,457.0$ & $1,472.2$ & $2,451.3$ & $3,304.5$ & 48.8 & $5,804.6$ \\
\hline January 1995 .................. & - & - & - & - & - & - & $1,032.1$ & $1,037.6$ & $w$ & $2,435.6$ & $w$ & $4,656.3$ \\
\hline
\end{tabular}

Dash $(-)=$ No data reported.

$N A=$ Not avallable.

$W=$ Witheld to avold disclosure of individual company data.

a Inciudes sales through retall outlets as well as all direct sales to end users that were not made through company-operated retail outlets, e.g., sales to agricultural customers, commercial sales, and industrial sales.

Notes: Motor gasoline averages and totals prior to October 1993 include leaded gasoline.

Notes: Values shown for the current month are preliminary. Values shown for previous months are revised. Data are final upon publication in the Petroleum Marketing Annual.

Source: Energy Information Administration Form ElA-782A, "Refiners'/Gas Plant Operators' Monthly Petroleum Product Sales Report." 
Table 45. Refiner Volumes of Aviation Fuels, Kerosene, No. 1 Distillate, and Propane (Consumer Grade) by PAD District and State

(Thousand Gallons per Day)

\begin{tabular}{|c|c|c|c|c|c|c|c|c|c|c|}
\hline \multirow{2}{*}{$\begin{array}{c}\text { Geographic Area } \\
\text { Month }\end{array}$} & \multicolumn{2}{|c|}{ Aviation Gasoline } & \multicolumn{2}{|c|}{$\begin{array}{l}\text { Kerosene-Type } \\
\text { Jet Fuel }\end{array}$} & \multicolumn{2}{|c|}{ Kerosene } & \multicolumn{2}{|c|}{ No. 1 Distillate } & \multicolumn{2}{|c|}{$\begin{array}{c}\text { Propane } \\
\text { (Consumer Grade) }\end{array}$} \\
\hline & $\begin{array}{l}\text { Sales to } \\
\text { End Users }\end{array}$ & \begin{tabular}{c|c} 
Sales for \\
Resale
\end{tabular} & $\begin{array}{c}\text { Sales to } \\
\text { End Users }\end{array}$ & $\begin{array}{l}\text { Sales for } \\
\text { Resale }\end{array}$ & \begin{tabular}{|c|} 
Sales to \\
End Users
\end{tabular} & $\begin{array}{l}\text { Sales for } \\
\text { Resale }\end{array}$ & $\begin{array}{c}\text { Sales to } \\
\text { End Users }\end{array}$ & $\begin{array}{l}\text { Sales for } \\
\text { Resale }\end{array}$ & $\begin{array}{c}\text { Sales to } \\
\text { End Users }\end{array}$ & $\begin{array}{l}\text { Sales for } \\
\text { Resale }\end{array}$ \\
\hline \multicolumn{11}{|l|}{ United States } \\
\hline January 1996 ............. & 158.2 & 405.7 & $46,703.6$ & $12,447.7$ & 789.0 & $6,174.5$ & 648.4 & $4,283.7$ & $3,323.1$ & $44,033.4$ \\
\hline December 1995 ......... & 168.5 & 409.4 & $44,598.5$ & $12,705.2$ & 725.3 & $5,622.9$ & 539.0 & $3,883.2$ & $4,110.1$ & $41,617.1$ \\
\hline January 1995 .............. & 154.8 & 419.0 & $45,446.7$ & $8,843.9$ & 604.1 & $4,505.7$ & 616.1 & $3,730.7$ & $3,891.0$ & $37,878.8$ \\
\hline \multicolumn{11}{|l|}{ PAD District I } \\
\hline January 1996 ............. & 55.4 & 82.8 & $12,367.6$ & $2,394.5$ & 332.3 & $4,138.7$ & w & 81.1 & 97.5 & $7,113.1$ \\
\hline December 1995 ........ & 58.4 & 83.7 & $12,881.2$ & $2,902.6$ & 341.3 & $3,647.3$ & w & 56.6 & 83.4 & $6,677.8$ \\
\hline \multirow{2}{*}{\multicolumn{11}{|c|}{ Subdistrict IA }} \\
\hline & & & & & & & & & & \\
\hline January 1996 .............. & w & 6.4 & $1,136.3$ & 158.2 & 33.7 & 373.0 & w & 11.3 & w & 107.7 \\
\hline December 1995 ........ & w & w & 989.3 & 142.2 & 18.2 & 350.0 & $w$ & 8.1 & $w$ & 103.3 \\
\hline January 1995 ............. & $w$ & $\mathbf{w}$ & $1,079.5$ & 149.2 & 10.4 & 251.0 & $w$ & W & $w$ & 143.1 \\
\hline \multicolumn{11}{|l|}{ Connecticut } \\
\hline January 1996 ............. & w & 1.3 & 323.0 & 13.1 & w & $w$ & w & 3.6 & w & w \\
\hline December 1995 ........ & w & w & 216.8 & 13.2 & w & $w$ & $w$ & 3.5 & $w$ & 34.0 \\
\hline January 1995 .............. & W & $\mathbf{w}$ & 350.6 & 26.0 & w & 53.9 & 3.1 & W & $w$ & 21.2 \\
\hline \multicolumn{11}{|l|}{ Maine } \\
\hline January 1996 ............. & $w$ & - & w & $w$ & w & 196.1 & $w$ & $w$ & - & 18.4 \\
\hline December 1995 ........ & W & - & $w$ & 51.2 & w & 158.7 & 0.2 & 2.1 & - & 19.2 \\
\hline January 1995 ............. & $w$ & - & $w$ & $w$ & w & 130.2 & $w$ & 4.2 & - & $w$ \\
\hline Massachusetts & & & & & & & & & & \\
\hline January 1996 ............. & $w$ & W & $w$ & 48.8 & 7.7 & 69.5 & $w$ & w & $w$ & 8.9 \\
\hline December 1995 ........ & $w$ & w & 609.3 & 64.5 & 11.2 & 80.4 & $w$ & $w$ & $w$ & $w$ \\
\hline January 1995 ............. & $w$ & $w$ & 601.2 & 49.7 & 6.0 & 47.0 & NA & $w$ & - & 27.4 \\
\hline New Hampshire & & & & & & & & & & \\
\hline January 1996 ............. & w & w & w & 2.5 & 1.9 & $w$ & w & w & $w$ & 22.5 \\
\hline December 1995 ........ & $w$ & w & $w$ & 5.8 & w & $w$ & w & w & $w$ & 18.7 \\
\hline January 1995 .............. & $w$ & w & . 22.8 & 2.0 & W & W & w & $w$ & $w$ & 49.2 \\
\hline Rhode Iśland & & & & & & & & & & \\
\hline January 1996 ............. & $w$ & $w$ & 48.6 & w & - & w & $w$ & w & - & $w$ \\
\hline Decernber 1995 ........ & $w$ & W & 46.1 & $w$ & - & $w$ & $\ddot{w}$ & $w$ & - & $\ddot{w}$ \\
\hline January 1995 ............. & $w$ & $w$ & 46.0 & 2.7 & - & w & - & w & - & $\mathbf{w}$ \\
\hline Vermont & & & & & & & & 1 & & \\
\hline January 1996 ............. & w & - & 10.5 & 1.4 & - & w & $\mathbf{w}$ & w & - & 21.1 \\
\hline December 1995 ......... & $w$ & W & 9.5 & $w$ & - & w & w & w & - & 24.3 \\
\hline January 1995 .............. & $\mathbf{w}$ & - & $w$ & $w$ & - & $w$ & $w$ & $\dot{w}$ & - & 30.7 \\
\hline Subdistrict IB & & & & & & & & & & \\
\hline January 1996 ............. & $w$ & 14.6 & $5,551.5$ & $1,265.9$ & 241.7 & $1,924.5$ & 24.6 & 62.0 & $w$ & $2,311.5$ \\
\hline December 1995 ........ & $w$ & $w$ & $6,243.3$ & $1,902.2$ & 276.7 & $1,731.5$ & 19.3 & 41.9 & $w$ & $2,227.7$ \\
\hline January 1995 ............. & $w$ & w & $6,038.2$ & 847.9 & 148.5 & $1,044.4$ & 22.1 & 56.6 & $w$ & $1,625,8$ \\
\hline Delaware & & & & & & & & & & \\
\hline January 1996 ............. & w & w & w & $\mathbf{w}$ & w & w & w & w & - & $w$ \\
\hline December 1995 ........ & w & w & w & $w$ & $w$ & $w$ & - & - & - & $w$ \\
\hline January 1995 ............. & $w$ & w & 1.5 & w & $w$ & W & - & - & - & $w$ \\
\hline District of Columbia & & & & & & & & & & \\
\hline January 1996 .............. & - & - & - & - & - & - & - & - & - & - \\
\hline December 1995 ........ & w & - & - & - & - & - & - & - & - & - \\
\hline January 1995 ............. & - & - & - & - & - & w & - & _ & - & - \\
\hline Maryland & & & & & & & & & & \\
\hline January 1996 ............ & w & w & w & w & $w$ & $w$ & 5.1 & 5.8 & - & w \\
\hline December 1995 ........ & w & w & w & W & W & $w$ & 3.2 & $w$ & - & $w$ \\
\hline January 1995 .............. & $w$ & $w$ & 330.3 & W & $w$ & 108.0 & 6.2 & w & - & $w$ \\
\hline New Jersey & & & & & & & & & & \\
\hline January 1996 ............. & w & 5.8 & $3,646.6$ & 794.1 & 87.7 & 661.5 & w & w & w & 699.8 \\
\hline December 1995 ......... & w & $w$ & $4,181.9$ & $1,341.4$ & w & 500.4 & $w$ & $w$ & - & 704.9 \\
\hline 1995 .............. & W & 9.5 & $4,267.8$ & 523.5 & 34.2 & 158.9 & $w$ & $w$ & $w$ & 249.6 \\
\hline New York & & & & & & & & & & \\
\hline January 1996 .............. & w & $w$ & 754.2 & 177.5 & 73.2 & 481.6 & $w$ & 27.9 & w & 389.7 \\
\hline December 1995 ......... & $w$ & w & 732.9 & 166.7 & 66.7 & 401.1 & w & 19.2 & - & 415.3 \\
\hline January 1995 ............. & w & 5.6 & 489.9 & 96.1 & 59.4 & 308.6 & w & 25.8 & - & 352.5 \\
\hline Pennsylvania & & & & & & & & & & \\
\hline January $1996 . . . . . . . . . . . .$. & 3.6 & 3.7 & 799.5 & 260.9 & 43.4 & & 8.4 & 25.0 & w & 804.1 \\
\hline December 1995 ........ & w & 1.6 & 913.0 & 375.2 & 43.6 & 679.4 & 5.7 & 17.0 & $w$ & 749.9 \\
\hline January 1995 ....... & w & w & 948.6 & 217.1 & 45.5 & 468.5 & 7.8 & 22.7 & w & 710.5 \\
\hline
\end{tabular}

See footnotes at end of table. 
Table 45. Refiner Volumes of Aviation Fuels, Kerosene, No. 1 Distillate, and Propane (Consumer Grade) by PAD District and State

(Thousand Gallons per Day) - Continued

\begin{tabular}{|c|c|c|c|c|c|c|c|c|c|c|}
\hline \multirow{2}{*}{$\begin{array}{l}\text { Geographic Area } \\
\text { Month }\end{array}$} & \multicolumn{2}{|c|}{ Aviation Gasoline } & \multicolumn{2}{|c|}{$\begin{array}{l}\text { Kerosene-Type } \\
\text { Jet Fuel }\end{array}$} & \multicolumn{2}{|c|}{ Kerosene } & \multicolumn{2}{|c|}{ No. 1 Distillate } & \multicolumn{2}{|c|}{$\begin{array}{c}\text { Propane } \\
\text { (Consumer Grade) }\end{array}$} \\
\hline & $\begin{array}{l}\text { Sales to } \\
\text { End Users }\end{array}$ & $\begin{array}{l}\text { Sales for } \\
\text { Resale }\end{array}$ & $\begin{array}{c}\text { Sales to } \\
\text { End Users }\end{array}$ & $\begin{array}{l}\text { Sales for } \\
\text { Resale }\end{array}$ & $\begin{array}{l}\text { Sales to } \\
\text { End Users }\end{array}$ & $\begin{array}{l}\text { Sales for } \\
\text { Resale }\end{array}$ & $\begin{array}{l}\text { Sales to } \\
\text { End Users }\end{array}$ & $\begin{array}{l}\text { Sales for } \\
\text { Resale }\end{array}$ & $\begin{array}{l}\text { Sales to } \\
\text { End Users }\end{array}$ & $\begin{array}{l}\text { Sales for } \\
\text { Resale }\end{array}$ \\
\hline \multicolumn{11}{|l|}{ Subdistrict IC } \\
\hline January 1996 .............. & 37.9 & 61.8 & $5,679.8$ & 970.4 & 56.9 & $1,841.2$ & 7.0 & 7.8 & 87.5 & $4,693.9$ \\
\hline December 1995 ........ & 40.0 & 59.2 & $5,648.6$ & 858.3 & 46.3 & $1,565.8$ & 4.4 & 6.6 & 74.1 & $4,346.7$ \\
\hline January 1995 ............. & 46.9 & 69.7 & $6,124.9$ & 901.4 & 57.3 & $1,456.0$ & 7.0 & $w$ & 96.7 & $4,214.5$ \\
\hline \multicolumn{11}{|l|}{ Florida } \\
\hline January 1996 ............. & 24.4 & 33.2 & $2,873.8$ & 269.0 & 9.1 & 113.2 & - & - & w & $1,129.5$ \\
\hline December 1995 ........ & 25.0 & 31.9 & $2,788.1$ & 212.8 & 8.7 & 112.9 & - & - & $w$ & 868.2 \\
\hline January 1995 .............. & 32.0 & 38.4 & $3,249.0$ & 381.8 & 8.3 & 116.4 & - & - & $\ddot{w}$ & 915.9 \\
\hline \multicolumn{11}{|l|}{ Georgia } \\
\hline January 1996 .............. & 3.4 & 13.0 & $1,523.7$ & 463.2 & 10.8 & 111.4 & w & - & $w$ & 846.4 \\
\hline December 1995 ........ & 4.3 & 10.8 & $1,507.9$ & 431.5 & 9.0 & 82.8 & w & - & $\ddot{w}$ & 826.2 \\
\hline January 1995 .............. & 4.9 & 12.5 & $1,395.4$ & 344.5 & 8.6 & 98.3 & - & - & $w$ & 888.2 \\
\hline \multicolumn{11}{|l|}{ North Carolina } \\
\hline January 1996 ............. & w & 10.3 & 414.7 & 156.2 & 8.9 & 762.5 & 2.3 & w & 48.7 & $1,248.4$ \\
\hline December 1995 ........ & W & 11.5 & 452.2 & 139.2 & 5.7 & 653.4 & $w$ & $w$ & 41.7 & $1,229.8$ \\
\hline January 1995 ................. & $w$ & $w$ & 366.7 & 86.0 & 11.9 & 579.9 & $w$ & - & 44.0 & $1,130.6$ \\
\hline \multicolumn{11}{|l|}{ South Carolina } \\
\hline January 1996 .............. & 2.3 & 3.9 & w & $w$ & 8.7 & 354.7 & - & NA & $w$ & 713.3 \\
\hline December 1995 ........ & 2.3 & $w$ & w & $w$ & 6.9 & 299.4 & - & $w$ & $w$ & 715.2 \\
\hline January 1995 ............... & 2.7 & $w$ & $w$ & $w$ & 8.4 & 277.8 & - & - & $w$ & 683.1 \\
\hline Virginia & & & & & & & & & & \\
\hline January 1996 ............ & $w$ & w & 778.5 & 56.4 & 4.0 & 425.2 & $w$ & 5.9 & w & $w$ \\
\hline December 1995 ........ & $w$ & w & 820.6 & 48.9 & 2.6 & 364.3 & 1.3 & 5.4 & $w$ & $w$ \\
\hline January 1995 .............. & $w$ & 4.5 & $1,016.5$ & 74.1 & 5.2 & 336.3 & NA & $w$ & $w$ & $w$ \\
\hline West Virginja & & & & & & & & & & \\
\hline January 1996 .............. & $w$ & w & w & w & 15.4 & 74.3 & 1.6 & $w$ & - & $w$ \\
\hline December 1995 ......... & $w$ & - & $w$ & $w$ & 13.4 & 53.2 & 1.8 & $w$ & - & $w$ \\
\hline January 1995 .............. & $w$ & 0.8 & $w$ & $w$ & 14.8 & 47.4 & $w$ & - & - & $\ddot{w}$ \\
\hline PAD District II & & & & & & & & & & \\
\hline January 1996 ............. & 47.0 & 82.7 & $8,971.2$ & 858.4 & 240.6 & $1,515.3$ & 480.2 & $3,079.4$ & $w$ & $17,220.5$ \\
\hline December 1995 ......... & 55.5 & 98.5 & $8,540.0$ & 853.4 & 112.2 & $1,353.3$ & 414.9 & $2,849.3$ & $w$ & $16,662.0$ \\
\hline January 1995 .............. & 35.5 & 101.2 & $8,240.2$ & 806.8 & 180.1 & $1,274.3$ & 476.7 & $2,691.1$ & $1,427.6$ & $13,256.3$ \\
\hline Illinois & & & & & & & & & & \\
\hline January 1996 ............. & w & $w$ & $1,650.7$ & 84.7 & 12.3 & 96.4 & 122.6 & 265.5 & $w$ & $2,208.1$ \\
\hline December 1995 ........ & $w$ & W & $1,749.2$ & 81.1 & 8.4 & 106.3 & $w$ & 265.8 & $w$ & $1,824.1$ \\
\hline January 1995 ............. & $w$ & $w$ & $1,363.2$ & 79.4 & 13.3 & 61.8 & $w$ & 287.1 & 206.8 & $2,047.0$ \\
\hline Indlana & & & & & & & & & & \\
\hline January 1996 .............. & $w$ & 6.5 & $1,597.5$ & 66.6 & 13.4 & 146.6 & 51.3 & 168.1 & $w$ & 981.2 \\
\hline December 1995 ........ & $w$ & 12.2 & $1,488.4$ & 112.8 & 13.9 & 185.5 & 42.8 & 157.7 & $w$ & 973.5 \\
\hline January 1995 ............. & $w$ & 9.3 & $1,699.0$ & 69.1 & 10.1 & 223.0 & 47.8 & 183.4 & $w$ & 898.1 \\
\hline lowa & & & & & & & & & & \\
\hline January 1996 ............. & w & w & 69.6 & 16.5 & - & 3.7 & 20.5 & 333.9 & $w$ & 758.2 \\
\hline December 1995 ........ & - & $w$ & 93.4 & 14.5 & - & 5.2 & $w$ & 326.6 & $\ddot{w}$ & 672.3 \\
\hline January 1995 .............. & w & 5.9 & 100.3 & 14.4 & - & 5.1 & $w$ & 268.8 & $w$ & 760.6 \\
\hline Kansas & & & & & & & & & & \\
\hline January 1996 ............. & w & 8.1 & w & w & - & 13.6 & 13.8 & 299.5 & w & $4,352.6$ \\
\hline December 1995 ........ & $w$ & 8.4 & w & $w$ & - & 9.4 & 8.5 & 254.4 & $w$ & $5,138.2$ \\
\hline January 1995 ............... & w & 9.8 & $w$ & w & - & 10.2 & 14.6 & 257.2 & $\dddot{w}$ & $2,213.4$ \\
\hline Kentucky & & & & & & & & & & \\
\hline January 1996 .............. & $w$ & w & 383.9 & 161.0 & 15.7 & 211.8 & 10.4 & 16.1 & $w$ & 835.9 \\
\hline December 1995 ........ & 1.8 & W & 556.4 & 111.9 & 15.7 & 153.4 & 4.9 & $w$ & $w$ & 916.1 \\
\hline January 1995 .............. & $w$ & 2.2 & 428.0 & 97.7 & 20.9 & 155.7 & 14.7 & 24.2 & - & 705.2 \\
\hline Michigan & & & & & & & & & & \\
\hline January 1996 ............... & $w$ & & $1,116.4$ & 75.8 & 8.9 & 193.2 & 57.3 & 98.7 & $w$ & $1,138.5$ \\
\hline December 1995 ......... & W & $w$ & 692.6 & 97.2 & 8.8 & 170.4 & 50.4 & 97.8 & $w$ & $1,057.5$ \\
\hline January 1995 .............. & w & W & 833.8 & 58.2 & 8.5 & 139.6 & 60.9 & 107.7 & $w$ & 750.7 \\
\hline Minnesota & & & & & & & & & & \\
\hline January 1996 ............. & $w$ & w & 808.9 & 103.6 & w & 33.3 & 73.1 & 565.7 & $w$ & \\
\hline December 1995 ........ & $w$ & w & 846.7 & 102.3 & W & 34.6 & 62.4 & 466.0 & $w$ & $1,129.5$ \\
\hline January $1995 \ldots$ & $w$ & 8.7 & 781.0 & 88.4 & $w$ & 30.1 & 66.8 & 362.7 & $w$ & $1,002.4$ \\
\hline Missouri & & & & & & & & & & \\
\hline January 1996 ............ & 1.4 & $\begin{array}{r}8.3 \\
106\end{array}$ & 350.0 & 45.1 & - & 30.6 & $w$ & 91.9 & - & $1,515.9$ \\
\hline 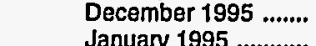 & $\begin{array}{l}W \\
w\end{array}$ & $\begin{array}{r}10.6 \\
89\end{array}$ & $\begin{array}{l}346.2 \\
355.5\end{array}$ & 44.3 & $\bar{w}$ & 23.8 & W & 79.4 & - & $1,219.9$ \\
\hline January 1995 & $w$ & 8.9 & 355.5 & 34.8 & W & 25.0 & w & 78.9 & - & $1,347.0$ \\
\hline
\end{tabular}

See footnotes at end of table. 
Table 45. Refiner Volumes of Aviation Fuels, Kerosene, No. 1 Distillate, and Propane (Consumer Grade) by PAD District and State

(Thousand Gallons per Day) - Continued

\begin{tabular}{|c|c|c|c|c|c|c|c|c|c|c|}
\hline \multirow{2}{*}{$\begin{array}{l}\text { Geographic Area } \\
\text { Month }\end{array}$} & \multicolumn{2}{|c|}{ Aviation Gasoline } & \multicolumn{2}{|c|}{$\begin{array}{l}\text { Kerosene-Type } \\
\text { Jet Fuel }\end{array}$} & \multicolumn{2}{|c|}{ Kerosene } & \multicolumn{2}{|c|}{ No. 1 Distillate } & \multicolumn{2}{|c|}{$\begin{array}{c}\text { Propane } \\
\text { (Consumer Grade) }\end{array}$} \\
\hline & $\begin{array}{l}\text { Sales to } \\
\text { End Users }\end{array}$ & $\begin{array}{l}\text { Sales for } \\
\text { Resale }\end{array}$ & $\begin{array}{l}\text { Sales to } \\
\text { End Users }\end{array}$ & $\begin{array}{l}\text { Sales for } \\
\text { Resale }\end{array}$ & $\begin{array}{l}\text { Sales to } \\
\text { End Users }\end{array}$ & $\begin{array}{l}\text { Sales for } \\
\text { Resale }\end{array}$ & $\begin{array}{l}\text { Sales to } \\
\text { End Users }\end{array}$ & $\begin{array}{l}\text { Sales for } \\
\text { Resale }\end{array}$ & $\begin{array}{l}\text { Sales to } \\
\text { End Users }\end{array}$ & $\begin{array}{l}\text { Sales for } \\
\text { Resale }\end{array}$ \\
\hline \multicolumn{11}{|l|}{ Nebraska } \\
\hline January 1996 ............. & w & $w$ & 83.7 & 20.6 & - & 3.1 & 8.4 & 219.1 & - & 369.9 \\
\hline December 1995 ........ & w & w & 78.3 & 18.9 & - & 0.8 & $w$ & 210.0 & - & 249.2 \\
\hline January 1995 .................. & w & 3.5 & 73.6 & 13.5 & - & 1.1 & $w$ & 154.5 & - & 306.3 \\
\hline \multicolumn{11}{|l|}{ North Dakota } \\
\hline January 1996 ................. & w & W & $w$ & 5.8 & - & $w$ & 9.4 & 189.9 & W & 357.2 \\
\hline December 1995 ........ & $\ddot{w}$ & $w$ & 19.0 & 6.8 & - & 0.4 & 15.1 & 165.2 & $w$ & 231.2 \\
\hline January 1995 .............. & 0.8 & W & 22.5 & $w$ & - & $w$ & $w$ & 125.5 & W & 362.1 \\
\hline \multicolumn{11}{|l|}{ Ohio } \\
\hline January 1996 ................... & w & 11.5 & 934.9 & 94.4 & 41.7 & 432.4 & 25.0 & 53.2 & w & $1,207.0$ \\
\hline December 1995 ........ & 10.4 & 11.1 & 919.0 & 109.5 & 38.7 & 356.2 & 15.6 & 40.1 & $w$ & $1,103.9$ \\
\hline $\begin{array}{l}\text { Uktanoma } \\
\text { January } 1996 \text {.............. }\end{array}$ & w & 8.0 & 675.0 & 86.1 & - & 7.4 & $W$ & 75.5 & W & 858.0 \\
\hline December 1995 ......... & $\ddot{w}$ & 8.8 & 438.3 & 47.2 & - & 1.3 & $w$ & 154.5 & w & 816.0 \\
\hline January 1995 .................. & $\ddot{w}$ & 6.3 & 600.1 & 171.2 & w & $w$ & 4.6 & 209.0 & $\ddot{w}$ & 745.9 \\
\hline \multicolumn{11}{|l|}{ South Dakota } \\
\hline January 1996 ............. & w & $w$ & 23.5 & w & - & $w$ & $w$ & 213.7 & $w$ & 246.8 \\
\hline December 1995 ......... & $w$ & $w$ & $w$ & $w$ & - & 1.1 & $w$ & 187.5 & W & 200.9 \\
\hline January 1995 .............. & $w$ & w & $w$ & w & - & $w$ & $W$ & 131.3 & w & 192.1 \\
\hline Tennessee & & & & & & & & & & \\
\hline January 1996 & 4.1 & 8.4 & 890.8 & 41.8 & 15.1 & 321.9 & 0.9 & 1.4 & $w$ & 375.1 \\
\hline December 1995 ........ & 5.3 & 7.5 & 911.8 & 54.8 & 12.5 & 286.9 & - & $w$ & W & 219.7 \\
\hline January 1995 .............. & $w$ & 10.1 & 695.9 & 31.6 & 13.9 & 290.2 & $w$ & 2.3 & - & 219.4 \\
\hline Wisconsin & & & & & & & & & & \\
\hline January 1996 .............. & $w$ & w & 158.3 & 30.6 & $w$ & 18.1 & 58.4 & 487.1 & w & 907.9 \\
\hline December 1995 ........ & $w$ & w & 147.5 & 26.0 & $\ddot{w}$ & 18.1 & 60.8 & 426.4 & $w$ & 910.1 \\
\hline January 1995 .............. & $w$ & 8.2 & 100.8 & 42.3 & $\ddot{w}$ & 11.0 & 39.5 & 433.8 & $\dddot{w}$ & 771.2 \\
\hline PAD District III & & & & & & & & & & \\
\hline January 1996 ............. & 21.7 & 103.5 & $9,620.5$ & $5,241,2$ & w & 369.4 & $w$ & 90.0 & $1,412.9$ & $16,313.4$ \\
\hline December 1995 ........ & 21.0 & 89.5 & $8,388.1$ & $6,308.3$ & $w$ & 500.9 & $w$ & 145.2 & $2,107.0$ & $15,256.6$ \\
\hline January 1995 .............. & 19.3 & 94.9 & $9,831.3$ & $3,833.4$ & w & 352.7 & $w$ & 131.4 & $2,159.1$ & $15,554.8$ \\
\hline Alabama & & & & & & & & & & \\
\hline January 1996 & 2.6 & 9.2 & 120.4 & 164.0 & $w$ & 58.6 & - & - & $w$ & 570.5 \\
\hline December 1995 ........ & 2.8 & 7.0 & 121.8 & 25.6 & w & 49.3 & - & - & $w$ & 596.8 \\
\hline January 1995 .............. & 2.8 & 6.1 & 120.0 & 47.2 & w & 59.4 & - & - & $w$ & 609.5 \\
\hline Arkansas & & & & & & & & & & \\
\hline January 1996 .............. & $w$ & $w$ & 80.3 & w & w & 15.5 & - & $w$ & $w$ & 506.2 \\
\hline December 1995 ........ & w & $w$ & 75.5 & 60.6 & - & w & - & - & $w$ & 454.3 \\
\hline January 1995 .............. & w & 7.5 & 82.1 & $w$ & $w$ & 27.9 & - & $w$ & $w$ & 326.8 \\
\hline Louisiana & & & & & & & & & & \\
\hline January 1996 .............. & 2.4 & 6.0 & $2,571.5$ & $1,407.2$ & w & 99.1 & - & - & 633.2 & $1,215.5$ \\
\hline December 1995 ........ & 3.3 & 5.5 & $2,099.5$ & $1,873.2$ & $w$ & 199.4 & - & w & $1,091.5$ & $1,423.7$ \\
\hline January 1995 & 3.4 & 4.8 & $2,185.3$ & $1,114.0$ & $\ddot{w}$ & 84.5 & - & $\ddot{w}$ & 962.5 & $1,346.6$ \\
\hline Mississippi & & & & & & & & & & \\
\hline January 1996 .............. & $w$ & $w$ & 954.9 & $w$ & w & w & - & - & $w$ & $1,228.5$ \\
\hline December 1995 ......... & W & $w$ & 693.1 & 385.0 & $w$ & 213.8 & - & - & $w$ & $1,335.4$ \\
\hline January 1995 ............... & w & $\ddot{w}$ & 661.1 & $w$ & $w$ & $w$ & - & - & $w$ & 900.1 \\
\hline New Mexico & & & & & & & & & & \\
\hline January 1996 .............. & $w$ & w & 146.5 & 34.8 & - & w & - & $w$ & w & 629.4 \\
\hline December 1995 ........ & $w$ & $w$ & 142.0 & 30.6 & - & $w$ & $w$ & w & - & 595.7 \\
\hline January 1995 ............. & $\ddot{w}$ & $\ddot{w}$ & 224.6 & 37.2 & $w$ & w & w & 29.6 & $w$ & 610.6 \\
\hline Texas & & & & & & & & & & \\
\hline January 1996 .............. & 11.7 & 72.1 & $5,746.9$ & $3,299.0$ & $w$ & 40.9 & w & 69.7 & 598.8 & $12,163.3$ \\
\hline December 1995 ........ & 9.6 & 61.7 & $5,256.2$ & $3,933.3$ & $w$ & 28.3 & $w$ & 63.0 & 854.0 & $10,850.7$ \\
\hline January 1995 ............. & 7.3 & 65.6 & $6,558.1$ & $2,309.4$ & w & 65.9 & w & 100.5 & $1,037.2$ & $11,761.3$ \\
\hline PAD Distr & & & & & & & & & & \\
\hline January 1996 ............. & 7.2 & 20.2 & $1,687.0$ & 159.6 & $w$ & 16.3 & 58.5 & 511.7 & 43.8 & $1,320.0$ \\
\hline December 1995 ........ & 6.4 & 21.3 & $1,543.0$ & 162.5 & w & 13.8 & 47.7 & 417.6 & 40.2 & $1,276.5$ \\
\hline January 1995 ............. & 7.3 & 18.3 & $1,163.3$ & 98.0 & w & 22.7 & 52.2 & 448.6 & w & $1,222.2$ \\
\hline Colorado & & & & & & & & & & \\
\hline January 1996 .............. & w & 10.6 & 839.5 & 94.5 & - & 12.0 & 9.6 & 103.6 & w & 436.7 \\
\hline December 1995 ........ & 1.6 & 10.5 & 598.9 & 84.2 & - & 8.8 & $w$ & 87.6 & w & 397.0 \\
\hline 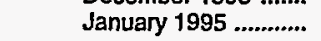 & 2.8 & 9.5 & 708.8 & 71.2 & - & 11.5 & $\ddot{w}$ & 117.8 & $w$ & 471.3 \\
\hline
\end{tabular}

See footnotes at end of table. 
Table 45. Refiner Volumes of Aviation Fuels, Kerosene, No. 1 Distillate, and Propane (Consumer Grade) by PAD District and State

(Thousand Gallons per Day) - Continued

\begin{tabular}{|c|c|c|c|c|c|c|c|c|c|c|}
\hline \multirow{2}{*}{$\begin{array}{c}\text { Geographic Area } \\
\text { Month }\end{array}$} & \multicolumn{2}{|c|}{ Aviation Gasoline } & \multicolumn{2}{|c|}{$\begin{array}{l}\text { Kerosene-Type } \\
\text { Jet Fuel }\end{array}$} & \multicolumn{2}{|c|}{ Kerosene } & \multicolumn{2}{|c|}{ No. 1 Distillate } & \multicolumn{2}{|c|}{$\begin{array}{c}\text { Propane } \\
\text { (Consumer Grade) }\end{array}$} \\
\hline & $\begin{array}{l}\text { Sales to } \\
\text { End Users }\end{array}$ & $\begin{array}{l}\text { Sales for } \\
\text { Resale }\end{array}$ & $\begin{array}{l}\text { Sales to } \\
\text { End Users }\end{array}$ & $\begin{array}{l}\text { Sales for } \\
\text { Resale }\end{array}$ & $\begin{array}{l}\text { Sales to } \\
\text { End Users }\end{array}$ & $\begin{array}{l}\text { Sales for } \\
\text { Resale }\end{array}$ & $\begin{array}{c}\text { Sales to } \\
\text { End Users }\end{array}$ & $\begin{array}{l}\text { Sales for } \\
\text { Resale }\end{array}$ & $\begin{array}{l}\text { Sales to } \\
\text { End Users }\end{array}$ & $\begin{array}{l}\text { Sales for } \\
\text { Resale }\end{array}$ \\
\hline \multicolumn{11}{|l|}{ Idaho } \\
\hline January 1996 & - & $w$ & 71.3 & $w$ & - & $W$ & 5.1 & 81.5 & $W$ & $W$ \\
\hline December 1995 ........ & $W$ & W & 74.8 & $W$ & - & $W$ & $w$ & 58.6 & W & $W$ \\
\hline January 1995 & $\mathbf{W}$ & $W$ & 52.4 & 5.7 & - & W & $w$ & 62.4 & $\dddot{W}$ & W \\
\hline \multicolumn{11}{|l|}{ Montana } \\
\hline January 1996 ............ & $W$ & W & 143.6 & W & - & 0.5 & 7.9 & 142.3 & $W$ & 249.5 \\
\hline December 1995 ........ & W & $W$ & 167.6 & 7.7 & - & 1.1 & 6.2 & 109.9 & $W$ & 222.3 \\
\hline January 1995 ........... & $w$ & $w$ & 56.1 & $w$ & - & 3.8 & 5.5 & 91.8 & W & 209.5 \\
\hline \multicolumn{11}{|l|}{ Utah } \\
\hline January 1996 & $W$ & 5.9 & 623.3 & 29.8 & - & $w$ & 18.1 & 90.7 & $w$ & $w$ \\
\hline December 1995 ....... & $w$ & 6.3 & 692.3 & 37.8 & - & $W$ & 16.5 & 84.7 & W & $w$ \\
\hline January 1995 ............ & $w$ & 5.3 & 338.2 & 16.7 & - & $W$ & 17.8 & 107.1 & W & W \\
\hline Wyoming & & & & & & & & & & \\
\hline January 1996 ............ & $w$ & $w$ & 9.2 & 26.5 & $w$ & - & 17.7 & 93.5 & W & 396.9 \\
\hline December 1995 ........ & $\ddot{w}$ & w & 9.3 & $W$ & $w$ & - & 11.3 & 76.7 & $W$ & 456.3 \\
\hline January 1995 ............ & $w$ & w & 7.8 & $w$ & $w$ & $W$ & 16.5 & 69.5 & 12.5 & 389.6 \\
\hline \multicolumn{11}{|l|}{ PAD District $V$} \\
\hline January 1996 & 26.8 & 116.4 & $14,057.2$ & $3,794.0$ & $w$ & 134.9 & 68.1 & 521.5 & $W$ & $2,066.4$ \\
\hline December 1995 ........ & 27.2 & 116.5 & $13,246.2$ & $2,478.5$ & $W$ & 107.7 & 45.4 & 414.4 & $W$ & $1,744.2$ \\
\hline January 1995 ............ & 27.6 & 110.7 & $12,969.4$ & $2,207.3$ & w & 104.5 & 52.5 & 386.5 & $w$ & $1,862.2$ \\
\hline \multicolumn{11}{|l|}{ Alaska } \\
\hline January 1996 & $W$ & 10.5 & $1,341.4$ & 223.4 & - & $w$ & 53.1 & 320.0 & $W$ & $W$ \\
\hline December 1995 ....... & $w$ & 11.8 & $1,478.1$ & 174.5 & - & $w$ & 30.4 & 265.5 & $W$ & $W$ \\
\hline January 1995 ............ & $w$ & 13.3 & $1,196.5$ & 143.5 & - & W & 44.8 & 214.1 & W & $W$ \\
\hline \multicolumn{11}{|l|}{ Arizona } \\
\hline January 1996 & 4.1 & 12.8 & 854.4 & 100.7 & w & $w$ & $w$ & $w$ & W & 79.9 \\
\hline December 1995 ....... & 4.4 & 11.3 & 768.6 & 127.0 & $W$ & $W$ & $w$ & $W$ & $\mathbf{W}$ & 24.4 \\
\hline January 1995 ............. & 4.4 & 11.4 & 670.6 & 108.0 & $\ddot{w}$ & w & $\ddot{w}$ & 3.3 & - & 47.5 \\
\hline \multicolumn{11}{|l|}{ California } \\
\hline January 1996 & 12.1 & 69.3 & $8,733.8$ & $2,719.7$ & $w$ & 37.8 & $w$ & $W$ & 4.0 & $1,253.3$ \\
\hline December 1995 ....... & 10.4 & 62.9 & $7,814.6$ & $1,483.4$ & $W$ & 24.4 & $w$ & w & 7.9 & $1,110.3$ \\
\hline January 1995 ............ & 10.0 & 56.5 & $7,945.2$ & $1,377.2$ & W & 38.4 & $w$ & $w$ & $w$ & $1,243.1$ \\
\hline \multicolumn{11}{|l|}{ Hawail } \\
\hline January 1996 & $W$ & $W$ & 637.3 & $w$ & - & - & - & W & $W$ & $W$ \\
\hline December 1995 ....... & $W$ & W & 576.9 & $w$ & - & - & - & $\mathbf{w}$ & $W$ & W \\
\hline January 1995 ............ & $w$ & W & 703.4 & w & - & - & - & W & $W$ & $w$ \\
\hline \multicolumn{11}{|l|}{ Novada } \\
\hline January 1996 & $w$ & 7.2 & 700.2 & 82.8 & - & - & W & $\mathbf{W}$ & $W$ & 113.8 \\
\hline December 1995 ....... & W & 2.3 & 610.9 & 53.4 & - & - & $W$ & 1.1 & $W$ & 66.5 \\
\hline January 1995 ............. & - & $w$ & 639.4 & 76.4 & - & $w$ & $w$ & 4.6 & - & 72.0 \\
\hline \multicolumn{11}{|l|}{ Oregon } \\
\hline January 1996 ............. & $w$ & $w$ & 433.8 & $W$ & - & 46.3 & $W$ & 54.8 & $W$ & 9.4 \\
\hline December 1995 ........ & - & W & 476.7 & $w$ & - & 35.3 & $w$ & 46.9 & $W$ & 17.0 \\
\hline January 1995 ............ & $w$ & 14.0 & 448.1 & $W$ & - & 40.7 & 1.6 & 51.3 & $W$ & $w$ \\
\hline Washington & & & & & & & & & & \\
\hline January 1996 & $W$ & $w$ & $1,356.3$ & 186.4 & - & 49.3 & 4.0 & 143.9 & $w$ & 524.3 \\
\hline December 1995 ........ & $W$ & $W$ & $1,520.3$ & 186.1 & - & 45.8 & 4.9 & 99.2 & $W$ & 445.6 \\
\hline January 1995 ............ & $w$ & $W$ & $1,366.3$ & 180.2 & - & 23.0 & 1.9 & 108.7 & $w$ & 413.0 \\
\hline
\end{tabular}

Dash $(-)=$ No data reported.

NA $=$ Not avallable.

$W=$ Withheld to avold disclosure of individual company data.

Notes: PAD District and U.S. totals equal the sum of the volumes for all States. In certain PAD Districts, however, volumes are not shown for every State.

Notes: Values shown for the current month are preliminary. Values shown for previous months are revised. Data are final upon publication in the Petroleum Marketing Annual.

Source: Energy Information Administration Form ElA-782A, "Refiners'/Gas Plant Operators' Monthly Petroleum Product Sales Report." 
Table 46. Refiner No. 2 Distillate, Diesel Fuel, and Fuel Oil Volumes by PAD District and State

(Thousand Gallons per. Day)

\begin{tabular}{|c|c|c|c|c|c|c|c|c|c|c|}
\hline \multirow{3}{*}{$\begin{array}{c}\text { Geographic Area } \\
\text { Month }\end{array}$} & \multicolumn{6}{|c|}{ No. 2 Diesel Fuel } & \multicolumn{2}{|c|}{ No. 2 Fuel Oil } & \multicolumn{2}{|c|}{ No. 2 Distillate } \\
\hline & \multicolumn{2}{|c|}{ Low-Sulfur } & \multicolumn{2}{|c|}{ High-Sulfur } & \multicolumn{2}{|c|}{ Total } & \multirow{2}{*}{$\begin{array}{c}\text { Sales to } \\
\text { End Users }\end{array}$} & \multirow{2}{*}{$\begin{array}{l}\text { Sales for } \\
\text { Resale. }\end{array}$} & \multirow{2}{*}{$\begin{array}{l}\text { Sales to } \\
\text { End Users }\end{array}$} & \multirow{2}{*}{$\begin{array}{l}\text { Sales for } \\
\text { Resale }\end{array}$} \\
\hline & $\begin{array}{c}\text { Sales to } \\
\text { End Users }\end{array}$ & $\begin{array}{c}\text { Sales for } \\
\text { Resale }\end{array}$ & $\begin{array}{l}\text { Sales to } \\
\text { End Users }\end{array}$ & $\begin{array}{l}\text { Sales for } \\
\text { Resale }\end{array}$ & $\begin{array}{l}\text { Sales to } \\
\text { End Users }\end{array}$ & $\begin{array}{l}\text { Sales for } \\
\text { Resale }\end{array}$ & & & & \\
\hline \multicolumn{11}{|l|}{ United States } \\
\hline $\begin{array}{l}\text { January } 1996 \ldots . . . . . . . \\
\text { December } 1995 \\
\text { January } 1995\end{array}$ & $\begin{array}{l}13,332.9 \\
13,026.2 \\
12,107.9\end{array}$ & $\begin{array}{l}56,791.7 \\
56,297.3 \\
52,137.1\end{array}$ & $\begin{array}{l}6,970.0 \\
7,550.1 \\
7,261.7\end{array}$ & $\begin{array}{l}15,563.5 \\
15,171.0 \\
12,420.7\end{array}$ & $\begin{array}{l}20,302.9 \\
20,576.4 \\
19,369.7\end{array}$ & $\begin{array}{l}72,355.2 \\
71,468.3 \\
64,557.8\end{array}$ & $\begin{array}{l}4,690.6 \\
4,137.9 \\
4,269.8\end{array}$ & $\begin{array}{l}46,067.6 \\
44,380.2 \\
36,947.8\end{array}$ & $\begin{array}{l}24,993.5 \\
24,714.2 \\
23,639.5\end{array}$ & $\begin{array}{l}118,422.8 \\
115,848.5 \\
101,505.6\end{array}$ \\
\hline \multicolumn{11}{|l|}{ PAD District I } \\
\hline $\begin{array}{l}\text { January } 1996 . . . . . . . . . \\
\text { December } 1995 \text {........ } \\
\text { January } 1995 \text {........... } \\
\text { Subdistrict IA }\end{array}$ & $\begin{array}{l}4,555.3 \\
4,403.6 \\
4,028.9\end{array}$ & $\begin{array}{l}14,388.6 \\
13,760.3 \\
13,083.0\end{array}$ & $\begin{array}{l}1,378.8 \\
1,432.3 \\
1,397.5\end{array}$ & $\begin{array}{l}3,475.7 \\
2,670.8 \\
2,290.6\end{array}$ & $\begin{array}{l}5,934.1 \\
5,836.0 \\
5,426.5\end{array}$ & $\begin{array}{l}17,864.3 \\
16,431.1 \\
15,373.6\end{array}$ & $\begin{array}{l}2,881.5 \\
2,303.3 \\
1,906.3\end{array}$ & $\begin{array}{l}31,307.8 \\
30,746.8 \\
23,926.7\end{array}$ & $\begin{array}{l}8,815.6 \\
8,139.3 \\
7,332.8\end{array}$ & $\begin{array}{l}49,172.1 \\
47,177.9 \\
39,300.4\end{array}$ \\
\hline $\begin{array}{l}\text { January } 1996 . . . . . . . . . \\
\text { December } 1995 \text {........ } \\
\text { January } 1995 \\
\text { Connecticut }\end{array}$ & $\begin{array}{c}W \\
294.3 \\
W\end{array}$ & $\begin{array}{c}W \\
W \\
1,256.3\end{array}$ & $\begin{array}{c}W \\
10.9 \\
W\end{array}$ & $\begin{array}{c}W \\
W \\
94.7\end{array}$ & $\begin{array}{l}294.4 \\
305.2 \\
231.0\end{array}$ & $\begin{array}{l}1,836.0 \\
1,442.6 \\
1,350.9\end{array}$ & $\begin{array}{c}W \\
322.7 \\
252.1\end{array}$ & $\begin{array}{l}7,158.0 \\
7,136.9 \\
5,305.4\end{array}$ & $\begin{array}{l}524.5 \\
628.0 \\
483.1\end{array}$ & $\begin{array}{l}8,994.1 \\
8,579.5 \\
6,656.3\end{array}$ \\
\hline $\begin{array}{l}\text { January } 1996 \ldots . . . . . . . . . \\
\text { December } 1995 \text {........ } \\
\text { January } 1995 \text {........... } \\
\text { Maine }\end{array}$ & $\begin{array}{l}W \\
W \\
W\end{array}$ & $\begin{array}{l}W \\
W \\
W\end{array}$ & $\begin{array}{l}W \\
W \\
W\end{array}$ & $\begin{array}{l}w \\
w \\
W\end{array}$ & $\begin{array}{l}90.0 \\
99.5 \\
69.7\end{array}$ & $\begin{array}{l}298.4 \\
435.8 \\
509.0\end{array}$ & $\begin{array}{r}39.2 \\
40.2 \\
7.9\end{array}$ & $\begin{array}{l}2,175.9 \\
1,849.3 \\
1,265.9\end{array}$ & $\begin{array}{r}129.2 \\
139.7 \\
77.6\end{array}$ & $\begin{array}{l}2,474.2 \\
2,285.1 \\
1,774.9\end{array}$ \\
\hline $\begin{array}{l}\text { January } 1996 \ldots . . . . . . . . . \\
\text { December } 1995 . . . . . . \\
\text { January } 1995 \ldots . . . . . . . . . \\
\text { Massachusetts }\end{array}$ & $\begin{array}{c}W \\
11.2 \\
W\end{array}$ & $\begin{array}{l}W \\
W \\
W\end{array}$ & $\begin{array}{l}\bar{w} \\
-\end{array}$ & $\begin{array}{l}W \\
W \\
W\end{array}$ & $\begin{array}{l}W \\
W \\
W\end{array}$ & $\begin{array}{l}134.9 \\
146.2 \\
156.0\end{array}$ & $\begin{array}{l}W \\
W \\
W\end{array}$ & $\begin{array}{l}729.9 \\
875.6 \\
821.2\end{array}$ & $\begin{array}{c}13.1 \\
W \\
W\end{array}$ & $\begin{array}{r}864.8 \\
1,021.9 \\
977.2\end{array}$ \\
\hline $\begin{array}{l}\text { January } 1996 \ldots . . . . . . . . . \\
\text { December } 1995 \\
\text { January } 1995 \text {............. } \\
\text { New Hampshire }\end{array}$ & $\begin{array}{c}W \\
129.0 \\
W\end{array}$ & $\begin{array}{c}W \\
567.8 \\
492.6\end{array}$ & $\begin{array}{l}W \\
6.4 \\
W\end{array}$ & $\begin{array}{l}W \\
9.5 \\
9.1\end{array}$ & $\begin{array}{l}137.8 \\
135.4 \\
123.5\end{array}$ & $\begin{array}{r}1,088.8 \\
577.4 \\
501.7\end{array}$ & $\begin{array}{c}W \\
224.4 \\
181.9\end{array}$ & $\begin{array}{l}2,768.3 \\
2,999.4 \\
2,262.5\end{array}$ & $\begin{array}{l}284.5 \\
359.8 \\
305.5\end{array}$ & $\begin{array}{l}3,857.1 \\
3,576.7 \\
2,764.3\end{array}$ \\
\hline $\begin{array}{l}\text { January } 1996 \text {........... } \\
\text { December } 1995 \text {........ } \\
\text { January } 1995 \text {........... } \\
\text { Rhode Island }\end{array}$ & $\begin{array}{c}13.1 \\
W \\
W\end{array}$ & $\begin{array}{c}W \\
W \\
41.9\end{array}$ & $\begin{array}{l}- \\
-\end{array}$ & $\begin{array}{l}- \\
-\end{array}$ & $\begin{array}{c}13.1 \\
W \\
W\end{array}$ & $\underset{41.9}{W}$ & $\begin{array}{l}W \\
W \\
W\end{array}$ & $\begin{array}{l}W \\
W \\
W\end{array}$ & $\begin{array}{c}49.7 \\
W \\
W\end{array}$ & $\begin{array}{c}W \\
239.1 \\
183.2\end{array}$ \\
\hline $\begin{array}{l}\text { January } 1996 \ldots . . . . . . . . . \\
\text { December } 1995 \text {....... } \\
\text { January } 1995 . . . . . . . . .\end{array}$ & $\begin{array}{l}24.2 \\
26.3 \\
18.4\end{array}$ & $\begin{array}{l}w \\
w \\
w\end{array}$ & $\begin{array}{l}- \\
- \\
-\end{array}$ & $\begin{array}{l}W \\
W \\
W\end{array}$ & $\begin{array}{l}24.2 \\
26.3 \\
18.4\end{array}$ & $\begin{array}{l}243.1 \\
223.9 \\
112.0\end{array}$ & $\begin{array}{c}W \\
23.1 \\
20.7\end{array}$ & $\begin{array}{r}1,161.0 \\
1,141.3 \\
747.5\end{array}$ & $\begin{array}{l}28.6 \\
49.4 \\
39.1\end{array}$ & $\begin{array}{r}1,404.1 \\
1,365.2 \\
859.5\end{array}$ \\
\hline $\begin{array}{l}\text { January } 1996 \ldots . . . . . . . . . \\
\text { December } 1995 \text {........ } \\
\text { January } 1995 \text {........... } \\
\text { Subdistrict IB }\end{array}$ & $\begin{array}{l}W \\
W \\
W\end{array}$ & $\begin{array}{l}w \\
w \\
w\end{array}$ & $\begin{array}{l}w \\
w \\
w\end{array}$ & $\bar{w}$ & $\begin{array}{l}W \\
W \\
W\end{array}$ & $\begin{array}{l}W \\
W \\
30.3\end{array}$ & $\begin{array}{l}W \\
W \\
W\end{array}$ & $\begin{array}{l}W \\
W \\
W\end{array}$ & $\begin{array}{r}19.4 \\
19.7 \\
6.1\end{array}$ & $\begin{array}{c}W \\
91.5 \\
97.3\end{array}$ \\
\hline $\begin{array}{l}\text { January } 1996 \ldots . . . . . . . \\
\text { December } 1995 \text {........ } \\
\text { January } 1995 \text {.......... } \\
\text { Delaware }\end{array}$ & $\begin{array}{r}W \\
1,595.0 \\
W\end{array}$ & $\begin{array}{c}W \\
W \\
5,019.4\end{array}$ & $\begin{array}{c}W \\
197.3 \\
W\end{array}$ & $\begin{array}{c}W \\
W \\
590.1\end{array}$ & $\begin{array}{l}1,805.8 \\
1,792.2 \\
1,741.2\end{array}$ & $\begin{array}{l}6,488.3 \\
6,286.4 \\
5,609.5\end{array}$ & $\begin{array}{c}W \\
1,350.3 \\
1,040.2\end{array}$ & $\begin{array}{l}19,596.5 \\
19,320.6 \\
14,352.4\end{array}$ & $\begin{array}{l}3,619.3 \\
3,142.5 \\
2,781.4\end{array}$ & $\begin{array}{l}26,084.8 \\
25,607.0 \\
19,961.9\end{array}$ \\
\hline $\begin{array}{l}\text { January } 1996 \ldots . . . . . . . . . \\
\text { December } 1995 \text {....... } \\
\text { January } 1995 \text {.......... } \\
\text { District of Columbia }\end{array}$ & $\begin{array}{c}14.0 \\
W \\
W\end{array}$ & $\begin{array}{l}W \\
W \\
W\end{array}$ & $\overline{-}$ & $\begin{array}{l}W \\
W \\
W\end{array}$ & $\begin{array}{c}14.0 \\
W \\
23.7\end{array}$ & $\begin{array}{c}W \\
W \\
155.0\end{array}$ & $\begin{array}{l}W \\
W \\
W\end{array}$ & $\begin{array}{l}W \\
W \\
W\end{array}$ & $\begin{array}{l}37.8 \\
17.3 \\
39.8\end{array}$ & $\begin{array}{c}W \\
W \\
564.2\end{array}$ \\
\hline $\begin{array}{l}\text { January } 1996 \ldots . . . . . . . . \\
\text { December } 1995 \text {....... } \\
\text { January } 1995 \text {........... } \\
\text { Maryland }\end{array}$ & $\begin{array}{c}77.7 \\
W \\
17.1\end{array}$ & $\begin{array}{l}w \\
W \\
w\end{array}$ & $\begin{array}{l}- \\
-\end{array}$ & $\overline{-}$ & $\begin{array}{c}77.7 \\
W \\
17.1\end{array}$ & $\begin{array}{l}W \\
W \\
7.6\end{array}$ & $\begin{array}{l}55.3 \\
W \\
W\end{array}$ & $\begin{array}{l}W \\
-w\end{array}$ & $\begin{array}{r}133.0 \\
46.8 \\
21.4\end{array}$ & $\begin{array}{c}W \\
W \\
43.6\end{array}$ \\
\hline $\begin{array}{l}\text { January } 1996 \ldots . . . . . . . . . \\
\text { December } 1995 . . . . . . \\
\text { January } 1995 . . . . . . . . . . \\
\text { New Jersey }\end{array}$ & $\begin{array}{r}W \\
250.5 \\
193.9\end{array}$ & $\begin{array}{l}500.5 \\
496.5 \\
450.5\end{array}$ & $\begin{array}{c}W \\
20.2 \\
86.5\end{array}$ & $\begin{array}{l}237.4 \\
205.3 \\
111.5\end{array}$ & $\begin{array}{l}273.5 \\
270.7 \\
280.4\end{array}$ & $\begin{array}{l}737.9 \\
701.8 \\
562.0\end{array}$ & $\begin{array}{l}321.6 \\
211.5 \\
127.8\end{array}$ & $\begin{array}{c}1,215.7 \\
W \\
764.4\end{array}$ & $\begin{array}{l}595.1 \\
482.2 \\
408.2\end{array}$ & $\begin{array}{l}1,953.6 \\
1,626.8 \\
1,326.4\end{array}$ \\
\hline $\begin{array}{l}\text { January } 1996 \ldots . . . . . . . . . \\
\text { December } 1995 \text {........ } \\
\text { January } 1995 \text {........... } \\
\text { New York }\end{array}$ & $\begin{array}{l}312.0 \\
342.6 \\
294.8\end{array}$ & $\begin{array}{l}1,859.8 \\
2,001.8 \\
1,725.7\end{array}$ & $\begin{array}{r}92.1 \\
109.7 \\
122.7\end{array}$ & $\begin{array}{l}582.8 \\
349.9 \\
250.1\end{array}$ & $\begin{array}{l}404.1 \\
452.3 \\
417.5\end{array}$ & $\begin{array}{l}2,442.6 \\
2,351.7 \\
1,975.7\end{array}$ & $\begin{array}{l}308.2 \\
222.0 \\
136.8\end{array}$ & $\begin{array}{l}7,658.7 \\
8,924.2 \\
5,922.6\end{array}$ & $\begin{array}{l}712.3 \\
674.3 \\
554.3\end{array}$ & $\begin{array}{r}10,101.4 \\
11,275.9 \\
7,898.3\end{array}$ \\
\hline $\begin{array}{l}\text { January } 1996 . . . . . . . . . \\
\text { December } 1995 \text {........ } \\
\text { January } 1995\end{array}$ & $\begin{array}{l}296.2 \\
272.0 \\
240.1\end{array}$ & $\begin{array}{l}W \\
W \\
W\end{array}$ & $\begin{array}{l}11.8 \\
12.1 \\
11.2\end{array}$ & $\begin{array}{l}W \\
W \\
W\end{array}$ & $\begin{array}{l}308.0 \\
284.1 \\
251.4\end{array}$ & $\begin{array}{l}909.9 \\
957.2 \\
747.9\end{array}$ & $\begin{array}{l}606.9 \\
442.0 \\
424.8\end{array}$ & $\begin{array}{l}5,536.1 \\
5,121.7 \\
3,881.1\end{array}$ & $\begin{array}{l}914.9 \\
726.1 \\
676.2\end{array}$ & $\begin{array}{l}6,446.0 \\
6,079.0 \\
4,629.0\end{array}$ \\
\hline $\begin{array}{l}\text { Pennsylvania } \\
\text { January } 1996 \ldots . . . . . . . . . \\
\text { December } 1995 \text {........ } \\
\text { January } 1995 \text {........... }\end{array}$ & $\begin{array}{l}671.9 \\
676.5 \\
627.8\end{array}$ & $\begin{array}{c}1,872.0 \\
W \\
1,950.7\end{array}$ & $\begin{array}{r}56.5 \\
55.4 \\
123.4\end{array}$ & $\begin{array}{c}316.7 \\
W \\
210.5\end{array}$ & $\begin{array}{l}728.5 \\
731.9 \\
751.2\end{array}$ & $\begin{array}{l}2,188.7 \\
2,061.8 \\
2,161.2\end{array}$ & $\begin{array}{l}497.7 \\
463.9 \\
330.3\end{array}$ & $\begin{array}{l}4,732.1 \\
4,038.1 \\
3,339.2\end{array}$ & $\begin{array}{l}1,226.2 \\
1,195.8 \\
1,081.5\end{array}$ & $\begin{array}{l}6,920.8 \\
6,099.9 \\
5,500.4\end{array}$ \\
\hline
\end{tabular}

See footnotes at end of table. 
Table 46. Refiner No. 2 Distillate, Diesel Fuel, and Fuel Oil Volumes by PAD District and State

(Thousand Gallons per Day) - Continued

\begin{tabular}{|c|c|c|c|c|c|c|c|c|c|c|}
\hline \multirow{3}{*}{$\begin{array}{c}\text { Geographic Area } \\
\text { Month }\end{array}$} & \multicolumn{6}{|c|}{ No. 2 Diesel Fuel } & \multicolumn{2}{|c|}{ No. 2 Fuel Oil } & \multicolumn{2}{|c|}{ No. 2 Distillate } \\
\hline & \multicolumn{2}{|c|}{ Low-Sulfur } & \multicolumn{2}{|c|}{ High-Sulfur } & \multicolumn{2}{|c|}{ Total } & \multirow{2}{*}{$\begin{array}{l}\text { Sales to } \\
\text { End Users }\end{array}$} & \multirow{2}{*}{$\begin{array}{l}\text { Sales for } \\
\text { Resale }\end{array}$} & \multirow{2}{*}{$\begin{array}{l}\text { Sales to } \\
\text { End Users }\end{array}$} & \multirow{2}{*}{$\begin{array}{l}\text { Sales for } \\
\text { Resale }\end{array}$} \\
\hline & $\begin{array}{c}\text { Sales to } \\
\text { End Users }\end{array}$ & $\begin{array}{l}\text { Sales for } \\
\text { Resale }\end{array}$ & $\begin{array}{c}\text { Sales to } \\
\text { End Users }\end{array}$ & $\begin{array}{c}\text { Sales for } \\
\text { Resale }\end{array}$ & $\begin{array}{c}\text { Sales to } \\
\text { End Users }\end{array}$ & $\begin{array}{c}\text { Sales for } \\
\text { Resale }\end{array}$ & & & & \\
\hline \multicolumn{11}{|l|}{ Subdistrict IC } \\
\hline $\begin{array}{l}\text { January } 1996 . . . . . . . . . . \\
\text { December } 1995 . . . . . \\
\text { January } 1995 \text {............ }\end{array}$ & $\begin{array}{l}2,642.9 \\
2,514.4 \\
2,408.7\end{array}$ & $\begin{array}{l}7,440.7 \\
6,899.8 \\
6,807.4\end{array}$ & $\begin{array}{l}1,191.0 \\
1,224.1 \\
1,045.6\end{array}$ & $\begin{array}{l}2,099.2 \\
1,802.4 \\
1,605.8\end{array}$ & $\begin{array}{l}3,833.9 \\
3,738.5 \\
3,454.3\end{array}$ & $\begin{array}{l}9,539.9 \\
8,702.1 \\
8,413.2\end{array}$ & $\begin{array}{l}838.0 \\
630.3 \\
614.0\end{array}$ & $\begin{array}{l}4,553.3 \\
4,289.4 \\
4,269.0\end{array}$ & $\begin{array}{l}4,671.9 \\
4,368.8 \\
4,068.3\end{array}$ & $\begin{array}{l}14,093.2 \\
12,991.5 \\
12,682.2\end{array}$ \\
\hline January 1996 ............. & 903.7 & $2,150.3$ & 641.4 & 428.2 & $1,545.1$ & $2,578.5$ & $w$ & 603.0 & $1,634.5$ & $3,181,5$ \\
\hline December 1995 ........ & 848.7 & $1,921.3$ & 702.5 & 501.9 & $1,551.2$ & $2,423.3$ & $w$ & 587.4 & $1,643.3$ & $3,010.6$ \\
\hline $\begin{array}{l}\text { January } 1995 \ldots . . . . . . . . . . \\
\text { Georgia }\end{array}$ & 827.4 & $1,790.1$ & 550.2 & 414.7 & $1,377.6$ & $2,204.8$ & 78.0 & 650.2 & $1,455.6$ & $2,855.0$ \\
\hline January 1996 ................ & 729.7 & $1,619.5$ & 180.3 & 277.0 & 910.0 & $1,896.5$ & 262.6 & 539.7 & $1,172.7$ & $2,436.2$ \\
\hline December 1995 ........ & 748.2 & $1,436.1$ & 151.9 & 212.0 & 900.1 & $1,648.1$ & 117.1 & 532.6 & $1,017.2$ & $2,180.7$ \\
\hline January 1995 ............. & 663.0 & $1,455.0$ & 114.7 & 169.7 & 777.7 & $1,624.7$ & 53.3 & 611.2 & 831.1 & $2,235.9$ \\
\hline North Carolina & & & & & & & & & & \\
\hline January 1996 .............. & 381.1 & $1,422.4$ & 79.0 & 671.2 & 460.1 & $2,093.5$ & 272.0 & $1,138.3$ & 732.1 & $3,231.8$ \\
\hline December 1995 ........ & 362.9 & $1,442.9$ & 73.5 & 522.4 & 436.4 & $1,965.3$ & 246.8 & $1,194.0$ & 683.2 & $3,159.3$ \\
\hline $\begin{array}{l}\text { January } 1995 \\
\text { South Carolina............. }\end{array}$ & 363.4 & $1,409.2$ & 54.0 & 569.5 & 417.4 & $1,978.7$ & 271.0 & 947.8 & 688.3 & $2,926.5$ \\
\hline January 1996 ............. & 249.3 & $w$ & 23.6 & $w$ & 272.8 & 760.9 & 62.3 & 463.9 & 335.1 & $1,224.8$ \\
\hline December 1995 ........ & 232.4 & $w$ & 14.5 & $w$ & 246.8 & 761.5 & 60.9 & 361.9 & 307.7 & $1,123.3$ \\
\hline January 1995 ................ & $w$ & $w$ & $w$ & $w$ & 315.4 & 703.0 & $w$ & 371.7 & 374.4 & $1,074.7$ \\
\hline Virginia & & & & & & & & & & \\
\hline January $1996 \ldots . . . . . . . . . . . .$. & 289.2 & $1,344.1$ & 148.9 & 554.9 & 438.1 & $1,899.1$ & 100.0 & $1,542.9$ & 538.1 & $3,441.9$ \\
\hline December 1995 ........ & 233.0 & $1,194.2$ & 144.0 & 378.0 & 377.0 & $1,572.1$ & 48.2 & $1,366.5$ & 425.3 & $2,938.7$ \\
\hline January 1995 .............. & 165.5 & $1,299.7$ & 162.5 & 296.4 & 327.9 & $1,596.1$ & 95.0 & $1,433.4$ & 422.9 & $3,029.5$ \\
\hline West Virginla & & & & & & & & & & \\
\hline January 1996 ............. & 90.0 & $w$ & 117.8 & w & 207.7 & 311.5 & w & 265.5 & 259.4 & 577.1 \\
\hline December 1995 ........ & 89.2 & W & 137.8 & $w$ & 227.0 & 331.9 & $W$ & 246.9 & 292.2 & 578.8 \\
\hline January 1995 ............. & w & w & w & W & 238.3 & 305.8 & W & 254.7 & 296.1 & 560.5 \\
\hline PAD District II & & & & & & & & & & \\
\hline January 1996 & $5,156.9$ & $16,107.4$ & $1,667.7$ & $3,047.8$ & $6,824.6$ & $19,155.2$ & $1,452.8$ & $8,863.5$ & $8,277.4$ & $28,018.7$ \\
\hline December 1995 ........ & $5,078.2$ & $15,992.1$ & $2,255.3$ & $2,994.9$ & $7,333.5$ & $18,987.0$ & $1,506.8$ & $8,421.6$ & $8,840.2$ & $27,408.7$ \\
\hline January 1995 ............. & $4,520.9$ & $14,891.0$ & $1,913.9$ & $2,595.2$ & $6,434.8$ & $17,486.2$ & $1,944.2$ & $7,515.7$ & $8,379.0$ & $25,001.9$ \\
\hline lllinois & & & & & & & & & & \\
\hline January 1996 .............. & 482.8 & 973.1 & 107.9 & 376.9 & 590.7 & $1,350.0$ & $w$ & $1,343.5$ & 690.3 & $2,693.5$ \\
\hline December 1995 ......... & 495.1 & $1,388.5$ & 92.5 & 481.3 & 587.6 & $1,869.7$ & w & $1,456.4$ & 683.9 & $3,326.1$ \\
\hline January 1995 .............. & 437.7 & $1,153.3$ & 139.9 & 166.6 & 577.6 & $1,320.0$ & $w$ & $1,244.9$ & 775.5 & $2,564.9$ \\
\hline Indiana & & & & & & & & & & \\
\hline January 1996 .............. & 782.1 & $1,612.2$ & 162.5 & 176.6 & 944.6 & $1,788.8$ & w & 850.2 & $1,141.3$ & $2,639.0$ \\
\hline December 1995 ........ & 734.5 & $1,663.9$ & 139.5 & 170.9 & 873.9 & $1,834.8$ & $w$ & 842.1 & $1,098.7$ & $2,676.9$ \\
\hline January 1995 .............. & 636.4 & $1,167.8$ & 142.6 & 251.3 & 779.0 & $1,419.0$ & 332.2 & 927.8 & $1,111.2$ & $2,346.8$ \\
\hline lowa & & & & & & & & & & \\
\hline January 1996 ............. & 136.8 & 935.5 & - & 80.4 & 136.8 & $1,015.9$ & w & 166.2 & 297.7 & $1,182.1$ \\
\hline December 1995 ......... & $w$ & 872.1 & $w$ & 64.3 & 139.3 & 936.4 & $w$ & 152.4 & 272.3 & $1,088.8$ \\
\hline January 1995 ............. & $w$ & 919.5 & $w$ & 43.9 & 159.2 & 963.4 & $w$ & 164.1 & 290.6 & $1,127.5$ \\
\hline Kansas & & & & & & & & & & \\
\hline January 1996 .............. & w & $1,104.5$ & w & 116.7 & 155.1 & $1,221.2$ & w & 187.4 & 218.2 & $1,408.6$ \\
\hline December 1995 ........ & $w$ & 986.2 & $w$ & 219.7 & 808.9 & $1,206.0$ & $w$ & 113.0 & 910.3 & $1,319.0$ \\
\hline January 1995 ................... & 160.4 & 995.4 & 144.5 & 284.2 & 304.9 & $1,279.6$ & $\ddot{w}$ & 127.6 & 391.4 & $1,407.2$ \\
\hline Kentucky & & & & & & & & & & \\
\hline January 1996 .............. & 302.1 & 699.2 & 356.4 & 210.9 & 658.5 & 910.1 & 53.9 & 741.9 & 712.5 & $1,652.0$ \\
\hline December 1995 ........... & 297.0 & 616.6 & 366.6 & 158.1 & 663.6 & 774.6 & 78.9 & 680.9 & 742.5 & $1,455.5$ \\
\hline January 1995 .............. & 231.9 & 657.8 & 429.8 & 155.3 & 661.8 & 813.1 & 169.2 & 658.0 & 830.9 & $1,471.1$ \\
\hline Michigan & & & & & & & & & & \\
\hline January 1996 ............. & 498.4 & $1,244.4$ & 13.4 & 249.3 & 511.8 & $1,493.7$ & w & 951.8 & 555.2 & $2,445.5$ \\
\hline December 1995 ........ & $w$ & $1,172.1$ & w & 238.4 & 599.4 & 1.410 .5 & w & 960.7 & 694.9 & $2,371.2$ \\
\hline January 1995 .............. & $w$ & $1,160.1$ & W & 221.5 & 383.5 & $1,381.6$ & 104.4 & 819.6 & 487.9 & $2,201.2$ \\
\hline Minnesota & & & & & & & & & & \\
\hline January 1996 .............. & & 804.9 & & 283.4 & 169.8 & $1,088.3$ & 206.9 & 666.7 & 376.8 & $1,755.0$ \\
\hline December 1995 ........ & 156.9 & 705.1 & 5.7 & 213.8 & 162.6 & 918.9 & 175.5 & 503.9 & 338.1 & $1,422.8$ \\
\hline January 1995 ............. & 168.9 & 800.3 & 16.1 & 172.7 & 185.1 & 973.0 & W & 393.9 & 523.1 & $1,366.9$ \\
\hline Missouri & & & & & & & & & & \\
\hline January 1996 ............. & 216.6 & $1,394.4$ & - & 116.9 & 216.6 & $1,511.3$ & w & 191.9 & 264.2 & $1,703.2$ \\
\hline December 1995 ........ & 294.5 & $1,439.3$ & - & 103.0 & 294.5 & $1,542.3$ & $w$ & 195.1 & 304.1 & $1,737.4$ \\
\hline January 1995 ............. & 291.7 & $1,121.5$ & - & 87.6 & 291.7 & $1,209.1$ & $\mathbf{w}$ & 159.0 & 332.0 & $1,368.1$ \\
\hline
\end{tabular}

See footnotes at end of table. 
Table 46. Refiner No. 2 Distillate, Diesel Fuel, and Fuel Oil Volumes by PAD District and State

(Thousand Gallons per Day) - Continued

\begin{tabular}{|c|c|c|c|c|c|c|c|c|c|c|}
\hline \multirow{3}{*}{$\begin{array}{c}\text { Geographic Area } \\
\text { Month }\end{array}$} & \multicolumn{6}{|c|}{ No. 2 Diesel Fuel } & \multicolumn{2}{|c|}{ No. 2 Fuel Oil } & \multicolumn{2}{|c|}{ No. 2 Distillate } \\
\hline & \multicolumn{2}{|c|}{ Low-Sulfur } & \multicolumn{2}{|c|}{ High-Sulfur } & \multicolumn{2}{|c|}{ Total } & \multirow{2}{*}{$\begin{array}{l}\text { Sales to } \\
\text { End Users }\end{array}$} & \multirow{2}{*}{$\begin{array}{l}\text { Sales for } \\
\text { Resale }\end{array}$} & \multirow{2}{*}{$\begin{array}{l}\text { Sales to } \\
\text { End Users }\end{array}$} & \multirow{2}{*}{$\begin{array}{l}\text { Sales for } \\
\text { Resale }\end{array}$} \\
\hline & $\begin{array}{l}\text { Sales to } \\
\text { End Users }\end{array}$ & $\begin{array}{l}\text { Sales for } \\
\text { Resale }\end{array}$ & $\begin{array}{l}\text { Sales to } \\
\text { End Users }\end{array}$ & $\begin{array}{l}\text { Sales for } \\
\text { Resale }\end{array}$ & $\begin{array}{l}\text { Sales to } \\
\text { End Users }\end{array}$ & $\begin{array}{l}\text { Sales for } \\
\text { Resale }\end{array}$ & & & & \\
\hline Nebraska & & & & & & & & & & \\
\hline January 1996 & $w$ & 557.2 & $w$ & 60.4 & 55.4 & 617.5 & $w$ & 71.7 & 55.8 & 689.2 \\
\hline December 1995 & W & 592.7 & $\ddot{W}$ & 50.9 & 39.8 & 643.6 & $w$ & 45.8 & 40.0 & 689.5 \\
\hline January 1995 .............. & $w$ & 534.8 & $\dddot{W}$ & 56.0 & 32.6 & 590.8 & $w$ & 54.8 & 44.6 & 645.5 \\
\hline North Dakota & & & & & & & & & & \\
\hline January 1996 & $W$ & 268.6 & - & 24.1 & $W$ & 292.7 & $w$ & 137.0 & $w$ & 429.8 \\
\hline December 1995 ........ & 7.5 & 276.5 & $w$ & 29.0 & $w$ & 305.5 & $w$ & 79.1 & $w$ & 384.6 \\
\hline January 1995 .............. & $w$ & 289.4 & - & 28.3 & w & 317.7 & $\ddot{W}$ & 79.2 & $W$ & 396.8 \\
\hline January 1996 ............. & $1,456.9$ & $1,574.4$ & 297.5 & 380.1 & $1,754.3$ & $1,954.4$ & 81.6 & 1.810 .5 & 1.835 .9 & $3,764.9$ \\
\hline December 1995 & W & $1,456.2$ & W & 374.5 & $1,660.3$ & $1,830.6$ & 143.7 & $1,575.1$ & $1,803.9$ & $3,405.7$ \\
\hline $\begin{array}{l}\text { January } 1995 \text {............. } \\
\text { Oklahoma }\end{array}$ & $1,108.5$ & $1,354.5$ & 355.5 & 260.6 & $1,463.9$ & $1,615.1$ & 320.8 & $1,372.4$ & $1,784.7$ & $2,987.5$ \\
\hline January 1996 & 148.8 & $1,872.8$ & 462.6 & 217.1 & 611.4 & $2,089.9$ & $w$ & 337.1 & 917.0 & $2,427.1$ \\
\hline December 1995 & $W$ & $1,875.0$ & $W$ & 142.5 & 430.5 & $2,017.5$ & W & 424.0 & 640.5 & $2,441.6$ \\
\hline January 1995 ............ & $W$ & $1,934.2$ & $w$ & 232.7 & 596.0 & $2,166.8$ & $\ddot{W}$ & 255.3 & 600.1 & $2,422.2$ \\
\hline South Dakota & & & & & & & & & & \\
\hline January 1996 ............ & $W$ & 308.1 & W & 25.5 & $w$ & 333.6 & $W$ & 69.7 & $w$ & 403.4 \\
\hline December 1995 ........ & $w$ & 348.2 & - & 17.9 & $w$ & 366.1 & $w$ & 58.7 & $w$ & 424.8 \\
\hline January 1995 ............. & $W$ & 345.4 & - & 22.2 & $w$ & 367.6 & $w$ & 59.7 & $w$ & 427.4 \\
\hline Tennessee & & & & & & & & & & \\
\hline January 1996 ............. & 586.6 & $1,577.8$ & 195.5 & 326.3 & 782.1 & $1,904.1$ & 21.7 & 577.5 & 803.8 & $2,481.6$ \\
\hline December 1995 ........ & 521.6 & $1,509.6$ & 210.2 & 350.7 & 731.8 & $1,860.4$ & 37.8 & 616.8 & 769.6 & $2,477.2$ \\
\hline January 1995 ............. & 530.8 & $1,387.2$ & 228.5 & 298.9 & 759.3 & $1,686.0$ & 56.2 & 604.0 & 815.5 & $2,290.0$ \\
\hline Wisconsin & & & & & & & & & & \\
\hline January 1996 ............ & $w$ & $1,180.3$ & $w$ & 403.2 & 224.3 & $1,583.5$ & $w$ & 760.4 & 252.8 & $2,343.8$ \\
\hline December 1995 ........ & $W$ & $1,090.1$ & $w$ & 379.9 & 329.6 & $1,470.0$ & W & 717.5 & 367.2 & $2,187.5$ \\
\hline January 1995 .............. & $W$ & $1,069.8$ & $W$ & 313.5 & 227.4 & $1,383.3$ & 37.9 & 595.4 & 265.2 & $1,978.7$ \\
\hline PAD District III & & & & & & & & & & \\
\hline January 1996 & $1,569.0$ & $13,207.1$ & $2,179.9$ & $6,042.1$ & $3,748.9$ & $19,249.3$ & 105.9 & $5,754.2$ & $3,854.8$ & $25,003.5$ \\
\hline December 1995 ........ & $1,434.0$ & $13,785.7$ & $2,140.5$ & $6,755.5$ & $3,574.5$ & $20,541.2$ & 109.5 & $4,595.1$ & $3,684.1$ & $25,136.4$ \\
\hline January 1995 ............ & $1,563.6$ & $12,268.9$ & $2,438.7$ & $4,260.4$ & $4,002.4$ & $16,529.3$ & 152.9 & $5,301.5$ & $4,155.3$ & $21,830.8$ \\
\hline Alabama & & & & & & & & & & \\
\hline January 1996 & 192.4 & $1,020.0$ & 178.2 & 251.9 & 370.6 & $1,271.9$ & 10.5 & 275.6 & 381.1 & $1,547.5$ \\
\hline December 1995 ......... & 170.0 & 913.1 & 171.8 & 223.5 & 341.8 & $1,136.6$ & 8.5 & 247.5 & 350.3 & $1,384.1$ \\
\hline January 1995 ............ & 221.6 & 888.3 & 214.5 & 242.5 & 436.1 & $1,130.9$ & 47.0 & 343.8 & 483.1 & $1,474.6$ \\
\hline Arkansas & & & & & & & & & & \\
\hline January 1996 ............ & $W$ & 844.7 & $W$ & 505.3 & 171.0 & $1,349.9$ & $w$ & $w$ & 172.7 & $1,440.9$ \\
\hline December 1995 ........ & 149.9 & 859.5 & 77.5 & 418.4 & 227.4 & $1,277.9$ & $w$ & $w$ & 230.8 & $1,329.4$ \\
\hline January 1995 & $w$ & 893.5 & $w$ & 289.6 & 230.8 & $1,183.1$ & W & $\ddot{w}$ & 231.0 & $1,327.1$ \\
\hline Louisiana & & & & & & & & & & \\
\hline January 1996 ............ & 210.3 & $2,430.3$ & 547.0 & $2,818.5$ & 757.3 & $5,248.7$ & $w$ & $1,925.9$ & 762.4 & $7,174.6$ \\
\hline December 1995 ........ & 201.0 & $1,868.3$ & 448.7 & $3,565.9$ & 649.7 & $5,434.2$ & w & $1,153.5$ & 657.8 & $6,587.7$ \\
\hline January 1995 & 125.9 & $1,370.2$ & 548.3 & $1,487.5$ & 674.2 & $2,857.7$ & $w$ & 831.7 & 688.1 & $3,689.4$ \\
\hline Mississippi & & & & & & & & & & \\
\hline January 1996 ............ & 122.5 & $1,094.8$ & 74.3 & 359.6 & 196.7 & $1,454.4$ & $w$ & 103.4 & 197.3 & $1,557.8$ \\
\hline December 1995 ........ & $w$ & $1,111.5$ & $W$ & 833.8 & 171.7 & $1,945.3$ & $W$ & 88.9 & 172.6 & $2,034.2$ \\
\hline January 1995 ............ & 160.5 & $1,215.3$ & 89.0 & 332.3 & 249.5 & $1,547.6$ & 3.7 & 593.9 & 253.2 & $2,141.5$ \\
\hline New Mexico & & & & & & & & & & \\
\hline January 1996 ............ & $W$ & 635.8 & $W$ & 35.0 & 126.7 & 670.9 & - & $w$ & 126.7 & 672.2 \\
\hline December 1995 ........ & $w$ & 640.5 & $W$ & 20.8 & 119.4 & 661.3 & - & $w$ & 119.4 & 661.6 \\
\hline January 1995 ............ & $W$ & 545.7 & $W$ & 34.9 & 132.5 & 580.6 & - & $W$ & 132.5 & 581.4 \\
\hline Texas & & & & & & & & & & \\
\hline January 1996 ............ & 785.5 & $7,181.5$ & $1,341.0$ & $2,071.9$ & $2,126.5$ & $9,253.4$ & $w$ & $3,357.1$ & $2,214.6$ & $12,610.5$ \\
\hline December 1995 ........ & 696.4 & $8,392.9$ & $1,368.1$ & $1,693.0$ & $2,064.5$ & $10,085.9$ & $w$ & $3,053.5$ & $2,153.2$ & $13,139.4$ \\
\hline January 1995 ............. & 720.7 & $7,355.9$ & $1,558.6$ & $1,873.5$ & $2,279.3$ & $9,229.4$ & $w$ & $3,387.5$ & $2,367.3$ & $12,616.8$ \\
\hline PAD District IV & & & & & & & & & & \\
\hline January 1996 & 613.6 & $2,670.1$ & 514.5 & 281.1 & $1,128.0$ & $2,951.2$ & $w$ & $w$ & $1,191.1$ & $2,977.3$ \\
\hline December 1995 ........ & 589.4 & $2,455.6$ & 572.5 & 269.9 & $1,161.9$ & $2,725.5$ & $w$ & W & $1,213.7$ & $2,751.9$ \\
\hline January 1995 ............. & 659.6 & $2,562.5$ & 530.6 & 328.1 & $1,190.3$ & $2,890.6$ & $\mathbf{W}$ & W & $1,266.0$ & $2,955.5$ \\
\hline Colorado & & & & & & & & & & \\
\hline January 1996 ............. & $W$ & $W$ & $w$ & $w$ & 308.7 & 847.5 & - & W & 308.7 & 872.6 \\
\hline December 1995 ........ & $W$ & $W$ & $W$ & $w$ & 288.8 & 768.5 & - & $w$ & 288.6 & 791.8 \\
\hline January 1995 ............ & 125.8 & 649.0 & 217.6 & 24.7 & 343.4 & 673.7 & - & W & 343.4 & 714.4 \\
\hline
\end{tabular}

See footnotes at end of table. 
Table 46. Refiner No. 2 Distillate, Diesel Fuel, and Fuel Oil Volumes by PAD District and State

(Thousand Gallons per Day) - Continued

\begin{tabular}{|c|c|c|c|c|c|c|c|c|c|c|}
\hline \multirow{3}{*}{$\begin{array}{l}\text { Geographic Area } \\
\text { Month }\end{array}$} & \multicolumn{6}{|c|}{ No. 2 Diesel Fuel } & \multicolumn{2}{|c|}{ No. 2 Fuel Oil } & \multicolumn{2}{|c|}{ No. 2 Distillate } \\
\hline & \multicolumn{2}{|c|}{ Low-Sulfur } & \multicolumn{2}{|c|}{ High-Sulfur } & \multicolumn{2}{|c|}{ Total } & \multirow{2}{*}{$\begin{array}{l}\text { Sales to } \\
\text { End Users }\end{array}$} & \multirow{2}{*}{$\begin{array}{l}\text { Sales for } \\
\text { Resale }\end{array}$} & \multirow{2}{*}{$\begin{array}{c}\text { Sales to } \\
\text { End Users }\end{array}$} & \multirow{2}{*}{$\begin{array}{l}\text { Sales for } \\
\text { Resale }\end{array}$} \\
\hline & $\begin{array}{c}\text { Sales to } \\
\text { End Users }\end{array}$ & $\begin{array}{c}\text { Sales for } \\
\text { Resale }\end{array}$ & $\begin{array}{c}\text { Sales to } \\
\text { End Users }\end{array}$ & $\begin{array}{c}\text { Sales for } \\
\text { Resale }\end{array}$ & $\begin{array}{c}\text { Sales to } \\
\text { End Users }\end{array}$ & $\begin{array}{c}\text { Sales for } \\
\text { Resale }\end{array}$ & & & & \\
\hline \multicolumn{11}{|l|}{ Idaho } \\
\hline January 1996 ............. & w & 292.5 & w & 121.3 & 100.5 & 413.8 & - & w & 100.5 & 414.3 \\
\hline December 1995 ........ & w & 236.1 & $w$ & 100.5 & 141.5 & 336.6 & - & w & 141.5 & 337.0 \\
\hline January 1995 ............. & $w$ & 313.9 & $w$ & 154.0 & 104.2 & 467.9 & - & $w$ & 104.2 & 468.0 \\
\hline \multicolumn{11}{|l|}{ Montaná } \\
\hline January 1996 ............. & 154.2 & 413.3 & - & - & 154.2 & 413.3 & w & w & 154.4 & 413.4 \\
\hline December 1995 ......... & 148.7 & 390.9 & - & - & 148.7 & 390.9 & w & w & 148.9 & 391.2 \\
\hline January 1995 ............. & 184.8 & $w$ & - & $w$ & 184.8 & 474.5 & $w$ & $\ddot{w}$ & 184.9 & 474.5 \\
\hline \multicolumn{11}{|l|}{ Utah } \\
\hline January 1996 ............. & 164.1 & 672.6 & 30.5 & 147.1 & 194.5 & 819.7 & w & - & 257.4 & 819.7 \\
\hline December 1995 ......... & 154.3 & 587.7 & 39.7 & 148.6 & 193.9 & 736.3 & w & w & 245.6 & 737.2 \\
\hline January 1995 .............. & 163.0 & 664.4 & 12.7 & 142.8 & 175.7 & 807.2 & w & $w$ & 251.3 & 830.5 \\
\hline \multicolumn{11}{|l|}{ Wyoming } \\
\hline January 1996 ............. & w & w & $w$ & w & 370.1 & 456.9 & - & w & 370.1 & 457.4 \\
\hline December 1995 ........ & W & W & $w$ & w & 388.9 & 493.3 & - & w & 388.9 & 494.8 \\
\hline January 1995 ............ & $w$ & $w$ & $w$ & $W$ & 382.2 & 467.4 & - & $w$ & 382.2 & 468.1 \\
\hline \multicolumn{11}{|l|}{ PAD District V } \\
\hline January 1996 .............. & $1,438.2$ & $10,418.5$ & $1,229.1$ & $2,716.7$ & $2,667.2$ & $13,135.2$ & w & w & $2,854.5$ & $13,251.1$ \\
\hline December 1995 ........ & $1,521.0$ & $10,303.6$ & $1,149.5$ & $2,479.8$ & $2,670.5$ & $12,783.4$ & w & w & $2,836.9$ & $13,373.6$ \\
\hline January 1995 ............. & $1,334.9$ & $9,331.7$ & 980.9 & $2,946.4$ & $2,315.8$ & $12,278.1$ & w & w & $2,506.4$ & $12,417.0$ \\
\hline \multicolumn{11}{|l|}{ Alaska } \\
\hline January 1996 .............. & w & - & $w$ & 385.8 & 17.6 & 385.8 & 96.8 & w & 114.4 & 476.6 \\
\hline December 1995 ........ & $w$ & w & $w$ & w & 60.4 & 263.5 & 88.8 & $w$ & 149.2 & 844.3 \\
\hline January 1995 ............. & $w$ & w & $w$ & $w$ & $w$ & 338.6 & 86.6 & 102.2 & w & 440.8 \\
\hline \multicolumn{11}{|l|}{ Arizona } \\
\hline January 1996 .............. & 208.6 & 866.5 & 85.6 & 85.3 & 294.2 & 951.7 & - & - & 294.2 & 951.7 \\
\hline December 1995 ......... & w & w & w & $w$ & 273.9 & 954.1 & - & w & 273.9 & 954.3 \\
\hline January 1995 .............. & 158.2 & 878.6 & 89.5 & 172.9 & 247.6 & $1,051.5$ & - & - & 247.6 & $1,051.5$ \\
\hline California & & & & & & & & & & \\
\hline January 1996 ............. & 923.1 & $6,587.7$ & 401.4 & 408.6 & $1,324.5$ & $6,996.3$ & - & - & $1,324,5$ & $6,996.3$ \\
\hline December 1995 ........ & $1,023.0$ & $6,673.5$ & 393.4 & 545.6 & $1,416.5$ & $7,219.1$ & - & - & $1,416.5$ & $7,219.1$ \\
\hline January 1995 ............... & 840.2 & $5,401.7$ & 160.5 & 376.5 & $1,000.6$ & $5,778.3$ & - & - & $1,000.6$ & $5,778.3$ \\
\hline Hawail & & & & & & & & & & \\
\hline January $1996 . . . . . . . . . . .$. & W & $w$ & w & w & 335.1 & 148.8 & w & - & 343.5 & 148.8 \\
\hline December 1995 ......... & 39.9 & 58.0 & $w$ & 138.0 & w & 196.0 & $w$ & - & w & 196.0 \\
\hline January 1995 ............. & $w$ & W & $w$ & $w$ & $w$ & 189.4 & $w$ & - & $w$ & 189.4 \\
\hline Nevada & & & & & & & & & & \\
\hline January 1996 .............. & $W$ & $w$ & w & w & 97.1 & 505.9 & $w$ & - & 121.0 & 505.9 \\
\hline December 1995 ........ & 78.1 & w & w & $w$ & $w$ & 464.9 & w & - & w & 464.9 \\
\hline January 1995 ............. & $w$ & 475.9 & w & 36.0 & 91.8 & 511.9 & w & - & 123.7 & 511.9 \\
\hline Oregon & & & & & & & & & & \\
\hline & & 936.0 & 190.7 & 589.5 & 263.2 & $1,525.5$ & $w$ & - & 276.1 & $1,525.5$ \\
\hline December 1995 ........ & 64.6 & $1,111.8$ & 210.9 & 482.6 & 275.5 & $1,584.4$ & $w$ & $w$ & 286.3 & $1,594.5$ \\
\hline January 1995 ............. & 86.8 & $1,110.8$ & 255.8 & 523.4 & 342.6 & $1,634.2$ & $w$ & $w$ & 358.4 & $1,634.6$ \\
\hline Washington & & & & & & & & & & \\
\hline January 1996 ............. & 107.0 & $1,511.1$ & 228.5 & $1,110.1$ & 335.5 & $2,621.2$ & w & 25.2 & 380.9 & $2,646.4$ \\
\hline December 1995 ........ & 109.5 & $1,186.8$ & 112.5 & 904.6 & 222.0 & $2,091.5$ & $\mathbf{w}$ & 9.1 & 260.2 & $2,100.6$ \\
\hline January 1995 ............. & 122.0 & $1,392.2$ & 167.4 & $1,382.1$ & 289.3 & $2,774.3$ & w & 36.3 & 341.0 & $2,810.6$ \\
\hline
\end{tabular}

$\operatorname{Dash}(-)=$ No data reported.

$W=$ Withheld to avoid dlsclosure of individual company data.

Notes: PAD District and U.S. totals equal the sum of the volumes for all States, In certain PAD Districts, however, volumes are not shown for every State.

Notes: Values shown for the current month are preliminary. Values shown for previous months are revised. Data are final upon publication in the Petroleum Marketling Annual.

Source: Energy Information Administration Form EIA-782A, "Reliners'/Gas Plant Operators' Monthly Petroleum Product Sales Report." 
Table 47. Refiner Residual Fuel Oil and No. 4 Fuel Volumes by PAD District (Thousand Gallons per Day)

\begin{tabular}{|c|c|c|c|c|c|c|c|c|}
\hline \multirow{3}{*}{$\begin{array}{c}\text { Geographic Area } \\
\text { Month }\end{array}$} & \multicolumn{6}{|c|}{ Residual Fuel Oil } & \multicolumn{2}{|c|}{ No. 4 Fuela } \\
\hline & \multicolumn{2}{|c|}{$\begin{array}{l}\text { Sulfur Less Than } \\
\text { or Equal to } 1 \text { Percent }\end{array}$} & \multicolumn{2}{|c|}{$\begin{array}{c}\text { Sulfur Greater Than } \\
1 \text { Percent }\end{array}$} & \multicolumn{2}{|c|}{ Total } & \multirow{2}{*}{$\begin{array}{l}\text { Sales to } \\
\text { End Users }\end{array}$} & \multirow{2}{*}{$\begin{array}{l}\text { Sales for } \\
\text { Resale }\end{array}$} \\
\hline & $\begin{array}{l}\text { Sales to } \\
\text { End Users }\end{array}$ & $\begin{array}{l}\text { Sales for } \\
\text { Resale }\end{array}$ & $\begin{array}{l}\text { Sales to } \\
\text { End Users }\end{array}$ & $\begin{array}{l}\text { Sales for } \\
\text { Resale }\end{array}$ & $\begin{array}{l}\text { Sales to } \\
\text { End Users }\end{array}$ & $\begin{array}{l}\text { Sales for } \\
\text { Resalo }\end{array}$ & & \\
\hline \multicolumn{9}{|l|}{ United States } \\
\hline $\begin{array}{l}\text { January } 1996 \ldots . . . . . \\
\text { December } 1995 \ldots . \\
\text { January } 1995 . . . . .\end{array}$ & $\begin{array}{l}3,999.5 \\
3,508.9 \\
3,628.0\end{array}$ & $\begin{array}{l}9,784.6 \\
9,510.1 \\
7,604.0\end{array}$ & $\begin{array}{l}9,417.0 \\
8,740.5 \\
9,342.4\end{array}$ & $\begin{array}{l}6,650.9 \\
8,647.1 \\
7,465.2\end{array}$ & $\begin{array}{l}13,416.5 \\
12,249.4 \\
12,970.4\end{array}$ & $\begin{array}{l}16,435.5 \\
18,157.2 \\
15,069.2\end{array}$ & $\begin{array}{l}898.5 \\
589.0 \\
933.4\end{array}$ & $\begin{array}{l}500.1 \\
642.2 \\
674.2\end{array}$ \\
\hline \multicolumn{9}{|l|}{ PAD District I } \\
\hline $\begin{array}{l}\text { January } 1996 . . . . . . \\
\text { December } 1995 \ldots . \\
\text { January } 1995 \ldots . . . \\
\text { Subdistrict IA }\end{array}$ & $\begin{array}{r}\text { NA } \\
2,560.5 \\
2,829.5\end{array}$ & $\begin{array}{l}7,884.4 \\
7,482.1 \\
4,336.9\end{array}$ & $\begin{array}{l}3,984.5 \\
3,735.2 \\
4,163.3\end{array}$ & $\begin{array}{l}1,813.7 \\
1,741.9 \\
1,226.7\end{array}$ & $\begin{array}{l}6,749.3 \\
6,295.6 \\
6,992.8\end{array}$ & $\begin{array}{l}9,698.1 \\
9,224.0 \\
5,563.6\end{array}$ & $\begin{array}{l}657.6 \\
496.3 \\
692.7\end{array}$ & $\begin{array}{l}460.1 \\
446.7 \\
477.0\end{array}$ \\
\hline $\begin{array}{l}\text { January } 1996 \ldots . . . . . \\
\text { December } 1995 \text {.... } \\
\text { January } 1995 \text {....... } \\
\text { Subdistrict IB }\end{array}$ & $\begin{array}{l}W \\
W \\
W\end{array}$ & $\begin{array}{r}W \\
2,507.4 \\
W\end{array}$ & $\begin{array}{l}W \\
W \\
W\end{array}$ & $\begin{array}{c}W \\
572.2 \\
W\end{array}$ & $\begin{array}{r}W \\
W \\
198.9\end{array}$ & $\begin{array}{r}3,099.5 \\
3,079.5 \\
W\end{array}$ & $\begin{array}{l}w \\
W \\
w\end{array}$ & $\begin{array}{l}W \\
W \\
W\end{array}$ \\
\hline $\begin{array}{l}\text { January } 1996 . . . . . . \\
\text { December } 1995 \ldots \\
\text { January } 1995 . . . . . . \\
\text { Subdistrict IC }\end{array}$ & $\begin{array}{r}\text { NA } \\
2,364.3 \\
2,527.8\end{array}$ & $\begin{array}{l}4,603.4 \\
4,644.1 \\
2,671.5\end{array}$ & $\begin{array}{r}W \\
1,193.1 \\
1,439.6\end{array}$ & $\begin{array}{l}643.6 \\
785.4 \\
889.6\end{array}$ & $\begin{array}{c}W \\
3,557.4 \\
3,967.4\end{array}$ & $\begin{array}{l}5,247.0 \\
5,429.5 \\
3,561.1\end{array}$ & $\begin{array}{l}568.1 \\
434.7 \\
620.0\end{array}$ & $\begin{array}{l}321.7 \\
296.7 \\
325.7\end{array}$ \\
\hline $\begin{array}{l}\text { January } 1996 \ldots . . . . . \\
\text { December } 1995 \ldots . \\
\text { January } 1995 . . . . .\end{array}$ & $\begin{array}{l}W \\
W \\
W\end{array}$ & $\begin{array}{c}W \\
330.6 \\
W\end{array}$ & $\begin{array}{l}W \\
W \\
W\end{array}$ & $\begin{array}{c}W \\
384.4 \\
W\end{array}$ & $\begin{array}{c}3,040.8 \\
W \\
2,826.4\end{array}$ & $\begin{array}{r}1,351.6 \\
715.0 \\
W\end{array}$ & $\begin{array}{l}W \\
W \\
W\end{array}$ & $\begin{array}{l}W \\
W \\
W\end{array}$ \\
\hline \multicolumn{9}{|l|}{ PAD District II } \\
\hline $\begin{array}{l}\text { January } 1996 . . . . . \\
\text { December } 1995 \ldots \\
\text { January } 1995 . . . . .\end{array}$ & $\begin{array}{l}W \\
W \\
W\end{array}$ & $\begin{array}{l}W \\
W \\
W\end{array}$ & $\begin{array}{l}W \\
W \\
W\end{array}$ & $\begin{array}{l}W \\
W \\
W\end{array}$ & $\begin{array}{c}W \\
W \\
91.8\end{array}$ & $\begin{array}{l}664.6 \\
343.5 \\
412.3\end{array}$ & $\begin{array}{c}W \\
18.4 \\
W\end{array}$ & $\begin{array}{l}W \\
W \\
W\end{array}$ \\
\hline \multicolumn{9}{|l|}{ PAD District III } \\
\hline $\begin{array}{l}\text { January } 1996 \ldots . . . . . \\
\text { December } 1995 \ldots . \\
\text { January } 1995 . . . . . .\end{array}$ & $\begin{array}{l}W \\
W \\
W\end{array}$ & $\begin{array}{l}1,415.8 \\
1,523.9 \\
2,772.8\end{array}$ & $\begin{array}{l}W \\
W \\
W\end{array}$ & $\begin{array}{l}2,857.3 \\
5,242.7 \\
4,486.0\end{array}$ & $\begin{array}{l}2,108.6 \\
1,759.5 \\
2,696.5\end{array}$ & $\begin{array}{l}4,273.1 \\
6,766.5 \\
7,258.9\end{array}$ & $\begin{array}{l}\bar{w} \\
w\end{array}$ & $\begin{array}{r}126.1 \\
W\end{array}$ \\
\hline $\begin{array}{l}\text { PAD District IV } \\
\text { January } 1996 \ldots . . . . . \\
\text { December } 1995 \text {... } \\
\text { January } 1995 \ldots . . . .\end{array}$ & $\overline{-}$ & $\begin{array}{l}W \\
W \\
W\end{array}$ & $\begin{array}{c}W \\
W \\
16.7\end{array}$ & $\begin{array}{l}W \\
W \\
W\end{array}$ & $\begin{array}{c}W \\
W \\
16.7\end{array}$ & $\begin{array}{l}74.0 \\
79.4 \\
79.2\end{array}$ & $\begin{array}{l}- \\
-\end{array}$ & $\begin{array}{l}- \\
-\end{array}$ \\
\hline $\begin{array}{l}\text { PAD District } Y \\
\text { January } 1996 \ldots . . . . . \\
\text { December } 1995 \ldots . \\
\text { January } 1995 \ldots . . . .\end{array}$ & $\begin{array}{r}1,214.5 \\
936.1 \\
778.6\end{array}$ & $\begin{array}{c}W \\
444.7 \\
412.5\end{array}$ & $\begin{array}{l}3,111.9 \\
3,117.6 \\
2,394.1\end{array}$ & $\begin{array}{r}W \\
1,299.0 \\
1,342.7\end{array}$ & $\begin{array}{l}4,326.4 \\
4,053.7 \\
3,172.7\end{array}$ & $\begin{array}{l}1,725.7 \\
1,743.7 \\
1,755.2\end{array}$ & $\begin{array}{l}W \\
W \\
W\end{array}$ & $\begin{array}{l}W \\
W \\
W\end{array}$ \\
\hline
\end{tabular}

Dash $(-)=$ No data reported.

NA $=$ Not available.

$W=$ Withheld to avold disclosure of individual company data.

a Includes No. 4 fuel oil and No. 4 diesel fuel.

Notes: Values shown for the current month are preliminary. Values shown for previous months are revised. Data are final upon publication in the $P$ etroleum Marketing Annual.

Source: Energy Information Administration Form ElA-782A, "Refiners'/Gas Plant Operators' Monthly Petroleum Product Sales Report." 


\section{PrimeSupplier Sales Volumes of Petroleum Products for Local Consumption}


Table 48. Prime Supplier Sales Volumes of Motor Gasoline by Grade, Formulation, PAD District, and State

(Thousand Gallons per Day)

\begin{tabular}{|c|c|c|c|c|c|c|c|c|}
\hline \multirow{2}{*}{$\begin{array}{l}\text { Geographic Area } \\
\text { Month }\end{array}$} & \multicolumn{4}{|c|}{ Regular } & \multicolumn{4}{|c|}{ Midgrade } \\
\hline & Conventional ${ }^{a}$ & Oxygenated & Reformulated & Total & Conventional & Oxygenated & Reformulated & Total \\
\hline \multicolumn{9}{|l|}{ United States } \\
\hline January 1996 ...................... & $136,842.2$ & $22,690.3$ & $48,823.7$ & $208,356.2$ & - $22,221.3$ & $5,051.0$ & $13,743.9$ & $41,016.3$ \\
\hline December 1995 ................... & $144,472.5$ & $23,807.5$ & $52,756.1$ & $221,036.1$ & $23,882.8$ & $5,352.4$ & $14,853.3$ & $44,088.5$ \\
\hline 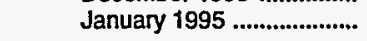 & $131,758.9$ & $24,336.1$ & $50,225.5$ & $206,320.5$ & $21,418.2$ & $3,586.0$ & $13,571.4$ & $38,575.6$ \\
\hline \multicolumn{9}{|l|}{ PAD District I } \\
\hline January 1996 & $41,768.3$ & - & $24,669.9$ & $66,438.3$ & $9,325.3$ & - & $6,325.5$ & $15,650.7$ \\
\hline December 1995 ................... & $44,747.3$ & - & $27,913.5$ & $72,660.9$ & $9,887.9$ & - & $7,083.7$ & $16,971.6$ \\
\hline 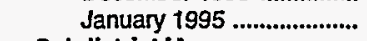 & $40,150.0$ & 487.9 & $26,124.8$ & $66,762.6$ & $9,277.5$ & 166.8 & $6,861,4$ & $16,305.7$ \\
\hline Subdistrict IA & & & & & & & & \\
\hline January 1996 ........................ & $1,402.1$ & - & $8,515.2$ & $9,917.3$ & 262.7 & - & $1,906.7$ & $2,169.4$ \\
\hline December 1995 ................... & $1,454.9$ & - & $9,568.7$ & $11,023.6$ & 272.9 & - & $2,062.2$ & $2,335.1$ \\
\hline January 1995 ........................ & $1,253.1$ & - & $8,302.5$ & $9,555.6$ & 244.5 & - & $1,967.8$ & $2,212.2$ \\
\hline \multicolumn{9}{|l|}{ Connecticut } \\
\hline January 1996 ...................... & NA & - & $2,289.6$ & $2,289.7$ & - & - & 529.5 & 529.5 \\
\hline December 1995 .................. & NA & - & $2,601.2$ & $2,601.3$ & - & - & 603.8 & 603.8 \\
\hline 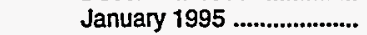 & w & - & & $2,220.5$ & - & - & 578.6 & 578.6 \\
\hline \multicolumn{9}{|l|}{ Maíne } \\
\hline 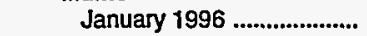 & 712.8 & - & 845.8 & $1,558.7$ & 127.1 & - & 150.2 & 277.3 \\
\hline December 1995 ...................... & 794.8 & - & 923.0 & $1,717.8$ & 140.4 & - & 163.5 & 303.9 \\
\hline January $1995 \ldots$ & 666.3 & - & 774.2 & $1,440.5$ & 128.3 & - & 139.3 & 267.6 \\
\hline \multicolumn{9}{|l|}{ Massachusetts } \\
\hline 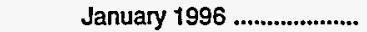 & - & - & $4,049.5$ & $4,049.5$ & - & - & 946.8 & 946.8 \\
\hline December 1995 .................. & - & - & $4,544.3$ & $4,544.3$ & - & - & 980.8 & 980.8 \\
\hline January 1995 ........................... & w & - & & $4,054.2$ & - & - & 963.8 & 963.8 \\
\hline \multicolumn{9}{|l|}{ New Hampshire } \\
\hline 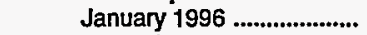 & 237.5 & - & 546.1 & 783.5 & 51.1 & - & 109.6 & 160.6 \\
\hline December 1995 ..................... & $w$ & - & w & 763.6 & $w$ & - & $w$ & 162.6 \\
\hline January 1995 & 208.5 & - & 441.6 & 650.2 & 42.9 & - & 101.6 & 144.5 \\
\hline \multicolumn{9}{|l|}{ Rhode Island } \\
\hline January 1996 ....................... & NA & - & 784.1 & 784.2 & w & - & $w$ & 170.9 \\
\hline December 1995 ................... & NA & - & 937.4 & 937.5 & - & - & 196.0 & 196.0 \\
\hline January $1995 \ldots$ & w & - & w & 808.0 & w & - & w & 185.0 \\
\hline \multicolumn{9}{|l|}{ Vermont } \\
\hline January 1996 & 451.6 & - & - & 451.6 & w & - & w & 84.4 \\
\hline December 1995 ................... & w & - & $w$ & 459.0 & $w$ & - & $\ddot{w}$ & 88.0 \\
\hline \multirow{2}{*}{\multicolumn{9}{|c|}{ Subdistrict IB }} \\
\hline & & & & & & & & \\
\hline January 1996 & $11,082.6$ & - & $13,539.0$ & $24,621.6$ & $1,791.1$ & - & $3,566.3$ & $5,357.4$ \\
\hline December 1995 & $12,439.4$ & - & $15,333.0$ & $27,772.4$ & $1,936.4$ & - & $4,033.8$ & $5,970,1$ \\
\hline 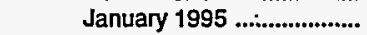 & $10,891.7$ & 3.1 & $15,154.5$ & $26,049.3$ & $1,850.3$ & 0.7 & $3,996.0$ & $5,847,1$ \\
\hline Delaware & & & & & & & & \\
\hline 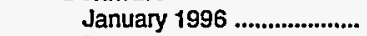 & - & - & 523.3 & 523.3 & - & - & 150.2 & 150.2 \\
\hline December 1995 ...................... & $=$ & - & 589.2 & 589.2 & - & - & 165.5 & 165.5 \\
\hline January 1995 .......................... & w & $w$ & W & 688.3 & w & - & $w$ & 199.5 \\
\hline District of Columbia & & & & & & & & \\
\hline 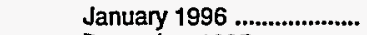 & - & - & 99.1 & 99.1 & - & - & 67.4 & 67.4 \\
\hline December 1995 ................... & - & - & 116.9 & 116.9 & - & - & 81.1 & 81.1 \\
\hline January 1995 & - & - & 126.9 & 126.9 & - & - & 86.3 & 86.3 \\
\hline Maryland & & & & & & & & \\
\hline January 1996 ........................... & w & - & w & $2,561.5$ & 82.1 & - & 772.2 & 854.2 \\
\hline December 1995 .................. & w & - & & $3,073.6$ & 96.8 & - & 913.0 & $1,009.8$ \\
\hline January 1995 & 301.2 & 0.5 & $2,536.4$ & $2,838.0$ & 83.0 & $w$ & W & 977.1 \\
\hline New Jersey & & & & & & & & \\
\hline January 1996 & w & - & w & $5,454.6$ & - & - & $1,165.6$ & $1,165.6$ \\
\hline December 1995 ................... & w & - & w & $6,093.1$ & - & - & $1,308.6$ & $1,308.6$ \\
\hline January 1995 ...................... & w & - & w & $6,013.7$ & $w$ & - & & $1,294.8$ \\
\hline New York & & & & & & & & \\
\hline 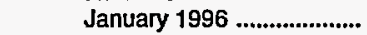 & $4,679.0$ & - & $3,433.0$ & $8,112.0$ & 605.4 & - & 894.9 & $1,500.3$ \\
\hline December $1995 \ldots . . . . . . . . . . . . .$. & $5,053.3$ & - & $3,826.1$ & $8,879.4$ & 629.7 & - & 978.7 & $1,608.4$ \\
\hline 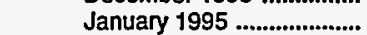 & $w$ & $w$ & $3,838.3$ & $8,428.4$ & 602.5 & w & W & $1,574.0$ \\
\hline $\begin{array}{l}\text { Pennsylvania } \\
\text { Pand }\end{array}$ & & & & & & & & \\
\hline January 1996 & & - & $1,846.0$ & $7,871.1$ & $1,103.6$ & - & & $1,619.7$ \\
\hline December 1995 ...................... & $6,938.0$ & - & $2,082.2$ & $9,020.2$ & $1,209.8$ & - & 586.9 & $1,796.7$ \\
\hline January 1995 ......................... & $5,984.0$ & 1.1 & $1,968.9$ & $7,954.0$ & $1,163.7$ & w & w & $1,715.3$ \\
\hline
\end{tabular}

See footnotes at end of table. 
Table 48. Prime Supplier Sales Volumes of Motor Gasoline by Grade, Formulation, PAD District, and State

(Thousand Gallons per Day) — Continued

\begin{tabular}{|c|c|c|c|c|c|c|c|c|}
\hline \multirow{2}{*}{$\begin{array}{l}\text { Geographic Area } \\
\text { Month }\end{array}$} & \multicolumn{4}{|c|}{ Premium } & \multicolumn{4}{|c|}{ All Grades } \\
\hline & Conventional & Oxygenated & Reformulated & Total & Conventional & Oxygenated & Reformulated & Total \\
\hline \multicolumn{9}{|l|}{ United States } \\
\hline January 1996 & $35,640.0$ & $5,414.0$ & $21,219.2$ & $62,273.2$ & $194,703.5$ & $33,155.3$ & $83,786.8$ & $311,645.6$ \\
\hline December 1995 ................. & $38,957.7$ & $5,822.0$ & $23,597.7$ & $68,377.4$ & $207,313.0$ & $34,981.9$ & $91,207.2$ & $333,502.0$ \\
\hline January 1995 ........................ & $35,021.9$ & $5,380.5$ & $21,572.7$ & $61,975.1$ & $188,199.1$ & $33,302.6$ & $85,369.6$ & $306,871.3$ \\
\hline \multicolumn{9}{|l|}{ PAD District I } \\
\hline January 1996 & $14,579.2$ & - & $12,152.8$ & $26,732.0$ & $65,672.8$ & - & $43,148.2$ & $108,821.0$ \\
\hline December 1995 .................. & $16,017.2$ & - & $14,203.0$ & $30,220.2$ & $70,652.5$ & - & $49,200.3$ & $119,852.7$ \\
\hline January 1995 ....................... & $14,210.2$ & 200.8 & $12,861.3$ & $27,272.3$ & $63,637.7$ & 855.5 & $45,847.5$ & $110,340.6$ \\
\hline Subdistrict IA & & & & & & & & \\
\hline 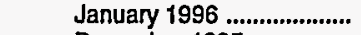 & 348.6 & - & $2,846.2$ & $3,194.8$ & $2,013.5$ & - & $13,268.1$ & $15,281.5$ \\
\hline December 1995 ................... & 342.2 & - & $3,187.5$ & $3,529.8$ & $2,070.1$ & - & $14,818.5$ & $16,888.5$ \\
\hline January 1995 ........................ & 314.2 & - & $2,940.5$ & $3,254.7$ & $1,811.7$ & - & $13,210.8$ & $15,022.5$ \\
\hline \multicolumn{9}{|l|}{ Connecticut } \\
\hline January 1996 ......................... & - & - & 805.8 & 805.8 & NA & - & $3,624.8$ & $3,624.9$ \\
\hline December 1995 ................... & - & - & 931.0 & 931.0 & NA & - & $4,135.9$ & $4,136.0$ \\
\hline January 1995 ......................... & $w$ & - & W & 821.6 & $w$ & - & $W$ & $3,620.7$ \\
\hline \multicolumn{9}{|l|}{ Maine } \\
\hline 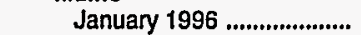 & 156.8 & - & 193.7 & 350.5 & 996.8 & - & $1,189.7$ & $2,186.5$ \\
\hline December 1995 & 162.2 & - & 213.3 & 375.5 & $1,097.3$ & - & $1,299.8$ & $2,397.1$ \\
\hline January 1995 ...................... & 145.5 & - & 201.0 & 346.5 & 940.0 & - & $1,114.5$ & $2,054.5$ \\
\hline \multicolumn{9}{|l|}{ Massachusetts } \\
\hline 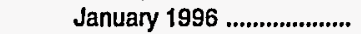 & - & - & $1,417.9$ & $1,417.9$ & - & - & $6,414.3$ & $6,414.3$ \\
\hline December 1995 & - & - & $1,547.9$ & $1,547.9$ & - & - & $7,073.1$ & $7,073.1$ \\
\hline January 1995 .............................. & - & - & $1,466.4$ & $1,466.4$ & w & - & w & $6,484.4$ \\
\hline \multicolumn{9}{|l|}{ New Hampshire } \\
\hline January 1996 & 59.8 & - & 153.0 & 212.8 & 348.4 & - & 808.6 & $1,157.0$ \\
\hline December 1995 ...................... & $w$ & - & W & 208.6 & $w$ & - & W & $1,134.9$ \\
\hline January 1995 & 51.7 & - & 133.2 & 184.9 & 303.1 & - & 676.5 & 979.6 \\
\hline \multicolumn{9}{|l|}{ Rhode Island } \\
\hline 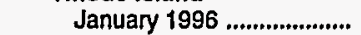 & - & - & 275.8 & 275.8 & w & - & $w$ & $1,230.9$ \\
\hline December 1995 ................ & - & - & 335.9 & 335.9 & NA & - & $1,469.3$ & $1,469.4$ \\
\hline \multirow{2}{*}{\multicolumn{9}{|c|}{$\begin{array}{l}\text { January } 1995 . . . . \\
\text { Vermont }\end{array}$}} \\
\hline & & & & & & & & \\
\hline January 1996 & 132.0 & - & - & 132.0 & w & - & $w$ & 668.0 \\
\hline December 1995 .................... & $w$ & - & w & 130.9 & $\ddot{w}$ & - & $\ddot{w}$ & 678.0 \\
\hline January 1995 ........................ & \multicolumn{3}{|c|}{ Subdistrict IB } & 118.2 & 565.6 & - & 7.5 & 573.2 \\
\hline $\begin{array}{l}\text { Subdistrict IB } \\
\text { January } 1996\end{array}$ & $3,032.0$ & - & $8,038.5$ & $11,070.5$ & $15,905.7$ & - & & \\
\hline 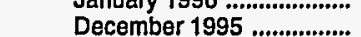 & $3,376.8$ & - & $9,501.8$ & $12,878.6$ & $17,752.6$ & $=$ & $\begin{array}{l}25,143.8 \\
28.868 .6\end{array}$ & $\begin{array}{l}41,049.5 \\
46,6212\end{array}$ \\
\hline January 1995 ......................... & $3,023.8$ & 0.9 & $8,681.2$ & $11,705.9$ & $15,765.9$ & 4.7 & $27,831.6$ & $43,602.2$ \\
\hline Delaware & & & & & & & & \\
\hline January 1996 & - & - & 153.5 & 153.5 & - & - & 826.9 & 826.9 \\
\hline December 1995 ................... & - & - & 177.6 & 177.6 & - & - & 932.3 & 932.3 \\
\hline January 1995 ........................ & $w$ & $w$ & W & 200.9 & w & w & $1,082.2$ & $1,088.6$ \\
\hline District of Columbia & & & & & & & & \\
\hline 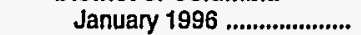 & - & - & 150.1 & 150.1 & - & - & 316.6 & 316.6 \\
\hline December 1995 .................... & - & - & 190.2 & 190.2 & - & - & 388.2 & 388.2 \\
\hline January 1995 ........................ & - & - & 172.0 & 172.0 & - & - & 385.2 & 385.2 \\
\hline Maryland & & & & & & & & \\
\hline January 1996 ....................... & 92.1 & - & $1,132.7$ & $1,224.7$ & $w$ & _- & $w$ & $4,640.5$ \\
\hline December 1995 ................... & 108.9 & - & $1,405.2$ & $1,514.2$ & $w$ & - & $\ddot{w}$ & $5,597,6$ \\
\hline January 1995 ...................... & 92.7 & w & $w$ & $1,313.9$ & 476.9 & 0.6 & $4,651.5$ & $5,129.0$ \\
\hline New Jersey & & & & & & & & \\
\hline January 1996 & - & - & $3,060.5$ & $3,060.5$ & w & - & $w$ & $9,680.8$ \\
\hline December 1995 .................. & - & - & $3,523.2$ & $3,523.2$ & $w$ & - & $\ddot{w}$ & $10,924.9$ \\
\hline January 1995 & $w$ & - & $W$ & $3,380.6$ & $w$ & - & $\ddot{w}$ & $10,689.2$ \\
\hline New York & & & & & & & & \\
\hline & & - & & $3,790.9$ & $6,354.3$ & - & & $13,403.3$ \\
\hline December 1995 .................. & $1,157.6$ & - & $3,213.8$ & $4,371.4$ & $6,840.6$ & - & $8,018.5$ & $14,859.2$ \\
\hline January 1995 ........................ & $w$ & w & $2,867.3$ & $3,886.8$ & $6,211.8$ & w & & $13,889.3$ \\
\hline Pennsylvania & & & & & & & & \\
\hline January 1996 ......................... & $1,870.0$ & - & 820.6 & $2,690.6$ & $8,998.7$ & - & $3,182.7$ & $12,181.5$ \\
\hline December 1995 .................... & $2,110.3$ & - & 991.7 & $3,102.0$ & $10,258.1$ & - & $3,660.8$ & $13,918.9$ \\
\hline January 1995 & $1,910.8$ & $w$ & $w$ & $2,751.6$ & $9,058.6$ & 2.0 & $3,360.3$ & $12,420.9$ \\
\hline
\end{tabular}

See foolnotes at end of table. 
Table 48. Prime Supplier Sales Volumes of Motor Gasoline by Grade, Formulation, PAD District, and State

(Thousand Gallons per Day) - Continued

\begin{tabular}{|c|c|c|c|c|c|c|c|c|}
\hline \multirow{2}{*}{$\begin{array}{l}\text { Geographic Area } \\
\text { Month }\end{array}$} & \multicolumn{4}{|c|}{ Regular } & \multicolumn{4}{|c|}{ Midgrade } \\
\hline & Conventionala & Oxygenated & Reformulated & Total & Conventional & Oxygenated & Roformulated & Total \\
\hline \multicolumn{9}{|l|}{ Subdistrict IC } \\
\hline January 1996 & $29,283.6$ & - & $2,615.7$ & $31,899.4$ & $7,271.5$ & - & 852.5 & $8,123.9$ \\
\hline December 1995 ................... & $30,853.0$ & - & $3,011.9$ & $33,864.8$ & $7,678.7$ & - & 987.7 & $8,666.4$ \\
\hline January 1995 ...................... & $28,005.2$ & 484.8 & $2,667.8$ & $31,157.7$ & $7,182.7$ & 166.1 & 897.6 & $8,246.4$ \\
\hline \multicolumn{9}{|l|}{ Florida } \\
\hline January 1996 ......................... & $10,592.6$ & - & - & $10,592.6$ & $3,091.6$ & - & - & $3,091.6$ \\
\hline December 1995 ...................... & $10,485.2$ & - & - & $10,485.2$ & $3,092.8$ & - & - & $3,092.8$ \\
\hline January 1995 & $9,807.5$ & - & - & $9,807.5$ & $3,018.5$ & - & - & $3,018,5$ \\
\hline \multicolumn{9}{|l|}{ Georgia } \\
\hline January 1996 ........................ & $6,618.5$ & - & - & $6,618.5$ & $1,593.8$ & - & - & $1,593.8$ \\
\hline December 1995 ................... & $6,924.4$ & - & - & $6,924.4$ & $1,693.5$ & - & - & $1,693.5$ \\
\hline January 1995 & $6,346.7$ & - & - & $6,346.7$ & $1,576.6$ & - & - & $1,576.6$ \\
\hline \multicolumn{9}{|l|}{ North Carolina } \\
\hline January 1996 ......................... & $5,726.2$ & - & - & $5,726.2$ & $1,288.7$ & - & - & $1,288.7$ \\
\hline December 1995 ........................ & $6,432.3$ & - & - & $6,432.3$ & $1,418.8$ & - & - & $1,418.8$ \\
\hline January 1995 & W & 484.8 & w & $5,793.2$ & $w$ & $w$ & w & $1,351.8$ \\
\hline \multicolumn{9}{|l|}{ South Carolina } \\
\hline January 1996 ....................... & $3,304.7$ & - & - & $3,304.7$ & 613.2 & - & - & 613.2 \\
\hline December 1995 & $3,485.7$ & - & - & $3,485.7$ & 687.3 & - & - & 687.3 \\
\hline 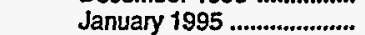 & $3,259.6$ & - & - & $3,259.6$ & 672.4 & - & - & 672.4 \\
\hline \multicolumn{9}{|l|}{ Virginia } \\
\hline January 1996 ....................... & $2,259.6$ & - & $2,615.7$ & $4,875.4$ & 516.2 & - & 852.5 & $1,368.6$ \\
\hline December 1995 & $2,638.3$ & - & $3,011.9$ & $5,650.2$ & 591.7 & - & 987.7 & $1,579.5$ \\
\hline 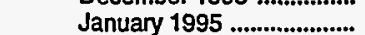 & $2,356.5$ & - & $2,664.2$ & $5,020.7$ & $w$ & - & $w$ & $1,402.9$ \\
\hline \multicolumn{9}{|l|}{ West Virginia } \\
\hline January 1996 & 782.0 & - & - & 782.0 & 167.9 & - & - & 167.9 \\
\hline December 1995 ................... & 887.0 & - & - & 887.0 & 194.5 & - & - & 194.5 \\
\hline January 1995 ........................ & $w$ & - & $\mathbf{w}$ & 930.1 & 224.3 & - & - & 224.3 \\
\hline \multicolumn{9}{|l|}{ PAD District II } \\
\hline January 1996 & $59,025.4$ & $2,163.4$ & $6,279.0$ & $67,467.8$ & $7,269.9$ & 353.3 & $1,648.0$ & $9,271.1$ \\
\hline 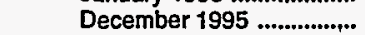 & $62,827.0$ & $2,221.4$ & $6,596.8$ & $71,645.1$ & $8,005.6$ & 376.2 & $1,752.5$ & $10,134.2$ \\
\hline January 1995 ....................... & $56,544.4$ & $2,119.4$ & $6,013.2$ & $64,677.0$ & $7,063.4$ & 342.9 & $1,510.3$ & $8,916.6$ \\
\hline \multicolumn{9}{|l|}{ fllinois } \\
\hline 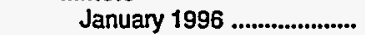 & $4,475.5$ & - & $3,894.1$ & $8,369.6$ & 547.0 & - & $1,147.2$ & $t, 694.2$ \\
\hline December 1995 ................. & $4,359.5$ & - & $4,046.8$ & $8,406.4$ & 599.5 & - & $1,211.6$ & $1,811.1$ \\
\hline January 1995 .............................. & $4,382.9$ & - & $3,583.1$ & $7,966.0$ & 504.7 & - & $1,068.2$ & $1,573.0$ \\
\hline Indiana & & & & & & & & \\
\hline January 1996 ....................... & $4,681.5$ & - & 462.5 & $5,143.9$ & 696.9 & - & 130.0 & 826.9 \\
\hline December 1995 & $4,857.3$ & - & 510.8 & $5,368.1$ & 731.6 & - & 142.1 & 873.7 \\
\hline January 1995 & $4,446.0$ & - & 468.7 & $4,914.6$ & 744.4 & - & 94.1 & 838.5 \\
\hline lowa & & & & & & & & \\
\hline January 1996 & $3,179.1$ & - & - & $3,179.1$ & 106.6 & - & - & 106.6 \\
\hline December 1995 ....................... & $3,502.0$ & - & - & $3,502.0$ & 100.8 & - & - & 100.8 \\
\hline January 1995 ......................... & $3,174.2$ & - & - & $3,174.2$ & 41.8 & - & - & 41.8 \\
\hline Kansas & & & & & & & & \\
\hline January 1996 ........................ & $3,209.0$ & - & - & $3,209.0$ & 138.8 & - & - & 138.8 \\
\hline December 1995 & $3,202.6$ & - & - & $3,202.6$ & 144.9 & - & - & 144.9 \\
\hline January 1995 ............................ & $3,166.8$ & - & - & $3,166.8$ & 129.1 & - & - & 129.1 \\
\hline Kentucky & & & & & & & & \\
\hline January 1996 .......................... & $2,472.3$ & - & 691.4 & $3,163.7$ & 394.3 & - & 186.3 & 580.5 \\
\hline December 1995 & $2,707.9$ & - & 773.5 & $3,481.4$ & 426.4 & - & 202.7 & 629.1 \\
\hline January 1995 & $2,349.5$ & - & 723.2 & $3,072.6$ & $w$ & - & $\ddot{w}$ & 554.5 \\
\hline Michigan & & & & & & & & \\
\hline January 1996 ........................ & $8,952.5$ & - & - & $8,952.5$ & $1,156.9$ & - & - & $1,156.9$ \\
\hline December 1995 ....................... & $9,429.3$ & - & - & $9,429.3$ & $1,227.6$ & - & - & $1,227.6$ \\
\hline January 1995 & $8,395.0$ & - & - & $8,395.0$ & $1,118.0$ & - & - & $1,118.0$ \\
\hline Minnesota & & & & & & & & \\
\hline & $2,614.2$ & $2,163.4$ & - & $4,777.6$ & 55.1 & 353.3 & - & 408.4 \\
\hline December 1995 ...................... & $3,007.6$ & $2,221.4$ & - & $5,229.0$ & 56.5 & 376.2 & - & 432.7 \\
\hline January 1995 & $2,348.6$ & $2,119,4$ & - & $4,468.0$ & 41.7 & 342.9 & - & 384.6 \\
\hline Missouri & & & & & & & & \\
\hline January 1996 & $5,198.5$ & - & - & $5,198.5$ & 509.6 & - & - & 509.6 \\
\hline December 1995 .................... & $5,679.4$ & - & - & $5,679.4$ & 552.3 & - & - & 552.3 \\
\hline January 1995 ......................... & $5,211,2$ & - & - & $5,211.2$ & 520.0 & - & - & 520.0 \\
\hline
\end{tabular}

See footnotes at end of table. 
Table 48. Prime Supplier Sales Volumes of Motor Gasoline by Grade, Formulation, PAD District, and State

(Thousand Gallons per Day) - Continued

\begin{tabular}{|c|c|c|c|c|c|c|c|c|}
\hline \multirow{2}{*}{$\begin{array}{l}\text { Geographic Area } \\
\text { Month }\end{array}$} & \multicolumn{4}{|c|}{ Premium } & \multicolumn{4}{|c|}{ All Grades } \\
\hline & Conventional & Oxygenated & Reformulated & Total & Conventional & Oxygenated & Reformulated & Total \\
\hline \multicolumn{9}{|l|}{ Subdistrict IC } \\
\hline January 1996 ....................... & $11,198.6$ & - & $1,268.1$ & $12,466.7$ & $47,753.6$ & - & $4,736.3$ & $52,490.0$ \\
\hline December 1995 .................... & $12,298.2$ & - & $1,513.6$ & $13,811.8$ & $50,829.8$ & - & $5,513.2$ & $56,343.1$ \\
\hline January 1995 & $10,872.2$ & 199.9 & $1,239.6$ & $12,311.7$ & $46,060.1$ & 850.7 & $4,805.0$ & $51,715.9$ \\
\hline \multicolumn{9}{|l|}{ Florida } \\
\hline January $1996 \ldots$ & $4,864.4$ & - & - & $4,864.4$ & $18,548.6$ & - & - & $18,548.6$ \\
\hline December 1995 ..................... & $5,047.7$ & - & - & $5,047.7$ & $18,625.8$ & - & - & $18,625.8$ \\
\hline January 1995 ........................... & $4,723.5$ & - & - & $4,723.5$ & $17,549.4$ & - & - & $17,549.4$ \\
\hline \multicolumn{9}{|l|}{ Georgía } \\
\hline January 1996 ........................ & $2,369.4$ & - & - & $2,369.4$ & 10.581 .7 & - & - & $10,581.7$ \\
\hline December 1995 .................... & $2,583.3$ & - & - & $2,583.3$ & $11,201.2$ & - & - & $11,201.2$ \\
\hline January 1995 ......................... & $2,263.6$ & - & - & $2,263.6$ & $10,187.0$ & - & - & $10,187.0$ \\
\hline \multicolumn{9}{|l|}{ North Carolina } \\
\hline January 1996 ......................... & $1,992.1$ & - & - & $1,992.1$ & $9,007.0$ & - & - & $9,007.0$ \\
\hline December 1995 ................... & $2,383.5$ & - & - & $2,383.5$ & $10,234.6$ & - & - & $10,234.6$ \\
\hline January 1995 ......................... & $w$ & $w$ & $w$ & $1,963.2$ & & 850.7 & w & $9,108.1$ \\
\hline \multicolumn{9}{|l|}{ South Carolina } \\
\hline January 1996 ........................ & $1,010.7$ & - & - & $1,010.7$ & $4,928.5$ & - & - & $4,928.5$ \\
\hline December 1995 .................... & $1,145.5$ & - & - & $1,145.5$ & $5,318.5$ & - & - & $5,318.5$ \\
\hline January 1995 ....................... & 989.3 & - & - & 989.3 & $4,921.2$ & - & - & $4,921.2$ \\
\hline \multicolumn{9}{|l|}{ Virginia } \\
\hline January 1996 ........................ & 754.7 & - & $1,268.1$ & $2,022.9$ & $3,530.5$ & - & $4,736.3$ & $8,266.9$ \\
\hline December 1995 .................... & 910.1 & - & $1,513.6$ & $2,423.7$ & $4,140.1$ & - & $5,513.2$ & $9,653.4$ \\
\hline January 1995 ....................... & $w$ & - & w & $2,111.4$ & $3,738.1$ & - & $4,796.8$ & $8,534.9$ \\
\hline \multicolumn{9}{|l|}{ West Virginia } \\
\hline January 1996 ........................ & 207.2 & - & - & 207.2 & $1,157.2$ & - & - & $1,157.2$ \\
\hline December 1995 ................... & 228.1 & - & - & 228.1 & $1,309.6$ & - & - & $1,309.6$ \\
\hline 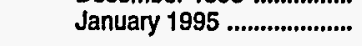 & $w$ & - & $w$ & 260.9 & $W$ & - & $\vec{W}$ & $1,415.2$ \\
\hline \multicolumn{9}{|l|}{ PAD District II } \\
\hline January 1996 & $11,964.9$ & 360.7 & $2,118.3$ & $14,444.0$ & $78,260.2$ & $2,877.4$ & $10,045.3$ & $91,182.9$ \\
\hline December 1995 ................... & $13,300.4$ & 389.4 & $2,243.8$ & $15,933.6$ & $84,133.0$ & $2,986.9$ & $10,593.1$ & $97,713.0$ \\
\hline January 1995 ......................... & $12,066.9$ & 374.5 & $2,099.7$ & $14,541.1$ & $75,674.7$ & $2,836.8$ & $9,623.2$ & $88,134.7$ \\
\hline \multicolumn{9}{|l|}{ Illinols } \\
\hline January 1996 ....................... & 692.7 & - & $1,493.5$ & $2,186.3$ & $5,715.3$ & - & $6,534.8$ & $12,250.1$ \\
\hline December 1995 ................... & 776.3 & - & $1,558.0$ & $2,334.3$ & $5,735.4$ & - & $6,816.4$ & $12,551.7$ \\
\hline January 1995 ...................... & 739.9 & - & $1,412.7$ & $2,152.6$ & $5,627.5$ & - & $6,064.0$ & 11.691 .5 \\
\hline Indiana & & & & & & & & \\
\hline January 1996 ........................ & $1,105.4$ & - & 163.0 & $1,268.3$ & $6,483.8$ & - & 755.4 & $7,239.2$ \\
\hline December 1995 ......................... & $1,232.5$ & - & 177.0 & $1,409.6$ & $6,821.5$ & - & 829.9 & $7,651.4$ \\
\hline January 1995 .......................... & $1,131.1$ & - & 182.9 & $1,314.0$ & $6,321.4$ & - & 745.7 & $7,067.1$ \\
\hline lowa & & & & & & & & \\
\hline January 1996 .......................... & 292.7 & - & - & 292.7 & $3,578.4$ & - & - & $3,578.4$ \\
\hline December 1995 ................... & 327.2 & - & - & 327.2 & $3,930.0$ & - & - & $3,930.0$ \\
\hline January 1995 ......................... & 270.8 & - & - & 270.8 & $3,486.8$ & - & - & $3,486.8$ \\
\hline Kansas & & & & & & & & \\
\hline January 1996 ......................... & 378.0 & - & - & 378.0 & $3,725.8$ & - & - & $3,725.8$ \\
\hline December 1995 .................. & 413.8 & - & - & 413.8 & $3,761.4$ & - & - & $3,761.4$ \\
\hline 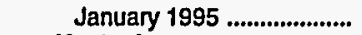 & 367.6 & - & - & 367.6 & $3,663.5$ & - & - & $3,663.5$ \\
\hline Kentucky & & & & & & & & \\
\hline January 1996 ........................ & 739.3 & - & 209.9 & 949.2 & $3,605.9$ & - & $1,087.5$ & $4,693.5$ \\
\hline December 1995 ........................ & 820.2 & - & 233.2 & $1,053.4$ & $3,954.5$ & - & $1,209.4$ & $5,163.8$ \\
\hline January 1995 ......................... & 790.9 & - & 258.0 & $1,048.9$ & $w$ & - & $w$ & $4,676.1$ \\
\hline Michigan & & & & & & & & \\
\hline January 1996 ....................... & $1,836.0$ & - & - & $1,836.0$ & $11,945.4$ & - & - & $11,945.4$ \\
\hline December 1995 .................. & $2,023.3$ & - & - & $2,023.3$ & $12,680.2$ & - & - & $12,680.2$ \\
\hline January 1995 ......................... & $1,980.4$ & - & - & $1,980.4$ & $11,493.4$ & - & - & $\$ 1,493.4$ \\
\hline Minnesota & & & & & & & & \\
\hline January 1996 ........................ & 245.1 & 360.7 & - & 605.8 & $2,914.4$ & $2,877.4$ & - & $5,791.8$ \\
\hline December 1995 ................... & 272.3 & 389.4 & - & 661.7 & $3,336.5$ & $2,986.9$ & - & $6,323.4$ \\
\hline January 1995 ........................ & 216.5 & 374.5 & - & 591.0 & $2,606.8$ & $2,836.8$ & - & $5,443.6$ \\
\hline Missouri & & & & & & & & \\
\hline January 1996 ........................... & $1,002.8$ & - & - & $1,002.8$ & $6,710.9$ & - & - & $6,710.9$ \\
\hline December 1995 ................... & 1.152 .1 & - & - & $1,152.1$ & $7,383.8$ & - & - & $7,383.8$ \\
\hline January 1995 .............................. & $1,023.6$ & - & - & $1,023.6$ & $6,754.8$ & - & - & $6,754.8$ \\
\hline
\end{tabular}

See footnotes at end of table. 
Table 48. Prime Supplier Sales Volumes of Motor Gasoline by Grade, Formulation, PAD District, and State

(Thousand Gallons per Day) - Continued

\begin{tabular}{|c|c|c|c|c|c|c|c|c|}
\hline \multirow{2}{*}{$\begin{array}{c}\text { Geographic Area } \\
\text { Month }\end{array}$} & \multicolumn{4}{|c|}{ Regular } & \multicolumn{4}{|c|}{ Midgrade } \\
\hline & Conventionala & Oxygenated & Reformulated & Total & Conventional & Oxygenated & Reformulated & Total \\
\hline \multicolumn{9}{|l|}{ Nebraska } \\
\hline 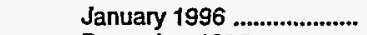 & $1,868.1$ & - & - & $1,868.1$ & 51.7 & - & - & 51.7 \\
\hline December 1995 ....................... & $1,932.6$ & - & - & $1,932.6$ & 44.7 & - & - & 44.7 \\
\hline 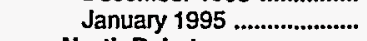 & $1,783.8$ & - & - & $1,783.8$ & 8.8 & - & - & 8.8 \\
\hline \multicolumn{9}{|l|}{ North Dakota } \\
\hline January 1996 ........................ & 759.5 & - & - & 759.5 & w & - & - & w \\
\hline December 1995 ...................... & 843.2 & - & - & 843.2 & w & - & - & w \\
\hline 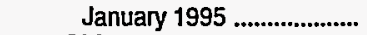 & 734.1 & - & - & 734.1 & $w$ & - & - & $w$ \\
\hline \multicolumn{9}{|l|}{ Ohio } \\
\hline 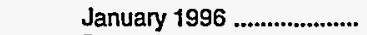 & $8,926.3$ & - & - & $8,926.3$ & $1,751.5$ & - & - & $1,751.5$ \\
\hline December 1995 ................... & $9,582.7$ & - & - & $9,582.7$ & $1,970.8$ & - & - & $1,970.8$ \\
\hline January 1995 & w & - & $w$ & $8,277.4$ & w & - & $w$ & $1,815.7$ \\
\hline \multicolumn{9}{|l|}{ Oklahoma } \\
\hline 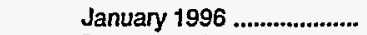 & $3,729.1$ & - & - & $3,729.1$ & 222.8 & - & - & 222.8 \\
\hline December 1995 ................... & $3,876.1$ & - & - & $3,876.1$ & 237.5 & - & - & 237.5 \\
\hline January 1995 ........................... & $3,683.9$ & - & - & $3,683.9$ & 194.7 & - & - & 194.7 \\
\hline \multicolumn{9}{|l|}{ South Dakota } \\
\hline 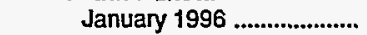 & 898.0 & - & - & 898.0 & $w$ & - & - & w \\
\hline 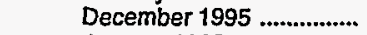 & 964.7 & - & - & 964.7 & $w$ & - & - & $\ddot{w}$ \\
\hline January 1995 ....................... & 826.7 & - & - & 826.7 & w & - & - & w \\
\hline \multicolumn{9}{|l|}{ Tennessee } \\
\hline 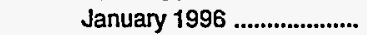 & $4,730.4$ & - & - & $4,730.4$ & $1,313.8$ & - & - & $1,313.8$ \\
\hline December 1995 .................... & $5,455.1$ & - & - & $5,455.1$ & $1,526.6$ & - & - & $1,526.6$ \\
\hline 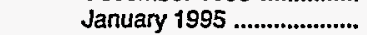 & $4,599.0$ & - & - & $4,599.0$ & $1,293.7$ & - & - & $1,293.7$ \\
\hline \multicolumn{9}{|l|}{ Wisconsin } \\
\hline January 1996 ....................... & $3,331.2$ & - & $1,231.1$ & $4,562.3$ & 285.2 & - & 184.6 & 469.7 \\
\hline December 1995 ..................... & $3,426.9$ & - & $1,265.8$ & $4,692.6$ & 344.2 & - & 196.1 & 540.3 \\
\hline January 1995 & w & - & W & $4,403.6$ & 244.0 & - & 162.5 & 406.5 \\
\hline \multicolumn{9}{|l|}{ PAD District III } \\
\hline January 1996 & $24,368.4$ & $1,071.8$ & $6,564.9$ & $32,005.0$ & $4,223.7$ & 115.5 & $1,722.7$ & $6,061.8$ \\
\hline December 1995 ................... & $25,444.6$ & $1,129.2$ & $6,586.9$ & $33,160.6$ & $4,467,3$ & 119.9 & $1,819.0$ & $6,406.1$ \\
\hline January 1995 ...................... & $23,192.4$ & $1,126.9$ & $6,322.2$ & $30,641.5$ & $4,246.2$ & 93.5 & $1,705.3$ & $6,045.0$ \\
\hline \multicolumn{9}{|l|}{ Alabama } \\
\hline January 1996 ....................... & $3,459.5$ & - & - & $3,459.5$ & 717.5 & - & - & 717.5 \\
\hline December 1995 ................... & $3,546.0$ & - & - & $3,546.0$ & 744.5 & - & - & 744.5 \\
\hline 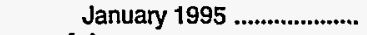 & $3,311.7$ & - & - & $3,311.7$ & 752.3 & - & - & 752.3 \\
\hline Arkansas & & & & & & & & \\
\hline January 1996 ............................ & $2,608.0$ & - & - & $2,608.0$ & 365.8 & - & - & 365.8 \\
\hline December 1995 .................. & $2,801.0$ & - & - & $2,801.0$ & 379.0 & - & - & 379.0 \\
\hline January 1995 ....................... & $2,586.1$ & - & - & $2,586.1$ & 384.2 & - & - & 384.2 \\
\hline Louisiana & & & & & & & & \\
\hline 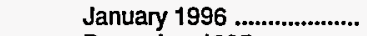 & w & - & w & $3,273.4$ & 788.6 & - & - & 788.6 \\
\hline December $1995 \ldots . . . . . . . . . . . . .$. & & - & $w$ & $3,377.0$ & 868.0 & - & - & 868.0 \\
\hline January 1995 ...................... & $3,114.0$ & - & - & $3,114.0$ & 793.1 & - & - & 793.1 \\
\hline Mississippi & & & & & & & & \\
\hline January 1996 ....................... & $2,271.6$ & - & - & $2,271.6$ & 419.4 & - & - & 419.4 \\
\hline December 1995 ................... & $2,350.1$ & -. & - & $2,350.1$ & 459.4 & - & - & 459.4 \\
\hline January 1995 ............................ & $2,198.8$ & - & - & $2,198.8$ & 425.9 & - & - & 425.9 \\
\hline New Mexico & & & & & & & & \\
\hline January 1996 & w & w & - & $1,811.0$ & 90.4 & 46.0 & - & 136.5 \\
\hline December 1995 .................. & w & w & - & $1,945.2$ & 89.5 & 50.5 & - & 140.0 \\
\hline 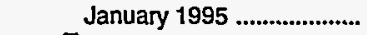 & $1,320.8$ & 603.5 & - & $1,924.3$ & 29.3 & 33.9 & - & 63.1 \\
\hline Texas & & & & & & & & \\
\hline January 1996 ........................ & $11,506.1$ & w & w & $18,581.7$ & $1,841.9$ & 69.5 & $1,722.7$ & $3,634.0$ \\
\hline December 1995 ................... & $12,020.8$ & $w$ & w & $19,141.4$ & $1,926.9$ & 69.4 & $1,819.0$ & $3,815.3$ \\
\hline 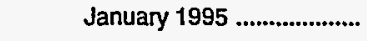 & $10,660.9$ & 523.5 & $6,322.2$ & $17,506.6$ & $1,861.5$ & 59.7 & $1,705.3$ & $3,626.4$ \\
\hline PAD District IV & & & & & & & & \\
\hline 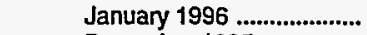 & $4,854.7$ & $2,173.3$ & - & $7,028.0$ & 687.0 & 580.2 & - & $1,267.2$ \\
\hline December 1995 ................... & $4,906.6$ & $2,365.8$ & - & $7,272.5$ & 745.8 & 593.2 & - & $1,339.0$ \\
\hline 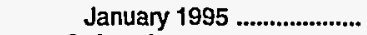 & $4,748.8$ & $2,274.3$ & - & $7,023.1$ & 554.1 & 553.5 & - & $1,107.7$ \\
\hline Colorado & & & & & & & & \\
\hline 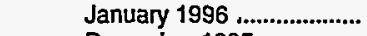 & $1,166.8$ & $2,055.4$ & - & $3,222.2$ & 90.0 & 568.2 & - & 658.2 \\
\hline December 1995 .................. & $1,113.0$ & $2,142.9$ & - & $3,255.9$ & 117.8 & 568.2 & - & 686.0 \\
\hline 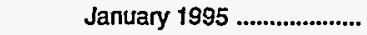 & 978.0 & $2,138.5$ & - & $3,116.5$ & 80.8 & 536.8 & - & 617.6 \\
\hline
\end{tabular}

See footnotes at end of table. 
Table 48. Prime Supplier Sales Volumes of Motor Gasoline by Grade, Formulation, PAD District, and State

(Thousand Gallons per Day) — Continued

\begin{tabular}{|c|c|c|c|c|c|c|c|c|}
\hline \multirow{2}{*}{$\begin{array}{l}\text { Geographic Area } \\
\text { Month }\end{array}$} & \multicolumn{4}{|c|}{ Premium } & \multicolumn{4}{|c|}{ All Grades } \\
\hline & Conventional & Oxygenated & Reformulated & Total & Conventional & Oxygenated $\mathrm{F}$ & Reformulated & Total \\
\hline \multicolumn{9}{|l|}{ Nebraska } \\
\hline January 1996 ........................ & 139.9 & - & - & 139.9 & $2,059.7$ & - & - & $2,059.7$ \\
\hline December 1995 .................... & 151.8 & - & - & 151.8 & $2,129.1$ & - & - & $2,129.1$ \\
\hline January 1995 .............................. & 125.2 & - & - & 125.2 & $1,917.7$ & - & - & $1,917.7$ \\
\hline \multicolumn{9}{|l|}{ North Dakota } \\
\hline January 1996 ........................ & w & - & - & $w$ & 854.4 & - & - & 854.4 \\
\hline December 1995 ....................... & $w$ & - & - & w & 950.0 & - & - & 950.0 \\
\hline 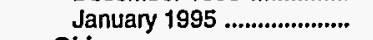 & $w$ & - & - & w & 821.7 & - & - & 821.7 \\
\hline \multicolumn{9}{|l|}{ Ohio } \\
\hline January 1996 ......................... & $2,050.6$ & - & - & $2,050.6$ & $12,728.5$ & - & - & $12,728.5$ \\
\hline December 1995 ................... & $2,270.3$ & - & - & $2,270.3$ & $13,823.8$ & - & - & $13,823.8$ \\
\hline 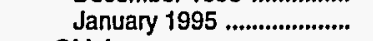 & $w$ & - & w & $2,129.0$ & w & - & w & $12,222.2$ \\
\hline \multicolumn{9}{|l|}{ Oklahoma } \\
\hline 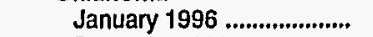 & 802.5 & - & - & 802.5 & $4,754.4$ & - & - & $4,754.4$ \\
\hline December 1995 .................. & 816.4 & - & - & 816.4 & $4,929.9$ & - & - & $4,929.9$ \\
\hline 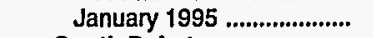 & 744.9 & - & - & 744.9 & $4,623.5$ & - & - & $4,623.5$ \\
\hline \multicolumn{9}{|l|}{ South Dakota } \\
\hline 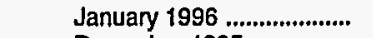 & $w$ & - & - & w & 976.4 & - & - & 976.4 \\
\hline 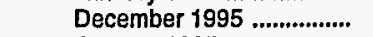 & $w$ & - & - & w & $1,047.9$ & - & - & $1,047.9$ \\
\hline 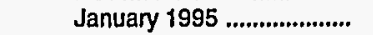 & w & - & - & w & 892.9 & - & - & 892.9 \\
\hline \multicolumn{9}{|l|}{ Tennessee } \\
\hline January 1996 ........................ & $1,944.8$ & - & - & $1,944.8$ & $7,989.0$ & - & - & $7,989.0$ \\
\hline December 1995 ........................ & $2,242.7$ & - & - & $2,242.7$ & $9,224.3$ & - & - & $9,224.3$ \\
\hline 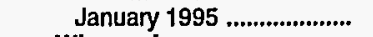 & $1,855.3$ & - & - & $1,855.3$ & $7,747.9$ & - & - & $7,747.9$ \\
\hline \multicolumn{9}{|l|}{ Wisconsin } \\
\hline January 1996 ........................ & 601.5 & - & 251.9 & 853.5 & $4,217 . \dot{9}$ & - & $1,667.6$ & $5,885.5$ \\
\hline December 1995 .................... & 653.7 & - & 275.6 & 929.3 & $4,424.8$ & - & 1.737 .4 & $6,162.2$ \\
\hline January 1995 ....................... & W & - & $w$ & 821.7 & $3,986.0$ & - & $1,645.8$ & $5,631.8$ \\
\hline \multicolumn{9}{|l|}{ PAD District III } \\
\hline January 1996 ........................ & $6,554.8$ & 196.7 & $2,426.5$ & $9,178.0$ & $35,146.8$ & $1,384.0$ & $10,714.1$ & $47,244.8$ \\
\hline December 1995 ................... & 7.043 .7 & 219.0 & $2,507.2$ & $9,770.0$ & $36,955.6$ & $1,468.1$ & $10,913.1$ & $49,336.8$ \\
\hline January 1995 ................................ & $6,419.5$ & 177.7 & $2,245.4$ & $8,842.6$ & $33,858.1$ & $1,398.2$ & $10,272.9$ & $45,529.2$ \\
\hline \multicolumn{9}{|l|}{ Alabama } \\
\hline January 1996 ........................ & $1,300.6$ & - & - & $1,300.6$ & $5,477.6$ & - & - & $5,477.6$ \\
\hline December 1995 .................. & $1,397.0$ & - & - & $1,397.0$ & $5,687.5$ & - & - & $5,687.5$ \\
\hline January 1995 ....................... & $1,223.3$ & - & - & $1,223.3$ & $5,287.4$ & - & - & $5,287.4$ \\
\hline Arkansas & & & & & & & & \\
\hline January 1996 .......................... & 676.4 & - & - & 676.4 & $3,650.2$ & - & - & $3,650.2$ \\
\hline December 1995 ..................... & 763.8 & - & - & 763.8 & $3,943.8$ & - & - & $3,943.8$ \\
\hline January 1995 .......................... & 729.3 & - & - & 729.3 & $3,699.6$ & - & - & $3,699.6$ \\
\hline Louisiana & & & & & & & & \\
\hline January 1996 ........................ & $1,332.1$ & - & - & $1,332.1$ & $\mathbf{w}$ & - & $w$ & $5,394.1$ \\
\hline December 1995 ................... & $1,412.9$ & - & - & $1,412.9$ & w & - & w & $5,657.9$ \\
\hline January 1995 ....................... & $1,289.3$ & - & - & $1,289.3$ & $5,196.3$ & - & - & $5,196.3$ \\
\hline Mississippi & & & & & & & & \\
\hline January 1996 ........................ & 763.9 & - & - & 763.9 & $3,454.8$ & - & - & $3,454.8$ \\
\hline December 1995 ................... & 821.5 & - & - & 821.5 & $3,630.9$ & - & - & $3,630.9$ \\
\hline January 1995 .............................. & 691.8 & - & - & 691.8 & $3,316.5$ & - & - & $3,316.5$ \\
\hline New Mexico & & & & & & & & \\
\hline January 1996 ........................ & 209.5 & 95.5 & - & 305.0 & w & w & - & $2,252.5$ \\
\hline December 1995 ................... & 227.0 & 106.3 & - & 333.3 & w & $w$ & - & $2,418.4$ \\
\hline 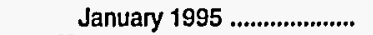 & 179.4 & 77.2 & - & 256.6 & $1,529.4$ & 714.6 & - & $2,244.0$ \\
\hline Texas & & & & & & & & \\
\hline January 1996 ........................... & $2,272.3$ & 101.2 & $2,426.5$ & $4,800.0$ & $15,620.3$ & $w$ & w & $27,015.7$ \\
\hline December 1995 ..................... & $2,421.6$ & 112.7 & $2,507.2$ & $5,041.6$ & $16,369.4$ & w & w & $27,998.3$ \\
\hline 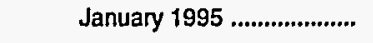 & $2,306.5$ & 100.5 & $2,245.4$ & $4,652.4$ & $14,828.9$ & 683.6 & $10,272.9$ & $25,785.4$ \\
\hline PAD District IV & & & & & & & & \\
\hline January 1996 ............................. & & & - & $1,810.8$ & $6,815.2$ & $3,290.8$ & - & $10,106.1$ \\
\hline December 1995 ................... & $1,322.6$ & 588.2 & - & $1,910.8$ & $6,975.0$ & $3,547.2$ & - & $10,522.2$ \\
\hline January 1995 ...................... & $1,222.3$ & 517.9 & - & $1,740.3$ & $6,525.3$ & $3,345.8$ & - & $9,871.1$ \\
\hline Colorado & & & & & & & & \\
\hline January $1996 \ldots . . . . . . . . . . . . . . . . . .$. & 214.4 & 491.1 & - & 705.5 & $1,471.1$ & $3,114.7$ & - & $4,585.8$ \\
\hline December 1995 ................. & 237.7 & 509.2 & - & 746.9 & $1,468.5$ & $3,220.3$ & - & $4,688.8$ \\
\hline January 1995 ........................ & 159.6 & 470.8 & - & 630.4 & $1,218.4$ & $3,146.1$ & - & $4,364.5$ \\
\hline
\end{tabular}

See footnotes at end of table. 
Table 48. Prime Supplier Sales Volumes of Motor Gasoline by Grade, Formulation, PAD District, and State

(Thousand Gallons per Day) - Continued

\begin{tabular}{|c|c|c|c|c|c|c|c|c|}
\hline \multirow{2}{*}{$\begin{array}{l}\text { Geographic Area } \\
\text { Month }\end{array}$} & \multicolumn{4}{|c|}{ Regular } & \multicolumn{4}{|c|}{ Midgrade } \\
\hline & Conventional ${ }^{\mathrm{a}}$ & Oxygenated & Reformulated & Total & Conventional & Oxygenated & Reformulated & Total \\
\hline \multicolumn{9}{|l|}{$\begin{array}{l}\text { Idaho } \\
\text { January } 1996\end{array}$} \\
\hline 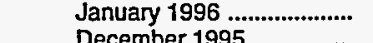 & 935.9 & - & - & 935.9 & 150.2 & - & - & 150.2 \\
\hline $\begin{array}{l}\text { December } 1995 \text {..................... } \\
\text { January } 1995\end{array}$ & 941.5 & - & - & 941.5 & 157.2 & - & - & 157.2 \\
\hline $\begin{array}{l}\text { January } 1995 \\
\text { Montana }\end{array}$ & 928.5 & - & - & 928.5 & 99.5 & - & - & 99.5 \\
\hline \multicolumn{9}{|l|}{ Montana } \\
\hline $\begin{array}{l}\text { January } 1996 \\
\text { December } 1995\end{array}$ & 802.9 & 51.5 & - & 854.4 & $w$ & $W$ & - & 75.4 \\
\hline 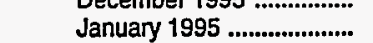 & 809.6 & 47.9 & - & 857.5 & $w$ & $w$ & - & w \\
\hline \multicolumn{9}{|l|}{ Utah } \\
\hline 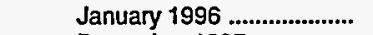 & w & w & - & $1,533.3$ & w & w & - & 378.5 \\
\hline December 1995 ................... & $1,477.4$ & 171.5 & - & $1,648.8$ & w & W & - & 407.3 \\
\hline January $1995 \ldots . . . . . . . . . . . . . . . . .$. & $1,501.7$ & 87.9 & - & $1,589.6$ & w. & w & - & 378.5 \\
\hline \multicolumn{9}{|l|}{ Wyoming } \\
\hline 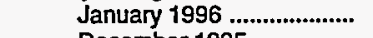 & W & w & - & 541.6 & 10.2 & - & - & 10.2 \\
\hline 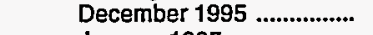 & 571.9 & - & - & 571.9 & 13.1 & - & - & 13.1 \\
\hline 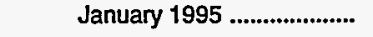 & 531.1 & - & - & 531.1 & w & - & - & w \\
\hline \multicolumn{9}{|l|}{ PAD District V } \\
\hline January 1996 ....................... & $6,825.4$ & $17,281.8$ & $11,309.8$ & $35,417.0$ & 715.5 & $4,002.1$ & $4,047.8$ & $8,765.4$ \\
\hline December 1995 .................. & $6,546.9$ & $18,091.2$ & $11,658.9$ & $36,297.0$ & 776.2 & $4,263.2$ & $4,198.2$ & $9,237.5$ \\
\hline January 1995 ......................... & $7,123.3$ & $18,327.5$ & $11,765.4$ & $37,216.2$ & 277.0 & $2,429.2$ & $3,494.5$ & $6,200.7$ \\
\hline \multicolumn{9}{|l|}{ Alaska } \\
\hline January 1996 & 358.8 & 123.1 & - & 481.9 & w & w & - & 49.1 \\
\hline December 1995 ......................... & 347.6 & 118.4 & - & 466.0 & w & w & - & 52.3 \\
\hline January 1995 ............................ & 353.5 & 102.6 & - & 456.2 & w & w & - & w \\
\hline \multicolumn{9}{|l|}{ Arizona } \\
\hline 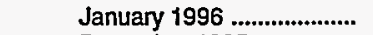 & w & $2,209.2$ & $w$ & $3,428.1$ & $\mathbf{w}$ & W & W & 565.5 \\
\hline December 1995 ..................... & $w$ & $2,284.5$ & W & $3,423.3$ & W & w & $\mathbf{w}$ & $\mathbf{5 7 9 . 5}$ \\
\hline January $1995 \ldots$ & $1,361.6$ & $2,517.7$ & - & $3,879.3$ & 35.5 & 102.9 & - & 138.3 \\
\hline \multicolumn{9}{|l|}{ California } \\
\hline 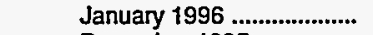 & 755.9 & $9,772.1$ & $11,289.3$ & $21,817.3$ & 53.8 & $2,502.7$ & $4,041.2$ & $6,597.7$ \\
\hline December 1995 .................. & 664.2 & $10,130.5$ & $11,640.3$ & $22,435.1$ & 64.4 & $2,650.8$ & $4,191.9$ & $6,907.1$ \\
\hline 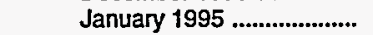 & 494.1 & $9,904.6$ & $11,765.4$ & $22,164.0$ & 38.1 & $2,077.6$ & $3,494.5$ & $5,610.2$ \\
\hline \multicolumn{9}{|l|}{ Hawaii } \\
\hline January 1996 & 555.4 & - & - & 555.4 & 127.3 & - & - & 127.3 \\
\hline 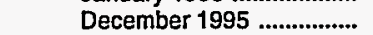 & 540.1 & - & - & 540.1 & 133.7 & - & - & 133.7 \\
\hline January 1995 ............................ & 520.3 & - & - & 520.3 & 116.0 & - & - & 116.0 \\
\hline \multicolumn{9}{|l|}{ Nevada } \\
\hline 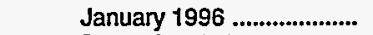 & w & 864.2 & W & $1,425.7$ & W & 182.9 & w & 228.0 \\
\hline December 1995 ................... & w & 926.2 & w & $1,420.5$ & $w$ & 188.8 & w & 238.3 \\
\hline 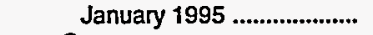 & 430.5 & 967.0 & - & $1,397.4$ & 32.5 & 139.6 & - & 172.1 \\
\hline \multicolumn{9}{|l|}{ Oregon } \\
\hline January 1996 ....................... & $1,433.0$ & $1,462.6$ & - & $2,895.6$ & 106.9 & 145.7 & - & 252.6 \\
\hline December 1995 .................... & $1,364.0$ & $1,548.2$ & - & $2,912.2$ & 108.1 & 171.4 & - & 279.5 \\
\hline 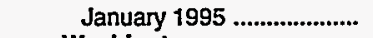 & $1,941.3$ & $1,469.0$ & - & $3,410.4$ & $w$ & w & - & w \\
\hline \multicolumn{9}{|l|}{ Washington } \\
\hline January 1996 ........................ & $1,962.5$ & $2,850.5$ & - & $4,813.1$ & 257.0 & 688.1 & - & 945.1 \\
\hline December 1995 .................. & $2,016.5$ & $3,083.4$ & - & $5,099.9$ & 289.0 & 758.3 & - & $1,047.3$ \\
\hline January 1995 ............................ & $2,022.0$ & $3,366.7$ & - & $5,388.6$ & w & w & - & w \\
\hline
\end{tabular}

See footnotes at end of table. 
Table 48. Prime Supplier Sales Volumes of Motor Gasoline by Grade, Formulation, PAD District, and State

(Thousand Gallons per Day) - Continued

\begin{tabular}{|c|c|c|c|c|c|c|c|c|}
\hline \multirow{2}{*}{$\begin{array}{l}\text { Geographic Area } \\
\text { Month }\end{array}$} & \multicolumn{4}{|c|}{ Premium } & \multicolumn{4}{|c|}{ All Grades } \\
\hline & Conventional & Oxygenated & Reformulated & Total & Conventional & Oxygenated & Reformulated & Total \\
\hline \multicolumn{9}{|l|}{ Idaho } \\
\hline January 1996 ......................... & 225.7 & - & - & 225.7 & $1,311.8$ & - & - & $1,311.8$ \\
\hline December 1995 ................... & 230.3 & - & - & 230.3 & $1,329.0$ & - & - & $1,329.0$ \\
\hline 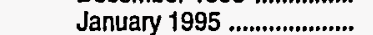 & 186.4 & - & - & 186.4 & $1,214.4$ & - & - & $1,214.4$ \\
\hline \multicolumn{9}{|l|}{ Montana } \\
\hline January 1996 .......................... & 191.4 & 15.1 & - & 206.5 & w & w & - & $1,071.6$ \\
\hline December 1995 .................. & $w$ & $w$ & - & 217.5 & $1,072.8$ & 74.5 & - & $1,147.3$ \\
\hline January 1995 ............................ & W & W & - & w & 986.5 & 63.2 & - & $1,049.6$ \\
\hline \multicolumn{9}{|l|}{ Utah } \\
\hline January 1996 ............................. & 514.7 & 31.2 & - & 545.9 & $2,346.8$ & 111.0 & - & $2,457.7$ \\
\hline December 1995 ................... & $w$ & w & - & 583.9 & $2,387.6$ & 252.4 & - & $2,640.0$ \\
\hline January 1995 ............................. & $w$ & w & - & 621.1 & $2,452.7$ & 136.5 & - & $2,589.2$ \\
\hline \multicolumn{9}{|l|}{ Wyoming } \\
\hline 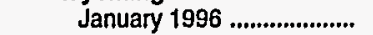 & 127.2 & - & - & 127.2 & $\mathbf{w}$ & $\mathbf{w}$ & - & 679.1 \\
\hline December 1995 ................... & 132.2 & - & - & 132.2 & 717.2 & - & - & 717.2 \\
\hline January 1995 ........................ & $w$ & - & - & w & 653.4 & - & - & 653.4 \\
\hline \multicolumn{9}{|l|}{ PAD District V } \\
\hline 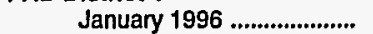 & $1,267.5$ & $4,319.2$ & $4,521.6$ & $10,108.4$ & $8,808.5$ & $25,603.1$ & $19,879.3$ & $54,290.8$ \\
\hline December 1995 ................... & $1,273.8$ & $4,625.4$ & $4,643.7$ & $10,542.8$ & $8,596.8$ & $26,979.7$ & $20,500.8$ & $56,077.3$ \\
\hline 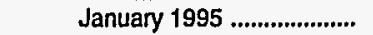 & $1,103.0$ & $4,109.5$ & $4,366.3$ & $9,578.8$ & $8,503.4$ & $24,866.3$ & $19,626.1$ & $52,995.7$ \\
\hline \multicolumn{9}{|l|}{ Alaska } \\
\hline January 1996 & $w$ & w & - & 78.7 & 443.3 & 166.4 & - & 609.7 \\
\hline 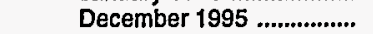 & W & $w$ & - & 79.8 & 436.9 & 161.2 & - & 598.0 \\
\hline January 1995 & w & $w$ & - & W & 403.2 & 135.5 & - & 538.6 \\
\hline \multicolumn{9}{|l|}{ Arizona } \\
\hline January 1996 & 157.5 & w & w & 766.9 & w & $3,282.9$ & $\mathbf{w}$ & $4,760.5$ \\
\hline December 1995 ................. & 145.4 & $w$ & $w$ & 797.7 & W & $3,411.8$ & $w$ & $4,800.4$ \\
\hline 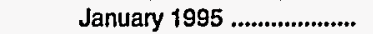 & 137.0 & 581.8 & - & 718.8 & $1,534.0$ & $3,202.4$ & - & $4,736.4$ \\
\hline \multicolumn{9}{|l|}{ California } \\
\hline January 1996 & 101.9 & $2,670.5$ & $4,517.1$ & $7,289.6$ & 911.7 & $14,945.3$ & $19,847.5$ & $35,704.6$ \\
\hline December 1995 .................... & 74.1 & $2,824.1$ & $4,639.1$ & $7,537.4$ & 802.7 & $15,605.5$ & $20,471.3$ & $36,879.5$ \\
\hline January 1995 ........................... & 68.1 & $2,464.7$ & $4,366.3$ & $6,899.1$ & 600.3 & $14,446.9$ & $19,626.1$ & $34,673.2$ \\
\hline \multicolumn{9}{|l|}{ Hawali } \\
\hline January 1996 & 303.2 & - & - & 303.2 & 985.9 & - & - & 985.9 \\
\hline December 1995 ................... & 312.9 & - & - & 312.9 & 986.7 & - & - & 986.7 \\
\hline January 1995 ............................ & 291.7 & - & - & 291.7 & 928.1 & - & - & 928.1 \\
\hline \multicolumn{9}{|l|}{ Nevada } \\
\hline January 1996 ........................ & W & 239.3 & $w$ & 326.2 & $w$ & $1,286.4$ & w & $1,979.9$ \\
\hline December 1995 .................... & 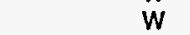 & 247.2 & $w$ & 362.3 & $w$ & $1,362.2$ & w & $2,021.0$ \\
\hline 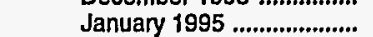 & 64.2 & 229.1 & - & 293.3 & 527.2 & $1,335.6$ & - & $1,862.8$ \\
\hline \multicolumn{9}{|l|}{ Oregon } \\
\hline January 1996 ......................... & 221.9 & 228.0 & - & 449.8 & $1,761.7$ & $1,836.3$ & - & $3,598.0$ \\
\hline December 1995 ................... & 186.9 & 253.7 & - & 440.6 & $1,659.0$ & $1,973.3$ & - & $3,632.3$ \\
\hline January 1995 & $w$ & W & - & W & $2,136.6$ & $1,711.3$ & - & $3,847.9$ \\
\hline \multicolumn{9}{|l|}{ Washington } \\
\hline January 1996 ............................ & 346.8 & 547.2 & - & 894.0 & $2,566.3$ & $4,085.8$ & - & $6,652.2$ \\
\hline December 1995 ................... & 388.1 & 624.1 & - & $1,012.2$ & $2,693.6$ & $4,465.8$ & - & $7,159.4$ \\
\hline January 1995 .......................... & w & w & - & w & $2,374.0$ & $4,034.6$ & - & $6,408.6$ \\
\hline
\end{tabular}

Dash $(-)=$ No data reported.

NA $=$ Not available.

$W=$ Withheld to avoid disclosure of individual company data.

a Includes leaded gasoline data.

Notes: Values shown for the current month are preliminary. Values shown for previous months are revised. Data are final upon publication in the Petroleum Marketing Annual. Totals may not equal the sum of the components due to rounding.

Source: Energy Information Administration, Form EIA-782C, "Monthly Report of Prime Supplier Sales of Petroleum Products Sold for Local Consumption." 
Table 49. Prime Supplier Sales Volumes of Aviation Fuels, Propane, and Residual Fuel Oil by PAD District and State

(Thousand Gallons per Day)

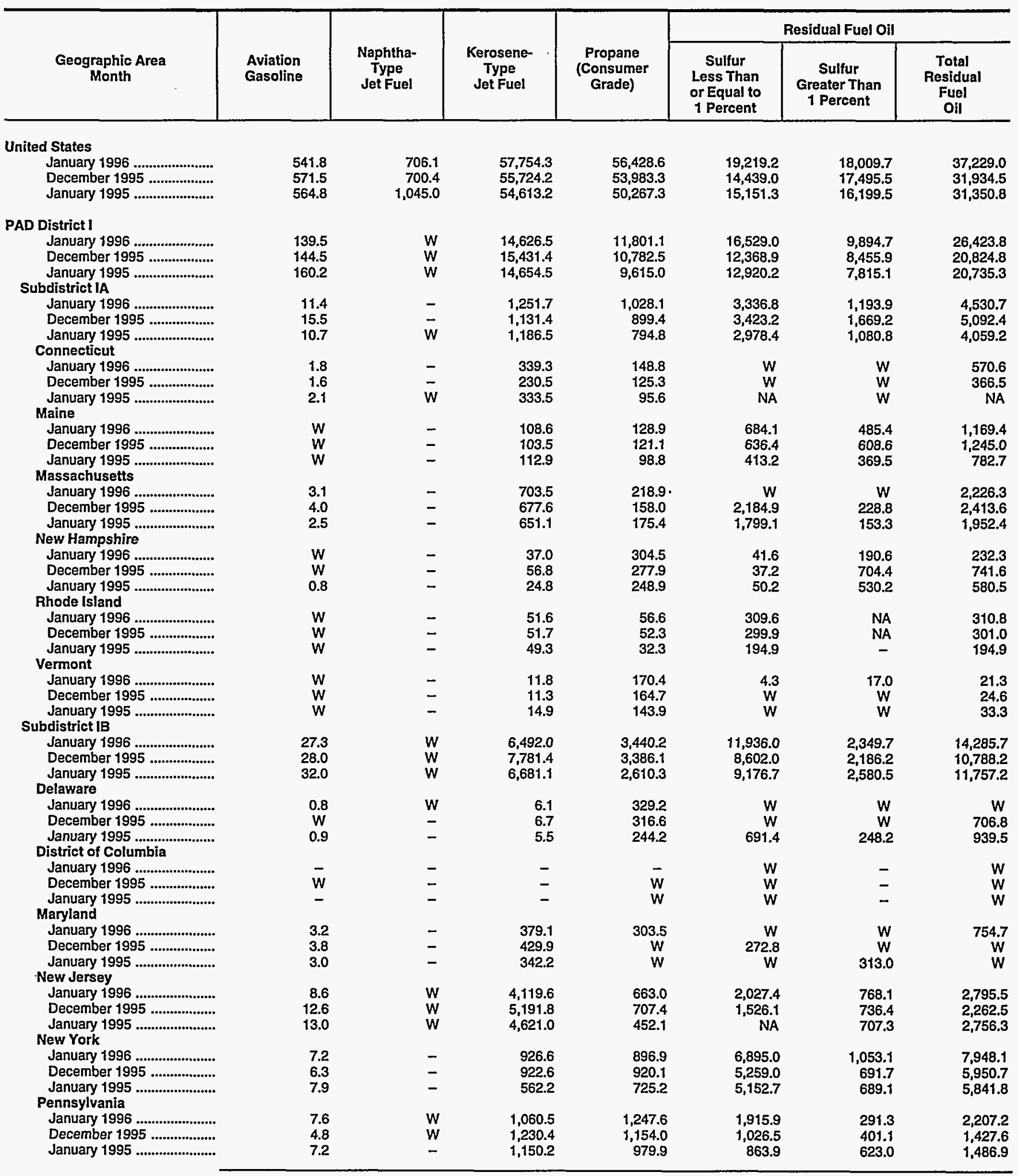

See footnotes at end of table. 
Table 49. Prime Supplier Sales Volumes of Aviation Fuels, Propane, and Residual Fuel Oil by PAD District and State

(Thousand Gallons per Day) - Continued

\begin{tabular}{|c|c|c|c|c|c|c|c|}
\hline \multirow[b]{2}{*}{$\begin{array}{l}\text { Geographic Area } \\
\text { Month }\end{array}$} & \multirow[b]{2}{*}{$\begin{array}{l}\text { Aviation } \\
\text { Gasoline }\end{array}$} & \multirow[b]{2}{*}{$\begin{array}{l}\text { Naphtha- } \\
\text { Type } \\
\text { Jet Fuel }\end{array}$} & \multirow[b]{2}{*}{$\begin{array}{l}\text { Kerosene- } \\
\text { Type } \\
\text { Jet Fuel }\end{array}$} & \multirow[b]{2}{*}{$\begin{array}{c}\text { Propane } \\
\text { (Consumer } \\
\text { Grade) }\end{array}$} & \multicolumn{3}{|c|}{ Residual Fuel Oil } \\
\hline & & & & & $\begin{array}{c}\text { Sulfur } \\
\text { Less Than } \\
\text { or Equal to } \\
1 \text { Percent }\end{array}$ & $\begin{array}{c}\text { Sulfur } \\
\text { Greater Than } \\
1 \text { Percent }\end{array}$ & $\begin{array}{c}\text { Total } \\
\text { Residual } \\
\text { Fuel } \\
\text { Oil } \\
\end{array}$ \\
\hline \multicolumn{8}{|l|}{ Subdistrict IC } \\
\hline January 1996 & 100.7 & - & $6,882.8$ & $7,332.8$ & $1,256.3$ & $6,351.1$ & $7,607.4$ \\
\hline December 1995 ..................... & 101.0 & w & $6,518.6$ & $6,497.0$ & 343.7 & $4,600.6$ & $4,944.3$ \\
\hline 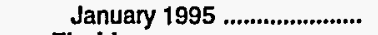 & 117.5 & - & $6,786.8$ & $6,209.8$ & 765.2 & $4,153.8$ & $4,918.9$ \\
\hline \multicolumn{8}{|l|}{ Florlda } \\
\hline January 1996 ........................... & 57.7 & - & $3,088.3$ & $1,767.3$ & 987.0 & $3,100.0$ & $4,087.0$ \\
\hline December 1995 ........................ & 57.2 & - & $2,998.4$ & $1,399.8$ & 206.5 & $2,360.8$ & $2,567.3$ \\
\hline January 1995 ............................. & & - & $3,335.3$ & $1,421.3$ & W & & $2,255.2$ \\
\hline \multicolumn{8}{|l|}{ Georgla } \\
\hline January 1996 ............................ & 16.3 & - & $1,981.2$ & $1,505.8$ & - & 611.6 & 611.6 \\
\hline December 1995 ........................ & 15.2 & w & $1,940.4$ & $1,352.8$ & - & 470.7 & 470.7 \\
\hline January 1995 .............................. & 17.4 & - & $1,758.5$ & $1,432.4$ & w & w & 466.5 \\
\hline \multicolumn{8}{|l|}{ North Carolina } \\
\hline 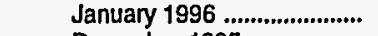 & 14.6 & - & 850.7 & $1,966.7$ & W & W & $1,092.2$ \\
\hline December 1995 ...................... & 17.1 & - & 593.7 & $1,828.7$ & w & $w$ & 829.9 \\
\hline January 1995 ............................. & 10.3 & - & 474.8 & $1,587.3$ & w & $w$ & 856.4 \\
\hline \multicolumn{8}{|l|}{ South Carolina } \\
\hline January 1996 ............................ & 6.3 & - & 101.0 & 849.6 & w & $W$ & $w$ \\
\hline December 1995 ..................... & 6.5 & - & 91.3 & 820.7 & $\mathbf{w}$ & w & $\mathbf{w}$ \\
\hline January 1995 ........................... & 8.4 & - & 100.8 & 777.8 & w & W & W \\
\hline \multicolumn{8}{|l|}{ Virginia } \\
\hline 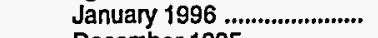 & w & - & 846.5 & $1,071.1$ & 180.0 & 831.0 & $1,011.0$ \\
\hline December 1995 .................... & w & - & 879.8 & 971.9 & 51.8 & 612.1 & 663.9 \\
\hline January 1995 ............................... & 9.2 & - & $1,104.4$ & 874.4 & 38.3 & 818.4 & 856.7 \\
\hline West Virginla & & & & & & & \\
\hline 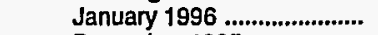 & $\mathbf{w}$ & - & 15.2 & 172.3 & w & 156.4 & w \\
\hline December 1995 ...................... & w & w & 15.0 & 123.0 & $w$ & $w$ & w \\
\hline January 1995 ........................... & 1.5 & - & 13.0 & 116.5 & w & $w$ & w \\
\hline PAD District II & & & & & & & \\
\hline January 1996 ............................... & 129.7 & w & $9,433.5$ & $22,540.9$ & w & $w$ & 707.3 \\
\hline December 1995 ...................... & 154.9 & w & $9,409.1$ & $21,814.9$ & w & $\mathbf{w}$ & 623.9 \\
\hline January 1995 .......................... & 136.5 & 292.4 & $9,033.4$ & $18,436.7$ & w & w & 554.5 \\
\hline Illinois & & & & & & & \\
\hline 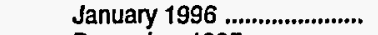 & w & - & $1,114.8$ & $2,716.2$ & w & w & w \\
\hline December 1995 ........................ & W & - & $1,023.0$ & $2,223.8$ & w & $w$ & 39.0 \\
\hline January $1995 \ldots . . . . . . . . . . . . . . . . . . . . . . .$. & W & - & 870.6 & $2,054.5$ & $w$ & - & w \\
\hline Indiana & & & & & & & \\
\hline January $1996 \ldots$ & 8.5 & - & $1,256.3$ & $1,592.2$ & $w$ & $w$ & 69.4 \\
\hline 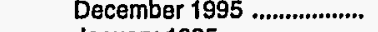 & 13.9 & - & $1,566.7$ & $1,534.7$ & w & w & 64.5 \\
\hline January 1995 .............................. & 10.9 & w & $1,766.8$ & $1,444.7$ & w & - & w \\
\hline lowa & & & & & & & \\
\hline 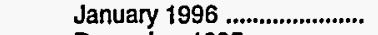 & W & - & 86.2 & $1,595.5$ & - & $w$ & W \\
\hline December 1995 ...................... & w & - & 107.8 & $1,445.2$ & - & w & w \\
\hline January 1995 ........................... & 6.3 & - & 114.7 & $1,506.0$ & - & w & w \\
\hline Kansas & & & & & & & \\
\hline 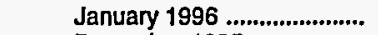 & 9.0 & - & 230.8 & $2,816.5$ & - & - & - \\
\hline December 1995 .................... & 8.9 & $\overline{-}$ & 259.6 & $3,608.0$ & - & - & - \\
\hline 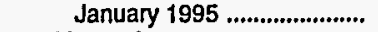 & 10.4 & w & 297.9 & $1,573.0$ & - & - & - \\
\hline Kentucky & & & & & & & \\
\hline 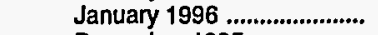 & 2.9 & - & 548.5 & $1,186.9$ & w & $\mathbf{w}$ & 23.9 \\
\hline December 1995 ...................... & 4.2 & - & 676.5 & $1,183.2$ & w & w & 18.0 \\
\hline January 1995 ............................. & 3.6 & - & 530.3 & $1,056.6$ & w & w & 4.9 \\
\hline Michigan & & & & & & & \\
\hline January $1996 \ldots . . . . . . . . . . . . . . . . . . . . .$. & 27.1 & $\bar{u}$ & $1,202.1$ & $2,082.2$ & - & 18.3 & 18.3 \\
\hline December 1995 .................... & 37.6 & W & 797.3 & $2,071.1$ & $\overline{-}$ & 32.4 & 32.4 \\
\hline January 1995 ............................. & 19.1 & - & 895.9 & $1,731.5$ & w & w & 50.6 \\
\hline Minnesota & & & & & & & \\
\hline January 1996 & W & - & 934.0 & $1,853.9$ & $\mathbf{w}$ & w & 230.5 \\
\hline December 1995 ........................ & w & - & 949.0 & $1,820.6$ & $w$ & $w$ & 267.1 \\
\hline 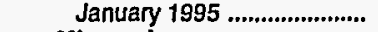 & 9.2 & - & 869.5 & $1,594.9$ & w & w & 173.3 \\
\hline Missouri & & & & & & & \\
\hline January 1996 ........................ & 9.6 & - & $1,135.2$ & $2,045.5$ & w & - & w \\
\hline December 1995 ..................... & 11.7 & - & $1,179.6$ & $1,945.3$ & $\overline{-}$ & - & $\overline{-}$ \\
\hline January 1995 ................................ & 9.5 & - & 956.8 & $2,138.8$ & w & - & w \\
\hline
\end{tabular}

See footnotes at end of table. 
Table 49. Prime Supplier Sales Volumes of Aviation Fuels, Propane, and Residual Fuel Oil by PAD District and State

(Thousand Gallons per Day) - Continued

\begin{tabular}{|c|c|c|c|c|c|c|c|}
\hline \multirow[b]{2}{*}{$\begin{array}{l}\text { Geographic Area } \\
\text { Month }\end{array}$} & \multirow[b]{2}{*}{$\begin{array}{l}\text { Aviation } \\
\text { Gasoline }\end{array}$} & \multirow[b]{2}{*}{$\begin{array}{l}\text { Naphtha- } \\
\text { Type } \\
\text { Jet Fuel }\end{array}$} & \multirow[b]{2}{*}{$\begin{array}{l}\text { Kerosene- } \\
\text { Type } \\
\text { Jet Fuel }\end{array}$} & \multirow[b]{2}{*}{$\begin{array}{l}\text { Propane } \\
\text { (Consumer } \\
\text { Grade) }\end{array}$} & \multicolumn{3}{|c|}{ Residual Fuel Oil } \\
\hline & & & & & $\begin{array}{l}\text { Sulfur } \\
\text { Less Than } \\
\text { or Equal to } \\
1 \text { Percent }\end{array}$ & $\begin{array}{c}\text { Sulfur } \\
\text { Greater Than } \\
1 \text { Percent }\end{array}$ & $\begin{array}{l}\text { Total } \\
\text { Residual } \\
\text { Fue! } \\
\text { Oil }\end{array}$ \\
\hline \multicolumn{8}{|l|}{ Nebraska } \\
\hline 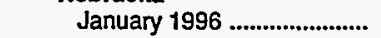 & 3.9 & - & 104.4 & 696.0 & - & $w$ & $w$ \\
\hline 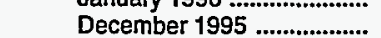 & 6.7 & - & 97.2 & 485.2 & - & $W$ & $\ddot{w}$ \\
\hline 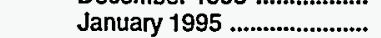 & 4.5 & - & 87.0 & 552.0 & - & $w$ & $w$ \\
\hline \multicolumn{8}{|l|}{ North Dakota } \\
\hline 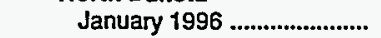 & 2.3 & - & 27.0 & 496.7 & - & - & - \\
\hline 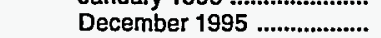 & 1.2 & - & 25.3 & 395.7 & - & - & - \\
\hline 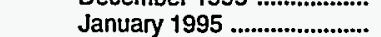 & 1.9 & - & 26.9 & 424.4 & - & - & - \\
\hline \multicolumn{8}{|l|}{ Ohio } \\
\hline January 1996 ....................... & 21.9 & $w$ & $1,045.5$ & $1,828.5$ & $w$ & $w$ & 76.5 \\
\hline 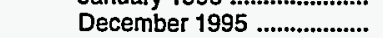 & 21.5 & $\dddot{W}$ & $1,047.3$ & $1,663.3$ & $W$ & $W$ & 36.0 \\
\hline 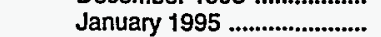 & 18.1 & $w$ & $1,072.9$ & $1,445.1$ & $w$ & $w$ & 45.0 \\
\hline \multicolumn{8}{|l|}{ Oklahoma } \\
\hline January 1996 & 8.3 & $w$ & 574.4 & 901.5 & - & $w$ & $w$ \\
\hline December 1995 ...................... & 9.1 & $\mathrm{~W}$ & 493.4 & 951.3 & - & $w$ & $w$ \\
\hline January 1995 ......................... & 7.4 & $w$ & 650.2 & 783.9 & $w$ & $w$ & $w$ \\
\hline \multicolumn{8}{|l|}{ South Dakota } \\
\hline January 1996 ....................... & $w$ & - & 52.3 & 470.1 & - & $w$ & $w$ \\
\hline 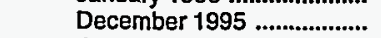 & W & - & 49.5 & 377.5 & - & $w$ & $w$ \\
\hline January 1995 ........................ & $w$ & - & 22.8 & 353.5 & - & - & - \\
\hline \multicolumn{8}{|l|}{ Tennessee } \\
\hline January 1996 ....................... & 12.5 & - & 933.1 & 694.8 & $\mathbf{w}$ & $w$ & 54.6 \\
\hline December 1995 ........................ & 13.5 & - & 963.7 & 493.4 & $w$ & $w$ & 22.0 \\
\hline 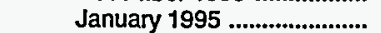 & 13.9 & - & 728.2 & 472.2 & $w$ & $w$ & 51.3 \\
\hline Wisconsin & & & & & & & \\
\hline January 1996 ....................... & $w$ & - & 189.0 & $1,564.2$ & - & 158.7 & 158.7 \\
\hline December 1995 ..................... & $w$ & - & 173.0 & $1,616.7$ & - & 131.8 & 131.8 \\
\hline 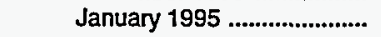 & 8.2 & - & 142.9 & $1,305.5$ & $w$ & $W$ & W \\
\hline PAD District III & & & & & & & \\
\hline January 1996 ........................ & 111.0 & 160.5 & $14,338.1$ & $17,182.2$ & 950.3 & $3,033.0$ & $3,983.3$ \\
\hline December 1995 .................... & 107.2 & 142.7 & $12,745.6$ & $16,860.6$ & 624.6 & $3,803.8$ & $4,428.4$ \\
\hline 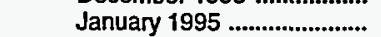 & 110.5 & 198.4 & $13,972.6$ & $17,627.8$ & $1,072.2$ & $4,460.0$ & $5,532.1$ \\
\hline Alabama & & & & & & & \\
\hline January 1996 ........................ & 11.8 & $W$ & 381.5 & 786.7 & W & $W$ & 285.9 \\
\hline December 1995 ................... & 9.8 & $\ddot{w}$ & 321.0 & 829.7 & $w$ & $w$ & 251.8 \\
\hline 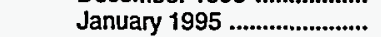 & 8.9 & $\ddot{W}$ & 477.6 & 806.4 & $w$ & $w$ & 283.6 \\
\hline Arkansas & & & & & & & \\
\hline 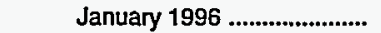 & 7.5 & - & 107.8 & 860.9 & - & W & $w$ \\
\hline December 1995 .................... & W & - & 136.8 & 723.0 & - & - & - \\
\hline January 1995 ........................ & 8.4 & - & 107.6 & 708.2 & - & - & - \\
\hline Louisiana & & & & & & & \\
\hline 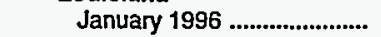 & 8.4 & - & $2,909.3$ & $1,974.8$ & $w$ & $w$ & $1,687.0$ \\
\hline 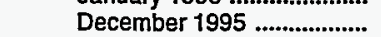 & 8.7 & - & $2,403.5$ & $2,413.4$ & $w$ & $w$ & $2,237.3$ \\
\hline 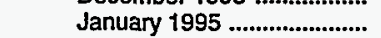 & 8.2 & - & $3,163.0$ & $2,192.6$ & - & $2,273.0$ & $2,273.0$ \\
\hline Mississippi & & & & & & & \\
\hline January 1996 ...................... & 4.4 & $w$ & 930.2 & $1,542.1$ & - & $w$ & $w$ \\
\hline December 1995 ................... & $\ddot{w}$ & $w$ & 908.7 & $1,575.3$ & - & $w$ & $w$ \\
\hline 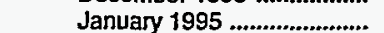 & 7.3 & $w$ & 568.1 & $1,200.4$ & - & $w$ & $\bar{w}$ \\
\hline New Mexico & & & & & & & \\
\hline January 1996 & 9.9 & $w$ & 164.9 & 670.2 & $w$ & - & $w$ \\
\hline December 1995 & 9.4 & $\ddot{w}$ & 161.2 & 590.7 & $w$ & - & $\ddot{w}$ \\
\hline 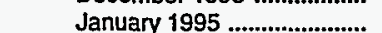 & 8.8 & $\dddot{w}$ & 262.1 & 676.0 & $w$ & - & $w$ \\
\hline Texas & & & & & & & \\
\hline 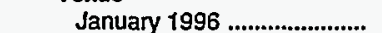 & 69.1 & $w$ & $9,844.5$ & $11,347.5$ & 824.8 & $1,140.8$ & $1,965.6$ \\
\hline December 1995 .................... & 67.9 & $W$ & $8,814.5$ & $10,728.4$ & 489.8 & $1,410.5$ & $1,900.3$ \\
\hline January 1995 & 68.8 & w & $9,394.2$ & $12,044.1$ & $1,035.9$ & $1,893.5$ & $2,929.4$ \\
\hline PAD District IV & & & & & & & \\
\hline January 1996 & 27.5 & $w$ & $1,788.8$ & $1,621.7$ & $w$ & $w$ & 24.3 \\
\hline December 1995 ................... & 27.1 & NA & $1,668.6$ & $1,525.5$ & $w$ & $w$ & 45.1 \\
\hline 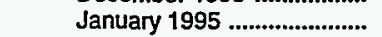 & 25.8 & 272.1 & $1,246.9$ & $1,507.8$ & W & $w$ & 84.2 \\
\hline Colorado & & & & & & & \\
\hline January 1996 ......................... & 13.8 & - & 899.7 & 564.3 & - & - & - \\
\hline December 1995 .................... & 13.2 & - & 670.5 & 478.6 & - & - & - \\
\hline January 1995 ........................ & 13.1 & - & 765.4 & 539.0 & - & - & - \\
\hline
\end{tabular}

See footnotes at end of table. 
Table 49. Prime Supplier Sales Volumes of Aviation Fuels, Propane, and Residual Fuel Oil by PAD District and State

(Thousand Gallons per Day) - Continued

\begin{tabular}{|c|c|c|c|c|c|c|c|}
\hline \multirow[b]{2}{*}{$\begin{array}{c}\text { Geographic Area } \\
\text { Month }\end{array}$} & \multirow[b]{2}{*}{$\begin{array}{l}\text { Aviation } \\
\text { Gasoline }\end{array}$} & \multirow[b]{2}{*}{$\begin{array}{l}\text { Naphtha- } \\
\text { Type } \\
\text { Jet Fuel }\end{array}$} & \multirow[b]{2}{*}{$\begin{array}{c}\text { Kerosene- } \\
\text { Type } \\
\text { Jet Fuel }\end{array}$} & \multirow[b]{2}{*}{$\begin{array}{c}\text { Propane } \\
\text { (Consumer } \\
\text { Grade) }\end{array}$} & \multicolumn{3}{|c|}{ Residual Fuel Oil } \\
\hline & & & & & $\begin{array}{l}\text { Sulfur } \\
\text { Less Than } \\
\text { or Equal to } \\
1 \text { Percent }\end{array}$ & $\begin{array}{c}\text { Sulfur } \\
\text { Greater Than } \\
1 \text { Percent }\end{array}$ & $\begin{array}{c}\text { Total } \\
\text { Residual } \\
\text { Fuel } \\
\text { Oil }\end{array}$ \\
\hline \multicolumn{8}{|l|}{ Idaho } \\
\hline January 1996 & $w$ & - & 74.9 & 107.7 & - & - & - \\
\hline December 1995 ................... & 2.0 & - & 81.4 & 113.4 & - & - & - \\
\hline January 1995 ...................... & 0.9 & - & 58.1 & 116.2 & - & - & - \\
\hline \multicolumn{8}{|l|}{ Montana } \\
\hline January 1996 ...................... & $W$ & - & 148.8 & 275.3 & - & $w$ & $w$ \\
\hline December 1995 .................. & $w$ & $\rightarrow$ & 175.3 & 249.1 & - & $w$ & W \\
\hline January 1995 .......................... & W & $W$ & 59.1 & 201.2 & - & $w$ & $w$ \\
\hline \multicolumn{8}{|l|}{ Utah } \\
\hline January 1996 ...................... & 5.2 & $w$ & 653.2 & 317.5 & NA & $w$ & $w$ \\
\hline December 1995 .................... & 5.5 & NA & 730.3 & 266.0 & $W$ & $w$ & 10.0 \\
\hline January 1995 ........................ & 4.9 & 175.0 & 354.9 & 284.0 & W & $W$ & W \\
\hline \multicolumn{8}{|l|}{ Wyoming } \\
\hline January 1996 ..................... & 1.9 & - & 12.2 & 356.9 & $W$ & - & $w$ \\
\hline December 1995 ................... & $w$ & - & 11.1 & 418.5 & W & $w$ & w \\
\hline January 1995 ......................... & W & $W$ & 9.5 & 367.5 & $W$ & W & $w$ \\
\hline \multicolumn{8}{|l|}{ PAD District $V$} \\
\hline January 1996 & 134.1 & 61.3 & $17,567.4$ & $3,282.7$ & $1,644.3$ & $4,446.0$ & $6,090.4$ \\
\hline December 1995 ................... & 137.8 & 48.1 & $16,469.5$ & $2,999.7$ & $1,379.1$ & $4,633.2$ & $6,012.3$ \\
\hline January 1995 ....................... & 131.7 & W & $15,705.8$ & $3,080.0$ & $1,070.3$ & $3,374.4$ & $4,444.7$ \\
\hline \multicolumn{8}{|l|}{ Alaska } \\
\hline January 1996 & 18.8 & $W$ & $1,564.6$ & $W$ & - & - & - \\
\hline December 1995 .................. & 22.1 & $W$ & $1,652.5$ & $W$ & - & - & - \\
\hline January 1995 ....................... & 23.2 & $W$ & $1,285.3$ & $W$ & - & - & - \\
\hline \multicolumn{8}{|l|}{ Arizona } \\
\hline January 1996 ....................... & 16.9 & - & 955.2 & 257.7 & $w$ & $w$ & 10.2 \\
\hline December 1995 .................... & 15.6 & $W$ & 885.3 & 158.5 & $W$ & $W$ & W \\
\hline January 1995 ...................... & 15.7 & - & 765.0 & 247.5 & $W$ & $w$ & 9.1 \\
\hline \multicolumn{8}{|l|}{ California } \\
\hline January 1996 ........................ & 72.3 & $W$ & $10,799.0$ & $1,742.3$ & 453.5 & $2,124.8$ & $2,578.2$ \\
\hline December 1995 .................... & 67.7 & $W$ & $9,421.8$ & $1,662.7$ & 428.5 & $1,771.2$ & $2,199.7$ \\
\hline January 1995 ....................... & 59.9 & $w$ & $9,436.5$ & $1,762.8$ & W & $W$ & $1,844.0$ \\
\hline \multicolumn{8}{|l|}{ Hawail } \\
\hline January 1996 ....................... & $W$ & - & $1,085.4$ & $W$ & $W$ & $W$ & $W$ \\
\hline December 1995 .................... & $W$ & - & 988.2 & $W$ & $W$ & $W$ & $1,151.5$ \\
\hline January 1995 ........................ & $w$ & - & 988.5 & $W$ & W & $w$ & $1,042.5$ \\
\hline \multicolumn{8}{|l|}{ Nevada } \\
\hline January 1996 ....................... & 7.5 & - & 783.0 & 216.8 & - & - & - \\
\hline December 1995 .................... & 2.3 & - & 670.4 & 158.9 & $w$ & - & $W$ \\
\hline January 1995 ......................... & $W$ & - & 714.7 & 162.9 & $W$ & - & $w$ \\
\hline \multicolumn{8}{|l|}{ Oregon } \\
\hline January 1996 ...................... & 9.3 & - & 466.9 & 153.7 & $W$ & NA & $w$ \\
\hline December 1995 ..................... & $w$ & - & 519.8 & 167.8 & w & $W$ & 379.2 \\
\hline January 1995 ....................... & 14.7 & - & 464.6 & 149.3 & - & $w$ & W \\
\hline \multicolumn{8}{|l|}{ Washington } \\
\hline January 1996 & W & - & $1,913.4$ & 652.4 & - & $1,757.2$ & $1,757.2$ \\
\hline December 1995 ...................... & 17.0 & W & $2,331.5$ & 585.3 & $w$ & $W$ & $2,267.2$ \\
\hline January 1995 ......................... & 6.8 & - & $2,051.1$ & 527.7 & - & $1,247.6$ & $1,247.6$ \\
\hline
\end{tabular}

Dash $(-)=$ No data reported.

NA $=$ Not available.

$W=$ Withheld to avoid disclosure of individual company data

Notes: Values shown for the current month are preliminary. Values shown for previous months are revised. Data are final upon publication in the Petroleum Marketing Annual. Totals may not equal the sum of the components due to rounding.

Source: Energy Information Administration, Form ElA-782C, "Monthly Report of Prime Supplier Sales of Pelroleum Products Sold for Local Consumption." 
Table 50. Prime Supplier Sales Volumes of Distillate Fuel Oils and Kerosene by PAD District and State

(Thousand Gallons per Day)

\begin{tabular}{|c|c|c|c|c|c|c|c|c|c|}
\hline \multirow{3}{*}{$\begin{array}{c}\text { Geographic Area } \\
\text { Month }\end{array}$} & \multirow{3}{*}{ Kerosene } & \multirow{3}{*}{$\begin{array}{c}\text { No. } 1 \\
\text { Distillate }\end{array}$} & \multicolumn{5}{|c|}{ No. 2 Distillate } & \multirow{3}{*}{ No. 4 Fuela } & \multirow{3}{*}{$\begin{array}{c}\text { Total } \\
\text { Distillate } \\
\text { and } \\
\text { Kerosene }\end{array}$} \\
\hline & & & \multirow{2}{*}{$\begin{array}{l}\text { No. } 2 \text { Fuel } \\
\text { Oil }\end{array}$} & \multicolumn{3}{|c|}{ No. 2 Diesel Fuel } & \multirow{2}{*}{$\begin{array}{c}\text { No. } 2 \\
\text { Distillate }\end{array}$} & & \\
\hline & & & & Low-Sulfur & High-Sulfur & Total & & & \\
\hline \multicolumn{10}{|l|}{ United States } \\
\hline January 1996 & $7,569.6$ & $5,084.5$ & $58,012.7$ & $71,253.2$ & $20,688.2$ & $91,941.4$ & $149,954.0$ & $2,299.7$ & $164,907.9$ \\
\hline December 1995 ............. & $6,421.1$ & $4,363.1$ & $53,052.8$ & $69,969.0$ & $20,459.6$ & $90,428.6$ & $143,481.4$ & $1,837.7$ & $156,103.3$ \\
\hline January 1995 ................. & $5,977.6$ & $4,393.7$ & $48,276.7$ & $66,012.5$ & $18,769.3$ & $84,781.8$ & $133,058.5$ & $2,091.0$ & $145,520.9$ \\
\hline \multicolumn{10}{|l|}{ PAD District I } \\
\hline January 1996 & $5,361.1$ & 304.5 & $46,542.1$ & $21,688.8$ & $5,301.5$ & $26,990.4$ & $73,532.5$ & $1,986.4$ & $81,184.5$ \\
\hline December 1995 ............. & $4,663.3$ & 257.8 & $42,109.3$ & $21,186.9$ & $4,693.2$ & $25,880.1$ & $67,989.4$ & $1,706.2$ & $74,616.7$ \\
\hline January 1995 .................. & $4,103.7$ & 305.5 & $36,734.4$ & $19,945.7$ & $3,872.9$ & $23,818.6$ & $60,553.0$ & $1,794.9$ & $66,757.2$ \\
\hline Subdistrict IA & & & & & & & & & \\
\hline January 1996 ................. & 781.4 & 19.4 & $14,093.2$ & $2,342.3$ & 256.1 & $2,598.4$ & $16,691.6$ & 460.2 & $17,952.6$ \\
\hline December 1995 ............. & 681.9 & 15.1 & $13,796.8$ & $2,461.4$ & 108.5 & $2,569.9$ & $16,366.7$ & 378.9 & $17,442.5$ \\
\hline January 1995 & 551.6 & 27.1 & $11,417.5$ & $2,037.1$ & 34.4 & $2,071.4$ & $13,489.0$ & 442.4 & $14,510.0$ \\
\hline Connecticut & & & & & & & & & \\
\hline January 1996 & 116.7 & $W$ & $3,717.7$ & $W$ & $W$ & 498.6 & $4,216.3$ & $W$ & $4,464.6$ \\
\hline December 1995 ............. & 110.5 & w & $3,492.8$ & $w$ & w & 522.9 & $4,015.7$ & W & $4,224.8$ \\
\hline January 1995 ................ & 70.6 & W & $2,678.4$ & $w$ & W & 466.8 & $3,145.3$ & $W$ & $3,282,5$ \\
\hline Maine & & & & & & & & & \\
\hline January 1996 & 333.5 & 2.0 & $1,880.7$ & 407.8 & 7.3 & 415.1 & $2,295.7$ & 27.1 & $2,658.3$ \\
\hline December 1995 ............. & 272.2 & 1.7 & $1,807.6$ & 395.1 & 7.0 & 402.1 & $2,209.7$ & 20.5 & $2,504.2$ \\
\hline January 1995 & 223.4 & 4.4 & $1,558.7$ & 364.8 & 5.3 & 370.1 & $1,928.8$ & 31.0 & $2,187.5$ \\
\hline Massachusetts & & & & & & & & & \\
\hline January 1996 & 129.2 & 1.8 & $5,177.3$ & 820.9 & 123.0 & 943.9 & $6,121.3$ & 185.5 & $6,437.7$ \\
\hline December 1995 .............. & 122.5 & 2.9 & $5,176.5$ & 829.7 & 41.2 & 870.9 & $6,047.4$ & 150.8 & $6,323.7$ \\
\hline January 1995 ................. & $w$ & W & $4,509.3$ & 671.3 & 22.2 & 693.5 & $5,202.8$ & 260.8 & $5,582.4$ \\
\hline New Hampshire & & & & & & & & & \\
\hline January 1996 & 149.9 & 3.2 & $1,380.5$ & $w$ & $w$ & 293.6 & $1,674.1$ & 56.1 & $1,883.4$ \\
\hline December 1995 ............ & 135.3 & $\bar{W}$ & $1,485.8$ & w & $\mathbf{w}$ & 328.4 & $1,814.1$ & $W$ & $2,001.7$ \\
\hline January 1995 ................ & 94.6 & $w$ & $1,101.9$ & w & $w$ & 290.1 & $1,392.0$ & $W$ & $1,523.8$ \\
\hline Rhode Island & & & & & & & & & \\
\hline January 1996 & NA & $w$ & $1,618.6$ & $w$ & $W$ & 353.7 & $1,972.3$ & $w$ & $2,061.3$ \\
\hline December 1995 ............. & NA & $W$ & $1,551.6$ & W & W & 354.1 & $1,905.7$ & $W$ & $1,979.6$ \\
\hline January 1995 ................. & W & $\mathbf{W}$ & $1,313.2$ & W & W & 168.4 & $1,481.5$ & 45.8 & $1,549.2$ \\
\hline Vermont & & & & & & & & & \\
\hline January 1996 .................. & 26.4 & W & 318.4 & 84.8 & 8.7 & 93.5 & 411.8 & $W$ & 447.3 \\
\hline December 1995 ............. & 25.1 & $W$ & 282.5 & 82.5 & 9.1 & 91.5 & 374.1 & w & 408.5 \\
\hline January 1995 & 33.2 & 0.8 & 256.1 & 77.2 & 5.4 & 82.5 & 338.6 & 12.0 & 384.7 \\
\hline Subdistrict IB & & & & & & & & & \\
\hline January 1996 ................ & $2,434.5$ & 264.4 & $26,072.2$ & $7,551.1$ & $1,278.5$ & $8,829.6$ & $34,901.8$ & $1,456.8$ & $39,057.6$ \\
\hline December 1995 .............. & $2,189.6$ & 225.5 & $22,957.6$ & $7,657.4$ & $1,206.7$ & $8,864.2$ & $31,821.8$ & $1,256.2$ & $35,493.2$ \\
\hline January 1995 ................ & $1,805.9$ & 262.2 & $19,944.3$ & $7,144.7$ & $1,047.8$ & $8,192.6$ & $28,136.9$ & $1,255.1$ & $31,460.0$ \\
\hline Delaware & & & & & & & & & \\
\hline January 1996 ................. & 67.4 & $w$ & 560.6 & $w$ & $w$ & 286.0 & 846.6 & $w$ & 914.4 \\
\hline December 1995 ............. & $w$ & - & 386.8 & w & W & 258.6 & 645.4 & w & 676.5 \\
\hline January 1995 ................. & W & - & 559.2 & $W$ & W & 215.5 & 774.7 & W & 809.2 \\
\hline District of Columbia & & & & & & & & & \\
\hline January 1996 & $\mathbf{W}$ & - & 293.0 & $w$ & $w$ & 91.9 & 384.8 & $w$ & 515.6 \\
\hline December 1995 ............. & $\ddot{w}$ & - & 195.1 & W & W & 55.2 & 250.3 & $\ddot{w}$ & 308.6 \\
\hline 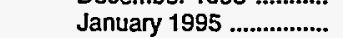 & $w$ & - & 176.5 & W & $\ddot{W}$ & 32.5 & 209.0 & $w$ & 283.3 \\
\hline Maryland & & & & & & & & & \\
\hline January 1996 ................ & 234.5 & $W$ & $2,273.3$ & 896.4 & 268.9 & $1,165.3$ & $3,438.6$ & W & $3,708.6$ \\
\hline December 1995 ............. & 200.8 & $w$ & $1,653.0$ & 933.9 & 251.7 & $1,185.6$ & $2,838.6$ & w & $3,072.4$ \\
\hline January 1995 .................. & 165.9 & $w$ & $1,428.6$ & 816.1 & 212.2 & $1,028.4$ & $2,457.0$ & W & $2,655.5$ \\
\hline New Jersey & & & & & & & & & \\
\hline January 1996 ................. & 515.5 & 10.5 & $7,895.6$ & $1,944.0$ & 431.3 & $2,375.3$ & $10,270.9$ & 219.1 & $11,016.1$ \\
\hline December 1995 ............. & 500.1 & 13.5 & $7,500.6$ & $1,981.1$ & 486.5 & $2,467.7$ & $9,968.3$ & 156.4 & $10,638.3$ \\
\hline 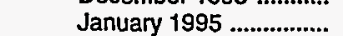 & 331.2 & 28.1 & $5,927.7$ & $1,930.4$ & 376.8 & $2,307.1$ & $8,234.8$ & 160.3 & $8,754.5$ \\
\hline New York & & & & & & & & & \\
\hline January 1996 ................. & 811.6 & 206.6 & $9,058.8$ & $1,537.0$ & 68.1 & $1,605.1$ & $10,663.9$ & $1,097.3$ & $12,779.5$ \\
\hline December 1995 ............. & 681.4 & 178.5 & $8,150.3$ & $1,552.7$ & 63.4 & $1,616.1$ & $9,766.4$ & $1,028.5$ & $11,654.7$ \\
\hline January 1995 ................. & 621.8 & 147.5 & $7,477.0$ & $1,501.8$ & 62.1 & $1,563.8$ & $9,040.8$ & 992.0 & $10,802.2$ \\
\hline Pennsylvania & & & & & & & & & \\
\hline January 1996 ................. & $w$ & 35.4 & $5,990.9$ & $2,813.6$ & 492.5 & $3,306.1$ & $9,297.0$ & $w$ & $10,123.6$ \\
\hline December 1995 ............ & 754.0 & $w$ & $5,071.7$ & $2,881.8$ & 399.2 & $3,281.0$ & $8,352.7$ & $w$ & $9,142.8$ \\
\hline January 1995 .................. & 652.7 & W & $4,375.3$ & $2,665.3$ & 379.9 & $3,045.2$ & $7,420.5$ & $w$ & $8,155.3$ \\
\hline
\end{tabular}

See footnotes at end of table. 
Table 50. Prime Supplier Sales Volumes of Distillate Fuel Oils and Kerosene by PAD District and State

(Thousand Gallons per Day) - Continued

\begin{tabular}{|c|c|c|c|c|c|c|c|c|c|}
\hline \multirow{3}{*}{$\begin{array}{c}\text { Geographic Area } \\
\text { Month }\end{array}$} & \multirow{3}{*}{ Kerosene } & \multirow{3}{*}{$\begin{array}{c}\text { No. } 1 \\
\text { Distillate }\end{array}$} & \multicolumn{5}{|c|}{ No. 2 Distillate } & \multirow{3}{*}{ No. 4 Fuela } & \multirow{3}{*}{$\begin{array}{c}\text { Total } \\
\text { Distillate } \\
\text { and } \\
\text { Kerosene }\end{array}$} \\
\hline & & & \multirow{2}{*}{$\begin{array}{c}\text { No. } 2 \text { Fuel } \\
\text { Oil }\end{array}$} & \multicolumn{3}{|c|}{ No. 2 Diesel Fuel } & \multirow{2}{*}{$\begin{array}{c}\text { No. } 2 \\
\text { Distillate }\end{array}$} & & \\
\hline & & & & Low-Sulfur & High-Suifur & Total & & & \\
\hline \multicolumn{10}{|l|}{ Subdistrict IC } \\
\hline January 1996 & $2,145.2$ & 20.7 & $6,376.6$ & $11,795.4$ & $3,767.0$ & $15,562.4$ & $21,939.0$ & 69.4 & $24,174.3$ \\
\hline December 1995 ............ & $1,791.7$ & 17.2 & $5,354.9$ & $11,068.1$ & $3,378.0$ & 14.446 .1 & $19,801.0$ & 71.1 & $21,681.0$ \\
\hline \multicolumn{10}{|l|}{ Florida } \\
\hline January 1996 & $w$ & $w$ & 764.4 & $3,399.9$ & $1,273.3$ & $4,673.2$ & $5,437.6$ & $w$ & $5,571.3$ \\
\hline December 1995 & 117.2 & $w$ & 705.2 & $3,068.3$ & $1,342.5$ & $4,410.8$ & $5,115.9$ & $w$ & $5,246.5$ \\
\hline January 1995 ................. & 124.3 & W & 760.2 & $2,899.0$ & $1,082.1$ & $3,981.0$ & $4,741.2$ & W & $4,876.5$ \\
\hline \multicolumn{10}{|l|}{ Georgia } \\
\hline January 1996 & 154.0 & $w$ & 650.5 & $2,987.5$ & 596.5 & $3,583.9$ & $4,234.5$ & W & $4,392.5$ \\
\hline December 1995 ............ & 112.9 & 1.2 & 598.7 & $2,630.8$ & 459.3 & $3,090.0$ & $3,688.7$ & - & $3,802.8$ \\
\hline January 1995 ................ & 120.1 & $\vec{W}$ & 673.7 & $2,534.4$ & 298.0 & $2,832.4$ & $3,506.1$ & $W$ & $3,627.8$ \\
\hline North Carolina & & & & & & & & & \\
\hline January 1996 ................ & 802.3 & $w$ & $1,521.9$ & $2,010.9$ & 824.9 & $2,835.7$ & $4,357.6$ & W & $5,173.8$ \\
\hline December 1995 ............ & 701.9 & $w$ & $1,382.3$ & $2,016.1$ & 649.9 & $2,666.0$ & $4,048.3$ & W & $4,760.5$ \\
\hline January 1995 ................. & 650.6 & $w$ & $1,318.7$ & $2,045.4$ & 627.7 & $2,673.2$ & $3,991.9$ & $\ddot{W}$ & $4,652.6$ \\
\hline South Carolina & & & & & & & & & \\
\hline January 1996 ................ & $w$ & - & 607.7 & $1,101.6$ & 176.0 & $1,277.6$ & $1,885.3$ & $w$ & $2,263.1$ \\
\hline December 1995 ........... & 316.7 & $w$ & 462.8 & $1,011.3$ & 194.3 & $1,205.6$ & $1,668.4$ & $W$ & $1,986.7$ \\
\hline January 1995 ................. & 314.2 & W & 478.2 & $1,058.6$ & 151.5 & $1,210.1$ & $1,688.3$ & $\ddot{W}$ & $2,003.9$ \\
\hline Virginia & & & & & & & & & \\
\hline January 1996 ................ & 569.3 & 8.8 & $2,411.7$ & $1,882.9$ & 720.6 & $2,603.5$ & $5,015.2$ & 42.0 & $5,635.3$ \\
\hline December 1995 ........... & 446.6 & 6.8 & $1,827.1$ & $1,903.5$ & 549.2 & $2,452.7$ & $4,279.8$ & 51.3 & $4,784.4$ \\
\hline 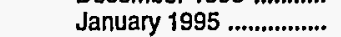 & 436.4 & 2.8 & $1,735.8$ & $1,806.8$ & 461.7 & $2,268.5$ & $4,004.4$ & 83.1 & $4,526.6$ \\
\hline West Virginia & & & & & & & & & \\
\hline January 1996 ................ & 126.7 & $w$ & 420.4 & 412.7 & 175.7 & 588.4 & $1,008.8$ & $W$ & $1,138.2$ \\
\hline 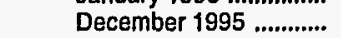 & 96.5 & $\ddot{w}$ & 378.9 & 438.1 & 182.9 & 621.0 & 999.9 & W & $1,100.2$ \\
\hline January 1995 ................. & 100.7 & W & 405.9 & 419.7 & 169.6 & 589.4 & 995.3 & W & $1,099.7$ \\
\hline PAD District II & & & & & & & & & \\
\hline January 1996 & $W$ & $3,468.7$ & $9,551.8$ & $22,022.5$ & $4,710.3$ & $26,732.8$ & $36,284.6$ & W & $41,625.4$ \\
\hline December 1995 ............ & $w$ & $3,125.2$ & $9,122.1$ & $22,062.9$ & $5,087.5$ & $27,150.4$ & $36,272.5$ & W & $40,863.1$ \\
\hline January 1995 ................. & W & $3,002.9$ & $9,066.2$ & $20,316.4$ & $4,666.4$ & $24,982.8$ & $34,049.0$ & $W$ & $38,552.0$ \\
\hline Illinois & & & & & & & & & \\
\hline January 1996 ................ & 89.3 & 399.5 & $1,162.2$ & $1,672.0$ & 400.2 & $2,072.2$ & $3,234.4$ & - & $3,723.2$ \\
\hline December 1995 ............ & 115.3 & 391.7 & $1,292.4$ & $2,159.1$ & 486.2 & $2,645.3$ & $3,937.6$ & - & $4,444.6$ \\
\hline January 1995 .................. & W & 415.8 & $1,233.9$ & $1,667.5$ & 356.4 & $2,023.8$ & $3,257.7$ & W & $3,780.8$ \\
\hline $\begin{array}{l}\text { Indiana } \\
\quad \text { January } 1996\end{array}$ & & & & & & & & & \\
\hline $\begin{array}{l}\text { January } 1996 \text {................. } \\
\text { December } 1995 \text {............ }\end{array}$ & $\begin{array}{r}W \\
2219\end{array}$ & 249.5 & $\begin{array}{l}1,224.3 \\
1218.1\end{array}$ & $2,450.8$ & 398.0 & $2,848.8$ & $4,073.1$ & $W$ & $4,576.1$ \\
\hline $\begin{array}{l}\text { December } 1995 \text {............. } \\
\text { January } 1995 \text {............... }\end{array}$ & $\begin{array}{r}221.9 \\
W\end{array}$ & 231.4 & $\begin{array}{l}1,218.1 \\
1,245.3\end{array}$ & $\begin{array}{l}2,347.1 \\
2,2375\end{array}$ & $\begin{array}{l}350.1 \\
5024\end{array}$ & $2,697.3$ & $3,915.3$ & $\bar{w}$ & $4,368.6$ \\
\hline 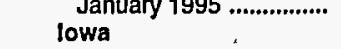 & W & 263.3 & $1,245.3$ & $2,237.5$ & 502.4 & $2,739.9$ & $3,985.2$ & W & $4,438.9$ \\
\hline January 1996 & 4.4 & 370.3 & 339.3 & $1,159.0$ & 101.9 & $1,260.9$ & $1,600.2$ & - & $1,974.8$ \\
\hline December 1995 ........... & 5.6 & 362.3 & 295.5 & $1,122.9$ & 78.1 & $1,201.0$ & $1,496.6$ & - & $1,864.5$ \\
\hline January 1995 ................. & 13.6 & 309.1 & 296.6 & $1,163.0$ & 54.4 & $1,217.4$ & $1,514.0$ & - & $1,836.8$ \\
\hline Kansas & & & & & & & & & \\
\hline January 1996 ................ & 13.7 & 201.6 & 213.7 & 987.9 & 252.3 & $1,240.2$ & $1,453.9$ & - & $1,669.2$ \\
\hline December 1995 ........... & 9.5 & 154.5 & 210.4 & 920.1 & 733.8 & $1,653.9$ & $1,864.3$ & - & $2,028.2$ \\
\hline January 1995 ................. & 12.9 & 166.5 & 184.5 & 906.5 & 312.9 & $1,219.4$ & $1,403.9$ & - & $1,583.4$ \\
\hline Kentucky & & & & & & & & & \\
\hline January 1996 ................. & 268.4 & $w$ & 822.7 & $1,305.3$ & 594.5 & $1,899.8$ & $2,722.5$ & $w$ & $3,013.6$ \\
\hline December 1995 ............ & 209.9 & $w$ & 758.3 & $1,258.9$ & 534.4 & $1,793.3$ & $2,551.6$ & W & $2,778.7$ \\
\hline January 1995 ................. & 224.5 & W & 866.6 & $1,202.6$ & 633.8 & $1,836.5$ & $2,703.1$ & $W$ & $2,964.0$ \\
\hline Michigan & & & & & & & & & \\
\hline January 1996 ................ & 204.7 & 158.8 & 959.0 & $1,782.8$ & 304.0 & $2,086.8$ & $3,045.8$ & - & $3,409.3$ \\
\hline December 1995 ............ & 186.3 & 148.0 & $1,025.6$ & $1,825.6$ & 350.7 & $2,176.3$ & $3,201.9$ & - & $3,536.1$ \\
\hline January 1995 ................. & 153.6 & 168.1 & 895.5 & $1,601.3$ & 267.7 & $1,869.1$ & $2,764.5$ & - & $3,086.2$ \\
\hline Minnesota & & & & & & & & & \\
\hline January 1996 ................. & $w$ & 701.5 & 872.5 & 991.7 & 358.2 & $1,349.8$ & $2,222.4$ & $W$ & $3,093.9$ \\
\hline December 1995 .............. & W & 584.9 & 679.0 & 895.5 & 295.9 & $1,191.5$ & $1,870.5$ & $W$ & $2,505.5$ \\
\hline January 1995 ................. & 107.3 & 523.9 & 734.2 & 999.7 & 286.0 & $1,285.7$ & $2,019.9$ & - & $2,651.1$ \\
\hline Missouri & & & & & & & & & \\
\hline January 1996 ................ & 34.7 & 136.3 & 265.1 & $1,989.5$ & 125.8 & $2,115.3$ & $2,380.4$ & - & $2,551.4$ \\
\hline December 1995 ............ & 29.7 & 112.1 & 203.5 & $2,091.9$ & 115.5 & $2,207.4$ & $2,410,8$ & - & $2,552.6$ \\
\hline January 1995 ................ & 29.9 & 119.8 & 247.9 & $1,784.8$ & 182.3 & $1,967.1$ & $2,215.0$ & - & $2,364.7$ \\
\hline
\end{tabular}


Table 50. Prime Supplier Sales Volumes of Distillate Fuel Oils and Kerosene by PAD District and State

(Thousand Gallons per Day) - Continued

\begin{tabular}{|c|c|c|c|c|c|c|c|c|c|}
\hline \multirow{3}{*}{$\begin{array}{c}\text { Geographic Area } \\
\text { Month }\end{array}$} & \multirow{3}{*}{ Kerosene } & \multirow{3}{*}{$\begin{array}{c}\text { No. } 1 \\
\text { Distillate }\end{array}$} & \multicolumn{5}{|c|}{ No. 2 Distillate } & \multirow{3}{*}{ No. 4 Fuela } & \multirow{3}{*}{$\begin{array}{c}\text { Total } \\
\text { Distillate } \\
\text { and } \\
\text { Kerosene }\end{array}$} \\
\hline & & & \multirow{2}{*}{$\begin{array}{l}\text { No. } 2 \text { Fuel } \\
\text { Oil }\end{array}$} & \multicolumn{3}{|c|}{ No. 2 Diesel Fuel } & \multirow{2}{*}{$\begin{array}{c}\text { No. } 2 \\
\text { Distillate }\end{array}$} & & \\
\hline & & & & Low-Sulfur & High-Sulfur & Total & & & \\
\hline \multicolumn{10}{|l|}{ Nebraska } \\
\hline January 1996 & 3.1 & 226.6 & 70.5 & 608.7 & 323.0 & 931.7 & $1,002.2$ & - & $1,232.0$ \\
\hline December 1995 ............ & 0.8 & 213.7 & 46.1 & 637.1 & 386.1 & $1,023.2$ & $1,069.3$ & - & $1,283.8$ \\
\hline January 1995 ................... & 2.5 & 170.6 & 71.0 & 577.4 & 273.2 & 850.6 & 921.6 & - & $1,094.7$ \\
\hline \multicolumn{10}{|l|}{ North Dakota } \\
\hline January 1996 & $\mathbf{w}$ & $W$ & 265.8 & 302.1 & 31.0 & 333.1 & 598.9 & - & 794.7 \\
\hline December 1995 ............. & 0.4 & 176.6 & 241.3 & 277.7 & 37.0 & 314.7 & 556.0 & - & 732.9 \\
\hline January 1995 .................. & 1.3 & 132.6 & 193.9 & 305.8 & 35.5 & 341.3 & 535.2 & - & 669.1 \\
\hline Ohio & & & & & & & & & \\
\hline January 1996 & 450.1 & W & $1,697.7$ & $3,136.0$ & 695.7 & $3,831.7$ & $5,529.4$ & W & $6,059.0$ \\
\hline December 1995 ............. & 359.8 & $W$ & $1,525.8$ & $2,971.0$ & 690.6 & $3,661.5$ & $5,187.4$ & $w$ & $5,604.0$ \\
\hline January 1995 ................... & 344.5 & $W$ & $1,682.4$ & $2,632.2$ & 637.9 & $3,270.0$ & $4,952.4$ & $w$ & $5,393.9$ \\
\hline Oklahoma & & & & & & & & & \\
\hline January 1996 & 7.3 & 42.1 & 411.4 & $1,409.0$ & 208.1 & $1,617.1$ & $2,028.5$ & - & $2,077.8$ \\
\hline December 1995 ............. & $\mathbf{W}$ & 54.5 & W & $1,408.1$ & 132.0 & $1,540.1$ & $1,893.9$ & - & $1,949.6$ \\
\hline January 1995 .................. & 2.0 & 82.1 & 208.3 & $1,381.7$ & 393.4 & $1,775.1$ & $1,983.4$ & - & $2,067.5$ \\
\hline South Dakota & & & & & & & & & \\
\hline January 1996 & 2.3 & 211.0 & 83.8 & 369.1 & 25.2 & 394.3 & 478.1 & - & 691.4 \\
\hline December 1995 ............. & 1.1 & 186.7 & $W$ & 365.5 & 30.0 & 395.5 & 454.7 & - & 642.6 \\
\hline January 1995 .................. & $W$ & $w$ & 59.2 & 356.4 & 23.1 & 379.5 & 438.6 & - & 574.2 \\
\hline Tennessee & & & & & & & & & \\
\hline January 1996 & 275.8 & $W$ & 386.6 & $2,393.7$ & 522.2 & $2,915.9$ & $3,302.5$ & $w$ & $3,582.2$ \\
\hline December 1995 ............ & 238.3 & $w$ & 465.6 & $2,331.6$ & 458.5 & $2,790.2$ & $3,255.8$ & $w$ & $3,497.7$ \\
\hline January 1995 .................. & 236.7 & $w$ & 427.3 & $2,122.7$ & 444.4 & $2,567.1$ & $2,994.4$ & $\ddot{W}$ & $3,240.0$ \\
\hline Wisconsin & & & & & & & & & \\
\hline January 1996 .................. & $\mathbf{W}$ & 473.0 & 777.2 & $1,464.8$ & 370.4 & $1,835.2$ & $2,612.4$ & $w$ & $3,176.9$ \\
\hline December 1995 ............ & W & 435.9 & 747.5 & $1,450.6$ & 408.6 & $1,859.2$ & $2,606.7$ & $w$ & $3,073.7$ \\
\hline 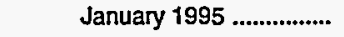 & $W$ & 386.4 & 719.7 & $1,377.4$ & 262.9 & $1,640.3$ & $2,360.0$ & $w$ & $2,806.7$ \\
\hline PAD District II! & & & & & & & & & \\
\hline January 1996 & 328.4 & 139.8 & $1,521.7$ & $12,251.2$ & $5,983.0$ & $18,234.1$ & $19,755.9$ & - & $20,224.1$ \\
\hline December 1995 ............. & W & 53.4 & $1,451.6$ & $12,147.7$ & $6,167.9$ & $18,315.6$ & $19,767.2$ & $w$ & $20,000.4$ \\
\hline January 1995 & W & 144.2 & $1,986.7$ & $11,323.9$ & $5,922.5$ & $17,246.4$ & $19,233.1$ & W & $19,759.7$ \\
\hline Alabama & & & & & & & & & \\
\hline January 1996 .................. & W & $w$ & 312.3 & $1,399.8$ & 433.7 & $1,833.5$ & $2,145.8$ & - & $2,209.9$ \\
\hline December 1995 ............. & 52.0 & - & 268.5 & $1,203.0$ & 394.8 & $1,597.8$ & $1,866.3$ & - & $1,918.3$ \\
\hline January 1995 ................. & 65.0 & - & 412.7 & $1,188.6$ & 458.3 & $1,646.8$ & $2,059.6$ & - & $2,124.5$ \\
\hline Arkansas & & & & & & & & & \\
\hline January 1996 .................. & 17.4 & 6.1 & 76.5 & $1,300.9$ & 365.3 & $1,666.2$ & $1,742.7$ & - & $1,766.2$ \\
\hline December 1995 ............. & 9.1 & 3.4 & 55.0 & $1,273.9$ & 471.5 & $1,745.4$ & $1,800.5$ & - & $1,813.0$ \\
\hline January 1995 & $w$ & W & 131.7 & $1,199.5$ & 332.8 & $1,532.3$ & $1,664.0$ & - & $1,701.0$ \\
\hline Louisiana & & & & & & & & & \\
\hline January 1996 .................. & $W$ & $w$ & 238.0 & $1,412.2$ & $2,196.2$ & $3,608.3$ & $3,846.3$ & - & $4,034.0$ \\
\hline December 1995 .............. & 75.9 & - & 329.6 & $1,458.3$ & $2,314.4$ & $3,772.6$ & $4,102.2$ & - & $4,178.2$ \\
\hline January 1995 & $W$ & $W$ & 277.1 & $1,298.7$ & $1,721.2$ & $3,019.9$ & $3,296.9$ & - & $3,326.8$ \\
\hline Mississippi & & & & & & & & & \\
\hline January 1996 ................ & W & W & $W$ & $1,041.9$ & 423.4 & $1,465.3$ & $1,565.5$ & - & $1,658.8$ \\
\hline December 1995 ............. & $W$ & - & W & $1,130.4$ & 389.0 & $1,519.4$ & $1,605.1$ & W & $1,617.3$ \\
\hline January 1995 ................. & W & - & $W$ & 974.0 & 438.1 & $1,412.1$ & $1,567.9$ & $w$ & $1,582.3$ \\
\hline New Mexico & & & & & & & & & \\
\hline January 1996 ................. & W & 17.8 & $W$ & 701.1 & 86.8 & 787.9 & 789.2 & - & 812.0 \\
\hline December 1995 ............. & W & 14.8 & $W$ & 696.0 & 66.1 & 762.1 & 762.5 & - & 779.2 \\
\hline January 1995 .................. & $W$ & 33.1 & $W$ & 678.2 & 38.1 & 716.4 & 719.7 & - & 757.2 \\
\hline Texas & & & & & & & & & \\
\hline January 1996 ................. & 38.4 & 38.7 & 793.4 & $6,395.3$ & $2,477.6$ & $8,872.9$ & $9,666.3$ & - & $9,743.4$ \\
\hline December 1995 ............. & 28.5 & 35.2 & 712.4 & $6,386.2$ & $2,532.1$ & $8,918.2$ & $9,630.6$ & - & $9,694.4$ \\
\hline January 1995 ................. & 237.2 & 105.8 & $1,006.1$ & $5,984.9$ & $2,934.0$ & $8,918.9$ & $9,925.0$ & - & $10,268.0$ \\
\hline PAD District IV & & & & & & & & & \\
\hline January 1996 ................. & $w$ & 580.3 & $W$ & $3,554.0$ & 795.2 & $4,349.2$ & $4,438.4$ & - & $5,039.5$ \\
\hline December 1995 .............. & W & 468.8 & $W$ & $3,251.5$ & 843.2 & $4,094.7$ & $4,172.7$ & - & $4,661.9$ \\
\hline January 1995 & $W$ & 505.9 & $W$ & $3,277.1$ & 852.8 & $4,130.0$ & $4,271.0$ & - & $4,811.5$ \\
\hline Colorado & & & & & & & & & \\
\hline January 1996 ................. & $w$ & 117.8 & $W$ & $w$ & W & $1,179.0$ & $1,204.2$ & - & $1,334.0$ \\
\hline December 1995 ............. & $w$ & 96.1 & $W$ & $w$ & W & $1,051.8$ & $1,075.2$ & - & $1,180.1$ \\
\hline January 1995 .................. & $\ddot{w}$ & 139.6 & $W$ & 777.5 & 242.5 & $1,020.1$ & $1,061.1$ & - & $1,212.2$ \\
\hline
\end{tabular}

See footnotes at end of table. 
Table 50. Prime Supplier Sales Volumes of Distillate Fuel Oils and Kerosene by PAD District and State

(Thousand Gallons per Day) - Continued

\begin{tabular}{|c|c|c|c|c|c|c|c|c|c|}
\hline \multirow{3}{*}{$\begin{array}{l}\text { Geographic Area } \\
\text { Month }\end{array}$} & \multirow{3}{*}{ Kerosene } & \multirow{3}{*}{$\begin{array}{c}\text { No. } 1 \\
\text { Distillate }\end{array}$} & \multicolumn{5}{|c|}{ No. 2 Distillate } & \multirow{3}{*}{ No. 4 Fuel $^{a}$} & \multirow{3}{*}{$\begin{array}{c}\text { Total } \\
\text { Distillate } \\
\text { and } \\
\text { Kerosene }\end{array}$} \\
\hline & & & \multirow{2}{*}{$\begin{array}{l}\text { No. } 2 \text { Fuel } \\
\text { Oil }\end{array}$} & \multicolumn{3}{|c|}{ No. 2 Diesel Fuel } & \multirow{2}{*}{$\begin{array}{c}\text { No. } 2 \\
\text { Distillate }\end{array}$} & & \\
\hline & & & & Low-Sulfur & High-Sulfur & Total & & & \\
\hline \multicolumn{10}{|l|}{ Idaho } \\
\hline January 1996 ................. & W & 90.7 & W & 420.1 & 197.6 & 617.6 & 618.1 & - & 710.2 \\
\hline December 1995 ............ & $W$ & 66.0 & W & 345.2 & 222.6 & 567.8 & 568.1 & - & 637.4 \\
\hline January 1995 ................. & W & 73.6 & W & 374.3 & 236.6 & 610.9 & 611.1 & - & 690.4 \\
\hline \multicolumn{10}{|l|}{ Montana } \\
\hline January 1996 .................. & $W$ & 148.8 & $W$ & W & $W$ & 560.3 & 560.5 & - & 710.0 \\
\hline & $W$ & 114.4 & $W$ & W & $W$ & 529.4 & 529.6 & - & 645.1 \\
\hline $\begin{array}{l}\text { January } 1995 \text {................. } \\
\text { Utah }\end{array}$ & 2.7 & 93.1 & 0.2 & W & $W$ & 634.6 & 634.8 & - & 730.6 \\
\hline January 1996 & $w$ & 106.5 & W & 900.1 & 176.9 & $1,077.0$ & $1,139.9$ & - & $1,249.0$ \\
\hline December 1995 ............ & $w$ & 98.0 & $W$ & 777.9 & 189.0 & 966.9 & $1,019.5$ & - & $1,118.7$ \\
\hline January 1995 ................. & $W$ & 109.0 & $W$ & 807.3 & 149.0 & 956.3 & $1,055.3$ & - & $1,173.2$ \\
\hline \multicolumn{10}{|l|}{ Wyoming } \\
\hline January 1996 ................ & $W$ & 116.4 & $W$ & W & W & 915.3 & 915.7 & - & $1,036.5$ \\
\hline December 1995 ............ & W & 94.4 & $W$ & $W$ & $W$ & 978.9 & 980.4 & - & $1,080.6$ \\
\hline January 1995 ................ & W & 90.6 & $W$ & W & W & 908.1 & 908.8 & - & $1,005.1$ \\
\hline \multicolumn{10}{|l|}{ PAD District V } \\
\hline January 1996 .................. & $W$ & 591.2 & $W$ & $11,736.6$ & $3,898.2$ & $15,634.8$ & $15,942.8$ & $W$ & $16,834.4$ \\
\hline December 1995 ........... & $W$ & 457.8 & $W$ & $11,320.0$ & $3,667.9$ & $14,987.9$ & $15,279.6$ & 112.9 & $15,961.2$ \\
\hline January 1995 .................. & $W$ & 435.2 & $W$ & $11,149.3$ & $3,454.7$ & $14,604.1$ & $14,952.5$ & 147.0 & $15,640.5$ \\
\hline \multicolumn{10}{|l|}{ Alaska } \\
\hline January 1996 ................. & $W$ & 373.3 & 187.5 & 12.6 & 401.6 & 414.3 & 601.8 & $W$ & $1,071.8$ \\
\hline December 1995 ............ & $W$ & 295.8 & 204.8 & $W$ & $W$ & 331.4 & 536.3 & $W$ & 910.5 \\
\hline January 1995 ................. & W & 258.8 & 188.8 & $w$ & W & 369.7 & 558.5 & W & 914.3 \\
\hline \multicolumn{10}{|l|}{ Arizona } \\
\hline January 1996 ................. & $W$ & $W$ & $\bar{u}$ & $1,308.9$ & 170.8 & $1,479.7$ & $1,479.7$ & - & $1,481.6$ \\
\hline December 1995 ............ & $W$ & $W$ & W & $1,219.9$ & 194.1 & $1,413.9$ & $1,414.1$ & - & $1,416.6$ \\
\hline January 1995 ................. & W & W & - & $1,165.1$ & 262.4 & $1,427.5$ & $1,427.5$ & - & $1,432.4$ \\
\hline \multicolumn{10}{|l|}{ California } \\
\hline January 1996 ................. & 39.1 & $W$ & - & $7,142.6$ & 816.7 & $7,959.4$ & $7,959.4$ & W & $8,005.8$ \\
\hline December 1995 ........... & 27.2 & $W$ & - & $6,833.3$ & 923.4 & $7,756.7$ & $7,756.7$ & W & $7,798.1$ \\
\hline January 1995 ................. & 46.0 & $W$ & W & $6,387.7$ & 535.7 & $6,923.5$ & $6,924.0$ & W & $7,013.8$ \\
\hline \multicolumn{10}{|l|}{ Hawail } \\
\hline January 1996 ................. & - & $W$ & W & 101.6 & 386.3 & 487.9 & 496.3 & - & 496.4 \\
\hline December 1995 ............ & - & $W$ & $W$ & W & W & 524.7 & 530.1 & - & 530.1 \\
\hline January 1995 ................ & - & $W$ & W & $W$ & W & 515.8 & 520.5 & - & 520.5 \\
\hline \multicolumn{10}{|l|}{ Nevada } \\
\hline January 1996 ................. & - & $W$ & W & 677.4 & 64.2 & 741.6 & 765.5 & - & 775.3 \\
\hline December 1995 ............ & - & $W$ & $W$ & 640.1 & 57.4 & 697.5 & 720.8 & - & 731.3 \\
\hline January 1995 ................ & $W$ & 8.3 & W & 621.5 & 55.2 & 676.7 & 708.5 & - & 717.8 \\
\hline Oregon & & & & & & & & & \\
\hline January 1996 ................. & 46.9 & 62.1 & W & $1,138.3$ & 737.0 & $1,875.3$ & $1,888.3$ & $W$ & $2,029.9$ \\
\hline December 1995 ............ & 35.6 & 47.8 & W & $1,200.5$ & 686.5 & $1,887.0$ & $1,897.8$ & W & $1,994.9$ \\
\hline January 1995 ................. & W & 52.9 & W & $1,316.2$ & 743.5 & $2,059.7$ & $2,094.3$ & - & $2,182.2$ \\
\hline Washington & & & & & & & & & \\
\hline January 1996 ................. & 50.0 & 143.9 & 75.1 & $1,355.2$ & $1,321.5$ & $2,676.7$ & $2,751.8$ & NA & $2,973.6$ \\
\hline December 1995 ............ & W & 101.8 & 47.3 & $1,304.1$ & $1,072.5$ & $2,376.6$ & $2,423.9$ & W & $2,579.6$ \\
\hline January 1995 ................. & W & 107.3 & 88.0 & $1,523.6$ & $1,107.5$ & $2,631.2$ & $2,719.2$ & W & $2,859.5$ \\
\hline
\end{tabular}

$\operatorname{Dash}(-)=$ No data reported.

NA $=$ Not available.

$W=$ Withheld to avoid disclosure of individual company data.

Notes: Total Distillate $=$ No. 1 Distillate + No. 2 Distillate + No. 4 Fuel Oil.

Notes: Values shown for the current month are preliminary. Values shown for previous months are revised. Data are final upon publication in the Petroleum Marketing Annual. Totals may not equal the sum of the components due to rounding.

a Includes No. 4 fuel oil and No. 4 diesel fuel.

Source: Energy Information Administration, Form ElA-782C, "Monthly Report of Prime Supplier Sales of Petroleum Products Sold for Local Consumption." 

Explanatory Notes 


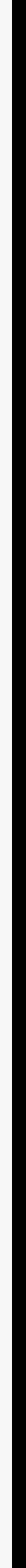




\section{Explanatory Notes}

\section{The EIA-782 Survey}

\section{Background}

The ELA-782 surveys were implemented in 1983 to fulfill the data requirements necessary to meet Energy Information Administration (EIA) legislative mandates and user community data needs. The requirements include petroleum product price, market distribution, demand (or sales), and product supply data, which are needed for a complete evaluation of petroleum market performance. The EIA-782 series includes the Form EIA-782A, "Refiners'/Gas Plant Operators' Monthly Petroleum Product Sales Report"; Form EIA-782B, "Resellers'/Retailers' Monthly Petroleum Product Sales Report"; and Form EIA782C, "Monthly Report of Prime Supplier Sales of Petroleum Products Sold for Local Consumption."

The Form EIA-782A collects refiner and gas plant operator monthly price and volume data at a State level on 14 petroleum products for various retail and wholesale marketing categories. The Form EIA-782B collects reseller/retailer monthly price and volume data at a State level for gasoline, No. 2 distillate, propane, and residual fuel. The Form EIA-782C collects prime supplier monthly volume data on 15 petroleum products. The EIA-782 forms were modified in October 1993 to reflect the changes in refined petroleum products arising out of the requirements of the Clean Air Act Amendments of 1990 (CAAA). The CAAA require that oxygenated gasoline be sold during the winter months in carbon monoxide nonattainment areas beginning October 1, 1992. They require that reformulated gasoline be sold in ozone nonattainment areas beginning January 1, 1995. Beginning October 1, 1993, diesel fuel sold for on-highway use must be low-sulfur diesel fuel (i.e., diesel fuel containing less than or equal to 0.05 percent sulfur). As a result of these environmental regulations, gasoline data collected on the EIA-782 forms were divided into conventional, oxygenated, and reformulated categories. Diesel fuel sales were separated into low- and high-sulfur categories. The wholesale gasoline categories on the EIA-782A and EIA-782B forms were also modified to include dealer tank wagon, rack, and bulk sales. The retail categories for propane on the EIA-782A and EIA-782B were expanded to include residential, commercial/institutional, industrial, sales through company-operated retail outlets, petrochemical, and other end user sales.

\section{Discussion of Sample Design}

The Form EIA-782A is sent to a census of refiners and gas plant operators. Respondents are selected with certainty due to their small number and because of the relative size of their sales volume.

The Form EIA-782B is sent to a scientifically selected sample of motor gasoline resellers, and distillate, propane, and residual fuel oil resellers and retailers. The Form EIA-863, "Petroleum Product Sales Identification Survey," served as the basis of the sampling frame of dealers. Information obtained from the Form EIA-863 is supplemented with information from the Form EIA-821, "Annual Fuel Oil and Kerosene Sales Report." The sales volumes obtained from these surveys are used to create separate stratification schemes that vary by product and State. Dealers selling in more than four States and company/State units comprising 5 percent or more of sales in a State were selected with certainty. The remaining company/State units on the frame were stratified within geographic area and sales type by urban/rural designation and volume of product sales. The geographic areas were defined as (a) the 24 States in which No. 2 distillate was a significant heating source and 50 States and the District of Columbia for residual and motor gasoline, or as (b) the PAD Districts for districts where not all State estimates are provided. The type-of-sale classifications were retail and resale for motor gasoline and residual fuel oil, and residential and nonresidential retail and wholesale for distillate. Three volume-of-sales classifications (zero, low, and high) were defined with volume boundaries and 
numbers of strata, differing by State, sales type, and product.

The design of the EIA-782B sample was based on seven target variables: total retail motor gasoline, total wholesale motor gasoline, residential No. 2 fuel oil, other retail No. 2 fuel oil, total wholesale No. 2 fuel oil, total retail residual fuel oil, and total wholesale residual fuel oil. The required level of accuracy for each target variable was defined by a volume coefficient of variation (CV) of 15 percent for No. 2 distillate and 10 percent for motor gasoline and residual fuel oil, determined at the publishable State level (24 States for distillate, 50 States and the District of Columbia for motor gasoline and residual). Studies on the relationship of volume $\mathrm{CV}$ to price $\mathrm{CV}$ have shown that this will produce price CVs of less than 1 percent. The reliability of current month estimates will vary from these goals due to the deterioration of the frame over time and the changing distributions of price and volume.

Beginning in October 1993, the sample design was modified to include a supplemental sample of propane dealers. The name and address list of propane dealers was constructed by extracting those companies on the EIA-863 who had indicated that they sold propane. This list was supplemented by: (1) respondents on other surveys who reported propane sales, (2) names and addresses of propane dealers furnished by industry associations and State Energy Offices, and (3) commercial lists. Since no information existed to predetermine the sales volumes of propane dealers, two strata for propane dealers were used. A certainty stratum of the known, large, multiState dealers was created. These companies were identified using establishment lists and industry surveys. All other companies were assigned to the second stratum and sample weights were calculated as the inverse sample was selected. Sample weights were calculated as the inverse of the probability of selection $(\mathrm{N} / \mathrm{n})$.

The samples resulting from the separate stratification schemes for the products other than propane were combined by means of a linked-selection procedure designed to maximize the overlap among samples. This procedure produced a sample size of approximately 3,500 companies. Each company selected was required to file completed survey forms for all States in which it had petroleum sales.

The Form EIA-782C was sent to all prime suppliers of any of the selected products on the EIA-782C. A prime supplier is a firm that produces, imports, or transports any of the selected petroleum products across State boundaries and local marketing areas and sells the product to local distributors, local retailers, or end users. They were selected with certainty due to their small number and the relative size of their sales volumes.

\section{Discussion of the Sampling Frame}

The EIA-782A survey consists of a census of respondents who either directly or indirectly control a refinery or gas plant facility. The EIA-782A form collects sales data on 14 refined petroleum products. Currently, 155 companies respond to the ELA-782A survey.

The EIA-863 data base provided the sampling frame for the EIA-782B survey. The Form EIA-863, "Petroleum Product Sales Identification Survey," was mailed to approximately 22,000 companies in January 1992, in order to collect 1991 State-level sales volume data for No. 2 distillate, residual, and motor gasoline. The No. 2 distillate data were further identified by residential/nonresidential end-use and non-end-use sales, while the residual and motor gasoline data were identified by end-use and nonend-use sales. The mailing list for the EIA-863 survey was constructed by merging and unduplicating the previous master frame file and approximately 71 State and commercial lists.

Data from the 1991 EIA-821, "Annual Fuel Oil and Kerosene Sales Report" survey were merged with data from the EIA-863 survey to yield a combined file. A transformed and edited version of this file was created to form the sample file used to design and select the EIA-782B sample.

NOTE: Truck stops selling No. 2 diesel fuel were not specifically included in the frame. Therefore, the EIA-782B end-use category, "sales through company outlets," does not incorporate all sales of No. 2 distillate.

The EIA-782C survey consists of a census of suppliers who produce, import, or transport any of the 15 refined petroleum products listed on the form across State boundaries and local marketing areas, and who sell the product to local distributors, local retailers, or end users. Currently, 237 firms respond to the EIA$782 \mathrm{C}$ survey. 


\section{Reliability of Data}

There are two types of errors possible in an estimate based on a sample survey: sampling and nonsampling. Sampling errors occur because observations are made only on a sample, not on the entire population. Non-sampling errors can be attributed to many sources in the collection and processing of data. The accuracy of survey results is determined by the joint effects of sampling and nonsampling errors.

\section{Measures of Sampling Variability}

Tables 14 through 18, 31 through 34, and 38 through 41 utilize a sample of nonrefiners and, therefore, have sampling error. The remainder of the tables published are based on census data; therefore, there is no error due to sampling. The particular sample used for the ELA-782B is one of a large number of all possible samples that could have been selected using the same design. Estimates derived from the different samples would differ from each other. The average of these estimates would be close to the estimate derived from a complete enumeration of the population (a census), assuming that a complete enumeration has the same nonsampling errors as the sample survey.

The sampling error, or standard error of the estimate, is a measure of the variability among the estimates from all possible samples of the same size and design and, thus, is a measure of the precision with which an estimate from a particular sample approximates the results of a complete enumeration.

\section{Nonsampling Errors}

Nonsampling errors can be attributed to many sources: (1) inability to obtain complete information about all cases in the sample (i.e., nonresponse), (2) response errors, (3) definitional difficulties, (4) differences in the interpretation of questions, (5) mistakes in recording or coding the data obtained, and (6) other errors of collection, response, coverage, and estimation for missing data. These nonsampling errors also occur in complete censuses.

Although no direct measurement of the biases due to nonsampling errors can be obtained, precautionary steps were taken in all phases of the frame development and data collection, processing, and tabulation processes, in an effort to minimize their influence. In addition, the close cooperative consultation between
EIA and the EIA-782 survey respondents and data users results in a more accurate information gathering and reporting process.

\section{Imputation and Estimation}

Survey data gathered from the respondents invariably contain incomplete reporting, nonresponse, and values that fail editing. These missing data are estimated, or imputed for, as follows. First, for all survey units, the previous month's reported value and the previous month's predicted value are weighted together to yield a predicted value for the current month. The sum of the weighted, predicted values for nonrespondents in the current month is then multiplied by a chain link multiplier (the ratio of the sum of the weighted, reported values for respondents in the current month to the sum of the weighted, predicted values for respondents in the current month). The resulting estimate for nonreported values is then added to the reported values. That is,

$$
\hat{V}_{t .}=\sum_{R} W_{i}^{*} V_{i, t}+\sum_{N R} W_{j}^{*} V_{j, t}^{\prime}
$$

and similarly

$$
\hat{Q}_{t .}=\sum_{R} W_{i}^{*} V_{i, t} P_{i, t}+\sum_{N R} W_{j}^{*} V^{\prime}{ }_{j, t} P^{\prime}{ }_{j, t}
$$

where

$$
\begin{gathered}
V_{j, t}^{\prime}=\frac{\sum_{R, P A D D} W_{i}^{*} V_{i}}{\sum_{R, P A D D} W_{i}^{*} \tilde{V}_{i, t}} \tilde{V}_{j, t} \\
P_{j, t}^{\prime}=\frac{\sum_{R, P A D D} P_{i, t}}{\sum_{R, P A D D} \tilde{P}_{i, t}} \tilde{P}_{j, t}
\end{gathered}
$$

and,

$$
W_{i}^{*}=\frac{N}{\sum_{i=1}^{n} W_{i}}\left(W_{i}\right)
$$


$W_{i}=$ the weight for company i. For resellers/retailers responding to EIA-782B, $W i$ is inversely proportional to the probability of inclusion. For all certainty units $W i=1$. The certainty units are all respondents to the EIA-782A, the EIA$782 \mathrm{C}$, and the units selected with certainty for the EIA-782B.

$N=$ total number of population units,

$n=$ number of sampled units, $\sum_{R}=\begin{aligned} & \text { summation across current month respon- } \\ & \text { dents } \mathrm{i},\end{aligned}$ $\sum_{N R}=\begin{aligned} & \text { summation across current month nonre- } \\ & \text { spondents } \mathrm{j},\end{aligned}$

$V_{i, t}=$ current month $(\mathrm{t})$ reported volume for company $i$,

$P_{i, t}=$ current month $(\mathrm{t})$ reported price for company $i$,

$\hat{V}_{t}=$ current month $(t)$ estimated total volume,

$\hat{Q}_{t}=$ current month $(t)$ estimated total revenue,

$\tilde{V}_{i, t}=$ current month $(t)$ predicted volume for company $i$, respondent,

$\tilde{P}_{i, t}=$ current month $(t)$ predicted price for company $i$, respondent,

$\tilde{V}_{i, t}=\alpha V^{\prime}{ }_{i, t-1}+(1-\alpha) V_{i, t-1}$

$\tilde{P}_{i, t}=\alpha P_{i, t-1}^{\prime}+(1-\alpha) P_{i, t-1}$

where

$V_{i, t-1}=$ previous month $(\mathrm{t}-1)$ reported volume for company $i$,

$P_{i, t-1}=\quad$ previous month $(\mathrm{t}-1)$ reported price for company i,

$\alpha=$ constant between 0 and 1 , set by form, product, type of sale and price or volume, and

$$
\hat{P}_{t .}=\frac{\hat{Q}_{t .}}{\hat{V}_{t .}}
$$

the resulting estimate of price at the published level for month $\mathrm{t}$.

Multiple product data collection and linked sample selection yield two types of respondents: basic and supplemental. Both types are used for imputation, estimation, and standard errors.

The variance estimate is :

$$
\operatorname{VAR} \hat{\left(P_{t .}\right)}=\frac{1}{\hat{V}_{t .}{ }^{2}} \sum_{k} N_{k}^{2} n_{k}(1-f k) \frac{M_{k}}{\left(\sum_{i} W_{i k}\right)^{2}}
$$

where

$$
\begin{aligned}
& N_{k}=\text { the number of population units in group } k, \\
& n_{k}=\begin{array}{l}
\text { the number of basic and volunteer re- } \\
\text { spondents in group } k,
\end{array} \\
& W_{i k}=\text { the sampling weight for respondent } i \text { in } \\
& \text { group } k, \\
& f_{k}=\frac{n_{k}}{N_{k}}
\end{aligned}
$$

and $\hat{P}_{t .}$ and $\hat{V}_{t}$ are previously defined.

The term $M_{k}$ is computed as follows:

$$
M_{k}=\frac{\sum_{i}\left(M_{i k}\right)^{2}}{n_{k}-1}
$$

where

$$
M_{i k}=W_{i k} V_{i k} D_{i k}-\frac{W_{i k}}{\left(\sum_{i} W_{i k}\right)} \times\left(\sum_{i} W_{i k} V_{i k} D_{i k}\right)
$$


and

$$
\begin{aligned}
D_{i k}= & P_{i k}-\hat{P}_{t .} \\
V_{i k}= & \begin{array}{l}
\text { reported volume for respondent } \mathrm{i} \text { in } \\
\text { group } \mathrm{k}
\end{array} \\
P_{i k}= & \begin{array}{l}
\text { reported price for respondent } \mathrm{i} \text { in } \\
\text { group } \mathrm{k} .
\end{array}
\end{aligned}
$$

\section{Data Continuity}

When the EIA-782 series was implemented in 1983, it replaced prior surveys that had been used to meet the Energy Information Administration's data requirements. The Form EIA-782A replaced the refiner and gas plant operator portions of the Form EIA-460, "Petroleum Industry Monthly Report for Product Prices"; and Form EIA-9A, "No. 2 Distillate Price Monitoring Report"; the Form EIA-782B replaced the nonrefiner portions of the Form EIA-460 and Form EIA-9A; and the Form EIA-782C replaced Form EIA25, "Prime Supplier's Monthly Report."

Since the transition from the EIA-460, the EIA-9A, and the EIA-25 to the EIA-782 took place over a period of 4 months, rather than occurring at one time, it was possible to compare data from the predecessor surveys with data from the new survey during the transition period for some data elements. This comparative analysis yielded adjustment factors which reflected the estimated overall effect of the changes.

These adjustment factors were applied to the appropriate predecessor survey prices to yield a backcast estimate. A complete description of the estimation of historical data prior to January 1983 is contained in the feature article of the December 1983(3) issue of the PMM.

The backcast price estimation employed the predecessor survey published price as the initial approximation. The initial approximation, however, frequently represented less aggregated product categories and more aggregated seller/sales categories. Therefore, more comparable product categories were formed by volume weighting the disaggregated predecessor survey product prices. For the EIA-9A, comparable categories were formed by subtracting from the price the average taxes reported. Comparable seller/sales categories were formed by multiplying the predecessor price by the ratio of the EIA-782 price for the category to be estimated divided by the volume weighted prices for the aggregate of the EIA-
782 categories most comparable to the predecessor category. That is,

$$
\hat{P}_{460, i}=\stackrel{\wedge}{P}_{460, j} \frac{\hat{P}_{782, i}}{\hat{P}_{782, j}}
$$

where $i$ represents the EIA-782 category to be backcast and $j$ represents the most similar category on the predecessor survey.

The backcast price series were estimated by multiplying the estimate for the previous time period from the predecessor survey by an adjustment factor:

$$
\hat{P}_{782, i, t}=\hat{P}_{\text {Predecessor }, i, t} \times(\text { Adjustment Factor })
$$

where $\mathrm{t}=$ reference month.

Adjustment factors were computed by dividing the EIA-782 December price by the derived December predecessor price for comparable categories:

$$
\text { Adjustment Factor }=\frac{\hat{P}_{782, i, \text { December }}}{\hat{\hat{P}}_{\text {Predecessor, }, \text {,December }}}
$$

The EIA-782 December 1982 price for all respondents had to be estimated since not all of the EIA-782 respondents were reporting in December. This estimate was based on the average of the ratios of the prices for the December respondents to the prices for all respondents in January, February, and March of 1982. That is,

$$
\hat{\hat{P}}_{782, i, \text { December }}=\hat{P}_{782, i, r, \text { December }} \div \frac{\sum_{m} \frac{\hat{P}_{782, i, r} m}{\hat{P}_{782, i, m}}}{3}
$$

where $r=$ respondents who reported in the December reference month and $\mathrm{m}=$ the months of January, February, and March.

Starting with the September 1990 final estimates, prices published are derived using the sample described under "Discussion of Sample Design." Prices published for January 1984 through August 1990 were derived using different samples and slightly different designs (refer to the 1987 PMA for a further description). Also, the monthly price estimates from January through December 1983 were derived using another sample design (see the December 1983(3) issue of the PMM). Therefore, there may be some minor discontinuity in price estimates between $\mathrm{Au}$ - 
Table EN1. Federal and State Motor Fuel Taxes ${ }^{1}$

(Cents per Gallon)

\begin{tabular}{|c|c|c|c|c|c|c|c|}
\hline & $\begin{array}{c}\text { Motor } \\
\text { Gasoline }\end{array}$ & $\begin{array}{l}\text { Diesel } \\
\text { Fuel }\end{array}$ & Gasohol & & $\begin{array}{c}\text { Mofor } \\
\text { Gasoline }\end{array}$ & $\begin{array}{c}\text { Diesel } \\
\text { Fuel }\end{array}$ & Gasohol \\
\hline Federal ${ }^{2}$. & 18.40 & 24.40 & 13.00 & Mississippi ${ }^{4}$. & 18.40 & 18.40 & 18.40 \\
\hline \multirow[t]{2}{*}{ Average State Tax . . . } & 19.77 & 19.58 & 19.60 & Missouri $^{4}$ & 15.04 & 15.04 & 15.04 \\
\hline & & & & Montana ${ }^{4}$. & 27.00 & 27.75 & 27.00 \\
\hline Alabama ${ }^{4}$ & 18.00 & 19.00 & 18.00 & Nebraska & 25.70 & 25.70 & 25.70 \\
\hline Alaska $^{4}$ & 8.00 & 8.00 & 8.00 & $\mathrm{Nevada}^{4} \ldots \ldots$ & 23.60 & 27.60 & 23.60 \\
\hline$\ldots \ldots$ & 18.00 & 18.00 & 18.00 & New Hampshire . . . & 18.80 & 18.80 & 18.80 \\
\hline Arkansas $^{4}$ & 18.70 & 18.70 & 18.70 & New Jersey . . . . . . & 14.50 & 17.50 & 14.50 \\
\hline California $^{34}$. & 18.60 & 18.60 & 18.60 & New Mexico ...... & 18.00 & 19.00 & 18.00 \\
\hline Colorado . . . . . . . . & 22.00 & 20.50 & 22.00 & New York ${ }^{34} \ldots \ldots$ & 22.46 & 24.46 & 22.46 \\
\hline Connecticut $^{3} \ldots \ldots$ & 35.00 & 18.00 & 34.00 & North Carolina . . . . . & 22.00 & 22.00 & 22.00 \\
\hline Delaware $\ldots \ldots \ldots$ & 23.00 & 22.00 & 23.00 & North Dakota . . . . . . & 20.03 & 20.03 & 20.03 \\
\hline District of Columbia ${ }^{3}$. & 20.00 & 20.00 & 20.00 & Ohio .. & 22.00 & 22.00 & 22.00 \\
\hline Florida ${ }^{4}$ & 12.50 & 12.50 & 12.50 & Oklahoma $a^{4} \ldots \ldots$ & 17.00 & 14.00 & 17.00 \\
\hline Georgia $^{34}$ & 7.70 & 7.70 & 7.70 & Oregon $^{4} \ldots \ldots \ldots$ & 24.00 & 24.00 & 24.00 \\
\hline Hawail $^{34} \ldots \ldots$ & 16.00 & 16.00 & 16.00 & Pennsylvania . . . . . & 22.35 & 22.35 & 22.35 \\
\hline$\ldots \ldots \ldots$ & 22.00 & 22.00 & 22.00 & Rhode Island . . . . . & 28.00 & 28.00 & 28.00 \\
\hline lllinois ${ }^{34}$ & 19.30 & 21.80 & 19.30 & South Carolina ${ }^{4} \ldots$ & 16.75 & 16.75 & 16.75 \\
\hline Indiana ${ }^{3}$. & 15.80 & 16.80 & 15.80 & South Dakota . . . . . & 20.00 & 20.00 & 18.00 \\
\hline lowa ${ }^{3} \ldots \ldots \ldots$ & 20.00 & 22.50 & 19.00 & Tennessee . . . . . . . & 21.40 & 18.40 & 21.40 \\
\hline Kansas & 18.02 & 20.02 & 18.02 & Texas. . . . . . . . . & 20.00 & 20.00 & 20.00 \\
\hline Kentucky & 15.10 & 12.10 & 15.10 & Utah $\ldots \ldots \ldots$ & 19.50 & 19.50 & 19.50 \\
\hline Louisiana $^{3}$. & 20.03 & 20.03 & 20.03 & Vermont. . . . . . . . & 16.00 & 17.00 & 16.00 \\
\hline Maine ... . & 19.00 & 20.00 & 19.00 & Virginia $^{3} \ldots \ldots \ldots$ & 17.70 & 16.20 & 17.70 \\
\hline Maryland . . . . . . . & 23.50 & 24.25 & 23.50 & Washington ${ }^{4}$ & 23.12 & 23.12 & 23.12 \\
\hline Massachusetts . . . . & 21.00 & 21.00 & 21.00 & West Virginia ${ }^{3}$. & 20.50 & 20.50 & 20.50 \\
\hline Michigan $^{3} \ldots$ & 15.88 & 15.88 & 15.88 & Wisconsin . . . . . . & 26.40 & 26.40 & 26.40 \\
\hline Minnesota ${ }^{3}$ & 20.00 & 20.00 & 20.00 & Wyoming . . . . . . & 9.00 & 9.00 & 9.00 \\
\hline
\end{tabular}

${ }^{l}$ This figure lists rates of general application (including, but not limited to, excise taxes, environmental taxes, special taxes, and inspection fees), exclusive of county and local taxes. Rates are also exclusive of any State taxes based on gross or net receipts. The State rates are effective January 1, 1996.

2 The Federal tax on motor gasoline and diesel fuel increased 4.3 cents, to 18.4 and 24.4 cents, respectively, on October $1,1993$.

${ }^{3}$ Additional State taxes are levied as follows: California: 7.25 percent salestax; Connecticut: 5 percent gross earningstax; Georgia: 3 percent sales tax; Hawail: 4 percent sales tax; llinois: 6.25 percent sales tax; Indiana: 5 percent sales tax; lowa: 1 percent environmental protection tax; Michigan: 6 percent sales tax; Minnesota: clean-up fund rate of 1.5 - 2.0 cents per gallon based on storage size: New York: 4 percent salestax; Virginia: 2 percent salestax in areas where mass transit systems exist; West Virglnia: Consumer and sales tax of 4.85 cents per gallon.

${ }^{4}$ Local option taxes (LOTS) are allowed. In Florida, the State assesses a State Comprehensive Enhanced Transportation System (SCETS) tax which is two-thirds of each county's rate. In addition, the State collects a "ninth cent tax" and a second local tax. These taxes add an average of 11.5 cents to the motor fuel State tax. In Hawaii, LOTS are as follows: Honolulu: 16.5 cents per gallon; Maul: 13.0 cents per gallon; Hawaii: 8.8 cents per gallon; Kauai: 10.0 cents per gallon. 
Table EN 2. U.S. Posial Two-Letter State Abbreviations

\begin{tabular}{l|l|l|l|l|l}
\hline $\begin{array}{l}\text { State } \\
\text { Code }\end{array}$ & \multicolumn{1}{c|}{ State } & $\begin{array}{l}\text { State } \\
\text { Code }\end{array}$ & Stale & $\begin{array}{l}\text { State } \\
\text { Code }\end{array}$ & Stale \\
\hline AL & Alabama & KY & Kentucky & ND & North Dakota \\
AK & Alaska & LA & Louisiana & OH & Ohio \\
AZ & Arizona & ME & Maine & OK & Oklahoma \\
AR & Arkansas & MD & Maryland & OR & Oregon \\
CA & California & MA & Massachusetts & PA & Pennsylvania \\
CO & Colorado & MI & Michigan & Rl & Rhode Island \\
CT & Connecticut & MN & Minnesota & SC & South Carolina \\
DE & Delaware & MS & Mississippi & SD & South Dakota \\
DC & District of Columbia & MO & Missouri & TN & Tennessee \\
FL & Florida & MT & Montana & TX & Texas \\
GA & Georgia & NE & Nebraska & UT & Utah \\
HI & Hawail & NV & Nevada & VT & Vermont \\
ID & Idaho & NH & New Hampshire & VA & Virginia \\
IL & Illinois & NJ & New Jersey & WA & Washington \\
IN & Indiana & NM & New Mexico & WI & Wisconsin \\
IA & lowa & NY & New York & WV & West Virginia \\
KS & Kansas & NC & North Carolina & WY & Wyoming \\
\hline \hline & & & & & \\
\hline
\end{tabular}

gust 1988 and September 1988 and between December 1983 and January 1984.

\section{Collection Methods}

Survey data are collected by mail every month. It is mandatory for each respondent to submit completed forms to EIA within the specified time allotted. For the EIA-782A and B, completed forms must be submitted no later than 30 calendar days after the close of each reference month. For the EIA-782C, completed forms must be submitted no later than 20 calendar days after the close of the reference month. Telephone follow-up calls to nonrespondents begin the day after the established due date in order to collect all outstanding data. Late submissions and resubmissions are processed when received.

\section{Data Processing}

As EIA-782 forms are received, they are logged into an automated Survey Control File which maintains monthly status codes for each company. The data are reviewed manually and then eritered onto the computer files. They are then processed through an automated edit program which detects missing data, inconsistent prices, volumes and prices that significantly differ from those previously reported by the company, and outlying values that will affect published estimates. Data that fail the edits are resolved through telephone calls to the data reporters, and corrections and verification codes are entered onto the computer files. Statistical reports, including publication tables, are then generated using only acceptable and verified data. 


\section{Nondisclosure}

The data contained in this publication are subject to statistical nondisclosure procedures. The objective of the disclosure-avoidance procedures, as stated in the Energy Information Administration Standard 88-0506, Subject: "Nondisclosure of Company Identifiable Data in Aggregate Cells," is to ensure that confidential, company-identifiable data are not disclosed in tables where "company specific responses may be proprietary and prohibited from public disclosure by 18 U.S.C. 1905." Statistics representing data aggregated from fewer than three companies or that are dominated by input from one or two companies are withheld. EIA identifies cells that are sensitive according to these criteria by applying a statistical formula to the data contained in each cell to determine if a few companies "dominate" the cell.

If a cell is sensitive, the data in that cell are suppressed and $\mathrm{a}$ "W" is placed in the publication cell. Also, since many tables include row or column totals, some nonsensitive data cells have been suppressed to prevent the reader from calculating the suppressed numbers by simply subtracting the published numbers from the total.

\section{Relationship of Refiner and Prime Supplier Sales Volumes}

The refiner sales volumes collected on the EIA-782A are related to the prime supplier sales volumes collected on the EIA-782C, but conceptual differences exist that cause variations between these data. In general, EIA-782A volumes are intended to reflect refiner sales of petroleum products into all secondary and tertiary markets, while ELA-782C volumes are designed to measure prime supplier sales into only the local markets of final consumption. Specifically:

The reporting universe for the EIA-782C survey is significantly larger than that of the EIA-782A. While nearly all refiners and gas plant operators report on both surveys (a small number do not qualify as prime suppliers), some large, inter-State distributors and retailers, as well as some importers, report only on the EIA-782C.

EIA-782A respondents are asked only to exclude sales to other refiners (that is, other respondents that comprise the primary market), while EIA-782C respondents are asked to exclude sales to any company that is not a local distributor, local retailer, or end user (DRE). Therefore, EIA-782C respondents are asked not only to exclude sales to refiners, but also to most large inter-State resellers, importers, traders, and retailers who transport products across State boundaries.

The EIA-782A is designed to gather data on the sales of selected petroleum products made in each State, regardless of where the products are physically located or will be consumed. In contrast, the EIA-782C is designed to collect data reflecting only delivered sales of selected petroleum products into those States where the products are expected to be locally consumed.

Consequently, EIA-782A and EIA-782C volumetric data generally vary at national, regional, and State levels. In particular, differences are expected in States and regions in which major supply origination, pipeline distribution, or transfer points are located. In these States, large volumes of products may change hands many times, often for eventual shipment outside the State. Since the EIA-782C is intended to measure only those sales into the final local markets of consumption (sales to DREs), all preceding sales are excluded. Furthermore, sales by EIA-782C respondents are reported wherever the product was delivered, which may differ from the State where title transferred. In contrast, the ELA-782A reflects all sales made to secondary resellers, wherever title transfers.

Additionally, the EIA-782C reflects imports by firms that are neither refiners nor gas plant operators, that would not be measured on the EIA-782A unless they were transferred to a distribution chain. This mostly affects regions with a high level of product imports, such as the New England or Mid-Atlantic States.

Therefore, States with major refining areas, such as Texas or California, generally show higher volumes on the EIA-782A survey than the EIA-782C survey, since some of the volumes reported on the EIA-782A are excluded on the EIA-782C or are reported in different States. Conversely, net consuming States (e.g., most PAD District I and PAD District II States) may show larger prime supplier sales on the EIA$782 \mathrm{C}$ due to inter-State movements or imports by resellers and/or differences in State of delivery versus title transfer. However, this may be partially or entirely offset by some refiners reporting larger sales volumes on the EIA-782A than on the EIA-782C (due to fewer exclusions taken on the EIA-782A).

In summary, caution should be exercised when comparing sales volumes between refiners and prime 


\section{Table EN3. Revision Error in Selected 1994 U.S. Average Price Data}

(Cents per Gallon Excluding Taxes)

\begin{tabular}{|c|c|c|c|c|c|c|c|c|c|}
\hline \multirow{2}{*}{ Dale } & \multicolumn{3}{|c|}{$\begin{array}{l}\text { Refiner/Reseller Unleaded } \\
\text { Regular Sales to End Users }\end{array}$} & \multicolumn{3}{|c|}{$\begin{array}{l}\text { No. } 2 \text { Distillate Sales } \\
\text { to Residential Customers }\end{array}$} & \multicolumn{3}{|c|}{$\begin{array}{l}\text { Residual Fuel Oil Sales } \\
\text { to End Users }\end{array}$} \\
\hline & PMM & Final & Difference & PMM & Final & Difference & PMM & Final & Difference \\
\hline January. . & 62.3 & 62.3 & 0.0 & 89.7 & 89.6 & 0.1 & 32.5 & 33,4 & -0.9 \\
\hline February ... & 63.1 & 63.3 & -0.2 & 92.8 & 92.9 & -0.1 & 37.5 & 37.3 & 0.2 \\
\hline March ....... & 62.9 & 63.0 & -0.1 & 91.4 & 91.4 & 0.0 & 34.0 & 33.9 & 0.1 \\
\hline 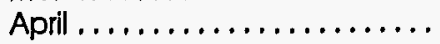 & 64.9 & 65.0 & -0.1 & 88.0 & 88.2 & -0.2 & 31.6 & 31.3 & 0.3 \\
\hline May ........... & 66.6 & 66.7 & -0.1 & 85.8 & 86.1 & -0.3 & 32.6 & 32.4 & 0.2 \\
\hline June........ & 69.6 & 69.6 & 0.0 & 84.9 & 85.2 & -0.3 & 34.8 & 34.8 & 0.0 \\
\hline July. ... . . & 72.6 & 72.7 & -0.1 & 82.2 & 82.7 & -0.5 & 37.6 & 37.8 & -0.2 \\
\hline August... & 76.7 & 76.7 & 0.0 & 82.0 & 82.1 & -0.1 & 38.3 & 38.3 & 0.0 \\
\hline September . & 75.2 & 75.1 & 0.1 & 81.5 & 83.2 & -1.7 & 34.8 & 34.8 & 0.0 \\
\hline October ... & 72.8 & 72.8 & 0.0 & 84.5 & 84.7 & -0.2 & 34.8 & 34.8 & 0.0 \\
\hline November . & 72.9 & 73.0 & -0.1 & 85.7 & 85.7 & 0.0 & 36.9 & 37.2 & -0.3 \\
\hline December .... & 70.5 & 70.4 & 0.1 & 86.3 & 86.8 & -0.5 & 38.7 & 38.9 & -0.2 \\
\hline
\end{tabular}

Sources: PMM data are from Tables 15, 31, and 42 of the Petroleum Marketing Monthly. Final data are from Tables 15, 31, and 42 of the Petroleum Marketing Annual, 1994.

\section{Table EN4. Revision Error in Selected 1994 Refiner Sales Volume Data} (Million Gallons)

\begin{tabular}{|c|c|c|c|c|c|c|c|c|c|}
\hline \multirow{2}{*}{ Date } & \multicolumn{3}{|c|}{$\begin{array}{l}\text { Motor Gasoline } \\
\text { Sales for Resale }\end{array}$} & \multicolumn{3}{|c|}{$\begin{array}{l}\text { No. } 2 \text { Distillate } \\
\text { Sales for Resale }\end{array}$} & \multicolumn{3}{|c|}{$\begin{array}{l}\text { Residual Fuel Oil } \\
\text { Sales to End Users }\end{array}$} \\
\hline & PMM & Final & $\begin{array}{l}\text { Percent } \\
\text { Change }\end{array}$ & PMM & Final & $\begin{array}{l}\text { Percent } \\
\text { Change }\end{array}$ & PMM & Final & $\begin{array}{l}\text { Percent } \\
\text { Change }\end{array}$ \\
\hline January & 253.3 & 254.5 & -0.5 & 109.4 & 109.9 & -0.5 & 16.7 & 17.5 & -4.8 \\
\hline 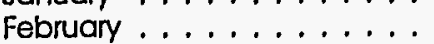 & 257.5 & 261.2 & -1.4 & 111.9 & 112.5 & -0.5 & 15.2 & 15.5 & -2.0 \\
\hline 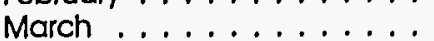 & 267.0 & 269.0 & -0.7 & 109.4 & 110.4 & -0.9 & 14.2 & 15.2 & -7.0 \\
\hline April . . & 274.7 & 276.2 & -0.5 & 103.2 & 104.1 & -0.9 & 32.5 & $\cdot 12.6$ & -0.8 \\
\hline May... & 277.6 & 279.4 & -0.6 & 101.5 & 101.5 & 0.0 & 10.6 & 12.2 & -15.1 \\
\hline June & 285.8 & 287.7 & -0.7 & 107.5 & 108.9 & -1.3 & 13.4 & 14.0 & -4.5 \\
\hline$\ldots$ & 277.4 & 279.2 & -0.6 & 94.1 & 94.9 & -0.9 & 12.0 & 11.5 & 4.2 \\
\hline August & 287.9 & 288.4 & -0.2 & 103.5 & 103.5 & 0.0 & 11.6 & 11.6 & 0.0 \\
\hline September & 281.4 & 282.0 & -0.2 & 106.1 & 107.2 & -1.0 & 12.6 & 12.5 & 0.8 \\
\hline October. & 274.8 & 276.1 & -0.5 & 103.4 & 103.9 & -0.5 & 13.1 & 13.1 & 0.0 \\
\hline November & 273.4 & 275.9 & -0.9 & 100.0 & 100.0 & 0.0 & 13.9 & 12.9 & 7.2 \\
\hline December . & 290.3 & 290,0 & 0.1 & 107.6 & 107.5 & 0.1 & 14.5 & 14.0 & 3.4 \\
\hline
\end{tabular}

Sources: PMM data are from Tables 7, 46, and 47 of the Petroleum Marketing Monthly. Final data are from Tables 7, 46, and 47 of the Petroleum Marketing Annual, 1994. 
Table EN5. Revision Error in Selected 1994 Prime Supplier Volumes Data (Million Gallons)

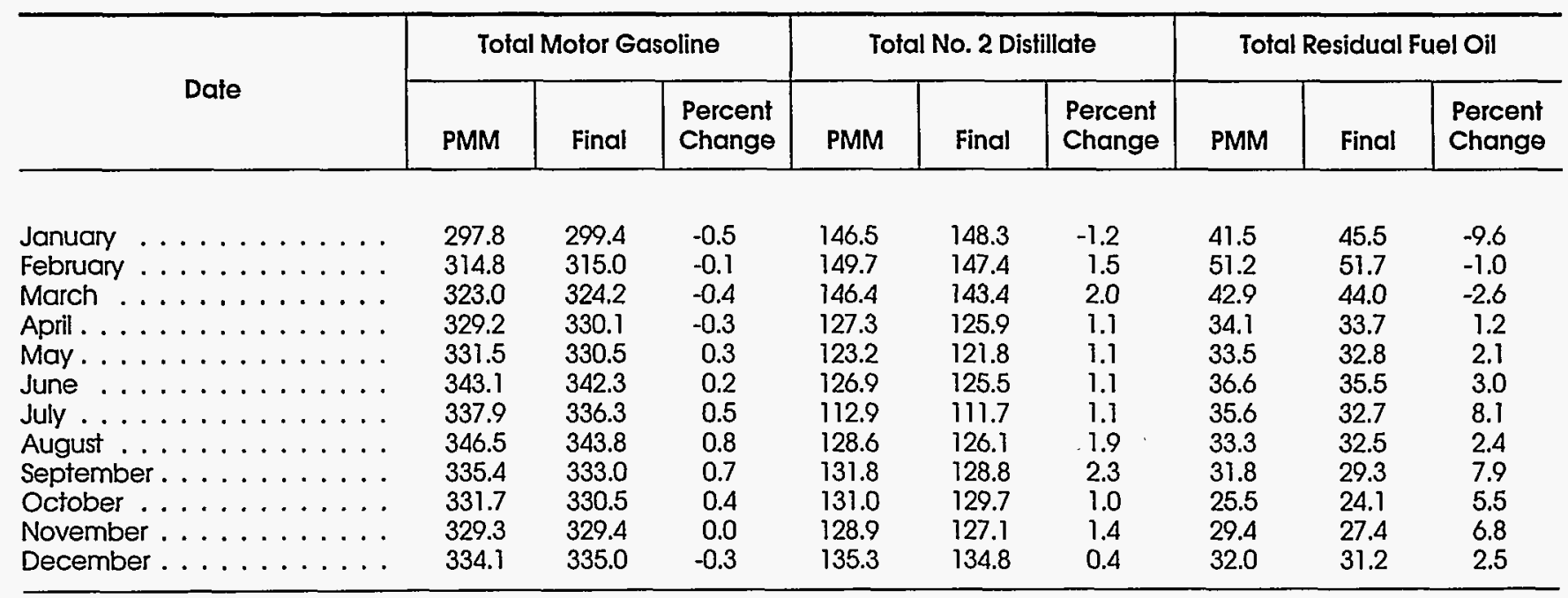

Sources: PMM data are from Tables 48, 49, and 50 of the Petroleum Marketing Monthly. Final data are from Tables 48, 49, and 50 of the Petroleum Marketing Annual, 1994.

suppliers. Whereas EIA-782A data reflect the marketing of products by refiners to non-refiners where the sale occurs, EIA-782C data reflect prime supplier sales to local distributors, local retailers, and end users where the product is delivered. Therefore, the EIA-782A and EIA-782C surveys differ by the respondents reporting (refiners versus prime suppliers), the types of sales reported (sales to non-refiners versus sales to DREs), and the location of the reported sales (point of title transfer versus destination of the sale).

\section{Revision Error}

The petroleum product price and volume data shown for the current month are preliminary. These numbers may be revised in the next month's publication based on data received late or revisions received. For example, if the latest data shown are for the month of February, the February data are preliminary and the January data may have been revised due to the receipt of late or revised data. The data are final upon publication in the Petroleum Marketing Annual (PMA). The difference between the data when they appear in the Petroleum Marketing Monthly (PMM) and when they appear in the PMA is called the revision error. The amount of revision error for some selected EIA782 data series is shown in Tables EN3 - EN5.

\section{The Crude Oil Price Surveys}

\section{Background}

\section{Form EIA-182: "Domestic Crude Oil First Purchase Report"}

Each month, the Form EIA-182 collects data from the buyers on first purchases of domestic crude oil. A "first purchase" constitutes a transfer of ownership of crude oil during or immediately after the physical removal of the crude oil from a production property for the first time. Transactions between affiliated companies are reported as if they were "arms-length" transactions. (This definition is consistent with the Windfall Profits Tax (WPT) concepts of "first sale" and "removal price.") The primary objective is to calculate an average first purchase price at various levels of aggregation. A company's monthly average first purchase prices are volume weighted across given geographical areas for selected crude streams and gravity bands. Prices are computed from the following reported data elements:

Area of production. The producing State or non-State production "area" (i.e., Alaska North Slope, Alaska Other Mainland, Federal Offshore California and Federal Offshore Gulf--about one-fifth off Texas and the remainder off Louisiana). 
Average cost. Reported f.o.b. the lease boundary and based on the actual purchase expenditures, including discounts or premiums paid.

Total volume purchased. The amount of crude bought and paid for as it is measured at the lease boundary (usually at a lease automatic custody transfer unit--a LACT unit), adjusted for basic sediment and water (BS\&W) and temperature.

Prices published from data collected on Form EIA182 are calculated by dividing the sum of the total average costs paid by the sum of the total volumes purchased.

\section{Form EIA-856: "Monthly Foreign Crude Oil Acquisition Report"}

The Form EIA-856 collects monthly price and volume data for about 90 percent of all crude oil imported into the United States. It also collects classification data that enable EIA to determine the terms of an acquisition. The data are reported for the parent company and all the affiliates controlled by the parent. Under this definition, the acquisition price reported for each cargo is the one paid to an unaffiliated seller, in principle an "arms-length" price, which is consistent with use of the data to represent market trends, rather than monitoring internal company transfer pricing policies.

Each month, respondents report the following for cargos acquired for U.S. importation:

Offshore inventories. Crude oil owned by the respondent that is intended for importation into the United States. These inventories include oil in tankers enroute to the United States and floating or on-land storage outside the United States.

Crude type. Includes the country of origin of the cargo of crude, the stream or type of crude oil (e.g., Saudi Light), and the API gravity.

Volume acquired. The number of 42 U.S. gallon barrels in the cargo.

Dates. The date of loading/acquisition and the expected date of landing.

Transportation. Ports of loading and landing and the name of the vessel.
Prices. Acquisition cost, landed cost, and other costs such as demurrage, agent's fees, import tariffs and fees, etc. (all costs are reported in dollars per barrel).

Days credit. The number of days credit is extended to the purchaser by the seller.

Purchase classifying information. Type of transaction (e.g., purchase from host government), terms of transaction (spot or contract), and point of transaction (f.o.b. (free on board), country of origin or CIF (cost, insurance, and freight), U.S. port of entry).

Published prices are calculated by first multiplying the purchase volume by a price to obtain a total cost, then the sums of the total costs are divided by the sums of the purchase volumes.

The prices associated with data collected on Form EIA-856 are aggregated within the month of acquisition, which can be the month of loading, the month of landing, or sometime between those events. By design, the prices are not aggregated for the month in which they are determined, unless the acquisition and price determination month are the same. EIA856 data reflect types of trades occurring over the entire spectrum of international crude oil markets, ranging from continuing supply agreements to spot market purchases. Prices can be determined at time of loading or at time of landing. Prices can be negotiated between the parties involved or tied to spot or futures market price levels. The methodology chosen for the EIA-856 provides a consistent historical series even though its prices may not always agree with measures of prices from other sources.

International crude oil markets are complex and dynamic. For example, a cargo of Saudi Arabian crude oil could be acquired in June at a loading port in Saudi Arabia. The cargo may land in the United States in August. The price for the crude oil could be determined by spot crude oil prices in effect during the 5 days before and after landing. For the PMM, the price for this cargo will be aggregated in the month of June, when it was acquired. Conversely, a cargo of Brent crude may be acquired in June, but its price may have been determined in the forward Brent market in April. This cargo's price will also be aggregated in June, when the purchaser took title to the crude.

In the early 1980's, most crude oil prices were set by the country selling the crude. Gradually, as the supply of crude oil became more abundant, markets became more competitive. A robust spot market for 
crude evolved, in which prices for crude oil were determined by demand and supply. Frequently, the official sales price set by the selling government was considerably different than spot market assessments. As buyers began to purchase more crude oil on the spot market, the control that sellers had theretofore exercised eroded.

In order to protect their market share, crude oil producing governments began to tie prices for their crude to market-related prices. When these marketrelated pricing formulas came into prominence in late 1985, many crude oil prices were tied to a "netback realization," wherein a crude oil's value was determined by volume weighted spot market prices of products derivable from that crude. The weights essentially reflected the relative yield of selected products from a given crude stream. These netbackbased formulas gradually gave way to formulas based on spot crude oil assessments.

The formulas and terms used by sellers of crude oil continue to change. Since the ELA-856 prices are aggregated by month of acquisition-not necessarily the same as month of price determination--they may not always show the same pattern as a series from another source (e.g., trade-press publications). During periods of dramatic change in crude oil prices, aggregate prices derived from EIA-856 data will tend to "lead" the market. That is, these prices will show the emerging trend earlier, reach the inflection point sooner, and then return to the underlying trend. When averaged over longer periods of time, however, EIA-856 prices show the same relative price movements as exogenous sources.

\section{Form EIA-14: "Refiners' Monthly Cost Report"}

The EIA-14 is a monthly census of all U.S. refiners. It collects the net acquisition costs and volumes of crude oil, both domestic and imported, on a corporate national basis (i.e., not for individual refineries). Included in the costs are all charges associated with the acquisition, transportation, and storage of crude incurred by respondents up to the time the oil is booked into their refineries.

Each month, refiners report the volume (in thousands of barrels) and costs (in thousands of dollars) for:

Domestic crude oil. Oil produced in the United States or from its outer continental shelf.
Imported crude oil. Oil produced outside the United States and brought into the United States for domestic processing.

Unfinished oil. All other oils, both domestic and imported, requiring further refining, except those requiring only mechanical blending.

Average prices are calculated by dividing the sum of the costs by the sum of the volumes.

\section{Respondent Frame}

\section{Form EIA-182:}

All firms that buy domestic crude oil at the lease boundary, acquiring ownership of the crude in a first purchase transaction. The list initially was compiled from the 1974 Federal Energy Administration (FEA) Oil and Gas Survey of Producers and Operators. Collection of data from first purchasers began in February 1976. By 1978, the frame consisted of 340 respondents. Of these, 198 purchased more than 150,000 barrels per year and together represented 99.9 percent of the total reported volume.

Adjustments to the frame have mostly been "deaths," with relatively few "births." Following decontrol in January 1981, there was a major contraction of the list of active first purchasers. Many small firms went out of business or were absorbed by larger companies. More recent changes include several mergers among majors and one breakup of a major company. Currently, the EIA-182 survey collects data from 102 active respondents.

\section{Form EIA-856:}

All companies that were reporting data on the ERA51, "Transfer Pricing Report," as of June 1982, regardless of the total volumes of crude oil that are imported. In addition, all other companies that acquire more than 500,000 barrels of foreign crude oil in the report month for importation into the United States are required to prepare and submit an EIA-856 for that month.

\section{Form EIA-14:}

All refiners of crude oil in the United States, including its territories and possessions. There are currently 107 active respondents to the EIA- 14 . 
The list of respondents to the EIA-14 is updated annually by supplementation from the EIA-782A, "Refiners' / Gas Plant Operators' Monthly Petroleum Product Sales Report," and the EIA-810, "Monthly Refinery Report."

\section{Data Collection Processing}

All three crude oil data collection systems are operated independently. Each performs similar data collection and processing functions that are outlined below.

Survey data are collected by mail every month. It is mandatory for each respondent to submit completed forms to EIA no later than 30 calendar days after the close of each reference month. Telephone follow-up calls to nonrespondents begin 2 days after the established due date in order to collect all outstanding data. Late submissions and resubmissions are processed when received.

The forms are logged and reviewed manually. The data are then entered onto computer files. The files are then processed through an automated edit program which detects missing data, inconsistent prices, and outlying values that affect published estimates. Data that fail the edits are resolved through telephone calls to data reporters, and corrections and verification codes are entered onto computer files. Statistical reports, including publication tables, are then generated using only acceptable and verified data. Response rates are normally 100 percent by the time final statistics are calculated.

\section{Nondisclosure}

The data contained in this publication are subject to statistical nondisclosure procedures. The objective of the disclosure-avoidance procedures, as stated in the Energy Information Administration Standard 88-0506, Subject: "Nondisclosure of Company Identifiable Data in Aggregate Cells," is to ensure that confidential, company-identifiable data are not disclosed in tables where "company specific responses may be proprietary and prohibited from public disclosure by 18 U.S.C. 1905." Statistics representing data aggregated from fewer than three companies or that are dominated by input from one or two companies are withheld. EIA identifies cells that are sensitive according to these criteria by applying a statistical formula to the data contained in each cell to determine if a few companies "dominate" the cell.
If a cell is sensitive, the data in that cell are suppressed and $\mathrm{a}$ "W" is placed in the publication cell. Also, since many tables include row or column totals, some nonsensitive data cells have been suppressed to prevent the reader from calculating the suppressed numbers by simply subtracting the published numbers from the total.

\section{Data Continuity}

The crude oil statistics published in the Petroleum Marketing Monthly constitute both a republishing of numbers that already appear in the Monthly Energy Review (MER) and the Annual Energy Review (AER), and a simple extension of the detail of such statistics. These statistics have been published for a number of years in the MER and AER. The data currently collected through the crude oil surveys are compatible with data used to derive statistics for the historical series. The definitions, respondents, and processing have not changed substantially over the years the data have been collected. The target populations and the computational algorithms have remained virtually unchanged.

\section{Reliability of Data}

There are two types of errors possible in an estimate based on a sample survey: sampling and nonsampling. Sampling errors occur because observations are made only on a sample, not on the entire population. Since the crude oil surveys are based on a census of the population, these surveys contain no sampling error.

Nonsampling errors can be attributed to many sources: (1) inability to obtain complete information from all respondents in the survey (i.e., nonresponse), (2) response errors, (3) definitional difficulties, (4) differences in the interpretation of questions, (5) mistakes in recording or coding the data obtained, and (6) other errors of collection, response, coverage, and estimation for missing data.

Although no direct measurement of the biases due to nonsampling errors can be obtained, precautionary steps were taken in all phases of the frame development and data collection, processing, and tabulation processes, in an effort to minimize their influence. In addition, the close cooperative consultation between EIA and the survey respondents and data users results in a more accurate information gathering and reporting process. 
Table EN6. Revision Error in 1994 Refiner Acquisition Cost Data

(Dollars per Barrel)

\begin{tabular}{|c|c|c|c|c|c|c|c|c|c|}
\hline \multirow{3}{*}{ Date } & \multicolumn{9}{|c|}{ Refiner Acquisition Costs } \\
\hline & \multicolumn{3}{|c|}{ Domestic } & \multicolumn{3}{|c|}{ Imporfed } & \multicolumn{3}{|c|}{ Composite } \\
\hline & PMM & Final & Difference & PMM & Final & Difference & PMM & Final & Difference \\
\hline January & 12.72 & 12.73 & -0.01 & 12.97 & 12.93 & 0.04 & 12.85 & 12.83 & 0.02 \\
\hline February ...... & 13.24 & 13.24 & 0.00 & 12.90 & 12.90 & 0.00 & 13.07 & 13.07 & 0.00 \\
\hline March . . & 13.14 & 13.14 & 0.00 & 13.18 & 13.18 & 0.00 & 13.16 & 13.16 & 0,00 \\
\hline April . & 14.71 & 14.74 & -0.03 & 14.63 & 14.54 & 0.09 & 14.67 & 14.64 & 0.03 \\
\hline May & 15.95 & 15.86 & 0.09 & 15.79 & 15.74 & 0.05 & 15.87 & 15.80 & 0.07 \\
\hline June .. & 17.34 & 17.38 & -0.04 & 17.03 & 17.04 & -0.01 & 17.18 & 17.21 & -0.03 \\
\hline July & 17.74 & 17.74 & 0.00 & 17.50 & 17.52 & -0.02 & 17.61 & 17.62 & -0.01 \\
\hline August . & 17.23 & 17.22 & 0.01 & 16.68 & 16.66 & 0.02 & 16.93 & 16.92 & 0.01 \\
\hline September. . & 16.46 & 16.46 & 0.00 & 15.88 & 15.91 & -0.03 & 16.17 & 16.18 & -0.01 \\
\hline October.. & 16.35 & 16.35 & 0.00 & 16.23 & 16.27 & -0.04 & 16.29 & 16.31 & -0.02 \\
\hline November & 16.63 & 16.63 & 0.00 & 16.44 & 16.46 & -0.02 & 16.53 & 16.54 & -0.01 \\
\hline December. & 16.22 & 16.22 & 0.00 & 15.78 & 15.78 & 0.00 & 16.03 & 16.03 & 0.00 \\
\hline
\end{tabular}

Sources: PMM data are from Table 1 of the Petroleum Marketing Monthly. Final data are from Table 1 of the Petroleum Marketing Annual, 1994.

\section{Table EN7. Revision Error in 1994 Domestic First Purchase Price Data} (Dollars per Barrel)

\begin{tabular}{|c|c|c|c|}
\hline Month & PMM & Final & Difference \\
\hline January. . & 10.51 & 10.49 & 0.02 \\
\hline February $\ldots \ldots \ldots \ldots \ldots \ldots \ldots \ldots \ldots$ & 10.73 & 10.71 & 0.02 \\
\hline March $\ldots \ldots \ldots \ldots \ldots \ldots \ldots \ldots \ldots \ldots \ldots$ & 10.81 & 10.94 & -0.13 \\
\hline April $\ldots \ldots \ldots \ldots \ldots \ldots \ldots \ldots \ldots \ldots \ldots \ldots$ & 12.33 & 12.31 & 0.02 \\
\hline 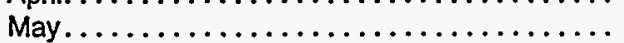 & 14.03 & 14.02 & 0.01 \\
\hline 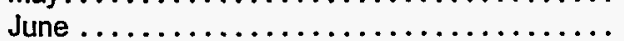 & 14.94 & 14.93 & 0.01 \\
\hline July . . . . . . . . . . & 15.32 & 15.34 & -0.02 \\
\hline 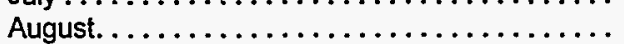 & 14.50 & 14.50 & 0.00 \\
\hline September.$\ldots \ldots \ldots \ldots \ldots \ldots \ldots \ldots \ldots$ & 13.62 & 13.62 & 0.00 \\
\hline October.$\ldots \ldots \ldots \ldots \ldots \ldots \ldots \ldots \ldots \ldots$ & 13.85 & 13.84 & 0.01 \\
\hline November........................ & 14.14 & 14.14 & 0.00 \\
\hline 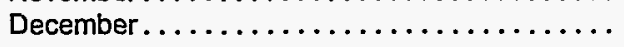 & 13.43 & 13.43 & 0.00 \\
\hline
\end{tabular}

Sources: Preliminary data are from Table 1 of the Petroleum Marketing Monthly for each respective month. Final data are from Table 1 of the Petroleum Marketing Annual, 1994. 


\section{Table EN8. Revision Error in 1994 Foreign Crude Oil Acquisition Cost Data}

(Dollars per Barrel)

\begin{tabular}{|c|c|c|c|c|c|c|}
\hline \multirow{2}{*}{ Month } & \multicolumn{3}{|c|}{ FOB Cost of Imports } & \multicolumn{3}{|c|}{ Landed Cost of Imports } \\
\hline & PMM & Final & Difference & PMM & Final & Difference \\
\hline January. & 12.01 & 12.07 & -0.06 & 12.67 & 12.74 & -0.13 \\
\hline February . & 11.95 & 12.05 & -0.10 & 12.57 & 12.71 & -0.14 \\
\hline March... & 12.04 & 12.38 & -0.34 & 12.66 & 13.00 & -0.34 \\
\hline April.... & 13.38 & 13.55 & -0.17 & 14.08 & 14.30 & -0.22 \\
\hline May............ & 14.54 & 14.67 & -0.13 & 15.46 & 15.62 & -0.16 \\
\hline June............ & 15.49 & 15.44 & 0.05 & 16.51 & 16.51 & 0.00 \\
\hline July....... & 16.21 & 16.10 & 0.11 & 17.22 & 17.15 & 0.07 \\
\hline August... & 15.03 & 14.94 & 0.09 & 16.18 & 16.07 & 0.11 \\
\hline September. & 14.26 & 14.32 & -0.06 & 15.39 & 15.47 & -0.08 \\
\hline ,............ & 14.61 & 14.74 & -0.13 & 15.59 & 15.66 & -0.07 \\
\hline November ....... & 14.84 & 14.88 & -0.04 & 15.98 & 15.98 & 0.00 \\
\hline December ........ & 14.30 & 14.46 & -0.16 & 15.48 & 15.61 & -0.13 \\
\hline
\end{tabular}

Sources: PMM data are from Table 1 of the Petroleum Marketing Monthly for each respective month. Final data are from Table 1 of the Petroleum Marketing Annual, 1994.

\section{Imputation}

Since the response rates for the crude oil survey are virtually 100 percent, there are no imputation procedures in the PMM data for nonresponse to these surveys. Imputation is performed, however, on EIA182 volume data used in estimating crude oil production published in the Petroleum Supply Monthly (PSM). Since production estimates for the PSM are required on an expedited schedule, some responses are imputed for the PSM. However, all responses are received prior to the publication of the PMM, thus no imputation is required for the price data published in the PMM. See Note 4 in the Explanatory Notes in the PSM for additional information on the use of EIA-182 data in estimating domestic crude oil production.

\section{Revision Error}

The crude oil values shown for Domestic First Purchase Prices and Refiner Acquisition Cost (RAC) for the current month and for Average Landed Costs for the current 2 months are preliminary. These numbers are revised in the month after the preliminary month(s) based on data received late or revisions received. For example, in the February publication, the February RAC data are preliminary and the January RAC data may have been revised due to receipt of late or revised data. The data are final upon publication in the Petroleum Marketing Annual (PMA). In the above example, the difference between the January RAC data in the Petroleum Marketing Monthly (PMM) and when they appear in the PMA is called the revision error. The amount of revision error for some selected crude oil data series is shown in Tables EN6 through EN8. 


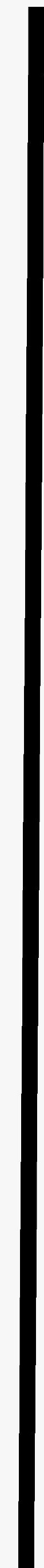


$+$

Product Guide 


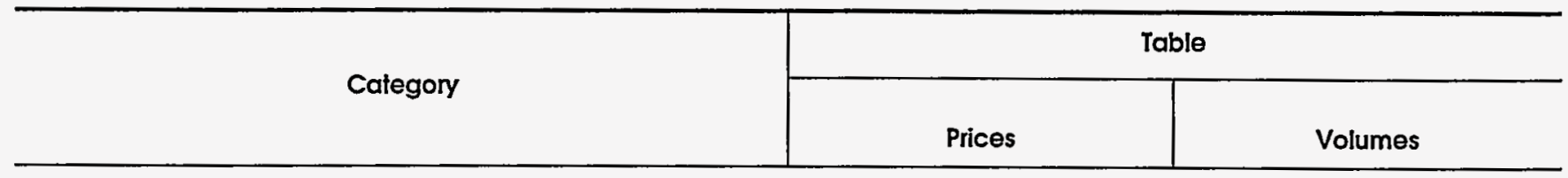

\section{Crude Oil}

Refiner Acquisition Cost

Domestic First Purchases. ....................

from selected States. . . . . . . . . . . . . . . . .

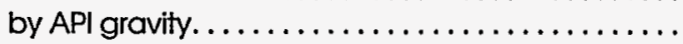
Imporis

F.O.B. Costs . . . . . . . . . . . . . . . . . . .

from selected countries. . . . . . . . . . . . . . . .

by API gravity. . . . . . . . . . . . . . . . . . . .

for selected crude streams $\ldots \ldots \ldots \ldots \ldots \ldots \ldots$.

Landed Costs . . . . . . . . . . . . . . . . . . . . .

from selected countries..................

by API gravity. . . . . . . . . . . . . . . . . . . . .

for selected crude streams .................

Percentage by Gravity Band . . . . . . . . . . . . . .

Motor Gasoline

all sellers...............................

refiners.$\ldots \ldots \ldots \ldots \ldots \ldots \ldots \ldots \ldots \ldots \ldots \ldots \ldots \ldots$.

prime suppliers..$\ldots \ldots \ldots \ldots \ldots \ldots \ldots \ldots \ldots$.

Conventional

all sellers.$\ldots \ldots \ldots \ldots \ldots \ldots \ldots \ldots \ldots \ldots \ldots \ldots \ldots$

refiners..$\ldots \ldots \ldots \ldots \ldots \ldots \ldots \ldots \ldots \ldots \ldots \ldots$.

prime suppliers $\ldots \ldots \ldots \ldots \ldots \ldots \ldots \ldots \ldots \ldots$

Oxygenated

all sellers..$\ldots \ldots \ldots \ldots \ldots \ldots \ldots \ldots \ldots \ldots \ldots \ldots$

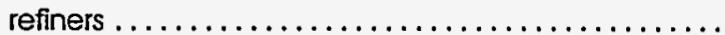

prime suppliers $\ldots \ldots \ldots \ldots \ldots \ldots \ldots \ldots \ldots \ldots$.

Reformulated

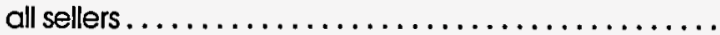

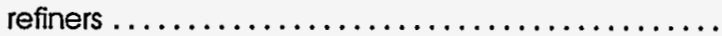

prime suppliers

Aviafion Gasoline

refiners

Kerosene-Type Jet Fuel

refiners ...............................

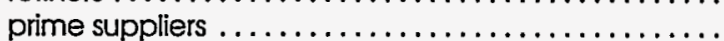

Naphtha-Type Jet Fuel

prime suppliers

Propane, Consumer Grade

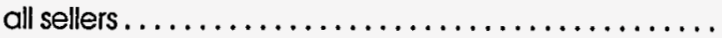

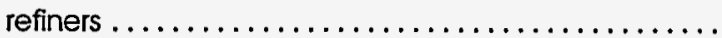

prime suppliers

\begin{tabular}{|c|c|}
\hline 1 & -- \\
\hline 1 & -- \\
\hline 21 & - \\
\hline 23 & - \\
\hline 22 & - \\
\hline 1 & - \\
\hline 24 & - \\
\hline 26 & - \\
\hline 29 & - \\
\hline 1 & - \\
\hline 25 & - \\
\hline 27 & - \\
\hline 30 & - \\
\hline 28 & - \\
\hline $\begin{array}{c}31 \\
2,4,6,35\end{array}$ & $\overline{-}$ \\
\hline- & 48 \\
\hline 32 & - \\
\hline 8 & 9,44 \\
\hline- & 48 \\
\hline 33 & - \\
\hline 10 & 11,44 \\
\hline- & 48 \\
\hline 34 & - \\
\hline 12 & 13,44 \\
\hline- & 48 \\
\hline $2,4,36$ & $3,5,45$ \\
\hline- & 49 \\
\hline $2,4,36$ & $3,5,45$ \\
\hline- & 49 \\
\hline- & 49 \\
\hline 14,38 & - \\
\hline 2,4 & $3,5,45$ \\
\hline- & 49 \\
\hline
\end{tabular}




\begin{tabular}{|c|c|c|}
\hline \multirow{2}{*}{ Calegory } & \multicolumn{2}{|c|}{ Table } \\
\hline & Prices & Volumes \\
\hline $\begin{array}{l}\text { Kerosene } \\
\quad \begin{array}{l}\text { refiners } \\
\text { prime suppliers } \ldots \ldots \ldots \ldots \ldots \ldots \ldots \ldots \ldots \ldots \ldots \ldots \ldots \ldots \ldots \ldots\end{array}\end{array}$ & $\begin{array}{c}2,4,36 \\
-\end{array}$ & $\begin{array}{c}3,5,45 \\
50\end{array}$ \\
\hline 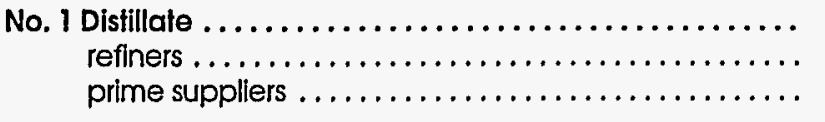 & $\begin{array}{c}2,4,37 \\
-\end{array}$ & $\begin{array}{c}3,5,45 \\
50\end{array}$ \\
\hline 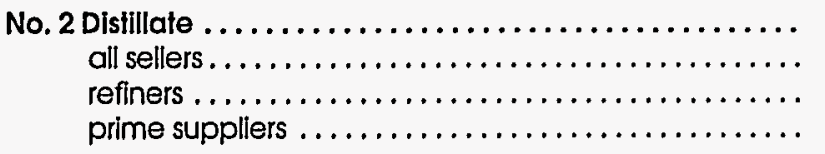 & $\begin{array}{c}15,18,39 \\
2,4,37 \\
-\end{array}$ & $\begin{array}{l}3 . \overline{5.46} \\
50\end{array}$ \\
\hline 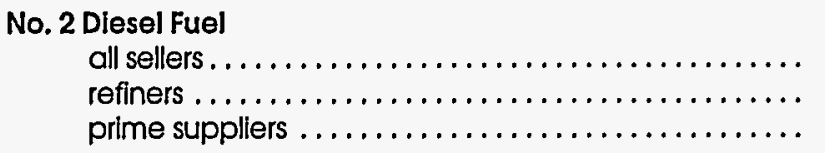 & $\begin{array}{l}16,17,40 \\
2,4 \\
-\end{array}$ & $\begin{array}{l}3, \overline{5,46} \\
50\end{array}$ \\
\hline 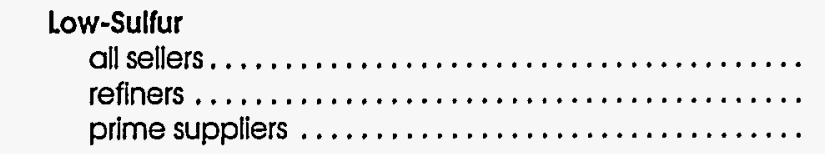 & $\begin{array}{l}17,41 \\
- \\
-\end{array}$ & $\begin{array}{l}3, \overline{5,46} \\
50\end{array}$ \\
\hline 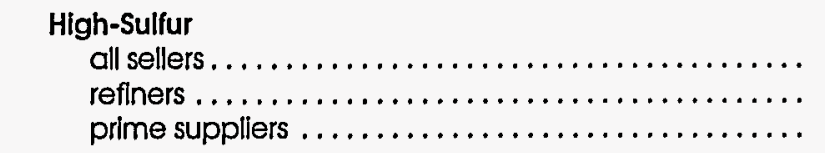 & $\begin{array}{l}17,41 \\
- \\
-\end{array}$ & $\begin{array}{l}-\overline{5,46} \\
50\end{array}$ \\
\hline 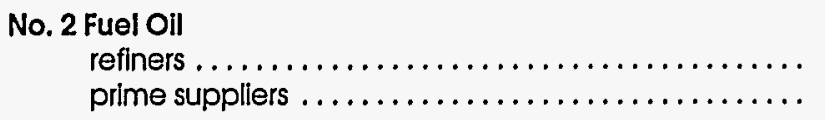 & $\begin{array}{c}2,4 \\
-\end{array}$ & $\begin{array}{c}3,5,46 \\
50\end{array}$ \\
\hline 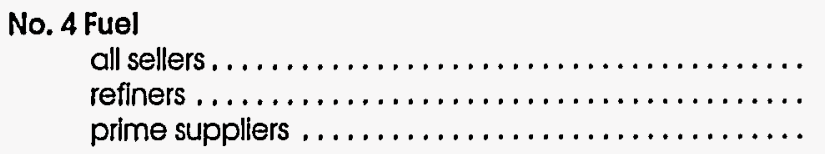 & $\begin{array}{l}37 \\
2,4 \\
-\end{array}$ & $\begin{array}{l}3, \overline{5,47} \\
50\end{array}$ \\
\hline 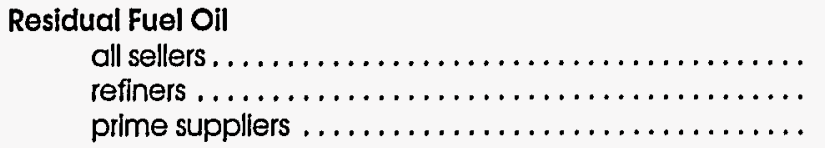 & $2,4,19$ & $\begin{array}{l}3, \overline{5,20,47} \\
49\end{array}$ \\
\hline 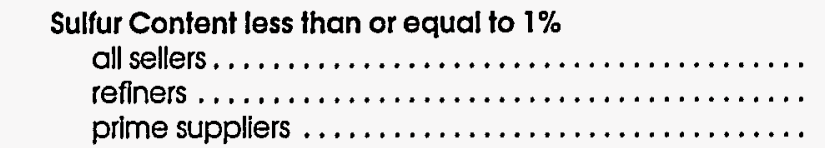 & $\begin{array}{l}42 \\
19 \\
-\end{array}$ & $\begin{array}{c}- \\
20,47\end{array}$ \\
\hline 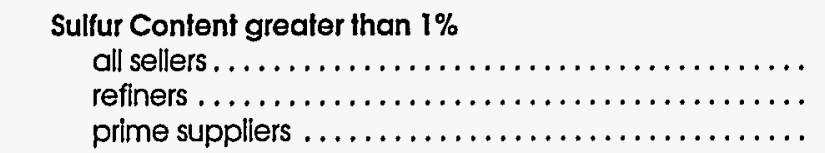 & $\begin{array}{l}42 \\
19 \\
-\end{array}$ & $\begin{array}{c}- \\
20,47\end{array}$ \\
\hline
\end{tabular}




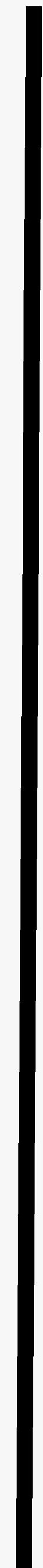


Glossary 



\section{Glossary}

API Gravity: An arbitrary scale expressing the gravity or density of liquid petroleum products. The measuring scale is calibrated in terms of degrees API; it may be calculated in terms of the following formula:

$$
\operatorname{Deg} A P I=\frac{141.5}{s p g r 60 \operatorname{deg} F / 60 \operatorname{deg} F}-131.5
$$

The higher the API gravity, the lighter the compound. Light crudes generally exceed 38 degrees API and heavy crudes are commonly labeled as all crudes with an API gravity of 22 degrees or below. Intermediate crudes fall in the range of 22 degrees to 38 degrees API gravity.

ASTM: American Society for Testing and Materials.

Aviation Gasoline (Finished): All special grades of gasoline for use in aviation reciprocating engines, as given in ASTM Specification D 910 and Military Specification MIL-G-5572. Excludes blending components which will be used in blending or compounding into finished aviation gasoline.

Barrel: A volumetric unit of measure for crude oil and petroleum products equivalent to 42 U.S. gallons.

Bulk Sales: Wholesale sales of gasoline in individual transactions which exceed the size of a truckload.

CIF: Cost, insurance, and freight. A type of sale in which the buyer of the product agrees to pay a unit price that includes the f.o.b. value of the product at the point of origin plus all costs of insurance and transportation. This type of transaction differs from a "delivered" purchase, in that the buyer accepts the quantity as determined at the loading port (as certified by the bill of loading and quality report) rather than pay based on the quantity and quality ascertained at the unloading port. It is similar to the terms of an f.o.b. sale, except that the seller, as a service for which he is compensated, arranges for transportation and insurance.
Commercial/Institutional: Firms engaged in transportation, wholesale or retail trade, finance, insurance, and real estate. Also included are apartment buildings/complexes and other multifamily dwellings, hotels and office buildings or complexes, local, State, or Federal facilities or organizations including the military, schools, hospitals, religious institutions, universities, and all other government-supported organizations.

\section{Conventional Gasoline: See Motor Gasoline.}

Crude Oil (including lease condensate): A mixture of hydrocarbons that existed in liquid phase in underground reservoirs and remains liquid at atmospheric pressure after passing through surface separating facilities. Includes lease condensate and drip gas, as well as liquid hydrocarbons produced from tar sands, gilsonite, and oil shale. Excludes topped crude oil, residual oil, other unfinished oils, and liquids produced at natural gas processing plants and mixed with crude oil, where identifiable. Crude oil is considered as either domestic or imported according to the following:

1. Domestic Crude Oil: Crude oil produced in the United States or from its "outer continental shelf" as defined in 43 U.S.C. 1331.

2. Imported Crude Oil: Crude oil produced outside the United States and brought into the United States.

3. First purchase volume and cost data for crude oil are classified in accordance with what the product was sold as, regardless of the actual specifications. Hence, its volumes may include some of the excluded liquids discussed above.

Crude Oil Acquisitions (unfinished oil acquisitions): The volume of crude oil either (1) acquired by the respondent for processing for its own account in accordance with accounting procedures generally accepted and consistently and historically applied by 
the refiner concerned, or (2) in the case of a processing agreement, delivered to another refinery for processing for the respondent's own account.

Crude oil which has been added by a refiner to inventory, and which is thereafter sold or otherwise disposed of without processing for the account of that refiner, shall be deducted from its crude oil purchases at the time when the related cost is deducted from refinery inventory in accordance with accounting procedures generally applied by the refiner concerned.

Dealer Tank Wagon (DTW) Sales: Wholesale sales of gasoline priced on a delivered basis to a retail outlet.

Distillate Fuel Oil: A general classification for one of the petroleum fractions produced in conventional distillation operations. It is used primarily for space heating, on- and off-highway diesel engine fuel (including railroad engine fuel and fuel for agricultural machinery), and electric power generation. Included are products known as No. 1, No. 2, and No. 4 fuel oils and No. 1, No. 2, and No. 4 diesel fuels.

1. No. 1 Distillate: A petroleum distillate which meets the specifications for No. 1 heating or fuel oil as defined in ASTM D 396 and/or the specifications for No. 1 diesel fuel as defined in ASTM Specification D 975, with distillation temperatures of 420 degrees Fahrenheit at the 10-percent recovery point and 550 degrees Fahrenheit at the 90-percent recovery point, and kinematic viscosities between 1.4 and 2.2 centistokes at 100 degrees Fahrenheit.

a. No. 1 Diesel Fuel: A volatile distillate fuel oil with a boiling range between 300-575 degrees Fahrenheit and used in high-speed diesel engines generally operated under wide variations in speed and load. Includes type C-B diesel fuel used for city buses and similar operations. Properties are defined in ASTM Specification D 975.

b. No. 1 Fuel Oil: A light distillate fuel oil intended for use in vaporizing pot-type burners. ASTM Specification D 396 specifies for this grade maximum distillation temperatures of 400 degrees Fahrenheit at the 10percent recovery point and 550 degrees Fahrenheit at the 90-percent point, and kinematic viscosities between 1.4 and 2.2 centistokes at 100 degrees Fahrenheit.
2. No. 2 Distillate: A petroleum distillate which meets the specifications for No. 2 heating or fuel oil as defined in ASTM D 396 and/or the specifications for No. 2 diesel fuel as defined in ASTM Specification D 975.

a. No. 2 Diesel Fuel: A gas oil type distillate of lower volatility with distillation temperatures at the 90-percent point between 540-640 degrees Fahrenheit for use in high speed diesel engines generally operated under uniform speed and load conditions. Includes Type $R-R$ diesel fuel used for railroad locomotive engines, and Type $T-T$ for diesel-engine trucks. Properties are defined in ASTM Specification D 975.

- Low Sulfur: The sulfur level does not exceed 0.05 percent by weight.

- High Sulfur: The sulfur level is above 0.05 percent by weight.

b. No. 2 Fuel Oil: A distillate fuel oil for use in atomizing type burners for domestic heating or for moderate capacity commercial-industrial burner units. ASTM Specification D 396 specifies for this grade distillation temperatures at the 90-percent point between 540 and 640 degrees Fahrenheit, and kinematic viscosities between 2.0 and 3.6 centistokes at 100 degrees Fahrenheit.

NOTE: Published volume and price data for No. 2 diesel fuel and No. 2 fuel oil are classified in accordance to what the product was sold as, regardless of the actual specifications of that product; i.e., if a No. 2 distillate was sold as a heating or fuel oil, the volume and price would be published in the category "No. 2 Fuel Oil" even if the product conformed to the higher specifications of a diesel fuel.

3. No. 4 Fuel: A fuel oil for commercial burner installations not equipped with preheating facilities: It is used extensively in industrial plants. This grade is a blend of distillate fuel oil and residual fuel oil stocks that conforms to ASTM Specification D 396 or Federal Specification VV-F-815C; its kinematic viscosity is between 5.8 and 26.4 centistokes at 100 degrees Fahrenheit. Also included is No. 4-D, a fuel oil for low- and medium-speed diesel en- 
gines that conforms to ASTM Specification D 975.

First Purchase (of crude oil): An equity (not custody) transaction involving an arms-length transfer of ownership of crude oil associated with the physical removal of the crude oil from a property (lease) for the first time. A first purchase normally occurs at the time and place of ownership transfer where the crude oil volume sold is measured and recorded on a run ticket or other similar physical evidence of purchase. The reported cost is the actual amount paid by the purchaser, allowing for any adjustments (deductions or premiums) passed on to the producer or royalty owner.

F.o.b. Price (free on board): The f.o.b. price is the price actually charged at the producing country's port of loading. The reported price includes deductions for any rebates and discounts or additions of premiums where applicable and should be the actual price paid with no adjustment for credit terms.

Gas Plant Operator: Any firm, including a gas plant owner, which operates a gas plant and keeps the gas plant records. A gas plant is a facility in which natural gas liquids are separated from natural gas, or in which natural gas liquids are fractionated or otherwise separated into natural gas liquid products or both. For the purposes of this publication, gas plant operator data are contained in the refiner categories.

Gasohol: A blend of finished motor gasoline and alcohol (generally ethanol but sometimes methanol) in which 10 percent or less of the product is alcohol. For the purposes of this publication, gasohol may be included in any of the types of gasoline, depending on how it was marketed.

Industrial: Firms engaged in mining, construction, or manufacturing.

Kerosene: A petroleum distillate that has a maximum distillation temperature of 401 degrees Fahrenheit at the 10-percent recovery point, a final boiling point of 572 degrees Fahrenheit, and a minimum flash point of 100 degrees Fahrenheit. Included are the two grades designated in ASTM D3699: No. 1-K and No. 2-K, and all grades of kerosene called range or stove oil which have properties similar to No. 1 fuel oil, but with a gravity of about 43 degrees API and a maximum endpoint of 625 degrees Fahrenheit. Kerosene is used in space heaters, cook stoves, and water heaters; it is suitable for use as an illuminant when burned in wick lamps.

Kerosene-Type Jet Fuel: A quality kerosene product with maximum distillation temperature of $400 \mathrm{de}-$ grees Fahrenheit at the 10-percent recovery point and a final maximum boiling point of 572 degrees Fahrenheit. The fuel is designated in ASTM Specification D 1655 and Military Specifications MIL-T-5624P and MIL-T-83133D (Grades JP-5 and JP-8). A relatively low freezing point distillate of the kerosene type used primarily for commercial turbojet and turboprop aircraft engines.

Landed Cost: Landed cost represents the dollar per barrel price of crude oil at the port of discharge. Includes charges associated with the purchase, transporting, and insuring of a cargo from the purchase point to the port of discharge. Does not include charges incurred at the discharge port (e.g., import tariffs or fees, wharfage charges, and demurrage).

Motor Gasoline (Finished): A complex mixture of relatively volatile hydrocarbons, with or without small quantities of additives, blended to form a fuel suitable for use in spark-ignition engines. Specifications for motor gasoline, as given in ASTM Specification D 439-88 or Federal Specification VV-G-1690B, include a boiling range of 122 to 158 degrees Fahrenheit at the 10-percent recovery point to 365 to 374 degrees Fahrenheit at the 90-percent recovery point. "Motor Gasoline" includes conventional gasoline, oxygenated gasoline (EPA approved), and reformulated gasoline. Blendstock (including ethanol and MTBE) are excluded until blending has been completed.

1. Conventional Gasoline: Motor gasoline not included in the oxygenated or reformulated gasoline categories. Excludes reformulated gasoline blendstock for oxygenate blending (RBOB).

2. Oxygenated Gasoline: Gasoline formulated for use in motor vehicles that is intended for use in EPA approved carbon monoxide (CO) nonattainment State programs. Excludes reformulated gasoline, oxygenated fuels program reformulated gasoline (OPRG) and reformulated gasoline blendstock for oxygenate blending ( $R B O B)$.

3. Reformulated Gasoline: Gasoline formulated for use in motor vehicles, the composition and properties of which meet the requirements of 
the reformulated gasoline regulations promulgated by the U.S. Environmental Protection Agency under Section 211(k) of the Clean Air Act. Includes oxygenated fuels program reformulated gasoline (OPRG). Excludes reformulated gasoline blendstock for oxygenate blending (RBOB).

Within each of these three types of gasoline are the following three grades:

a. Regular Gasoline: Gasoline having an antiknock index $((R+M) / 2)$ greater than or equal to 85 and less than 88 .

b. Midgrade Gasoline: Gasoline having an antiknock index $((\mathrm{R}+\mathrm{M}) / 2)$ greater than or equal to 88 and less than or equal to 90 .

c. Premium Gasoline: Gasoline having an antiknock index $((R+M) / 2)$ greater than 90 .

NOTE: For this publication, gasoline sales are reported by grade in accordance with their classification at the time of sale. In general, automotive octane requirements are lower at high altitudes. Therefore, in some areas of the United States, such as the Rocky Mountain States, the octane ratings for the gasoline grades above may be 2 or more octane points lower.

MTBE (methyl tertiary butyl ether): An ether eligible for gasoline blending, blends up to 15.0 percent by volume MTBE which must meet the ASTMD 4814 Specifications. Blenders must take precautions that the blends are not used as base gasolines for other oxygenated blends.

Naphtha: A generic term applied to a petroleum fraction with an approximate boiling range between 122 and 400 degrees Fahrenheit.

Naphtha-Type Jet Fuel: A fuel in the heavy naphtha boiling range with an average gravity of 52.8 degrees API and 20 to 90 percent distillation temperatures of 290 degrees to 470 degrees F., meeting Military Specification MIL-T-5624L (Grade JP-4). JP-4 is used for turbojet and turboprop aircraft engines, primarily by the military. Excludes ram-jet and petroleum rocket fuels.

OPEC: Organization of Petroleum Exporting Countries, oil-producing and exporting countries that have organized for the purpose of negotiating with oil companies on matters of oil production, prices, and future concession rights. Current members are
Algeria, Gabon, Indonesia, Iran, Iraq, Kuwait, Libya, Nigeria, Qatar, Saudi Arabia, United Arab Emirates, and Venezuela. Prior to January 1,1993, Ecuador was a member of OPEC.

OPRG: "Oxygenated Fuels Program Reformulated Gasoline" is reformulated gasoline which is intended for use in an oxygenated fuels program control area during an oxygenated fuels program control period.

Other End Users: For motor gasoline, all direct sales to end users other than those made through company outlets. For No. 2 distillate, all direct sales to end users other than residential, commercial/institutional, industrial sales, and sales through company outlets. Included in the "other end users" category are sales to utilities and agriculture.

Oxygenated Gasoline: See Motor Gasoline.

Oxygenates: Any substance which, when added to gasoline, increases the amount of oxygen in that gasoline blend.

PAD District: Petroleum Administration for Defense Districts

\section{PAD District I:}

Subdistrict IA: Connecticut, Maine, Massachusetts, New Hampshire, Rhode Island, Vermont.

Subdistrict IB: Delaware, District of Columbia, Maryland, New Jersey, New York, Pennsylvania.

Subdistrict IC: Florida, Georgia, North Carolina, South Carolina, Virginia, West Virginia.

\section{PAD District II:}

Illinois, Indiana, Iowa, Kansas, Kentucky, Michigan, Minnesota, Missouri, Nebraska, North Dakota, Ohio, Oklahoma, South Dakota, Tennessee, Wisconsin.

\section{PAD District III:}

Alabama, Arkansas, Louisiana, Mississippi, New Mexico, Texas, Federal Offshore Gulf.

\section{PAD District IV:}

Colorado, Idaho, Montana, Utah, Wyoming. 


\section{PAD District V:}

Alaska (North Slope and Other Mainland), Arizona, California, Hawaii, Nevada, Oregon, Washington, Federal Offshore California.

Petrochemical Sales: Sales of propane to a manufacturer of chemicals derived from petroleum or natural gas, or from raw materials derived from petroleum or natural gas.

Petroleum Products: Petroleum products are obtained from the processing of crude oil (including lease condensate), natural gas, and other hydrocarbon compounds. Petroleum products include unfinished oils, liquefied petroleum gases, pentanes, plus aviation gasoline, motor gasoline, naphtha-type jet fuel, kerosene, distillate fuel oil, residual fuel oil, petrochemical feedstocks, special naphthas, lubricants, waxes, petroleum coke, asphalt, road oil, still gas, and miscellaneous products.

Prime Supplier: A firm that produces, imports, or transports selected petroleum products across State boundaries and local marketing areas, and sells the product to local distributors, local retailers, or end users.

Propane, Consumer Grade: A normally gaseous paraffinic compound $\left(\mathrm{C}_{3} \mathrm{H}_{8}\right)$, which includes all products covered by Natural Gas Policy Act (NGPA) Specifications for commercial use and HD-5 propane and ASTMSpecification D 1835. It is a colorless paraffinic gas that boils at a temperature of -43.67 degrees Fahrenheit. It does not include the propane portion of any natural gas liquids (NGL) mixes; i.e., butanepropane mix.

Rack Sales: Wholesale truckload sales or smaller of gasoline where title transfers at a terminal.

RBOB: "Reformulated Gasoline Blendstock for Oxygenate Blending" is a motor gasoline blending component which, when blended with a specified type and percentage of oxygenate, meets the definition of reformulated gasoline.

Reference Month: The calendar month and year to which the reported cost, price, and volume information relates.

Refiner: A firm or the part of a firm that refines products or blends and substantially changes products, or refines liquid hydrocarbons from oil and gas field gases, or recovers liquefied petroleum gases incident to petroleum refining and sells those products to resellers, retailers, resellers/retailers, or ultimate consumers. "Refiner" includes any owner of products which contracts to have those products refined and then sells the refined products to resellers, retailers, or ultimate consumers. For the purposes of this publication, gas plant operator data are included in this category.

\section{Reformulated Gasoline: See Motor Gasoline.}

Reseller: A firm (other than a refiner) that carries on the trade or business of purchasing refined petroleum products and reselling them to purchasers other than ultimate consumers.

Reseller/Retailer: A firm (other than a refiner) that carries on the trade or business activities of both a reseller and a retailer; i.e., purchasing refined petroleum products and reselling them to purchasers who may be either ultimate or other than ultimate consumers.

Residential: Sales of No. 2 distillate and propane to individual customers or households (as opposed to businesses or institutions) who ostensibly use the fuel in a residence for space heating, cooking, etc. Sales to apartment buildings/complexes or to other multifamily dwellings are excluded from the "Residential Sales" category and are included in the "Commercial/Institutional Sales" category.

Residual Fuel Oils: The topped crude of refinery operations, which includes No. 5 and No. 6 fuel oils as defined in ASTM Specification D 396 and Federal Specification VV-F-815C, Navy Special fuel oil as defined in Military Specification MIL-F-859E including Amendment 2 (NATO Symbol F-77), and Bunker $C$ fuel oil. Residual fuel oil is used for the production of electric power, space heating, vessel bunkering, and various industrial purposes.

Retailer: A firm (other than a refiner, reseller, or reseller/retailer) that carries on the trade or business of purchasing refined petroleum products and reselling them to ultimate consumers.

Retail Outlet: Any company-owned outlet (e.g., service station) selling gasoline, on-highway low-sulfur diesel fuel, or propane for on-highway vehicle use which is under the direct control of the firm filing the EIA-782 by virtue of the ability to set the retail product price and directly collect all or part of the retail margin. This category includes retail outlets: (1) be- 
ing operated by salaried employees of the company and/or its subsidiaries and affiliates, and/or (2) involving personnel services contracted by the firm.

Sale: The transfer of title from the seller to a buyer for a price. Excludes intrafirm transfers, products consumed directly by the reporting firm, or sales of bonded fuel. Also excludes products delivered/loaned to exchange partners, except where the amount supplied exceeds the amount received and the differential is invoiced as a sale during the reference month.

Sales for Resale: Sales of refined petroleum products to purchasers who are other-than-ultimate consumers; wholesale sales.

Sales to End Users: Sales made directly to the consumer of the product. Includes bulk consumers such as agriculture, industry, and utilities, as well as residential and commercial consumers.

Sales Type: Sales categories of sales to end users and sales for resale.
Unit Price: Total revenue derived from the sale of product during the reference month divided by the total volume sold; also known as the weighted average price. Total revenue excludes all taxes but includes transportation costs that were paid as part of the purchase price.

United States: For the crude oil statistics, the United States includes the 50 States, the District of Columbia, Puerto Rico, the Virgin Islands, and all American Territories and Possessions. For the petroleum products data, United States includes the 50 States and the District of Columbia.

Wellhead: The point at which the crude (and/or natural gas) exits the ground. Following historical precedent, the volume and price for crude oil production are labeled as "wellhead," even though the cost and volume are now generally measured at the lease boundary. In the context of domestic crude price data, the term "wellhead" is the generic term used to reference the production site or lease property. 
List of

Articles 


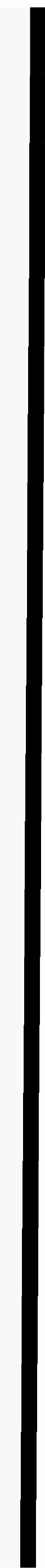




\section{Articles}

Featurearticlesonenergy-related subjects arefrequentlyincludedinthispublication. Thefollowing articles and special focus items have appeared in previous issues.

1995 Reformulated Gasoline Market Affected Refiners Differently . . . . . . . . . . . . January 1996

Distillate Fuel Oil Assessment for Winter 1995-1996 . . . . . . . . . . . . . November 1995

Propane Assessment for Winter $1995-1996$. . . . . . . . . . . . . . . . . . November 1995

Environmental Regulations and Changes in the Petroleum Refining Operations . . . . . . S September 1995

What Drives Motor Gasoline Prices? . . . . . . . . . . . . . . . . June 1995

1995 Motor Gasoline Assessment . . . . . . . . . . . . . . . . . . May 1995

Results of the Office of Oil and Gas "Outlook Survey" . . . . . . . . . . . . March 1995

Distillate Fuel Oil Assessment for Winter 1994-1995 . . . . . . . . . . . . . N November 1994

Propane Assessment for Winter 1994-1995 . . . . . . . . . . . . . . . . . N November 1994

Sales of Fuel Oil and Kerosene in $1993 . \ldots \ldots \ldots \ldots \ldots$. . . . . . . . November 1994

Impact of New Low-Sulfur Diesel Requirements, October through December 1993 . . . . . November 1994

Reformulated Gasoline Supply Issues . . . . . . . . . . . . . . . . . . November 1994

A Comparison of Selected EIA-782 Data With Other Data Sources. . . . . . . . . October1994

Demand, Supply, and Price Outlook for Reformulated Motor Gasoline 1995 . . . . . . . A August 1994

The Second Oxygenated Gasoline Season . . . . . . . . . . . . . . . June 1994

Changes to the 1994 PetroleumMarketingMonthly . . . . . . . . . . . . May 1994

Summer 1994 Motor Gasoline Outlook . . . . . . . . . . . . . . . . . . . . May 1994

Sales of Fuel Oil and Kerosene in $1992 \ldots \ldots$. . . . . . . . . . . . . December1993

Low-Sulfur Diesel: Requirements and Impacts . . . . . . . . . . . . . . D December1993

Distillate Fuel Oil Outlook for Winter $1993-94 \ldots \ldots \ldots \ldots \ldots \ldots \ldots$. . . . . November 1993

Propane Outlook for Winter $1993-1994 \ldots \ldots \ldots \ldots$. . . . . . . . . . . . November 1993 
A Comparison of Selected EIA-782 Data with Other Data Sources . . . . . . . . . . . .

August 1993

The Economics of the Clean Air Act Amendments of 1990:

Review of the 1992-1993 Oxygenated Motor Gasoline Season . . . . . . . . . . . . .

August 1993

Changes to Form EIA-782C "Monthly Report of Petroleum Products

Sold into States for Consumption" . . . . . . . . . . . . . . . .

May 1993

Summer 1993 Motor Gasoline Outlook

May 1993

Economics of Energy Futures Markets

September1991

Economics of Gasoline Pool Octane Growth . . . . . . . . . . . . . . . .

March 1990

A Comparison of Selected EIA-782 Data with Other Data Sources

August 1989

The Introduction of Unleaded Midgrade Gasoline $\ldots \ldots \ldots \ldots \ldots$

April 1989

A Review of Valdez Oil Spill Market Impacts . . . . . . . . . . . . . . .

March 1989

California Crude Oil Price Levels . . . . . . . . . . . . . . . . . . . .

April 1987

Determining Minimum Acceptable Bid Prices for the Test Sale of

Strategic Petroleum Reserve Crude Oil . . . . . . . . . . . . . . . . .

Commercial/Industrial Sales of Residual Fuel Oil . . . . . . . . . . . . .

August 1986

Crude Oil High-Seas Stocks . . . . . . . . . . . . . . . . . .

December1985

August 1985

Foreign Crude Oil Prices: The Differential Dilemma ...............

May 1985

A Short-Term Analysis of the Effects of Concentration on Price

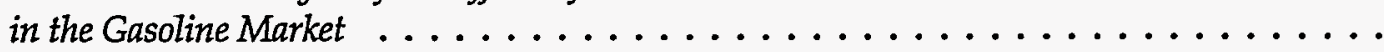

Petroleum Marketing Monthly Initiates Crude Oil Data Series

April 1985

............. February 1985

Estimated Historic Time Series for the ELA-782 . . . . . . . . . . . . . . . December1983[3]

No. 2 Distillate Price and Sales Reflect a Period of Adjustment to

Changing Market Conditions . . . . . . . . . . . . . . . . . . . . November 1983

The Consolidation of Petroleum Marketing Surveys . . . . . . . . . . . . . . . April 1983 


\section{Introducing the Energy InfoDisc ... .}

\section{How much oil does the U.S. import from the Persian Gulf?}

\section{How many U.S. households use personal computers? \\ What's the long range outlook for nuclear power, and how will it affect other fuels?}

\section{How are clean air regulations affecting emissions in the electric power industry?}

Find answers to all your energy questions with Energy InfoDisc, the new CD-ROM providing the most comprehensive collection of energy information and analysis tools available today. Energy InfoDisc is a valuable resource for industry and government analysts, forecasters, journalists, and academic researchers - almost anyone needing access to current or historical energy information.

The Energy InfoDisc comes with advanced search capability to find the information you need in seconds - from over 180 of the most current periodicals and analytical publications of the Energy Information Administration (EIA), the independent statistical and analytical agency within the U.S. Department of Energy. With Energy InfoDisc, you also have instant access to ElA's wide range of national energy databases and special analysis applications. Don't be without this important resource; subscribe today.

\section{STAT-USA Order Form}

Yes, enter my order for the Energy InfoDisc CD-ROM:

Number of copies:

Single issue (U.S., Canada, \& Mexico): $\$ 45$

Single issue outside the U.S., Canada \& Mexico: $\$ 60$

Quarterly subscription in the U.S., Canada, \& Mexico: $\$ 100$

Quarterly subscription outside the U.S., Canada

\& Mexico: $\$ 160$

Contact or personal name

(please type or print)

Company

Street address

Additional address

City, State, ZIP Code

Daytime phone

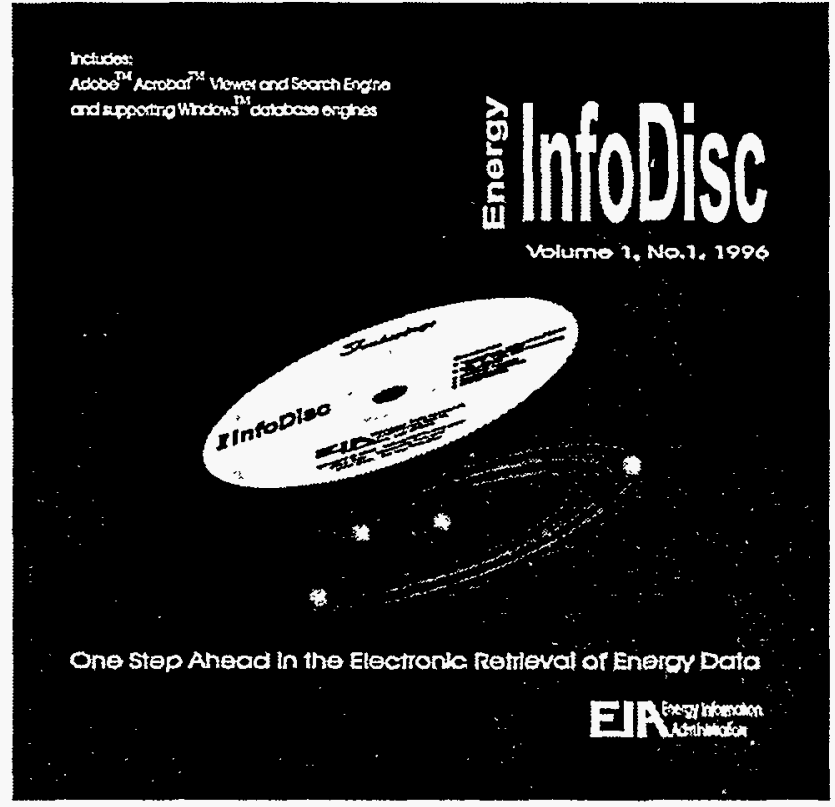

NOTE: Documents can be accessed using either Microsoft Windows or Macintosh System 7 operating systems. Databases and applications can only be accessed using Microsoft Windows.
Charge your order

It's easy!

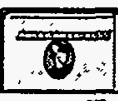

reolit

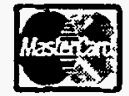

VISA

To fax your orders (202) 482-2164

To phone your orders (202)482-1986

For privacy protection, check the box below:

Do not make my name available to requesters.

Check method of payment:

IAMEX

$\square$ Discover $\square$ MasterCard

VISA

(expiration date)

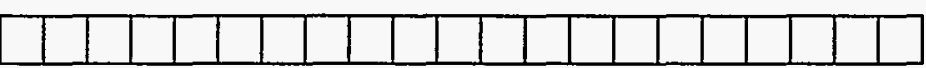

Thank you for your order!

Authorizing signature

Check payable to STAT-USA

Mail to: U.S. Department of Commerce

STAT-USA, Room H-4885

Washington, DC 20230 
